In cooperation with PacifiCorp

\title{
Development of New Information to Inform Fish Passage Decisions at the Yale and Merwin Hydro Projects on the Lewis River, Washington-Final Report, 2018
}

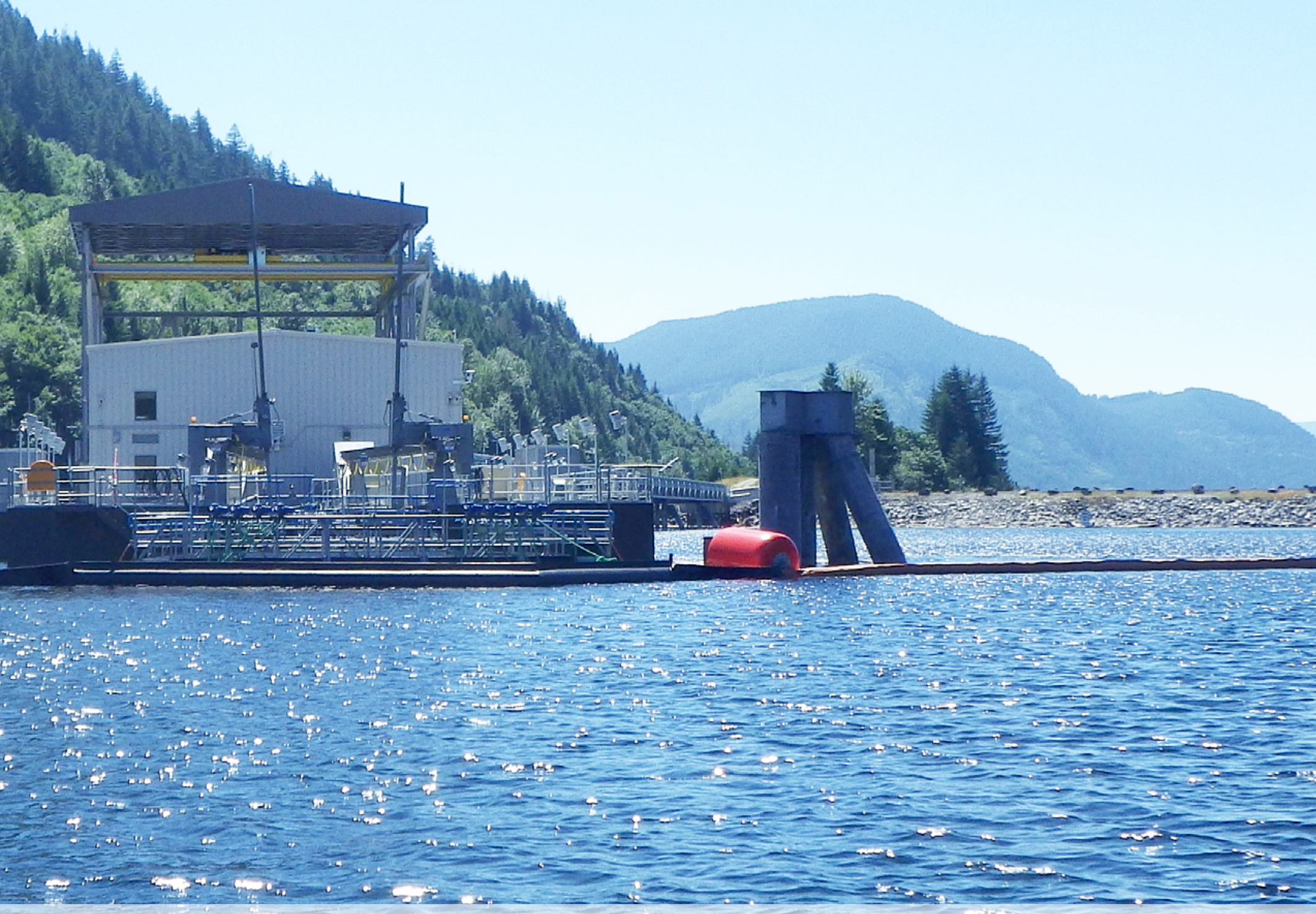

Open-File Report 2018-1190

U.S. Department of the Interior U.S. Geological Survey 
Front cover. A view from the floating surface collector at Swift Dam on Swift Reservoir in the upper Lewis River, Washington.

Back cover. An adult Oncorhynchus kisutch (coho salmon) after spawning in Clear Creek in the upper Lewis River, Washington. 


\section{Development of New Information to Inform Fish Passage Decisions at the Yale and Merwin Hydro Projects on the Lewis River, Washington-Final Report, 2018}

By Robert Al-Chokhachy, Christopher L. Clark, Mark H. Sorel, and

David A. Beauchamp

In cooperation with PacifiCorp

Open-File Report 2018-1190 


\title{
U.S. Department of the Interior \\ RYAN K. ZINKE, Secretary
}

\author{
U.S. Geological Survey \\ James F. Reilly II, Director
}

U.S. Geological Survey, Reston, Virginia: 2018

For more information on the USGS - the Federal source for science about the Earth, its natural and living resources, natural hazards, and the environment-visit https://www.usgs.gov or call 1-888-ASK-USGS.

For an overview of USGS information products, including maps, imagery, and publications,

visit https://store.usgs.gov.

Any use of trade, firm, or product names is for descriptive purposes only and does not imply endorsement by the U.S. Government.

Although this information product, for the most part, is in the public domain, it also may contain copyrighted materials as noted in the text. Permission to reproduce copyrighted items must be secured from the copyright owner.

Suggested citation:

Al-Chokhachy, R., Clark, C.L., Sorel, M.H., and Beauchamp, D.A., 2018, Development of new information to inform fish passage decisions at the Yale and Merwin hydro projects on the Lewis River, Washington-Final report, 2018: U.S.

Geological Survey Open-File Report 2018-1190, 206 p., https://doi.org/10.3133/ofr20181190.

ISSN 2331-1258 (online) 


\section{Acknowledgments}

We would like to thank Adam Sepulveda (USGS) and Shane Vatland (Nez Perce Tribe) for reviewing an earlier version of this document. Funding for this study was provided by PacifiCorp. Readers should contact PacifiCorp for information about the data generated during this study.

\section{Contents}

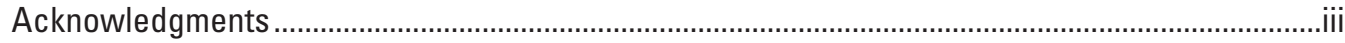

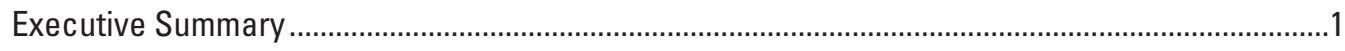

Chapter A. Review of Information Relevant to Anadromous Fish Reintroduction to and

Fish Passage in the Lewis River, Washington....................................................................5

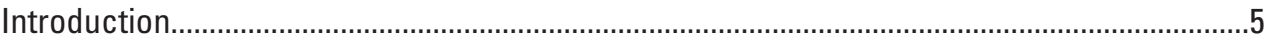

Effects of Smolt Acclimation Facilities.........................................................................

Smolt Collection and Injury and Survival at Downstream Collection Facilities ................7

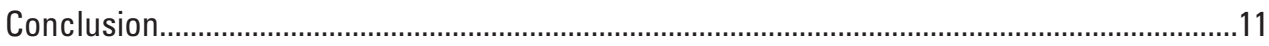

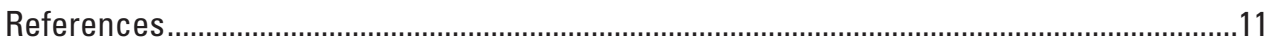

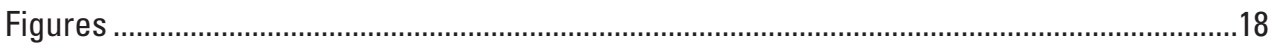

Tables

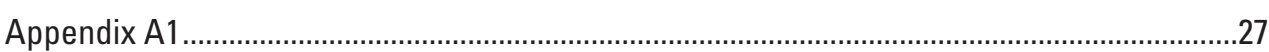

Chapter B. Habitat Assessment of Tributaries to Swift Reservoir, Yale Lake, and

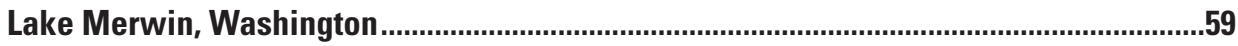

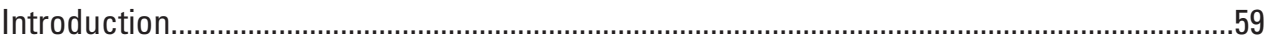

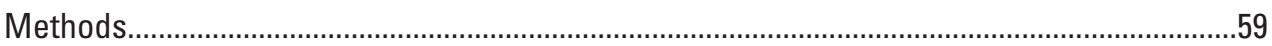

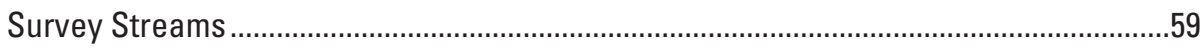

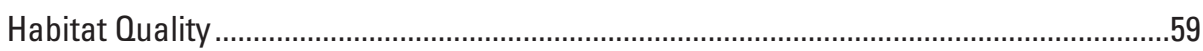

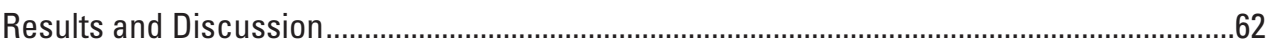

Extent of Tributary Habitat in Lake Merwin and Yale Lake ............................................62

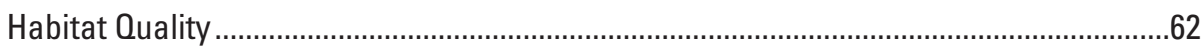

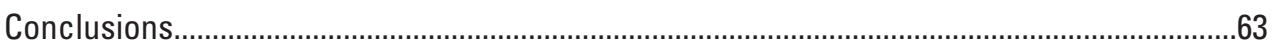

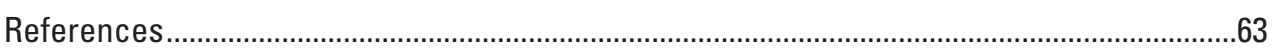

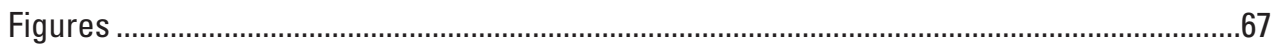

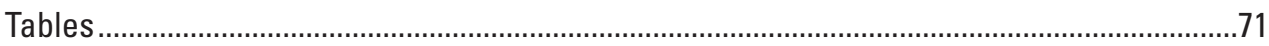

Chapter C. Studies Assessing Adult Potential for Spawning Successin Yale Lake and Lake Merwin, Washington ..........................................................................................79

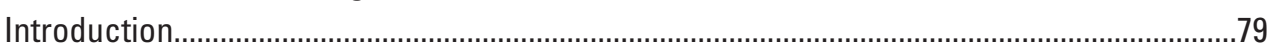

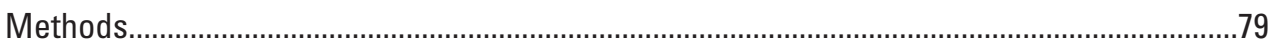

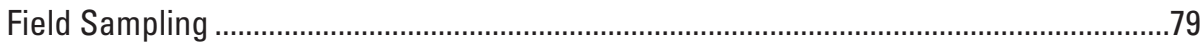

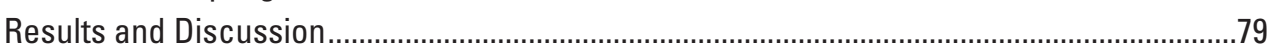

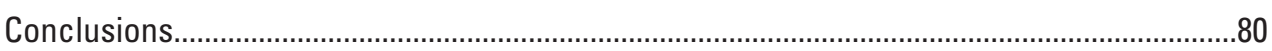

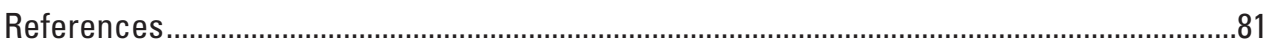

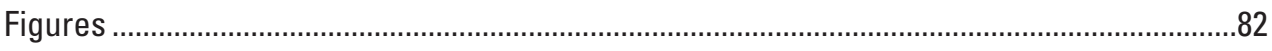

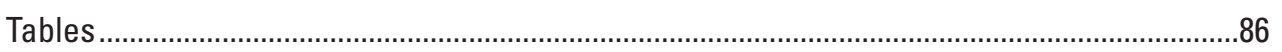




\section{Chapter D. Assessment of Juvenile Production and Outmigration Success on the}

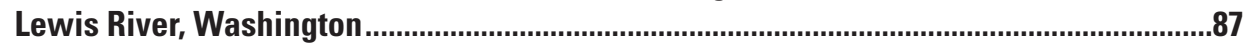

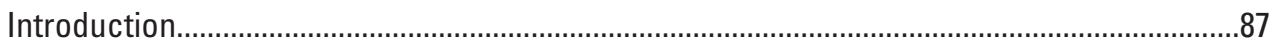

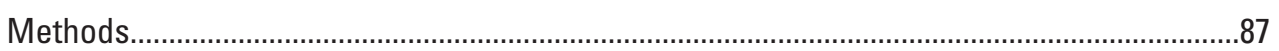

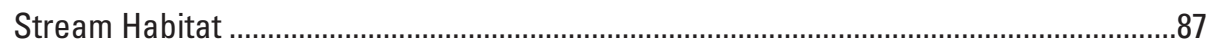

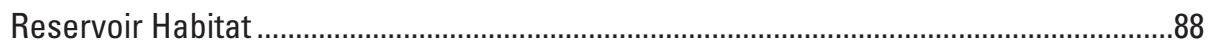

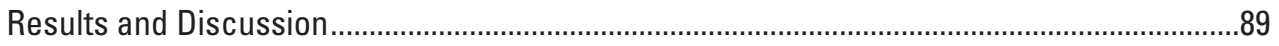

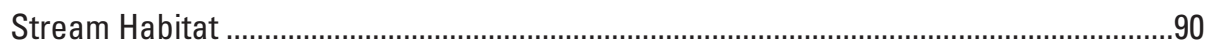

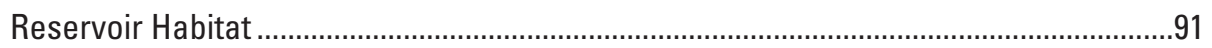

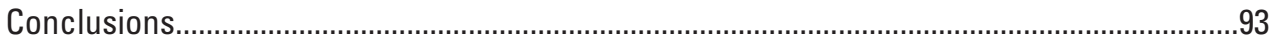

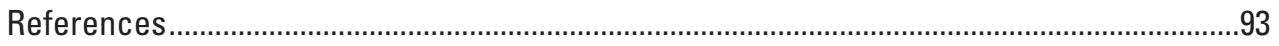

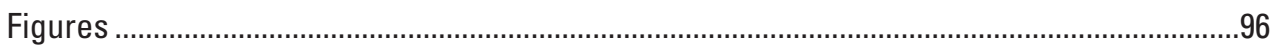

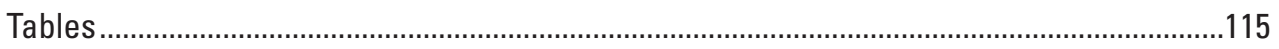

Chapter E. Piscine Predator Impact Studies 2013-2014, Lake Merwin, Washington....................117

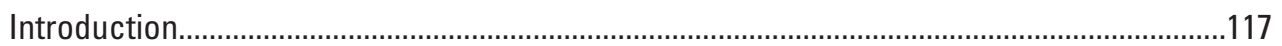

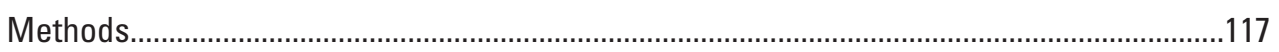

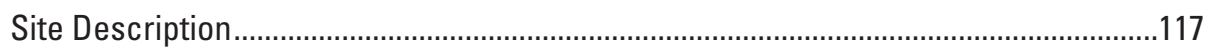

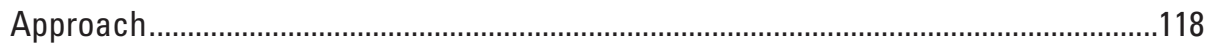

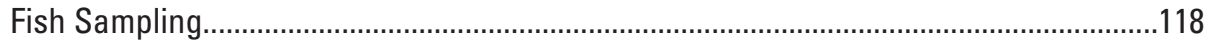

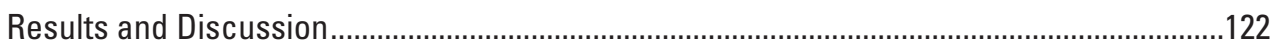

Seasonal Distribution and Thermal Experience of Northern Pikeminnow ...................122

Bioenergetics Inputs and Model Simulations................................................................123

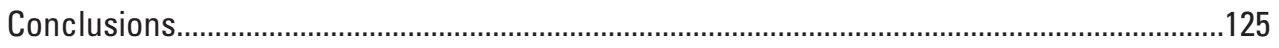

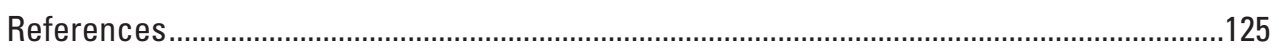

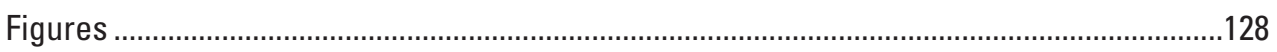

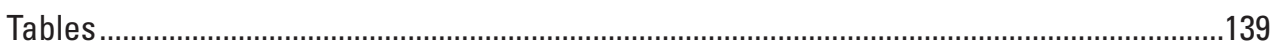

Chapter F. Studies Assessing Anadromous-Resident Fish Interactions in the Lewis River,

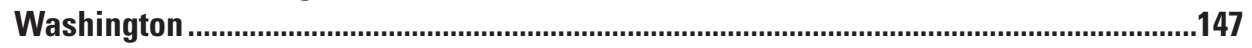

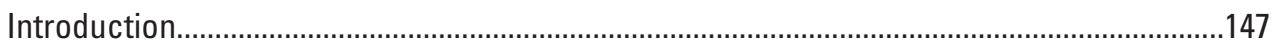

Literature Review and Potential Community-Level Interactions ...........................................147

Review of Anadromous-Resident Interactions........................................................... 147

Effects of Anadromous Reintroductions on Native Species ........................................147

Effects of Native Species on Anadromous Reintroductions .......................................150

Effects of Native and Nonnative Salmonid Predators to Reintroduction Efforts ..........150

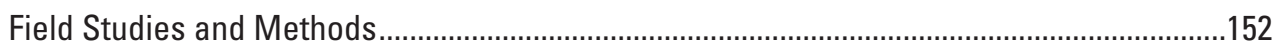

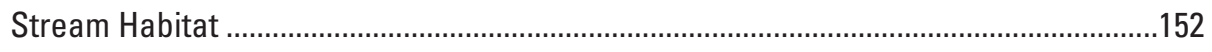

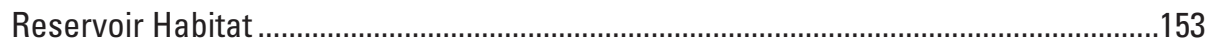

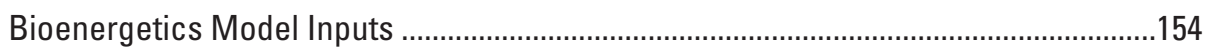

Assessing the Effects of Anadromous Reintroductions on Bull Trout-Simulation

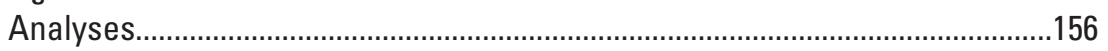

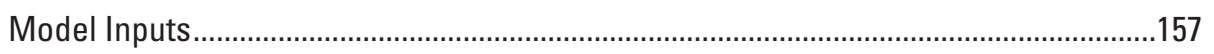

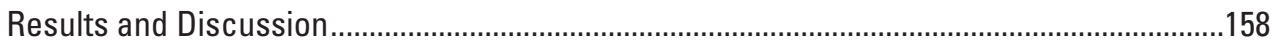

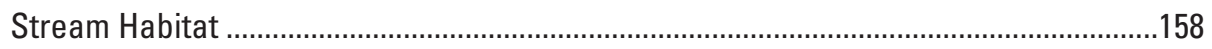

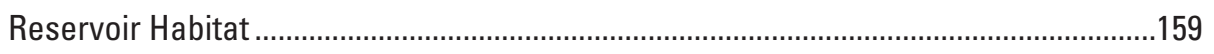

Assessing the Effects of Anadromous Reintroductions on Bull Trout............................163

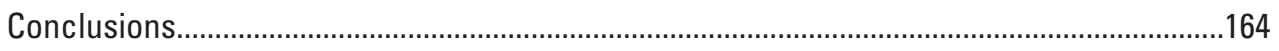




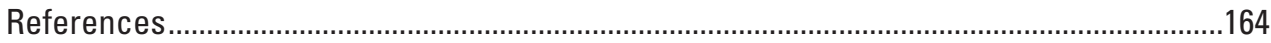

Figures

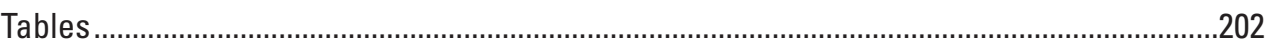

\section{Figures}

A1. The North Fork of the Lewis River and the three hydropower impoundments: Lake Merwin, Yale Lake, and Swift Reservoir in the Lewis River Basin, Washington .............18

A2. Interannual comparison of collection efficiencies for juvenile Chinook salmon, coho salmon, and steelhead at the Cowlitz Falls collection facility in Washington........19

B1. The North Fork of the Lewis River and the three hydropower impoundments: Lake Merwin, Yale Lake, and Swift Reservoir in the Lewis River Basin, Washington

B2. Upper Lewis River tributaries, Washington, where updated habitat data were needed for assessments of anadromous salmon reintroductions.

B3. Stream habitat potentially available to anadromous salmon from upstream barriers in tributaries to Yale Lake and Lake Merwin, Washington.

B4. Predicted average August stream temperatures in degrees Celsius from the NorWeST stream temperature model for tributaries to Swift Reservoir, Washington ...70

C1. The North Fork of the Lewis River and the three hydropower impoundments: Lake Merwin, Yale Lake, and Swift Reservoir, in the Lewis River Basin, Washington.

C2. The location of the coho salmon release site, existing barriers to anadromous species, and spawning activity including observed carcass locations, adults observed staging, and redds during 2015 fall spawning surveys in Lake Merwin tributaries.

C3. Length frequency histograms for juvenile coho salmon from early summer electrofishing surveys in 2013-2015 for Clear Creek (Swift Reservoir) and in 2015 for Brooks Creek.

C4. Catch-per-unit-effort in fish per second estimates from Brooks Creek (Lake Merwin) and sample reaches from Clear Creek (Swift Reservoir) from 2015

D1. Study area map showing the North Fork of the Lewis River and the three hydropower impoundments: Lake Merwin, Yale Lake, and Swift Reservoir in the Lewis River Basin, Washington.

D2. Sample reaches used in the systematic sampling design for passive integrated transponder-tagging coho salmon in Clear Creek (2013-2015).

D3. Yale Lake, Washington, with labeled regions that were used to evaluate densities of coho salmon smolt-sized targets in hydroacoustic surveys and the release location of smolts.

D4. Echograms from the sidelooking transducer showing smolt-sized targets in Yale Lake, Washington

D5. Length frequency of coho salmon captured in Clear Creek, Washington, during surveys in 2013, 2014, and 2015 .

D6. The number of passive integrated transponder-tagged coho salmon by reach in 2013, 2014, and 2015.

D7. Outmigration timing of passive integrated transponder-tagged wild coho salmon downstream past the fixed antennas on Clear Creek, Washington and average daily hydrologic stage and stream temperature at the Clear Creek antennas (2013-2015)

D8. Julian day of outmigration past the fixed passive integrated transponder-tag antenna for wild coho salmon marked with PIT-tags in different sampling reaches ...103 
D9. Year day that passive integrated transponder-tagged individual wild coho salmon (2013-2015) and hatchery-reared coho salmon (2013 only) juveniles were collected at the floating surface collector in Swift Reservoir, Washington.

D10. Total coho salmon smolt counts by month for 2014 and 2015 at the floating surface collector in Swift Reservoir, Lewis River, Washington. 105

D11. Proportion of coho salmon and steelhead passive integrated transponder-tagged at the Eagle Cliff screw trap collected at the floating surface collector by month.......106

D12. A frequency distribution and a cumulative frequency of the number of days passive integrated transponder-tagged spring Chinook salmon spent between outmigration and collection in Swift Reservoir, Washington (2013-2015).

D13. Total travel time for acclimation spring Chinook salmon from release in tributaries to the floating surface collector in Swift Reservoir, Washington (2013-2015) 108

D14. Total number of days between release and collection at the floating surface collector in Swift Reservoir for acclimation spring Chinook salmon across different release locations and years

D15. Year day that acclimation spring Chinook salmon released in 2013, 2014, and 2015 were captured at the floating surface collector in Swift Reservoir, Washington .........110

D16. The total number of spring Chinook salmon collected at the floating surface collector in Swift Reservoir, Washington

D17. Coho salmon smolt sized targets per 1,000 cubic meters observed by the sidelooking transducer in Yale Lake, Washington, prerelease, the day of elease, and postrelease of 5,000 test smolts

D18. Smolt-sized targets per 1,000 cubic meters observed by the downlooking transducer in 10-meter depth bins in Yale Lake, Washington, prerelease, the day of release, and postrelease of 5,000 test smolts

D19. Coho salmon smolt-sized targets per 1,000 observed by the downlooking transducer in 10-meter depth bins during zig-zag transects conducted in the lower quarter of Yale Lake, Washington, prerelease, day of release, and postrelease of 5,000 coho salmon smolts at Yale Park

E1. Study area map showing the reservoirs in the North Fork of the Lewis River, Washington.

E2. Limnological stations and Merwin trap locations within Lake Merwin, Washington, with numbers categorizing the different quadrants

E3. Length at age in years and von Bertalanffy growth curves for male and female Ptychocheilus oregonensis (northern pikeminnow) in Lake Merwin, Washington for years 2013-2014

E4. Isoclines of average water temperature from two stations one-third and two-thirds along the length of Swift Reservoir and Lake Merwin during April-0ctober 2014, and November 2014 in Yale Lake, Washington.

E5. Catch-per-unit-effort for Ptychocheilus oregonensis (northern pikeminnow) greater than or equal to 200 millimeters caught in sinking horizontal gill nets by depth and season in Lake Merwin, Washington, in 2013 and 2014.

E6. Stable isotope values of individual Ptychocheilus oregonensis (northern pikeminnow) and Esox lucius $\mathrm{x}$ masquinongy (tiger muskellunge) as a function of fork length, Lake Merwin, Washington, for 2013-2014 ..

E7. Average $\delta^{15} \mathrm{~N}$ and $\delta^{13} \mathrm{C}$ signatures for organisms sampled in Lake Merwin, Washington, in 2013-2014

E8. The relationship between Ptychocheilus oregonensis (northern pikeminnow) fork length and prey fish fork length for 2014-2014, Lake Merwin, Washington.. 
E9. The relationship between Esox lucius $\mathrm{x}$ masquinongy (tiger muskellunge) fork length and prey fish's fork length for 2013-2014, Lake Merwin, Washington.

E10. Length frequency distributions of male and female Ptychocheilus oregonensis (northern pikeminnow) captured in gill nets and traps in Lake Merwin, Washington, between 2013-2014 and 1958-1963..

E11. Length frequency histograms from raw catch data and data adjusted for size selectivity, Lake Merwin, Washington, from 2013-2014...

E12. Estimates from bioenergetics simulations of monthly biomass of prey consumed by size-structured populations of 1,000 Ptychocheilus oregonensis (northern pikeminnow)

E13. Estimate of potential number of subyearling spring Oncorhynchus tshawytscha (Chinook salmon) that could be consumed monthly by a size-structured population unit of 1,000 large Ptychocheilus oregonensis (northern pikeminnow), and the expected monthly masses of reservoir-rearing subyearling Chinook salmon, Lake Merwin, Washington.

E14. Estimates from bioenergetics simulations of seasonal biomass of prey consumed by an age-structured population of 5,488 Esox lucius $\mathrm{x}$ masquinongy (tiger muskellunge), Lake Merwin, Washington

F1. Map of North Fork of the Lewis River reservoirs in Washington with respect to the hydrologic basin.

F2. Isoclines of mean water temperature with respect to depth in Swift Reservoir, Yale Lake, and Lake Merwin, Washington, during March-December, 2013.

F3. Annual individual bull trout growth data for fish individually marked by Washington Department of Fish and Wildlife and PacifiCorp biologists from 1993-2016 in Swift Reservoir, Washington

F4. The location of coho salmon redds in Pine Creek and a tributary to Pine Creek, Washington; snorkel surveys used to identify overlap in the distribution of bull trout and coho salmon during 2013 and 2014; and the number of juvenile coho salmon detected in surveys.

F5. The number of juvenile coho salmon observed during daytime snorkel surveys during early summer in Pine Creek, P8, P3, and Rush Creek, Washington.

F6. The number of juvenile coho salmon observed during daytime snorkel surveys during mid-late summer in Pine Creek, P8, P3, and Rush Creek, Washington ...

F7. The number of juvenile coho salmon observed during snorkel surveys in early (June 2015) and mid-late summer (July-September 2015) in Pine Creek, P8, P3, and Rush Creek, Washington.

F8. The proportion of bull trout, coastal cutthroat trout, young-of-year coho salmon, mountain whitefish, and young-of-year Oncorhynchus spp. (rainbow trout/steelhead and coastal cutthroat trout) observed during snorkel surveys in different reaches in Pine Creek and P8 during 2013 and 2014

F9. The proportion of bull trout, coastal cutthroat trout, young-of-year coho salmon, mountain whitefish, and Oncorhynchus spp. (rainbow trout/steelhead and coastal cutthroat trout) observed during snorkel surveys in different reaches in Pine Creek and P8 during 2015.

F10. The proportion of bull trout, coastal cutthroat trout, young-of-year coho salmon, mountain whitefish, and young-of-year Oncorhynchus spp. (rainbow trout/steelhead and coastal cutthroat trout) observed during snorkel surveys in different reaches in P3 and Rush Creek during 2015.

F11. The cumulative frequency of the days between release and emigration past the Clear Creek, Washington, passive integrated transponder-tag antenna for acclimation spring Chinook salmon released during the spring of 2014 and 2015 
F12. Stable isotope results with 95-percent confidence intervals during late spring-summer in 2009 and 2014 for different size classes of fish from P8, a main tributary to Pine Creek and the major bull trout spawning tributary in the Swift Reservoir portion of the upper Lewis River, Washington.

F13. Stable isotope results with 95-percent confidence intervals during late spring-summer in 2009 and 2014 for different size classes of fish from Rush Creek, one of the major bull trout spawning tributaries in the Swift Reservoir portion of the upper Lewis River, Washington.

F14. The proportion of bull trout, juvenile coho salmon, coastal cutthroat trout/rainbow trout by size class as measured in millimeters and sculpin diets during the late spring-summer

F15. The proportion of bull trout, juvenile coho salmon, coastal cutthroat trout/rainbow trout and sculpin diets during the late fall

F16. The distribution of coho salmon redds in the upper Lewis River, Washington, from surveys conducted in 2012 to 2014

F17. The cumulative frequency distributions of the timing of coho salmon and bull trout redds in the upper Lewis River, Washington.

F18. Day, dusk, and night sequence of hydroacoustic target depth distributions in June, August, and November 2013 in Swift Reservoir, Washington.

F19. Day, dusk, and night sequence of hydroacoustic target depth distributions in June, August, and November 2013 in Yale Lake, Washington ..

F20. Day, dusk, and night sequence of hydroacoustic target depth distributions in June, August, and November 2013 in Lake Merwin, Washington............................................192

F21. Mean $\delta^{15} \mathrm{~N}$ and $\delta^{13} \mathrm{C}$ values for organisms sampled in Swift Reservoir .........................193

F22. Mean $\delta^{15} \mathrm{~N}$ versus $\delta^{13} \mathrm{C}$ for organisms sampled in Yale Lake ......................................194

F23. Mean $\delta^{15} \mathrm{~N}$ and $\delta^{13} \mathrm{C}$ for organisms sampled in Lake Merwin .....................................195

F24. The mean monthly density of Daphnia in three depth strata from two stations located approximately one-third and two-thirds along the length of Swift Reservoir, Yale Lake, and Lake Merwin between April and November 2014

F25. Estimates for Swift Reservoir, Washington, monthly consumption demand of Daphnia by a cohort of hatchery-reared rainbow trout.

F26. Estimates for Yale Lake, Washington, monthly consumption demand of Daphnia by three age-classes of kokanee salmon

F27. Estimates for Lake Merwin, Washington, monthly consumption demand of Daphnia by three age-classes of kokanee salmon.

F28. Simulation results indicating the number of adult bull trout in each simulation and the mean for the 20 years of model runs under the baseline bull trout population model with no effects of salmon reintroductions.

F29. The mean number of adult bull trout across all simulation years under the baseline population model, where a 20 percent loss of egg survival occurs through coho salmon redd superimposition, a 20 percent reduction in reservoir growth, a 20 percent reduction in reservoir growth and survival, and a 20 percent reduction in bull trout tributary carrying capacity

F30. Simulation results indicating the number of adult bull trout in each simulation and the mean for the 20 years of model runs where carrying capacity of tributary juvenile bull trout rearing is reduced by 20 percent of the baseline levels as a result of increased distribution and abundance of juvenile salmon

F31. Simulation results indicating the number of adult bull trout in each simulation and the mean for the 20 years of model runs where bull trout growth and survival in the reservoir are reduced by 20 percent through reductions in reservoir carrying capacity as a result of increased abundance of juvenile and residualized salmon .....201 


\section{Tables}

A1. Smolt collection efficiencies and standard deviations by river, name of hydropower facility, collector type, species, and sample size...

A2. Survival rates and standard deviations by river, smolt collection facility,species, and sample size.

A3. Average estimates of smolt survival and standard deviations by river, collection facility, collector type, and sample size.

A4. Descaling rates and standard deviations by river, facility, species, and sample size ....24

A5. The percent of injured fish and survival rates for different species at different life stages as measured at the floating surface collector in Swift Reservoir on the Upper Lewis River, Washington

A6. The type of injury by percent for all fish species for the 1.1 percent of fish injured during collection efforts at the floating surface collector in Swift Reservoir on the Upper Lewis River, Washington, in 2014 and 2015

A7. Survival rates and standard deviations for nontarget species at downstream fish collection facilities by river, facility, species, and sample size.

A8. Average mortality rates and standard deviations during adult trap and haul operations for different species, runs, and sources of salmon in the Pacific Northwest

A9. Average fallback rates and standard deviations by anadromous species and runs......26

A1.1. Estimates of smolt survival in acclimation facilities by river, acclimation facility, year, species, run type, age class, acclimation facility type, and reference.

A1.2. Estimates of collection efficiency by river, facility, collection method, year, species, and reference.

A1.3. Estimates of survival through collectors by river, facility, collection method, year, species, origin, and reference.

A1.4. Descaling estimates by river, facility, type of collector, year, species, and reference

A1.5. Mortality estimates for nontarget species at downstream fish collection facilities by river, facility, year, species, and reference...

A1.6. Estimates of adult mortality during trap and haul operations for different species by river, facility, year, species, run, source, and references

A1.7. Fallback rates over dam facilities for adult anadromous species by river, dam, year, species, run, and reference

B1. The total stream length surveyed, average bankfull width, wetted width, and number of sample reaches in each tributary stream to Swift Reservoir, Washington, during surveys in 2015

B2. The total stream length, average bankfull width, wetted width, and number of sample reaches in each tributary stream to Lake Merwin and Yale Lake, Washington, during surveys in 2014.

B3. The total surface area by reach and the average surface area in pool habitat, pool tailouts, and glides in each tributary stream to Swift Reservoir in 2015 and Lake Merwin and Yale Lake in 2014 in Washington.

B4. Average depth for nonpool habitat and residual pool depth for pools by habitat type and stream in each tributary stream to Swift Reservoir, Washington, from surveys in 2015 .....

B5. Average depth for nonpool habitat and residual pool depth for pools by habitat type and stream in each tributary stream to Lake Merwin and Yale Lake, Washington, from surveys in 2014 
B6. The average density of large woody debris delineated by large and small pieces, and percent surface fines in pool tailouts in each tributary stream to Swift Reservoir in 2014 and Lake Merwin and Yale Lake in 2014 in Washington

B7. Percent of substrate estimated as bedrock, boulder, large cobbles, small cobbles, gravel, and fines in fast-water habitat in tributaries to Swift Reservoir in 2015 and Lake Merwin and Yale Lake in 2014 in Washington

B8. The average and range of stream temperatures by month from July through October 2013 for Clear Creek and in each tributary stream to Lake Merwin and Yale Lakein Washington in 2014

B9. The average and range of stream temperatures by month from June through October for Clear Creek and in each tributary stream to Lake Merwin and Yale Lake in 2014 in Washington.

B10. The average measures of dissolved oxygen, reach gradient, and riparian function in each tributary stream to Lake Merwin and Yale Lake, Washington, in 2014.

B11. The average measures of riparian function in sampled tributary stream to Swift Reservoir, Washington, in 2015.

C1. Field estimates from 2014 surveys of the percent of fine sediment in pool tailouts in available tributary habitat in Lake Merwin and Yale Lake.

C2. Percent of substrate estimated as bedrock, boulder, large cobbles, small cobbles, gravel, and fines in fast-water habitat from 2014 field surveys in tributaries to Lake Merwin and Yale Lake, Washington.

D1. Number of passive integrated transponder-tagged acclimation coho salmon and spring Chinook salmon released by location and year in the Lewis River upstream from Swift Dam, Washington, 2013-2015.

D2. Hydroacoustic transects conducted in 2014 to evaluate coho salmon smolt movement and distribution in Yale Lake, Washington, including release status, date, location, start and end times, and diel period.

D3. Total numbers of spring Chinook salmon, coho salmon, and juvenile steelhead passive integrated transponder-tagged by year at the screw trap at Eagle Cliff on the Lewis River at the head of Swift Reservoir, Washington, and the percent of fish collected at the floating surface collector, 2014-2015

E1. Limnological and morphometric characteristics of Lake Merwin, Washington............139

E2. Relative abundance of fish species present in Lake Merwin, Washington .. 139

E3. Abundance estimates of Ptychocheilus oregonensis (northern pikeminnow) by size class in Lake Merwin, Washington for 2013..

E4. Thermal experiences of Ptychocheilus oregonensis (northern pikeminnow) and Esox lucius $\mathrm{x}$ masquinongy (tiger muskellunge) in Lake Merwin, Washington...

E5. Energy density of prey items of Ptychocheilus oregonensis (northern pikeminnow) and Esox lucius $\mathrm{x}$ masquinongy (tiger muskellunge) in Lake Merwin, Washington .....141

E6. Bioenergetics inputs of growth and spawning losses and output of percent maximum consumption and total Oncorhynchus nerka (Kokanee salmon), Ptychocheilus oregonensis (northern pikeminnow), Cottus spp. (sculpins); and Pacifastacus leniusculus (signal crayfish) annual consumption by individual male and female northern pikeminnow age 3-14 years, Lake Merwin, Washington.

E7. Bioenergetics inputs of growth-at-age and outputs of percent maximum consumption, total consumption, and growth efficiency by individual Esox lucius $\mathrm{x}$ masquinongy (tiger muskellunge) aged 1-10 years, Lake Merwin, Washington

E8. Mean $\boldsymbol{\delta}^{15} \mathrm{~N}$ and $\boldsymbol{\delta}^{13} \mathrm{C}$ stable isotope values for fish and invertebrates sampled in the Lake Merwin, Yale Lake, and Swift Reservoir of the Lewis River, Washington, by location and size class for years 2013-2014 
E9. Diet proportions of two size classes of Ptychocheilus oregonensis (northern pikeminnow) in Lake Merwin, Washington, for the years 2013-2014

E10. Diet proportions of tiger muskellunge including Ptychocheilus oregonensis (northern pikeminnow) in Lake Merwin, Washington for years 2013-2014 146

F1. Estimates of water temperatures for optimum and maximum growth from existing literature for bull trout, Chinook salmon, coho salmon, steelhead, and coastal cutthroat trout

F2. Percent of fish in bull trout diets by lake or river, life-history forms, and size classes.

F3. Thermal experience of pelagic planktivore consumption demands across different dates and simulation days within a bioenergetics modeling framework in Swift Reservoir, Yale Lake, and Lake Merwin, Washington... .204

F4. Energy density of prey items consumed by pelagic planktivores .................................204

F5. Starting and ending dates and consumer weights used in bioenergetics simulations and outputs of feeding rate and consumption in biomass

F6. Model inputs of survival, growth, and percent maturity by age for bull trout in the Swift Reservoir, Washington

F7. Relative abundance of fish species observed in sampling conducted in this study and predominant summer crustacean zooplankton found in the Swift Reservoir, Yale Lake, and Lake Merwin, Washington.

F8. Seasonal diet proportions for invertebrate-feeding salmonids including spring Chinook salmon, hatchery-reared rainbow trout, and kokanee salmon captured in Swift Reservoir, Yale Lake, and Lake Merwin, June-November 2013 and MayNovember 2014 


\section{Conversion Factors}

International System of Units to U.S. customary units

\begin{tabular}{|c|c|c|}
\hline Multiply & By & To obtain \\
\hline \multicolumn{3}{|c|}{ Length } \\
\hline centimeter $(\mathrm{cm})$ & 0.3937 & inch (in.) \\
\hline micrometer $(\mu \mathrm{m})$ & 0.0000393701 & inch (in.) \\
\hline millimeter (mm) & 0.03937 & inch (in.) \\
\hline meter $(\mathrm{m})$ & 3.281 & foot $(\mathrm{ft})$ \\
\hline kilometer (km) & 0.6214 & mile (mi) \\
\hline kilometer (km) & 0.5400 & mile, nautical (nmi) \\
\hline meter $(\mathrm{m})$ & 1.094 & yard (yd) \\
\hline \multicolumn{3}{|c|}{ Area } \\
\hline square meter $\left(\mathrm{m}^{2}\right)$ & 0.0002471 & acre \\
\hline square kilometer $\left(\mathrm{km}^{2}\right)$ & 247.1 & acre \\
\hline square meter $\left(\mathrm{m}^{2}\right)$ & 10.76 & square foot $\left(\mathrm{ft}^{2}\right)$ \\
\hline square kilometer $\left(\mathrm{km}^{2}\right)$ & 0.3861 & square mile $\left(\mathrm{mi}^{2}\right)$ \\
\hline \multicolumn{3}{|c|}{ Volume } \\
\hline cubic meter $\left(\mathrm{m}^{3}\right)$ & 6.290 & barrel (petroleum, 1 barrel $=42$ gal) \\
\hline cubic meter $\left(\mathrm{m}^{3}\right)$ & 264.2 & gallon (gal) \\
\hline cubic meter $\left(\mathrm{m}^{3}\right)$ & 0.0002642 & million gallons (Mgal) \\
\hline liter $(\mathrm{L})$ & 61.02 & cubic inch $\left(\mathrm{in}^{3}\right)$ \\
\hline cubic meter $\left(\mathrm{m}^{3}\right)$ & 35.31 & cubic foot $\left(\mathrm{ft}^{3}\right)$ \\
\hline cubic meter $\left(\mathrm{m}^{3}\right)$ & 1.308 & cubic yard $\left(\mathrm{yd}^{3}\right)$ \\
\hline cubic meter $\left(\mathrm{m}^{3}\right)$ & 0.0008107 & acre-foot (acre-ft) \\
\hline \multicolumn{3}{|c|}{ Flow rate } \\
\hline cubic meter per second $\left(\mathrm{m}^{3} / \mathrm{s}\right)$ & 70.07 & acre-foot per day (acre-ft/d) \\
\hline cubic meter per second $\left(\mathrm{m}^{3} / \mathrm{s}\right)$ & 35.31 & cubic foot per second $\left(\mathrm{ft}^{3} / \mathrm{s}\right)$ \\
\hline cubic meter per day $\left(\mathrm{m}^{3} / \mathrm{d}\right)$ & 264.2 & gallon per day (gal/d) \\
\hline \multicolumn{3}{|c|}{ Mass } \\
\hline gram $(\mathrm{g})$ & 0.03527 & ounce, avoirdupois (oz) \\
\hline kilogram (kg) & 2.205 & pound avoirdupois (lb) \\
\hline metric ton $(\mathrm{t})$ & 1.102 & ton, short $[2,000 \mathrm{lb}]$ \\
\hline metric ton $(\mathrm{t})$ & 0.9842 & ton, long $[2,240 \mathrm{lb}]$ \\
\hline microgram $(\mu \mathrm{g})$ & 0.00000003527 & ounce, avoirdupois (oz) \\
\hline \multicolumn{3}{|c|}{ Energy } \\
\hline joule $(\mathrm{J})$ & 0.0000002 & kilowatthour (kWh) \\
\hline
\end{tabular}

Temperature in degrees Celsius $\left({ }^{\circ} \mathrm{C}\right)$ may be converted to degrees Fahrenheit $\left({ }^{\circ} \mathrm{F}\right)$ as ${ }^{\circ} \mathrm{F}=(1.8 \times$ $\left.{ }^{\circ} \mathrm{C}\right)+32$. 


\section{Datum}

Horizontal coordinate information is referenced to the North American Datum of 1983 (NAD 1983).

\section{Supplemental Information}

Specific conductance is given in microsiemens per centimeter at 25 degrees Celsius $(\mu \mathrm{S} / \mathrm{cm}$ at $\left.25^{\circ} \mathrm{C}\right)$.

Concentrations of chemical constituents in water are given in either milligrams per liter (mg/L) or micrograms per liter $(\mu \mathrm{g} / \mathrm{L})$.

Results for measurements of stable isotopes of an element (with symbol E) in water, solids, and dissolved constituents commonly are expressed as the relative difference in the ratio of the number of the less abundant isotope ( $\mathrm{E}$ ) to the number of the more abundant isotope of a sample with respect to a measurement standard.

Note to USGS users: Use of liter (L) as a special name for cubic decimeter $\left(\mathrm{dm}^{3}\right)$ is restricted to the measurement of liquids and gases. No prefix other than milli should be used with liter.

\section{Abbreviations}

AICc Akaike Information Criteria corrected for small sample size

CHaMP Columbia Habitat Monitoring Program

CPUE catch-per-unit effort

CV coefficient of variation

DFW Department of Fish and Wildlife

EDT Ecosystem Diagnosis and Treatment

FSC floating surface collector

LWD large woody debris

PIT passive integrated transponder

PUD public utility district

RSW removable spillway weir

SA surface area

SAS smolt-to-adult survival rates

SBC surface bypass collector

SD standard deviation

SE standard error

USGS U.S. Geological Survey

UW-IACUC University of Washington Institutional Animal Care and Use Committee

YOY young-of-year 


\section{Species}

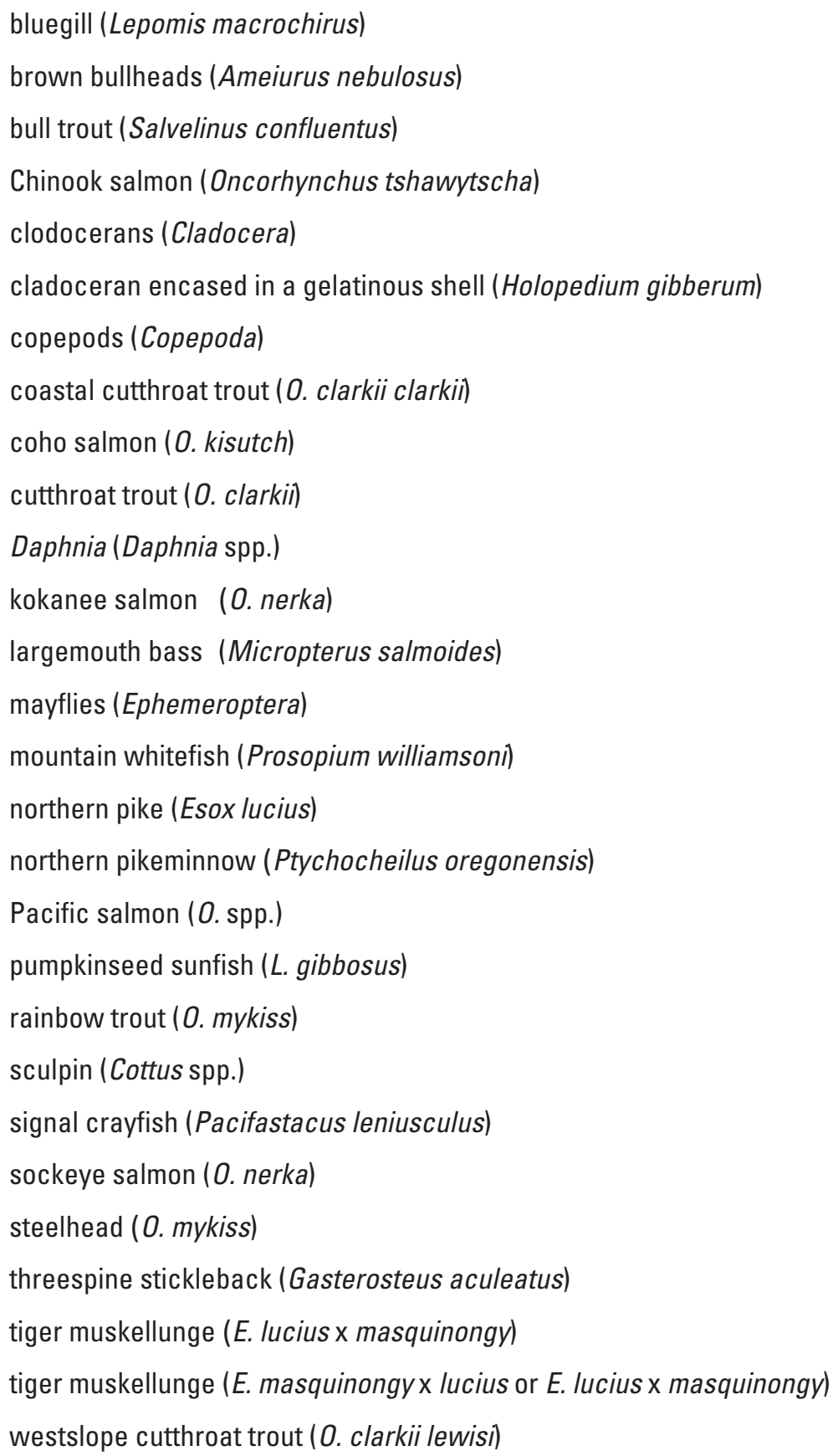




\section{Scientific Notation}

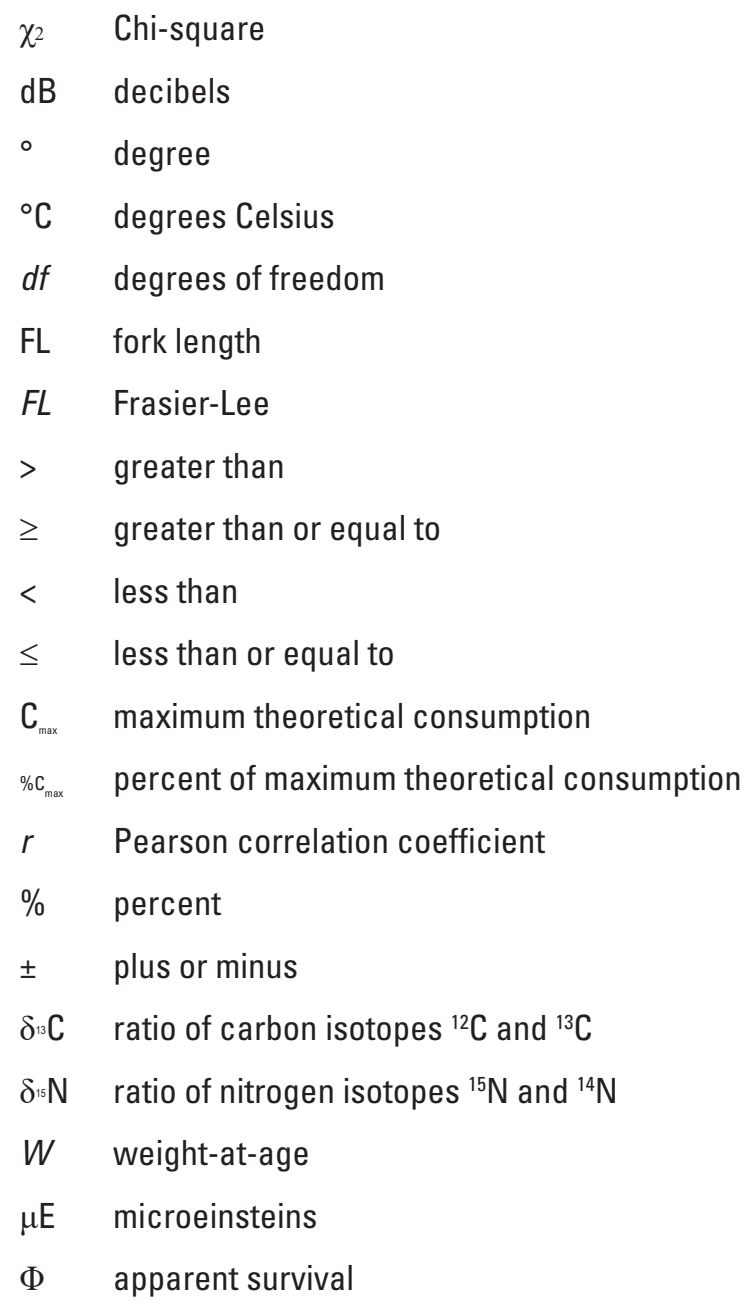





\title{
Development of New Information to Inform Fish Passage Decisions at the Yale and Merwin Hydro Projects on the Lewis River, Washington-Final Report, 2018
}

\author{
By Robert Al-Chokhachy, ${ }^{1}$ Christopher L. Clark, ${ }^{2}$ Mark H. Sorel, ${ }^{3}$ and \\ David A. Beauchamp ${ }^{1}$
}

\section{Executive Summary}

The reintroduction of extirpated salmonids to historically occupied areas is becoming increasingly common as a conservation and recovery strategy. Often, reintroductions are implemented after the factors that originally led to species extirpation have been reduced, eliminated, or mitigated. For anadromous Oncorhynchus spp. (Pacific salmon) and O. mykiss (steelhead), addressing barriers to migration, which have been a primary factor in the decline and extirpation of many populations, has been an integral component of recovery efforts. Mitigation has included barrier removal, developing fish passage opportunities, and (or) actively trapping and hauling juvenile and adult anadromous salmonids around barriers.

With any reintroduction, there are a number of concerns regarding the ecological impact of the reintroduction efforts. Three of the main tenets to consider when assessing reintroductions are (1) the potential benefits if reintroduction is successful, (2) the biological risk through interactions of reintroduced strains with existing populations, and (3) the factors potentially limiting a successful reintroduction. This report focuses on information and data to address the second and third factors as they apply to the upper Lewis River in Washington.

The upper Lewis River historically contained wild populations of $O$. tshawytscha (Chinook salmon), O. kisutch (coho salmon), and steelhead. These populations were extirpated after completion of hydropower facilities on Lake Merwin in 1932, Yale Lake in 1953, and Swift Reservoir in 1958, which prevented fish from migrating to and from ocean environments. However, recent licenses issued by the Federal Energy Regulatory Commission require the installation and operation of an upstream fish passage facility at Lake Merwin and a downstream fish passage facility at Swift Reservoir. The licenses were developed in consultation with the National Marine Fisheries Service and the U.S. Fish and Wildlife Service. The overarching goal of this fish reintroduction project is to establish viable, self-sustaining, naturally reproducing, harvestable populations of spring Chinook salmon, winter steelhead, and coho salmon at levels higher than minimum viable populations.

This report uses a combination of field data and existing information to address six key objectives related to the reintroduction in order to inform decisions about passage at the Yale Lake and Lake Merwin hydropower projects. The objectives are (1) a review of information relevant to anadromous fish reintroduction and full fish passage; (2) a habitat assessment of tributaries to Swift Reservoir, Yale Lake, and Lake Merwin; (3) a field study to assess adult potential for spawning success; (4) an assessment of juvenile production and outmigration success; (5) a Lake Merwin predator impact study; and (6) a set of studies assessing interactions between anadromous and resident fish.

${ }^{1}$ U.S. Geological Survey.

${ }^{2}$ Montana State University, Department of Ecology, Bozeman, Montana.

${ }^{3}$ University of Washington, School of Aquatic and Fishery Sciences, Seattle, Washington. 


\section{Chapter A. Review of Information Relevant to Anadromous Fish Reintroduction to and Fish Passage in the Lewis River, Washington}

Comparing the success of collection operations for juvenile and adult fish at different locations is challenging because of different collection designs, hydrologic conditions, and reservoir bathymetry. In general, juvenile and adult injuries and direct mortalities appear to be generally low regardless of species and location; the extent of delayed mortality, however, is less clear. Juvenile collection efficiencies are highly variable between locations and species, likely because of the inherent differences at the various locations. Fallback rates of adult Pacific salmon and steelhead in trap and haul operations are poorly studied, but available data from such operations and other hydropower facilities show that fallback rates can be moderate (up to 24 percent) but variable. The review highlights the need to continue to evaluate designs and operations that can increase the effectiveness of trap and haul operations (for example, increase the collection efficiency of juveniles) while minimizing the effects to target and nontarget taxa.

\section{Chapter B. Habitat Assessment of Tributaries to Swift Reservoir, Yale Lake, and Lake Merwin, Washington}

Aside from a finite number of locations, empirical habitat data suggest little evidence that habitat quality will limit salmon and steelhead introductions. Overall, stream temperatures vary extensively within the upper Lewis River Basin, but do not appear to be a limiting factor in the near term for anadromous species. The extent of available habitat varies across reservoirs from primarily natural barriers, with particularly low amounts of habitat (8.2 kilometers) available to anadromous species in Lake Merwin.

\section{Chapter C. Studies Assessing Adult Potential for Spawning Success in Yale Lake and Lake Merwin, Washington}

The ability to thoroughly evaluate spawning success in Yale Lake and Lake Merwin was constrained to a single year in Lake Merwin by low numbers of available salmon. Field results with coho salmon did show that adults use a variety of habitat types. Young-of-year coho salmon recruitment was lower than observed in Swift Reservoir based on measures of catch-per-unit effort, but size distributions of fish were similar. The lower recruitment was likely driven by the proximity of coho salmon spawning to the reservoirs and migration patterns, which were not documented herein. The robust habitat in the tributaries and spawning and recruitment patterns suggest high potential for successful spawning in Lake Merwin and Yale Lake.

\section{Chapter D. Assessment of Juvenile Fish Production and Outmigration Success on the Lewis River, Washington}

Using a combination of field methods, results suggested outmigration success may be limited by collection efficiencies. Test releases of hatchery-reared juvenile salmon suggested fish migrated relatively quickly though the reservoir environments. However, median travel time for individually tagged coho salmon and steelhead indicated movements through Swift Reservoir to 
the floating surface collector at Swift Dam were 37 days and 21 days, respectively. Most salmon and steelhead were collected at the floating surface collector in the spring (April 15-June 30) and survival through the reservoir environment was high (54 percent). Among spring Chinook salmon, coho salmon, and steelhead collection efficiencies were low and did not exceed 18.7 percent. The low proportions of juvenile salmon and steelhead reaching the downstream collector suggests a considerable number of fish are likely residualizing in the reservoir environment.

\section{Chapter E. Piscine Predator Impact Studies 2013-2014, Lake Merwin, Washington}

The population of Ptychocheilus oregonensis (northern pikeminnow) in Lake Merwin is extensive suggesting considerable predation on juvenile anadromous salmon and steelhead. Northern pikeminnow were increasingly piscivorous with size and diet studies suggested major prey items included pelagic and benthic fish and crayfish. The current size distribution of northern pikeminnow suggests extensive predation by large (greater than or equal to 300 millimeters) northern pikeminnow and Esox lucius x masquinongy (tiger muskellunge). Bioenergetics model estimates of northern pikeminnow predation on salmonids indicated the potential for 39,250 Chinook salmon smolts to be preyed upon each year. The predation during peak salmon migration (that is, April-June) was markedly lower. Predation by tiger muskellunge on northern pikeminnow would likely continue to reduce the overall predation on salmonids by northern pikeminnow.

\section{Chapter F. Studies Assessing Anadromous-Resident Fish Interactions in the Lewis River, Washington}

A review of existing literature addressing anadromous-resident fish interactions suggests high potential for interactions given the diverse life-history patterns of resident fish such as O. clarkii clarkii (coastal cutthroat trout) and Salvelinus fontinalis (bull trout). Although some differences in thermal requirements between salmon and steelhead and resident salmonids exist, overlap in juvenile rearing (all species) and spawning of coho salmon and bull trout is likely. Bull trout predation on salmon and steelhead would be expected, given that bull trout is a toplevel predator in the Pacific Northwest. While population-level effects are unknown, bull trout historically overlapped with salmon and steelhead suggesting limited implications to salmon and steelhead populations.

Field studies from Swift Reservoir show that the distribution of coastal cutthroat trout, bull trout, and coho salmon varied spatially across tributaries. Where sympatric, the results from diet studies in stream habitat indicate similar diets for juvenile coastal cutthroat trout, bull trout, and coho salmon. Coho salmon were found to superimpose on bull trout spawning redds, and the later timing of coho salmon spawing indicates a high potential for further superimposition. Diet studies indicated bull trout to be the top predator in Yale Lake and Swift Reservoir, yet there were few indications that bull trout preyed upon juvenile coho or Chinook salmon. The lack of predation on juvenile salmon may be from bull trout gape limitations and the larger size of juvenile salmon currently in Swift Reservoir. Pelagic studies illustrate that food abundance will limit the number of planktivorous fish in reservoir environment, and modeling results indicate that shifts in the amount of forage for juvenile bull trout may be detrimental to the population in the long term. The large changes in the physical template of the upper Lewis River Basin stemming from habitat alterations (for example, building of hydropower facilities, the eruption of Mount Saint Helens) means uncertainty in how anadromous reintroductions will influence native salmonids such as bull trout. 



\section{Chapter A. Review of Information Relevant to Anadromous Fish Reintroduction to and Fish Passage in the Lewis River, Washington}

\section{Introduction}

The complex life cycle of Oncorhynchus spp. (Pacific salmon) predicates the need to understand fish passage at different life stages during reintroduction efforts (Anderson and others, 2014), particularly in trap and haul operations. The extirpation of populations of O. tshawytscha (Chinook salmon), O. kisutch (coho salmon), and O. mykiss (steelhead) after completion of hydropower facilities (Merwin Dam, Yale Dam, and Swift Dam; fig. A1) in the upper North Fork of the Lewis River predicated the need for the installation and operation of an upstream fish passage facility at Lake Merwin and a downstream fish passage facility at Swift Reservoir. This report is a complement to previous documents (PacifiCorp and Cowlitz Public Utility District [PUD], 2002) but with specific objectives of reviewing different aspects of anadromous species reintroductions above hydropower facilities, particularly where trap and haul operations are used. As such, the specific objectives of this report are to assess key attributes related to reintroductions including (1) the effects of juvenile acclimation facilities on salmon performance; (2) downstream collection efficiency and injury and survival at downstream collection facilities; (3) adult fallback, injury, and survival; and (4) collection at adult fish upstream collection facilities. Each of these attributes (for example, downstream collection efficiencies) is likely to be particularly relevant in considering the overall success and effects of the anadromous reintroductions. For example, collection efficiency, which is the proportion of fish within the zone of influence that are collected, will have profound effects on factors such as overall capacity, reservoir capacity, and anadromous-resident interactions. Thus, the direct and indirect implications of each of the attributes described in this study needs to be considered in assessing the reintroduction of anadromous salmon in each of the reservoir environments.

\section{Effects of Smolt Acclimation Facilities}

Rearing and release.-Smolt acclimation facilities are commonly used to reduce stress associated with fish transport (Maule and others, 1989; Schreck and others, 1989) and for imprinting natal waters to induce adults to return to specific acclimation streams (Dittman and others, 2010). Acclimation facilities that integrate local surface-water sources also provide more natural stream temperatures, particularly where source hatcheries maintain relatively constant stream temperatures. A variety of acclimation facilities are currently being employed in anadromous species supplementation and reintroduction programs including aluminum tanks (for example, McLeod, 2008), off-channel raceways (for example, Zollman and others, 2009), and seminatural stream channels and ponds (for example, PacifiCorp and Cowlitz PUD, 2011).

Survival within the acclimation facilities is high (typically greater than [>] 98 percent; table A1.1 in appendix A1), excluding any disease (not covered herein). In most facilities, smolts are allowed volitional movements out of the acclimation facilities to natural systems; the time allowed for volitional release varies by systems, but typically ranges from 1-8 weeks (Cleary, 2005; Clarke and others, 2011), after which remaining smolts are often forced out of the acclimation facilities. The percentage of smolts to volitionally release from acclimation facilities can vary considerably between systems. Cleary (2005) found high (88-97 percent for all releases) volitional release of spring Chinook salmon smolts within 10 days of acclimation in the Lostine River in Oregon, which is consistent with the pattern of 100 percent volitional release for spring Chinook salmon in the Hood River, Oregon (Gerstenberger, 2009). Osborne and Rhine (1999) and Gerstenberger (2009) have reported high volitional releases ( $>85$ percent) for steelhead. However, Cleary and others (2006) found considerable annual variability in proportion of volitional releases of Chinook salmon during 1999-2004 with values as low as 10.9 percent, and low percentages of volitionally migrating smolts have been reported elsewhere (for example, McLean and others, 2003). 
A. Review of Information Relevant to Anadromous Fish Reintroduction to and Fish Passage in the Lewis River, Wash.

Of concern are the effects of the length of acclimation and volitional emigration as opposed to forced emigration of smolts from acclimation facilities. In a recent study, Clark and others (2012) found the amount of time spring Chinook salmon were acclimated (either 2 or 4 months) had little effect on smolt survival or stray rates, but longer acclimation (4-month acclimation from November through early March) resulted in significantly slower travel times and 27 percent higher smolt-to-adult survival rates (SAS). However, identifying the relative effects of a longer acclimation period and over-winter acclimation on parameters such as survival, stray rates, and SAS was not possible because of the limited available information.

The effects of volitional as opposed to forced release of smolts from acclimation facilities appear to be inconsistent for the different studies. In a comparison of juvenile steelhead in Washington, Gale and others (2009) found no consistent differences in survival rates or travel times of volitional and forced-migrant smolts. In contrast, Clarke and others (2011) found smolts that migrated volitionally from acclimation facilities had slower travel times and significantly higher survival rates but the higher smolt survival rate did not result in higher SAS rates for volitional migrants. The uncertainty in the effects of release strategies suggests additional research is needed with paired, experimental approaches.

Acclimation and smolt migration patterns.-Studies have shown inconsistent effects of acclimation on anadromous migration patterns. In a 10-year paired study, Clarke and others (2010) found travel times for acclimated summer steelhead to be 10 percent ( 2.9 days) longer than for fish directly released into streams. In contrast, acclimated fall Chinook salmon have demonstrated faster travel times than direct release hatchery-reared smolts (3-11 days), which more closely resembled migration patterns for naturally produced smolts (Rosenberger and others, 2013). In other studies, no consistent differences have been observed in the migration timing of direct and acclimated steelhead or Chinook salmon smolts (Fast and others, 1991; Whitesel and others, 1994; Cameron and others, 2013). Such inconsistencies highlight the need for additional studies to better understand how acclimation facilities affect travel times and the effects to long-term measures of fitness (for example, SAS).

An additional concern is the effect of acclimation facilities on residualization, which occurs when anadromous fish remain within the freshwater. When acclimated, smolts demonstrate a lower residualization rate than direct-release fish (Viola and Schuck, 1995; Hausch and Melnychuk, 2012). There continues to be uncertainty as to how residualization interferes with smolts that do not volitionally release from acclimation facilities (Hausch and Melnychuk, 2012), particularly when considering the results of Clarke and others (2011) that demonstrated more rapid travel times for smolts that did not volitionally migrate from acclimation facilities. Given the potential ecological effects of residualized smolts, additional research is needed to identify migration patterns of smolts that do not volitionally release from acclimation facilities.

Acclimation and juvenile survival and SAS.-Similar to other performance metrics, there is extensive variability in the influence of acclimation facilities on juvenile survival (see table A1.1 for individual estimates) and SAS. When acclimated, fall Chinook salmon smolts have higher survival rates than direct-release hatchery-reared smolts (Rosenberger and others, 2013), which is similar to the pattern for spring Chinook salmon (Fast and others, 1991; Cameron and others, 2013) and steelhead (Whitesel and others, 1994). These results contradict earlier studies with winter and summer steelhead, which found no difference in acclimated and direct-release smolt survival (Tipping, 1998; Kenaston and others, 2001; Appleby and others, 2002; Clarke and others, 2010). In contrast, Clarke and others (2010) found SAS for acclimated smolts to be $>11$ percent higher than observed for direct-release summer steelhead smolts, a pattern consistent in most years (6 out of the 7 years, where estimates were comparable, 2002-2010) for summer steelhead in the Umatilla River in Oregon (Cameron and others, 2013). Variability in the effects of acclimation is demonstrated in the Umatilla River, where SAS of fall Chinook salmon was lower than directrelease smolts in 4 of the 6 years that were monitored (Cameron and others, 2013).

At least some of the discrepancy between studies may be the lack of consistency in study methods. For example, Tipping (1998) found no difference in steelhead survival rates between direct release smolts and smolts allowed to rest for 24 hours. Comparing these results with longer duration acclimation practices (for example, 20-23 days; Rosenberger and others, 2013) may not be appropriate, suggesting the need for consistent experimental tests to allow for more informed comparisons.

Acclimation and adult stray rates. - In this study, stray rates are specifically considered for studies where direct (for example, experimental) comparisons were performed between acclimated and nonacclimated (direct release) groups. Again, results from most studies suggest moderate to high variability in stray rates. Early studies of $O$. kisutch (coho salmon), and Chinook salmon found no differences in homing rates within Lake Michigan in Illinois (Savitz and others, 1993). An experiment with spring Chinook salmon in the lower Willamette River in Oregon using net pens as acclimation facilities, yielded a mixture of results that suggest no clear pattern of the effect of acclimation on natal homing (Schroeder and Kenaston, 2005). However, Clarke and others (2010) found 42 percent lower stray rates for acclimated steelhead compared to direct-release fish. Dittman and others (2010) found that, despite homing to acclimation streams, a large portion (55.1 percent) of adult Chinook salmon spawned at distances $>25$ kilometers to natal sites within these streams, which was similar to the distribution observed in wild Chinook salmon. The broad distribution of spawners in the Yakima River in Washington is likely to be influenced by local spawning habitat within and proximate to acclimation facilities (Cram and others, 2013). The results of Cram and others (2013) illustrate the need to consider spatial patterns in suitable habitat when assessing stray rates to reduce uncertainty when comparing studies.

In general, comparing stray rates from different studies appears to be problematic, particularly given differences in the timing and duration of acclimation (Keefer and Caudill, 2012). Furthermore, comparison of stray rates may not be meaningful 
given recent evidence of differences in stray rates among species and streams (Westley and others, 2013), suggesting the need for in situ measures of stray rates for all species.

\section{Smolt Collection and Injury and Survival at Downstream Collection Facilities}

The focus of this section is to summarize data from existing studies on smolt collection efficiencies and survival at collection facilities.

Smolt collection efficiency.-A variety of collector types are used to capture downstream-migrating anadromous fish in rivers with migration barriers. Table A1 provides smolt collection efficiencies and standard deviations (SDs) by river, facility, collector type, and species. Comparisons of collection efficiencies between different projects are difficult because of the inherently different dam operations and ambient conditions. This study considers collection efficiencies as the number of migratory fish captured at a collector and summarizes results from different rivers and sites for reference.

Substantial differences in collection efficiencies were found for different species, years, and collectors. For example, estimates of collection efficiency for steelhead during the period from 1996 to 2012 varied from a low of 5.4 percent to a high of 68 percent (Serl and Morrill 2010; Serl and Heimbigner, 2013). Although general trends are consistent for different species, interannual variability may not be strongly correlated for all species (Pearson correlation coefficient $[r]=0.57$; fig. A2).

At some facilities, collection efficiencies appear to be relatively consistent between species, such as at the Mayfield facility on the Cowlitz River where collection efficiencies for coho salmon, Chinook salmon, and steelhead ranged from 61.5 to 76.5 percent (table A1). At other facilities, collection efficiencies for different species differ substantially. For example, collection efficiencies using a forebay collector at Rocky Reach Dam on the Columbia River in Oregon were 17.7 percent for Chinook salmon and 47.8 percent for steelhead. There does not appear to be any consistent pattern of higher/lower collection efficiencies for the different species. For example, on the Cowlitz River, collection efficiencies for juvenile Chinook salmon (mean=21.5 percent) are half that observed for steelhead at Cowlitz Falls (mean=43.3 percent; 1996-2012), but little difference in average collection efficiencies occurs at Mayfield (the mean collection efficiency for Chinook salmon is 75 percent and for steelhead is 76.5 percent); however, the Mayfield studies were done from 1964 to 1965.

In part, the interannual differences in collection efficiencies may be a result of varying environmental conditions (for example, epilimnetic water tempertures and discharge; Kock and others, 2015). For example, Beeman and others (2016) found juvenile Chinook salmon preferred temperatures of 13-15 degrees Celsius $\left({ }^{\circ} \mathrm{C}\right)$ when migrating through Cougar Reservoir in Oregon; when temperatures were higher, fish went to deeper water and were less likely to be collected. The Lewis River, Lake Merwin, Yale Lake, and Swift Reservoir all have warm $\left(>20^{\circ} \mathrm{C}\right)$ near-surface temperatures during the summer (see "Piscine Predator Impact Studies 2013-2014, Lake Merwin, Washington," chap. E of this report) suggesting warmer temperatures may reduce collection, particularly if thermal barriers lead to increased residualization.

In recent years, state-of-the-art collection facilities have been constructed by hydropower entities on numerous rivers in the Pacific Northwest (for example, the Lewis River and Baker River in Washington and the Deschutes River in Oregon). However, the ability to compare collection efficiencies in different rivers is likely to require multiple years of monitoring. Early evaluations of collection efficiencies are likely to be biased because of the high interannual variability observed at many existing facilities and site-to-site differences. As such, an understanding of the factors associated with or influencing collection efficiencies (for example, variations in migration timing, in-reservoir conditions such as temperature) will be needed to establish collection-efficiency goals. That said, because the Upper Baker (Baker River) and Round Butte (Deschutes River) collectors are very similar to the floating surface collector (FSC) at the Swift Reservoir facility on the Lewis River in terms of entrance configuration, it is informative to look at how effective those two facilities are at capturing juvenile salmonid outmigrants. As shown in table A1.2, capture efficiencies for coho salmon at Upper Baker ranged from 82.6 to 99 percent (Jeanes and Verretto, 2012). At the Round Butte facility on the Deschutes River, collection efficiencies have been considerably lower for Chinook salmon (30.1-51.7 percent) and steelhead (12.4-24.2 percent, table A1.2; Hill and Quesada, 2011, 2012, 2013). However, the estimates for the surface collector at Round Butte include both hatchery-reared and wild fish. Although early results indicated no apparent difference between these groups, recent collection results suggest considerably lower collection efficiencies for naturally reared Chinook salmon and steelhead compared to hatchery-reared releases (Hill and Quesada, 2014, 2015). In addition, it is worth noting that the estimates of collection efficiencies at Round Butte include reservoir survival, a measure of downstream migrants (that is, compared to residualized fish), and collection efficiency of fish that make it to the collector. Studies at Lake Billy Chinook in Oregon indicate a large portion of the fish entering the reservoir did not make it to the surface collector at Round Butte (Hill and Quesada, 2013).

In the Lewis River, the proportion of marked to unmarked juvenile salmon collected at the FSC was relatively low through 2015. For coho salmon, the majority of data were from naturally produced juveniles (that is, subyearlings) that were tagged with passive integrated transponders (PIT) in Clear Creek (a tributary to the Muddy River in the upper Lewis River basin); other data were from hatchery-reared coho salmon juveniles that were released in 2013. To date, only 5.8 percent of the wild coho salmon 
A. Review of Information Relevant to Anadromous Fish Reintroduction to and Fish Passage in the Lewis River, Wash.

tagged in Clear Creek and 15.2 percent of the coho salmon marked in screw trap operations near the head of Swift Reservoir have been collected at the FSC. The differences in collection for coho salmon likely stem from the timing of marking, as the Clear Creek estimate includes overwinter mortality. Although data are limited, the percent of steelhead tagged at Eagle Cliff screw trap operations (on the Lewis River, immediately above the head of Swift Reservoir; see fig. D1 in chap. D of this report) and collected at the FSC is slightly higher (19 percent) than observed for coho salmon. For spring Chinook salmon, hatcheryreared smolts released into tributary streams that were PIT-tagged were included, as well as the total number of individuals released (that is, unmarked; see "Assessment of Juvenile Fish Production and Outmigration Success on the Lewis River, Washington," chap. D of this report). The percent of spring Chinook salmon collected at the FSC was extremely low for fish individually PIT-tagged (yearly average for 2013-2015 was 2.2 percent) and from large releases of unmarked plants (average is 3.7 percent). Overall, the low collection rates for spring Chinook salmon are generally consistent with results from Cowlitz Falls (fig. A2; Serl and Morrill, 2010; Serl and Heimbigner, 2013). The discrepancy between the estimates of collection at the Lewis River FSC and Cowlitz Falls may be because of natural recruitment of juvenile spring Chinook salmon from residualized individuals from previous releases in the Lewis River (for example, Romer and Monzyk, 2014; Perales and others, 2015), especially as any effects of PIT-tagging large, acclimation smolts were likely minimal (Prentice and others, 1993), and high estimates of reservoir survival are common (for example, Williams and others, 2001).

Recent behavioral studies in Swift Reservoir (Beeman and others, 2016) suggest the proportion of juvenile salmon collected is likely to be increased through modifications at the FSC. In particular, results indicate that a large proportion of test-marked juvenile salmon (90 percent) move within 150 meters of the FSC. Estimates of collection efficiency (that is, the proportion of fish within the zone of influence collected at the FSC) in a 2014-2015 study (PacifiCorp and Cowlitz PUD, 2016) were relatively low (12-29 percent for coho salmon, 0 percent for spring Chinook salmon, and 16-25 percent for steelhead) but may increase substantially with different orientations of the guide nets. Ultimately, adaptively modifying efforts to capture downstream migrating salmon and steelhead will be necessary to increase collection efficiencies and consequently, the overall numbers of adult returns (for example, as documented at Cowlitz Falls; Serl and Morrill, 2010).

\section{Juvenile Survival Through Collection Facilities}

Ample data exist to estimate survival rates for fish that go through hydropower facilities (see, for example, the Columbia Basin Fishery Agencies and Tribes Fish Passage Center webpage at http://www.fpc.org). However, this report is constrained to estimates of survival through collection facilities where complicating factors (for example, mortality during reservoir travel, large migration distances, and so on) are minimized. For all species, survival rates are generally high at collection facilities (mean range from 0.89 to 1; table A2). A comparison of the different types of collectors shows that survival estimates were lowest at the Bonneville Dam using the Bonneville FSC (0.86) and sampling from a barge (0.89), but mean survival at all other facilities was relatively high ( $>0.92$; table A3). Survival estimates at forebay collectors (that is, where trap and haul activities are used on the Baker and Cowlitz Rivers) suggest mortality rates are extremely low using those collectors.

Injury rates, measured through descaling rates ( $>20$ percent) also appear to be extremely low at collection facilities in the Pacific Northwest (table A4). Based on data in table A1.4, the average descaling rate is 2.7 percent $(\mathrm{SD}=3.0)$ for all species and facilities. No apparent patterns exist in average descaling rates of 2.3 percent for Chinook salmon, 1.8 percent for coho salmon, 3.6 percent for steelhead, and 0.7 percent for $O$. nerka [sockeye salmon]). Similar to survival rates, descaling rates from forebay collectors used in trap and haul operations comparable to the Upper Lewis (that is, the FSC at Swift Reservoir) were all less than $(<) 1$ percent.

Juvenile survival and injury rates at the FSC in the Lewis River (Swift Reservoir) have been generally consistent with rates at other structures in the Pacific Northwest. Injury rates in 2014-2015 were low for all species (0-2.4 percent), with highest injury rates for hatchery-reared spring Chinook smolts (2.4 percent; table A5; PacifiCorp and Cowlitz PUD, 2016). For the 1.1 percent of fish experiencing injuries at the FSC, descaling of $>20$ percent has been the most common injury to date (table A6). Survival rates were generally highest ( $>98.3$ percent) for all smolts (Chinook salmon, $O$. clarki [coastal cutthroat trout], coho salmon, steelhead; table A4). The lowest survival rates occurred for adult steelhead (average of 85.0 percent; small sample size of 5) and adult coho salmon (average of 88.3 percent; table A5).

In addition to high survival rates through collection facilities, reduced survival through delayed mortality effects are possible. To address this concern, trap and haul facilities on the Baker and Cowlitz Rivers are currently using stress-relief ponds for smolts transported downstream of hydropower facilities. A long-term study (1998-2009) of delayed mortality by Serl and Morrill (2010) has found mortality rates to be relatively low. For example, average delayed mortality rates were $<1$ percent for hatchery-reared and wild steelhead, coho salmon, hatchery Chinook salmon, and coastal cutthroat trout. Mortality rates did vary between species as delayed mortality rates for wild Chinook salmon were as high as 3.58 percent. Recent tests of delayed mortality in the Cowlitz River (2008-2012) found $<0.5$ percent mortality for all species (Serl and Heimbigner, 2013). The short duration of stress relief used in the Cowlitz River (24-48 hours after which fish were forced out) may underestimate the effects of stress (that is, additive stress) that may not be apparent until later in downstream migration (Budy and others, 2002; Schaller 
and Petrosky, 2007). Although overall mortality of fish that go through collection facilities and stress relief ponds is likely to be lower than fish that go through turbines (Keefer and others, 2012), further research and comparative studies are needed to determine site-specific mortality rates and the benefits of management actions (for example, stress relief ponds). Future studies of the effects of acclimation ponds should also consider using controls for direct comparisons of survival, as opposed to mortality assessments with just treatment groups (that is, where only acclimation ponds are used).

Effects of downstream collection facilities on other species.-In addition to salmon and steelhead smolts, collection facilities can collect potamodromous and other anadromous species (for example, coastal cutthroat trout), which raises concern for the effects of collection on these nontarget species. Numerous native and nonnative species, including game and nongame species, are often collected at downstream collection facilities (Portland General Electric Company [PGE], 2012; 2013) as a result of either random movements or natural, life-history movement patterns. Where evaluated, mortality estimates for nontarget species appear to be generally low for most species ( $<2$ percent; table A7). However, higher mortality estimates were observed for O. nerka (kokanee salmon) with a mean of 6.5 percent (table A1.5). In general, mortality rates for nontarget species at collection facilities are expected to be considerably lower than for species that pass through turbines and or spillways (for example, Muir and others, 2001).

Injury and mortality rates at the Swift Reservoir FSC for nontarget species appear to be low (table A4). Coastal cutthroat trout fry had the lowest survival rate (52.9 percent) at the Swift Reservoir FSC, but this estimate is derived from a small sample size of 8. However, adult mortality rates for species such as Salvelinus confluentus (bull trout) (10 percent in 2015), which have relatively small population sizes, should be monitored to identify ways to minimize the potential negative population-level effects.

\section{Adult Fish Upstream Collection Facilities}

The review of adult fish upstream collection facilities largely focuses on programs that use trapping and hauling methods of adults upstream of passage barriers. The use of trap and haul methods to increase the distribution of and create additional source populations for anadromous stocks has occurred for decades (for example, in the Baker and Cowlitz Rivers), and the use of this technique is currently increasing throughout the historic range of anadromous species (Vogel, 2007; Keefer and others, 2010). The report includes adult injury and survival rates, fallback rates, and upstream mortality rates for nontarget, native salmonids. Limited data currently exist for upstream capture efficiency in trap and haul projects, so they are not discussed further.

Adult injury and survival rates.-The collection facilities used for most trap and haul and long-term trapping facilities involves fish ladder systems where adults ascend fish ladders to separators, collectors, and so on (Zimmerman and Duke, 1993; Henning, 2010; PGE, 2013). For all species, runs (that is, life histories) and source (for example, hatchery, wild) mortalities from adult trapping and transport activities appear to be low (table A8). The majority of mortality events appear to occur during the trapping as opposed to during hauling events (Zimmerman and Duke, 1997). However, injury during hauling, as measured by descaling rates, can be substantial (Scully and Buettner, 1986). In addition, delayed mortality because of stress and (or) ambient conditions that result in prespawn mortality is rarely recorded.

The highest mortality rates from reports included herein were found for spring Chinook salmon (9.6 percent in 2000 and 6.8 percent in 1996) in the Tucannon River in Washington, and steelhead at the Mayfield facility on the Cowlitz River (6.7 percent; table A1.6). Aside from these high mortality events, average adult mortality for different species of salmon and steelhead at trapping locations was 0.4 percent; $(\mathrm{SD}=0.92$ percent; table A1.6). Although mortality associated with trapping is generally low, the occurrence of anomalous events affecting mortality, including density-independent factors (for example, ambient climate conditions) and density-dependent factors (for example, crowding; U.S. Army Corps of Engineers, 2015), can result in high mortality rates.

Stress and delayed mortality from instream conditions during freshwater migration are additional possible causes of upstream mortality for anadromous species. Exposure to warm water during migration and (or) during delayed migration at tailrace areas can affect migration timing, thus indirectly exposing fish to additional stress mechanisms and reduced fitness (Keefer and Caudill, 2010). For example, excessive warming has resulted in abnormally high prespawn mortality for spring Chinook salmon (Keefer and others, 2010; Mosser and others, 2013). Caudill and others (2007) found excessive delay times associated with high mortality of adult Chinook salmon and steelhead during passage at hydropower facilities. Although the exact mechanisms contributing to the high mortality rate are not known, the results suggest the need for monitoring and evaluation of delayed migration below adult traps to determine if management alternatives are needed to reduce mortality.

An additional concern in upstream trap and haul operations is thermal shock. For bottom-releasing reservoirs with relatively cold temperatures in reaches downstream of dams, transport and release into warm epilimnetic waters of reservoirs may result in thermal shock and lead to decreased fitness (Hovda and Linley, 2000). The differences in thermal regimes between tailrace and release locations should be monitored, and where needed, release methods that avoid drastic thermal differences during the warm, stratified periods of summer (for example, release mechanisms to lower depths; PGE, 2013) should be considered. 
Adult trapping studies below the dam at Lake Merwin (PacifiCorp and Cowlitz PUD, 2016) indicate that the success of adult trapping operations is generally consistent with previous studies. Operations at the Lake Merwin upstream adult trap have resulted in low injury rates (0-2.2 percent) (PacifiCorp and Cowlitz PUD, 2016). Results from this 2016 study also indicate the amount of time spent within the tailrace zone varied considerably for the different species and suggest residency time may be excessive for Chinook salmon and adult steelhead. Overall, collection efficiencies of adults were generally low (9-62 percent; (PacifiCorp and Cowlitz PUD, 2016), but the higher proportion of fish at the trap entrance (22-90 percent) suggests changes in trap designs and operations may improve collection efficiency. However, such data are preliminary and may be driven by the anomalous climatic conditions observed during the 2015 study (PacifiCorp and Cowlitz PUD, 2016), and continued studies and modifications are needed to improve collection efficiencies.

Fallback.-Fallback of adults during migration or transport above hydropower facilities can lead to increased stress, injury, and mortality, and considerably delayed migration times (Boggs and others, 2004). Fallback typically occurs when adults overshoot the natal spawning grounds or become disoriented (Naughton and others, 2006). How ambient conditions may contribute to fallback is poorly understood, but they likely influence fallback rates in any given year. For example, Holbrook and others (2009) found overall fallback rates for Salmo salar (Atlantic salmon) to range from 0.8 to 9.4 percent; however, fallback rates increased to more than 47 percent during periods of excessive stream warming $\left(>22^{\circ} \mathrm{C}\right)$. Understanding how conditions such as stream temperature and streamflow interact with management operations to reduce fallback warrants additional research.

Within the Pacific Northwest, we found moderate variability in fallback rates when comparing species and years (table A9; see table A1.7 for individual estimates). Overall, we found the highest fallback rates for Chinook salmon, with the highest estimates for spring Chinook salmon (mean=24.1 percent, $\mathrm{SD}=12.9$ percent). The fallback estimates were markedly lower for fall Chinook salmon (mean=4.9 percent, $\mathrm{SD}=4.0$ ) and spring-summer Chinook salmon (mean=12.4 percent, $\mathrm{SD}=8.0$ ). Albeit limited in number of estimates (sample size of 5), fallback rates for coho salmon (mean=5.0, $\mathrm{SD}=6.7$ ) were similar to those observed for fall Chinook salmon but with higher variability. Fallback estimates for steelhead (mean=10.0 percent, $\mathrm{SD}=7.8$ ) were similar to those for spring and spring-summer Chinook salmon; however, in many instances, fallback estimates include kelts and thus may be biased high (Keefer and Caudill, 2010). Estimates for sockeye salmon (mean=5.9 percent, $\mathrm{SD}=4.1$ ) were relatively low. The observed patterns indicate relatively lower fallback rates for fall spawners than for fish observed in spring and spring/summer species/runs.

Currently, few estimates of fallback are available for storage projects that utilize trap and haul methods, such as at Round Butte. Limited annual fallback rates are available from the Cowlitz facilities (Mayfield and Cowlitz Falls), and estimates are variable (table A1.7), thus rigorous comparisons between run-of-the-river projects and trap and haul are limited at this time. In Swift Reservoir, estimates of fallback are only available for adult coho salmon (hatchery) and data from 2014 (0.3 percent) and 2015 (0 percent) suggest attempted fallback (as adults are returned to the reservoir) is low. Low fallback rates are expected given that adults are released near the head of Swift Reservoir.

Upstream mortality of nontarget salmonids. - Estimates of mortality at upstream collection facilities for nontarget species do not appear to be common. Henning (2010) found numerous injuries to Esox masquinongy $\times$ lucius or Esox lucius $\times$ masquinongy (tiger muskellunge) but relatively low evidence of mortality. Studies with limited data for bull trout from the Pelton adult fish trap on the Deschutes River system have found no evidence of mortality (PGE, 2013), which is consistent with reports for bull trout migrating through hydropower facilities on the Columbia River (Chelan County PUD, 2012; Douglas County PUD, 2012). Studies specifically targeting handling effects for bull trout, however, have found mortality rates as high as 4 percent (PUD No. 1 of Douglas County, 2016). Little information currently exists on upstream passage effects on coastal cutthroat trout. Although survival estimates for anadromous salmon and steelhead are relatively low (table A5), additional data are needed to identify how upstream trap and haul operations may affect native salmonid survival rates.

Of particular importance for species that have narrow thermal tolerances, such as bull trout (Selong and others, 2001; McMahon and others, 2007), may be delayed migrations at upstream collection facilities and the potential for detrimental effects of thermal shock upon release into epilimnetic waters of stratified reservoirs. Temperature data from Swift Reservoir, where biweekly average temperatures during mid-July through mid-September exceeded $16{ }^{\circ} \mathrm{C}$ at depths to 9 meters (Sorel and others, 2016) suggest thermal regimes may be detrimental for species such as bull trout that are released in the epilimnion during these periods. Thermal regime monitoring may need to be integrated into the transport protocols, and alternative release strategies to avoid thermal regimes stressful to target species may need to be considered. 


\section{Conclusion}

Comparing the success of collection operations for juvenile and adult fish at different locations is challenging because of different collection designs, hydrologic conditions, and reservoir bathymetry. Juvenile and adult injuries and direct (that is, not delayed) mortalities appear to be generally low regardless of species and location, a pattern observed during the early stages of reintroduction in Swift Reservoir. Juvenile collection efficiencies appear to be highly variable between locations and species, likely because of the inherent differences at the various locations. In Swift Reservoir, the proportion of coho and Chinook salmon smolts collected at the downstream collector (that is, FSC) on Swift Reservoir appears to be relatively low compared to other collectors (table A1). Juvenile behavior near collection sites often drives such patterns (see the "Smolt collection efficiency" section in this chapter). Recent studies in Swift Reservoir suggest modifications to the netting placement are likely to substantially increase the proportion of juveniles collected, highlighting the need to continue to evaluate designs and operations that can yield substantial increases in collections of juveniles and the likely success of the reintroduction efforts.

\section{References}

AECOM, 2010, Willamette downstream fish passage design requirements study: Redmond, Wash., AECOM, Contract W9127N10-D-0002, T.O. 003.

Anderson, J.H., Pess, G.R., Carmichael, R.W., Ford, M.J., Cooney, T.D., Baldwin, C.M., and McClure, M.M., 2014, Planning Pacific salmon and steelhead reintroductions aimed at long-term viability and recovery: North American Journal of Fisheries Management, v. 34, no. 1, p. 72-93.

Appleby, A.E., Tipping, J.M., and Vander Haegen, G.E., 2002, Effects of surface water acclimitization on postrelease survival of yearling spring Chinook salmon: North American Journal of Aquaculture, v. 64, p. 301-304.

Beeman, J.W., Evans, R.E., Haner, P.V., Hansel, H.C., Hansen, A.C., Hansen, G.S., Hatton, T.W., Sprando, J.M., Smith, C.D., and Adams, N.S., 2016, Evalauation of the biological and hydraulic performance of the portable floating fish collector at Cougar Reservoir and Dam, Oregon, 2014: U.S. Geological Survey Open-File Report 2016-1003, 143 p., accessed November 8, 2018, at https://pubs.usgs.gov/of/2016/1003/ofr20161003.pdf.

Bjornn, T.C., Keefer, M.L., Peery, C.A., Tolotti, K.R., Ringe, R.R., and Stuehrenberg, L.C., 2000, Adult Chinook and sockeye salmon, and steelhead fallback rates at Bonneville Dam, 1996-1998: Moscow, Idaho, University of Idaho, project number MPE-P-95-1.

Boggs, C.T., Keefer, M.L., Peery, C.A., Bjornn, T.C., and Stuehrenberg, L.C., 2004, Fallback, reascension, and adjusted fishway escapement estimates for adult Chinook salmon and steelhead at Columbia and Snake River dams: Transactions of the American Fisheries Society, v. 133, no. 4, p. 932-949.

Budy, P., Thiede, G.P., Bouwes, N., Petrosky, C.E., and Schaller, H., 2002, Evidence linking delayed mortality of Snake River salmon to their earlier hydrosystem experience: North American Journal of Fisheries Management, v. 22, no. 1, p. 35-51.

Cameron, W.A., Clarke, L.R., Suchy, M.D., Carmichael, R.W., Onjukka, S.T., Keniry, J., and White, M.G., 2013, Umatilla hatchery monitoring and evaluation annual report, 1/1/2012-12/31/2012: Portland, Oreg., Bonneville Power Administration, project number 1990-005-00.

Caudill, C.C., Daigle, W.R., Keefer, M.L., Boggs, C.T., Jepson, M.A., Burke, B.J., Zabel, R.W., Bjornn, T.C., and Peery, C.A., 2007, Slow dam passage in adult Columbia River salmonids associated with unsuccessful migration-Delayed negative effects of passage obstacles or condition-dependent mortality?: Canadian Journal of Fisheries and Aquatic Sciences, v. 64, no. 7, p. 979-995.

Chelan County Public Utility District [PUD], 2012, Annual summary report on 2011 bull trout observations and incidental take at the Rocky Reach project: Wenatchee, Wash., Chelan County Public Utility District, FERC Project Number 2145.

Clarke, L.R., Flesher, M.W., Warren, S.M., and Carmichael, R.W., 2011, Survival and straying of hatchery steelhead following forced or volitional release: North American Journal of Fisheries Management, v. 31, no. 1, p. 116-123. 
Clarke, L.R., Flesher, M.W., Whitesel, T.A., Vonderohe, G.R., and Carmichael, R.W., 2010, Postrelease performance of acclimated and directly released hatchery summer steelhead into Oregon tributaries of the Snake River: North American Journal of Fisheries Management, v. 30, no. 5, p. 1098-1109.

Cleary, P., 2005, Evaluation of spring Chinook salmon (Oncorhynchus tshawytscha) supplementation in the Lostine River, Oregon-2005 annual report: Portland, Oreg., Bonneville Power Administration report DOE/BP-00004219-2, project number 199800702.

Cleary, P., Harbeck, J., and Bright, D., 2006, Evaluation of spring Chinook salmon (Oncorhynchus tshawytscha) supplementation in the Lostine River, Oregon-1997-2004 progress report: Portland, Oreg., Bonneville Power Adminstration, project number 199800702.

Clemens, B.J., Clements, S.P., Karnowski, M.D., Jepsen, D.B., Gitelman, A.I., and Schreck, C.B., 2009, Effects of transportation and other factors on survival estimates of juvenile salmonids in the unimpounded lower Columbia River: Transactions of the American Fisheries Society, v. 138, no. 1, p. 169-188.

Cram, J.M., Torgersen, C.E., Klett, R.S., Pess, G.R., May, D., Pearsons, T.N., and Dittman, A.H., 2013, Tradeoffs between homing and habitat quality for spawning site selection by hatchery-origin Chinook salmon: Environmental Biology of Fishes, v. 96, no. 1, p. 109-122.

Croze, O., 2008, Assessment of downstream fish bypasses for Atlantic salmon smolts at four hydroelectric facilities on the Ariege River (France): American Fisheries Society Symposium, v. 61, p. 123-140.

Dittman, A.H., May, D., Larsen, D.A., Moser, M.L., Johnston, M., and Fast, D., 2010, Homing and spawning site selection by supplemented hatchery- and natural-origin Yakima River spring Chinook salmon: Transactions of the American Fisheries Society, v. 139, no. 4, p. 1014-1028.

Douglas County Public Utility District [PUD], 2012, Bull trout monitoring and management plan, 2011 annual report: Wenatchee, Wash., Douglas County Public Utiltiy District, FERC Project Number 2149.

Fast, D., Hubble, J., Kohn, M., and Watson, B., 1991, Yakima River spring Chinook enhancement study: Portland, Oreg., Bonneville Power Administration, contract number DE-A179-83BP39461, project number 82-16.

Fish Passage Center [FPC], 2001, Fish Passage Center 2000 annual report: Portland, Oreg., Fish Passage Center, Bonneville Power Administration contract number 94-033.

Fish Passage Center [FPC], 2002, Fish Passage Center 2001 annual report: Portland, Oreg., Fish Passage Center, Bonneville Power Administration contract number 94-033.

Fish Passage Center [FPC], 2003, Fish Passage Center 2002 annual report: Portland, Oreg., Fish Passage Center, Bonneville Power Administration contract number 94-033.

Fish Passage Center [FPC], 2004, Fish Passage Center 2003 annual report: Portland, Oreg., Fish Passage Center, Bonneville Power Administration contract number 94-033.

Fish Passage Center [FPC], 2006, Fish Passage Center 2005 annual report: Portland, Oreg., Fish Passage Center, Bonneville Power Administration contract number 21186, project number 1994-033-00.

Fish Passage Center [FPC], 2007, Fish Passage Center 2007 annual report: Portland, Oreg., Fish Passage Center, Bonneville Power Administration contract number 35850, project number 1994-033-00.

Fish Passage Center [FPC], 2008, Fish Passage Center annual report: Portland, Oreg., Fish Passage Center, Bonneville Power Administration contract number 40288, project number 1994-033-00.

Fish Passage Center [FPC], 2009, Fish Passage Center 2008 annual report: Portland, Oreg., Fish Passage Center, Bonneville Power Administration contract number 40288, project number 1994-033-00.

Fish Passage Center [FPC], 2010, Fish Passage Center 2009 annual report: Portland, Oreg., Fish Passage Center, Bonneville Power Administration contract number 45525, project number 1994-033-00.

Fish Passage Center [FPC], 2011, Fish Passage Center 2010 annual report: Portland, Oreg., Fish Passage Center, Bonneville Power Administration contract number 50744, project number 1994-033-00. 
Gale, W.L., Pasley, C.R., Kennedy, B.M., and Ostrand, K.G., 2009, Juvenile steelhead release strategies-A comparison of volitional- and forced-release practices: North American Journal of Aquaculture, v. 71, no. 2, p. 97-106.

Gallinat, M.P., and Ross, L.A., 2012, Tucannon River spring Chinook salmon hatchery evaluation program: Olympia, Wash., Washington Department of Fish and Wildlife, Cooperative Agreement 14110-B-J012.

Gerstenberger, R., 2009, Hood River production program monitoring and evaluation (M\&E) - Confederated Tribes of Warm Springs: Parkdale, Oreg., Confederated Tribes of Warms Springs Reservation, project number 1988-053-03, contract number 00034865 .

Hanson, B.N., 1999, Effectiveness of two different surface bypass facilities on the Connecticut River to pass emigrating Atlantic salmon (Salmo salar) juvenile salmonids, in Odeh, M., ed., Innovations in fish passage technology: Bethesda, Md., American Fisheries Society, $212 \mathrm{p}$.

Hausch, S.J., and Melnychuk, M.C., 2012, Residualization of hatchery steelhead-A meta-analysis of hatchery practices: North American Journal of Fisheries Management, v. 32, no. 5, p. 905-921.

Heisey, P.G., Mathur, D., Fulmer, J.L., Adams, S.W., and Brush, T.D., 2002, Final report—Estimation of juvenile salmonid spillway passage survival at North Fork Dam: Prepared for Portland General Electric, Portland, Oreg., and Clackamas River Project Fisheries and Aquatics Workgroup.

Henning, J.A., 2010, Cowlitz River evaluation program annual report for 2009: Olympia, Wash., Washington Department of Fish and Wildlife, FPA 10-02.

Hill, M., and Quesada, C., 2011, 2010 annual test and verification report—Juvenile migration studies: Portland, Oreg., Portland General Electric Company.

Hill, M., and Quesada, C., 2012, 2011 juvenile migration test and verification study annual report: Portland, Oreg., Portland General Electric Company.

Hill, M., and Quesada, C., 2013, Test and verification study_Juvenile migration 2012 annual report: Portland, Oreg., Portland General Electric Company.

Hill, M., and Quesada, C., 2014, 2013 juvenile migration test and verification study annual report: Portland, Oreg., Portland General Electric Company.

Hill, M., and Quesada, C., 2015, 2014 juvenile migration test and verification study: Portland, Oreg., Portland General Electric Company.

Holbrook, C.M., Zydlewski, J., Gorsky, D., Shepard, S.L., and Kinnison, M.T., 2009, Movements of prespawn adult Atlantic salmon near hydroelectric dams in the lower Penobscot River, Maine: North American Journal of Fisheries Management, v. 29 , no. 2 , p. $495-505$.

Hovda, J., and Linley, T.J., 2000, The potential application of hypothermia for anesthesia in adult Pacific salmon: North American Journal of Aquaculture, v. 62, no. 1, p. 67-72.

Jeanes, E., and Verretto, N., 2012, Summary results of PIT-tag biological evaluations: Bellevue, Wash., Puget Sound Energy.

Karchesky, C.M., McDonald, R.D., Heisey, P.G., Adams, S.W., and Graver, S.N., 2008, Estimating survival and condition of juvenile salmonids after passage through the modified fish bypass system at the T.W. Sullivan Hydroelectric Project, Willamette River, OR: Prepared for Portland General Electric, Portland, Oreg.

Keefer, M.L., and Caudill, C.C., 2010, A review of adult salmon and steelhead life history and behavior in the Willamette River basin-Identification of knowledge gaps and research needs: Moscow, Idaho, University of Idaho, Technical Report 2010-8.

Keefer, M.L., and Caudill, C.C., 2012, A review of adult salmon and steelhead straying with an emphasis on Columbia River populations: Moscow, Idaho, University of Idaho, contract number W912EF-08-D-0007.

Keefer, M.L., Peery, C.A., Bjornn, T.C., Jepson, M.A., and Stuehrenberg, L.C., 2004, Hydrosystem, dam, and reservoir passage rates of adult Chinook salmon and steelhead in the Columbia and Snake Rivers: Transactions of the American Fisheries Society, v. 133, no. 6, p. 1413-1439. 
Keefer, M.L., Taylor, G.A., Garletts, D.F., Gauthier, G.A., Pierce, T.M., and Caudill, C.C., 2010, Prespawn mortality in adult spring Chinook salmon outplanted above barrier dams: Ecology of Freshwater Fish, v. 19, no. 3, p. 361-372.

Keefer, M.L., Taylor, G.A., Garletts, D.F., Helms, C.K., Gauthier, G.A., Pierce, T.M., and Caudill, C.C., 2012, Reservoir entrapment and dam passage mortality of juvenile Chinook salmon in the Middle Fork Willamette River: Ecology of Freshwater Fish, v. 21, no. 2, p. 222-234.

Kenaston, K.R., Lindsay, R.B., and Schroeder, R.K., 2001, Effect of acclimation on the homing and survival of hatchery winter steelhead: North American Journal of Fisheries Management, v. 21, no. 4, p. 765-773.

Kock, T.J., Beeman, J.W., Hansen, A.C., Hansel, H.C., Hansen, G.S., Hatton, T.W., Kofoot, E.E., Sholtis, M.D., and Sprando, J.M., 2015, Behavoir, passage, and downstream migration of juvenile Chinook salmon from Detroit Reservoir to Portland, Oregon, 2014-15: U.S. Geological Survey Open-File Report 2015-1220, 30 p., accessed November 19, 2018, at https://pubs.usgs.gov/of/2015/1220/ofr20151220.pdf.

Martinson, R., Kamps, J., Kovalchuk, G., and Ballinger, D., 2004, Monitoring of downstream salmon and steelhead at federal hydropower facilities: Portland, Oreg., Bonneville Power Administration, report DOE/BP-00003992-4.

Maule, A.G., Tripp, R.A., Kaattari, S.L., and Schreck, C.B., 1989, Stress alters immune function and disease resistance in Chinook salmon (Oncorhynchys tshawytscha): Journal of Endocrinology, v. 120, no. 1, p. 135-142.

McLean, M., Seeger, R., and Hewitt, L., 2003, Grande Ronde endemic spring Chinook salmon supplementation programFacility operations and maintenance: Portland, Oreg., Bonneville Power Administration, project number 199800703.

McLeod, B., 2007, Fall Chinook acclimation project: Lapwai, Idaho, Nez Perce Tribe, Project Number 1998-010-05-07, contract numbers 4235, 4186, 4297.

McLeod, B., 2008, Fall Chinook acclimation project: Lapwai, Idaho, Nez Perce Tribe, project number 1998-010-05, contract number 30385 .

McLeod, B., 2009, Fall Chinook acclimation project: Lapwai, Idaho, Nez Perce Tribe, project number 1998-010-05, contract number 36041 .

McMahon, T.E., Zale, A.V., Barrows, F.T., Selong, J.H., and Danehy, R.J., 2007, Temperature and competition between bull trout and brook trout - A test of the elevation refuge hypothesis: Transactions of the American Fisheries Society, v. 136, no. 5, p. 1313-1326.

McMichael, G.A., Skalski, J.R., and Deters, K.A., 2011, Survival of juvenile Chinook salmon during barge transport: North American Journal of Fisheries Management, v. 31, no. 6, p. 1187-1196.

Mosser, C.M., Thompson, L.C., and Strange, J.S., 2013, Survival of captured and relocated adult spring-run Chinook salmon Oncorhynchus tshawytscha in a Sacramento River tributary after cessation of migration: Environmental Biology of Fishes, v. 96 , no. 2-3, p. $405-417$.

Muir, W.D., Smith, S.G., Williams, J.G., and Sandford, B. P., 2001, Survival of juvenile salmonids passing through bypass systems, turbines, and spillways with and without flow deflectors at Snake River Dams: North American Journal of Fisheries Management, v. 21, p. 135-146.

Murdoch, K., Prevatte, S., and Kamphaus, C., 2005, Mid-Columbia reintroduction feasibility study—Monitoring and evaluation: Portland, Oreg., Bonneville Power Administration, project number 199604000.

Naughton, G.P., Caudill, C.C., Keefer, M.L., Bjornn, T.C., Peery, C.A., and Stuehrenberg, L.C., 2006, Fallback by adult sockeye salmon at Columbia River dams: North American Journal of Fisheries Management, v. 26, no. 2, p. 380-390.

Osborne, R.S., and Rhine, T.D., 1999, Steelhead volitional release experiment, Squaw Creek Pond, Idaho: Boise, Idaho, Idaho Department of Fish and Game, report number 00-18.

PacifiCorp and Cowlitz Public Utiltiy District [PUD], 2002, Summary of informtion available to assess potential aquatic species interactions in the Lewis River Basin: Portland, Oreg., PacifiCorp and Cowlitz Public Utitlity District, report AQU 16.

PacifiCorp and Cowlitz Public Utitlity District [PUD], 2011, Lewis River hydroelectric project—Lewis River acclimation pond project: Portland, Oreg., PacifiCorp and Cowlitz Public Utility District. 
PacifiCorp and Cowlitz Public Utility District [PUD], 2016, Lewis River fish passage program 2015 annual report: Portland, Oreg., PacifiCorp and Cowlitz Public Utility District.

Perales, K.M., Rowan, J., and Moyle, P.B., 2015, Evidence of landlocked Chinook salmon populations in California: North American Journal of Fisheries Management, v. 35, no. 6, p. 1101-1105.

Ploskey, G. R., Schilt, C.R., Kim, J., Escher, C.W., and Skalski, J.R., 2003, Hydroacoustic evaluation of fish passage through Bonneville Dam in 2002 - Annual report: Richland, Wash., U.S. Army Corps of Engineers, prepared under a Related Services Agreement with the U.S. Department of Energy, PNNL-14356.

Ploskey G.R., Weiland, M.A., Schilt, C.R., Johnson, P.N., Hanks, M.E., Patterson, D.S., Skalski, J.R., and Hedgepeth, J., 2005, Hydroacoustic evaluation of fish passage through Bonneville Dam in 2004: Richland, Wash., Pacific Northwest National Laboratory, PNNL-15249, prepared for the U. S. Army Corps of Engineers, Portland, Oregon.

Ploskey, G. R., Weiland, M.A., Zimmerman, S.A., Hughes, J.S., Bouchard, K., Fischer, E.S., Schilt, C.R., Hanks, M.E., Kim, J., Skalski, J.R., Hedgepeth, J., and Nagy, W.T., 2006, Hydroacoustic evaluation of fish passage through Bonneville Dam in 2005: Richland, Wash., Pacific Northwest Laboratory, PNNL-15994, prepared for the U.S. Army Corps of Engineers, Portland, Oregon.

Portland General Electric Company [PGE], 2011, Pelton Round Butte project (FERC 2030) 2010 fish passage annual report: Portland, Oreg., Portland General Electric Company and The Confederated Tribes of the Warm Springs Reservation of Oregon.

Portland General Electric Company [PGE], 2012, Pelton Round Butte Project (FERC 2030) 2011 fish passage annual report: Portland, Oreg., Portland General Electric Company and The Confederated Tribes of the Warm Springs Reservation of Oregon.

Portland General Electric Company [PGE], 2013, Pelton Round Butte project (FERC 2030) 2012 fish passage annual report: Portland, Oreg., Portland General Electric Company and Confederated Tribes of the Warms Springs Reservation.

Portland General Electric Company [PGE], 2014, Pelton Round Butte Project (FERC 2030) 2013 fish passage annual report: Portland, Oreg., Portland General Electric Company.

Portland General Electric Company [PGE], 2015, Pelton Round Butte Project (FERC 2030) fish passage annual report: Portland, Oreg., Portland General Electric Company.

Prentice, E., Waknitz, F., Maynard, D., Sparks-McConkey, P., McCutcheon, C., Steffens, W., Jensen, A., Stuehrenberg, L.C., Downing, S., Sandford, B.P., Newcomb, T., and Neff, D., 1993, Study to determine the biological feasibility of a new fish tagging system-Annual report 1989: Portland, Oreg., Bonneville Power Administration, report DOE/BP-11982-4, project number 1983-31900.

Public Utility District No. 1 of Douglas County, 2016, Bull trout passage and take monitoring at Wells Dam and Twisp River weir-Final study plan, Wells Hydroelectric Project (FERC number 2149): East Wenatchee, Wash., Public Utility District No. 1 of Douglas County, 47 p.

Puget Sound Energy [PSE], 2012, Downstream fish passage 2011 annual report: Puget Sound Energy, Baker River Hydroelectric Project FERC No. 2150.

Puget Sound Energy [PSE], 2015, Downstream fish passage 2014 annual report: Puget Sound Energy, Baker River Hydroelectric Project FERC No. 2150.

Reagan, R. E., Evans, S.D., Wright, L.S., Farley, M.J., Adams, N.S., and Rondorf, D.W., 2005, Passage behavior of radio-tagged yearling Chinook salmon and steelhead at Bonneville Dam, 2004: Cook, Wash., U.S. Geological Survey, Columbia River Research Laboratory, contract number W66QKZ40238289, submitted to U.S. Army Corps of Engineers, 69 p.

Romer, J.D., and Monzyk, F.R., 2014, Adfluvial life history in spring Chinook salmon from Quartzville Creek, Oregon: North American Journal of Fisheries Management, v. 34, no. 5, p. 885-891.

Rosenberger, S.J., Connor, W.P., Peery, C.A., Milks, D.J., Schuck, M.L., Hesse, J.A., and Smith, S.G., 2013, Acclimation enhances postrelease performance of hatchery fall Chinook salmon subyearlings while reducing the potential for interaction with natural fish: North American Journal of Fisheries Management, v. 33, no. 3, p. 519-528. 
Rowan, G. D., 1994, Confederated Tribes of the Umatilla Indian Reservation, Minthorn Springs Creek summer juvenile release and adult collection facility-Annual report 1993: Portland, Oreg., U.S. Department of Energy, Bonneville Power Administration, Division of Fish \& Wildlife, contract number 1984BP17622, project number 1998343500, $136 \mathrm{p}$.

Savitz, J., Bardygula, L.G., and Funk, G., 1993, Returns of cage-released and non-cage-released Chinook and coho salmon to Illinois harbors of Lake Michigan: North American Journal of Fisheries Management, v. 13, no. 3, p. 550-557.

Schaller, H.A., and Petrosky, C.E., 2007, Assessing hydrosystem influence on delayed mortality of Snake River stream-type Chinook salmon: North American Journal of Fisheries Management, v. 27, no. 3, p. 810-824.

Schreck, C.B., Solazzi, M.F., Johnson, S.L., and Nickelson, T.E., 1989, Transportation stress affects performance of coho salmon (Oncorhynchus kisutch): Aquaculture, v. 82, no. 1-4, p. 15-20.

Schroeder, R., Wade, M., Firman, J., Buckman, M., Cannon, B., Hogansen, M., Kenaston, K., and Krentz, L., 2006, Compliance with the biological opinion for hatchery programs in the Willamette Basin: Oregon Department of Fish and Wildlife, NWP-OP-FH-02-01.

Schroeder, R.K., and Kenaston, K.R., 2005, Spring Chinook salmon in the Willamette and Sandy Rivers: Salem, Oreg., Oregon Department of Fish and Wildlife, project number F-163-R-09.

Schroeder, R.K., Kenaston, K., and McLaughlin, L.K., 2007, Spring Chinook salmon in the Willamette and Sandy Rivers: Salem, Oreg., Oregon Department of Fish and Wildlife, project number F-163-R-11/12.

Scully, R.J., and Buettner, E., 1986, Smolt condition and timing of arrival at Lower Granite Reservoir: Boise, Idaho, Idaho Department of Fish and Game, project number 83-323B.

Selong, J.H., McMahon, T.E., Zale, A.V., and Barrows, F.T., 2001, Effect of temperature on growth and survival of bull trout, with application of an improved method for determining thermal tolerance in fishes: Transactions of the American Fisheries Society, v. 130, no. 6, p. 1026-1037.

Serl, J., and Heimbigner, D., 2013, Data summary for the 2012 operation of Cowlitz Falls fish facility and related activities: Olympia, Wash., Washington Depaterment of Fish and Wildlife.

Serl, J., and Morrill, C., 2010, Summary report for the 1996 to 2009 seasonal operation of the Cowlitz Falls fish facility and related Cowlitz Falls anadromous reintroduction program activities: Olympia, Wash., Washington Department of Fish and Wildlife.

Skalski, J. R., Johnson, G.E., Sullivan, C.M., Kudera, E., and Erho, M.W., 1996, Statistical evaluation of turbine bypass efficiency at Wells Dam on the Columiba River, Washington: Canadian Journal of Fisheries and Aquatic Sciences, v. 53, p. 2188-2198.

Sorel, M.H., Hansen, A.G., Connelly, K.A., and Beauchamp, D.A., 2016, Trophic feasibility of reintroducing anadromous salmonids in three reservoirs on the North Fork Lewis River, Washington-Prey supply and consumption demand of resident fishes: Transactions of the American Fisheries Society, v. 145, p. 1331-1347.

Steig, T. W., Skalksi, J.R., and Ransom, B.H., 2003, Comparison of acoustic and PIT tagged juvenile Chinook, steelhead and sockey salmon (Oncorhynchus, spp.) passing dams on the Columbia River, USA in Aquatic telemetry, advances and applications-Proceedings of the Fifth Conference on Fish Telemetry held in Europe, 2003: Ustica, Italy, Food and Agriculture Organization of the United Nations, p. 275-286.

Timko, M. A., Sullivan, L.S., Rizor, S.E., O’Connor, R.R., Wright, C.D., Hannity, J.L., Fitzgerald, C.A., Meagher, M.L., Stephenson J.D., Skalski, J.R., and Townsend, R.L., 2011, Behavior and survival analysis of juvenile steelhead and sockeye through the Priest Rapids Project in 2010: Ellensburg, Wash., Public Utility District No. 2 of Grant County, Washington, prepared by Blue Leaf Environmental, Inc., 116 p.

Timko, M.A., Sullivan, L.S., Wright, C.D., Rizor, S.E., O’Connor, R.R., Fitzgerald, C.A., Meagher, M.L., Kukes, T., and Stephenson, J.D., 2010, Survival and behavior analysis of steelhead and sockeye through the Priest Rapids Hydroelectric Project in 2009: Ellensburg, Wash., Blue Leaf Environmental to Public Utility District No. 2 of Grant Co., Ephrata, Washington.

Tipping, J.M., 1998, Return rates of transported steelhead smolts with and without a rest period before release: Progressive FishCulturist, v. 60, no. 4, p. 284-287. 
Travade, F., and Larinier, M., 2006, French experience in downstream migration devices in Free passage for aquatic fauna in rivers and other water bodies, Hannef, Germany, 2006, Proceedings: German Association for Water, Wastewater and Waste, p. 91-99.

U.S. Army Corps of Engineers [USACE], 2007, Surface bypass program comprehensive review report: Portland, Oreg., U.S. Army Corps of Engineers, contract number W9127N-06-D-0004, T.O. 012, prepared by ENSR Corporation, 494 p.

U.S. Army Corps of Engineers [USACE], 2015, Final environmental assessment for Mud Mountain Dam upstream fish passage, Pierce County, Washington: Seattle, Wash., U.S. Army Corps of Engineers, 143 p.

Viola, A.E., and Schuck, M.L., 1995, A method to reduce the abundance of residual hatchery steelhead in rivers: North American Journal of Fisheries Management, v. 15, p. 488-493.

Vogel, D.A., 2007, A feasibility investigation of reintroduction of anadromous salmonids above Crocker-Huffman Dam on the Merced River: Red Bluff, Calif., Natural Resource Scientists.

Westley, P.A.H., Quinn, T.P., and Dittman, A.H., 2013, Rates of straying by hatchery-produced Pacific salmon (Oncorhynchus spp.) and steelhead (Oncorhynchus mykiss) differ among species, life history types, and populations: Canadian Journal of Fisheries and Aquatic Sciences, v. 70, no. 5, p. 735-746.

Whitesel, T.A., Lofy, P.T., Carmichael, R.W., Messmer, R.T., Flesher, M.W., and Rondorf, D.W., 1994, A comparison of the performance of acclimated and direct stream released, hatchery-reared steelhead smolts in northeast Oregon, in MacKinlay, D.D., ed., High performance fish: Vancouver, Fish Physiology Association, p. 87-92.

Williams, J.G., Smith, S.G., and Muir, W.D., 2001, Survival estimates for downstream migrant yearling juvenile salmonids through the Snake and Columbia Rivers hydropower system, 1966-1980 and 1993-1999: North American Journal of Fisheries Management, v. 21, no. 2, p. 310-317.

Zimmerman, B., and Duke, B.B., 1993, Umatilla River basin trap and haul program: Portland, Oreg., Bonneville Power Administration, project number 88-022, contract number DE-Bi79-89BP98636.

Zimmerman, B., and Duke, B.B., 1995, Trapping and transportation of adult and juvenile salmon in the Lower Umatilla River in Northeast Oregon: Portland, Oreg., Bonneville Power Administration, contract number 1989BP98636, project number 198802200.

Zimmerman, B., and Duke, B.B., 1996, Trapping and transportation of adult and juvenile salmon in the Lower Umatilla River in Northeast Oregon, 1995-1996, Umatilla River basin trap and haul program: Portland, Oreg., Bonneville Power Administration, contract number 1989BP98636, project number 198802200.

Zimmerman, B., and Duke, B.B., 1997, Trapping and transportation of adult and juvenile salmon in the Lower Umatilla River in Northeast Oregon, 1997-1997: Portland, Oreg., Bonneville Power Administration, project number 88-022, contract number DE-BI79-89BP98636.

Zollman, R.L., Eschler, R., Sealey, S., Williams, J., and Johnson, B., 2009, Lostine River operations and maintenance-2007 smolt acclimation and adult return report: Enterprise, Oreg., Nez Perce Tribe, Bonneville Power Administration project number 1998-007-02, contract number 00004277.

Zymonas, N.D., and Hogansen, M.J., 2013, Adult Chinook salmon monitoring in the South Fork Mackenzie River relative to water temperature control and upstream passage facilities at Cougar Dam: Portland, Oreg., Oregon Department of Fish and Wildlife, task order W9127N-11-2-0002-0004. 


\section{Figures}

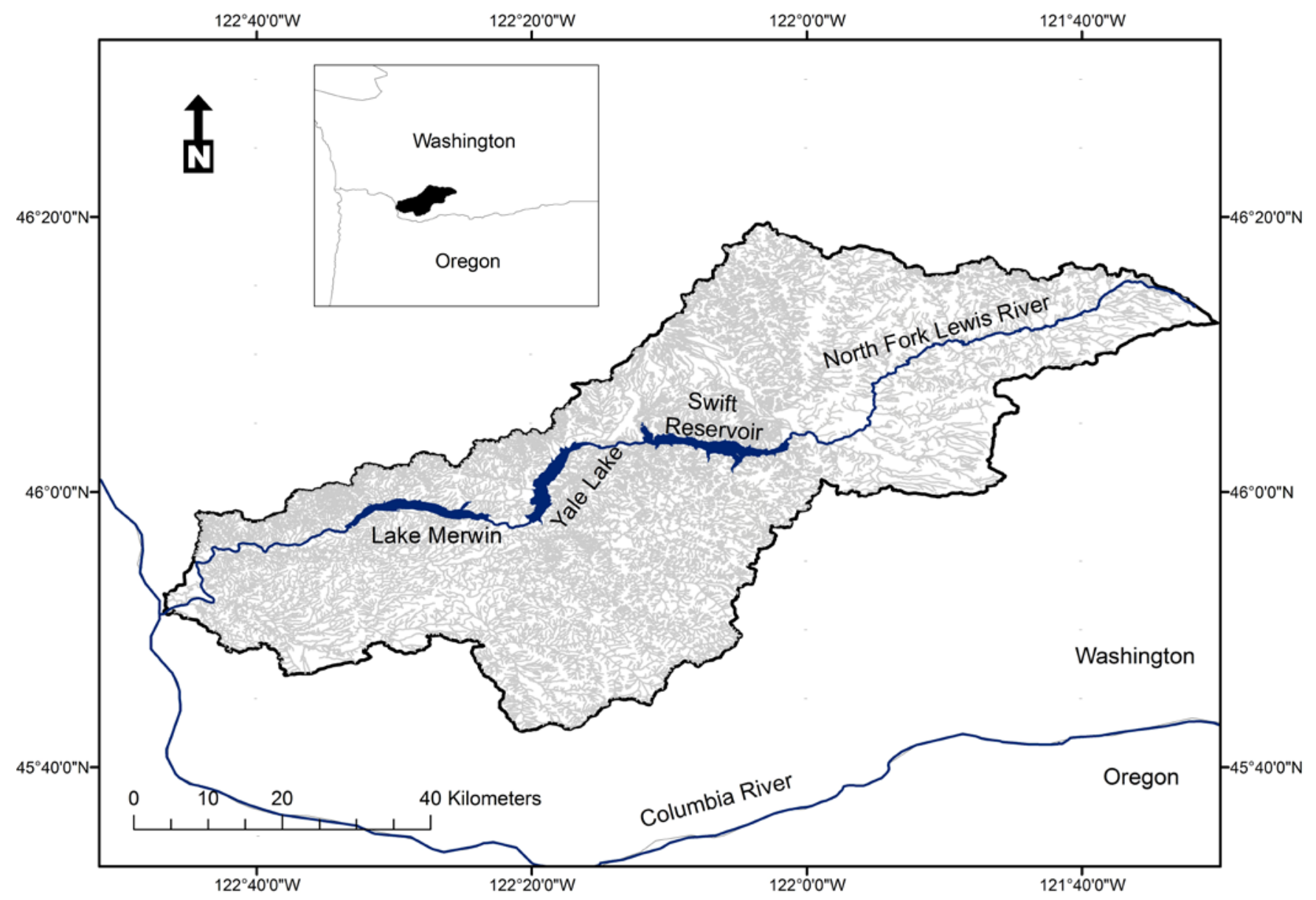

Figure A1. The North Fork of the Lewis River and the three hydropower impoundments: Lake Merwin, Yale Lake, and Swift Reservoir in the Lewis River Basin, Washington. 


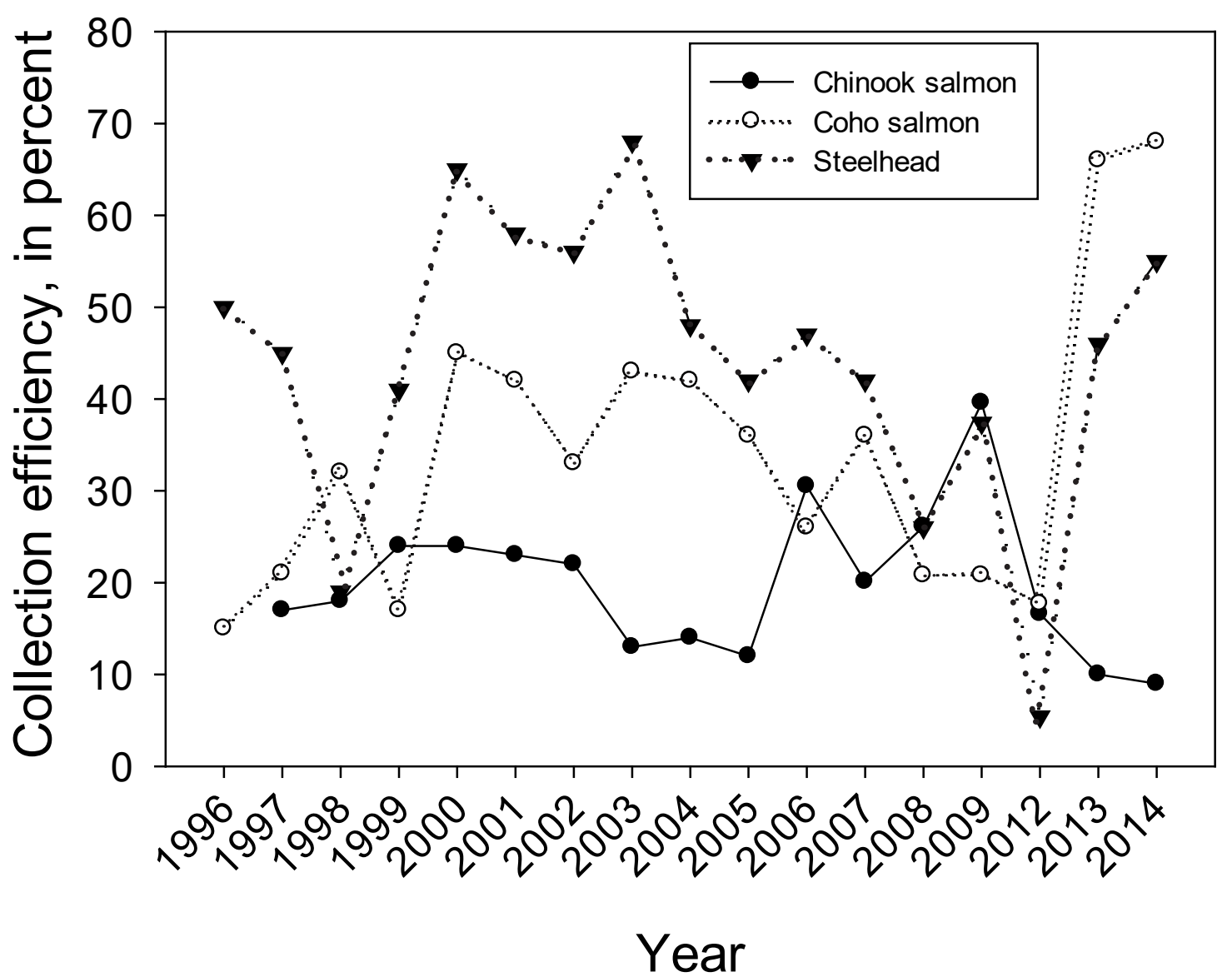

Figure A2. Interannual comparison of collection efficiencies for juvenile Chinook salmon, coho salmon, and steelhead at the Cowlitz Falls collection facility in Washington. [Data adapted from Serl and Heimbigner, 2013, and Serl and Morrill, 2010] 


\section{Tables}

Table A1. Smolt collection efficiencies and standard deviations by river, name of hydropower facility, collector type, species, and sample size.

[Estimates from individual studies, the years of the studies, and references for the data sources are available in table A1.2. U.S.A., United States of America; SS, Atlantic salmon; NA, not available; CO, coho salmon; OM, steelhead; $\mathrm{CH}$, Chinook salmon; B2CC, second powerhouse corner collector; >, greater than; $<$, less than]

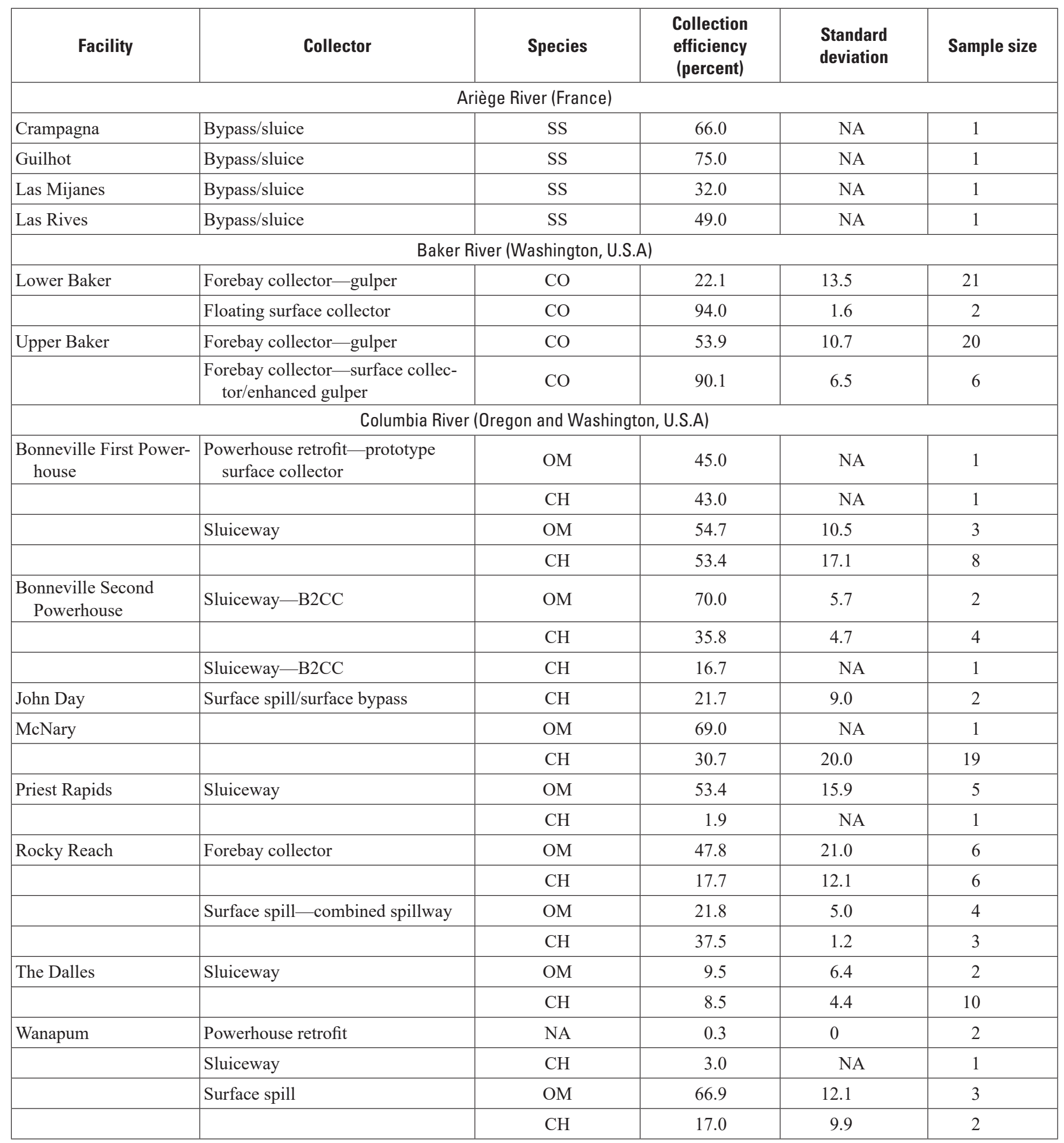


Table A1. Smolt collection efficiencies and standard deviations by river, name of hydropower facility, collector type, species, and sample size.-Continued

[Estimates from individual studies, the years of the studies, and references for the data sources are available in table A1.2. U.S.A., United States of America; SS, Atlantic salmon; NA, not available; $\mathrm{CO}$, coho salmon; OM, steelhead; $\mathrm{CH}$, Chinook salmon; B2CC, second powerhouse corner collector; >, greater than; $<$, less than]

\begin{tabular}{|c|c|c|c|c|c|}
\hline Facility & Collector & Species & $\begin{array}{l}\text { Collection } \\
\text { efficiency } \\
\text { (percent) }\end{array}$ & $\begin{array}{l}\text { Standard } \\
\text { deviation }\end{array}$ & Sample size \\
\hline Wells & $\begin{array}{l}\text { Powerhouse retrofit - surface } \\
\text { bypass units with retrofit baffle } \\
\text { bays }\end{array}$ & $\mathrm{CH} / \mathrm{OM}$ & 90.4 & 7.0 & 8 \\
\hline \multicolumn{6}{|c|}{ Connecticut River (New Hampshire, U.S.A.) } \\
\hline Vernon Station & & SS & 74.0 & NA & 1 \\
\hline \multicolumn{6}{|c|}{ Cowlitz River (Washington, U.S.A.) } \\
\hline \multirow[t]{2}{*}{ Cowlitz Falls } & Forebay collector-retrofit baffle & $\mathrm{CO}$ & 34.2 & 15.8 & 16 \\
\hline & & $\mathrm{OM}$ & 44.2 & 15.9 & 16 \\
\hline Mayfield & & $\mathrm{CH}$ & 75.0 & 1.4 & 2 \\
\hline \multicolumn{6}{|c|}{ Deschutes River (Oregon, U.S.A.) } \\
\hline \multirow[t]{2}{*}{ Petlon Round Butte } & Forebay collector & $\mathrm{CH}$ & 42.7 & 8.7 & 5 \\
\hline & & $\mathrm{OM}$ & 16.5 & 4.8 & 5 \\
\hline \multicolumn{6}{|c|}{ Garonne River (France) } \\
\hline Camon & Bypass/sluice & SS & 73.0 & NA & 1 \\
\hline \multicolumn{6}{|c|}{ Gave d' Aspe River (France) } \\
\hline Bedous & Bypass/sluice & SS & 55.0 & NA & 1 \\
\hline \multicolumn{6}{|c|}{ Lewis River (Washington, U.S.A.) } \\
\hline \multirow[t]{3}{*}{ Swift Reservoir } & Floating surface collector & $\mathrm{CO}$ & 11.8 & NA & 1 \\
\hline & & $\mathrm{CH}$ & 0 & NA & 1 \\
\hline & Floating surface collector & $\mathrm{OM}$ & 18.6 & NA & 1 \\
\hline \multicolumn{6}{|c|}{ Santiam River (Oregon, U.S.A.) } \\
\hline \multirow[t]{2}{*}{ Green Peter } & $\begin{array}{l}\text { Forebay collector-floating collec- } \\
\text { tion horn }\end{array}$ & $\mathrm{CH}$ & $>80$ & NA & 3 \\
\hline & $\begin{array}{l}\text { Forebay collector-floating collec- } \\
\text { tion horn }\end{array}$ & $\mathrm{OM}$ & $<57$ & NA & 4 \\
\hline \multicolumn{6}{|c|}{ Snake River (Washington, U.S.A.) } \\
\hline \multirow[t]{3}{*}{ Ice Harbor } & Sluiceway & NA & 31.8 & 15.2 & 4 \\
\hline & Surface spill—RSW & $\mathrm{OM}$ & 42.5 & 6.4 & 2 \\
\hline & & $\mathrm{CH}$ & 52.0 & 16.8 & 4 \\
\hline
\end{tabular}


Table A1. Smolt collection efficiencies and standard deviations by river, name of hydropower facility, collector type, species, and sample size.-Continued

[Estimates from individual studies, the years of the studies, and references for the data sources are available in table A1.2. U.S.A., United States of America; SS, Atlantic salmon; NA, not available; CO, coho salmon; OM, steelhead; $\mathrm{CH}$, Chinook salmon; B2CC, second powerhouse corner collector; >, greater than; $<$, less than]

\begin{tabular}{|c|c|c|c|c|c|}
\hline Facility & Collector & Species & $\begin{array}{l}\text { Collection } \\
\text { efficiency } \\
\text { (percent) }\end{array}$ & $\begin{array}{l}\text { Standard } \\
\text { deviation }\end{array}$ & Sample size \\
\hline \multirow[t]{2}{*}{ Little Goose } & & $\mathrm{OM}$ & 45.3 & 17.7 & 19 \\
\hline & & $\mathrm{CH}$ & 38.4 & 16.7 & 36 \\
\hline \multirow[t]{2}{*}{ Lower Granite } & $\begin{array}{l}\text { Powerhouse retrofit-surface } \\
\text { bypass collector }\end{array}$ & $\mathrm{OM}$ & 22.5 & 6.4 & 2 \\
\hline & $\begin{array}{l}\text { Surface spill—removable spillway } \\
\text { weir }\end{array}$ & $\mathrm{OM}$ & 46.6 & 20.4 & 36 \\
\hline All & & $\mathrm{CH}$ & 37.9 & 20.0 & 53 \\
\hline \multirow[t]{2}{*}{ Lower Monumental } & & $\mathrm{OM}$ & 33.4 & 17.6 & 19 \\
\hline & & $\mathrm{CH}$ & 26.1 & 17.0 & 36 \\
\hline Willamette Falls & & $\mathrm{CH}$ & 97.3 & NA & 3 \\
\hline
\end{tabular}

Table A2. Survival rates and standard deviations by river, smolt collection facility, species, and sample size.

[Estimates from individual studies, the years of the studies, and references for the data sources are available in table A1.3. CO, coho salmon; NA, not available; OM, steelhead; $\mathrm{CH}$, Chinook salmon; SO, sockeye salmon]

\begin{tabular}{|c|c|c|c|c|}
\hline Facility & Species & Survival (percent) & Standard deviation & Sample size \\
\hline \multicolumn{5}{|c|}{ Baker River (Washington) } \\
\hline Upper Baker & $\mathrm{CO}$ & 100 & 0 & 4 \\
\hline \multirow[t]{3}{*}{ North Fork } & $\mathrm{CO}$ & 100 & NA & 1 \\
\hline & $\mathrm{OM}$ & 96 & 6 & 5 \\
\hline & $\mathrm{CH} / \mathrm{CO}$ & 89 & 7 & 4 \\
\hline \multirow[t]{2}{*}{ River Mill } & $\mathrm{OM}$ & 99 & NA & 1 \\
\hline & $\mathrm{CH}$ & 99 & 1 & 2 \\
\hline \multicolumn{5}{|c|}{ Columbia River (Oregon and Washington) } \\
\hline \multirow[t]{3}{*}{ Cowlitz Falls } & $\mathrm{CO}$ & 99 & 2 & 13 \\
\hline & $\mathrm{OM}$ & 99 & 1 & 13 \\
\hline & $\mathrm{CH}$ & 98 & 3 & 22 \\
\hline \multirow[t]{2}{*}{ Mayfield } & $\mathrm{CO}$ & 95 & 0.1 & 11 \\
\hline & $\mathrm{OM}$ & 96 & 0.1 & 9 \\
\hline
\end{tabular}


Table A2. Survival rates and standard deviations by river, smolt collection facility, species, and sample size.-Continued

[Estimates from individual studies, the years of the studies, and references for the data sources are available in table A1.3. CO, coho salmon; NA, not available; OM, steelhead; $\mathrm{CH}$, Chinook salmon; SO, sockeye salmon]

\begin{tabular}{|c|c|c|c|c|}
\hline Facility & Species & Survival (percent) & Standard deviation & Sample size \\
\hline & $\mathrm{CH}$ & 95 & \multicolumn{3}{|c|}{ Deschutes River (Oregon) } \\
\hline \multicolumn{7}{|c|}{} & \multicolumn{1}{|c|}{ Willamette River (Oregon) } & 5 \\
\hline Pelton Round Butte & $\mathrm{OM}$ & 98 & 1 & 5 \\
\hline & $\mathrm{CH}$ & 98 & 1 & 4 \\
\hline & $\mathrm{SO}$ & 98 & 0 & 3 \\
\hline
\end{tabular}

Table A3. Average estimates of smolt survival and standard deviations by river, collection facility, collector type, and sample size.

[Estimates from individual studies, the years of the studies, and references for the data sources are available in table A1.3]

\begin{tabular}{|c|c|c|c|c|}
\hline Facility & Collector type & $\begin{array}{c}\text { Survival } \\
\text { (percent) }\end{array}$ & $\begin{array}{l}\text { Standard } \\
\text { deviation }\end{array}$ & Sample size \\
\hline \multicolumn{5}{|c|}{ Baker River (Washington) } \\
\hline \multicolumn{5}{|c|}{ Clackamas River (Oregon) } \\
\hline North Fork & Forebay collector & 92 & 7 & 8 \\
\hline River Mill & Surface spill—spillway weir & 99 & 1 & 3 \\
\hline \multicolumn{5}{|c|}{ Columbia River (Oregon and Washington) } \\
\hline \multirow[t]{2}{*}{ Bonneville } & Bonneville floating surface collector & 86 & 15 & 21 \\
\hline & Sampled from barge & 89 & 12 & 33 \\
\hline \multicolumn{5}{|c|}{ Cowlitz River (Washington) } \\
\hline \multicolumn{5}{|c|}{ Deschutes River (Oregon) } \\
\hline Pelton Round Butte & Guidance net/skimmer & 98 & 1 & 14 \\
\hline \multicolumn{5}{|c|}{ Willamette River (Oregon) } \\
\hline Willamette Falls & Mixed & 99 & 1 & 5 \\
\hline
\end{tabular}




\section{A. Review of Information Relevant to Anadromous Fish Reintroduction to and Fish Passage in the Lewis River, Wash.}

Table A4. Descaling rates and standard deviations by river, facility, species, and sample size.

[See table A1.4 for individual estimates, years, and references. PH, individual powerhouse at Bonneville Dam; CH, Chinook salmon; CO, coho salmon; OM, steelhead; NA, not available; SO, sockeye salmon]

\begin{tabular}{|c|c|c|c|c|}
\hline Facility & Species & $\begin{array}{c}\text { Descaling rate } \\
\text { (percent) }\end{array}$ & $\begin{array}{l}\text { Standard } \\
\text { deviation }\end{array}$ & $\begin{array}{l}\text { Sample } \\
\text { size }\end{array}$ \\
\hline \multicolumn{5}{|c|}{ Columbia River (Oregon and Washington) } \\
\hline Bonneville Dam PH1 & $\mathrm{CH}$ & 3.7 & 2.6 & 30 \\
\hline Bonneville Dam PH2 & $\mathrm{CO}$ & 3 & 1.6 & 15 \\
\hline Bonneville Dam PH3 & $\mathrm{OM}$ & 6.5 & 4 & 29 \\
\hline Bonneville Dam PH2 & $\mathrm{CH}$ & 1.6 & 1.1 & 8 \\
\hline Bonneville Dam PH3 & $\mathrm{CO}$ & 1.2 & 0.4 & 4 \\
\hline Bonneville Dam PH4 & $\mathrm{OM}$ & 4.1 & 2.2 & 8 \\
\hline John Day & $\mathrm{CH}$ & 2.6 & 2 & 12 \\
\hline John Day & $\mathrm{CO}$ & 3 & 1.5 & 6 \\
\hline John Day & $\mathrm{OM}$ & 4.4 & 2.6 & 12 \\
\hline \multicolumn{5}{|c|}{ Cowlitz River (Washington) } \\
\hline Cowlitz Falls & $\mathrm{CH}$ & 0.6 & 0.8 & 20 \\
\hline Cowlitz Falls & $\mathrm{CO}$ & 0.4 & 1 & 14 \\
\hline Cowlitz Falls & $\mathrm{OM}$ & 0.4 & 0.5 & 27 \\
\hline Mayfield & $\mathrm{CH}$ & 4.1 & NA & 1 \\
\hline \multicolumn{5}{|c|}{ Deschutes River (Oregon) } \\
\hline Fish transfer & $\mathrm{CH}$ & 0.1 & 0.1 & 3 \\
\hline Fish transfer & $\mathrm{OM}$ & 0.4 & 0.6 & 3 \\
\hline Fish transfer & $\mathrm{SO}$ & 0.7 & 0.4 & 3 \\
\hline
\end{tabular}


Table A5. The percent of injured fish and survival rates for different species at different life stages as measured at the floating surface collector in Swift Reservoir on the Upper Lewis River, Washington. Data from PacifiCorp and Cowlitz Public Utility District, 2016.

[NA, not available]

\begin{tabular}{|l|c|c|c|c|}
\hline \multirow{2}{*}{ Species } & \multicolumn{2}{c|}{ Injured (percent) } & \multicolumn{2}{c|}{ Survival (percent) } \\
\cline { 2 - 5 } & $\mathbf{2 0 1 4}$ & $\mathbf{2 0 1 5}$ & $\mathbf{2 0 1 4}$ & $\mathbf{2 0 1 5}$ \\
\hline Coho salmon (adult) & 0 & 0 & 88.0 & 88.6 \\
\hline Coho salmon (smolt) & 0.9 & 0.4 & 99.3 & 99.2 \\
\hline Coho salmon (fry) & 0 & 0 & 99.8 & 96.9 \\
\hline Coho salmon (parr) & NA & 0 & NA & 100 \\
\hline Chinook salmon (adult) & NA & NA & NA & NA \\
\hline Chinook salmon (smolt) & 2.4 & 2.1 & 98.3 & 97.2 \\
\hline Chinook salmon (parr) & NA & 0 & NA & 0 \\
\hline Steelhead (adult) & 0 & 0 & 82.4 & 87.5 \\
\hline Steelhead (smolt) & 2.2 & 0.6 & 100 & 99.4 \\
\hline Steelhead (fry) & 0 & 0 & 100 & 100 \\
\hline Steelhead (kelt) & 0 & 0 & 85.7 & 90.3 \\
\hline Steelhead (parr) & NA & 0 & NA & 100 \\
\hline Rainbow trout & 0 & 0 & 100 & 100 \\
\hline Bull trout & 0 & 0 & 100 & 90.0 \\
\hline Coastal cutthroat (smolt) & 0.8 & 0.2 & 100 & 99.2 \\
\hline Coastal cutthroat (fry) & 0 & 0 & 99.3 & 52.9 \\
\hline Average (percent) & 0.49 & 0.40 & 96.3 & 93.4 \\
\hline
\end{tabular}

Table A6. The type of injury by percent for all fish species for the 1.1 percent of fish injured during collection efforts at the floating surface collector in Swift Reservoir on the Upper Lewis River, Washington, in 2014 and 2015. Data from PacifiCorp and Cowlitz Public Utility District, 2016.

[>, greater than; \%, percent; cm, centimeter]

\begin{tabular}{|l|c|c|}
\hline \multirow{2}{*}{\multicolumn{1}{|c|}{ Type of injury }} & \multicolumn{2}{c|}{ Percent of injuries } \\
\cline { 2 - 3 } & $\mathbf{2 0 1 4}$ & $\mathbf{2 0 1 5}$ \\
\hline Descaling $>20 \%$ & 77.5 & 81.9 \\
\hline Hemorrhaging & 4.2 & 8.1 \\
\hline Bruising $>0.5$ cm diameter & 14.1 & 5.4 \\
\hline Open wound (no fungus) & 1.4 & 2.3 \\
\hline Gill damage & 0.7 & 0.5 \\
\hline Loss of equilibrium & 0.7 & 1.8 \\
\hline Fin damage (injury) & 1.4 & 0 \\
\hline
\end{tabular}


Table A7. Survival rates and standard deviations for nontarget species at downstream fish collection facilities by river, facility, species, and sample size.

[See table A1.5 for years and specific references. NA, not available]

\begin{tabular}{|l|l|c|c|c|}
\hline \multicolumn{1}{|c|}{ Facility } & \multicolumn{1}{|c|}{ Species } & Survival (percent) & Standard deviation & Sample size \\
\hline \multicolumn{5}{|c|}{ Cowlitz River (Washington) } \\
\hline Cowlitz Falls & Coastal cutthroat trout & 99.6 & 0.3 & 13 \\
\hline Mayfield & Coastal cutthroat trout & 99.9 & NA & 1 \\
\hline \multicolumn{5}{|c|}{ Deschutes River (Oregon) } \\
\hline Round Butte & Bull trout & 98.3 & 0.3 & 5 \\
\hline & Kokanee salmon & 93.5 & 1.5 & 5 \\
\hline & Mountain whitefish & 99.9 & 1.2 & 5 \\
\hline & Rainbow trout & 98.6 & 5 & 5 \\
\hline
\end{tabular}

Table A8. Average mortality rates and standard deviations during adult trap and haul operations for different species, runs (that is, spawning run type), and sources of salmon in the Pacific Northwest.

[See table A1.6 for individual estimates, years, and references. NA, not available]

\begin{tabular}{|c|c|c|c|c|}
\hline Run & Source & Mortality (percent) & Standard deviation & Sample size \\
\hline \multicolumn{5}{|c|}{ Chinook salmon } \\
\hline Fall & Mixed & 1.4 & 2.5 & 2 \\
\hline Fall & Wild & 0.6 & 0.7 & 3 \\
\hline Spring & Hatchery & 1.4 & 2.5 & 24 \\
\hline Spring & Mixed & 0.7 & NA & 1 \\
\hline Spring & Wild & 0.1 & 0.3 & 29 \\
\hline \multicolumn{5}{|c|}{ Coho salmon } \\
\hline NA & Hatchery/mixed & 0.3 & 0.2 & 2 \\
\hline NA & Wild & 0.2 & 0.3 & 4 \\
\hline \multicolumn{5}{|c|}{ Sockeye salmon } \\
\hline NA & NA & 0 & NA & 1 \\
\hline \multicolumn{5}{|c|}{ Steelhead } \\
\hline NA & Hatchery/mixed & 1 & 1.7 & 3 \\
\hline NA & Wild & 1.7 & 3.3 & 4 \\
\hline
\end{tabular}

Table A9. Average fallback rates and standard deviations by anadromous species and runs.

[See table A1.7 for individual estimates, years, and references. NA, not available]

\begin{tabular}{|l|r|r|c|}
\hline \multicolumn{7}{|c|}{ Run } & Fallback rate (percent) & Standard deviation & Number of estimates \\
\hline \multicolumn{5}{|c|}{ Chinook salmon } \\
\hline Fall & 4.9 & 4.1 & 21 \\
\hline Spring & 24.1 & 12.9 & 12 \\
\hline Spring-summer & 10.3 & 4.7 & 23 \\
\hline Summer & 12.4 & 8.0 & 10 \\
\hline \multicolumn{5}{|c|}{ Coho salmon } \\
\hline NA & 5.0 & 6.7 & 5 \\
\hline \multicolumn{7}{|c|}{ Sockeye salmon } \\
\hline NA & $5.9 \quad 4.1$ & 28 \\
\hline \multicolumn{7}{|c|}{7.9} & \\
\hline NA & 7.9 & \\
\hline
\end{tabular}




\section{Appendix A1}

Table A1.1. Estimates of smolt survival in acclimation facilities by river, acclimation facility, year, species, run type (that is, life history), age class, acclimation facility type, and reference.

[ND, not denoted; NA, not available]

\begin{tabular}{|c|c|c|c|c|c|c|c|}
\hline Acclimation facility & Year & Species & Run & Age (years) & Facility type & $\begin{array}{l}\text { Survival } \\
\text { (percent) }\end{array}$ & Reference \\
\hline \multicolumn{8}{|c|}{ Catherine Creek (Oregon) } \\
\hline Catherine Creek & 2002 & Chinook salmon & Spring & ND & $\begin{array}{l}\text { Aluminum raceways, } \\
\text { lined with vinyl }\end{array}$ & 99.7 & $\begin{array}{l}\text { McLean and others, } \\
2003\end{array}$ \\
\hline \multicolumn{8}{|c|}{ Clearwater River (Idaho) } \\
\hline Big Canyon & 2008 & Chinook salmon & Fall & $1+$ & Aluminum circular tanks & 98.5 & McLeod, 2009 \\
\hline Big Canyon & 2008 & Chinook salmon & Fall & $0+$ & Aluminum circular tanks & 99.5 & McLeod, 2009 \\
\hline Big Canyon & 2007 & Chinook salmon & Fall & $1+$ & Aluminum circular tanks & 96.9 & McLeod, 2008 \\
\hline Big Canyon & 2007 & Chinook salmon & Fall & $0+$ & Aluminum circular tanks & 98.7 & McLeod, 2008 \\
\hline Big Canyon & 2007 & Chinook salmon & Fall & $1+$ & Aluminum circular tanks & 97.8 & McLeod, 2007 \\
\hline Big Canyon & 2007 & Chinook salmon & Fall & $0+$ & Aluminum circular tanks & 98.5 & McLeod, 2007 \\
\hline \multicolumn{8}{|c|}{ Grand Ronde River (Oregon) } \\
\hline Upper Grand Ronde & 2002 & Chinook salmon & Spring & ND & $\begin{array}{l}\text { Aluminum raceways, } \\
\text { lined with vinyl }\end{array}$ & 99.7 & $\begin{array}{l}\text { McLean and others, } \\
2003\end{array}$ \\
\hline \multicolumn{8}{|c|}{ Hood River (Oregon) } \\
\hline Blackberry & 2008 & Steelhead & Summer & ND & Polypropylene-lined tanks & 99.7 & Gerstenberger, 2009 \\
\hline \multicolumn{8}{|c|}{ Lostine River (Oregon) } \\
\hline Lostine River & 2007 & Chinook salmon & Spring & ND & $\begin{array}{l}\text { Polyvinyl chloride-lined } \\
\text { raceways }\end{array}$ & 99.9 & $\begin{array}{l}\text { Zollman and others, } \\
2009\end{array}$ \\
\hline \multicolumn{8}{|c|}{ MF Hood River (Oregon) } \\
\hline Parkdale & 2008 & Steelhead & Winter & ND & Painted concrete & 99.9 & Gerstenberger, 2009 \\
\hline \multicolumn{8}{|c|}{ Snake River (Idaho and Washington) } \\
\hline Capt. John Rapids & 2008 & Chinook salmon & Fall & $1+$ & In-ground, lined pond & 99.8 & McLeod, 2009 \\
\hline Capt. John Rapids & 2008 & Chinook salmon & Fall & $0+$ & In-ground, lined pond & 99.8 & McLeod, 2009 \\
\hline Capt. John Rapids & 2007 & Chinook salmon & Fall & $1+$ & In-ground, lined pond & 99.9 & McLeod, 2008 \\
\hline Capt. John Rapids & 2007 & Chinook salmon & Fall & $0+$ & In-ground, lined pond & 99.7 & McLeod, 2008 \\
\hline Capt. John Rapids & 2007 & Chinook salmon & Fall & $1+$ & In-ground, lined pond & 99.1 & McLeod, 2007 \\
\hline Capt. John Rapids & 2007 & Chinook salmon & Fall & $0+$ & In-ground, lined pond & 99.9 & McLeod, 2007 \\
\hline Pittsburg Landing & 2008 & Chinook salmon & Fall & $1+$ & Aluminum circular tanks & 97.9 & McLeod, 2009 \\
\hline Pittsburg Landing & 2008 & Chinook salmon & Fall & $0+$ & Aluminum circular tanks & 99.7 & McLeod, 2009 \\
\hline Pittsburg Landing & 2007 & Chinook salmon & Fall & $1+$ & Aluminum circular tanks & 97.7 & McLeod, 2008 \\
\hline Pittsburg Landing & 2007 & Chinook salmon & Fall & $0+$ & Aluminum circular tanks & 98.4 & McLeod, 2008 \\
\hline Pittsburg Landing & 2007 & Chinook salmon & Fall & $1+$ & Aluminum circular tanks & 99.6 & McLeod, 2007 \\
\hline Pittsburg Landing & 2007 & Chinook salmon & Fall & $0+$ & Aluminum circular tanks & 99.7 & McLeod, 2007 \\
\hline \multicolumn{8}{|c|}{ Umatilla River (Oregon) } \\
\hline Bonifer & 1993 & Steelhead & Summer & ND & NA & 99.5 & Rowan, 1994 \\
\hline Bonifer & 1993 & Steelhead & Summer & ND & NA & 99.8 & Rowan, 1994 \\
\hline Minthorn & 1993 & Steelhead & Summer & ND & NA & 98.6 & Rowan, 1994 \\
\hline
\end{tabular}


Table A1.2. Estimates of collection efficiency by river, facility, collection method, year, species, and reference.

[NA, not available; SS, Atlantic salmon; U.S.A., United States of America; CO, coho salmon; PSE, Puget Sound Energy; PH, powerhouse; PSC, prototype surface collector; USACE, U.S. Army Corps of Engineers; CH, Chinook salmon; FPC, Fish Passage Center; OM, steelhead; B2CC, second power house corner collector; PUD, Public Utility District; >, greater than; <, less than; RSW, removable spillway weir; SBC, surface bypass collector]

\begin{tabular}{|c|c|c|c|c|c|}
\hline Facility & Method & Year & Species & $\begin{array}{l}\text { Collection } \\
\text { efficiency } \\
\text { (percent) }\end{array}$ & Reference \\
\hline \multicolumn{6}{|c|}{ Ariège River (France) } \\
\hline Crampagna & Bypass/sluice & NA & SS & 66 & Croze, 2008 \\
\hline Guilhot & Bypass/sluice & NA & SS & 70 & Croze, 2008 \\
\hline Las Mijanes & Bypass/sluice & NA & SS & 32 & Croze, 2008 \\
\hline Las Rives & Bypass/sluice & NA & SS & 39 & Croze, 2008 \\
\hline \multicolumn{6}{|c|}{ Baker River (Washington, U.S.A.) } \\
\hline Lower Baker & Forebay collector-gulper & 1992 & $\mathrm{CO}$ & 11.3 & PSE, 2012 \\
\hline Lower Baker & Forebay collector-gulper & 1993 & $\mathrm{CO}$ & 7.8 & PSE, 2012 \\
\hline Lower Baker & Forebay collector-gulper & 1994 & $\mathrm{CO}$ & 17.6 & PSE, 2012 \\
\hline Lower Baker & Forebay collector-gulper & 1995 & $\mathrm{CO}$ & 7.2 & PSE, 2012 \\
\hline Lower Baker & Forebay collector-gulper & 1996 & $\mathrm{CO}$ & 9.2 & PSE, 2012 \\
\hline Lower Baker & Forebay collector-gulper & 1997 & $\mathrm{CO}$ & 23.1 & PSE, 2012 \\
\hline Lower Baker & Forebay collector-gulper & 1998 & $\mathrm{CO}$ & 56.8 & PSE, 2012 \\
\hline Lower Baker & Forebay collector-gulper & 1999 & $\mathrm{CO}$ & 27.2 & PSE, 2012 \\
\hline Lower Baker & Forebay collector-gulper & 2000 & $\mathrm{CO}$ & 45.2 & PSE, 2012 \\
\hline Lower Baker & Forebay collector-gulper & 2001 & $\mathrm{CO}$ & 22.9 & PSE, 2012 \\
\hline Lower Baker & Forebay collector-gulper & 2002 & $\mathrm{CO}$ & 21.9 & PSE, 2012 \\
\hline Lower Baker & Forebay collector-gulper & 2003 & $\mathrm{CO}$ & 8.2 & PSE, 2012 \\
\hline Lower Baker & Forebay collector-gulper & 2004 & $\mathrm{CO}$ & 17.3 & PSE, 2012 \\
\hline Lower Baker & Forebay collector-gulper & 2005 & $\mathrm{CO}$ & 31.6 & PSE, 2012 \\
\hline Lower Baker & Forebay collector-gulper & 2006 & $\mathrm{CO}$ & 25.4 & PSE, 2012 \\
\hline Lower Baker & Forebay collector-gulper & 2007 & $\mathrm{CO}$ & 33.5 & PSE, 2012 \\
\hline Lower Baker & Forebay collector-gulper & 2008 & $\mathrm{CO}$ & 7.8 & PSE, 2012 \\
\hline Lower Baker & Forebay collector-gulper & 2009 & $\mathrm{CO}$ & 38.5 & PSE, 2012 \\
\hline Lower Baker & Forebay collector-gulper & 2010 & $\mathrm{CO}$ & 8.3 & PSE, 2012 \\
\hline Lower Baker & Forebay collector-gulper & 2011 & $\mathrm{CO}$ & 18.2 & PSE, 2012 \\
\hline Lower Baker & Forebay collector-gulper & 2012 & $\mathrm{CO}$ & 24.5 & PSE, 2012 \\
\hline Lower Baker & Forebay collector-gulper & 2013 & $\mathrm{CO}$ & 95.1 & PSE, 2015 \\
\hline Lower Baker & Forebay collector-gulper & 2014 & $\mathrm{CO}$ & 92.8 & PSE, 2015 \\
\hline Upper Baker & Forebay collector-gulper & 1988 & $\mathrm{CO}$ & 40.9 & PSE, 2012 \\
\hline Upper Baker & Forebay collector-gulper & 1989 & $\mathrm{CO}$ & 41.8 & PSE, 2012 \\
\hline Upper Baker & Forebay collector-gulper & 1990 & $\mathrm{CO}$ & 62.7 & PSE, 2012 \\
\hline
\end{tabular}


Table A1.2. Estimates of collection efficiency by river, facility, collection method, year, species, and reference.-Continued

[NA, not available; SS, Atlantic salmon; U.S.A., United States of America; CO, coho salmon; PSE, Puget Sound Energy; PH, powerhouse; PSC, prototype surface collector; USACE, U.S. Army Corps of Engineers; CH, Chinook salmon; FPC, Fish Passage Center; OM, steelhead; B2CC, second power house corner collector; PUD, Public Utility District; >, greater than; <, less than; RSW, removable spillway weir; SBC, surface bypass collector]

\begin{tabular}{|c|c|c|c|c|c|}
\hline Facility & Method & Year & Species & $\begin{array}{l}\text { Collection } \\
\text { efficiency } \\
\text { (percent) }\end{array}$ & Reference \\
\hline Upper Baker & Forebay collector-gulper & 1991 & $\mathrm{CO}$ & 48.5 & PSE, 2012 \\
\hline Upper Baker & Forebay collector-gulper & 1992 & $\mathrm{CO}$ & 59.3 & PSE, 2012 \\
\hline Upper Baker & Forebay collector-gulper & 1993 & $\mathrm{CO}$ & 27.2 & PSE, 2012 \\
\hline Upper Baker & Forebay collector-gulper & 1994 & $\mathrm{CO}$ & 73.2 & PSE, 2012 \\
\hline Upper Baker & Forebay collector-gulper & 1995 & $\mathrm{CO}$ & 58.8 & PSE, 2012 \\
\hline Upper Baker & Forebay collector-gulper & 1996 & $\mathrm{CO}$ & 42.5 & PSE, 2012 \\
\hline Upper Baker & Forebay collector-gulper & 1997 & $\mathrm{CO}$ & 48.4 & PSE, 2012 \\
\hline Upper Baker & Forebay collector-gulper & 1998 & $\mathrm{CO}$ & 64 & PSE, 2012 \\
\hline Upper Baker & Forebay collector-gulper & 1999 & $\mathrm{CO}$ & 62 & PSE, 2012 \\
\hline Upper Baker & Forebay collector-gulper & 2000 & $\mathrm{CO}$ & 56.8 & PSE, 2012 \\
\hline Upper Baker & Forebay collector-gulper & 2001 & $\mathrm{CO}$ & 54.9 & PSE, 2012 \\
\hline Upper Baker & Forebay collector-gulper & 2002 & $\mathrm{CO}$ & 55.3 & PSE, 2012 \\
\hline Upper Baker & Forebay collector-gulper & 2003 & $\mathrm{CO}$ & 45.1 & PSE, 2012 \\
\hline Upper Baker & Forebay collector-gulper & 2004 & $\mathrm{CO}$ & 55.6 & PSE, 2012 \\
\hline Upper Baker & Forebay collector-gulper & 2005 & $\mathrm{CO}$ & 54.1 & PSE, 2012 \\
\hline Upper Baker & Forebay collector-gulper & 2006 & $\mathrm{CO}$ & 61.5 & PSE, 2012 \\
\hline Upper Baker & Forebay collector-gulper & 2007 & $\mathrm{CO}$ & 65.5 & Jeanes and Verretto, 2012 \\
\hline Upper Baker & $\begin{array}{l}\text { Forebay collector-surface } \\
\text { collector/enhanced gulper }\end{array}$ & 2008 & $\mathrm{CO}$ & 92.7 & Jeanes and Verretto, 2012 \\
\hline Upper Baker & $\begin{array}{l}\text { Forebay collector-surface } \\
\text { collector/enhanced gulper }\end{array}$ & 2009 & $\mathrm{CO}$ & 99 & Jeanes and Verretto, 2012 \\
\hline Upper Baker & $\begin{array}{l}\text { Forebay collector-surface } \\
\text { collector/enhanced gulper }\end{array}$ & 2010 & $\mathrm{CO}$ & NA & Jeanes and Verretto, 2012 \\
\hline Upper Baker & $\begin{array}{l}\text { Forebay collector-surface } \\
\text { collector/enhanced gulper }\end{array}$ & 2011 & $\mathrm{CO}$ & 82.6 & Jeanes and Verretto, 2012 \\
\hline Upper Baker & $\begin{array}{l}\text { Forebay collector-surface } \\
\text { collector/enhanced gulper }\end{array}$ & 2013 & $\mathrm{CO}$ & 94.3 & PSE, 2015 \\
\hline Upper Baker & $\begin{array}{l}\text { Forebay collector-surface } \\
\text { collector/enhanced gulper }\end{array}$ & 2014 & $\mathrm{CO}$ & 83.3 & PSE, 2015 \\
\hline \multicolumn{6}{|c|}{ Columbia River (Oregon and Washington, U.S.A.) } \\
\hline Bonneville PH1 & Powerhouse retrofit-PSC & 2000 & All & 83 & Ploskey and others, 2000, cited in USACE, 2007 \\
\hline Bonneville PH1 & Powerhouse retrofit—PSC & 2000 & All & 84 & Ploskey and others, 2000, cited in USACE, 2007 \\
\hline Bonneville PH1 & Sluiceway & 2002 & All & 33 & Ploskey and others, 2003 \\
\hline
\end{tabular}


Table A1.2. Estimates of collection efficiency by river, facility, collection method, year, species, and reference.-Continued

[NA, not available; SS, Atlantic salmon; U.S.A., United States of America; CO, coho salmon; PSE, Puget Sound Energy; PH, powerhouse; PSC, prototype surface collector; USACE, U.S. Army Corps of Engineers; CH, Chinook salmon; FPC, Fish Passage Center; OM, steelhead; B2CC, second power house corner collector; PUD, Public Utility District; >, greater than; <, less than; RSW, removable spillway weir; SBC, surface bypass collector]

\begin{tabular}{|c|c|c|c|c|c|}
\hline Facility & Method & Year & Species & $\begin{array}{l}\text { Collection } \\
\text { efficiency } \\
\text { (percent) }\end{array}$ & Reference \\
\hline Bonneville PH1 & Sluiceway & 2002 & All & 29 & Ploskey and others, 2003 \\
\hline Bonneville PH1 & Sluiceway & 2004 & All & 33 & Ploskey and others, 2005 \\
\hline Bonneville PH1 & Sluiceway & 2004 & All & 38 & Ploskey and others, 2005 \\
\hline Bonneville PH1 & Sluiceway & 2005 & All & 37 & Ploskey and others, 2006 \\
\hline Bonneville PH1 & Sluiceway & 2005 & All & 71 & Ploskey and others, 2006 \\
\hline Bonneville PH1 & Sluiceway & 2000 & $\mathrm{CH}$ & 68 & Evans and others, 2006, cited in USACE, 2007 \\
\hline Bonneville PH1 & Sluiceway & 2000 & $\mathrm{CH}$ & 29 & Reagan and others, 2005 \\
\hline Bonneville PH1 & Powerhouse retrofit-PSC & 2000 & $\mathrm{CH}$ & 43 & Evans and others, 2001, cited in USACE, 2007 \\
\hline Bonneville PH1 & Sluiceway & 2001 & $\mathrm{CH}$ & 70 & Evans and others, 2006, cited in USACE, 2007 \\
\hline Bonneville PH1 & Sluiceway & 2001 & $\mathrm{CH}$ & 77 & Reagan and others, 2005 \\
\hline Bonneville PH1 & Sluiceway & 2002 & $\mathrm{CH}$ & 48 & Evans and others, 2006, cited in USACE, 2007 \\
\hline Bonneville PH1 & Sluiceway & 2002 & $\mathrm{CH}$ & 35 & Reagan and others, 2005 \\
\hline Bonneville PH1 & Sluiceway & 2004 & $\mathrm{CH}$ & 47 & Evans and others, 2006, cited in USACE, 2007 \\
\hline Bonneville PH1 & Sluiceway & 2004 & $\mathrm{CH}$ & 53 & Reagan and others, 2005 \\
\hline Bonneville PH1 & Sluiceway B2CC & 2008 & $\mathrm{CH}$ & 16.7 & FPC, 2008 \\
\hline Bonneville PH1 & Sluiceway & 2000 & $\mathrm{OM}$ & 44 & Reagan and others, 2005 \\
\hline Bonneville PH1 & Powerhouse retrofit—PSC & 2000 & $\mathrm{OM}$ & 45 & Evans and others, 2001, cited in USACE, 2007 \\
\hline Bonneville PH1 & Sluiceway & 2002 & $\mathrm{OM}$ & 65 & Reagan and others, 2005 \\
\hline Bonneville PH1 & Sluiceway & 2004 & $\mathrm{OM}$ & 55 & Reagan and others, 2005 \\
\hline Bonneville PH2 & Sluiceway-B2CC & 2004 & All & 31 & Ploskey and others, 2005 \\
\hline Bonneville PH2 & Sluiceway-B2CC & 2004 & All & 40 & Ploskey and others, 2005 \\
\hline Bonneville PH2 & Sluiceway-B2CC & 2005 & All & 32 & Ploskey and others, 2006 \\
\hline Bonneville PH2 & Sluiceway-B2CC & 2005 & All & 44 & Ploskey and others, 2006 \\
\hline Bonneville PH2 & Sluiceway-B2CC & 2004 & $\mathrm{CH}$ & 37 & Evans and others, 2005, cited in USACE, 2007 \\
\hline Bonneville PH2 & Sluiceway-B2CC & 2004 & $\mathrm{CH}$ & 37 & Evans and others, 2005, cited in USACE, 2007 \\
\hline Bonneville PH2 & Sluiceway-B2CC & 2005 & $\mathrm{CH}$ & 40 & Evans and others, 2005, cited in USACE, 2007 \\
\hline Bonneville $\mathrm{PH} 2$ & Sluiceway-B2CC & 2005 & $\mathrm{CH}$ & 29 & Evans and others, 2005, cited in USACE, 2007 \\
\hline Bonneville PH2 & Sluiceway-B2CC & 2004 & $\mathrm{OM}$ & 74 & Reagan and others, 2005 \\
\hline Bonneville PH2 & Sluiceway-B2CC & 2005 & $\mathrm{OM}$ & 66 & Reagan and others, 2005 \\
\hline John Day & Surface spill—surface bypass & 2001 & $\mathrm{CH}$ & 28 & FPC, 2001 \\
\hline John Day & Surface spill—surface bypass & 2008 & $\mathrm{CH}$ & 15.3 & FPC, 2008 \\
\hline
\end{tabular}


Table A1.2. Estimates of collection efficiency by river, facility, collection method, year, species, and reference.-Continued

[NA, not available; SS, Atlantic salmon; U.S.A., United States of America; CO, coho salmon; PSE, Puget Sound Energy; PH, powerhouse; PSC, prototype surface collector; USACE, U.S. Army Corps of Engineers; CH, Chinook salmon; FPC, Fish Passage Center; OM, steelhead; B2CC, second power house corner collector; PUD, Public Utility District; >, greater than; <, less than; RSW, removable spillway weir; SBC, surface bypass collector]

\begin{tabular}{|c|c|c|c|c|c|}
\hline Facility & Method & Year & Species & $\begin{array}{l}\text { Collection } \\
\text { efficiency } \\
\text { (percent) }\end{array}$ & Reference \\
\hline Priest Rapids & Sluiceway & 1992 & All & 2.7 & McFadden and others, 1993, cited in USACE, 2007 \\
\hline Priest Rapids & Sluiceway & 1992 & All & 3.8 & Ransom, 1997, cited in USACE, 2007 \\
\hline Priest Rapids & Sluiceway & 1994 & All & 2.9 & Ransom, 1997, cited in USACE, 2007 \\
\hline Priest Rapids & Sluiceway & 1995 & All & 8.3 & Ransom, 1997, cited in USACE, 2007 \\
\hline Priest Rapids & Sluiceway & 1995 & All & 5.7 & Ransom, 1997, cited in USACE, 2007 \\
\hline Priest Rapids & Sluiceway & 1996 & All & 3.2 & Ransom, 1997, cited in USACE, 2007 \\
\hline Priest Rapids & Sluiceway & 1996 & All & 2.8 & Ransom, 1997, cited in USACE, 2007 \\
\hline Priest Rapids & Sluiceway & 2001 & $\mathrm{CH}$ & 1.9 & Robichaud and others, 2003, cited in USACE, 2007 \\
\hline Priest Rapids & Sluiceway & 2006 & $\mathrm{OM}$ & 39 & Timko and others, 2011 \\
\hline Priest Rapids & Sluiceway & 2007 & $\mathrm{OM}$ & 34 & Timko and others, 2011 \\
\hline Priest Rapids & Sluiceway & 2008 & $\mathrm{OM}$ & 59 & Timko and others, 2011 \\
\hline Priest Rapids & Sluiceway & 2009 & $\mathrm{OM}$ & 66 & Timko and others, 2011 \\
\hline Priest Rapids & Sluiceway & 2010 & $\mathrm{OM}$ & 69 & Timko and others, 2011 \\
\hline Rocky Reach & $\begin{array}{l}\text { Surface spill-combined } \\
\text { spillway }\end{array}$ & 1998 & All & 27.7 & $\begin{array}{l}\text { Iverson and Birmingham, 1998, cited in USACE, } \\
2007\end{array}$ \\
\hline Rocky Reach & $\begin{array}{l}\text { Surface spill—combined } \\
\text { spillway }\end{array}$ & 1998 & All & 33.1 & $\begin{array}{l}\text { Iverson and Birmingham, 1998, cited in USACE, } \\
2007\end{array}$ \\
\hline Rocky Reach & Forebay collector & 2002 & $\mathrm{CH}$ & 5 & Steig and others, 2007, cited in USACE, 2007 \\
\hline Rocky Reach & Forebay collector & 2002 & $\mathrm{CH}$ & 2 & Steig and others, 2007, cited in USACE, 2007 \\
\hline Rocky Reach & Forebay collector & 2002 & $\mathrm{CH}$ & 23 & Steig and others, 2007, cited in USACE, 2007 \\
\hline Rocky Reach & Forebay collector & 2002 & $\mathrm{CH}$ & 17 & Steig and others, 2007, cited in USACE, 2007 \\
\hline Rocky Reach & Forebay collector & 2004 & $\mathrm{CH}$ & 27 & Steig and others, 2007, cited in USACE, 2007 \\
\hline Rocky Reach & $\begin{array}{l}\text { Surface spill-combined } \\
\text { spillway }\end{array}$ & 2004 & $\mathrm{CH}$ & 38.6 & Steig and others, 2007, cited in USACE, 2007 \\
\hline Rocky Reach & $\begin{array}{l}\text { Surface spill-combined } \\
\text { spillway }\end{array}$ & 2004 & $\mathrm{CH}$ & 37.6 & Steig and others, 2007, cited in USACE, 2007 \\
\hline Rocky Reach & Forebay collector & 2005 & $\mathrm{CH}$ & 32 & Steig and others, 2007, cited in USACE, 2007 \\
\hline Rocky Reach & $\begin{array}{l}\text { Surface spill—combined } \\
\text { spillway }\end{array}$ & 2005 & $\mathrm{CH}$ & 36.2 & Steig and others, 2007, cited in USACE, 2007 \\
\hline Rocky Reach & $\begin{array}{l}\text { Surface spill—combined } \\
\text { spillway }\end{array}$ & 1999 & $\mathrm{OM}$ & 28.5 & Steig and others, 2007, cited in USACE, 2007 \\
\hline Rocky Reach & Forebay collector & 2002 & $\mathrm{OM}$ & 27 & Steig and others, 2003 \\
\hline Rocky Reach & Forebay collector & 2002 & $\mathrm{OM}$ & 30 & Steig and others, 2007, cited in USACE, 2007 \\
\hline Rocky Reach & Forebay collector & 2002 & $\mathrm{OM}$ & 29 & Steig and others, 2007, cited in USACE, 2007 \\
\hline
\end{tabular}


Table A1.2. Estimates of collection efficiency by river, facility, collection method, year, species, and reference.-Continued

[NA, not available; SS, Atlantic salmon; U.S.A., United States of America; CO, coho salmon; PSE, Puget Sound Energy; PH, powerhouse; PSC, prototype surface collector; USACE, U.S. Army Corps of Engineers; CH, Chinook salmon; FPC, Fish Passage Center; OM, steelhead; B2CC, second power house corner collector; PUD, Public Utility District; >, greater than; <, less than; RSW, removable spillway weir; SBC, surface bypass collector]

\begin{tabular}{|c|c|c|c|c|c|}
\hline Facility & Method & Year & Species & $\begin{array}{l}\text { Collection } \\
\text { efficiency } \\
\text { (percent) }\end{array}$ & Reference \\
\hline Rocky Reach & Forebay collector & 2004 & $\mathrm{OM}$ & 67 & Steig and others, 2007, cited in USACE, 2007 \\
\hline Rocky Reach & $\begin{array}{l}\text { Surface spill—combined } \\
\text { spillway }\end{array}$ & 2004 & $\mathrm{OM}$ & 16.7 & Steig and others, 2007, cited in USACE, 2007 \\
\hline Rocky Reach & Forebay collector & 2005 & $\mathrm{OM}$ & 68 & Steig and others, 2007, cited in USACE, 2007 \\
\hline Rocky Reach & $\begin{array}{l}\text { Surface spill—combined } \\
\text { spillway }\end{array}$ & 2005 & $\mathrm{OM}$ & 20.1 & Steig and others, 2007, cited in USACE, 2007 \\
\hline Rocky Reach & $\begin{array}{l}\text { Surface spill—combined } \\
\text { spillway }\end{array}$ & 2005 & $\mathrm{OM}$ & 21.9 & Steig and others, 2007, cited in USACE, 2007 \\
\hline Rocky Reach & Forebay collector & 2006 & $\mathrm{OM}$ & 66 & Steig and others, 2007, cited in USACE, 2007 \\
\hline The Dalles & Sluiceway & 1999 & All & 13 & Ploskey and others, 2001, cited in USACE, 2007 \\
\hline The Dalles & Sluiceway & 1999 & All & 12 & Ploskey and others, 2001, cited in USACE, 2007 \\
\hline The Dalles & Sluiceway & 2000 & All & 6 & Moursund and others, 2001, cited in USACE, 2007 \\
\hline The Dalles & Sluiceway & 2000 & All & 7 & Moursund and others, 2001, cited in USACE, 2007 \\
\hline The Dalles & Sluiceway & 2001 & All & 18 & Moursund and others, 2002, cited in USACE, 2007 \\
\hline The Dalles & Sluiceway & 2001 & All & 5 & Moursund and others, 2002, cited in USACE, 2007 \\
\hline The Dalles & Sluiceway & 2002 & All & 25 & Johnson and others, 2003, cited in USACE, 2007 \\
\hline The Dalles & Sluiceway & 2002 & All & 11 & Johnson and others, 2003, cited in USACE, 2007 \\
\hline The Dalles & Sluiceway & 2004 & All & 7 & Johnson and others, 2005, cited in USACE, 2007 \\
\hline The Dalles & Sluiceway & 2004 & All & 4 & Johnson and others, 2005, cited in USACE, 2007 \\
\hline The Dalles & Sluiceway & 2002 & $\mathrm{CH}$ & 10 & $\begin{array}{l}\text { Hausman and others, 2004, Counihan and others, } \\
\text { 2006, cited in USACE, } 2007\end{array}$ \\
\hline The Dalles & Sluiceway & 2003 & $\mathrm{CH}$ & 12 & Hansel and others, 2004, cited in USACE, 2007 \\
\hline The Dalles & Sluiceway & 2003 & $\mathrm{CH}$ & 17 & Hansel and others, 2004, cited in USACE, 2007 \\
\hline The Dalles & Sluiceway & 2004 & $\mathrm{CH}$ & 7 & $\begin{array}{l}\text { Hansel and others, } 2005 \text { and Counihan and others, } \\
\text { 2006, cited in USACE, } 2007\end{array}$ \\
\hline The Dalles & Sluiceway & 2004 & $\mathrm{CH}$ & 1 & Cash and others, 2005, cited in USACE, 2007 \\
\hline The Dalles & Sluiceway & 2004 & $\mathrm{CH}$ & 7 & $\begin{array}{l}\text { Hansel and others, 2005, Counihan and others, } \\
\text { 2006, cited in USACE, } 2007\end{array}$ \\
\hline The Dalles & Sluiceway & 2004 & $\mathrm{CH}$ & 8 & Cash and others, 2005, cited in USACE, 2007 \\
\hline The Dalles & Sluiceway & 2005 & $\mathrm{CH}$ & 4 & Beeman and others, 2006a cited in USACE, 2007 \\
\hline The Dalles & Sluiceway & 2005 & $\mathrm{CH}$ & 11 & Beeman and others, 2006a cited in USACE, 2007 \\
\hline The Dalles & Sluiceway & 2002 & $\mathrm{OM}$ & 14 & Hausman and others, 2004, cited in USACE, 2007 \\
\hline The Dalles & Sluiceway & 2004 & $\mathrm{OM}$ & 5 & Cash and others, 2005, cited in USACE, 2007 \\
\hline Wanapum & Sluiceway & 1989 & All & 8.6 & Ransom, 1997, cited in USACE, 2007 \\
\hline
\end{tabular}


Table A1.2. Estimates of collection efficiency by river, facility, collection method, year, species, and reference.-Continued

[NA, not available; SS, Atlantic salmon; U.S.A., United States of America; CO, coho salmon; PSE, Puget Sound Energy; PH, powerhouse; PSC, prototype surface collector; USACE, U.S. Army Corps of Engineers; CH, Chinook salmon; FPC, Fish Passage Center; OM, steelhead; B2CC, second power house corner collector; PUD, Public Utility District; >, greater than; <, less than; RSW, removable spillway weir; SBC, surface bypass collector]

\begin{tabular}{|c|c|c|c|c|c|}
\hline Facility & Method & Year & Species & $\begin{array}{l}\text { Collection } \\
\text { efficiency } \\
\text { (percent) }\end{array}$ & Reference \\
\hline Wanapum & Sluiceway & 1990 & All & 5.7 & Ransom, 1997, cited in USACE, 2007 \\
\hline Wanapum & Sluiceway & 1991 & All & 4.2 & Ransom, 1997, cited in USACE, 2007 \\
\hline Wanapum & Sluiceway & 1994 & All & 5.8 & Ransom, 1997, cited in USACE, 2007 \\
\hline Wanapum & Sluiceway & 1995 & All & 10 & Ransom, 1997, cited in USACE, 2007 \\
\hline Wanapum & Sluiceway & 1995 & All & 9.9 & Ransom, 1997, cited in USACE, 2007 \\
\hline Wanapum & Sluiceway & 1996 & All & 3 & Ransom, 1997, cited in USACE, 2007 \\
\hline Wanapum & Sluiceway & 1996 & All & 3.7 & Ransom, 1997, cited in USACE, 2007 \\
\hline Wanapum & Powerhouse retrofit & 1996 & All & 0.3 & Kumagai and others, 1997, cited in USACE, 2007 \\
\hline Wanapum & Powerhouse retrofit & 1997 & All & 0.3 & Kumagai and others, 1997, cited in USACE, 2007 \\
\hline Wanapum & Sluiceway & 1996 & $\mathrm{CH}$ & 3 & Robichaud and others, 2003, cited in USACE, 2007 \\
\hline Wanapum & Surface spill & 2002 & $\mathrm{CH}$ & 10 & Robichaud and others, 2003, cited in USACE, 2007 \\
\hline Wanapum & Surface spill & 2004 & $\mathrm{CH}$ & 24 & USACE, 2007 \\
\hline Wanapum & Surface spill & 2008 & $\mathrm{OM}$ & 53.5 & Timko and others, 2010 \\
\hline Wanapum & Surface spill & 2009 & $\mathrm{OM}$ & 70.2 & Timko and others, 2010 \\
\hline Wanapum & Surface spill & 2010 & $\mathrm{OM}$ & 77 & Timko and others, 2011 \\
\hline Wells & $\begin{array}{l}\text { Powerhouse retrofit - surface } \\
\text { bypass units with retrofit } \\
\text { baffle bays }\end{array}$ & 1990 & $\mathrm{CH}, \mathrm{OM}$ & 84.3 & Skalski and others, 1996 \\
\hline Wells & $\begin{array}{l}\text { Powerhouse retrofit - surface } \\
\text { bypass units with retrofit } \\
\text { baffle bays }\end{array}$ & 1990 & $\mathrm{CH}, \mathrm{OM}$ & 76.5 & Skalski and others, 1996 \\
\hline Wells & $\begin{array}{l}\text { Powerhouse retrofit - surface } \\
\text { bypass units with retrofit } \\
\text { baffle bays }\end{array}$ & 1991 & $\mathrm{CH}, \mathrm{OM}$ & 95 & Skalski and others, 1996 \\
\hline Wells & $\begin{array}{l}\text { Powerhouse retrofit - surface } \\
\text { bypass units with retrofit } \\
\text { baffle bays }\end{array}$ & 1991 & $\mathrm{CH}, \mathrm{OM}$ & 97 & Skalski and others, 1996 \\
\hline Wells & $\begin{array}{l}\text { Powerhouse retrofit- - surface } \\
\text { bypass units with retrofit } \\
\text { baffle bays }\end{array}$ & 1992 & $\mathrm{CH}, \mathrm{OM}$ & 89 & Skalski and others, 1996 \\
\hline Wells & $\begin{array}{l}\text { Powerhouse retrofit- - surface } \\
\text { bypass units with retrofit } \\
\text { baffle bays }\end{array}$ & 1992 & $\mathrm{CH}, \mathrm{OM}$ & 93.4 & Skalski and others, 1996 \\
\hline \multicolumn{6}{|c|}{ Connecticut River (New Hampshire, U.S.A.) } \\
\hline Bellows Falls & Bypass/sluice & NA & SS & 94 & Hanson, 1999 \\
\hline Vernon Station & Bypass/sluice & NA & SS & 74 & Hanson, 1999 \\
\hline
\end{tabular}


Table A1.2. Estimates of collection efficiency by river, facility, collection method, year, species, and reference.-Continued

[NA, not available; SS, Atlantic salmon; U.S.A., United States of America; CO, coho salmon; PSE, Puget Sound Energy; PH, powerhouse; PSC, prototype surface collector; USACE, U.S. Army Corps of Engineers; CH, Chinook salmon; FPC, Fish Passage Center; OM, steelhead; B2CC, second power house corner collector; PUD, Public Utility District; >, greater than; <, less than; RSW, removable spillway weir; SBC, surface bypass collector]

\begin{tabular}{|c|c|c|c|c|c|}
\hline Facility & Method & Year & Species & $\begin{array}{l}\text { Collection } \\
\text { efficiency } \\
\text { (percent) }\end{array}$ & Reference \\
\hline \multicolumn{6}{|c|}{ Cowlitz River (Washington, U.S.A.) } \\
\hline Cowlitz Falls & $\begin{array}{l}\text { Forebay collector-retrofit } \\
\text { baffle }\end{array}$ & 1997 & $\mathrm{CH}$ & 17 & Serl and Morrill, 2010 \\
\hline Cowlitz Falls & $\begin{array}{l}\text { Forebay collector-retrofit } \\
\text { baffle }\end{array}$ & 1998 & $\mathrm{CH}$ & 18 & Serl and Morrill, 2010 \\
\hline Cowlitz Falls & $\begin{array}{l}\text { Forebay collector-retrofit } \\
\text { baffle }\end{array}$ & 1999 & $\mathrm{CH}$ & 24 & Serl and Morrill, 2010 \\
\hline Cowlitz Falls & $\begin{array}{l}\text { Forebay collector-retrofit } \\
\text { baffle }\end{array}$ & 2000 & $\mathrm{CH}$ & 24 & Serl and Morrill, 2010 \\
\hline Cowlitz Falls & $\begin{array}{l}\text { Forebay collector-retrofit } \\
\text { baffle }\end{array}$ & 2001 & $\mathrm{CH}$ & 23 & Serl and Morrill, 2010 \\
\hline Cowlitz Falls & $\begin{array}{l}\text { Forebay collector-retrofit } \\
\text { baffle }\end{array}$ & 2002 & $\mathrm{CH}$ & 22 & Serl and Morrill, 2010 \\
\hline Cowlitz Falls & $\begin{array}{l}\text { Forebay collector-retrofit } \\
\text { baffle }\end{array}$ & 2003 & $\mathrm{CH}$ & 13 & Serl and Morrill, 2010 \\
\hline Cowlitz Falls & $\begin{array}{l}\text { Forebay collector-retrofit } \\
\text { baffle }\end{array}$ & 2004 & $\mathrm{CH}$ & 14 & Serl and Morrill, 2010 \\
\hline Cowlitz Falls & $\begin{array}{l}\text { Forebay collector-retrofit } \\
\text { baffle }\end{array}$ & 2005 & $\mathrm{CH}$ & 12 & Serl and Morrill, 2010 \\
\hline Cowlitz Falls & $\begin{array}{l}\text { Forebay collector-retrofit } \\
\text { baffle }\end{array}$ & 2006 & $\mathrm{CH}$ & 30.5 & Serl and Morrill, 2010 \\
\hline Cowlitz Falls & $\begin{array}{l}\text { Forebay collector-retrofit } \\
\text { baffle }\end{array}$ & 2007 & $\mathrm{CH}$ & 20.1 & Serl and Morrill, 2010 \\
\hline Cowlitz Falls & $\begin{array}{l}\text { Forebay collector-retrofit } \\
\text { baffle }\end{array}$ & 2008 & $\mathrm{CH}$ & 26.1 & Serl and Morrill, 2010 \\
\hline Cowlitz Falls & $\begin{array}{l}\text { Forebay collector-retrofit } \\
\text { baffle }\end{array}$ & 2009 & $\mathrm{CH}$ & 39.6 & Serl and Morrill, 2010 \\
\hline Cowlitz Falls & $\begin{array}{l}\text { Forebay collector-retrofit } \\
\text { baffle }\end{array}$ & 2012 & $\mathrm{CH}$ & 16.6 & Serl and Heimbigner, 2013 \\
\hline Cowlitz Falls & $\begin{array}{l}\text { Forebay collector-retrofit } \\
\text { baffle }\end{array}$ & 1996 & $\mathrm{CO}$ & 15 & Serl and Morrill, 2010 \\
\hline Cowlitz Falls & $\begin{array}{l}\text { Forebay collector-retrofit } \\
\text { baffle }\end{array}$ & 1997 & $\mathrm{CO}$ & 21 & Serl and Morrill, 2010 \\
\hline Cowlitz Falls & $\begin{array}{l}\text { Forebay collector-retrofit } \\
\text { baffle }\end{array}$ & 1998 & $\mathrm{CO}$ & 32 & Serl and Morrill, 2010 \\
\hline Cowlitz Falls & $\begin{array}{l}\text { Forebay collector-retrofit } \\
\text { baffle }\end{array}$ & 1999 & $\mathrm{CO}$ & 17 & Serl and Morrill, 2010 \\
\hline Cowlitz Falls & $\begin{array}{l}\text { Forebay collector-retrofit } \\
\text { baffle }\end{array}$ & 2000 & $\mathrm{CO}$ & 45 & Serl and Morrill, 2010 \\
\hline Cowlitz Falls & $\begin{array}{l}\text { Forebay collector-retrofit } \\
\text { baffle }\end{array}$ & 2001 & $\mathrm{CO}$ & 42 & Serl and Morrill, 2010 \\
\hline Cowlitz Falls & $\begin{array}{l}\text { Forebay collector-retrofit } \\
\text { baffle }\end{array}$ & 2002 & $\mathrm{CO}$ & 33 & Serl and Morrill, 2010 \\
\hline
\end{tabular}


Table A1.2. Estimates of collection efficiency by river, facility, collection method, year, species, and reference.-Continued

[NA, not available; SS, Atlantic salmon; U.S.A., United States of America; CO, coho salmon; PSE, Puget Sound Energy; PH, powerhouse; PSC, prototype surface collector; USACE, U.S. Army Corps of Engineers; CH, Chinook salmon; FPC, Fish Passage Center; OM, steelhead; B2CC, second power house corner collector; PUD, Public Utility District; >, greater than; <, less than; RSW, removable spillway weir; SBC, surface bypass collector]

\begin{tabular}{|c|c|c|c|c|c|}
\hline Facility & Method & Year & Species & $\begin{array}{l}\text { Collection } \\
\text { efficiency } \\
\text { (percent) }\end{array}$ & Reference \\
\hline Cowlitz Falls & $\begin{array}{l}\text { Forebay collector-retrofit } \\
\text { baffle }\end{array}$ & 2003 & $\mathrm{CO}$ & 43 & Serl and Morrill, 2010 \\
\hline Cowlitz Falls & $\begin{array}{l}\text { Forebay collector-retrofit } \\
\text { baffle }\end{array}$ & 2004 & $\mathrm{CO}$ & 42 & Serl and Morrill, 2010 \\
\hline Cowlitz Falls & $\begin{array}{l}\text { Forebay collector-retrofit } \\
\text { baffle }\end{array}$ & 2005 & $\mathrm{CO}$ & 36 & Serl and Morrill, 2010 \\
\hline Cowlitz Falls & $\begin{array}{l}\text { Forebay collector-retrofit } \\
\text { baffle }\end{array}$ & 2006 & $\mathrm{CO}$ & 26 & Serl and Morrill, 2010 \\
\hline Cowlitz Falls & $\begin{array}{l}\text { Forebay collector-retrofit } \\
\text { baffle }\end{array}$ & 2007 & $\mathrm{CO}$ & 36 & Serl and Morrill, 2010 \\
\hline Cowlitz Falls & $\begin{array}{l}\text { Forebay collector-retrofit } \\
\text { baffle }\end{array}$ & 2008 & $\mathrm{CO}$ & 20.8 & Serl and Morrill, 2010 \\
\hline Cowlitz Falls & $\begin{array}{l}\text { Forebay collector-retrofit } \\
\text { baffle }\end{array}$ & 2009 & $\mathrm{CO}$ & 20.8 & Serl and Morrill, 2010 \\
\hline Cowlitz Falls & $\begin{array}{l}\text { Forebay collector-retrofit } \\
\text { baffle }\end{array}$ & 2012 & $\mathrm{CO}$ & 17.7 & Serl and Heimbigner, 2013 \\
\hline Cowlitz Falls & $\begin{array}{l}\text { Forebay collector-retrofit } \\
\text { baffle }\end{array}$ & 1996 & $\mathrm{OM}$ & 50 & Serl and Morrill, 2010 \\
\hline Cowlitz Falls & $\begin{array}{l}\text { Forebay collector-retrofit } \\
\text { baffle }\end{array}$ & 1997 & $\mathrm{OM}$ & 45 & Serl and Morrill, 2010 \\
\hline Cowlitz Falls & $\begin{array}{l}\text { Forebay collector-retrofit } \\
\text { baffle }\end{array}$ & 1998 & $\mathrm{OM}$ & 19 & Serl and Morrill, 2010 \\
\hline Cowlitz Falls & $\begin{array}{l}\text { Forebay collector-retrofit } \\
\text { baffle }\end{array}$ & 1999 & $\mathrm{OM}$ & 41 & Serl and Morrill, 2010 \\
\hline Cowlitz Falls & $\begin{array}{l}\text { Forebay collector-retrofit } \\
\text { baffle }\end{array}$ & 2000 & $\mathrm{OM}$ & 65 & Serl and Morrill, 2010 \\
\hline Cowlitz Falls & $\begin{array}{l}\text { Forebay collector-retrofit } \\
\text { baffle }\end{array}$ & 2001 & $\mathrm{OM}$ & 58 & Serl and Morrill, 2010 \\
\hline Cowlitz Falls & $\begin{array}{l}\text { Forebay collector-retrofit } \\
\text { baffle }\end{array}$ & 2002 & $\mathrm{OM}$ & 56 & Serl and Morrill, 2010 \\
\hline Cowlitz Falls & $\begin{array}{l}\text { Forebay collector-retrofit } \\
\text { baffle }\end{array}$ & 2003 & $\mathrm{OM}$ & 68 & Serl and Morrill, 2010 \\
\hline Cowlitz Falls & $\begin{array}{l}\text { Forebay collector-retrofit } \\
\text { baffle }\end{array}$ & 2004 & $\mathrm{OM}$ & 48 & Serl and Morrill, 2010 \\
\hline Cowlitz Falls & $\begin{array}{l}\text { Forebay collector-retrofit } \\
\text { baffle }\end{array}$ & 2005 & $\mathrm{OM}$ & 42 & Serl and Morrill, 2010 \\
\hline Cowlitz Falls & $\begin{array}{l}\text { Forebay collector-retrofit } \\
\text { baffle }\end{array}$ & 2006 & $\mathrm{OM}$ & 47 & Serl and Morrill, 2010 \\
\hline Cowlitz Falls & $\begin{array}{l}\text { Forebay collector-retrofit } \\
\text { baffle }\end{array}$ & 2007 & $\mathrm{OM}$ & 42 & Serl and Morrill, 2010 \\
\hline Cowlitz Falls & $\begin{array}{l}\text { Forebay collector-retrofit } \\
\text { baffle }\end{array}$ & 2008 & $\mathrm{OM}$ & 26 & Serl and Morrill, 2010 \\
\hline Cowlitz Falls & $\begin{array}{l}\text { Forebay collector-retrofit } \\
\text { baffle }\end{array}$ & 2009 & $\mathrm{OM}$ & 37.4 & Serl and Morrill, 2010 \\
\hline
\end{tabular}


Table A1.2. Estimates of collection efficiency by river, facility, collection method, year, species, and reference.-Continued

[NA, not available; SS, Atlantic salmon; U.S.A., United States of America; CO, coho salmon; PSE, Puget Sound Energy; PH, powerhouse; PSC, prototype surface collector; USACE, U.S. Army Corps of Engineers; CH, Chinook salmon; FPC, Fish Passage Center; OM, steelhead; B2CC, second power house corner collector; PUD, Public Utility District; >, greater than; <, less than; RSW, removable spillway weir; SBC, surface bypass collector]

\begin{tabular}{|c|c|c|c|c|c|}
\hline Facility & Method & Year & Species & $\begin{array}{l}\text { Collection } \\
\text { efficiency } \\
\text { (percent) }\end{array}$ & Reference \\
\hline Cowlitz Falls & $\begin{array}{l}\text { Forebay collector-retrofit } \\
\text { baffle }\end{array}$ & 2012 & $\mathrm{OM}$ & 5.4 & Serl and Heimbigner, 2013 \\
\hline Mayfield & Forebay collector & 1964 & $\mathrm{CH}$ & 76 & USACE, 2007 \\
\hline Mayfield & Forebay collector & 1965 & $\mathrm{CH}$ & 74 & USACE, 2007 \\
\hline Mayfield & Forebay collector & 1964 & $\mathrm{CO}$ & 50 & USACE, 2007 \\
\hline Mayfield & Forebay collector & 1965 & $\mathrm{CO}$ & 62 & USACE, 2007 \\
\hline Mayfield & Forebay collector & 2001 & $\mathrm{CO}$ & 67 & USACE, 2007 \\
\hline Mayfield & Forebay collector & 2012 & $\mathrm{CO}$ & 67 & Serl and Heimbigner, 2013 \\
\hline Mayfield & Forebay collector & 1964 & $\mathrm{OM}$ & 73.6 & USACE, 2007 \\
\hline Mayfield & Forebay collector & 1965 & $\mathrm{OM}$ & 79.3 & USACE, 2007 \\
\hline \multicolumn{6}{|c|}{ Deschutes River (Oregon, U.S.A.) } \\
\hline Round Butte & $\begin{array}{l}\text { Forebay collector-double } \\
-\mathrm{V} \text { screen }\end{array}$ & 2010 & $\mathrm{CH}$ & 46.9 & Hill and Quesada, 2011 \\
\hline Round Butte & $\begin{array}{l}\text { Forebay collector-double } \\
-\mathrm{V} \text { screen }\end{array}$ & 2011 & $\mathrm{CH}$ & 51.7 & Hill and Quesada, 2012 \\
\hline Round Butte & $\begin{array}{l}\text { Forebay collector-double } \\
-\mathrm{V} \text { screen }\end{array}$ & 2012 & $\mathrm{CH}$ & 46.9 & Hill and Quesada, 2013 \\
\hline Round Butte & $\begin{array}{l}\text { Forebay collector-double } \\
- \text {-V screen }\end{array}$ & 2013 & $\mathrm{CH}$ & 30.1 & Hill and Quesada, 2014 \\
\hline Round Butte & $\begin{array}{l}\text { Forebay collector-double } \\
-\mathrm{V} \text { screen }\end{array}$ & 2014 & $\mathrm{CH}$ & 37.7 & Hill and Quesada, 2015 \\
\hline Round Butte & $\begin{array}{l}\text { Forebay collector-double } \\
-\mathrm{V} \text { screen }\end{array}$ & 2010 & $\mathrm{OM}$ & 17.4 & Hill and Quesada, 2011 \\
\hline Round Butte & $\begin{array}{l}\text { Forebay collector-double } \\
-\mathrm{V} \text { screen }\end{array}$ & 2011 & $\mathrm{OM}$ & 24.2 & Hill and Quesada, 2012 \\
\hline Round Butte & $\begin{array}{l}\text { Forebay collector-double } \\
-\mathrm{V} \text { screen }\end{array}$ & 2012 & $\mathrm{OM}$ & 16 & Hill and Quesada, 2013 \\
\hline Round Butte & $\begin{array}{l}\text { Forebay collector-double } \\
-\mathrm{V} \text { screen }\end{array}$ & 2013 & $\mathrm{OM}$ & 12.7 & Hill and Quesada, 2014 \\
\hline Round Butte & $\begin{array}{l}\text { Forebay collector-double } \\
-\mathrm{V} \text { screen }\end{array}$ & 2014 & $\mathrm{OM}$ & 12.4 & Hill and Quesada, 2015 \\
\hline \multicolumn{6}{|c|}{ Garonne (France) } \\
\hline Camon & Bypass/sluice & NA & SS & 73 & Travade and Larinier 2006 \\
\hline \multicolumn{6}{|c|}{ Gave d'Aspe (France) } \\
\hline Bedous & Bypass/sluice & NA & SS & 55 & Travade and Larinier 2006 \\
\hline Soeix & Bypass/sluice & NA & SS & 61 & Travade and Larinier 2006 \\
\hline \multicolumn{6}{|c|}{ Gave d' Ossau (France) } \\
\hline St. Cricq & Bypass/sluice & NA & SS & 79 & Travade and Larinier 2006 \\
\hline
\end{tabular}


Table A1.2. Estimates of collection efficiency by river, facility, collection method, year, species, and reference.-Continued

[NA, not available; SS, Atlantic salmon; U.S.A., United States of America; CO, coho salmon; PSE, Puget Sound Energy; PH, powerhouse; PSC, prototype surface collector; USACE, U.S. Army Corps of Engineers; CH, Chinook salmon; FPC, Fish Passage Center; OM, steelhead; B2CC, second power house corner collector; PUD, Public Utility District; >, greater than; <, less than; RSW, removable spillway weir; SBC, surface bypass collector]

\begin{tabular}{|c|c|c|c|c|c|}
\hline Facility & Method & Year & Species & $\begin{array}{l}\text { Collection } \\
\text { efficiency } \\
\text { (percent) }\end{array}$ & Reference \\
\hline \multicolumn{6}{|c|}{ Gave de Pau (France) } \\
\hline Baigts & Bypass/sluice & NA & SS & 93 & Travade and Larinier 2006 \\
\hline Castetarbe & Bypass/sluice & NA & SS & 100 & Travade and Larinier 2006 \\
\hline \multicolumn{6}{|c|}{ Lewis River (Washington, U.S.A.) } \\
\hline Swift Reservoir & Floating surface collector & 2015 & $\mathrm{CO}$ & 11.8 & PacifiCorp and Cowlitz PUD, 2016 \\
\hline Swift Reservoir & Floating surface collector & 2015 & $\mathrm{CH}$ & 0 & PacifiCorp and Cowlitz PUD, 2016 \\
\hline Swift Reservoir & Floating surface collector & 2015 & $\mathrm{OM}$ & 18.6 & PacifiCorp and Cowlitz PUD, 2016 \\
\hline \multicolumn{6}{|c|}{ Santiam River (Oregon, U.S.A.) } \\
\hline Green Peter & $\begin{array}{l}\text { Forebay collector-Floating } \\
\text { collection horn }\end{array}$ & NA & $\mathrm{CH}$ & $>80$ & AECOM, 2010 \\
\hline Green Peter & $\begin{array}{l}\text { Forebay collector-Floating } \\
\text { collection horn }\end{array}$ & $\begin{array}{l}4 \text { years } \\
\text { in the } \\
\text { late } \\
1960 \mathrm{~s}\end{array}$ & $\mathrm{OM}$ & $<57$ & AECOM, 2010 \\
\hline \multicolumn{6}{|c|}{ Snake River (Idaho, Oregon, and Washington, U.S.A.) } \\
\hline Ice Harbor & Sluiceway & 1982 & All & 13 & Johnson and others, 1982, cited in USACE, 2007 \\
\hline Ice Harbor & Sluiceway & 1983 & All & 30 & Johnson and others, 1982, cited in USACE, 2007 \\
\hline Ice Harbor & Sluiceway & 1986 & All & 50 & Sullivan and others, 1986, cited in USACE, 2007 \\
\hline Ice Harbor & Sluiceway & 1987 & All & 34 & Ransom and Ouellette, 1988, cited in USACE, 2007 \\
\hline Ice Harbor & Surface Spill—RSW & 2005 & All & 28 & USACE, 2007 \\
\hline Ice Harbor & Surface Spill—RSW & 2005 & All & 38 & USACE, 2007 \\
\hline Ice Harbor & Surface Spill-RSW & 2005 & $\mathrm{CH}$ & 60 & USACE, 2007 \\
\hline Ice Harbor & Surface Spill-RSW & 2005 & $\mathrm{CH}$ & 29 & Axel and others, 2006, cited in USACE, 2007 \\
\hline Ice Harbor & Surface Spill—RSW & 2006 & $\mathrm{CH}$ & 68 & USACE, 2007 \\
\hline Ice Harbor & Surface Spill—RSW & 2006 & $\mathrm{CH}$ & 51 & USACE, 2007 \\
\hline Ice Harbor & Surface Spill—RSW & 2005 & $\mathrm{OM}$ & 47 & Axel and others, 2006, cited in USACE, 2007 \\
\hline Ice Harbor & Surface Spill-RSW & 2006 & $\mathrm{OM}$ & 38 & USACE, 2007 \\
\hline Lower Granite & Powerhouse retrofit $-\mathrm{SBC}$ & 2000 & All & 43 & Anglea and others, 2001, cited in USACE, 2007 \\
\hline Lower Granite & Surface Spill—RSW & 2002 & All & 65 & USACE, 2007 \\
\hline Lower Granite & Surface Spill-RSW & 2005 & All & 31 & Dawson and others, 2006, cited in USACE, 2007 \\
\hline Lower Granite & Surface Spill—RSW & 2005 & All & 25 & Dawson and others, 2006, cited in USACE, 2007 \\
\hline Lower Granite & Surface Spill—RSW & 1998 & $\mathrm{CH}$ & 49 & FPC, 2001 \\
\hline Lower Granite & Surface Spill—RSW & 1998 & $\mathrm{CH}$ & 49 & FPC, 2001 \\
\hline
\end{tabular}


Table A1.2. Estimates of collection efficiency by river, facility, collection method, year, species, and reference.-Continued

[NA, not available; SS, Atlantic salmon; U.S.A., United States of America; CO, coho salmon; PSE, Puget Sound Energy; PH, powerhouse; PSC, prototype surface collector; USACE, U.S. Army Corps of Engineers; CH, Chinook salmon; FPC, Fish Passage Center; OM, steelhead; B2CC, second power house corner collector; PUD, Public Utility District; >, greater than; <, less than; RSW, removable spillway weir; SBC, surface bypass collector]

\begin{tabular}{|c|c|c|c|c|c|}
\hline Facility & Method & Year & Species & $\begin{array}{l}\text { Collection } \\
\text { efficiency } \\
\text { (percent) }\end{array}$ & Reference \\
\hline Lower Granite & Surface Spill—RSW & 1999 & $\mathrm{CH}$ & 26 & FPC, 2001 \\
\hline Lower Granite & Surface Spill_-RSW & 1999 & $\mathrm{CH}$ & 26 & FPC, 2001 \\
\hline Lower Granite & Surface Spill—RSW & 2000 & $\mathrm{CH}$ & 38 & FPC, 2001 \\
\hline Lower Granite & Surface Spill—RSW & 2000 & $\mathrm{CH}$ & 38 & FPC, 2001 \\
\hline Lower Granite & Surface Spill—RSW & 2000 & $\mathrm{CH}$ & 38 & FPC, 2001 \\
\hline Lower Granite & Surface Spill—RSW & 2000 & $\mathrm{CH}$ & 55 & FPC, 2001 \\
\hline Lower Granite & Powerhouse retrofit $-\mathrm{SBC}$ & 2000 & $\mathrm{CH}$ & 29 & Plumb and others, 2002, cited in USACE, 2007 \\
\hline Lower Granite & Surface Spill—-RSW & 2001 & $\mathrm{CH}$ & 79 & FPC, 2001 \\
\hline Lower Granite & Surface Spill—RSW & 2001 & $\mathrm{CH}$ & 75 & FPC, 2006 \\
\hline Lower Granite & Surface Spill—RSW & 2001 & $\mathrm{CH}$ & 82 & FPC, 2006 \\
\hline Lower Granite & Surface Spill—RSW & 2001 & $\mathrm{CH}$ & 60 & FPC, 2001 \\
\hline Lower Granite & Surface Spill—RSW & 2002 & $\mathrm{CH}$ & 22 & FPC, 2006 \\
\hline Lower Granite & Surface Spill—RSW & 2002 & $\mathrm{CH}$ & 22 & FPC, 2006 \\
\hline Lower Granite & Surface Spill—RSW & 2002 & $\mathrm{CH}$ & 60 & FPC, 2002 \\
\hline Lower Granite & Surface Spill—RSW & 2002 & $\mathrm{CH}$ & 56 & Plumb and others, 2003, cited in USACE, 2007 \\
\hline Lower Granite & Surface Spill—RSW & 2003 & $\mathrm{CH}$ & 32 & FPC, 2008 \\
\hline Lower Granite & Surface Spill—RSW & 2003 & $\mathrm{CH}$ & 42 & FPC, 2006 \\
\hline Lower Granite & Surface Spill—RSW & 2003 & $\mathrm{CH}$ & 49 & FPC, 2004 \\
\hline Lower Granite & Surface Spill—RSW & 2003 & $\mathrm{CH}$ & 58 & Plumb and others, 2004, cited in USACE, 2007 \\
\hline Lower Granite & Surface Spill-RSW & 2004 & $\mathrm{CH}$ & 55 & FPC, 2008 \\
\hline Lower Granite & Surface Spill—RSW & 2004 & $\mathrm{CH}$ & 61 & FPC, 2006 \\
\hline Lower Granite & Surface Spill—RSW & 2005 & $\mathrm{CH}$ & 72 & FPC, 2008 \\
\hline Lower Granite & Surface Spill—RSW & 2005 & $\mathrm{CH}$ & 76 & FPC, 2008 \\
\hline Lower Granite & Surface Spill—RSW & 2005 & $\mathrm{CH}$ & 35 & FPC, 2006 \\
\hline Lower Granite & Surface Spill—RSW & 2005 & $\mathrm{CH}$ & 69 & USACE, 2007 \\
\hline Lower Granite & Surface Spill—RSW & 2005 & $\mathrm{CH}$ & 37 & USACE, 2007 \\
\hline Lower Granite & Surface Spill—RSW & 2006 & $\mathrm{CH}$ & 24 & FPC, 2011 \\
\hline Lower Granite & Surface Spill—RSW & 2006 & $\mathrm{CH}$ & 32 & FPC, 2011 \\
\hline Lower Granite & Surface Spill—RSW & 2006 & $\mathrm{CH}$ & 16 & FPC, 2006 \\
\hline Lower Granite & Surface Spill—RSW & 2006 & $\mathrm{CH}$ & 16 & FPC, 2006 \\
\hline Lower Granite & Surface Spill-RSW & 2006 & $\mathrm{CH}$ & 58 & USACE, 2007 \\
\hline Lower Granite & Surface Spill—RSW & 2006 & $\mathrm{CH}$ & 30 & USACE, 2007 \\
\hline
\end{tabular}


Table A1.2. Estimates of collection efficiency by river, facility, collection method, year, species, and reference.-Continued

[NA, not available; SS, Atlantic salmon; U.S.A., United States of America; CO, coho salmon; PSE, Puget Sound Energy; PH, powerhouse; PSC, prototype surface collector; USACE, U.S. Army Corps of Engineers; CH, Chinook salmon; FPC, Fish Passage Center; OM, steelhead; B2CC, second power house corner collector; PUD, Public Utility District; >, greater than; <, less than; RSW, removable spillway weir; SBC, surface bypass collector]

\begin{tabular}{|c|c|c|c|c|c|}
\hline Facility & Method & Year & Species & $\begin{array}{l}\text { Collection } \\
\text { efficiency } \\
\text { (percent) }\end{array}$ & Reference \\
\hline Lower Granite & Surface Spill—RSW & 2007 & $\mathrm{CH}$ & 25 & FPC, 2011 \\
\hline Lower Granite & Surface Spill—-RSW & 2007 & $\mathrm{CH}$ & 32 & FPC, 2011 \\
\hline Lower Granite & Surface Spill—RSW & 2007 & $\mathrm{CH}$ & 13 & FPC, 2007 \\
\hline Lower Granite & Surface Spill—RSW & 2007 & $\mathrm{CH}$ & 9 & FPC, 2007 \\
\hline Lower Granite & Surface Spill—RSW & 2008 & $\mathrm{CH}$ & 37 & FPC, 2011 \\
\hline Lower Granite & Surface Spill—RSW & 2008 & $\mathrm{CH}$ & 38 & FPC, 2011 \\
\hline Lower Granite & Surface Spill-RSW & 2008 & $\mathrm{CH}$ & 14 & FPC, 2008 \\
\hline Lower Granite & Surface Spill—RSW & 2008 & $\mathrm{CH}$ & 16 & FPC, 2008 \\
\hline Lower Granite & Surface Spill-RSW & 2009 & $\mathrm{CH}$ & 32 & FPC, 2011 \\
\hline Lower Granite & Surface Spill—RSW & 2009 & $\mathrm{CH}$ & 45 & FPC, 2011 \\
\hline Lower Granite & Surface Spill—-RSW & 2009 & $\mathrm{CH}$ & 17 & FPC, 2009 \\
\hline Lower Granite & Surface Spill—RSW & 2009 & $\mathrm{CH}$ & 15 & FPC, 2009 \\
\hline Lower Granite & Surface Spill-RSW & 2010 & $\mathrm{CH}$ & 17 & FPC, 2011 \\
\hline Lower Granite & Surface Spill—RSW & 2010 & $\mathrm{CH}$ & 26 & FPC, 2011 \\
\hline Lower Granite & Surface Spill—-RSW & 2010 & $\mathrm{CH}$ & 15 & FPC, 2010 \\
\hline Lower Granite & Surface Spill—RSW & 2010 & $\mathrm{CH}$ & 11 & FPC, 2010 \\
\hline Lower Granite & Surface Spill- RSW & 2011 & $\mathrm{CH}$ & 34 & FPC, 2011 \\
\hline Lower Granite & Surface Spill—RSW & 2011 & $\mathrm{CH}$ & 42 & FPC, 2011 \\
\hline Lower Granite & Surface Spill—RSW & 2011 & $\mathrm{CH}$ & 19 & FPC, 2011 \\
\hline Lower Granite & Surface Spill—RSW & 2011 & $\mathrm{CH}$ & 17 & FPC, 2011 \\
\hline Lower Granite & Surface Spill-RSW & 1998 & $\mathrm{OM}$ & 59 & FPC, 2001 \\
\hline Lower Granite & Surface Spill-RSW & 1998 & $\mathrm{OM}$ & 59 & FPC, 2001 \\
\hline Lower Granite & Surface Spill—-RSW & 1999 & $\mathrm{OM}$ & 36 & FPC, 2003 \\
\hline Lower Granite & Surface Spill—RSW & 1999 & $\mathrm{OM}$ & 37 & FPC, 2001 \\
\hline Lower Granite & Surface Spill—RSW & 1999 & $\mathrm{OM}$ & 31 & FPC, 2001 \\
\hline Lower Granite & Surface Spill—RSW & 2000 & $\mathrm{OM}$ & 59 & FPC, 2006 \\
\hline Lower Granite & Surface Spill—RSW & 2000 & $\mathrm{OM}$ & 63 & FPC, 2002 \\
\hline Lower Granite & Surface Spill-RSW & 2000 & $\mathrm{OM}$ & 53 & FPC, 2002 \\
\hline Lower Granite & Powerhouse retrofit- $\mathrm{SBC}$ & 2000 & $\mathrm{OM}$ & 27 & Plumb and others, 2002, cited in USACE, 2007 \\
\hline Lower Granite & Powerhouse retrofit- $\mathrm{SBC}$ & 2000 & $\mathrm{OM}$ & 18 & Plumb and others, 2002, cited in USACE, 2007 \\
\hline
\end{tabular}


Table A1.2. Estimates of collection efficiency by river, facility, collection method, year, species, and reference.-Continued

[NA, not available; SS, Atlantic salmon; U.S.A., United States of America; CO, coho salmon; PSE, Puget Sound Energy; PH, powerhouse; PSC, prototype surface collector; USACE, U.S. Army Corps of Engineers; CH, Chinook salmon; FPC, Fish Passage Center; OM, steelhead; B2CC, second power house corner collector; PUD, Public Utility District; >, greater than; <, less than; RSW, removable spillway weir; SBC, surface bypass collector]

\begin{tabular}{|c|c|c|c|c|c|}
\hline Facility & Method & Year & Species & $\begin{array}{l}\text { Collection } \\
\text { efficiency } \\
\text { (percent) }\end{array}$ & Reference \\
\hline Lower Granite & Surface Spill—RSW & 2001 & $\mathrm{OM}$ & 89 & FPC, 2006 \\
\hline Lower Granite & Surface Spill—RSW & 2001 & $\mathrm{OM}$ & 91 & FPC, 2002 \\
\hline Lower Granite & Surface Spill—RSW & 2001 & $\mathrm{OM}$ & 87 & FPC, 2002 \\
\hline Lower Granite & Surface Spill—RSW & 2002 & $\mathrm{OM}$ & 24 & FPC, 2006 \\
\hline Lower Granite & Surface Spill—RSW & 2002 & $\mathrm{OM}$ & 23 & FPC, 2002 \\
\hline Lower Granite & Surface Spill—RSW & 2002 & OM & 27 & FPC, 2002 \\
\hline Lower Granite & Surface Spill—RSW & 2002 & $\mathrm{OM}$ & 61 & Plumb and others, 2003, cited in USACE, 2007 \\
\hline Lower Granite & Surface Spill—RSW & 2002 & $\mathrm{OM}$ & 62 & Plumb and others, 2003, cited in USACE, 2007 \\
\hline Lower Granite & Surface Spill—RSW & 2003 & $\mathrm{OM}$ & 32 & FPC, 2008 \\
\hline Lower Granite & Surface Spill—RSW & 2003 & $\mathrm{OM}$ & 67 & Plumb and others, 2004, cited in USACE, 2007 \\
\hline Lower Granite & Surface Spill—RSW & 2003 & $\mathrm{OM}$ & 69 & Plumb and others, 2004, cited in USACE, 2007 \\
\hline Lower Granite & Surface Spill-RSW & 2004 & $\mathrm{OM}$ & 73 & FPC, 2008 \\
\hline Lower Granite & Surface Spill—-RSW & 2005 & $\mathrm{OM}$ & 68 & FPC, 2008 \\
\hline Lower Granite & Surface Spill—RSW & 2005 & $\mathrm{OM}$ & 49 & USACE, 2007 \\
\hline Lower Granite & Surface Spill—RSW & 2005 & OM & 41 & USACE, 2007 \\
\hline Lower Granite & Surface Spill—RSW & 2006 & $\mathrm{OM}$ & 35 & FPC, 2011 \\
\hline Lower Granite & Surface Spill—RSW & 2006 & $\mathrm{OM}$ & 37 & FPC, 2011 \\
\hline Lower Granite & Surface Spill—RSW & 2006 & $\mathrm{OM}$ & 27 & USACE, 2007 \\
\hline Lower Granite & Surface Spill—RSW & 2007 & OM & 22 & FPC, 2011 \\
\hline Lower Granite & Surface Spill—RSW & 2007 & $\mathrm{OM}$ & 24 & FPC, 2011 \\
\hline Lower Granite & Surface Spill—RSW & 2008 & $\mathrm{OM}$ & 28 & FPC, 2011 \\
\hline Lower Granite & Surface Spill—RSW & 2008 & $\mathrm{OM}$ & 35 & FPC, 2011 \\
\hline Lower Granite & Surface Spill—RSW & 2009 & $\mathrm{OM}$ & 44 & FPC, 2011 \\
\hline Lower Granite & Surface Spill—RSW & 2009 & $\mathrm{OM}$ & 46 & FPC, 2011 \\
\hline Lower Granite & Surface Spill—RSW & 2010 & $\mathrm{OM}$ & 19 & FPC, 2011 \\
\hline Lower Granite & Surface Spill—RSW & 2010 & $\mathrm{OM}$ & 22 & FPC, 2011 \\
\hline Lower Granite & Surface Spill_-RSW & 2011 & $\mathrm{OM}$ & 38 & FPC, 2011 \\
\hline Lower Granite & Surface Spill—RSW & 2011 & OM & 41 & FPC, 2011 \\
\hline
\end{tabular}


Table A1.3. Estimates of survival through collectors by river, facility, collection method, year, species, origin, and reference.

[CO, coho salmon; Mixed, hatchery and wild; OM, steelhead; CH, Chinook salmon; H, hatchery, NA, not available; W, wild; FPC, Fish Passage Center; PGE, Portland General Electric Company]

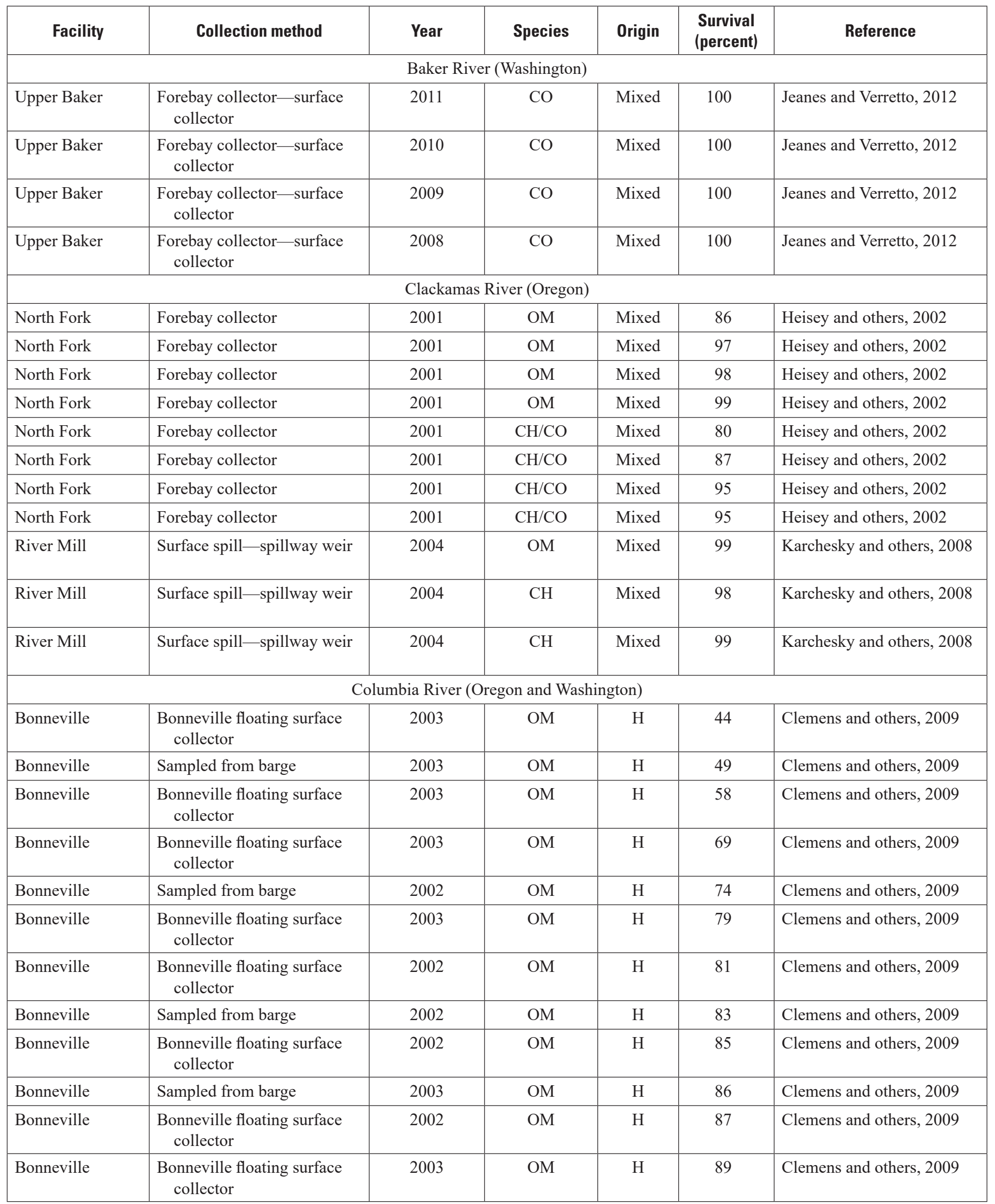


Table A1.3. Estimates of survival through collectors by river, facility, collection method, year, species, origin, and reference.-Continued [CO, coho salmon; Mixed, hatchery and wild; OM, steelhead; CH, Chinook salmon; H, hatchery, NA, not available; W, wild; FPC, Fish Passage Center; PGE, Portland General Electric Company]

\begin{tabular}{|c|c|c|c|c|c|c|}
\hline Facility & Collection method & Year & Species & Origin & $\begin{array}{c}\text { Survival } \\
\text { (percent) }\end{array}$ & Reference \\
\hline Bonneville & Sampled from barge & 2003 & $\mathrm{OM}$ & $\mathrm{H}$ & 89 & Clemens and others, 2009 \\
\hline Bonneville & Sampled from barge & 2003 & $\mathrm{OM}$ & $\mathrm{H}$ & 93 & Clemens and others, 2009 \\
\hline Bonneville & Sampled from barge & 2003 & $\mathrm{OM}$ & $\mathrm{H}$ & 94 & Clemens and others, 2009 \\
\hline Bonneville & $\begin{array}{l}\text { Bonneville floating surface } \\
\text { collector }\end{array}$ & 2003 & $\mathrm{OM}$ & $\mathrm{H}$ & 95 & Clemens and others, 2009 \\
\hline Bonneville & $\begin{array}{l}\text { Bonneville floating surface } \\
\text { collector }\end{array}$ & 2003 & $\mathrm{OM}$ & $\mathrm{H}$ & 95 & Clemens and others, 2009 \\
\hline Bonneville & $\begin{array}{l}\text { Bonneville floating surface } \\
\text { collector }\end{array}$ & 2003 & $\mathrm{OM}$ & $\mathrm{H}$ & 96 & Clemens and others, 2009 \\
\hline Bonneville & $\begin{array}{l}\text { Bonneville floating surface } \\
\text { collector }\end{array}$ & 2003 & $\mathrm{OM}$ & $\mathrm{H}$ & 96 & Clemens and others, 2009 \\
\hline Bonneville & Sampled from barge & 2003 & $\mathrm{OM}$ & $\mathrm{H}$ & 98 & Clemens and others, 2009 \\
\hline Bonneville & Sampled from barge & 2003 & $\mathrm{OM}$ & $\mathrm{H}$ & 98 & Clemens and others, 2009 \\
\hline Bonneville & Sampled from barge & 2003 & $\mathrm{OM}$ & $\mathrm{H}$ & 100 & Clemens and others, 2009 \\
\hline Bonneville & \begin{tabular}{|l}
$\begin{array}{l}\text { Bonneville floating surface } \\
\text { collector }\end{array}$ \\
\end{tabular} & 2003 & $\mathrm{OM}$ & $\mathrm{H}$ & 100 & Clemens and others, 2009 \\
\hline Bonneville & Sampled from barge & 2003 & $\mathrm{OM}$ & $\mathrm{H}$ & 100 & Clemens and others, 2009 \\
\hline Bonneville & Sampled from barge & 2004 & $\mathrm{CH}$ & $\mathrm{H}$ & 73 & Clemens and others, 2009 \\
\hline Bonneville & Sampled from barge & 2004 & $\mathrm{CH}$ & $\mathrm{H}$ & 77 & Clemens and others, 2009 \\
\hline Bonneville & Sampled from barge & 2003 & $\mathrm{CH}$ & Mixed & 78 & Clemens and others, 2009 \\
\hline Bonneville & Sampled from barge & 2003 & $\mathrm{CH}$ & Mixed & 87 & Clemens and others, 2009 \\
\hline Bonneville & Sampled from barge & 2003 & $\mathrm{CH}$ & Mixed & 87 & Clemens and others, 2009 \\
\hline Bonneville & \begin{tabular}{|l} 
Bonneville floating surface \\
collector
\end{tabular} & 2003 & $\mathrm{CH}$ & Mixed & 87 & Clemens and others, 2009 \\
\hline Bonneville & Sampled from barge & 2004 & $\mathrm{CH}$ & $\mathrm{H}$ & 88 & Clemens and others, 2009 \\
\hline Bonneville & Sampled from barge & 2004 & $\mathrm{CH}$ & $\mathrm{H}$ & 90 & Clemens and others, 2009 \\
\hline Bonneville & $\begin{array}{l}\text { Bonneville floating surface } \\
\text { collector }\end{array}$ & 2003 & $\mathrm{CH}$ & Mixed & 91 & Clemens and others, 2009 \\
\hline Bonneville & Sampled from barge & 2004 & $\mathrm{CH}$ & $\mathrm{H}$ & 92 & Clemens and others, 2009 \\
\hline Bonneville & Sampled from barge & 2004 & $\mathrm{CH}$ & $\mathrm{H}$ & 92 & Clemens and others, 2009 \\
\hline
\end{tabular}


Table A1.3. Estimates of survival through collectors by river, facility, collection method, year, species, origin, and reference.—Continued [CO, coho salmon; Mixed, hatchery and wild; OM, steelhead; CH, Chinook salmon; H, hatchery, NA, not available; W, wild; FPC, Fish Passage Center; PGE, Portland General Electric Company]

\begin{tabular}{|c|c|c|c|c|c|c|}
\hline Facility & Collection method & Year & Species & Origin & $\begin{array}{c}\text { Survival } \\
\text { (percent) }\end{array}$ & Reference \\
\hline Bonneville & $\begin{array}{l}\text { Bonneville floating surface } \\
\text { collector }\end{array}$ & 2003 & $\mathrm{CH}$ & Mixed & 96 & Clemens and others, 2009 \\
\hline Bonneville & Sampled from barge & 2004 & $\mathrm{CH}$ & $\mathrm{H}$ & 98 & Clemens and others, 2009 \\
\hline Bonneville & $\begin{array}{l}\text { Bonneville floating surface } \\
\text { collector }\end{array}$ & 2003 & $\mathrm{CH}$ & Mixed & 99 & Clemens and others, 2009 \\
\hline Bonneville & $\begin{array}{l}\text { Bonneville floating surface } \\
\text { collector }\end{array}$ & 2003 & $\mathrm{CH}$ & Mixed & 100 & Clemens and others, 2009 \\
\hline Bonneville & Sampled from barge & 2003 & $\mathrm{CH}$ & Mixed & 100 & Clemens and others, 2009 \\
\hline Bonneville & Sampled from barge & 2003 & $\mathrm{CH}$ & Mixed & 100 & Clemens and others, 2009 \\
\hline $\begin{array}{l}\text { Collected at } \\
\text { Lower Granite } \\
\text { Dam }\end{array}$ & NA & 2010 & $\mathrm{CH}$ & Mixed & 98 & McMichael and others, 2011 \\
\hline \multicolumn{7}{|c|}{ Cowlitz River (Washington) } \\
\hline Cowlitz Falls & $\begin{array}{l}\text { Forebay collector-retrofit } \\
\text { baffle }\end{array}$ & 1997 & $\mathrm{CO}$ & $\mathrm{H}$ & 93 & Serl and Morrill, 2010 \\
\hline Cowlitz Falls & $\begin{array}{l}\text { Forebay collector-retrofit } \\
\text { baffle }\end{array}$ & 1998 & $\mathrm{CO}$ & $\mathrm{H}$ & 98 & Serl and Morrill, 2010 \\
\hline Cowlitz Falls & $\begin{array}{l}\text { Forebay collector-retrofit } \\
\text { baffle }\end{array}$ & 1999 & $\mathrm{CO}$ & $\mathrm{H}$ & 98 & Serl and Morrill, 2010 \\
\hline Cowlitz Falls & $\begin{array}{l}\text { Forebay collector-retrofit } \\
\text { baffle }\end{array}$ & 2000 & $\mathrm{CO}$ & $\mathrm{H}$ & 100 & Serl and Morrill, 2010 \\
\hline Cowlitz Falls & $\begin{array}{l}\text { Forebay collector-retrofit } \\
\text { baffle }\end{array}$ & 2001 & $\mathrm{CO}$ & $\mathrm{H}$ & 100 & Serl and Morrill, 2010 \\
\hline Cowlitz Falls & $\begin{array}{l}\text { Forebay collector-retrofit } \\
\text { baffle }\end{array}$ & 2003 & $\mathrm{CO}$ & $\mathrm{H}$ & 100 & Serl and Morrill, 2010 \\
\hline Cowlitz Falls & $\begin{array}{l}\text { Forebay collector-retrofit } \\
\text { baffle }\end{array}$ & 2004 & $\mathrm{CO}$ & $\mathrm{H}$ & 100 & Serl and Morrill, 2010 \\
\hline Cowlitz Falls & $\begin{array}{l}\text { Forebay collector-retrofit } \\
\text { baffle }\end{array}$ & 2005 & $\mathrm{CO}$ & $\mathrm{H}$ & 100 & Serl and Morrill, 2010 \\
\hline Cowlitz Falls & $\begin{array}{l}\text { Forebay collector-retrofit } \\
\text { baffle }\end{array}$ & 2009 & $\mathrm{CO}$ & $\mathrm{H}$ & 100 & Serl and Morrill, 2010 \\
\hline Cowlitz Falls & $\begin{array}{l}\text { Forebay collector-retrofit } \\
\text { baffle }\end{array}$ & 2012 & $\mathrm{CO}$ & $\mathrm{H}$ & 100 & Serl and Heimbigner, 2013 \\
\hline
\end{tabular}


Table A1.3. Estimates of survival through collectors by river, facility, collection method, year, species, origin, and reference.-Continued [CO, coho salmon; Mixed, hatchery and wild; OM, steelhead; CH, Chinook salmon; H, hatchery, NA, not available; W, wild; FPC, Fish Passage Center; PGE, Portland General Electric Company]

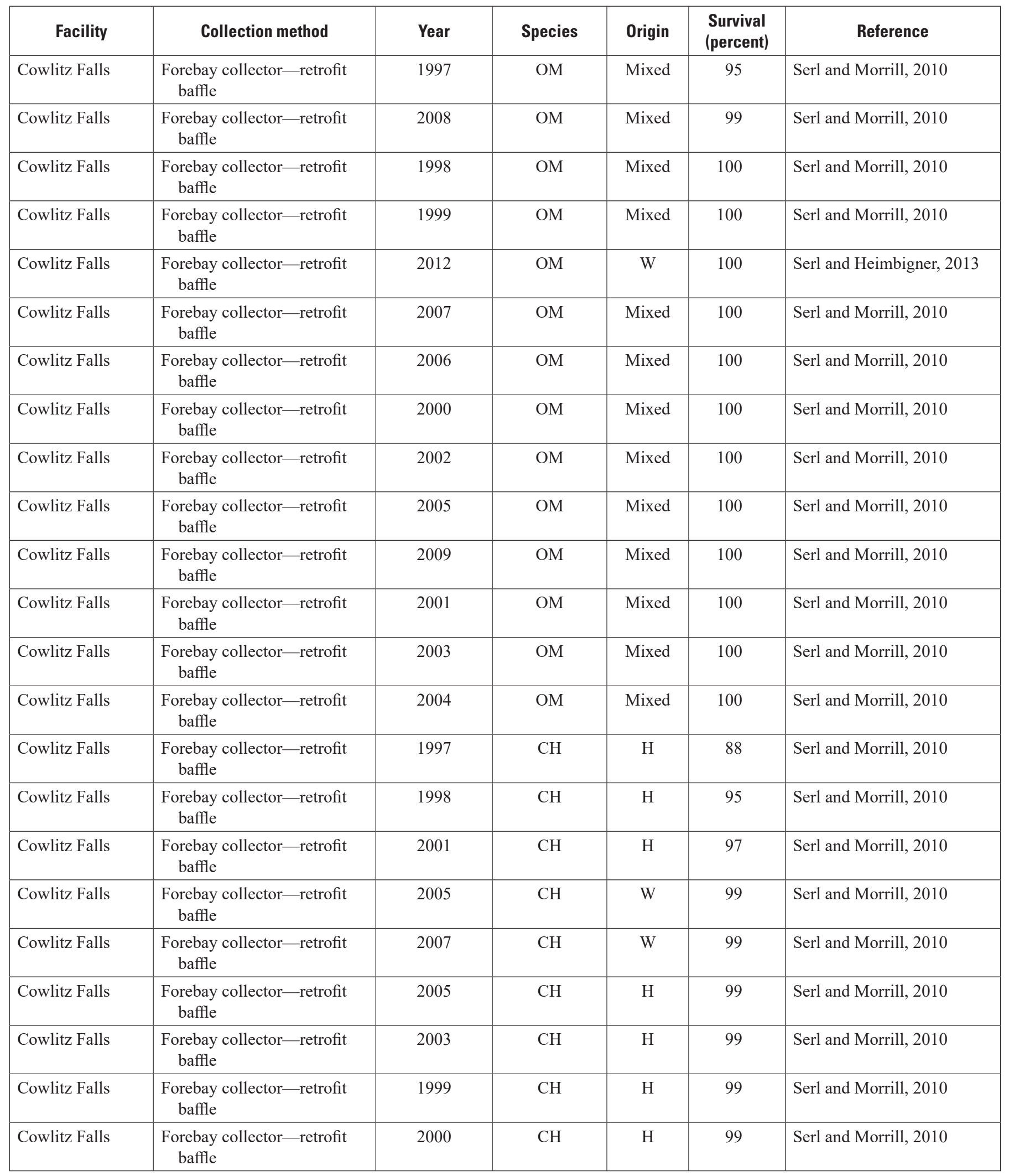


Table A1.3. Estimates of survival through collectors by river, facility, collection method, year, species, origin, and reference.—Continued [CO, coho salmon; Mixed, hatchery and wild; OM, steelhead; CH, Chinook salmon; H, hatchery, NA, not available; W, wild; FPC, Fish Passage Center; PGE, Portland General Electric Company]

\begin{tabular}{|c|c|c|c|c|c|c|}
\hline Facility & Collection method & Year & Species & Origin & $\begin{array}{c}\text { Survival } \\
\text { (percent) }\end{array}$ & Reference \\
\hline Cowlitz Falls & $\begin{array}{l}\text { Forebay collector-retrofit } \\
\text { baffle }\end{array}$ & 2008 & $\mathrm{CH}$ & W & 99 & Serl and Morrill, 2010 \\
\hline Cowlitz Falls & $\begin{array}{l}\text { Forebay collector-retrofit } \\
\text { baffle }\end{array}$ & 2004 & $\mathrm{CH}$ & $\mathrm{H}$ & 99 & Serl and Morrill, 2010 \\
\hline Cowlitz Falls & $\begin{array}{l}\text { Forebay collector-retrofit } \\
\text { baffle }\end{array}$ & 2006 & $\mathrm{CH}$ & W & 99 & Serl and Morrill, 2010 \\
\hline Cowlitz Falls & $\begin{array}{l}\text { Forebay collector-retrofit } \\
\text { baffle }\end{array}$ & 2002 & $\mathrm{CH}$ & $\mathrm{H}$ & 100 & Serl and Morrill, 2010 \\
\hline Cowlitz Falls & $\begin{array}{l}\text { Forebay collector-retrofit } \\
\text { baffle }\end{array}$ & 2006 & $\mathrm{CH}$ & $\mathrm{H}$ & 100 & Serl and Morrill, 2010 \\
\hline Cowlitz Falls & $\begin{array}{l}\text { Forebay collector-retrofit } \\
\text { baffle }\end{array}$ & 2007 & $\mathrm{CH}$ & $\mathrm{H}$ & 100 & Serl and Morrill, 2010 \\
\hline Cowlitz Falls & $\begin{array}{l}\text { Forebay collector-retrofit } \\
\text { baffle }\end{array}$ & 2009 & $\mathrm{CH}$ & $\mathrm{H}$ & 100 & Serl and Morrill, 2010 \\
\hline Cowlitz Falls & $\begin{array}{l}\text { Forebay collector-retrofit } \\
\text { baffle }\end{array}$ & 2009 & $\mathrm{CH}$ & $\mathrm{W}$ & 100 & Serl and Morrill, 2010 \\
\hline Cowlitz Falls & $\begin{array}{l}\text { Forebay collector-retrofit } \\
\text { baffle }\end{array}$ & 2012 & $\mathrm{CH}$ & W & 100 & Serl and Heimbigner, 2013 \\
\hline Mayfield & Louver system & 2008 & $\mathrm{CO}$ & $\mathrm{W}$ & 96 & FPC, 2011 \\
\hline Mayfield & Louver system & 2009 & $\mathrm{CO}$ & W & 98 & Henning, 2010 \\
\hline $\begin{array}{l}\text { Pelton Round } \\
\text { Butte }\end{array}$ & Guidance net/skimmer & 2010 & $\mathrm{SO}$ & $\mathrm{H}$ & 98 & PGE, 2012 \\
\hline $\begin{array}{l}\text { Pelton Round } \\
\text { Butte }\end{array}$ & Guidance net/skimmer & 2011 & $\mathrm{SO}$ & $\mathrm{H}$ & 97 & PGE, 2012 \\
\hline $\begin{array}{l}\text { Pelton Round } \\
\text { Butte }\end{array}$ & Guidance net/skimmer & 2011 & $\mathrm{CH}$ & $\mathrm{H}$ & 99 & PGE, 2012 \\
\hline $\begin{array}{l}\text { Pelton Round } \\
\text { Butte }\end{array}$ & Guidance net/skimmer & 2011 & $\mathrm{OM}$ & $\mathrm{H}$ & 99 & PGE, 2012 \\
\hline $\begin{array}{l}\text { Pelton Round } \\
\text { Butte }\end{array}$ & Guidance net/skimmer & 2012 & $\mathrm{SO}$ & $\mathrm{H}$ & 97 & PGE, 2012 \\
\hline $\begin{array}{l}\text { Pelton Round } \\
\text { Butte }\end{array}$ & Guidance net/skimmer & 2012 & $\mathrm{CH}$ & $\mathrm{H}$ & 98 & PGE, 2012 \\
\hline $\begin{array}{l}\text { Pelton Round } \\
\text { Butte }\end{array}$ & Guidance net/skimmer & 2012 & $\mathrm{OM}$ & $\mathrm{H}$ & 99 & PGE, 2012 \\
\hline
\end{tabular}


Table A1.3. Estimates of survival through collectors by river, facility, collection method, year, species, origin, and reference.-Continued [CO, coho salmon; Mixed, hatchery and wild; OM, steelhead; CH, Chinook salmon; H, hatchery, NA, not available; W, wild; FPC, Fish Passage Center; PGE, Portland General Electric Company]

\begin{tabular}{|c|c|c|c|c|c|c|}
\hline Facility & Collection method & Year & Species & Origin & $\begin{array}{l}\text { Survival } \\
\text { (percent) }\end{array}$ & Reference \\
\hline $\begin{array}{l}\text { Pelton Round } \\
\text { Butte }\end{array}$ & Guidance net/skimmer & 2013 & $\mathrm{CH}$ & Mixed & 98 & PGE, 2014 \\
\hline $\begin{array}{l}\text { Pelton Round } \\
\text { Butte }\end{array}$ & Guidance net/skimmer & 2013 & $\mathrm{OM}$ & Mixed & 99 & PGE, 2014 \\
\hline $\begin{array}{l}\text { Pelton Round } \\
\text { Butte }\end{array}$ & Guidance net/skimmer & 2013 & SO & $\mathrm{H}$ & 98 & PGE, 2014 \\
\hline $\begin{array}{l}\text { Pelton Round } \\
\text { Butte }\end{array}$ & Guidance net/skimmer & 2014 & $\mathrm{CH}$ & Mixed & 98 & PGE, 2015 \\
\hline $\begin{array}{l}\text { Pelton Round } \\
\text { Butte }\end{array}$ & Guidance net/skimmer & 2014 & $\mathrm{OM}$ & Mixed & 99 & PGE, 2015 \\
\hline \multicolumn{7}{|c|}{ Clackamas River (Oregon) } \\
\hline River Mill & Surface spill—-spillway weir & 2004 & $\mathrm{CH}$ & Mixed & 99 & Karchesky and others, 2008 \\
\hline \multicolumn{7}{|c|}{ Willamette River (Oregon) } \\
\hline Willamette Falls & From hatchery & 2008 & $\mathrm{OM}$ & $\mathrm{H}$ & 98 & Karchesky, 2008 \\
\hline Willamette Falls & From hatchery & 2008 & $\mathrm{OM}$ & $\mathrm{H}$ & 99 & Karchesky and others, 2008 \\
\hline Willamette Falls & From hatchery & 2008 & $\mathrm{OM}$ & $\mathrm{H}$ & 100 & Karchesky and others, 2008 \\
\hline Willamette Falls & From hatchery & 2008 & $\mathrm{CH}$ & $\mathrm{H}$ & 100 & Karchesky and others, 2008 \\
\hline Willamette Falls & From hatchery & 2008 & $\mathrm{CH}$ & $\mathrm{H}$ & 100 & Karchesky and others, 2008 \\
\hline
\end{tabular}

Table A1.4. Descaling estimates by river, facility, type of collector, year, species, and reference.

[CH, Chinook salmon; PH, power house; CO, coho salmon; NA, not available; OM, steelhead; PGE, Portland General Electric Company; SO, sockeye salmon]

\begin{tabular}{|c|c|c|c|c|c|c|}
\hline Facility & Collector & Year & Species & Age & $\begin{array}{c}\text { Descaling } \\
\text { rates (percent) }\end{array}$ & Reference \\
\hline \multicolumn{7}{|c|}{ Columbia River (Oregon and Washington) } \\
\hline John Day & Juvenile bypass & 1999 & $\mathrm{CH}$ & $1+$ & 6.2 & Martinson and others, 2004 \\
\hline John Day & Juvenile bypass & 2000 & $\mathrm{CH}$ & $1+$ & 2.4 & Martinson and others, 2004 \\
\hline John Day & Juvenile bypass & 2002 & $\mathrm{CH}$ & $1+$ & 3.1 & Martinson and others, 2004 \\
\hline John Day & Juvenile bypass & 2003 & $\mathrm{CH}$ & $1+$ & 4.6 & Martinson and others, 2004 \\
\hline John Day & Juvenile bypass & 1998 & $\mathrm{CH}$ & $0+$ & 2.2 & Martinson and others, 2004 \\
\hline John Day & Juvenile bypass & 1999 & $\mathrm{CH}$ & $0+$ & 0.9 & Martinson and others, 2004 \\
\hline
\end{tabular}


Table A1.4. Descaling estimates by river, facility, type of collector, year, species, and reference.—Continued

[CH, Chinook salmon; PH, power house; CO, coho salmon; NA, not available; OM, steelhead; PGE, Portland General Electric Company; SO, sockeye salmon]

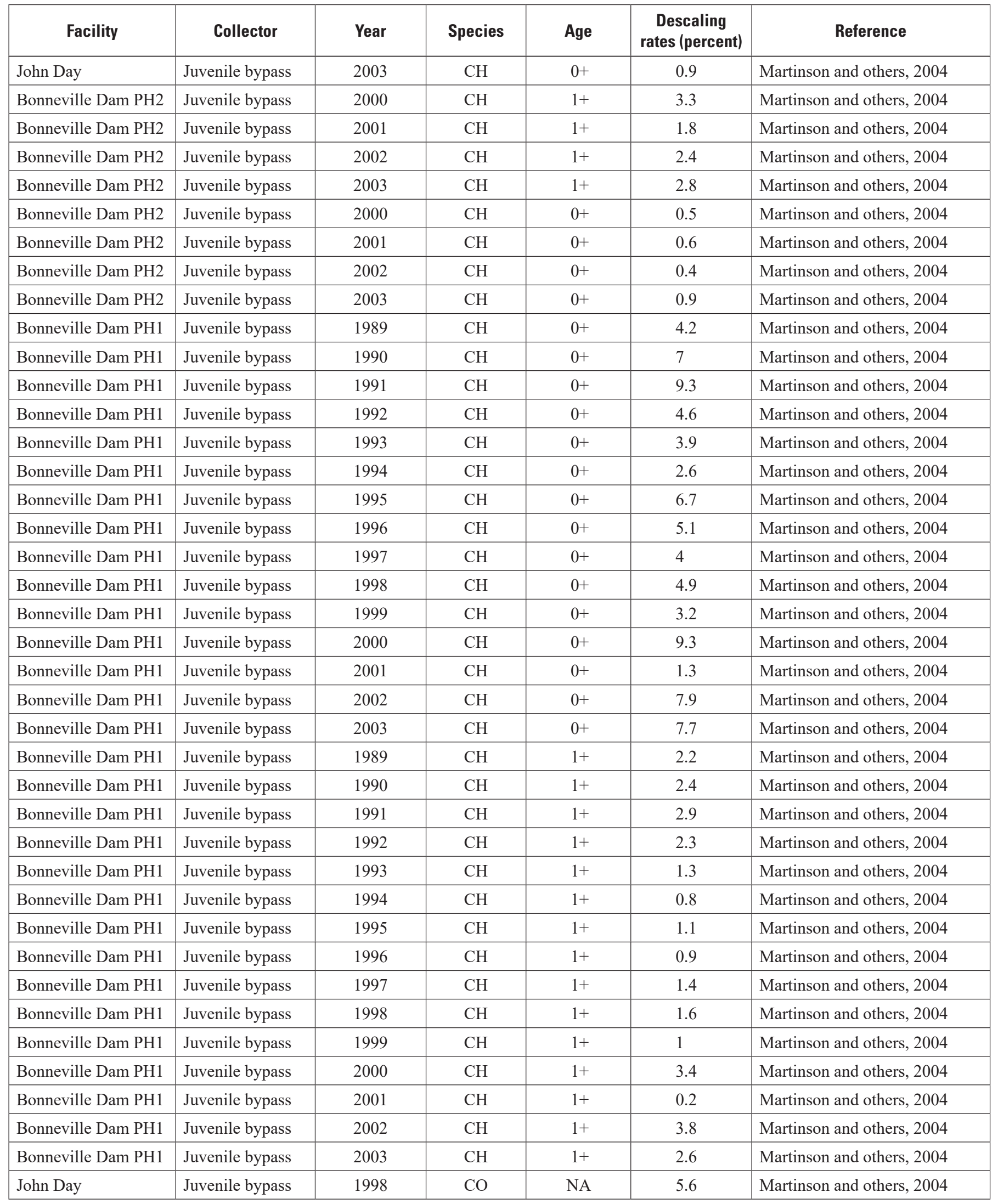


Table A1.4. Descaling estimates by river, facility, type of collector, year, species, and reference.—Continued

[CH, Chinook salmon; PH, power house; CO, coho salmon; NA, not available; OM, steelhead; PGE, Portland General Electric Company; SO, sockeye salmon]

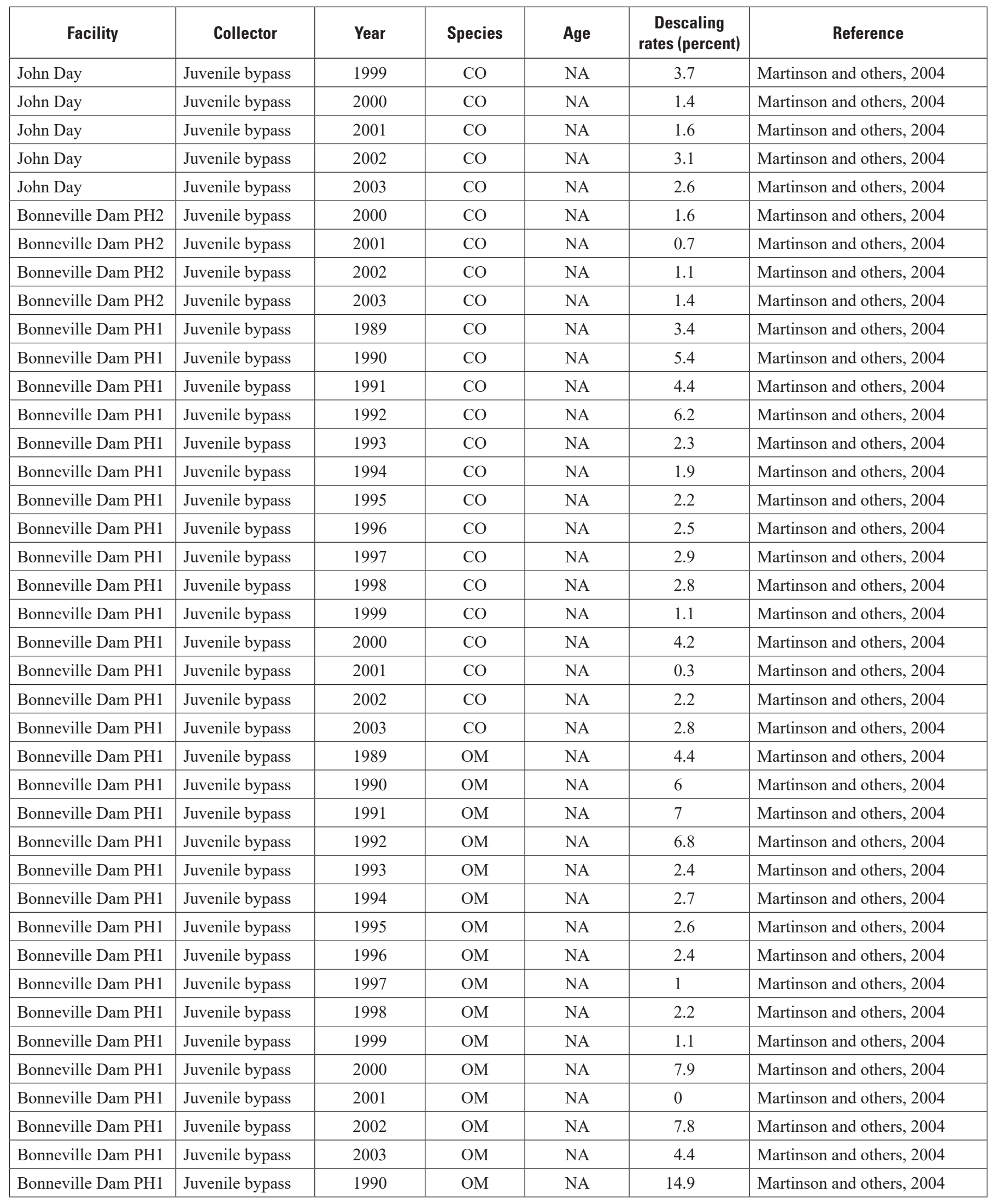


Table A1.4. Descaling estimates by river, facility, type of collector, year, species, and reference.—Continued

[CH, Chinook salmon; PH, power house; $\mathrm{CO}$, coho salmon; NA, not available; OM, steelhead; PGE, Portland General Electric Company; SO, sockeye salmon]

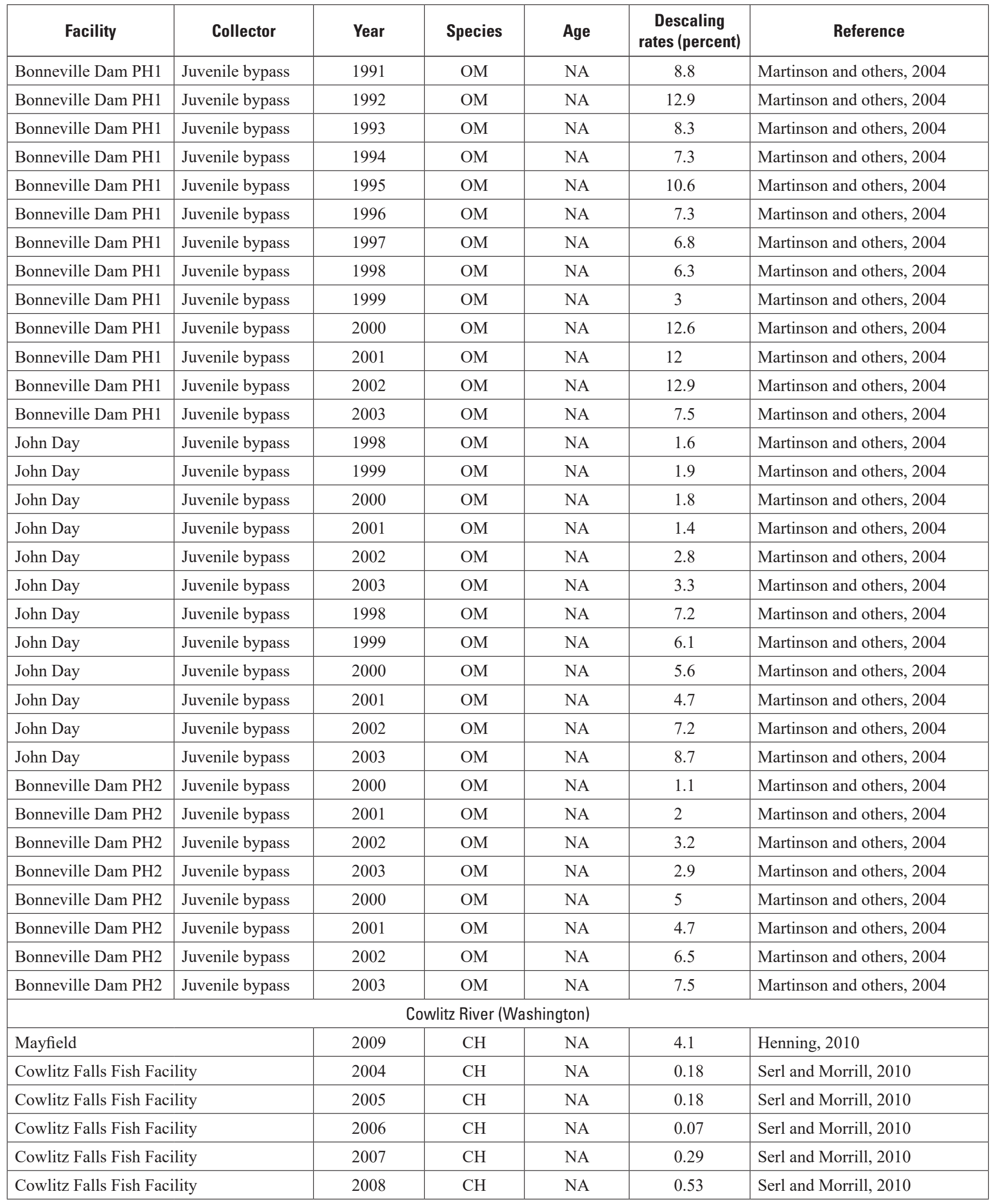


Table A1.4. Descaling estimates by river, facility, type of collector, year, species, and reference.—Continued

[CH, Chinook salmon; PH, power house; CO, coho salmon; NA, not available; OM, steelhead; PGE, Portland General Electric Company; SO, sockeye salmon]

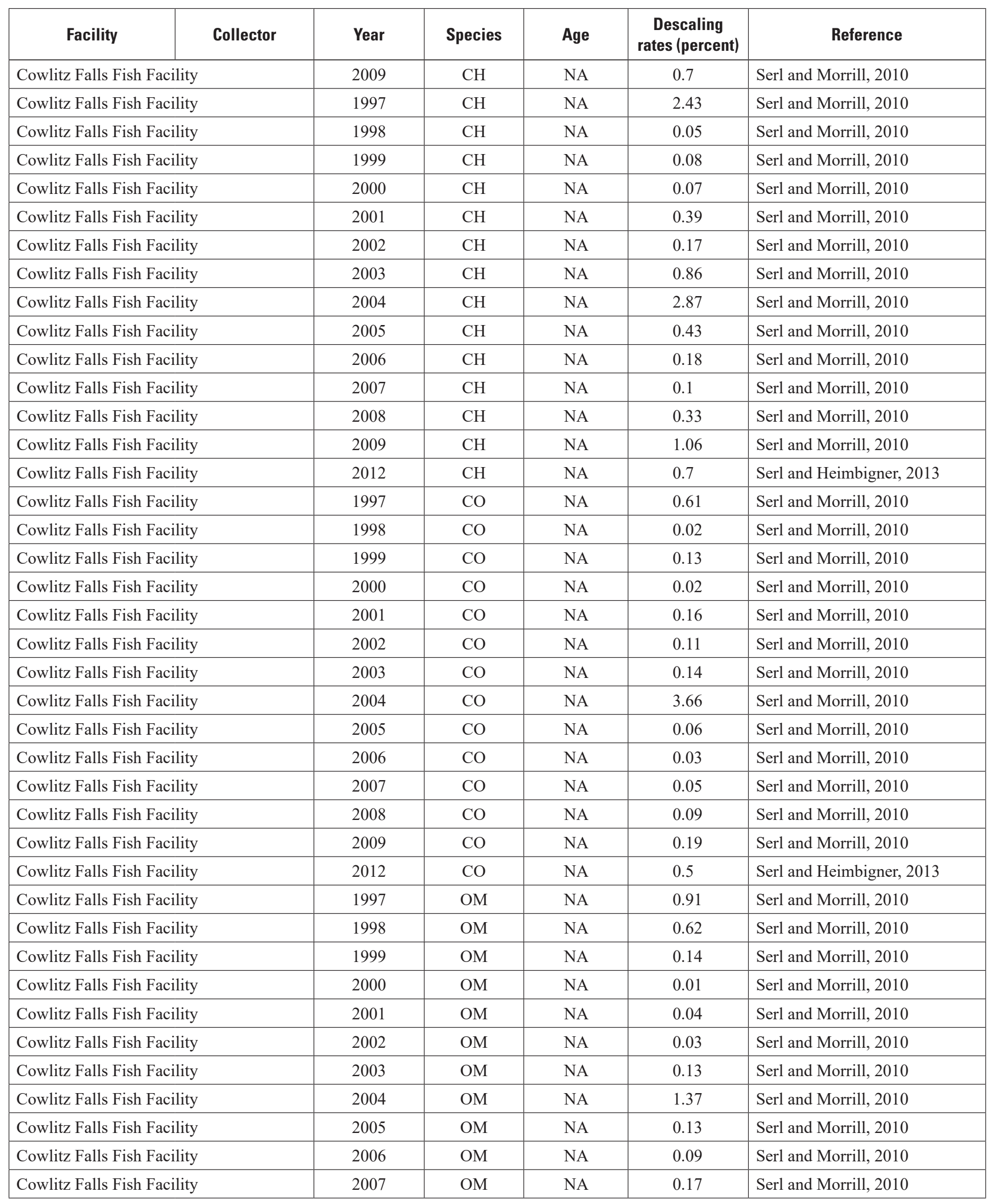


Table A1.4. Descaling estimates by river, facility, type of collector, year, species, and reference.-Continued

[CH, Chinook salmon; PH, power house; CO, coho salmon; NA, not available; OM, steelhead; PGE, Portland General Electric Company; SO, sockeye salmon]

\begin{tabular}{|c|c|c|c|c|c|c|}
\hline Facility & Collector & Year & Species & Age & $\begin{array}{c}\text { Descaling } \\
\text { rates (percent) }\end{array}$ & Reference \\
\hline \multicolumn{2}{|c|}{ Cowlitz Falls Fish Facility } & 2008 & $\mathrm{OM}$ & NA & 0.19 & Serl and Morrill, 2010 \\
\hline \multicolumn{2}{|c|}{ Cowlitz Falls Fish Facility } & 1997 & $\mathrm{OM}$ & NA & 2.34 & Serl and Morrill, 2010 \\
\hline \multicolumn{2}{|c|}{ Cowlitz Falls Fish Facility } & 1998 & $\mathrm{OM}$ & NA & 0.62 & Serl and Morrill, 2010 \\
\hline \multicolumn{2}{|c|}{ Cowlitz Falls Fish Facility } & 2000 & $\mathrm{OM}$ & NA & 0 & Serl and Morrill, 2010 \\
\hline \multicolumn{2}{|c|}{ Cowlitz Falls Fish Facility } & 2001 & $\mathrm{OM}$ & NA & 0.03 & Serl and Morrill, 2010 \\
\hline \multicolumn{2}{|c|}{ Cowlitz Falls Fish Facility } & 2002 & $\mathrm{OM}$ & NA & 0.02 & Serl and Morrill, 2010 \\
\hline \multicolumn{2}{|c|}{ Cowlitz Falls Fish Facility } & 2003 & $\mathrm{OM}$ & NA & 0.31 & Serl and Morrill, 2010 \\
\hline \multicolumn{2}{|c|}{ Cowlitz Falls Fish Facility } & 2007 & $\mathrm{OM}$ & NA & 0.23 & Serl and Morrill, 2010 \\
\hline \multicolumn{2}{|c|}{ Cowlitz Falls Fish Facility } & 2008 & $\mathrm{OM}$ & NA & 0.21 & Serl and Morrill, 2010 \\
\hline \multicolumn{2}{|c|}{ Cowlitz Falls Fish Facility } & 2009 & $\mathrm{OM}$ & NA & 0.16 & Serl and Morrill, 2010 \\
\hline \multicolumn{2}{|c|}{ Cowlitz Falls Fish Facility } & 2012 & $\mathrm{OM}$ & NA & 0.7 & Serl and Heimbigner, 2013 \\
\hline \multicolumn{7}{|c|}{ Deschutes River (Oregon) } \\
\hline \multicolumn{2}{|l|}{ Round Butte } & 2011 & $\mathrm{CH}$ & NA & 0 & PGE, 2013 \\
\hline \multicolumn{2}{|l|}{ Round Butte } & 2012 & $\mathrm{CH}$ & NA & 0.1 & PGE, 2013 \\
\hline \multicolumn{2}{|l|}{ Round Butte } & 2013 & $\mathrm{CH}$ & NA & 0.28 & PGE, 2014 \\
\hline \multicolumn{2}{|l|}{ Round Butte } & 2014 & SO & NA & 0.98 & PGE, 2015 \\
\hline
\end{tabular}




\section{A. Review of Information Relevant to Anadromous Fish Reintroduction to and Fish Passage in the Lewis River, Wash.}

Table A1.5. Mortality estimates for nontarget species at downstream fish collection facilities by river, facility, year, species, and reference.

[PGE, Portland General Electric Company]

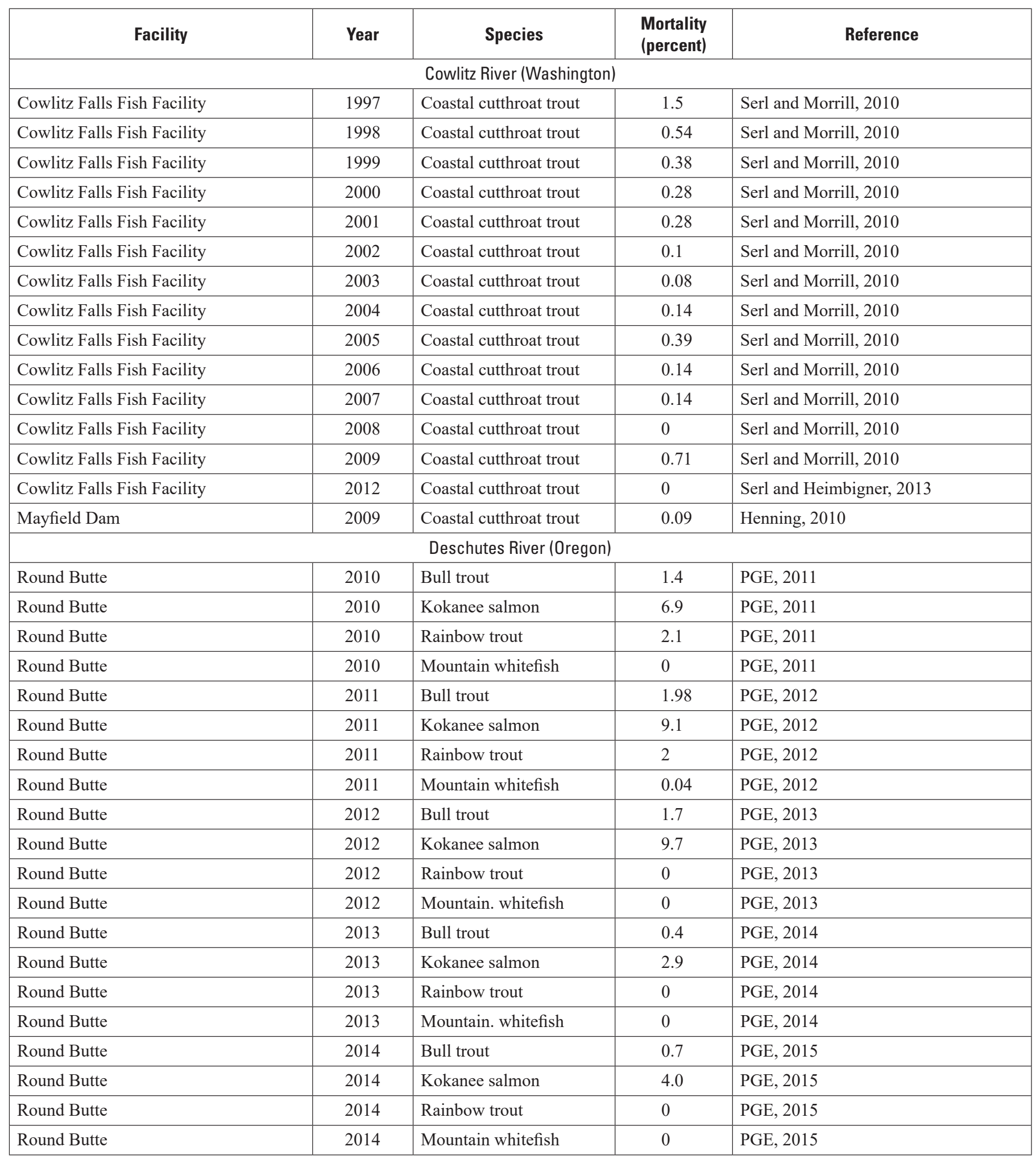


Table A1.6. Estimates of adult mortality during trap and haul operations for different species by river, facility, year, species, run, source, and references.

[CH, Chinook salmon; CO, coho salmon; NA, not available; OM, steelhead; PGE, Portland General Electric Company; SO, sockeye salmon; TFH, Tucannon Fish Hatchery]

\begin{tabular}{|c|c|c|c|c|c|c|}
\hline Facility & Year & Species & Run & Source & $\begin{array}{l}\text { Mortality } \\
\text { (percent) }\end{array}$ & Reference \\
\hline \multicolumn{7}{|c|}{ Cowlitz River (Washington) } \\
\hline Mayfield & 2009 & $\mathrm{CH}$ & Fall & Mixed & 1.6 & Henning, 2010 \\
\hline Mayfield & 2009 & $\mathrm{CO}$ & NA & Hatchery & 0.3 & Henning, 2010 \\
\hline Mayfield & 2009 & $\mathrm{CO}$ & NA & Wild & 0.6 & Henning, 2010 \\
\hline Mayfield & 2009 & $\mathrm{OM}$ & NA & Wild & 6.7 & Henning, 2010 \\
\hline Mayfield & 2009 & $\mathrm{OM}$ & NA & Hatchery & 3 & Henning, 2010 \\
\hline \multicolumn{7}{|c|}{ Deschutes River (Oregon) } \\
\hline Pelton Trap & 2012 & $\mathrm{CH}$ & Spring & Wild & 0 & PGE, 2013 \\
\hline Pelton Trap & 2012 & $\mathrm{OM}$ & NA & Wild & 0 & PGE, 2013 \\
\hline Pelton Trap & 2012 & SO & NA & Wild & 0 & PGE, 2013 \\
\hline \multicolumn{7}{|c|}{ South Fork Mackenzie River (Oregon) } \\
\hline Cougar Reservoir & 2010 & $\mathrm{CH}$ & Spring & Mixed & 0 & Zymonas and Hogansen, 2013 \\
\hline Cougar Reservoir & 2011 & $\mathrm{CH}$ & Spring & Mixed & 0.8 & Zymonas and Hogansen, 2013 \\
\hline \multicolumn{7}{|c|}{ Tucannon River (Washington) } \\
\hline TFH & 1986 & $\mathrm{CH}$ & Spring & Wild & 0 & Gallinat and Ross, 2012 \\
\hline TFH & 1987 & $\mathrm{CH}$ & Spring & Wild & 0 & Gallinat and Ross, 2012 \\
\hline TFH & 1988 & $\mathrm{CH}$ & Spring & Wild & 0 & Gallinat and Ross, 2012 \\
\hline TFH & 1989 & $\mathrm{CH}$ & Spring & Wild & 0 & Gallinat and Ross, 2012 \\
\hline TFH & 1990 & $\mathrm{CH}$ & Spring & Wild & 0 & Gallinat and Ross, 2012 \\
\hline TFH & 1991 & $\mathrm{CH}$ & Spring & Wild & 0 & Gallinat and Ross, 2012 \\
\hline TFH & 1992 & $\mathrm{CH}$ & Spring & Wild & 0 & Gallinat and Ross, 2012 \\
\hline TFH & 1993 & $\mathrm{CH}$ & Spring & Wild & 0 & Gallinat and Ross, 2012 \\
\hline TFH & 1994 & $\mathrm{CH}$ & Spring & Wild & 0 & Gallinat and Ross, 2012 \\
\hline TFH & 1995 & $\mathrm{CH}$ & Spring & Wild & 0 & Gallinat and Ross, 2012 \\
\hline TFH & 1996 & $\mathrm{CH}$ & Spring & Wild & 1.3 & Gallinat and Ross, 2012 \\
\hline TFH & 1997 & $\mathrm{CH}$ & Spring & Wild & 0 & Gallinat and Ross, 2012 \\
\hline TFH & 1998 & $\mathrm{CH}$ & Spring & Wild & 0 & Gallinat and Ross, 2012 \\
\hline TFH & 1999 & $\mathrm{CH}$ & Spring & Wild & 0 & Gallinat and Ross, 2012 \\
\hline TFH & 2000 & $\mathrm{CH}$ & Spring & Wild & 0 & Gallinat and Ross, 2012 \\
\hline TFH & 2001 & $\mathrm{CH}$ & Spring & Wild & 0 & Gallinat and Ross, 2012 \\
\hline TFH & 2002 & $\mathrm{CH}$ & Spring & Wild & 0 & Gallinat and Ross, 2012 \\
\hline TFH & 2003 & $\mathrm{CH}$ & Spring & Wild & 0 & Gallinat and Ross, 2012 \\
\hline TFH & 2004 & $\mathrm{CH}$ & Spring & Wild & 0 & Gallinat and Ross, 2012 \\
\hline TFH & 2005 & $\mathrm{CH}$ & Spring & Wild & 0 & Gallinat and Ross, 2012 \\
\hline TFH & 2006 & $\mathrm{CH}$ & Spring & Wild & 0 & Gallinat and Ross, 2012 \\
\hline TFH & 2007 & $\mathrm{CH}$ & Spring & Wild & 0 & Gallinat and Ross, 2012 \\
\hline TFH & 2008 & $\mathrm{CH}$ & Spring & Wild & 0 & Gallinat and Ross, 2012 \\
\hline TFH & 2009 & $\mathrm{CH}$ & Spring & Wild & 0 & Gallinat and Ross, 2012 \\
\hline TFH & 2010 & $\mathrm{CH}$ & Spring & Wild & 0 & Gallinat and Ross, 2012 \\
\hline TFH & 2011 & $\mathrm{CH}$ & Spring & Wild & 0 & Gallinat and Ross, 2012 \\
\hline TFH & 1988 & $\mathrm{CH}$ & Spring & Hatchery & 0 & Gallinat and Ross, 2012 \\
\hline
\end{tabular}


Table A1.6. Estimates of adult mortality during trap and haul operations for different species by river, facility, year, species, run, source, and references.-Continued

[CH, Chinook salmon; CO, coho salmon; NA, not available; OM, steelhead; PGE, Portland General Electric Company; SO, sockeye salmon; TFH, Tucannon Fish Hatchery]

\begin{tabular}{|c|c|c|c|c|c|c|}
\hline Facility & Year & Species & Run & Source & $\begin{array}{l}\text { Mortality } \\
\text { (percent) }\end{array}$ & Reference \\
\hline TFH & 1989 & $\mathrm{CH}$ & Spring & Hatchery & 0 & Gallinat and Ross, 2012 \\
\hline TFH & 1990 & $\mathrm{CH}$ & Spring & Hatchery & 0.5 & Gallinat and Ross, 2012 \\
\hline TFH & 1991 & $\mathrm{CH}$ & Spring & Hatchery & 0 & Gallinat and Ross, 2012 \\
\hline TFH & 1992 & $\mathrm{CH}$ & Spring & Hatchery & 1 & Gallinat and Ross, 2012 \\
\hline TFH & 1993 & $\mathrm{CH}$ & Spring & Hatchery & 0 & Gallinat and Ross, 2012 \\
\hline TFH & 1994 & $\mathrm{CH}$ & Spring & Hatchery & 0 & Gallinat and Ross, 2012 \\
\hline TFH & 1995 & $\mathrm{CH}$ & Spring & Hatchery & 0 & Gallinat and Ross, 2012 \\
\hline TFH & 1996 & $\mathrm{CH}$ & Spring & Hatchery & 6.8 & Gallinat and Ross, 2012 \\
\hline TFH & 1997 & $\mathrm{CH}$ & Spring & Hatchery & 0 & Gallinat and Ross, 2012 \\
\hline TFH & 1998 & $\mathrm{CH}$ & Spring & Hatchery & 0 & Gallinat and Ross, 2012 \\
\hline TFH & 1999 & $\mathrm{CH}$ & Spring & Hatchery & 0.7 & Gallinat and Ross, 2012 \\
\hline TFH & 2000 & $\mathrm{CH}$ & Spring & Hatchery & 9.6 & Gallinat and Ross, 2012 \\
\hline TFH & 2001 & $\mathrm{CH}$ & Spring & Hatchery & 0 & Gallinat and Ross, 2012 \\
\hline TFH & 2002 & $\mathrm{CH}$ & Spring & Hatchery & 0 & Gallinat and Ross, 2012 \\
\hline TFH & 2003 & $\mathrm{CH}$ & Spring & Hatchery & 0 & Gallinat and Ross, 2012 \\
\hline TFH & 2004 & $\mathrm{CH}$ & Spring & Hatchery & 0 & Gallinat and Ross, 2012 \\
\hline TFH & 2005 & $\mathrm{CH}$ & Spring & Hatchery & 2.6 & Gallinat and Ross, 2012 \\
\hline TFH & 2006 & $\mathrm{CH}$ & Spring & Hatchery & 3.8 & Gallinat and Ross, 2012 \\
\hline TFH & 2007 & $\mathrm{CH}$ & Spring & Hatchery & 5.4 & Gallinat and Ross, 2012 \\
\hline TFH & 2008 & $\mathrm{CH}$ & Spring & Hatchery & 0.3 & Gallinat and Ross, 2012 \\
\hline TFH & 2009 & $\mathrm{CH}$ & Spring & Hatchery & 0.8 & Gallinat and Ross, 2012 \\
\hline TFH & 2010 & $\mathrm{CH}$ & Spring & Hatchery & 1.1 & Gallinat and Ross, 2012 \\
\hline TFH & 2011 & $\mathrm{CH}$ & Spring & Hatchery & 1.6 & Gallinat and Ross, 2012 \\
\hline \multicolumn{7}{|c|}{ Umatilla River (Oregon) } \\
\hline Threemile & 1993 & $\mathrm{CH}$ & Spring & Wild & 0.7 & Zimmerman and Duke, 1993 \\
\hline Threemile & 1993 & $\mathrm{CH}$ & Fall & Wild & 0 & Zimmerman and Duke, 1993 \\
\hline Threemile & 1995 & $\mathrm{CH}$ & Spring & Wild & 0.8 & Zimmerman and Duke, 1995 \\
\hline Threemile & 1996 & $\mathrm{CH}$ & Spring & Wild & 0.4 & Zimmerman and Duke, 1996 \\
\hline Threemile & 1995 & $\mathrm{CH}$ & Fall & Wild & 0 & Zimmerman and Duke, 1995 \\
\hline Threemile & 1996 & $\mathrm{CH}$ & Fall & Wild & 0 & Zimmerman and Duke, 1996 \\
\hline Threemile & 1997 & $\mathrm{CH}$ & Fall & Mixed & 0 & Zimmerman and Duke, 1997 \\
\hline Threemile & 1997 & $\mathrm{CH}$ & Spring & Mixed & 0.7 & Zimmerman and Duke, 1997 \\
\hline Threemile & 1993 & $\mathrm{CO}$ & NA & Wild & 0 & Zimmerman and Duke, 1993 \\
\hline Threemile & 1995 & $\mathrm{CO}$ & NA & Wild & 0 & Zimmerman and Duke, 1995 \\
\hline Threemile & 1996 & $\mathrm{CO}$ & NA & Wild & 0 & Zimmerman and Duke, 1996 \\
\hline Threemile & 1993 & $\mathrm{OM}$ & NA & Wild & 0.1 & Zimmerman and Duke, 1993 \\
\hline Threemile & 1995 & $\mathrm{OM}$ & NA & Wild & 0 & Zimmerman and Duke, 1995 \\
\hline Threemile & 1996 & $\mathrm{OM}$ & NA & Mixed & 0 & Zimmerman and Duke, 1996 \\
\hline Threemile & 1997 & $\mathrm{OM}$ & NA & Mixed & 0 & Zimmerman and Duke, 1997 \\
\hline Threemile & 1997 & $\mathrm{OM}$ & NA & Mixed & 0 & Zimmerman and Duke, 1997 \\
\hline
\end{tabular}


Table A1.7. Fallback rates over dam facilities for adult anadromous species by river, dam, year, species, run, and reference.

[CH, Chinook salmon; NA, not available; $\mathrm{CO}$, coho salmon; SO, sockeye salmon; OM, steelhead]

\begin{tabular}{|c|c|c|c|c|c|}
\hline Dam & Year & Species & Run & $\begin{array}{l}\text { Fallback } \\
\text { (percent) }\end{array}$ & Reference \\
\hline \multicolumn{6}{|c|}{ Columbia River (Oregon and Washington) } \\
\hline Bonneville & 1996 & $\mathrm{CH}$ & Spring-summer & 13.8 & Boggs and others, 2004 \\
\hline Bonneville & 1997 & $\mathrm{CH}$ & Spring-summer & 14.6 & Boggs and others, 2004 \\
\hline Bonneville & 1998 & $\mathrm{CH}$ & Spring-summer & 11.2 & Boggs and others, 2004 \\
\hline Bonneville & 2000 & $\mathrm{CH}$ & Spring-summer & 13 & Boggs and others, 2004 \\
\hline Bonneville & 2001 & $\mathrm{CH}$ & Spring-summer & 4.1 & Boggs and others, 2004 \\
\hline Bonneville & 1996 & $\mathrm{CH}$ & Spring-summer & 16.4 & Bjornn and others, 2000 \\
\hline Bonneville & 1997 & $\mathrm{CH}$ & Spring-summer & 19.9 & Bjornn and others, 2000 \\
\hline Bonneville & 1998 & $\mathrm{CH}$ & Spring-summer & 15.9 & Bjornn and others, 2000 \\
\hline Bonneville & 1996 & $\mathrm{CH}$ & Fall & NA & Boggs and others, 2004 \\
\hline Bonneville & 1997 & $\mathrm{CH}$ & Fall & NA & Boggs and others, 2004 \\
\hline Bonneville & 1998 & $\mathrm{CH}$ & Fall & 3.5 & Boggs and others, 2004 \\
\hline Bonneville & 2000 & $\mathrm{CH}$ & Fall & 3.9 & Boggs and others, 2004 \\
\hline Bonneville & 2001 & $\mathrm{CH}$ & Fall & 4.8 & Boggs and others, 2004 \\
\hline The Dalles & 1996 & $\mathrm{CH}$ & Spring-summer & 13.3 & Boggs and others, 2004 \\
\hline The Dalles & 1997 & $\mathrm{CH}$ & Spring-summer & 14.4 & Boggs and others, 2004 \\
\hline The Dalles & 1998 & $\mathrm{CH}$ & Spring-summer & 11.5 & Boggs and others, 2004 \\
\hline The Dalles & 2000 & $\mathrm{CH}$ & Spring-summer & 9.6 & Boggs and others, 2004 \\
\hline The Dalles & 2001 & $\mathrm{CH}$ & Spring-summer & 5.5 & Boggs and others, 2004 \\
\hline The Dalles & 1996 & $\mathrm{CH}$ & Fall & NA & Boggs and others, 2004 \\
\hline The Dalles & 1997 & $\mathrm{CH}$ & Fall & NA & Boggs and others, 2004 \\
\hline The Dalles & 1998 & $\mathrm{CH}$ & Fall & 10.2 & Boggs and others, 2004 \\
\hline The Dalles & 2000 & $\mathrm{CH}$ & Fall & 8.5 & Boggs and others, 2004 \\
\hline The Dalles & 2001 & $\mathrm{CH}$ & Fall & 6.9 & Boggs and others, 2004 \\
\hline John Day & 1996 & $\mathrm{CH}$ & Spring-summer & 11.9 & Boggs and others, 2004 \\
\hline John Day & 1997 & $\mathrm{CH}$ & Spring-summer & 9.9 & Boggs and others, 2004 \\
\hline John Day & 1998 & $\mathrm{CH}$ & Spring-summer & 10.6 & Boggs and others, 2004 \\
\hline John Day & 2000 & $\mathrm{CH}$ & Spring-summer & 6.0 & Boggs and others, 2004 \\
\hline John Day & 2001 & $\mathrm{CH}$ & Spring-summer & 3.0 & Boggs and others, 2004 \\
\hline John Day & 1996 & $\mathrm{CH}$ & Fall & NA & Boggs and others, 2004 \\
\hline John Day & 1997 & $\mathrm{CH}$ & Fall & NA & Boggs and others, 2004 \\
\hline John Day & 1998 & $\mathrm{CH}$ & Fall & 3.7 & Boggs and others, 2004 \\
\hline John Day & 2000 & $\mathrm{CH}$ & Fall & 2.6 & Boggs and others, 2004 \\
\hline John Day & 2001 & $\mathrm{CH}$ & Fall & 2.6 & Boggs and others, 2004 \\
\hline McNary & 1996 & $\mathrm{CH}$ & Spring-summer & 9.3 & Boggs and others, 2004 \\
\hline McNary & 1997 & $\mathrm{CH}$ & Spring-summer & 8.0 & Boggs and others, 2004 \\
\hline McNary & 1998 & $\mathrm{CH}$ & Spring-summer & 9.2 & Boggs and others, 2004 \\
\hline McNary & 2000 & $\mathrm{CH}$ & Spring-summer & 4.3 & Boggs and others, 2004 \\
\hline McNary & 2001 & $\mathrm{CH}$ & Spring-summer & 1.4 & Boggs and others, 2004 \\
\hline McNary & 1998 & $\mathrm{CH}$ & Fall & 2.1 & Boggs and others, 2004 \\
\hline McNary & 2000 & $\mathrm{CH}$ & Fall & 2.0 & Boggs and others, 2004 \\
\hline McNary & 2001 & $\mathrm{CH}$ & Fall & 3.5 & Boggs and others, 2004 \\
\hline McNary & 1996 & $\mathrm{CH}$ & Spring & 22.6 & Keefer and others, 2004 \\
\hline
\end{tabular}


Table A1.7. Fallback rates over dam facilities for adult anadromous species by river, dam, year, species, run, and reference.-Continued [CH, Chinook salmon; NA, not available; CO, coho salmon; SO, sockeye salmon; OM, steelhead]

\begin{tabular}{|c|c|c|c|c|c|}
\hline Dam & Year & Species & Run & $\begin{array}{l}\text { Fallback } \\
\text { (percent) }\end{array}$ & Reference \\
\hline McNary & 1997 & $\mathrm{CH}$ & Spring & 27.5 & Keefer and others, 2004 \\
\hline McNary & 1998 & $\mathrm{CH}$ & Spring & 19.6 & Keefer and others, 2004 \\
\hline McNary & 2000 & $\mathrm{CH}$ & Spring & 20.9 & Keefer and others, 2004 \\
\hline McNary & 2001 & $\mathrm{CH}$ & Spring & 7.6 & Keefer and others, 2004 \\
\hline Lower Granite & 1996 & $\mathrm{CH}$ & Spring & 35.5 & Keefer and others, 2004 \\
\hline Lower Granite & 1997 & $\mathrm{CH}$ & Spring & 36.4 & Keefer and others, 2004 \\
\hline Lower Granite & 1998 & $\mathrm{CH}$ & Spring & 28.3 & Keefer and others, 2004 \\
\hline Lower Granite & 2000 & $\mathrm{CH}$ & Spring & 35.4 & Keefer and others, 2004 \\
\hline Lower Granite & 2001 & $\mathrm{CH}$ & Spring & 10.4 & Keefer and others, 2004 \\
\hline McNary & 1996 & $\mathrm{CH}$ & Summer & 10.7 & Keefer and others, 2004 \\
\hline McNary & 1997 & $\mathrm{CH}$ & Summer & 13.8 & Keefer and others, 2004 \\
\hline McNary & 1998 & $\mathrm{CH}$ & Summer & 10.6 & Keefer and others, 2004 \\
\hline McNary & 2000 & $\mathrm{CH}$ & Summer & 8.5 & Keefer and others, 2004 \\
\hline McNary & 2001 & $\mathrm{CH}$ & Summer & 0.6 & Keefer and others, 2004 \\
\hline Lower Granite & 1996 & $\mathrm{CH}$ & Summer & 22.2 & Keefer and others, 2004 \\
\hline Lower Granite & 1997 & $\mathrm{CH}$ & Summer & 12.5 & Keefer and others, 2004 \\
\hline Lower Granite & 1998 & $\mathrm{CH}$ & Summer & 25.7 & Keefer and others, 2004 \\
\hline Lower Granite & 2000 & $\mathrm{CH}$ & Summer & 17.4 & Keefer and others, 2004 \\
\hline Lower Granite & 2001 & $\mathrm{CH}$ & Summer & 2 & Keefer and others, 2004 \\
\hline Bonneville & 1998 & $\mathrm{CH}$ & Fall & 4.2 & Bjornn and others, 2000 \\
\hline McNary & 1996 & $\mathrm{CH}$ & Fall & NA & Keefer and others, 2004 \\
\hline McNary & 1997 & $\mathrm{CH}$ & Fall & NA & Keefer and others, 2004 \\
\hline McNary & 1998 & $\mathrm{CH}$ & Fall & 1.7 & Keefer and others, 2004 \\
\hline McNary & 2000 & $\mathrm{CH}$ & Fall & 3.8 & Keefer and others, 2004 \\
\hline McNary & 2001 & $\mathrm{CH}$ & Fall & 3.7 & Keefer and others, 2004 \\
\hline Lower Granite & 1996 & $\mathrm{CH}$ & Fall & NA & Keefer and others, 2004 \\
\hline Lower Granite & 1997 & $\mathrm{CH}$ & Fall & NA & Keefer and others, 2004 \\
\hline Lower Granite & 1998 & $\mathrm{CH}$ & Fall & 0 & Keefer and others, 2004 \\
\hline Lower Granite & 2000 & $\mathrm{CH}$ & Fall & 5.9 & Keefer and others, 2004 \\
\hline Lower Granite & 2001 & $\mathrm{CH}$ & Fall & 16.7 & Keefer and others, 2004 \\
\hline Wells & 2004 & $\mathrm{CO}$ & NA & 3.3 & Murdoch and others, 2005 \\
\hline Wanapum & 2004 & $\mathrm{CO}$ & NA & 16.7 & Murdoch and others, 2005 \\
\hline Bonneville & 1997 & SO & NA & 11.4 & Naughton and others, 2006 \\
\hline The Dalles & 1997 & SO & NA & 4.9 & Naughton and others, 2006 \\
\hline John Day & 1997 & SO & NA & 3.6 & Naughton and others, 2006 \\
\hline McNary & 1997 & SO & NA & 2 & Naughton and others, 2006 \\
\hline Priest Rapids & 1997 & SO & NA & 4.2 & Naughton and others, 2006 \\
\hline Wanapum & 1997 & SO & NA & 4 & Naughton and others, 2006 \\
\hline Rock Island & 1997 & SO & NA & 1.9 & Naughton and others, 2006 \\
\hline Rocky Reach & 1997 & SO & NA & 7.1 & Naughton and others, 2006 \\
\hline Bonneville & 1998 & $\mathrm{SO}$ & - & 13.7 & Bjornn and others, 2000 \\
\hline Bonneville & 1996 & $\mathrm{OM}$ & NA & 4.9 & Boggs and others, 2004 \\
\hline Bonneville & 1997 & $\mathrm{OM}$ & NA & 9.1 & Boggs and others, 2004 \\
\hline Bonneville & 2000 & $\mathrm{OM}$ & NA & 6.9 & Boggs and others, 2004 \\
\hline
\end{tabular}


Table A1.7. Fallback rates over dam facilities for adult anadromous species by river, dam, year, species, run, and reference.-Continued [CH, Chinook salmon; NA, not available; CO, coho salmon; SO, sockeye salmon; OM, steelhead]

\begin{tabular}{|c|c|c|c|c|c|}
\hline Dam & Year & Species & Run & $\begin{array}{l}\text { Fallback } \\
\text { (percent) }\end{array}$ & Reference \\
\hline Bonneville & 2001 & $\mathrm{OM}$ & NA & 4.3 & Boggs and others, 2004 \\
\hline The Dalles & 1996 & $\mathrm{OM}$ & NA & 6 & Boggs and others, 2004 \\
\hline The Dalles & 1997 & $\mathrm{OM}$ & NA & 6.6 & Boggs and others, 2004 \\
\hline The Dalles & 2000 & $\mathrm{OM}$ & NA & 6.3 & Boggs and others, 2004 \\
\hline The Dalles & 2001 & $\mathrm{OM}$ & NA & 6.1 & Boggs and others, 2004 \\
\hline John Day & 1996 & $\mathrm{OM}$ & NA & 10.1 & Boggs and others, 2004 \\
\hline John Day & 1997 & $\mathrm{OM}$ & NA & 7.9 & Boggs and others, 2004 \\
\hline John Day & 2000 & $\mathrm{OM}$ & NA & 4.3 & Boggs and others, 2004 \\
\hline John Day & 2001 & $\mathrm{OM}$ & NA & 5.3 & Boggs and others, 2004 \\
\hline McNary & 1996 & OM & NA & 7.4 & Boggs and others, 2004 \\
\hline McNary & 1997 & $\mathrm{OM}$ & NA & 10.7 & Boggs and others, 2004 \\
\hline McNary & 2000 & $\mathrm{OM}$ & NA & 9.8 & Boggs and others, 2004 \\
\hline McNary & 2001 & OM & NA & 7.1 & Boggs and others, 2004 \\
\hline Bonneville & 1996 & $\mathrm{OM}$ & NA & 5.2 & Bjornn and others, 2000 \\
\hline Bonneville & 1997 & $\mathrm{OM}$ & NA & 9.9 & Bjornn and others, 2000 \\
\hline McNary & 1996 & $\mathrm{OM}$ & Spring & 9.3 & Keefer and others, 2004 \\
\hline McNary & 1997 & $\mathrm{OM}$ & Spring & 7.8 & Keefer and others, 2004 \\
\hline McNary & 1998 & $\mathrm{OM}$ & Spring & NA & Keefer and others, 2004 \\
\hline McNary & 2000 & $\mathrm{OM}$ & Spring & 8.8 & Keefer and others, 2004 \\
\hline McNary & 2001 & $\mathrm{OM}$ & Spring & 4.8 & Keefer and others, 2004 \\
\hline Lower Granite & 1996 & $\mathrm{OM}$ & Spring & 12.1 & Keefer and others, 2004 \\
\hline Lower Granite & 1997 & $\mathrm{OM}$ & Spring & 14.7 & Keefer and others, 2004 \\
\hline Lower Granite & 1998 & $\mathrm{OM}$ & Spring & NA & Keefer and others, 2004 \\
\hline Lower Granite & 2000 & $\mathrm{OM}$ & Spring & 16.9 & Keefer and others, 2004 \\
\hline Lower Granite & 2001 & $\mathrm{OM}$ & Spring & 16.2 & Keefer and others, 2004 \\
\hline \multicolumn{6}{|c|}{ Cowlitz River (Washington) } \\
\hline Mayfield & 2009 & $\mathrm{CH}$ & Fall & 12.0 & Henning, 2010 \\
\hline Cowlitz Falls & 2012 & $\mathrm{CH}$ & Spring & 0.7 & Serl and Heimbigner, 2013 \\
\hline Mayfield & 2009 & $\mathrm{CO}$ & NA & 3.3 & Henning, 2010 \\
\hline Mayfield & 2009 & $\mathrm{CO}$ & NA & 1.5 & Henning, 2010 \\
\hline Mayfield & 2009 & $\mathrm{OM}$ & NA & 30.8 & Henning, 2010 \\
\hline Mayfield & 2009 & $\mathrm{OM}$ & NA & 38.7 & Henning, 2010 \\
\hline Cowlitz Falls & 2012 & $\mathrm{OM}$ & NA & 2.0 & Serl and Heimbigner, 2013 \\
\hline \multicolumn{6}{|c|}{ North Santiam River (Oregon) } \\
\hline Bennett Dams & 2003 & $\mathrm{CH}$ & Spring & 1.2 & Schroeder and others, 2007 \\
\hline Bennett Dams & 2005 & $\mathrm{CH}$ & NA & 2.9 & Schroeder and others, 2006 \\
\hline \multicolumn{6}{|c|}{ Umatilla River (Oregon) } \\
\hline Threemile & 1994-1995 & $\mathrm{CO}$ & NA & 0 & Zimmerman and Duke, 1995 \\
\hline Threemile & 1994-1995 & $\mathrm{CH}$ & Spring & 43.8 & Zimmerman and Duke, 1995 \\
\hline Threemile & 1994-1995 & $\mathrm{CH}$ & Fall & 0 & Zimmerman and Duke, 1995 \\
\hline Threemile & 1994-1995 & $\mathrm{OM}$ & NA & 1.3 & Zimmerman and Duke, 1995 \\
\hline
\end{tabular}





\section{Chapter B. Habitat Assessment of Tributaries to Swift Reservoir, Yale Lake, and Lake Merwin, Washington}

\section{Introduction}

Habitat portrays an important role in determining the distribution and abundance of biota in stream ecosystems (Minshall and others, 1983; Schlosser, 1991). For anadromous Oncorhynchus spp. (Pacific salmon) and O. mykiss (steelhead), freshwater habitat is strongly associated with fish growth and survival (for example, Tappel and Bjornn, 1983; Quinn and Peterson, 1996; Sommer and others, 2001), distribution (for example, Nickelson and others, 1992; Torgersen and others, 1999), and abundance (for example, Fausch and Northcote, 1992; Solazzi and others, 2000). Indeed, understanding the amount and quality of habitat is an important part of Pacific salmon reintroductions (Anderson and others, 2014).

In the upper Lewis River, characterizing the status and extent of habitat available to anadromous salmon and steelhead in tributary streams is needed for robust assessments of the reintroduction potential. From 2013 through 2015, we assessed the available habitat of tributaries to Swift Reservoir, Yale Lake, and Lake Merwin (fig. B1). In Swift Reservoir, assessments were targeted towards updating habitat information where recent surveys (within the last decade) were not available and (or) in tributaries where O. kisutch (coho salmon) have been observed during recent reintroductions. In tributaries to Lake Merwin and Yale Lake, we conducted surveys to quantify the extent and status of available habitat for potential reintroductions. Information regarding the extent and quality of habitat was subsequently integrated with the Ecosystem Diagnosis and Treatment (EDT) method (Lestelle and others, 1996) for species-specific estimates of production potential in the Swift Reservoir, Yale Lake, and Lake Merwin basins.

\section{Methods}

\section{Survey Streams}

Survey locations varied based on data needs to inform anadromous species reintroductions and data limitations identified by regional biologists and existing data collected by U.S. Department of Agriculture Forest Service biologists in tributaries to Swift Reservoir, Our habitat surveys included Chickoon Creek; Clear Creek; Curly Creek; Cussed Hollow Creek; Diamond Creek; the upper Lewis River (upstream of Little Creek to the lower Lewis River Falls); Little Creek; Range Creek; S15 and S20 (tributaries to Swift Reservoir); Spencer Creek; M1 (tributary to Muddy River); upper Clearwater Creek; Pepper Creek; and P1, P3, and P7 (tributaries to Pine Creek) (fig. B2). Curly Creek was found to be dry and was not surveyed. We inventoried all streams with at least 25 meters (m) of available habitat in (1) Lake Merwin including Brooks Creek, Buncombe Hollow Creek, Cape Horn Creek, Indian George Creek, Jim Creek, lower Speelyai Creek, and Rock Creek; and (2) Yale Lake including Cougar Creek, Dog Creek, North fork of Siouxon Creek, Ole Creek, Panamaker Creek, Siouxon Creek, Speelyai Creek, Swift Bypass Channel, and west tributary of Speelyai Creek (fig. B3). All surveys were conducted in 2014 and 2015. Given that Rain Creek was dry at the confluence with Ole Creek, except during extremely high discharge events, we did not sample this tributary. In addition, no habitat data were collected from Rock Creek, as the habitat was influenced by the reservoir elevation.

\section{Habitat Quality}

During late July and early August 2014, we conducted continuous habitat surveys from each tributary mouth to the upstream migration barrier. Features were considered barriers based on changes in elevation or continued high-gradient stretches that likely impede salmon passage and the habitat features below the barrier (in the sense of Washington State Department of Fish and Wildlife, 2007). We used a census sampling design to collect continuous habitat data for a comprehensive assessment of habitat and to minimize bias in assessments from site-to-site variability (Urquhart and others, 1998; Larsen and others, 2004; Anlauf and others, 2011). To ensure consistency with regional approaches, we conducted our surveys using protocols derived from the Columbia Habitat Monitoring Program (CHaMP, 2013) that are currently employed in anadromous salmon 
and steelhead research and monitoring in the Columbia River Basin. To allow for rapid continuous surveys, our approach was a modification from the original CHaMP 2013 method (for example, Blanchard, 2015). We georeferenced each habitat unit and the data associated with that unit using handheld global positioning devices while conducting our surveys.

Reach length.-We established reach lengths in each stream using an average measure of bankfull width. We determined the minimum reach length for each stream by taking the average bankfull width of the first 10 habitat units (table B1) and multiplying it by 20 (Kershner and others, 2004; CHaMP, 2013). The first habitat unit boundary encountered after this minimum length served as the start of the next reach length.

Habitat unit classification.-We identified habitat units using a two-tiered hierarchical system (Hawkins and others, 1993). Tier I units were distinguished by gradient, relative stream velocity, and turbulence and included three classes: fast-water turbulent, fast-water nonturbulent, and slow-water pool. Tier I fast-water turbulent units were further subdivided into Tier II classes. These subclasses were differentiated by hydraulic properties and included riffles, rapids, cascades, and falls. Tier I slow-water pools were further subdivided into Tier II classes based on the primary process that formed the unit and included scour pool, plunge pool, dam pool, beaver pool, and off-channel pool. Tier I fast-water nonturbulent units were referred to as glides.

During each sampling event, we evaluated stream physical habitat attributes that are important for anadromous salmon and steelhead. We georeferenced each habitat unit and estimated (1) unit dimensions consisting of wetted and bankfull width and length, (2) the total number of pieces of large woody debris (LWD) exceeding 10 centimeters $(\mathrm{cm})$ in diameter and 1 meter in length, (3) percent substrate composition of each size class to the nearest 5 percent, (4) percent fine sediment in pool tails, and (5) riparian condition according to Blair and others (2008).

Habitat unit dimensions.-We measured wetted and bankfull widths at two transects located approximately at one-third and two-thirds total unit length and unit length from bottom to top (Hankin and Reeves, 1988) using a laser range finder. We recorded three depth measurements spaced equidistantly across each transect for a total of six depth measurements, which we averaged for each unit.

We used the individual measurements from transects for an estimate of average wetted and bankfull width and depth for each habitat unit. Additionally, we calculated the surface area of each habitat unit through estimates of average width and unit length. Computing estimates of surface area enabled us to quantify the total surface area for each stream and the proportional surface area for any type of habitat unit.

For all pools, we also calculated the residual pool depth as a measure of pool volume (Lisle, 1987). We calculated the residual pool depth in each unit by taking the difference between the pool tail depth, located at pool tail crest, and the maximum depth (Kershner and others, 2004). We identified the pool crest visually as the point at which there is a break or transition in stream channel slope.

Large woody debris (LWD).- The importance of stream habitat diversity is undeniable for aquatic biota (Lonzarich and Quinn, 1995). Structural elements (for example, woody debris) often control geomorphic processes (Abbe and Montgomery, 2003; Montgomery and others, 2003) and can benefit salmonid communities (Cederholm and others, 1997; Johnson and others, 2005). We enumerated all LWD equal to or greater than ( $>$ ) 1 meter within the bankfull channel; we further delineated pieces based on diameter as either large (diameter $>50 \mathrm{~cm}$ ) or small $(10 \mathrm{~cm}-50 \mathrm{~cm}$ ). If the stem was cracked or broken, it was treated as one piece if any portions were still connected. If the piece was broken completely and both pieces met the minimum size criteria, then they were both counted. Where pieces extended outside of the bankfull prism (area between and below bankfull width and height, respectively), we only considered the portions of pieces within the prism. If a piece extended across multiple habitat unit boundaries, we considered these as separate pieces in our counts for each unit but only where the criteria for LWD were met.

We summarized LWD by obtaining reach-based estimates of pieces per unit surface area (count divided by average width multiplied by reach length) for both size categories. This approach enabled us to estimate the LWD density of each size category separately as well as a sum across the large and small categories for a total estimate of LWD density for a given reach.

Substrate composition.-We classified surface sediment composition using a two-tiered visual estimation approach (Buffington and Montgomery, 1999) to quantify the dominant and subdominant size classes of substrate in habitat units. First, we estimated the extent of each habitat unit (to the nearest 5 percent) with different sediment characteristics. We modified the size class categories from Lane (1947) and Church and others (1987), which were generally consistent with the CHaMP protocol, and included exposed bedrock surface, boulder ( $>256$ millimeters $[\mathrm{mm}])$, large cobbles $(127.1-256 \mathrm{~mm})$, small cobbles (64.1-127 mm), gravel (2-64 mm), and fines (less than $2 \mathrm{~mm}$ ).

Next, we used this information to quantify the dominant and subdominant sediment characteristics of each habitat unit (Buffington and Montgomery, 1999). Given concerns of bias for visual sediment estimates (for example, McHugh and Budy, 2005), we also conducted systematic pebble counts (Wolman, 1954) at transects in the first 10 habitat units of each stream. We placed transects at approximately one-half the total habitat length, and we selected particles at 10 equally spaced sampling points spanning the width of the wetted channel. Substrate particles were selected while turning eyes away and extending finger down and picking up the first particle touched at the tip of the surveyor's boot (Wolman, 1954; Kondolf, 2000) and measuring the b-axis of each particle (Kershner and others, 2004). We then regressed the sediment data from the ocular estimates against the sediment measurement from the pebble counts to quantify observer error and bias in the ocular estimates. The resulting regression equation $\left(\mathrm{R}^{2}=0.51\right)$ was then used to correct all ocular estimates of substrate across streams. 
For all pool units, we also quantified the substrate characteristics of pool tailouts for measures of the quality of salmonid spawning habitat (McHugh and Budy, 2005). We visually identified pool tailouts as 20 percent of the channel unit upstream of the pool tail crest. Pool tailouts were not measured individually for width or length. The surface area of a pool tailout was estimated to be on average 20 percent of the surface area of the pool per EDT instructions. We estimated the proportion of fine sediment within each pool tailout using the same two-tiered visual estimation approach as used in the unit substrate surveys. To ensure accurate summaries of pool tail fines at the reach scale, we used a weighted approach. The weighted average of percent fines over pool tails within a reach, with weights proportional to the surface area of each pool tail, were summed to obtain a reach-based estimate of percent fines in pool tails.

Riparian function.-Riparian forests exert strong controls on stream microclimates (Gregory and others, 1991; Caissie, 2006), in-channel morphology (Bilby and Ward, 1991; Montgomery and others, 1995), nutrient cycling (McClain and others, 2003), and water quality (Gilliam, 1994; Naiman and Decamps, 1997). We evaluated the riparian condition and assigned a riparian index rating for each habitat unit. We adopted an index rating from Blair and others (2008) ranging from 0 (no anthropogenic alterations with strong linkages to floodplain) to 4 (complete severing of floodplain stream linkages). Given the differences in channel unit lengths, we used a weighted approach for estimates of riparian condition at the reach scale. We first calculated the proportion of the total surface area within the channel unit relative to the total surface area of the reach and then multiplied the weighting proportion by the riparian condition rating of the channel unit. These weighted riparian condition ratings were then summed to the reach scale and rounded to the nearest whole number.

In addition to habitat characteristics, we also quantified attributes that drive habitat forming processes and water quality. At the stream level, we measured (1) stream gradient, (2) ambient stream temperature, (3) stage discharge relationships, and (4) dissolved oxygen, which are described next.

Longitudinal gradient survey.-Gradient profiles were collected for each tributary using a Trimble GeoXH 6000 that is capable of collecting high-resolution elevation data $(10 \mathrm{~cm})$. Before each gradient survey, we surveyed an established reference location with constant $\mathrm{x}, \mathrm{y}$, and $\mathrm{z}$ coordinates to use as a survey control station. This reference was Yale Lake monument (monument ID 6791; latitude 46.02615, longitude -122.31809; Washington State Department of Transportation; http://www.wsdot. wa.gov/monument/). We used the reference survey data to obtain estimates of bias and accuracy. By incorporating reference surveys estimates, we were able to evaluate and correct for potential bias in our elevation data. Our reference surveys deviated slightly from the control station (average $=0.72 \mathrm{~cm}$, standard deviation $=0.41$ ).

We analyzed the longitudinal gradient data in $\operatorname{ArcGIS}^{\circledR}$ to obtain estimates at the reach scale. We snapped the reach boundaries for each stream to the gradient profile and calculated gradient as the difference between reach boundary elevations divided by the length (that is, rise divided by run). Because of the timing of habitat data collection, gradient data were only collected in tributaries to Lake Merwin and Yale Lake.

Temperature and discharge.-Given the importance of streamflow and thermal profiles in driving fundamental biological processes (for example, Brett, 1971), we collected stream temperature and discharge data in each survey stream in 2013 and 2014. Where discharge data were not available from existing monitoring stations, we quantified stage discharge relationships for estimates of summer and early autumn hydrologic regimes (June-October). We installed pressure transducers (Solinst Levelogger model 3001) near the mouth of each stream and collected hourly measurements of the stage (that is, river height). In addition, we installed a barometric pressure logger to control for basin-specific changes in barometric pressure from ambient weather that can influence stage measurements. During the period of deployment (that is, June-October in 2013 and 2014), we measured discharge at least three times per stream to establish stream-specific, stage-discharge relationships.

Each pressure transducer also recorded stream temperatures, and we placed additional temperature loggers at the upstream barrier in all study streams to measure ambient stream temperature profiles. All temperature loggers were tested and calibrated before deployment (Dunham and others, 2005). Each year (2013-2014), stream temperature was collected in each study stream from June 1 through the October 31. Temperature data were collected at 1-hour intervals using Onset Tidbit v2 (ranges from -20 to 50 degrees Celsius $\left[{ }^{\circ} \mathrm{C}\right.$ ], accuracy $\pm 0.53{ }^{\circ} \mathrm{C}$ from 0 to $50{ }^{\circ} \mathrm{C}$ ), and Onset $\mathrm{HOBO}$ Pendant (range -20 to $70{ }^{\circ} \mathrm{C}$, accuracy $\pm 0.2{ }^{\circ} \mathrm{C}$ from 0 to $50^{\circ} \mathrm{C}$ ) data loggers. Data loggers were housed in white PVC housing to avoid bias from direct solar radiation (in the sense of Isaak and Horan, 2011).

For both the upstream and downstream temperature logging devices, we calculated the mean temperature for each day. We then averaged these two numbers to get an overall daily temperature and then calculated the mean and range from these numbers from June through October. Temperature and streamflow information was only collected in tributaries to Lake Merwin and Yale Lake because of the timing of habitat data collection.

Dissolved oxygen.-We measured dissolved oxygen during habitat sampling (August 14-31) using a YSI multimeter model (Pro 2030). Measurements were taken in fast-moving water at the top and bottom of each study stream. We summarized dissolved oxygen at the reach level by correcting for lapse change, which we estimated by differencing our top and bottom measurements then dividing by total length of the stream. This provided a per-meter rate of change that could then be multiplied by each reach length and added to the previous reach-based estimate of dissolved oxygen. Because of the timing of habitat data collection, dissolved oxygen data were only collected in tributaries to Lake Merwin and Yale Lake. 


\section{Results and Discussion}

\section{Extent of Tributary Habitat in Lake Merwin and Yale Lake}

Surveys of available habitat indicated a total of 36.2 kilometers $(\mathrm{km})$ of available habitat in tributaries to Yale Lake and Lake Merwin (tables B1, B2; fig. B3). The majority $(28.0 \mathrm{~km})$ were in tributaries to Yale Lake with Siouxon Creek (and tributary; total=6.4 km), Speelyai Creek (and tributaries; total=7.5 km), Swift Bypass Channel $(4.9 \mathrm{~km})$, and Cougar Creek $(2.7 \mathrm{~km})$ having the majority of available habitat. Within Lake Merwin, Brooks Creek (including a tributary to Brooks Creek [B1]; $4.1 \mathrm{~km})$ and Indian George Creek $(1.5 \mathrm{~km})$ contain the most available habitat. Brooks Creek (10,318 square meters [m²] in Lake Merwin and Cougar Creek $\left(35,565 \mathrm{~m}^{2}\right)$, Siouxon Creek $\left(96,753 \mathrm{~m}^{2}\right)$, Speelyai Creek $\left(45,346 \mathrm{~m}^{2}\right)$, and the Swift Bypass Channel $\left(119,180 \mathrm{~m}^{2}\right)$ in Yale Lake contained the most amount of habitat based on surface area in Yale Lake (table B3). Tributaries to Swift Reservoir $\left(>700,000 \mathrm{~m}^{2}\right)$ clearly contained the most amount of tributary habitat among the three reservoirs, particularly when compared to Lake Merwin $\left(27,389 \mathrm{~m}^{2}\right)$. The amount of habitat available is a strong predictor of salmon population sizes (Isaak and others, 2007), as density dependence limits freshwater survival (Jonsson and others, 1998; Milner and others, 2003; Walters and others, 2013). The small amounts of existing habitat likely controlling salmon production (that is, smolts) combined with extensive predation potential (see "Piscine Predator Impact Studies 2013-2014, Lake Merwin, Washington," chap. E of this report) suggest available habitat may limit the likelihood of developing self-sustaining populations within Lake Merwin (McElhany and others, 2000; Nickelson, 2000; Anderson and others, 2014).

\section{Habitat Quality}

The onset of early snow during the fall of 2015 prevented the completion of surveys in M1 (tributary to Muddy River) and the upper Clearwater Creek. Where surveys were completed, our data suggest the complexity of stream habitat based on stream habitat type and depth varied considerably across streams (tables B4, B5). LWD is an important driver of juvenile salmon distribution and abundance (Rosenfeld and others, 2000; Roni and Quinn, 2001). On average, tributaries to Lake Merwin contain the highest densities of LWD, and tributaries to Swift Reservoir contain the lowest densities (table B6). Much of these differences, however, were attributed to sizes of LWD present as the density of small LWD (diameter 10-50 cm) was substantially greater than large LWD (diameter $>50 \mathrm{~cm}$ ) across all streams (table B6). We observed the highest densities of LWD in Cape Horn, Jim, and lower Speelyai Creeks in Lake Merwin and Dog, North Fork Siouxon, Ole, and Panamaker Creeks in Yale Lake.

We found little evidence of sediment limitations in most tributaries that were evaluated, regardless of reservoir. Fine sediment values in pool tails were low in the most streams except for Little Creek and Pepper Creek (Swift Reservoir; table B6), both of which exceeded tolerant levels for salmonids (Bryce and others, 2010). These patterns were generally consistent with data from fast-water habitat (riffles, glides, cascades) where gravel, cobble, and boulders composed the majority of streambed substrate (table B7). Together, these results indicate that sediment is unlikely limiting salmonid populations in the upper Lewis River Basin (Tappel and Bjornn, 1983; McHugh and others, 2004).

Average temperatures differed across tributaries, and the differences across years varied by stream (tables B8, B9). The largest range of temperatures occurred during August (range 2013-2014=6.8-17.0 ${ }^{\circ} \mathrm{C}$ ). During 2013 and 2014, we found generally consistent temperatures during June and July, but September and October 2014 temperatures were dramatically warmer than 2013 temperatures in all streams except Cougar Creek. Average summer (July-August 2014) temperatures in Buncombe Hollow, Dog, Siouxon, and Speelyai Creeks were generally higher than preferred juvenile rearing temperatures for coho and Chinook salmon (Oregon Department of Environmental Quality, 1995). While empirical summer stream temperature data were not available for tributaries to Swift Reservoir at the time of this field study, recent estimates of average August stream temperature from the U.S. Department of Agriculture Forest Service NorWeST stream temperature model (http://www.fs.fed.us/rm/boise/AWAE/projects/NorWeST.html; Isaak and others, 2010) suggest excessively warm temperatures are unlikely to impede salmon or steelhead (fig. B4). As expected, measures of dissolved oxygen were largely correlated with observed temperatures in tributaries to Lake Merwin and Yale Lake (table B10).

Riparian conditions and stream gradients varied substantially across tributaries (table B11). Aside from Cougar Creek and the Swift Bypass Channel, average stream gradients in all tributaries exceeded 2 percent with the highest gradients (about 10 percent) in tributaries to Speelyai Creek. Overall, we found little evidence of excessive alteration in riparian conditions in most tributaries (tables B8, B9). However, moderate-to-high riparian degradation was found in lower Speelyai Creek in Lake Merwin; Dog Creek, Brooks Creek, and Swift Bypass Channel in Yale Lake; and Little Creek, P7, and S20 in Swift Reservoir. Stream riparia represent an important part of aquatic ecosystems in terms of controlling sediment inputs and geomorphology (Barling and Moore, 1994; Naiman and others, 2005). Riparian vegetation is also a key factor influencing terrestrial prey inputs, which are often a substantial component of juvenile salmon diets (Wipfli, 1997, 2005). Furthermore, riparian vegetation also serves as major contributors of LWD and in limiting solar inputs and stream warming (Caissie, 2006). Ultimately, the importance of riparia suggests the need to consider restoration actions as a means for increasing the overall capacity for anadromous species. Quantifying the effectiveness of such actions may be an important step in prioritizing and directing restoration efforts (Roni and others, 2012). 


\section{Conclusions}

Empirical habitat data suggest little evidence that habitat quality will limit anadromous salmon and steelhead reintroductions. Evidence of habitat degradation appears to be location specific and includes sediment degradation (for example, fine sediment in Little Creek, tables B6 and B7), some thermal constraints during the summer months in tributaries to Lake Merwin and Yale Lake, and riparian degradation. The data collected herein and other available data from State and Federal agencies can be used to specifically identify factors limiting salmonids and to help target further studies to quantify potential effectiveness of restoration.

The extent of available habitat is likely to be a major factor limiting anadromous salmonids in Lake Merwin. The observed habitat limitations appear to be largely natural (that is, natural geologic features), suggesting that opportunities to enhance the capacity in Lake Merwin are minimal. The relatively small amount of habitat may also influence habitat use and emigration patterns for juvenile salmon, as juveniles more proximate to large water bodies can exhibit earlier emigration (see "Studies Assessing Adult Potential for Spawning Success in Yale Lake and Lake Merwin, Washington," chap. C of this report) and suggests limited potential for establishing and maintaining viable populations of anadromous fishes in Lake Merwin. Ultimately, assessing the potential for viable populations will require consideration not only of habitat availability but also biotic interactions between anadromous fishes and extant species (see "Piscine Predator Impact Studies 2013-2014, Lake Merwin, Washington," chap. E of this report).

\section{References}

Abbe, T.B., and Montgomery, D.R., 2003, Patterns and processes of wood debris accumulation in the Queets river basin, Washington: Geomorphology, v. 51, no. 1-3, p. 81-107.

Anderson, J.H., Pess, G.R., Carmichael, R.W., Ford, M.J., Cooney, T.D., Baldwin, C.M., and McClure, M.M., 2014, Planning Pacific salmon and steelhead reintroductions aimed at long-term viability and recovery: North American Journal of Fisheries Management, v. 34, no. 1, p. 72-93.

Anlauf, K.J., Jensen, D.W., Burnett, K.M., Steel, E.A., Christiansen, K., Firman, J.C., Feist, B.E., and Larsen, D.P., 2011, Explaining spatial variability in stream habitats using both natural and management-influenced landscape predictors: Aquatic Conservation-Marine and Freshwater Ecosystems, v. 21, no. 7, p. 704-714.

Barling, R.D., and Moore, I.D., 1994, Role of buffer strips in management of waterway pollution-A review: Environmental Management, v. 18, no. 4, p. 543-558.

Bilby, R.E., and Ward, J.W., 1991, Characteristics and function of large woody debris in streams draining old-growth, clear-cut, and 2nd-growth forests in southwesetern Washington: Canadian Journal of Fisheries and Aquatic Sciences, v. 48, no. 12, p. 2499-2508.

Blair, G.R., Lastelle, L.C., and Mobrand, L.E., 2008, The ecosystem diagnosis and treatment model—A tool for assessing salmonid performance potential based on habitat conditions: American Fisheries Society Symposium, v. 71, p. 289-309.

Blanchard, M.R., 2015, Using network models to predict steelhead abundance, Middle Fork John Day, OR: Logan, Utah, Utah State University, M.S. thesis, 47 p.

Brett, J.R., 1971, Energetic responses of salmon to temperature-A study of some thermal relations in the physiology and freshwater ecology of sockeye salmon (Oncorhynchus nerka): American Zoologist, v. 11, p. 99-113.

Bryce, S.A., Lomnicky, G.A., and Kaufmann, P.R., 2010, Protecting sediment-sensitive aquatic specise in mountain streams through application of biologically based streambed sediment criteria: Journal of the North American Benthological Society, v. 29 , p. $657-672$.

Buffington, J.M., and Montgomery, D.R., 1999, A procedure for classifying textural facies in gravel-bed rivers: Water Resources Research, v. 35, no. 6, p. 1903-1914.

Caissie, D., 2006, The thermal regime of rivers-A review: Freshwater Biology, v. 51, no. 8, p. 1389-1406.

Cederholm, C.J., Bilby, R.E., Bisson, P.A., and Bumstead, B., 1997, Response of juvenile coho salmon and steelhead to placement of large woody debris in a coastal Washington stream: North American Journal of Fisheries Management, v. 17, p. 947-963. 
Church, M., McLean, D., and Wolcott, J.F., 1987, River bed gravels: sampling and analysis, in Thorne, C.R., Bathurst, J.C., and Hey, R.W., eds., Sediment transport in gravel bed rivers: Chichester, United Kingdom, Wiley, p. 43-79.

Columbia Habitat Monitoring Program [CHaMP], 2013, Scientific protocol for salmonid habitat surveys within the Columbia Habitat Monitoring Program: Prepared by the Integrated Status and Effectiveness Monitoring Program, Wauconda, Wash.

Dunham, J., Chandler, G., Rieman, B., and Martin, D., 2005, Measuring stream temperature with digital data loggers-A user's guide: U.S. Department of Agriculture, Forest Service, General Technical Report RMRS GTR-150WWW.

Fausch, K.D., and Northcote, T.G., 1992, Large woody debris and salmonid habitat in a small coastal British Columbia stream: Canadian Journal of Fisheries and Aquatic Sciences, v. 49, no. 4, p. 682-693.

Gilliam, J.W., 1994, Riparian wetlands and water quality: Journal of Environmental Quality, v. 23, no. 5, p. 896-900.

Gregory, S.V., Swanson, F.J., McKee, W.A., and Cummins, K.W., 1991, An ecosystem perspective of riparian zones: Bioscience, v. 41 , no. 8 , p. 540-551.

Hankin, D.G., and Reeves, G.H., 1988, Estimating total fish abundance and total habitat area in small streams based on visual estimation methods: Canadian Journal of Fisheries and Aquatic Sciences, v. 45, p. 1575-1591.

Hawkins, C.P., Kershner, J.L., Bisson, P.A., Bryant, M.D., Decker, L.M., Gregory, S.V., McCullough, D.A., Overton, C.K., Reeves, G.H., Steedman, R.J., and Young, M.K., 1993, A hierarchical approach to classifying stream habitat features: Fisheries, v. 18 , no. 6 , p. $3-12$.

Isaak, D.J., and Horan, D.L., 2011, An evaluation of underwater epoxies to permanently install temperature sensors in mountain streams: North American Journal of Fisheries Management, v. 31, no. 1, p. 134-137.

Isaak, D.J., Luce, C.H., Rieman, B.E., Nagel, D.E., Peterson, E.E., Horan, D.L., Parkes, S., and Chandler, G.L., 2010, Effects of climate change and wildfire on stream temperatures and salmonid thermal habitat in a mountain river network: Ecological Applications, v. 20, no. 5, p. 1350-1371.

Isaak, D.J., Thurow, R.F., Rieman, B.E., and Dunham, J.B., 2007, Chinook salmon use of spawning patches-Relative roles of habitat quality, size, and connectivity: Ecological Applications, v. 17, p. 352-364.

Johnson, S.L., Rodgers, J.D., Solazzi, M.F., and Nickelson, T.E., 2005, Effects of an increase in large wood on abundance and survival of juvenile salmonids (Oncorhynchus spp.) in an Oregon coastal stream: Canadian Journal of Fisheries and Aquatic Sciences, v. 62 , no. 2 , p. $412-424$.

Jonsson, N., Jonsson, B., and Hansen, L.P., 1998, The relative role of density-dependent and density-independent survival in the life cycle of Atlantic salmon Salmo salar: Journal of Animal Ecology, v. 67, no. 5, p. 751-762.

Kershner, J.L., Roper, B.B., Bouwes, N., Henderson, R., and Archer, E., 2004, An analysis of stream habitat conditions in reference and managed watersheds on some federal lands within the Columbia River basin: North American Journal of Fisheries Management, v. 24, no. 4, p. 1363-1375.

Kondolf, G.M., 2000, Assessing salmonid spawning gravel quality: Transactions of the American Fisheries Society, v. 129, no. 1, p. 262-281.

Lane, E.W., 1947, Report of the subcommittee on sediment terminology: Transactions of the American Geophysical Union, v. 28, p. 936-938.

Larsen, D.P., Kaufmann, P.R., Kincaid, T.M., and Urquhart, N.S., 2004, Detecting persistent change in the habitat of salmonbearing streams in the Pacific Northwest: Canadian Journal of Fisheries and Aquatic Sciences, v. 61, no. 2, p. 283-291.

Lestelle, L.C., Mobrand, L.E., Lichatowich, J.A., and Vogel, T.S., 1996, Applied ecosystem analysis-A primer: Portland, Oreg., Bonneville Power Administration, project number 9404600.

Lisle, T.E., 1987, Using "residual depths" to monitor pool depths independently of discharge: U.S. Department of Agriculture, Forest Service, Rearch Note PSW-394.

Lonzarich, D.G., and Quinn, T.P., 1995, Experimental evidence for the effect of depth and structure on the distribution, growth, and survival of stream fishes: Canadian Journal of Zoology, v. 73, p. 2223-2230. 
McClain, M.E., Boyer, E.W., Dent, C.L., Gergel, S.E., Grimm, N.B., Groffman, P.M., Hart, S.C., Harvey, J.W., Johnston, C.A., Mayorga, E., McDowell, W.H., and Pinay, G., 2003, Biogeochemical hot spots and hot moments at the interface of terrestrial and aquatic ecosystems: Ecosystems, v. 6, no. 4, p. 301-312.

McElhany, P., Ruckelshaus, M.H., Ford, M.J., Wainwright, T.C., and Bjorkstedt, E.P., 2000, Viable salmonid populations and the recovery of Evolutionary Significant Units: Seattle, Wash., National Marine Fisheries Service.

McHugh, P., and Budy, P., 2005, A comparison of visual and measurement-based techniques for quantifying cobble embeddedness and fine-sediment levels in salmonid-bearing streams: North American Journal of Fisheries Management, v. 25, no. 4, p. $1208-1214$.

McHugh, P., Budy, P., and Schaller, H., 2004, A model-based assessment of the potential response of Snake River springsummer Chinook salmon to habitat improvements: Transactions of the American Fisheries Society, v. 133, no. 3, p. 622-638.

Milner, N.J., Elliott, J.M., Armstrong, J.D., Gardiner, R., Welton, J.S., and Ladle, M., 2003, The natural control of salmon and trout populations in streams: Fisheries Research, v. 62, no. 2, p. 111-125.

Minshall, G.W., Petersen, R.C., Cummins, K.W., Bott, T.L., Sedell, J.R., Cushing, C.E., and Vannote, R.L., 1983, Interbiome comparison of stream ecosystem dynamics: Ecological Monographs, v. 53, p. 1-25.

Montgomery, D.R., Buffington, J.M., Smith, R.D., Schmidt, K.M., and Pess, G., 1995, Pool spacing in forest channels: Water Resources Research, v. 31, no. 4, p. 1097-1105.

Montgomery, D.R., Collins, B.D., Buffington, J.M., and Abbe, T.B., 2003, Geomorphic effects of wood in rivers, in Gregory, S.V., Boyer, K.L., and Gurnell, A.M., eds., Ecology and Management of Wood in World Rivers: Bethesda, Md., American Fisheries Society, Symposium 37, p. 21-47.

Naiman, R.J., and Decamps, H., 1997, The ecology of interfaces-Riparian zones: Annual Review of Ecology and Systematics, v. 28, p. 621-658.

Naiman, R.J., Decamps, H., and McClain, M.E., 2005, Riparia_Ecology, conservation and management of streamside communities: San Diego, Calif., Elsevier, 445 p.

Nickelson, T.E., 2000, Population assessment—Oregon Coast coho salmon ESU: Portland, Oreg., Oregon Department of Fish and Wildlife.

Nickelson, T.E., Rodgers, J.D., Johnson, S.L., and Solazzi, M.F., 1992, Seasonal changes in habitat use by juvenile coho salmon (Oncorhynchus kisutch) in Oregon coastal streams: Canadian Journal of Fisheries and Aquatic Sciences, v. 49, no. 4, p. $783-789$.

Oregon Department of Environmental Quality, 1995, Temperature, in 1992-1994 water quality standards review: Oregon Department of Environmental Quality, final issue paper, variously paged.

Quinn, T.P., and Peterson, N.P., 1996, The influence of habitat complexity and fish sire on over-winter survival and growth of individually marked juvenile coho salmon (Oncorhynchus kisutch) in Big Beef creek, Washington: Canadian Journal of Fisheries and Aquatic Sciences, v. 53, no. 7, p. 1555-1564.

Roni, P., Bennett, T., Holland, R., Pess, G., Hanson, K., Moses, R., McHenry, M., Ehinger, W., and Walter, J., 2012, Factors affecting migration timing, growth, and survival of juvenile coho salmon in two coastal Washington watersheds: Transactions of the American Fisheries Society, v. 141, no. 4, p. 890-906.

Roni, P., and Quinn, T.P., 2001, Density and size of juvenile salmonids in response to placement of large woody debris in western Oregon and Washington streams: Canadian Journal of Fisheries and Aquatic Sciences, v. 58, no. 2, p. $282-292$.

Rosenfeld, J., Porter, M., and Parkinson, E., 2000, Habitat factors affecting the abundance and distribution of juvenile cutthroat trout (Oncorhynchus clarki) and coho salmon (Oncorhynchus kisutch): Canadian Journal of Fisheries and Aquatic Sciences, v. 57, no. 4, p. 766-774.

Schlosser, I.J., 1991, Stream fish ecology_A landscape perspective: BioScience, v. 41, no. 10, p. 704-712.

Solazzi, M.F., Nickelson, T.E., Johnson, S.L., and Rodgers, J.D., 2000, Effects of increasing winter rearing habitat on abundance of salmonids in two coastal Oregon streams: Canadian Journal of Fisheries and Aquatic Sciences, v. 57, no. 5, p. 906-914. 
Sommer, T.R., Nobriga, M.L., Harrell, W.C., Batham, W., and Kimmerer, W.J., 2001, Floodplain rearing of juvenile chinook salmon-Evidence of enhanced growth and survival: Canadian Journal of Fisheries and Aquatic Sciences, v. 58, no. 2, p. 325-333.

Tappel, P.D., and Bjornn, T.C., 1983, A new method of relating size of spawning gravel to salmonid embryo survival: North American Journal of Fisheries Management, v. 3, p. 123-135.

Torgersen, C.E., Price, D.M., Li, H.W., and McIntosh, B.A., 1999, Multiscale thermal refugia and stream habitat associations of Chinook salmon in northeastern Oregon: Ecological Applications, v. 9, no. 1, p. 301-319.

Urquhart, N.S., Paulsen, S.G., and Larsen, D.P., 1998, Monitoring for policy-relevant regional trends over time: Ecological Applications, v. 8, no. 2, p. 246-257.

Walters, A.W., Copeland, T., and Venditti, D.A., 2013, The density dilemma-Limitations on juvenile production in threatened salmon populations: Ecology of Freshwater Fish, v. 22, no. 4, p. 508-519.

Washington State Department of Fish and Wildlife, 2007, WSDOT fish passage inventory: Olympia, Wash., Washington State Department of Fish and Wildlife, $51 \mathrm{p}$.

Wipfli, M.S., 1997, Terrestrial invertebrates as salmonid prey and nitrogen sources in streams - Contrasting old-growth and young-growth riparian forests in southeastern Alaska, USA: Canadian Journal of Fisheries and Aquatic Sciences, v. 54, no. 6, p. $1259-1269$.

Wipfli, M.S., 2005, Trophic linkages between headwater forests and downstream fish habitats-Implications for forest and fish management: Landscape and Urban Planning, v. 72, no. 1-3, p. 205-213.

Wolman, M.G., 1954, A method of sampling coarse river-bed material: Transactions American Geophysical Union, v. 35, p. $951-956$. 


\section{Figures}

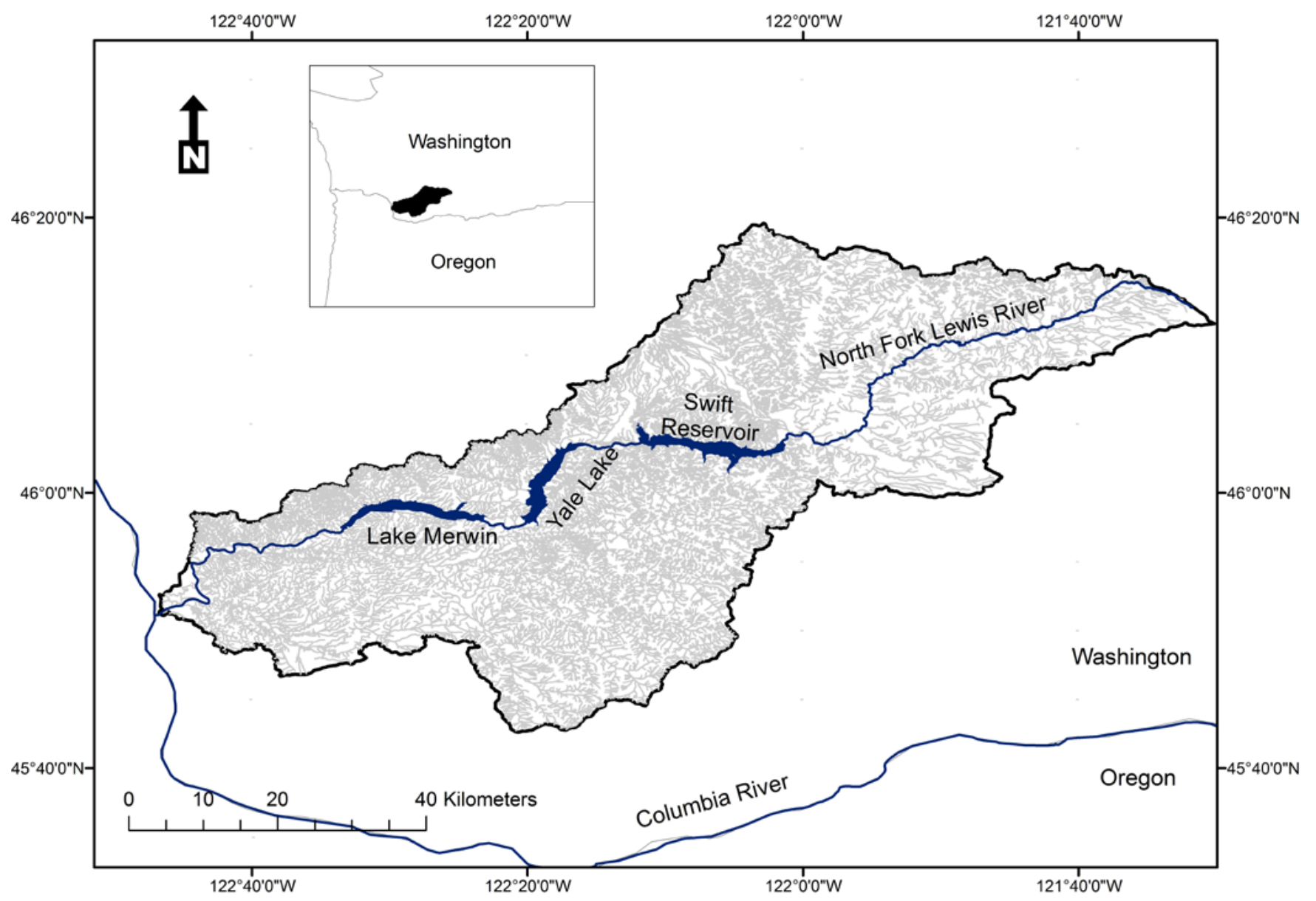

Figure B1. The North Fork of the Lewis River and the three hydropower impoundments: Lake Merwin, Yale Lake, and Swift Reservoir in the Lewis River Basin, Washington. 


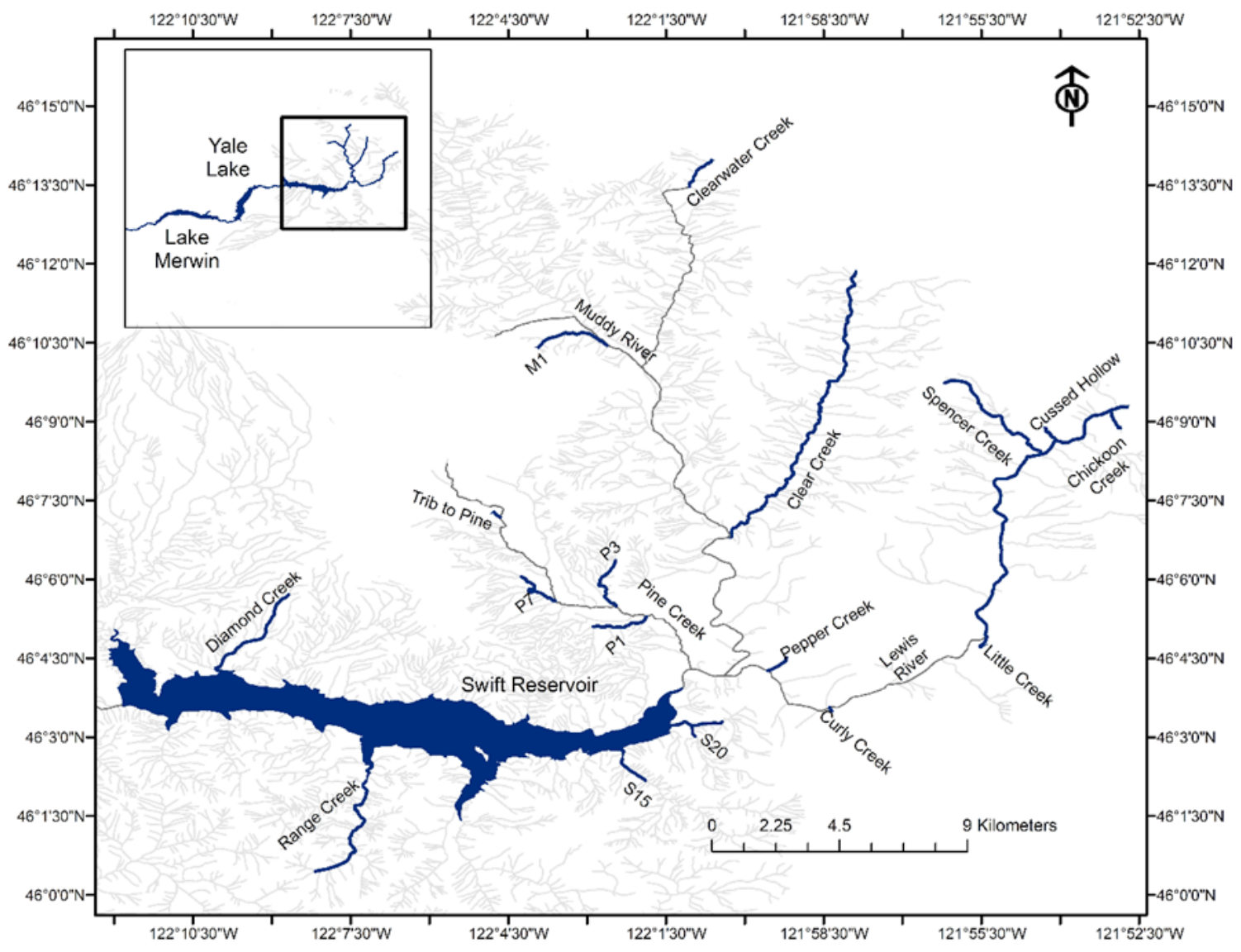

Figure B2. Upper Lewis River tributaries, Washington, where updated habitat data were needed (as noted by the thick blue lines) for assessments of anadromous salmon reintroductions. (trib., tributary; M1, tributary to Muddy River; P1, P3, and P7, tributaries to Pine Creek; S15 and S20, tributaries to Swift Reservoir) 


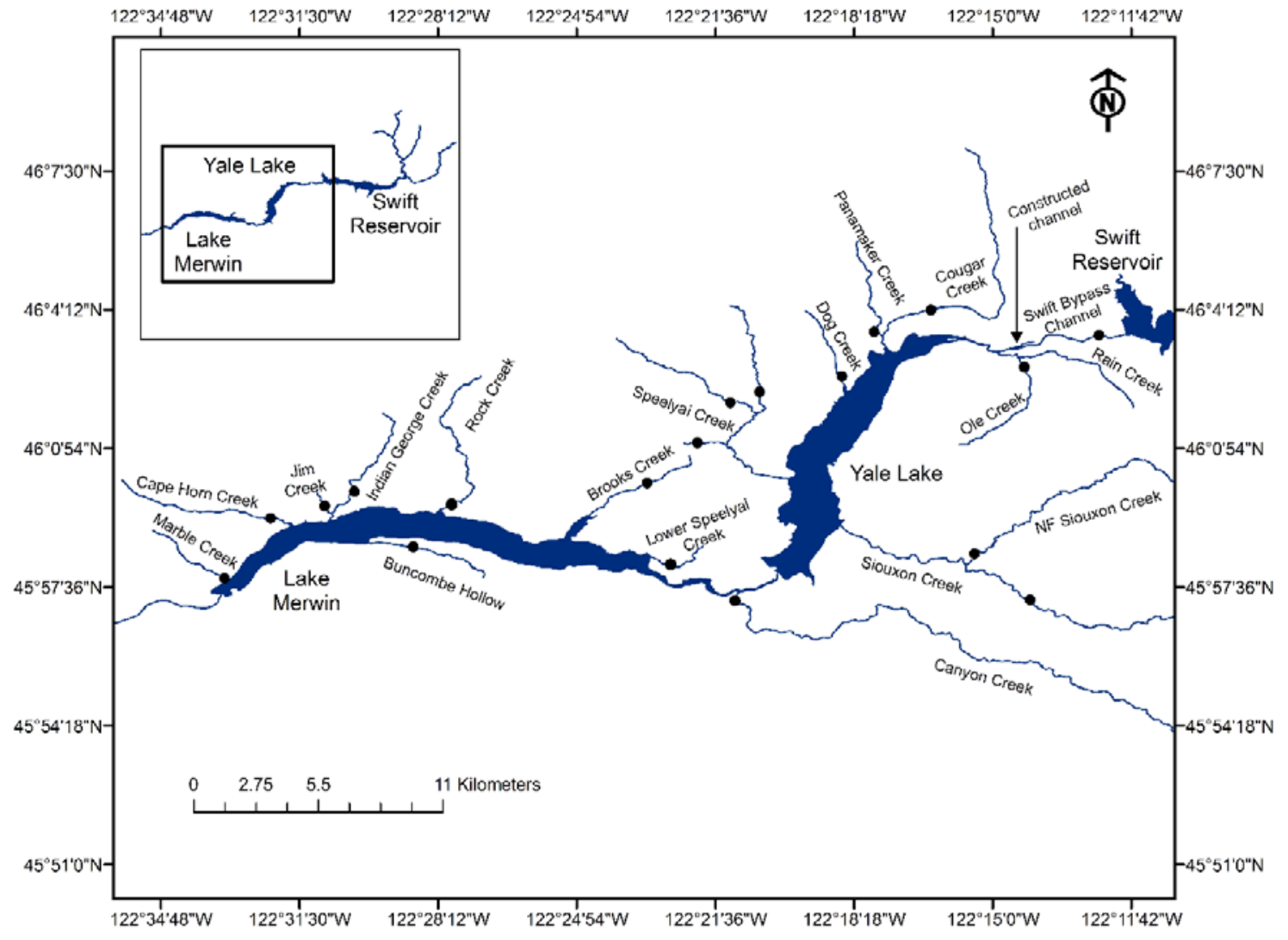

Figure B3. Stream habitat potentially available to anadromous salmon (blue) from upstream barriers (black circles) in tributaries to Yale Lake and Lake Merwin, Washington. (NF, north fork) 


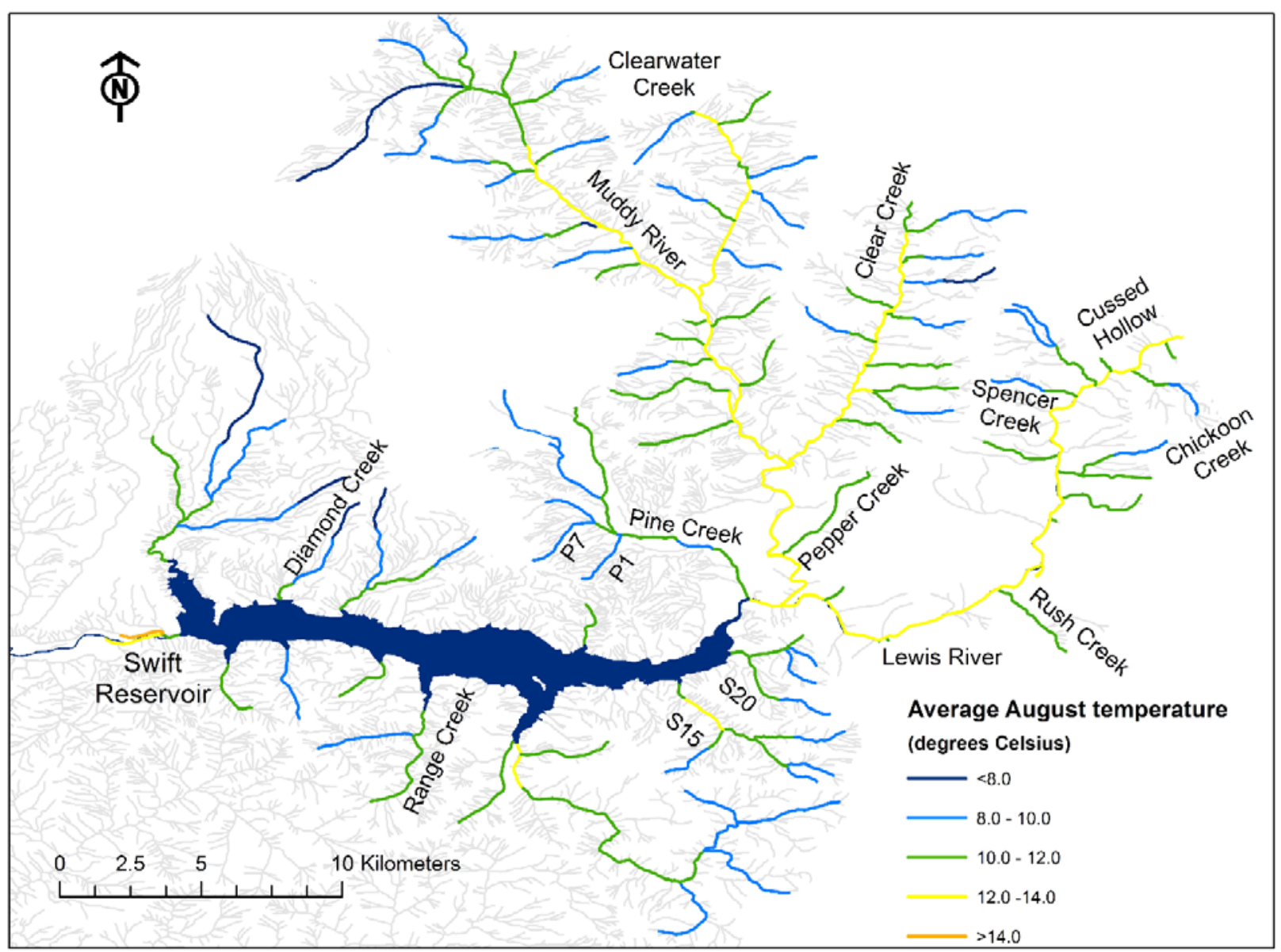

Figure B4. Predicted average August stream temperatures in degrees Celsius $\left({ }^{\circ} \mathrm{C}\right)$ from the NorWeST (http://www.fs.fed.us/ $\mathrm{rm} /$ boise/AWAE/projects/NorWeST.html; Isaak and others, 2010) stream temperature model for tributaries to Swift Reservoir, Washington. (P3 is not shown on the map because no estimates from the NorWeST stream temperature model were available. P1 and P7, tributaries to Pine Creek; S15 and S20, tributaries to Swift Reservoir) 
Tables

Table B1. The total stream length surveyed, average bankfull width (standard deviation [SD] in parentheses), wetted width (SD in parentheses), and number of sample reaches (n) in each tributary stream to Swift Reservoir, Washington, during surveys in 2015.

[The number of sample reaches corresponds to all subsequent tables. Curly Creek contained no streamflow below the falls at the time of the survey, and a barrier to upstream passage was identified at the mouth of $\mathrm{P} 1$ - as such no data were collected in these tributaries. km, kilometer, m, meter; NA, not available; P1, P3, and P7, tributaries to Pine Creek; S15 and S20, tributaries to Swift Reservoir]

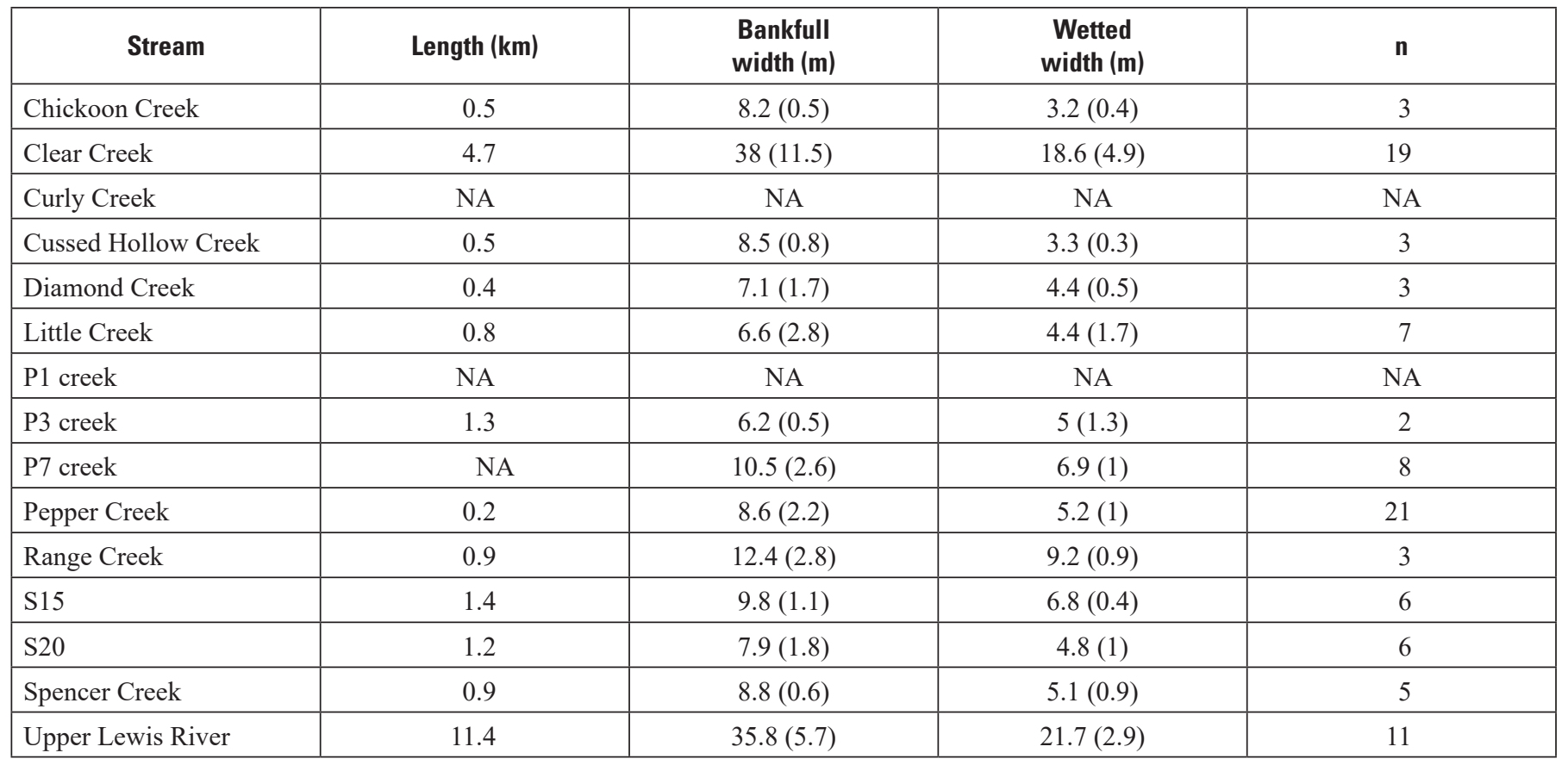

Table B2. The total stream length, average bankfull width (standard deviation [SD] in parentheses), wetted width (SD in parentheses), and number of sample reaches $(\mathrm{n})$ in each tributary stream to Lake Merwin and Yale Lake, Washington, during surveys in 2014.

[The number of sample reaches corresponds to all subsequent tables. km, kilometer; m, meter; NA, not available]

\begin{tabular}{|l|c|c|c|c|}
\hline \multicolumn{1}{|c|}{ Stream } & Length (km) & \multicolumn{2}{|c|}{$\begin{array}{c}\text { Bankfull } \\
\text { width (m) }\end{array}$} & Lake Merwin \\
\hline \multicolumn{3}{|c|}{ width (m) }
\end{tabular}


Table B2. The total stream length, average bankfull width (standard deviation [SD] in parentheses), wetted width (SD in parentheses), and number of sample reaches $(\mathrm{n})$ in each tributary stream to Lake Merwin and Yale Lake, Washington, during surveys in 2014. - Continued

[The number of sample reaches corresponds to all subsequent tables. km, kilometer; m, meter; NA, not available]

\begin{tabular}{|l|c|c|c|c|}
\hline \multicolumn{1}{|c|}{ Stream } & Length (km) & $\begin{array}{c}\text { Bankfull } \\
\text { width (m) }\end{array}$ & $\begin{array}{c}\text { Wetted } \\
\text { width (m) }\end{array}$ & n \\
\hline Panamaker Creek & 0.4 & $10.7(0.7)$ & $4.5(1.0)$ & 2 \\
\hline Siouxon Creek & 6.1 & $28.4(5.0)$ & $15.6(3.0)$ & 23 \\
\hline Speelyai Creek & 6.0 & $17.3(14.4)$ & $7.1(1.8)$ & 11 \\
\hline Swift Bypass Channel & 6.5 & $20.8(6.1)$ & $16.2(5.6)$ & 5 \\
\hline West Fork Speelyai Creek & 1.3 & $13.3(1.6)$ & $6.0(0.5)$ & 5 \\
\hline West tributary of Speelyai Creek & 1.1 & $9.9(1.9)$ & $5.4(0.6)$ & $\mathbf{7 1}$ \\
\hline Total & $\mathbf{2 8 . 0}$ & & & \\
\hline
\end{tabular}

Table B3. The total surface area (SA) by reach and the average SA (standard deviation in parentheses) in pool habitat, pool tailouts (that is, spawning habitat), and glides in each tributary stream to Swift Reservoir in 2015 and Lake Merwin and Yale Lake, Washington in 2014.

[In Swift Reservoir, not all tributary habitat was sampled (see table B1); $\mathrm{m}^{2}$, square meter; P3 and P7, tributaries to Pine Creek; S15 and S20, tributaries to Swift Reservoir]

\begin{tabular}{|c|c|c|c|c|}
\hline Stream & $\begin{array}{l}\text { Reach } \\
\text { SA }\left(m^{2}\right)\end{array}$ & $\begin{array}{c}\text { Pool } \\
\text { SA }\left(\mathrm{m}^{2}\right)\end{array}$ & $\begin{array}{c}\text { Pool tailout } \\
\text { SA }\left(\mathrm{m}^{2}\right)\end{array}$ & $\begin{array}{c}\text { Glide } \\
\text { SA }\left(\mathrm{m}^{2}\right)\end{array}$ \\
\hline \multicolumn{5}{|c|}{ Swift Reservoir } \\
\hline Chickoon Creek & 4,280 & $369(89)$ & $74(18)$ & $10(8)$ \\
\hline Clear Creek & 184,831 & $3,359(2,221)$ & $672(444)$ & $13(17)$ \\
\hline Cussed Hollow Creek & 4,159 & $434(141)$ & $87(28)$ & $18(7)$ \\
\hline Diamond Creek & 3,367 & $126(71)$ & $25(14)$ & $0(0)$ \\
\hline Little Creek & 5,130 & $136(139)$ & $27(28)$ & $37(22)$ \\
\hline P3 creek & 2,100 & $37(53)$ & $7(11)$ & $2(3)$ \\
\hline P7 creek & 19,040 & $350(99)$ & $70(20)$ & $4(7)$ \\
\hline Pepper Creek & 31,937 & $233(263)$ & $47(53)$ & $1(2)$ \\
\hline Range Creek & 11,326 & $634(131)$ & $127(26)$ & $0(0)$ \\
\hline S15 creek & 14,112 & $306(105)$ & $61(21)$ & $0(0)$ \\
\hline S20 creek & 8,941 & $176(202)$ & $35(40)$ & $0(0)$ \\
\hline Spencer Creek & 8,518 & $177(123)$ & $35(25)$ & $2(2)$ \\
\hline Upper Lewis River & 407,313 & $11,530(5,542)$ & $2,306(1,108)$ & $33(18)$ \\
\hline \multicolumn{5}{|c|}{ Lake Merwin } \\
\hline Brooks Creek & 10,318 & $90(40)$ & $18(8)$ & $69(53)$ \\
\hline Buncombe Hollow Creek & 3,373 & $119(114)$ & $24(23)$ & $7(10)$ \\
\hline Cape Horn Creek & 3,103 & $530(13)$ & $38(3)$ & $65(65)$ \\
\hline Indian George Creek & 6,165 & $142(57)$ & $74(11)$ & $64(48)$ \\
\hline Jim Creek & 2,692 & $604(89)$ & $46(18)$ & $90(26)$ \\
\hline Lower Speelyai Creek & 1,738 & $120(0)$ & $45(0)$ & $414(0)$ \\
\hline
\end{tabular}


Table B3. The total surface area (SA) by reach and the average SA (standard deviation in parentheses) in pool habitat, pool tailouts (that is, spawning habitat), and glides in each tributary stream to Swift Reservoir in 2015 and Lake Merwin and Yale Lake, Washington in 2014. - Continued

[In Swift Reservoir, not all tributary habitat was sampled (see table B1); $\mathrm{m}^{2}$, square meter; P3 and P7, tributaries to Pine Creek; S15 and S20, tributaries to Swift Reservoir]

\begin{tabular}{|c|c|c|c|c|}
\hline Stream & $\begin{array}{l}\text { Reach } \\
\mathrm{SA}\left(\mathrm{m}^{2}\right)\end{array}$ & $\begin{array}{c}\text { Pool } \\
\text { SA }\left(\mathrm{m}^{2}\right)\end{array}$ & $\begin{array}{c}\text { Pool tailout } \\
\text { SA }\left(\mathrm{m}^{2}\right)\end{array}$ & $\begin{array}{c}\text { Glide } \\
\mathrm{SA}\left(\mathrm{m}^{2}\right)\end{array}$ \\
\hline \multicolumn{5}{|c|}{ Yale Lake } \\
\hline Dog Creek & 1,647 & $386(0)$ & $77(0)$ & $27(0)$ \\
\hline North fork of Siouxon Creek & 9,688 & $1,147(473)$ & $229(95)$ & $0(0)$ \\
\hline Panamaker Creek & 1,866 & $285(161)$ & $57(32)$ & $120(120)$ \\
\hline Siouxon Creek & 96,753 & $3,452(1,622)$ & $690(324)$ & $1,052(729)$ \\
\hline Speelyai Creek & 45,346 & $329(198)$ & $66(40)$ & $299(349)$ \\
\hline Swift Bypass Channel & 119,180 & $2,683(2815)$ & $537(563)$ & $4,823(2,981)$ \\
\hline
\end{tabular}

Table B4. Average depth (standard deviation [SD] in parentheses) for nonpool habitat and residual pool depth (SD in parentheses) for pools by habitat type and stream in each tributary stream to Swift Reservoir, Washington, from surveys in 2015.

[m, meter; NA, not available; P3 and P7, tributaries to Pine Creek; S15 and S20, tributaries to Swift Reservoir]

\begin{tabular}{|c|c|c|c|c|c|c|c|c|}
\hline \multirow[b]{2}{*}{ Stream } & \multicolumn{4}{|c|}{ Average depth (m) } & \multicolumn{4}{|c|}{ Residual pool depth (m) } \\
\hline & Cascade & Glide & Rapid & Riffle & $\begin{array}{c}\text { Beaver } \\
\text { pool }\end{array}$ & $\begin{array}{l}\text { Dam } \\
\text { pool }\end{array}$ & $\begin{array}{c}\text { Plunge } \\
\text { pool }\end{array}$ & $\begin{array}{c}\text { Scour } \\
\text { pool }\end{array}$ \\
\hline Chickoon Creek & 0.17 (NA) & $0.19(0.05)$ & $0.15(0.02)$ & NA & NA & NA & $0.56(0.31)$ & $0.46(0.14)$ \\
\hline Clear Creek & 0.16 (NA) & $0.62(0.18)$ & $0.52(0.13)$ & $0.46(0.14)$ & NA & $1.36(0.61)$ & 1.35 (NA) & $0.99(0.26)$ \\
\hline Diamond Creek & $0.36(0.11)$ & 0.51 (NA) & $0.28(0.08)$ & 0.21 (NA) & NA & NA & $0.61(0.12)$ & $0.38(0.14)$ \\
\hline Little Creek & $0.3(0.11)$ & $0.48(0.11)$ & $0.27(0.09)$ & $0.21(0.06)$ & NA & $0.59(0.24)$ & NA & $0.5(0.12)$ \\
\hline P3 creek & $0.46(0.04)$ & 0.48 (NA) & 0.44 (NA) & 0.39 (NA) & NA & NA & NA & 0.23 (NA) \\
\hline P7 creek & $0.48(0.1)$ & $0.5(0.04)$ & $0.42(0.07)$ & $0.39(0.08)$ & NA & $0.8(0.28)$ & $0.99(0.69)$ & $0.82(0.41)$ \\
\hline S20 creek & $0.32(0.05)$ & $0.36(\mathrm{NA})$ & $0.33(0.06)$ & $0.32(0.06)$ & NA & NA & NA & $0.41(0.08)$ \\
\hline Spencer Creek & $0.4(0.09)$ & $0.44(0.16)$ & $0.42(0.04)$ & 0.41 (NA) & NA & NA & $0.4(0.07)$ & $0.5(0.07)$ \\
\hline Upper Lewis River & $0.57(0.07)$ & $0.61(0.13)$ & $0.47(0.11)$ & $0.43(0.07)$ & NA & NA & $1.3(0.91)$ & $1.45(0.33)$ \\
\hline
\end{tabular}


Table B5. Average depth (standard deviation [SD] in parentheses) for nonpool habitat and residual pool depth (SD in parentheses) for pools by habitat type and stream in each tributary stream to Lake Merwin and Yale Lake, Washington, from surveys in 2014.

[m, meter; NA, not available]

\begin{tabular}{|c|c|c|c|c|c|c|c|c|}
\hline \multirow[b]{2}{*}{ Stream } & \multicolumn{4}{|c|}{ Average depth (m) } & \multicolumn{4}{|c|}{ Residual pool depth (m) } \\
\hline & Cascade & Glide & Rapid & Riffle & $\begin{array}{c}\text { Beaver } \\
\text { pool }\end{array}$ & $\begin{array}{l}\text { Dam } \\
\text { pool }\end{array}$ & \begin{tabular}{|c|} 
Plunge \\
pool
\end{tabular} & $\begin{array}{c}\text { Scour } \\
\text { pool }\end{array}$ \\
\hline Brooks Creek. & $0.15(0.07)$ & $0.2(0.46)$ & $0.12(0.04)$ & $0.10(0.03)$ & $0.21(0.01)$ & $0.24(0.08)$ & $0.96(4.47)$ & $0.26(0.10)$ \\
\hline Buncombe Hollow Creek & $0.10(0.02)$ & $0.19(0.01)$ & $0.13(0.07)$ & $0.10(0.04)$ & 0 & $0.29(0.16)$ & $0.33(0.27)$ & $0.29(0.11)$ \\
\hline Indian George Creek & $0.18(0.06)$ & $0.16(0.03)$ & $0.16(0.04)$ & $0.12(0.07)$ & 0 & 0.28 (NA) & $0.55(0.51)$ & $0.32(0.12)$ \\
\hline Jim Creek & $0.22(0.05)$ & $0.2(0.03)$ & $0.17(0.08)$ & $0.13(0.02)$ & 0 & $0.33(0.21)$ & $0.33(0.28)$ & $0.50(0.37)$ \\
\hline Lower Speelyai Creek & $0.19(0.05)$ & $0.17(0.05)$ & $0.13(0.01)$ & 0.18 (NA) & 0 & 0 & $0.22(0.04)$ & $0.26(0.05)$ \\
\hline \multicolumn{9}{|c|}{ Yale Lake } \\
\hline Ole Creek & $0.20(0.05)$ & $0.28(0.31)$ & $0.16(0.05)$ & $0.10(0.02)$ & 0 & 0 & $0.72(1.0)$ & $0.53(0.33)$ \\
\hline Panamaker Creek & $0.20(0.09)$ & $0.20(0.01)$ & $0.20(0.06)$ & 0 & 0 & 0 & $1.15(0.89)$ & $0.51(0.41)$ \\
\hline Siouxon Creek & $0.67(0.25)$ & $0.52(0.1)$ & $0.42(0.1)$ & $0.34(0.1)$ & 0 & 0 & $0.56(10.08)$ & $1.44(1.37)$ \\
\hline Speelyai Creek & $0.26(0.06)$ & $0.39(0.54)$ & $0.27(0.32)$ & $0.24(0.07)$ & 0 & 0.98 (NA) & $0.53(0.48)$ & $0.70(0.54)$ \\
\hline Swift Bypass Channel & $0(0.25)$ & $0.67(0)$ & $0.48(0.18)$ & $0.28(0.15)$ & $1.37(1.06)$ & $0.49(0.31)$ & $0.69(0.40)$ & $0.70(0.69)$ \\
\hline West Fork Speelyai Creek & $0.26(0.06)$ & 0 & $0.24(0.04)$ & 0 & 0 & 0 & $1.07(1.93)$ & 0 \\
\hline West tributary of Speelyai Creek & $0.22(0.06)$ & $0.24(0.05)$ & $0.51(1.25)$ & $0.16(0.03)$ & 0 & 0 & $0.53(0.33)$ & $0.38(0.25)$ \\
\hline
\end{tabular}

Table B6. The average (standard deviation in parentheses) density (count multiplied by bankfull width divided by reach length as per Ecosystem Diagnosis and Treatment) of large woody debris (LWD) delineated by large (large: diameter greater than 50 centimeters [cm]) and small (small: diameter less than $[<] 10$ to $<50 \mathrm{~cm}$ ) pieces, and percent surface fines in pool tailouts in each tributary stream to Swift Reservoir in 2014 and Lake Merwin and Yale Lake in 2014 in Washington.

[\%, percent; NA, not available; P3 and P7, tributaries to Pine Creek; S15 and S20, tributaries to Swift Reservoir]

\begin{tabular}{|c|c|c|c|}
\hline Stream & LWD density (large) & LWD density (small) & $\%$ fines in tailout \\
\hline \multicolumn{4}{|c|}{ Swift Reservoir } \\
\hline Chickoon Creek & $0.22(0.09)$ & $0.55(0.24)$ & $7.0(12.2)$ \\
\hline Clear Creek & $0.45(0.23)$ & $0.67(0.36)$ & $11.2(16.5)$ \\
\hline Cussed Hollow Creek & $0.53(0.24)$ & $0.46(0.06)$ & $10.6(13.6)$ \\
\hline Diamond Creek & $0.17(0.08)$ & $0.18(0.03)$ & $12.1(17.7)$ \\
\hline Little Creek & $0.16(0.1)$ & $0.28(0.19)$ & $48.7(31.4)$ \\
\hline P3 creek & $0(0)$ & $0(0)$ & 68.5 (NA) \\
\hline P7 creek & $0.15(0.1)$ & $0.2(0.11)$ & $3.1(6.1)$ \\
\hline Pepper Creek & $0.2(0.16)$ & $0.3(0.25)$ & $28.4(23.5)$ \\
\hline Range Creek & $0.26(0.14)$ & $0.22(0.1)$ & $0(0)$ \\
\hline
\end{tabular}


Table B6. The average (standard deviation in parentheses) density (count multiplied by bankfull width divided by reach length as per Ecosystem Diagnosis and Treatment) of large woody debris (LWD) delineated by large (large: diameter greater than 50 centimeters [cm]) and small (small: diameter less than $[<] 10$ to $<50 \mathrm{~cm}$ ) pieces, and percent surface fines in pool tailouts in each tributary stream to Swift Reservoir in 2014 and Lake Merwin and Yale Lake in 2014 in Washington.-Continued

[\%, percent; NA, not available; P3 and P7, tributaries to Pine Creek; S15 and S20, tributaries to Swift Reservoir]

\begin{tabular}{|c|c|c|c|}
\hline Stream & LWD density (large) & LWD density (small) & $\%$ fines in tailout \\
\hline S15 creek & $0.09(0.06)$ & $0.16(0.09)$ & $0(0)$ \\
\hline S20 creek & $0.15(0.05)$ & $0.19(0.1)$ & $3.1(3.5)$ \\
\hline Spencer Creek & $0.23(0.08)$ & $0.25(0.08)$ & $5.3(9.0)$ \\
\hline Upper Lewis River & $0.33(0.09)$ & $0.38(0.13)$ & $0.6(1.5)$ \\
\hline \multicolumn{4}{|c|}{ Lake Merwin } \\
\hline Brooks Creek & $0.15(0.09)$ & $0.79(0.56)$ & $5.4(5.3)$ \\
\hline Buncombe Hollow Creek & $0.02(0.03)$ & $0.07(0.07)$ & $12.3(13.3)$ \\
\hline Cape Horn Creek & $0.31(0.09)$ & $1.91(0.18)$ & $2.0(0.5)$ \\
\hline Indian George Creek & $0.17(0.06)$ & $0.96(0.36)$ & $2.5(1.5)$ \\
\hline Jim Creek & $0.41(0.07)$ & $2.13(0.01)$ & $0.6(0.0)$ \\
\hline Lower Speelyai Creek & $0.40(0)$ & $1.59(0)$ & $4.9(0)$ \\
\hline \multicolumn{4}{|c|}{ Yale Lake } \\
\hline Cougar Creek & $0.22(0.18)$ & $0.42(0.32)$ & $0.2(0.4)$ \\
\hline Dog Creek & $0.10(0)$ & $2.97(0)$ & $1.0(0)$ \\
\hline North fork of Siouxon Creek & $0.39(0.22)$ & $1.05(0.57)$ & $2.3(2.3)$ \\
\hline Ole Creek & $0.16(0.17)$ & $0.99(1.09)$ & $0.2(0.4)$ \\
\hline Panamaker Creek & $0.40(0.20)$ & $1.68(0.12)$ & $5.2(4.0)$ \\
\hline Siouxon Creek & $0.16(0.08)$ & $0.43(0.19)$ & $0.3(0.6)$ \\
\hline Speelyai Creek & $0.17(0.34)$ & $0.40(0.54)$ & $3.8(9.9)$ \\
\hline Swift Bypass Channel & $0.03(0.08)$ & $0.12(0.12)$ & $0(0)$ \\
\hline West Fork Speelyai Creek & $0.22(0.18)$ & $0.44(0.26)$ & $2.0(3.0)$ \\
\hline West tributary of Speelyai Creek & $0.11(0.09)$ & $0.35(0.31)$ & $1.2(1.7)$ \\
\hline
\end{tabular}


Table B7. Percent of substrate estimated as bedrock, boulder (greater than 256 millimeters [mm]), large cobbles (127.1-256 mm), small cobbles (64.1-127 mm), gravel (2-64 mm), and fines (less than $2 \mathrm{~mm}$ ) in fast-water habitat (riffles, glides, cascades) in tributaries to Reservoir in 2015 and Lake Merwin and Yale Lake in 2014 in Washington.

[P3 and P7, tributaries to Pine Creek; S15 and S20, tributaries to Swift Reservoir]

\begin{tabular}{|c|c|c|c|c|c|}
\hline Stream & Bedrock & Boulder & Cobble & Gravel & Fines \\
\hline \multicolumn{6}{|c|}{ Swift Reservoir } \\
\hline Chickoon Creek & 31.4 & 11.8 & 48.3 & 8 & 0.5 \\
\hline Clear Creek & 0 & 4 & 60.4 & 28.1 & 5.7 \\
\hline Cussed Hollow Creek & 24.9 & 12.7 & 45.2 & 10.4 & 2.1 \\
\hline Diamond Creek & 0 & 36.4 & 46.3 & 17.2 & 0 \\
\hline Little Creek & 0 & 0 & 24.5 & 21.7 & 53.7 \\
\hline P3 creek & 0 & 50 & 22 & 16 & 12 \\
\hline P7 creek & 0 & 18.4 & 53.5 & 24.2 & 2 \\
\hline Pepper Creek & 6 & 9.2 & 52 & 28.3 & 3.2 \\
\hline Range Creek & 2.5 & 49.3 & 28.9 & 14.5 & 0 \\
\hline S15 creek & 7.8 & 60.9 & 28.6 & 2.7 & 0 \\
\hline S20 creek & 0 & 23.7 & 73.8 & 1.8 & 0.7 \\
\hline Spencer Creek & 5.9 & 30.6 & 56.5 & 7 & 0 \\
\hline Upper Lewis River & 6.2 & 17.8 & 60.7 & 14.5 & 0.6 \\
\hline \multicolumn{6}{|c|}{ Lake Merwin } \\
\hline Brooks Creek & 0.5 & 7.4 & 25.9 & 57.7 & 7.4 \\
\hline Buncombe Hollow Creek & 38.2 & 0 & 22 & 33.1 & 6.6 \\
\hline Cape Horn Creek & 27.4 & 15.4 & 31.1 & 19.8 & 6.4 \\
\hline Indian George Creek & 5.1 & 5.9 & 43.7 & 42.4 & 1.8 \\
\hline Jim Creek & 20.4 & 13.5 & 22.1 & 37.7 & 5.9 \\
\hline Lower Speelyai Creek & 0 & 6.4 & 15.5 & 53.6 & 15.5 \\
\hline \multicolumn{6}{|c|}{ Yale Lake } \\
\hline Cougar Creek & 0 & 0.9 & 34.4 & 52.7 & 3.5 \\
\hline Dog Creek & 0 & 23.6 & 31.8 & 35.5 & 9.1 \\
\hline North fork of Siouxon Creek & 16.7 & 58.3 & 13.3 & 11.7 & 0 \\
\hline Ole Creek & 0 & 14 & 52.7 & 32.3 & 1 \\
\hline Panamaker Creek & 10 & 12.4 & 57.1 & 20 & 0 \\
\hline Siouxon Creek & 13.3 & 46.1 & 33.3 & 7.2 & 0 \\
\hline Speelyai Creek & 4.7 & 38 & 40.8 & 11.3 & 0.6 \\
\hline Swift Bypass Channel & 0.4 & 26.7 & 57 & 10.8 & 4.2 \\
\hline West Fork Speelyai Creek & 2 & 59.5 & 30.6 & 7.9 & 0 \\
\hline West tributary of Speelyai Creek & 0.5 & 34.7 & 48.1 & 15.4 & 0.1 \\
\hline
\end{tabular}


Table B8. The average and range of stream temperatures by month from July through October 2013 for Clear Creek (Swift Reservoir) and in each tributary stream to Lake Merwin and Yale Lake in Washington in 2014.

[The logger and data for Siouxon Creek and Cape Horn Creeks were lost during the high flow event during September. avg., average; NA, not available]

\begin{tabular}{|c|c|c|c|c|c|c|c|c|}
\hline Stream & \multicolumn{2}{|c|}{ July' } & \multicolumn{2}{|c|}{ August } & \multicolumn{2}{|c|}{ September } & \multicolumn{2}{|c|}{ October } \\
\hline \multicolumn{9}{|c|}{ Lake Merwin } \\
\hline Buncombe Hollow & 16.3 & $14.2-18.7$ & 16.4 & $14.2-18.4$ & 14.7 & $11.3-17.6$ & 10.2 & $8.3-11.6$ \\
\hline Indian George & 14.3 & $11.5-16.5$ & 15.2 & $13.5-16.7$ & 13.8 & $9.7-16.7$ & 9.0 & $7.4-10.6$ \\
\hline \multicolumn{9}{|c|}{ Yale Lake } \\
\hline Cougar Creek & 6.8 & $6.2-7.9$ & 6.8 & $6.4-7.9$ & 6.8 & $6.2-8.1$ & 6.8 & $6.5-7.2$ \\
\hline Dog Creek & 13.0 & $10.5-14.9$ & 17.0 & $12.9-23.3$ & 14.6 & $9.1-20.5$ & 9.3 & $8.2-10.7$ \\
\hline Ole Creek & 13.8 & $11.6-15.8$ & 14.8 & $13.2-16.0$ & 13.2 & $8.9-15.6$ & 8.4 & $7.5-9.3$ \\
\hline Panamaker Creek & 12.8 & $10.5-14.9$ & 14.0 & $12.2-16.5$ & NA & NA & NA & NA \\
\hline
\end{tabular}

${ }^{1}$ July values include period from July 10-July 31 .

Table B9. The average and range of stream temperatures by month from June through October for Clear Creek (Swift Reservoir) and in each tributary stream to Lake Merwin and Yale Lake in 2014 in Washington.

[avg., average; NA, not available]

\begin{tabular}{|c|c|c|c|c|c|c|c|c|c|c|}
\hline Stream & \multicolumn{2}{|c|}{ June } & \multicolumn{2}{|c|}{ July } & \multicolumn{2}{|c|}{ August } & \multicolumn{2}{|c|}{ September } & \multicolumn{2}{|c|}{ October } \\
\hline \multicolumn{11}{|c|}{ Lake Merwin } \\
\hline Brooks Creek & 16.5 & $9.8-21.9$ & 13.8 & $12.4-19.4$ & 13.8 & $12.8-14.8$ & 12.4 & $11.9-13.3$ & 11.6 & $10.4-12.5$ \\
\hline Buncombe Hollow Creek & 13.7 & $11.4-15.1$ & 17.0 & $14.9-19.1$ & 17.5 & $15.6-19.0$ & 14.8 & $13.5-16.2$ & 12.6 & $11.4-14.0$ \\
\hline Cape Horn Creek & 11.2 & $9.5-12.4$ & 14.2 & $12.4-15.8$ & 14.9 & $13.5-16.2$ & 12.9 & $11.7-14.2$ & 11.3 & $9.7-13.0$ \\
\hline Jim Creek & 11.8 & $10.1-13.0$ & 14.9 & $13.1-16.4$ & 15.6 & $14.3-16.6$ & 13.6 & $12.6-14.7$ & 12.3 & $10.2-13.9$ \\
\hline Lower Speelyai Creek & NA & NA & NA & NA & NA & NA & NA & NA & NA & NA \\
\hline \multicolumn{11}{|c|}{ Yale Lake } \\
\hline Cougar Creek & 7.0 & $6.5-7.7$ & 7.3 & $7.1-7.7$ & 7.4 & $7.1-7.6$ & 7.0 & $6.7-7.2$ & 7.2 & $6.6-8.4$ \\
\hline Panamaker Creek & 10.6 & $9.4-11.7$ & 12.7 & $11.1-13.9$ & 14.4 & $13.7-14.9$ & 14.8 & $12.9-17.8$ & 11.5 & $8.7-15.2$ \\
\hline Siouxon Creek & 11.4 & $9.4-13.2$ & 15.4 & $12.4-17.7$ & 16.9 & $14.9-18.6$ & 14.0 & $12.5-15.2$ & 12.3 & $10.9-14.0$ \\
\hline Speelyai Creek & 11.6 & $9.6-13.3$ & 15.0 & $12.8-17.0$ & 16.8 & $15.0-17.8$ & 14.6 & $13.1-15.6$ & 11.6 & $9.1-14.0$ \\
\hline Swift Bypass Channel & 10.5 & $9.5-11.8$ & 12.3 & $11.0-13.1$ & 13.4 & $12.4-14.3$ & 13.3 & $12.4-14.0$ & 12.1 & $10.3-13.2$ \\
\hline West Fork Speelyai Creek & 10.8 & $9.0-12.1$ & 13.6 & $11.6-15.3$ & 15.0 & $13.4-15.9$ & 13.0 & $11.7-14.4$ & 10.8 & $8.7-13.0$ \\
\hline West tributary of Speelyai Creek & 11.3 & $9.1-12.8$ & 14.4 & $12.3-16.4$ & 15.5 & $13.9-16.9$ & 13.3 & $12.1-14.6$ & 12.2 & $10.6-14.0$ \\
\hline \multicolumn{11}{|c|}{ Swift Reservoir } \\
\hline Clear Creek & 10.4 & $8.1-14.4$ & 14.4 & $10.1-19.1$ & 15.6 & $13.2-19.3$ & 13.5 & $10.8-16.7$ & 10.7 & $6.8-14.8$ \\
\hline
\end{tabular}


Table B10. The average (standard deviation in parentheses) measures of dissolved oxygen, reach gradient, and riparian function in each tributary stream to Lake Merwin and Yale Lake, Washington, in 2014.

[ml/1, milliliter per liter; \%, percent; NA, not available]

\begin{tabular}{|c|c|c|c|}
\hline Stream & $\begin{array}{l}\text { Dissolved oxygen } \\
(\mathrm{ml} / \mathrm{l})\end{array}$ & Gradient (\%) & Riparian function \\
\hline \multicolumn{4}{|c|}{ Lake Merwin } \\
\hline Brooks Creek & $9.84(0.26)$ & $4.4(2.4)$ & $2.7(0.7)$ \\
\hline Buncombe Hollow Creek & $8.50(0.92)$ & $3.2(1.8)$ & $1.6(1.5)$ \\
\hline Cape Horn Creek & $9.54(0.15)$ & $5.2(0.9)$ & $0.1(0.1)$ \\
\hline Indian George Creek & $9.65(0.02)$ & $5.7(1.8)$ & $0.9(1.3)$ \\
\hline Jim Creek & $9.24(0.05)$ & $4.7(1.1)$ & $0(0)$ \\
\hline Lower Speelyai Creek & $9.89(0)$ & $2.8(0)$ & 4 (NA) \\
\hline \multicolumn{4}{|c|}{ Yale Lake } \\
\hline Cougar Creek & $12.29(0.04)$ & $1.6(0.9)$ & $1.1(1.6)$ \\
\hline Dog Creek & $8.83(0)$ & $4.9(0)$ & 2.8 (NA) \\
\hline North fork of Siouxon Creek & $9.75(0.08)$ & $7.0(2.7)$ & $1.8(0)$ \\
\hline Ole Creek & $9.46(0.25)$ & $2.0(1.7)$ & $0.9(1.1)$ \\
\hline Panamaker Creek & $9.80(0.16)$ & NA & $0(0)$ \\
\hline Siouxon Creek & $9.73(0.06)$ & $2.1(2.3)$ & $0.1(0.2)$ \\
\hline Speelyai Creek & $9.56(0.36)$ & $6.2(4.4)$ & $0.9(1.4)$ \\
\hline Swift Bypass Channel & NA & $0.7(0.5)$ & $2.1(0.2)$ \\
\hline West Fork Speelyai Creek & $9.68(0.05)$ & $10.7(2.5)$ & $0(0)$ \\
\hline West tributary of Speelyai Creek & $9.86(0.03)$ & $9.0(5.6)$ & $0(0)$ \\
\hline
\end{tabular}

Table B11. The average (standard deviation in parentheses) measures of riparian function in sampled tributary stream to Swift Reservoir, Washington, in 2015.

[P3 and P7, tributaries to Pine Creek; S15 and S20, tributaries to Swift Reservoir; NA, not available]

\begin{tabular}{|l|c|}
\hline \multicolumn{1}{|c|}{ Stream } & Riparian condition \\
\hline Chickoon Creek & $0(0)$ \\
\hline Clear Creek & $0.5(0.9)$ \\
\hline Cussed Hollow Creek & $0(0)$ \\
\hline Diamond Creek & $1.0(0.4)$ \\
\hline Little Creek & $2.3(1.2)$ \\
\hline P3 creek & $1(\mathrm{NA})$ \\
\hline P7 creek & $2.0(0.7)$ \\
\hline Pepper Creek & $0.2(0.6)$ \\
\hline Range Creek & $1.2(1.1)$ \\
\hline S15 creek & $1(\mathrm{NA})$ \\
\hline S20 creek & $2(\mathrm{NA})$ \\
\hline Spencer Creek & $0(0)$ \\
\hline Upper Lewis River & $0.1(0.3)$ \\
\hline
\end{tabular}




\section{Chapter C. Studies Assessing Adult Potential for Spawning Success in Yale Lake and Lake Merwin, Washington}

\section{Introduction}

Understanding the ability of reintroduced anadromous species to successfully reproduce in the tributaries to Yale Lake and Lake Merwin (fig. C1) is an essential component of a reintroduction program. Under current management plans, anadromous adults will be released into the reservoir systems, and with this, there remains considerable uncertainty in how these fish will disperse and utilize available stream habitat. We introduced a set of adult Oncorhynchus kisutch (coho salmon) into Lake Merwin (based on availability of surplus salmon) in the fall of 2014 to evaluate tributary use and potential for recruitment.

\section{Methods}

\section{Field Sampling}

Spawning surveys.-During 2014, we assessed the adult potential for spawning success in tributaries to Lake Merwin using a test release of adult coho salmon. On September 23, 2014, a set of 280 adults was released into the reservoir near Cresap Bay Campground. Beginning in the second week of October, which is the approximate start of coho salmon spawning observed in the Lewis River upstream of Merwin Dam (see "Studies Assessing Anadromous-Resident Fish Interactions in the Lewis River, Washington," chap. F of this report), and continuing through the first week of November, we tracked the adult spawning locations using biweekly redd and carcass surveys in each of the tributaries. Spawning surveys were continuous and included all habitats available in each tributary. During each survey, we georeferenced the occurrence of spawning activity and delineated observations into three categories: (1) carcasses, (2) observed redds, and (3) staging. We considered fish as "staging" where no redd was clearly observed near fish, and we did not delineate staging fish based on the presence of female only, male only, or male/female pairings.

Juvenile recruitment.- In the late spring and early summer of 2015, we conducted electrofishing and snorkel surveys in Cape Horn Creek, Jim Creek, Indian George Creek, and Brooks Creek to verify successful spawning. We sampled all the existing habitat in Cape Horn Creek, Jim Creek, and Indian George Creek using snorkel surveys. In Brooks Creek, we sampled continuously until at least 50 individuals were captured using a backpack electrofisher for an estimate of catch-per-unit effort (CPUE) in fish per second and measured the fork lengths of all individuals captured. We contrasted the capture information with field results for juvenile coho salmon in Clear Creek, a tributary in the Swift Basin, where reintroduction efforts have been ongoing since 2012 (see "Assessment of Juvenile Fish Production and Outmigration Success on the Lewis River, Washington," chap. D of this report). We used the size comparisons with Clear Creek as a measure of successful recruitment given the observed production of juvenile coho salmon in Clear Creek.

\section{Results and Discussion}

Spawning surveys.-We observed coho salmon spawning activity during surveys in Cape Horn Creek, Jim Creek, Indian George Creek, and Brooks Creek (fig. C2). Spawning activities were observed up to the existing barriers in Cape Horn Creek and Jim Creek but only partially upstream in Indian George Creek and Brooks Creek. No spawning activity was observed in Buncombe Hollow Creek. However, use of existing tributaries is likely to vary considerably given the lack of natal homing in the test fish used in this study (Quinn, 1993; Candy and Beacham, 2000). 
Juvenile recruitment.-During 2015 sampling surveys, no juvenile coho salmon were observed in Jim Creek and Indian George Creek, but low densities of coho salmon were observed in Cape Horn Creek and in Brooks Creek. For example, in Brooks Creek we sampled 1.2 kilometers to capture a total of 50 juvenile coho salmon. The size distribution of coho salmon in Brooks Creek was generally similar to that observed in Clear Creek (Swift Reservoir; fig. C3), suggesting that rearing conditions are suitable. Densities of juvenile coho salmon in Brooks Creek were relatively low (CPUE $=0.008$ fish per second) when compared to Clear Creek (average CPUE $=0.039$ fish per second; range $=0.012-0.066$; fig. C4), particularly given the low densities observed in Clear Creek in 2015 (see "Studies Assessing Anadromous-Resident Fish Interactions in the Lewis River, Washington" chap. F of this report) and the likely higher capture probabilities associated with stream size.

Habitat can be a factor limiting juvenile recruitment (Jensen and others, 2009), yet research suggests that coho salmon can use a variety of habitats, albeit to varying densities (Anlauf-Dunn and others, 2014). Habitat conditions in tributaries to Lake Merwin and Yale Lake are generally robust with relatively high large woody debris densities (see "Habitat Assessment of Tributaries to Swift Reservoir, Yale Lake, and Lake Merwin, Washington," chap. B of this report) and minimal indications of excessive levels of fine sediment (table C1; Bryce and others, 2008). In some tributaries, however (for example, Siouxon Creek; table C2), a considerable amount of existing substrate may be too large for coho salmon spawning (Kondolf, 2000; Mull and Wilzbach, 2007). The large substrate is likely from the ambient geology and the high stream power, which is driven by precipitation patterns and high gradients observed in many tributaries (table B10 in "Habitat Assessment of Tributaries to Swift Reservoir, Yale Lake, and Lake Merwin, Washington," chap. B of this report).

The low densities of juvenile coho salmon observed in tributary surveys in Lake Merwin, however, do not necessarily indicate that habitat is limiting juvenile production. For example, the observed low densities of juvenile coho salmon may also be a function of the location of observed redds, which were all within 1.15 kilometers of Lake Merwin (fig. C2), and the limited amount of existing habitat. Downstream emigration of coho salmon fry can be common in streams with limited habitat and locations proximate to larger water bodies (Roni and others, 2012; Rebenack and others, 2015). Coho salmon emigration at the fry stage appears to be relatively common in Swift Reservoir, as over 18 percent of the coho salmon captured at the floating surface collector in 2015 were fry. As such, the low densities of juvenile coho salmon may not be strong indicators of spawning capacity, as recruitment patterns can be complex, particularly given the variety of factors that can influence juvenile coho salmon life-history expressions (for example, Pess and others, 2011).

\section{Conclusions}

The lack of surplus coho salmon adults to mimic potential reintroduction scenarios somewhat hindered our ability to understand patterns of spawning behavior and the likely consequences of such patterns (that is, recruitment). However, coho salmon can utilize a variety of habitats, a pattern demonstrated in the variability of habitats and tributaries utilized by adult coho salmon in Swift Reservoir (see "Studies Assessing Anadromous-Resident Fish Interactions in the Lewis River, Washington" chap. F of this report). Furthermore, the colonization of tributaries that are spatially distant within the reservoir indicates that finding and accessing habitat is unlikely a limiting factor. For example, although adult coho salmon are released in the upper two-thirds of Swift Reservoir, redd count data from the first 3 years of reintroduction indicate individuals have been found spawning more than 10 kilometers downstream of the release site. Together, these results suggest coho salmon adults will be capable of finding and accessing a range of habitats in tributaries to Lake Merwin and Yale Lake.

Understanding juvenile salmon and steelhead recruitment patterns can be challenging given the variety of life-history expressions demonstrated by different species. Ultimately, the distribution of spawning coupled with the extent and condition of tributary habitat may lead to increased use of reservoir habitat by juveniles - particularly if progeny of redds proximate to the reservoir migrate earlier and have extended rearing in the reservoir environment. Longer periods of rearing in the reservoir environments may have profound influences on the potential predation of juvenile salmon by existing predators (see "Piscine Predator Impact Studies 2013-2014, Lake Merwin, Washington," chap. E of this report) and the seasonal carrying capacity of the reservoirs based on forage base (see "Studies Assessing Anadromous-Resident Fish Interactions in the Lewis River, Washington," chap. F of this report). 


\section{References}

Anlauf-Dunn, K.J., Ward, E.J., Strickland, M., and Jones, K., 2014, Habitat connectivity, complexity, and quality-Predicting adult coho salmon occupancy and abundance: Canadian Journal of Fisheries and Aquatic Sciences, v. 71, no. 12, p. 1864-1876.

Bryce, S.A., Lomnicky, G.A., Kaufmann, P.R., McAllister, L.S., and Ernst, T.L., 2008, Development of biologically based sediment criteria in mountain streams of the western United States: North American Journal of Fisheries Management, v. 28, no. 6, p. $1714-1724$

Candy, J.R., and Beacham, T.D., 2000, Patterns of homing and straying in southern British Columbia coded-wire tagged Chinook salmon (Oncorhynchus tshawytscha) populations: Fisheries Research, v. 47, no. 1, p. 41-56.

Jensen, D.W., Steel, E.A., Fullerton, A.H., and Pess, G., 2009, Impact of fine sediment on egg-to-fry survival of Pacific salmon-A meta-analysis of published studies: Reviews in Fisheries Science, v. 17, p. 348-359.

Kondolf, G.M., 2000, Assessing salmonid spawning gravel quality: Transactions of the American Fisheries Society, v. 129, no. 1, p. 262-281.

Mull, K.E., and Wilzbach, M.A., 2007, Selection of spawning sites by coho salmon in a northern California stream: North American Journal of Fisheries Management, v. 27, no. 4, p. 1343-1354.

Pess, G.R., Kiffney, P.M., Liermann, M.C., Bennett, T.R., Anderson, J.H., and Quinn, T.P., 2011, The influences of body size, habitat quality, and competition on the movement and survival of juvenile coho salmon during the early stages of stream recolonization: Transactions of the American Fisheries Society, v. 140, no. 4, p. 883-897.

Quinn, T.P., 1993, A review of homing and straying of wild and hatchery-produced salmon: Fisheries Research, v. 18, p. 29-44.

Rebenack, J.J., Ricker, S., Anderson, C., Wallace, M., and Ward, D.M., 2015, Early emigration of juvenile coho salmonImplications for population monitoring: Transactions of the American Fisheries Society, v. 144, no. 1, p. 163-172.

Roni, P., Bennett, T., Holland, R., Pess, G., Hanson, K., Moses, R., McHenry, M., Ehinger, W., and Walter, J., 2012, Factors affecting migration timing, growth, and survival of juvenile coho salmon in two coastal Washington watersheds: Transactions of the American Fisheries Society, v. 141, no. 4, p. 890-906. 


\section{Figures}

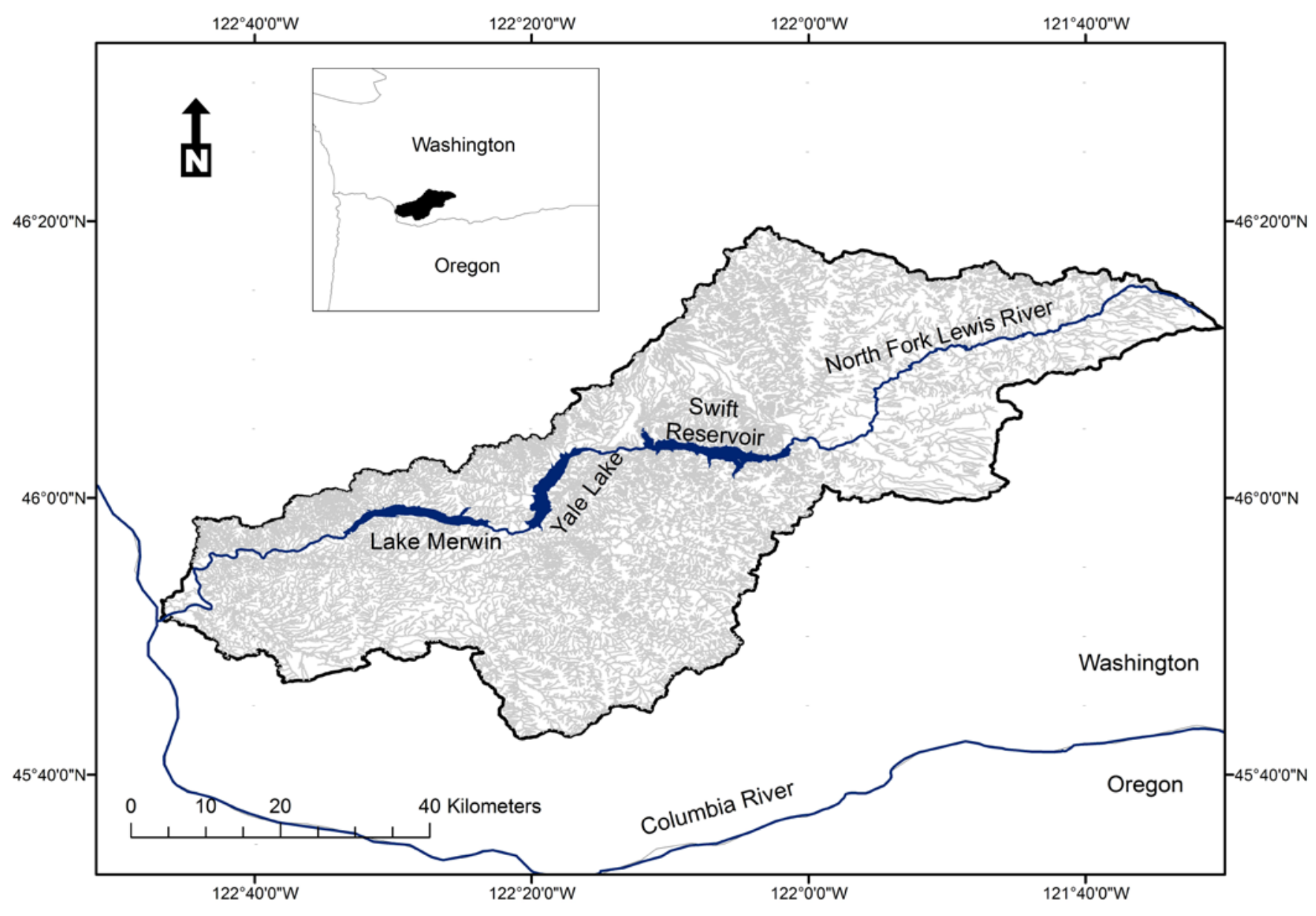

Figure C1. The North Fork of the Lewis River and the three hydropower impoundments: Lake Merwin, Yale Lake, and Swift Reservoir in the Lewis River Basin, Washington. 


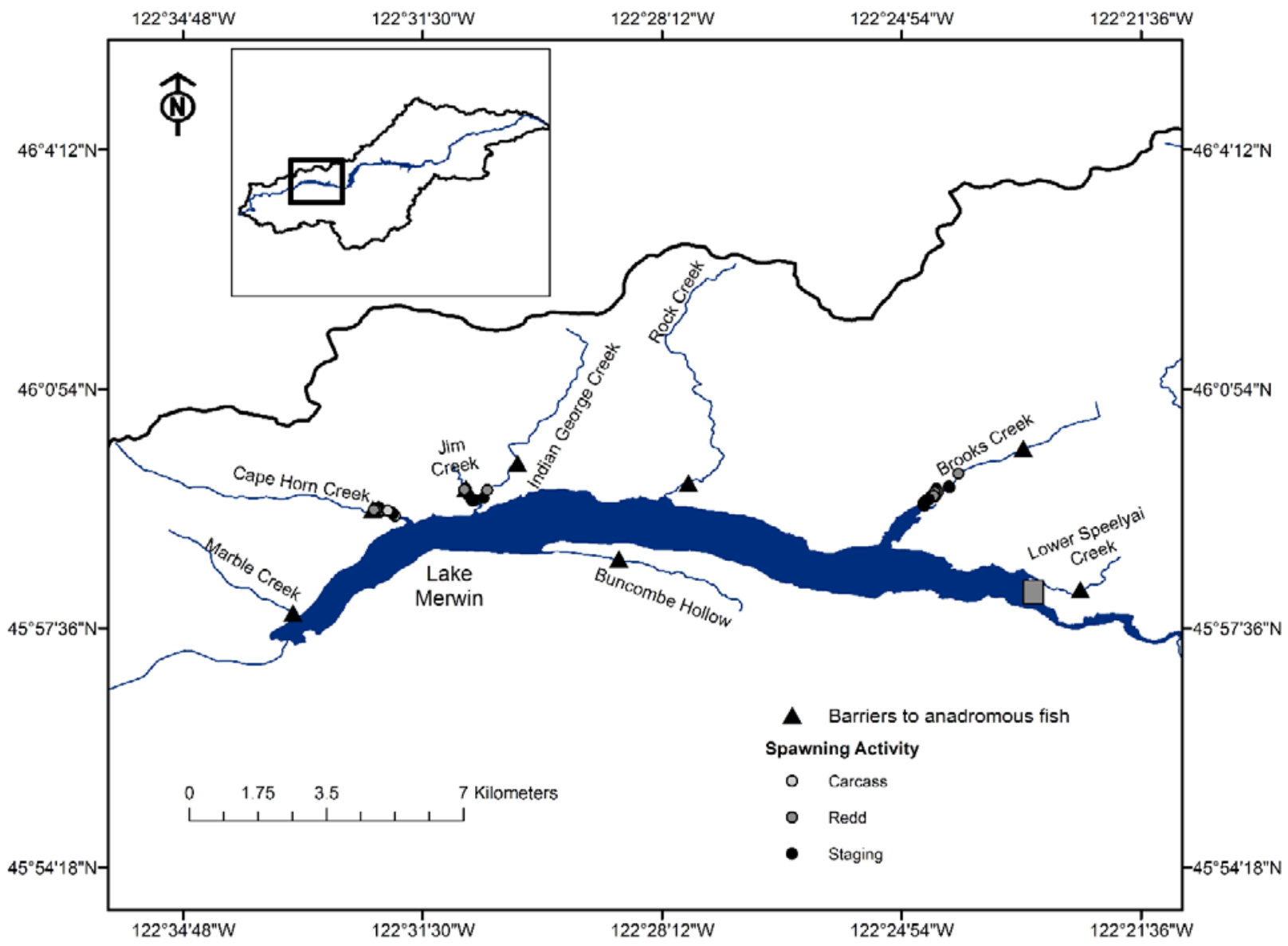

Figure C2. The location of the coho salmon release site (gray square), existing barriers to anadromous species (black triangles), and spawning activity including observed carcass locations (hollow circles), adults observed staging (gray circles), and redds (black circles) during 2015 fall spawning surveys in Lake Merwin tributaries. The inset map shows the location of Lake Merwin study area in relation to the Lewis River Basin, Washington. 

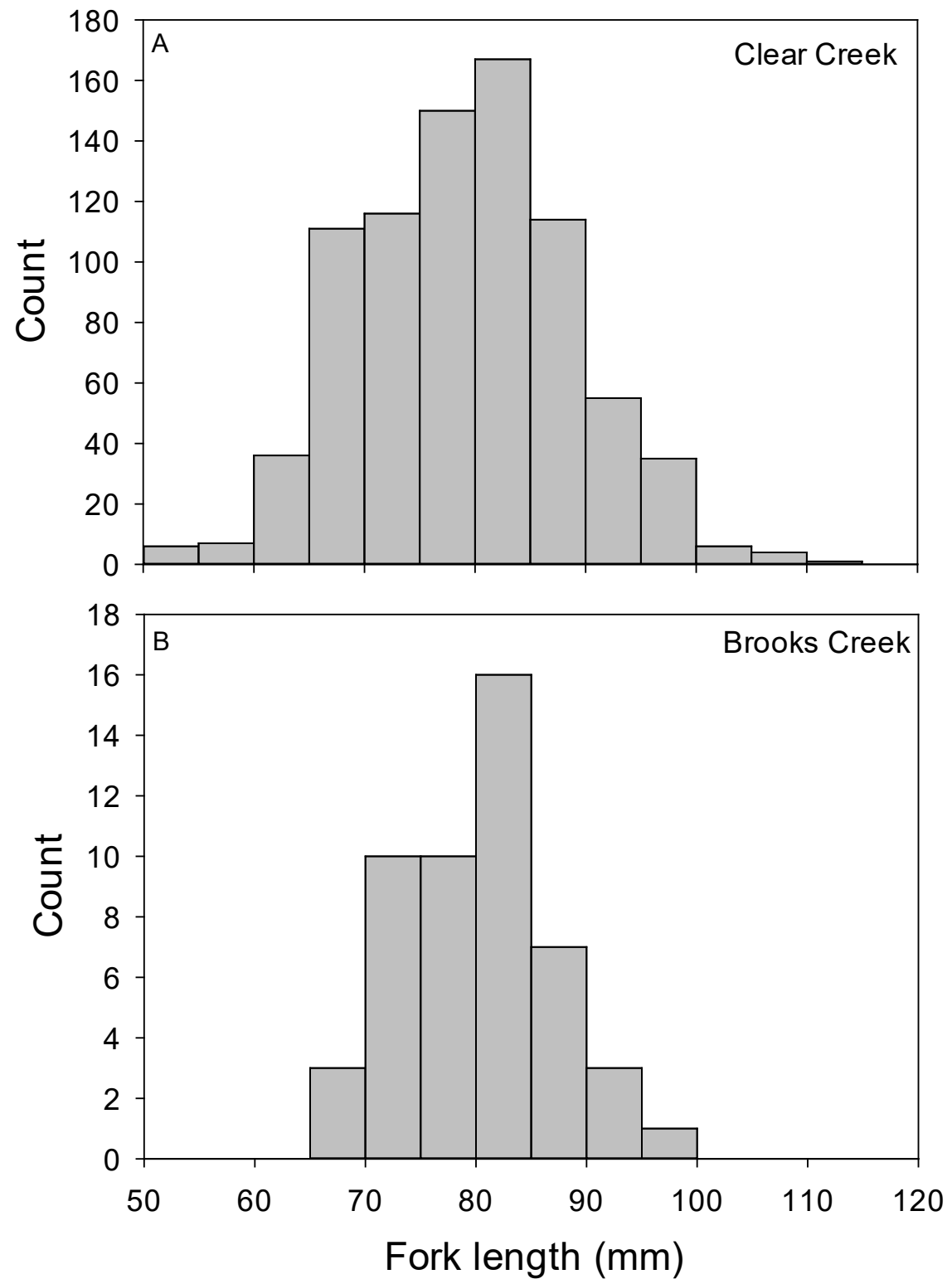

Figure C3. Length frequency histograms for juvenile coho salmon from early summer electrofishing surveys in 2013-2015 for $A$, Clear Creek (Swift Reservoir) and in 2015 for B, Brooks Creek demonstrating generally consistent sizes. mm, millimeter. 


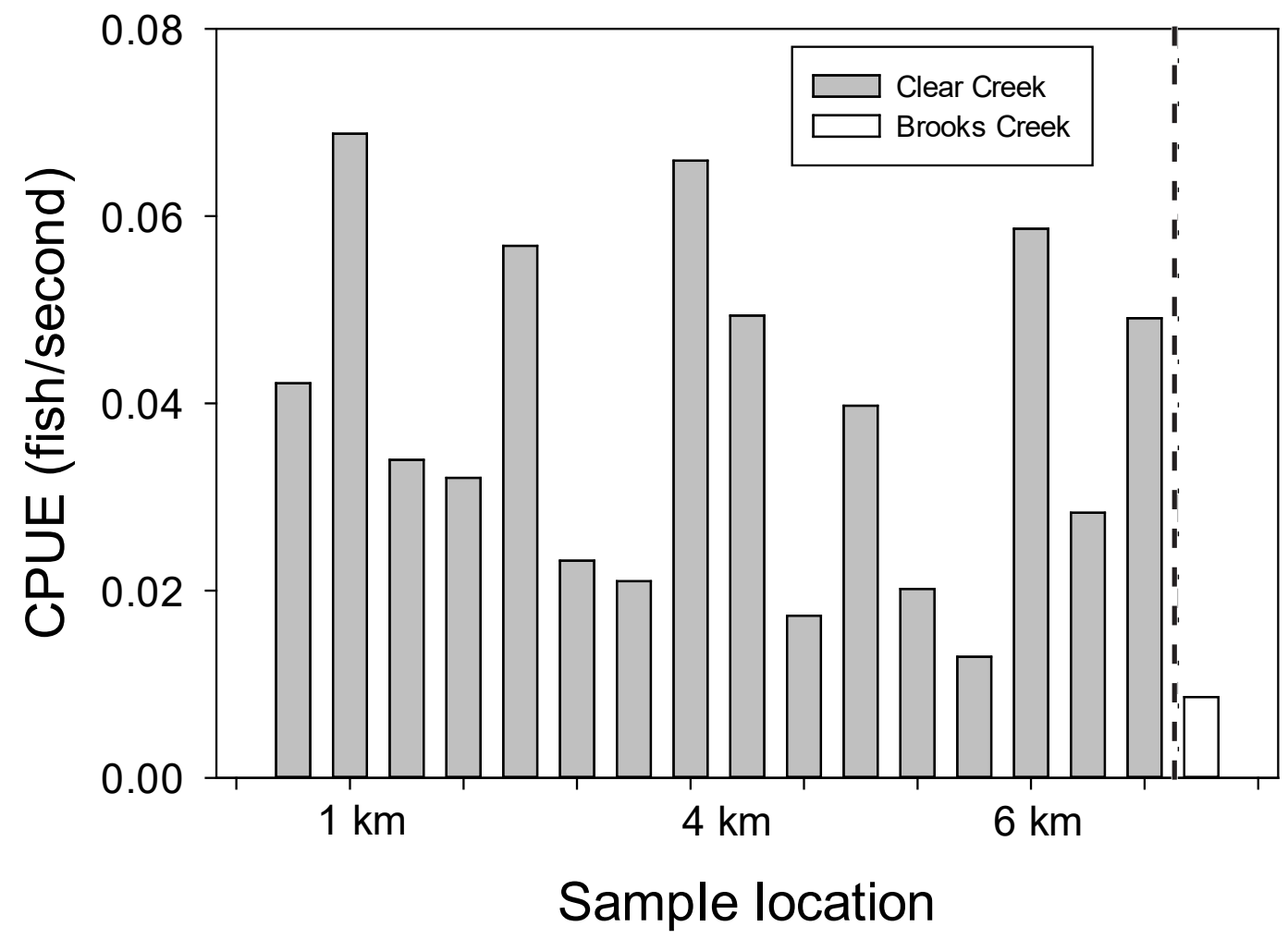

Figure C4. Catch-per-unit-effort (CPUE) in fish per second estimates from Brooks Creek (hollow; Lake Merwin) and sample reaches from Clear Creek (gray; Swift Reservoir) from 2015. Data from Clear Creek are organized from the most downstream (far left, near mouth of Clear Creek) upstream to the barrier based on systematic surveys (greater than 7 kilometers [km] total), with approximate distances upstream noted on $\mathrm{x}$-axis. 


\section{Tables}

Table C1. Field estimates from 2014 surveys of the percent of fine sediment (less than 6 millimeters) in pool tailouts in available tributary habitat in Lake Merwin and Yale Lake, Washington (see "Habitat Assessment of Tributaries to Swift Reservoir, Yale Lake, and Lake Merwin, Washington," chap. B of this report).

$[\%$, percent]

\begin{tabular}{|l|c|}
\hline \multicolumn{1}{|c|}{ Stream Lake Merwin } & \% fines in pool tailouts \\
\hline \multicolumn{2}{|c|}{} \\
\hline Brooks Creek & $5.4(5.3)$ \\
\hline Buncombe Hollow Creek & $12.3(13.3)$ \\
\hline Cape Horn Creek & $2.0(0.5)$ \\
\hline Indian George Creek & $2.5(1.5)$ \\
\hline Jim Creek & $0.6(0)$ \\
\hline Lower Speelyai Creek & $4.9(0)$ \\
\hline & \\
\hline Cougar Creek & $0.2(0.4)$ \\
\hline Dog Creek & $1.0(0)$ \\
\hline North fork of Siouxon Creek & $2.3(2.3)$ \\
\hline Ole Creek & $0.2(0.4)$ \\
\hline Panamaker Creek & $5.2(4.0)$ \\
\hline Siouxon Creek & $0.3(0.6)$ \\
\hline Speelyai Creek & $3.8(9.9)$ \\
\hline Swift Bypass Channel & $0(0)$ \\
\hline West Fork Speelyai Creek & $2.0(3.0)$ \\
\hline West tributary of Speelyai Creek & $1.2(1.7)$ \\
\hline & \\
\hline
\end{tabular}

Table C2. Percent of substrate estimated as bedrock, boulder (greater than 256 millimeters [mm]), large cobbles (127.1-256 mm), small cobbles (64.1-127 mm), gravel (2-64 mm), and fines (less than $2 \mathrm{~mm}$ ) in fast-water habitat (riffles, glides, cascades) from 2014 field surveys in tributaries to Lake Merwin and Yale Lake, Washington (see "Habitat Assessment of Tributaries to Swift Reservoir, Yale Lake, and Lake Merwin, Washintgon," chap. B of this report).

\begin{tabular}{|l|c|c|c|c|c|c|}
\hline \multicolumn{1}{|c|}{ Stream } & Bedrock & Boulder & Cobble & Gravel & Fines \\
\hline \multicolumn{7}{|c|}{ Lake Merwin } \\
\hline Brooks Creek & 0.5 & 7.4 & 25.9 & 57.7 & 7.4 \\
\hline Buncombe Hollow Creek & 38.2 & 0 & 22 & 33.1 & 6.6 \\
\hline Cape Horn Creek & 27.4 & 15.4 & 31.1 & 19.8 & 6.4 \\
\hline Indian George Creek & 5.1 & 5.9 & 43.7 & 42.4 & 1.8 \\
\hline Jim Creek & 20.4 & 13.5 & 22.1 & 37.7 & 5.9 \\
\hline Lower Speelyai Creek & 0 & 6.4 & 15.5 & 53.6 & 15.5 \\
\hline \multicolumn{7}{|c|}{ Yale Lake } \\
\hline Cougar Creek & 0 & 0.9 & 34.4 & 52.7 & 3.5 \\
\hline Dog Creek & 0 & 23.6 & 31.8 & 35.5 & 9.1 \\
\hline North Fork of Siouxon Creek & 16.7 & 58.3 & 13.3 & 11.7 & 0 \\
\hline Ole Creek & 0 & 14 & 52.7 & 32.3 & 1 \\
\hline Panamaker Creek & 10 & 12.4 & 57.1 & 20 & 0 \\
\hline Siouxon Creek & 13.3 & 46.1 & 33.3 & 7.2 & 0 \\
\hline Speelyai Creek & 4.7 & 38 & 40.8 & 11.3 & 0.6 \\
\hline Swift Bypass Channel & 0.4 & 26.7 & 57 & 10.8 & 4.2 \\
\hline West Fork Speelyai Creek & 2 & 59.5 & 30.6 & 7.9 & 0 \\
\hline West Tributary of Speelyai Creek & 0.5 & 34.7 & 48.1 & 15.4 & 0.1 \\
\hline
\end{tabular}




\section{Chapter D. Assessment of Juvenile Fish Production and Outmigration Success on the Lewis River, Washington}

\section{Introduction}

Given the differences in habitat and resident fish in Lake Merwin, Yale Lake, and Swift Reservoir, Washington (fig. D1), an important step of the Lewis River anadromous Oncorhynchus spp. (Pacific salmon) and O. mykiss (steelhead) reintroduction is to evaluate the survival and behavior of smolts in both stream and reservoir environments. Furthermore, understanding the timing of anadromous species outmigration to reservoirs, particularly as it relates to ambient conditions (for example, streamflow), will provide critical insight into the strength of these factors relative to intraannual cycles (that is, seasons). Because of the lack of anadromous fish currently present in Yale Lake and Lake Merwin, it was necessary to thoroughly evaluate these patterns by assessments in Swift Reservoir in combination with data collected from test smolts in Yale Lake. Integrating the information from ongoing data collection regarding outmigration timing, travel times within reservoirs, and results understanding of potential predation effects (see "Piscine Predator Impact Studies 2013-2014, Lake Merwin, Washington," chap. E of this report, and community interactions and capacity (see "Studies Assessing Adult Potential for Spawning Success in Yale Lake and Lake Merwin, Washington," chap. C of this report) allows for a more comprehensive assessment of the factors that may be limiting successful reintroductions.

\section{Methods}

To meet our objectives, we used a combination of field studies involving wild (naturally produced) and hatchery-reared salmon in the stream and reservoir habitat in the upper Lewis River Basin. We combined the data from our empirical field studies with additional data from the Washington Department of Fish and Wildlife (DFW) and PacifiCorp biologists for a more comprehensive assessment of juvenile salmon and steelhead production and outmigration.

\section{Stream Habitat}

Sampling of wild O. kisutch (coho salmon). - In 2013, we initiated mark-recapture methods in Clear Creek, a tributary to the Muddy River, to understand juvenile production and outmigration success. We selected Clear Creek as a focal study stream because of its current population of wild coho salmon, the potential for the installment of a passive instream antenna, and identification as a location for juvenile, acclimation spring $O$. tshawytscha (Chinook salmon) releases each year. In late August 2013, we installed a pass-by passive integrated transponder (PIT) tag antenna system with Biomark biologists on Clear Creek (latitude 46.11818, longitude -121.9995 , in decimal degrees), approximately 0.75 kilometers $(\mathrm{km})$ upstream from the confluence with Muddy Creek. The antenna spanned the entire stream channel, and the reader was able to detect fish tagged with both half duplex and full duplex that passed by the antenna. At the antenna site, we also installed a pressure transducer for real-time, continuous measurements of streamflow (that is, stage) and stream temperature and a modem to allow for cellular connection to the system for real-time information gathering. All equipment was powered by solar panels installed adjacent to the site, and all PIT-tag data, antenna and receiver diagnostics, streamflow, and stream temperature can be remotely accessed.

The initial study area in Clear Creek encompassed $11.7 \mathrm{~km}$ from the confluence with Muddy Creek upstream to the first known upstream barrier. We used a systematic sampling design with reach lengths of a minimum of 250 meters $(\mathrm{m})$ and a minimum target of 33 percent sampling rate for 2013. Reach lengths varied, however, as we began each reach at a pool tail and continued sampling until the first pool beyond $250 \mathrm{~m}$. We contracted our study area in 2014 by restricting our efforts to reaches that were above the Biomark PIT-tag antenna system to allow for detection of fish moving downstream past the antennas. This 
resulted in a study area of approximately $10.5 \mathrm{~km}$, which we maintained in 2015 . We continued using a systematic sampling design with reach lengths of a minimum of $250 \mathrm{~m}$ and a minimum target of 33 percent sampling rate (fig. D2). Reach lengths varied, however, as we began each reach at a pool tail and continued sampling until the first pool beyond $250 \mathrm{~m}$. In 2014 and 2015, we only surveyed those reaches above our PIT-tag antenna.

During 2013, we used a variety of sampling techniques to capture fish including backpack electrofishing, minnow traps, seining, and herding fish with a snorkeler into a seine. After evaluating the variety of sampling techniques used in 2013, we determined backpack electrofishing to be the most efficient technique, and this method was used exclusively during 20142015. Each year, all sampling occurred during the summer and early fall, and when captured, we sedated fish using the anesthetic AQUI-S (approximately 1.5 milliliters to 8 liters of water) and recorded weights and lengths (fork length). We marked all salmonids greater than 80 millimeters $(\mathrm{mm})$ with a $12-\mathrm{mm}$ full duplex PIT-tag in the dorsal sinus. Upon completion of marking, we placed fish in recovery pens within the sample reach; after equilibrium was fully regained, we returned fish to the sample reach. We spatially referenced each marked fish with a specific sampling reach to allow for analyses of spatial and temporal movement patterns.

We used the individual PIT-tag information in combination with recapture information at the fixed PIT-tag antenna to quantify spatial patterns of outmigration and potential factors affecting outmigration. In addition to our sampling data, we summarized information from individual fish PIT-tagged by Washington DFW and PacifiCorp biologists at a rotary screw trap located just upstream from the head of the reservoir at a pool (that is, Eagle Cliff; fig. D2). We integrated mark and recapture information from Clear Creek and the rotary screw trap with recaptures at the floating surface collector (FSC) on Swift Reservoir to estimate timing of outmigration and reservoir rearing and the proportion of fish marked collected at the FSC (that is, collection efficiency).

Ultimately integrating data from individual marking and recapture events will allow for robust estimates of production to the collector in Swift Reservoir. We computed initial survival estimates for wild coho salmon during the tributary phase using individual mark-recapture information including marking, antenna recapture events, and recaptures at the FSC in Swift Reservoir. We used a Cormack-Jolly-Seber mark-recapture model, which is commonly used for anadromous species survival estimates (Achord and others, 2007; Brakensiek and Hankin, 2007). We estimated apparent survival ( $\Phi)$ for fish marked in tributaries in 2013 and 2014, but we did not include fish marked in 2015 as outmigration and rearing continued in 2016. With only three sampling events (marking, antenna recaptures, and FSC), we were unable to estimate capture probability at the FSC and estimate survival in the reservoir environment. To overcome this hurdle, we used the proportion of fish marked at the rotary screw trap that were collected at the FSC $(0.15$; see "Wild coho salmon outmigration patterns" in the "Results and Discussion" section of this chapter) as the recapture probability at the FSC (fixed) with the modeling analysis. We consider this estimate to be appropriate as the rotary screw trap is located proximate to the reservoir (fig. D2), providing a more accurate estimate of recapture through the reservoir environment.

Acclimation Chinook salmon and coho salmon.-Hatchery-reared acclimation salmon remain an important part of the reintroduction efforts in the upper Lewis River. Starting in 2013, a portion of acclimation smolts have been PIT-tagged (table D1), providing information on outmigration patterns and success. Only in 2013 were hatchery-reared coho salmon and spring Chinook salmon released in Swift Reservoir tributaries, and only spring Chinook salmon were released in subsequent years. Most of the fish have been yearlings released during spring (45,000 in 2014 and 109,666 in 2015); however, an October release of 48,000 juvenile spring Chinook salmon (also yearlings) occurred in 2015. We used date of release, Clear Creek antenna recapture information, and date at which fish are captured at the FSC to estimate residence time and timing of outmigration from tributaries, travel times across the reservoir to the collector, and proportion of individuals reaching the collector.

\section{Reservoir Habitat}

To evaluate smolt movement behavior and migration timing through Yale Lake and to locate smolt aggregations in the forebay to guide the siting of a future smolt collector, coho salmon smolt movements were assessed before, during, and after a test release using a hydroacoustic system. A total of 5,000 hatchery-reared coho salmon smolts were released into Yale Lake at Yale Park (middle of reservoir; fig. D3) on April 8, 2014, at 09:50 (24-hour).

Hydroacoustic surveys of 2014 smolt test release in Yale Lake.-The hydroacoustics system used for all surveys consisted of two multiplexed split-beam 200 kilohertz transducers towed in sidelooking (12.8 degrees $\left[{ }^{\circ}\right]$ full beam angle) and downlooking ( $6.8^{\circ}$ full beam angle) orientations. The transducers were connected to a Biosonics DE -6000 scientific echo sounder with the following data acquisition parameters: bottom threshold $=70 \mathrm{~m}$, ping rate $=2-3$ pulses per second, pulse width $=0.3$ millisecond, and target strength detection threshold $=-65$ decibels $(\mathrm{dB})$. Surveys consisted of transects extending the length of the east and west banks and along the center axis of the reservoir, as well as a zig-zag transect going between banks through the forebay and within the lower quarter of the reservoir. We divided transects running the length of the reservoir into five distinct analysis regions based on minutes of latitude (fig. D3). These divisions were designed to obtain finer spatial resolution in fish distribution 
yet maintain enough sampling power within each spatial unit to obtain reliable estimates of fish densities. Most transects were surveyed during daylight, but some regions of the reservoir were surveyed during dusk (defined as 1 hour after sunset) and at night. Light levels (lux and microeinsteins: $[\mathrm{uE}] \cdot \mathrm{s}^{-1} \cdot \mathrm{m}^{-2}$ ) were recorded at the start, end, and periodically throughout each transect. Light intensity is important, as fish like Oncorhynchus nerka (kokanee salmon), the primary pelagic planktivore in Yale Lake, often exhibit diel vertical migrations, and their distribution is highly sensitive to time of day and light conditions (for example, Eggers, 1978).

Prerelease surveys were conducted in Yale Lake to establish the background distribution and density of resident fish targets on April 7, 2014, and on the morning of April 8, 2014. The daylight part of the survey conducted on April 7, 2014 consisted of transects along both banks and a zig-zag transect near the dam (table D2, fig. D3). Also, on April 7, 2014, a survey of the middle axis was conducted at dusk, and a second zig-zag transect was conducted at night. A short transect along the middle axis was conducted before the release on the morning of April 8, 2014, to examine the daytime distribution of fish, and these daylight surveys were used in place of the dusk surveys for comparison with postrelease transects conducted in these areas during daylight.

The boat launch at Yale Park and surrounding areas was surveyed while fish were being released to directly assess the target strengths of smolts as registered by the hydroacoustic survey system. Numerous direct sightings of the released smolts swimming through water while being sampled by the sidelooking transducer were linked to specific locations on the corresponding echograms (fig. D4). Postrelease surveys were conducted along the same tracks as the prerelease surveys, along both banks, the center axis, and in a zig-zag pattern within the forebay and lower quarter of the reservoir. We surveyed transects following the smolt release on April 8, 2014, during daylight beginning at 13:58 (24-hour time) and concluding at 17:06 (table D2). We surveyed an additional zig-zag transect in the lower quarter of the reservoir from 20:31 to 21:01 to examine the density of fish near the dam at night.

A third survey was conducted on April 9, 2014, the day after the smolts were released. All regions were surveyed during daylight beginning at 07:25 and concluding at 10:43 (table D3). An additional transect was conducted at dusk along the middleaxis in Region 4 (middle to upper reservoir) from 19:28 to 19:47 to qualitatively examine whether smolts had left the vicinity of the release site. Lastly, we surveyed the same zig-zag transect within the lower quarter of the reservoir during the night from 20:45 to 21:18 to assess whether smolts shifted to lower regions of the reservoir or were aggregated near the dam.

All echograms were visually examined, and regions of background noise and nonfish targets were excluded from the analysis. Standard echo counting techniques (Beauchamp and others, 1997, 2009) with EchoView 5.4 software (Myriax Pty. Ltd.) were used to evaluate depth specific densities of fish targets. Targets measured by the sidelooking transducer were used to estimate fish density within the upper 5-20 $\mathrm{m}$ of the water column over a range of 0-40 m from the survey vessel. Target strength frequency histograms were examined for groups of smolts sampled during the release at the boat launch to determine the range of target strengths corresponding to the smolts as seen by the sidelooking transducer. Fish densities were calculated for targets with strengths within the range determined for the smolts $(-55$ to $-43 \mathrm{~dB})$ on the sidelooker. Water depths were too shallow to effectively sample smolts during the release with the downlooking transducer. Therefore, we used the average fork length of test released coho salmon smolts in 2013 (data were unavailable for 2014) to determine the likely range of target strengths expected on the downlooking transducer based on the fish length-to-target strength relation of Love (1977). The average fork length of test released Coho in 2013 was $127 \mathrm{~mm}(\mathrm{n}=663$, ranged $84-178 \mathrm{~mm}, \mathrm{SD}=10.3 \mathrm{~mm})$, so densities were calculated for targets with strengths equivalent of $75-175 \mathrm{~mm}$ total length fish ( -42 to $-49 \mathrm{~dB}$ ). Echograms from both the sidelooking and downlooking transducers were visually examined for aggregations of smolt-sized targets, especially within the vicinity of the forebay.

Without real-time direct fish sampling during these hydroacoustic surveys to ground truth observed fish targets, or telemetry data from individually tracked coho salmon smolts, the interpretation of these data were limited to only qualitative descriptions of how smolt-sized fish targets are distributed in the reservoir across time and space. Any noticeable increase in the density of smolt-sized targets within different regions or depths after their release would only be suggestive that coho salmon smolts are occupying or moving through those areas.

\section{Results and Discussion}

By combining empirical field data in both stream and reservoir habitat, our results illustrate clear patterns of relatively rapid movement within the reservoir environment, high reservoir survival, but generally low collection efficiencies of juvenile anadromous salmon and steelhead at the FSC. We consider our results in the context of previous studies in the Pacific Northwest and potential limitations of our results. 


\section{Stream Habitat}

Wild coho salmon juveniles.-Our sampling intensity varied across years to achieve different project objectives. During 2013 and 2014, we sampled primarily in late August and early September, but in 2015 we sampled all reaches in mid-summer (July) and in late September and early October to provide additional recapture information for growth and survival. During 2013, we sampled a total of 20 reaches on Clear Creek for a total of $5.42 \mathrm{~km}$, and in 2014, we sampled 17 reaches totaling $4.65 \mathrm{~km}$. During 2015, we sampled a similar number of reaches and length of stream that were collected in 2014 but with two sampling collections, our sample length of stream was doubled $(9.3 \mathrm{~km})$. Within the sample reaches, we PIT-tagged 357 individual coho salmon in 2013, 883 individuals in 2014, and 780 individuals in 2015. Thus, with double effort in 2015, catch rate for wild coho salmon was roughly half those observed in 2014.

The average size of wild coho salmon captured differed across years and seasons. The average size of coho salmon captured in 2013 (average $=88.5 \mathrm{~mm}$; standard deviation $[\mathrm{SD}]=8.3$ ) was nearly $10 \mathrm{~mm}$ larger than fish captured in 2014 (average $=79.6 \mathrm{~mm} ; \mathrm{SD}=8.1$; fig. D5). The different timing of sampling during 2015 (that is, July and late September) led to shifts in size of sampled coho salmon, as fish captured during July $(78.2 \mathrm{~mm}, \mathrm{SD}=11.8)$ were smaller than those fish captured during late September (average $=85.1, \mathrm{SD}=13.0$ ). The number of coho salmon captured by reach varied considerably within and across years, and juvenile coho salmon were captured as high as $7.0 \mathrm{~km}$ upstream from the confluence with the Muddy River (fig. D6).

Wild coho salmon outmigration patterns. - An important part of the reintroduction of coho salmon is to understand the diversity and overall patterns of outmigration and rearing. Through December 31, 2015, antenna recapture data suggest considerable differences in migration of wild coho salmon across years. In 2013, most marked fish emigrated during September (fig. D7), which coincided with a large precipitation event. On the contrary, fish marked in 2014 demonstrated diffused outmigration throughout much of the winter with the peak outmigration during April-June 2015. Across years, however, our results are generally similar to previous studies (Bennett and others, 2011; Pess and others, 2011) as we found the largest numbers of fish emigrating during the fall and late spring (figs. D6, D7). Overall, the two-season outmigration pattern and differences across years suggests use of screw trap methods to monitor outmigration should consider sampling during both spring and fall.

Across both years, most fish emigrating in early fall were marked in the downstream reaches (fig. D8). Nearly identical to Pess and others, (2011), we found no outmigration from mid-May (year day 140) through early August (year day 212; fig. D8), perhaps a result of low streamflow patterns typical during this period and avoidance of shallow riffle habitat. Coho salmon emigrating during the period of October 1 through May displayed a wider range of location of origin (that is, tagging). In contrast to Pess and others (2011), however, we observed no clear trends in outmigration date when considering coho salmon size (not shown).

Considering individual PIT-tag data and PIT-tag antenna, and screw trap data along with the FSC data provides insight into the rearing behavior of juvenile coho salmon in the upper Lewis River. Individual marking data in Clear Creek suggest coho salmon reside in Swift Reservoir for nearly 4 months, with the median number of days between emigrating from Clear Creek and collecting at the FSC as 121 days (range $=21-347$ days). These outmigration patterns are substantially longer than patterns observed for coho salmon PIT-tagged at the Eagle Cliff Screw Trap (median reservoir travel time $=37$ days, range $=8-391$ ). The observed differences in reservoir rearing are largely driven by the variability in the timing of outmigration from Clear Creek, as fish emigrate throughout much of the year, and the sampling duration of screw trap information (spring and early summer).

We find general consistent patterns of timing of juvenile coho salmon to the FSC across data sources. The majority (93 percent) of wild (fig. D9A) and hatchery-reared (fig. D9B) coho salmon PIT-tagged in Clear Creek and collected at the FSC have occurred during the spring (April 15-June 30). This timing of outmigration to the collector is consistent with total counts of coho salmon smolts at the FSC (fig. D10) and from individuals marked at the Eagle Creek screw trap (2013-2014; fig. D11). The predominant outmigration through spring is consistent with coho salmon outmigration patterns elsewhere in the Pacific Northwest (Miller and Sadro 2003; Pess and others, 2011; Rebenack and others, 2015).

Collection rates at the FSC of juvenile coho salmon have been relatively low (see "Review of Information Relevant to Anadromous Fish Reintroduction to and Fish Passage in the Lewis River, Washington," chap. A of this report) across all data sources. Overall, relatively few (3.4 percent) of the PIT-tagged wild coho salmon (2,020 individuals; 2013-2015) from Clear Creek have reached the FSC through December 31, 2015. However, the percent of coho salmon PIT-tagged at a rotary screw trap (Eagle Cliff; latitude 46.0654, longitude -122.01998, in decimal degrees) that were successfully collected at the FSC during 2014 and 2015 was considerably higher (15.2 percent; table D3). Such differences are likely a result of the timing of marking, as fish in Clear Creek are marked during late September, while fish marked at the Eagle Cliff screw trap occurred during late spring, nearly 7 months later. The timing of sampling coupled with overwinter survival rates, which can be less than 50 percent (Ford and Lonzarich, 2000), likely explain the different collection rates at the FSC.

Juvenile coho salmon survival.-Initial estimates of coho salmon survival from mark-recapture analysis (2013 and 2014 data only) suggest apparent survival is high $(\Phi=0.54,0.48-0.61)$. The high summer and early fall survival is consistent with recent apparent survival estimates (Brakensiek and Hankin, 2007). However, we were unable to delineate time periods (Clear Creek survival compared to reservoir survival) at the time of this report because of data limitations. Updated apparent survival estimates will be possible after fish marked in 2015 have emigrated. 
Acclimation Chinook salmon and coho salmon.-Outmigration patterns for spring Chinook salmon include a relatively rapid downstream movement from release sites and a reservoir rearing period found to be longer and more variable than observed for coho salmon. As outlined in "Studies Assessing Adult Potential for Spawning Success in Yale Lake and Lake Merwin, Washington," chap. C of this report, the majority (60 percent) of acclimation spring Chinook salmon emigrated from Clear Creek within the first 7 days after release and 98 percent within 60 days. For acclimation Chinook salmon released in Clear Creek, we found the median residence time in Swift Reservoir, which we calculated as the time since emigrating from Clear Creek, as 70 days (range $=5-320$; fig. D12A). However, nearly 30 percent of the spring Chinook salmon resided in Swift Reservoir for more than 170 days (fig. D12B). When considering all PIT-tagged spring Chinook salmon, there has been a bimodal distribution of the total time since release to the FSC with an initial, substantially larger peak at 75 days after release and a second, smaller peak at approximately 9 months after release (fig. D13). The interannual variability in outmigration is not uncommon and may be linked with hydrologic regimes (Romer and others, 2014). The observed rearing times in Swift Reservoir are considerably shorter than found in Willamette Valley reservoirs (Oregon) where spring Chinook salmon appear to spend 5-7 months in reservoir environments (Romer and others, 2014). Such differences, however, are likely driven by size at outmigration into the reservoir environment as spring Chinook salmon in the Willamette Valley reservoirs enter the reservoir at size typically less than $100 \mathrm{~mm}$, which are considerably smaller than the hatchery-reared acclimation spring Chinook salmon in Swift Reservoir (greater than $120 \mathrm{~mm}$ ). Extended use of reservoir environments for rearing is common in anadromous juveniles (Hegg and others, 2013; Bourret and others, 2014) and can result in pronounced growth. The median days between initial release and collection at the FSC were generally consistent across release locations for 2013 (Clear Creek=67 days, Crab Creek=57 days, Muddy River=75 days; fig. D14), but we observed considerable differences in the median travel time in 2014 for Clear Creek (259 days). Travel times during 2015 were consistent with 2013 for Clear Creek (69 days) and Crab Creek (70 days). The differences in reservoir rearing time are largely driven by fish released in 2014, which demonstrated considerably more variability than releases during 2013 and 2015 (fig. D15).

Overall, the proportion of juvenile salmon collected as the FSC has been relatively low across species and years. Nearly 14 percent of the hatchery-reared juvenile coho salmon released in Clear Creek in 2013 successfully emigrated to the FSC. However, only 1.4 percent (2013), 2 percent (2014), and 1.7 percent (2015) of the acclimation spring Chinook salmon (PITtagged) have successfully emigrated to the FSC (fall 2015 release not included). Based on the total number of spring Chinook salmon released in the spring $2014(45,000)$, spring $2015(109,666)$, and fall $2015(48,000)$, we estimate that as of December 31, 2015, 3.7 percent of the acclimation spring Chinook salmon have been collected at the FSC. Although still low, this is markedly higher than inferences from PIT-tags. Given the likely minimal effects of PIT-tagging on large, acclimation smolts (Prentice and others, 1993) and high estimates of reservoir survival (for example, Williams and others, 2001), the discrepancy between estimates of the proportion of salmon collected at the FSC may be due to the increased number of juvenile spring Chinook salmon because of natural recruitment from residualized individuals from previous releases (for example, Romer and Monzyk, 2014; Perales and others, 2015).

Steelhead outmigration.-Because of the relatively low numbers of wild steelhead present in Swift Reservoir tributaries, no targeted field studies were conducted to assess outmigration success. However, information from screw trap data suggest median travel time for steelhead juveniles (21 days, range $=1-342$ ) was considerably lower than coho salmon (see "Wild coho salmon outmigration patterns" in the "Results and Discussion" section of this chapter). Albeit small sample sizes (table D3), we find a higher proportion of marked steelhead (18.7 percent) than either coho or spring Chinook salmon juveniles, a pattern requiring additional monitoring.

\section{Reservoir Habitat}

Targets identified in hydroacoustic surveys in Yale Lake were predominantly moving downstream after initial release. Smolt-sized targets were found to be using open water (mid-channel) with some skew toward the west bank, the same side as the release. The densities of "smolt-like" targets detected in the surface-oriented sidelooking data and downlooking data were approximately equal.

Density of smolt-sized targets from sidelooking hydroacoustic surveys. - We used sidelooking hydroacoustic surveys to estimate fish densities in the upper portions of the water column (Gangl and Whaley, 2004). When compared to the prerelease surveys in Yale Lake, densities of surface-oriented, smolt-sized targets increased in the afternoon following the morning test release of coho salmon smolts on April 8, 2014. Specifically, the densities of smolt-sized targets increased in the lower half of the reservoir (Regions 1-3) along the west bank (fig. D16). Densities also increased by a factor of nine along the middle axis in the region closest to the dam (Region 1) and in the zig-zig transect in the lower quarter of the reservoir by a factor of 13 during daylight and by a factor of 3 at night. Densities on the day after the release increased relative to prerelease densities in Regions 2 and 3 along the west bank. Densities also increased relative to prerelease in the lower quarter zig-zag transects during both daylight and night. 
On the day following release, densities of surface-oriented smolt-sized targets were similar or slightly higher than the previous day along the west bank in Regions 3 and 2 but were generally lower in the middle axis and other regions of the west bank with no notable pattern on the east bank (fig. D17). Densities estimated from the zig-zig transect in the lower quarter of the reservoir declined by 68 percent during the daylight survey, and the night zig-zag transect remained at similar levels to the day of the release. Aggregations of smolt-sized targets were observed on hydroacoustic echograms on the day after the release near the boat launch in Region 4 during the daylight survey and the additional dusk survey, although the densities in this area did not increase. Aggregations were also observed near Yale Dam during daylight on the day following the release, with the appearance of higher concentrations along the west shoreline in Region 1, directly in front of Saddle Dam, and directly in front of the spillway (fig. D4). Similar patterns were observed in echograms from nighttime near the dam on the day after the release.

Densities of smolt-sized targets from the sidelooking hydroacoustic surveys largely provide inference for the upper $20 \mathrm{~m}$ of the reservoir and suggest relatively rapid downstream movements through Yale Lake. Our results from Yale Lake are consistent with previous efforts in Swift Reservoir, where acoustically tagged coho salmon smolts were detected most commonly in the upper portions of the water column near Swift Dam (PacifiCorp and Cowlitz Public Utility District [PUD], 2004).

Density of smolt-sized targets from downlooking hydroacoustic surveys.-In contrast to sidelooking surveys, we employed downlooking hydroacoustic surveys to evaluate fish densities deeper than $20 \mathrm{~m}$ (that is, the portion of the water column not effectively sampled during sidelooking surveys). Densities of deeper coho salmon smolt-sized targets detected by the downlooking transducer increased during daylight surveys in the afternoon on the day of the release, compared to the prerelease surveys in different depths and regions. Densities increased from 0 to approximately 0.5 targets $/ 1,000$ cubic meters along the east bank in Regions 4 and 5 (fig. D18). We observed an increase of coho salmon smolt-sized targets by a factor of 24 in Region 2 at 50-60 m depths along the west bank. Densities also increased along the middle axis in the region closest to the dam (Region 1) by 210 percent at $20-30 \mathrm{~m} ; 202$ percent at $30-40 \mathrm{~m} ; 251$ percent at $40-50 \mathrm{~m}$; and by a factor of 6 at $50-60 \mathrm{~m}$. The lower quarter zig-zag transect increased by 134 percent at $30-40 \mathrm{~m}$ in the daylight survey, and by 115 percent in the night survey; however, densities in most other depths decreased in the zig-zag transects (fig. D19).

Densities on the day after the release increased relative to the prerelease in Regions 1 and 2 along the middle axis at depths below $30 \mathrm{~m}$, at 10-40 m in Region 3 along the west bank, and at 40-50 m in Region 2 along the east bank (fig. D18). Coho salmon smolt densities also increased at 30-40 m depths in the lower quarter zig-zag transect during daylight (fig. D16).

Densities on the day after the release changed relative to the afternoon and night of the day of release in several regions as well. We observed an increase in densities in Region 3 along the west bank from 0 to 1.3 targets $/ 1,000$ cubic meters at $30-40 \mathrm{~m}$, and Region 2 along the west bank increased by a factor of 4 at 30-40 m (fig. D18). We observed a 135 percent increase at $30-40 \mathrm{~m}$ in Region 1 along the middle axis, as well as a 145 percent increase at $40-50 \mathrm{~m}$ in this area. The lower quarter zig-zag transects during daylight increased by 153 percent at 20-30 m, 127 percent at $30-40 \mathrm{~m}$, and 184 percent at 50-60 m (fig. D19). In contrast, densities decreased by approximately 50 percent in most depths in the lower quarter zig-zag transects at night. Visual observation of aggregations of smolt-sized targets detected in Region 4 near the boat launch and increases in densities in Regions 4 and 5 along the east bank suggest that a portion of the smolts remained in the upper reservoir until at least the day following the release.

The results from monitoring Yale Lake indicate that coho salmon smolts have a relatively short migration time through the reservoir, a pattern similar to previous studies (PacifiCorp and Cowlitz PUD, 2001). The increase in densities of smolt-sized targets in lower regions on the day of the release suggests that smolts were capable of transiting the lower half of Yale Lake in less than a day. A portion of the test release smolts likely moved down reservoir along the west bank (the bank where they were released) immediately upon release, based on the increased densities of smolt-sized targets. It appears many smolts were able to reach the dam and distributed to the middle axis in Region 1 and the east bank in Region 2 within 12 hours of being released. Further increases in densities along the west bank and lower reservoir regions on the day following the release suggest that smolts continued to migrate down the reservoir. Smolts appeared to migrate to the lower quarter of the reservoir where they were detected on the sidelooker in both postrelease surveys during night.

The findings of this study (approximately $7 \mathrm{~km}$ per day) are consistent with the results of radio telemetry tracking of coho salmon smolts in Swift Reservoir, which found that more than half of tagged fish released in the upper reservoir were detected at the dam within 4 days, and the greatest number of detections by time interval was for the period of 1-2 days after release (5.2 km per day [PacifiCorp and Cowlitz PUD, 2001]). However, we acknowledge that migration rates can vary across species and reservoirs with minimal river effect (Miller and others, 2004). Most detections in mobile surveys were along the shore where they were released, generally near river inlets, in coves, and near woody debris.

Detections on the antenna array at Swift Dam revealed that virtually all smolts approached the intake structure in the upper $13.8 \mathrm{~m}$ of the water column, typically from the north side at first and then resided on the south side (PacifiCorp and Cowlitz PUD, 2001). However, the depth of reservoir used by juvenile Chinook salmon can vary considerably by season based on thermal regimes (Beeman and Maule, 2006; Kock and others, 2015; Beeman and others, 2016), suggesting the need to integrate information on the outmigration timing through Swift Reservoir. Results from telemetry of Chinook salmon smolts revealed that they also approached the intake in the upper $13.8 \mathrm{~m}$ of the water column, with a mean travel time of 5.5 days from release at 
Eagle Cliff Park to detection at the project intake (PacifiCorp and Cowlitz PUD, 2002). These results indicate that the densities observed by the sidelooking transducer may be more important for identifying smolt distribution than densities observed by the downlooking transducer.

We acknowledge several limitations to the hydroacoustic survey analyses. Several environmental conditions potentially biased our results or limited our ability to interpret results within the context of smolt movement through the reservoir. The prerelease survey along the middle axis in Regions 3-5 represented crepuscular fish densities, whereas the postrelease surveys represent daylight densities, making direct comparison difficult. The propensity of kokanee salmon to migrate vertically across the diel sequence likely added some variability into our results, particularly during dusk and night periods, which could have masked the signal of the relatively small release of 5,000 test smolts. Furthermore, noise from wind and rain, as well as the tops of standing timber masked the signal of fish targets for portions of the sidelooking data.

Despite these limitations, our results are consistent with other studies indicating use of relatively shallow habitats in reservoirs (Durkin and others, 1970; Dilley, 1994). Concomitantly, our data from hydroacoustic surveys in Yale Lake with coho salmon, findings from previous radiotelemetry studies with Chinook salmon in Swift Reservoir (PacifiCorp and Cowlitz PUD, 2002), and other studies (for example, Aitken and others, 1996) suggest reservoir travel times to be relatively rapid. Furthermore, we have consistently found considerably longer residence times for wild coho salmon and acclimation spring Chinook salmon in the Swift Reservoir than observed in test releases. Indeed, recent studies in Swift Reservoir (PacifiCorp and Cowlitz PUD, 2017) illustrated that a large portion of test-tagged juvenile salmon emigrated within $150 \mathrm{~m}$ of the zone of influence of the FSC but that most fish were not collected resulting in low collection efficiencies. Synthesizing our data with these previous studies suggest difficulties of fish "finding" the FSC, a pattern supported by studies elsewhere (Beeman and others, 2016).

We also acknowledge that collection efficiencies may be affected by summer thermal regimes within the reservoir. During the summer months, epilimnetic temperatures in Swift Reservoir can exceed 18 degrees Celsius. The effect of temperature on juvenile salmon behavior (Tiffan and others, 2009) and collection in trap and haul operations (Beeman and others, 2016) suggest temperatures during July through early September in Swift Reservoir may act as a thermal barrier during these months. However, the relative short duration of warm surface temperatures is unlikely to disproportionately explain the low collection rates of juvenile salmon in Swift Reservoir. Together, these results highlight the need to consider alternative measures to enhance collection at the FSC, particularly as delays in capture at the FSC may affect juvenile mortality (for example, Buchanan and others, 2009) and the lack of understanding of the effects of residualized populations of coho salmon and Chinook salmon on recovery efforts (Romer and Monzyk, 2014).

\section{Conclusions}

Together, our results suggest outmigration success appears to be limited by collection at the FSC. A critical next step in enhancing the reintroduction success will be to identify those factors affecting delays and overall capture rates of outmigrating juvenile salmon and steelhead. Such information may be particularly important as the proportion of juvenile fish collected at the FSC (across species and data sources-PIT-tags, screw trap, and so on) is generally low when compared to data from similar trap and haul operations (see "Review of Information Relevant to Anadromous Fish Reintroduction to and Fish Passage in the Lewis River, Washington," chap. A of this report). Furthermore, low proportions of juvenile salmon collected at the FSC are likely to have profound effects on community interactions and the overall capacity to support rearing in the reservoir environment (see "Studies Assessing Adult Potential for Spawning Success in Yale Lake and Lake Merwin, Washington," chap. C of this report).

\section{References}

Achord, S., Zabel, R.W., and Sandford, B.P., 2007, Migration timing, growth, and estimated parr-to-smolt survival rates of wild snake river spring-summer Chinook salmon from the salmon river basin, Idaho, to the Lower Snake River: Transactions of the American Fisheries Society, v. 136, no. 1, p. 142-154.

Aitken, J.K., Cook-Tabor, C.K., and Wunderlich, R.C., 1996, Travel time of coho salmon and steelhead smolts emigrating through Howard Hanson Reservoir, King County, Washington: Olympia, Wash., U.S. Fish and Wildlife Service, 64 p.

Beauchamp, D.A., Luecke, C., Wurtsbaugh, W.A., Gross, H.G., Budy, P.E., Spaulding, S., Dillenger, R., and Gubala, C.P., 1997, Hydroacoustic assessment of abundance and diel distribution of sockeye salmon and kokanee in the Sawtooth Valley Lakes, Idaho: North American Journal of Fisheries Management, v. 17, p. 253-267. 
Beauchamp, D.A., Parrish, D., and Whaley, P., 2009, Salmonids/coldwater species in large standing waters, in Bonar, S.A., Willis, D., and Hubert, W.A., eds., Standard sampling methods for North American freshwater fishes: Bethesda, Md., American Fisheries Society, p. 97-117.

Beeman, J.W., Evans, R.E., Haner, P.V., Hansel, H.C., Hansen, A.C., Hansen, G.S., Hatton, T.W., Sprando, J.M., Smith, C.D., and Adams, N.S., 2016, Evalauation of the biological and hydraulic performance of the portable floating fish collector at Cougar Reservoir and Dam, Oregon, 2014: U.S. Geological Survey Open-File Report 2016-1003, http://pubs.er.usgs.gov/ publication/ofr20161197.

Beeman, J.W., and Maule, A.G., 2006, Migration depths of juvenile Chinook salmon and steelhead relative to total dissolved gas supersaturation in a Columbia River reservoir: Transactions of the American Fisheries Society, v. 135, p. 584-594.

Bennett, T.R., Wissmar, R.C., and Roni, P., 2011, Fall and spring emigration timing of juvenile coho salmon from East Twin River, Washington: Northwest Science, v. 85, no. 4, p. 562-570.

Bourret, S.L., Kennedy, B.P., Caudill, C.C., and Chittaro, P.M., 2014, Using otolith chemical and structural analysis to investigate reservoir habitat use by juvenile Chinook salmon Oncorhynchus tshawytscha: Journal of Fish Biology, v. 85, no. 5, p. $1507-1525$.

Brakensiek, K.E., and Hankin, D.G., 2007, Estimating overwinter survival of juvenile Coho salmon in a northern California stream-Accounting for effects of passive integrated transponder tagging mortality and size-dependent survival: Transactions of the American Fisheries Society, v. 136, no. 5, p. 1423-1437.

Buchanan, R.A., Skalski, J.R., and McMichael, G.A., 2009, Differentiating mortality from delayed migration in subyearling fall Chinook salmon (Oncorhynchus tshawytscha): Canadian Journal of Fisheries and Aquatic Sciences, v. 66, no. 12, p. 22432255 .

Dilley, S.J., 1994, Horizontal and vertical distribution of juvenile salmonids in Howard Hanson Reservoir: Olympia, Wash., U.S. Fish and Wildlife Service, $49 \mathrm{p}$.

Durkin, J.T., Park, D.L., and Raleigh, R.F., 1970, Distribution and movement of juvenile salmon in Brownlee Reservoir, 1962-1965: Fishery Bulletin, v 68, p. 219-243.

Eggers, D.M., 1978, Limnetic feeding behavior of juvenile sockeye salmon in Lake Washington and predator avoidance: Limnology and Oceanography, v. 23, p. 1114-1125.

Ford, J.E., and Lonzarich, D.G., 2000, Over-winter survival and habitat use by juvenile coho salmon (Oncorhynchus kisutch) in two Lake Superior tributaries: Journal of Great Lakes Research, v. 26, p. 94-101.

Gangl, R.S., and Whaley, R.A., 2004, Comparison of fish density estimates from repeated hydroacoustic surveys on two Wyoming waters: North American Journal of Fisheries Management, v. 24, no. 4, p. 1279-1287.

Hegg, J.C., Kennedy, B.P., Chittaro, P.M., and Zabel, R.W., 2013, Spatial structuring of an evolving life-history strategy under altered environmental conditions: Oecologia, v. 172, no. 4, p. 1017-1029.

Kock, T.J., Beeman, J.W., Hansen, A.C., Hansel, H.C., Hansen, G.S., Hatton, T.W., Kofoot, E.E., Sholtis, M.D., and Sprando, J.M., 2015, Behavoir, passage, and downstream migration of juvenile Chinook salmon from Detroit Reservoir to Portland, Oregon, 2014-15: U.S. Geological Survey Open-File Report 2015-1220, 30 p., accessed November 19, 2018, at https://pubs. usgs.gov/of/2015/1220/ofr20151220.pdf.

Love, R.H., 1977, Target strength of an individual fish from any aspect: Journal of the Acoustical Society of America, v. 62, p. $1397-1403$.

Miller, B.A., and Sadro, S., 2003, Residence time and seasonal movements of juvenile coho salmon in the ecotone and lower estuary of Winchester Creek, South Slough, Oregon: Transactions of the American Fisheries Society, v. 132, no. 3, p. 546-559.

Miller, M., Giorgi, A., Snyder, D., Mikkelsen, N., and Nishitani, B., 2004, Description of migratory behavior of juvenile salmon smolts through California reservoirs using radio-telemetry techniques in the Klamath Basin: Portland, Oreg., PacifiCorp, prepared by BioAnalysts, Eagle, Idaho, 80 p.

PacifiCorp and Cowlitz Public Utility District [PUD], 2001, Migratory behavoir of radio-tagged juvenile coho salmon through Swift Reservoir, 2001: Portland, Oreg., PacifiCorp and Cowlitz Public Utility District, 47 p. 
PacifiCorp and Cowlitz Public Utility District [PUD], 2002, Migratory behavoir of radio-tagged juvenile Chinook salmon through Swift Reservoir, 2002: Portland, Oreg., PacifiCorp and Cowlitz Public Utility District, 34 p.

PacifiCorp and Cowlitz Public Utility District [PUD], 2004, Report on life history, habitat requirements, and distribution of aquatic analysis species: Portland, Oreg., PacifiCorp and Cowlitz Public Utility District, report AQU 1, 104 p.

PacifiCorp and Cowlitz Public Utility District [PUD], 2017, Lewis River fish passage program 2016 annual report: Portland, Oreg., PacifiCorp and Cowlitz Public Utility District, 176 p.

Perales, K.M., Rowan, J., and Moyle, P.B., 2015, Evidence of landlocked Chinook salmon populations in California: North American Journal of Fisheries Management, v. 35, no. 6, p. 1101-1105.

Pess, G.R., Kiffney, P.M., Liermann, M.C., Bennett, T.R., Anderson, J.H., and Quinn, T.P., 2011, The influences of body size, habitat quality, and competition on the movement and survival of juvenile coho salmon during the early stages of stream recolonization: Transactions of the American Fisheries Society, v. 140, no. 4, p. 883-897.

Prentice, E., Waknitz, F., Maynard, D., Sparks-McConkey, P., McCutcheon, C., Steffens, W., Jensen, A., Stuehrenberg, L.C., Downing, S., Sandford, B.P., Newcomb, T., and Neff, D., 1993, Study to determine the biological feasibility of a new fish tagging system-Annual report 1989: Portland, Oreg., Bonneville Power Administration, report DOE/BP-11982-4, project number 1983-31900, $193 \mathrm{p}$.

Rebenack, J.J., Ricker, S., Anderson, C., Wallace, M., and Ward, D.M., 2015, Early emigration of juvenile coho salmonImplications for population monitoring: Transactions of the American Fisheries Society, v. 144, no. 1, p. 163-172.

Romer, J.D., and Monzyk, F.R., 2014, Adfluvial life history in spring Chinook salmon from Quartzville Creek, Oregon: North American Journal of Fisheries Management, v. 34, no. 5, p. 885-891.

Romer, J.D., Monzyk, F.R., Emig, R., and Friesen, T.A., 2014, Juvenile salmonid outmigration monitoring at Willamette Valley project reservoirs: Corvallis, Oreg., Oregon Department of Fish and Wildlife, task order number W9127N-10-2-0008-0019, $61 \mathrm{p}$.

Tiffan, K.F., Kock, T.J., Connor, W.P., Steinhorst, R.K., and Rondorf, D.W., 2009, Behavioural thermoregulation by subyearling fall (autumn) Chinook salmon Oncorhynchus tshawytscha in a reservoir: Journal of Fish Biology, v. 74, no. 7, p. 1562-1579.

Williams, J.G., Smith, S.G., and Muir, W.D., 2001, Survival estimates for downstream migrant yearling juvenile salmonids through the Snake and Columbia rivers hydropower system, 1966-1980 and 1993-1999: North American Journal of Fisheries Management, v. 21, no. 2, p. 310-317. 


\section{Figures}

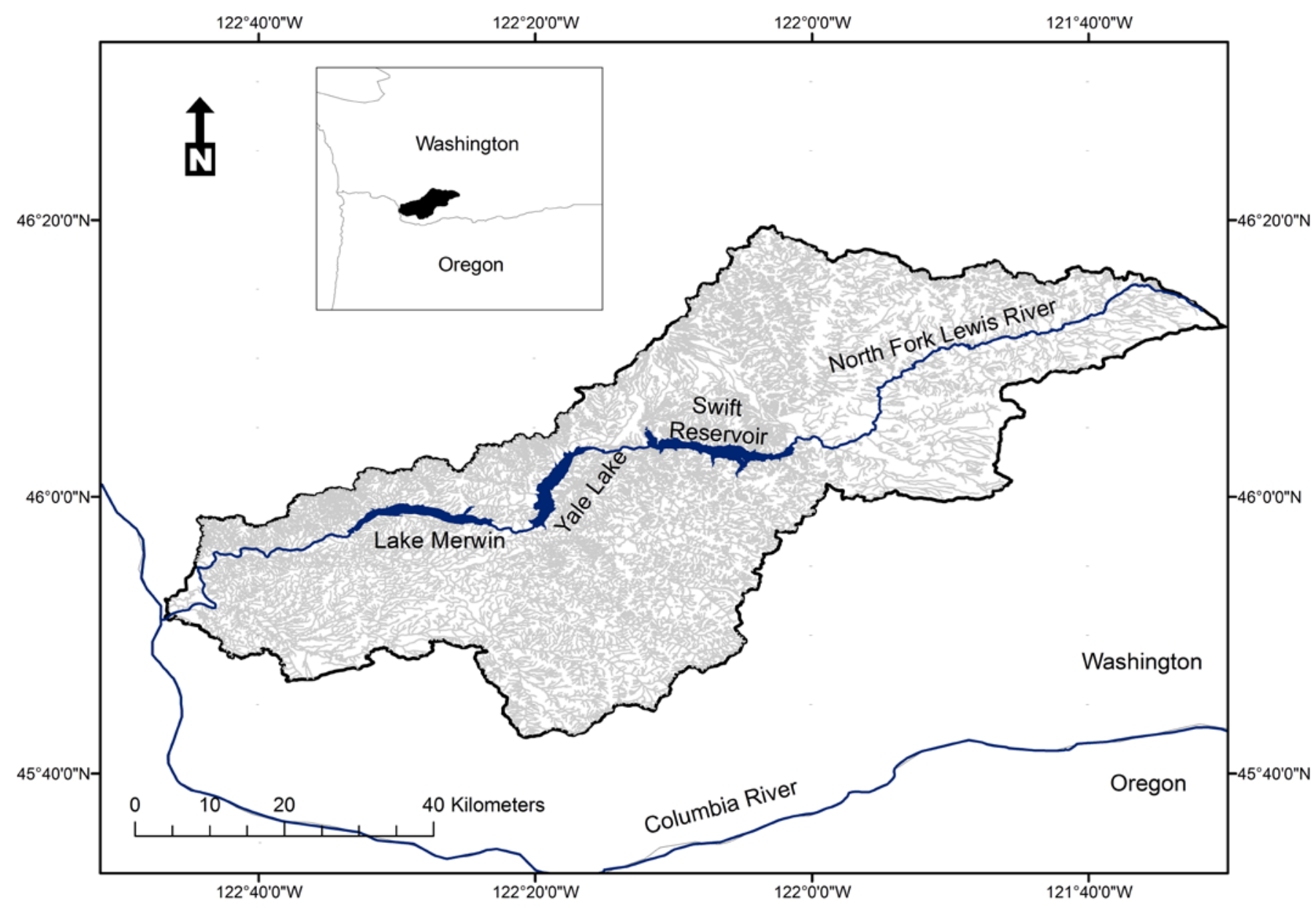

Figure D1. Study area map. The North Fork of the Lewis River and the three hydropower impoundments: Lake Merwin, Yale Lake, and Swift Reservoir in the Lewis River Basin, Washington. 


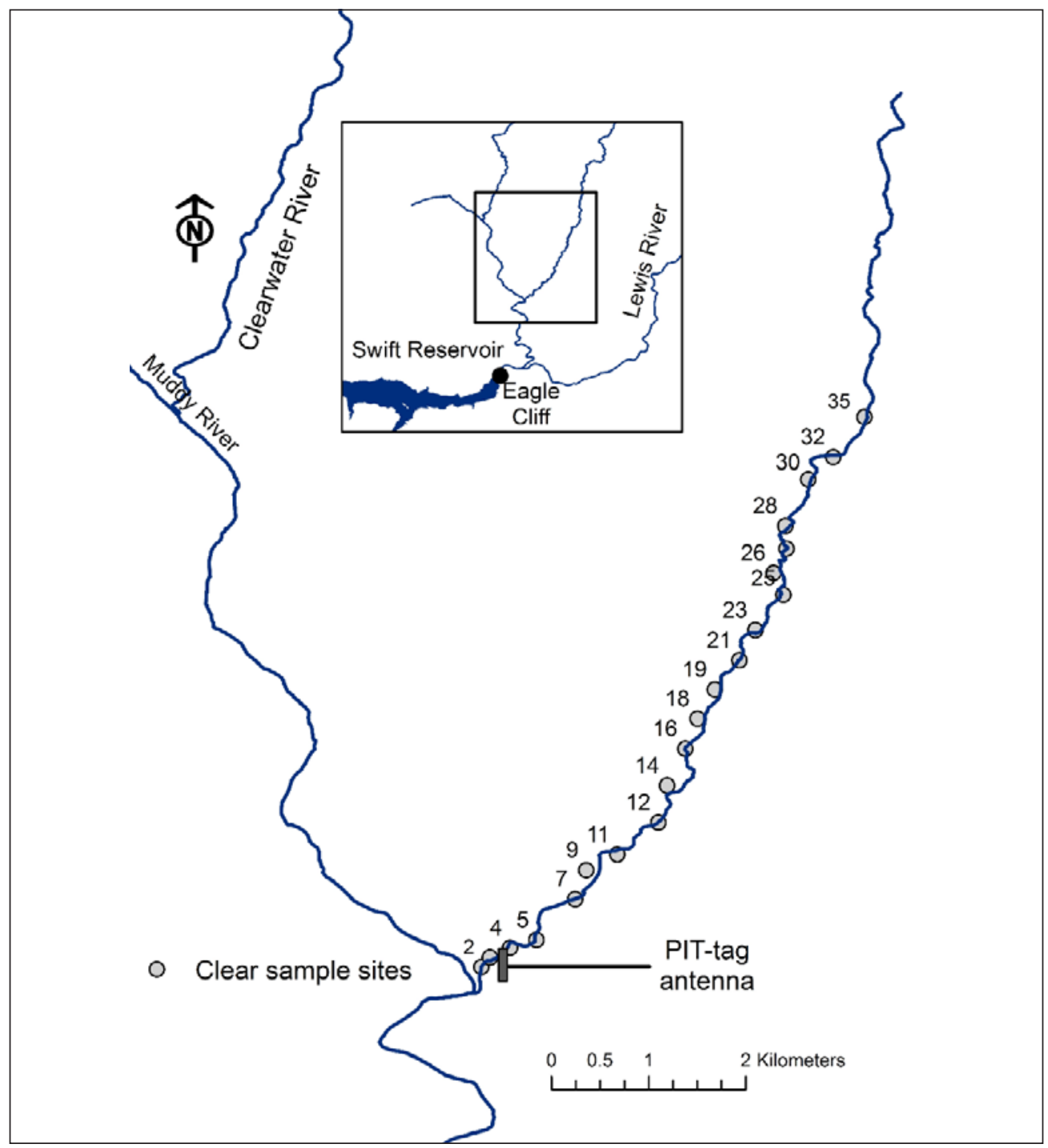

Figure D2. Sample reaches (light gray circles) used in the systematic sampling design for passive integrated transponder (PIT)-tagging coho salmon in Clear Creek (2013-2015) with location of the PIT-tag antenna (gray box). Inset map shows the location of the sampling area in relation to Swift Reservoir, Washington, and the location of Eagle Cliff where the rotary screw trap was operated (black circle). 


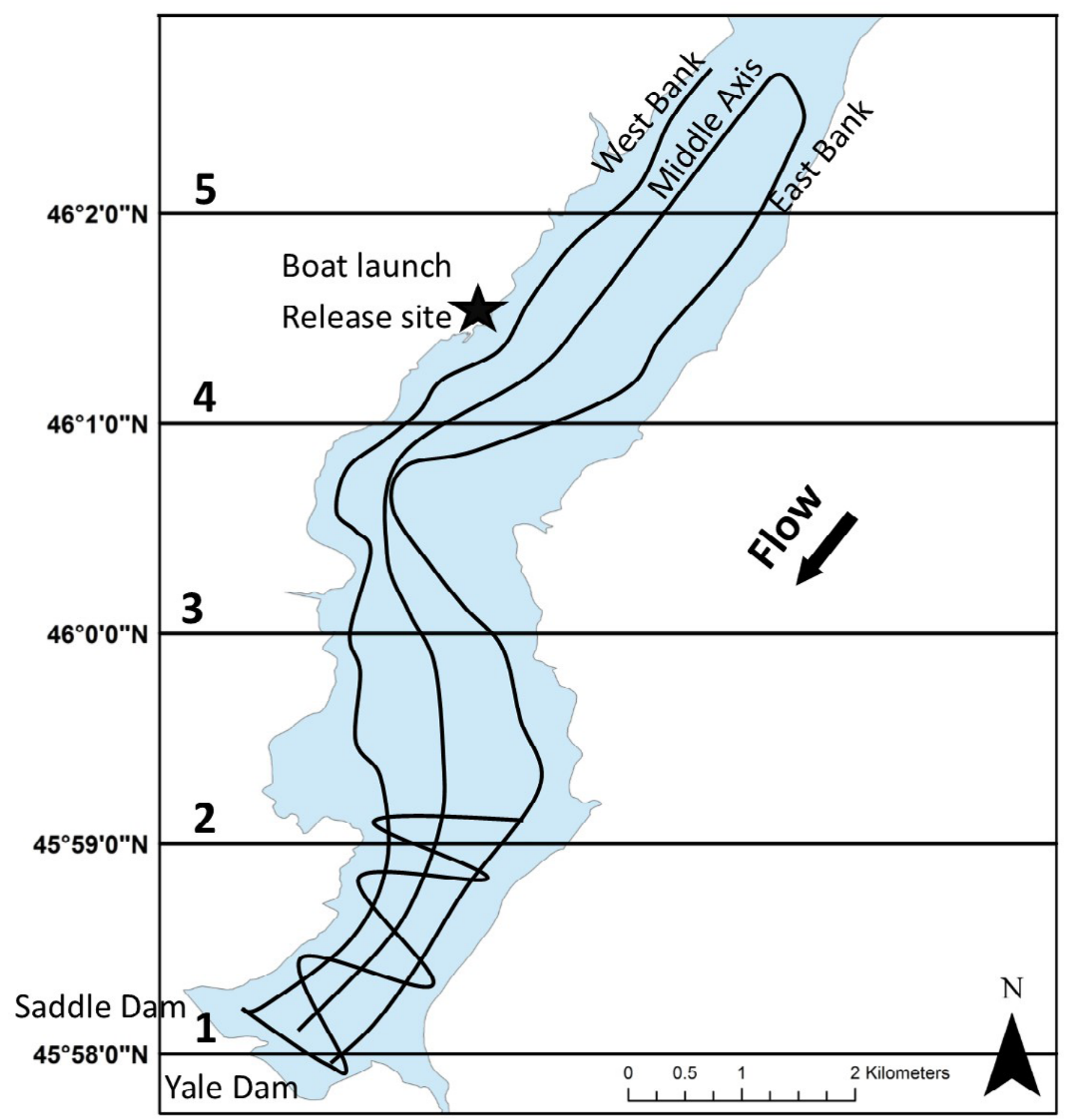

Figure D3. Yale Lake, Washington, with labeled regions that were used to evaluate densities of coho salmon smolt-sized targets in hydroacoustic surveys (black lines) and the release location of smolts (star; Yale Park). Region 1 contained all parts of the reservoir below $45^{\circ} 59^{\prime} \mathrm{N}$. Hydroacoustic survey transects were replicated for the prerelease, day of release, and day after release surveys. 


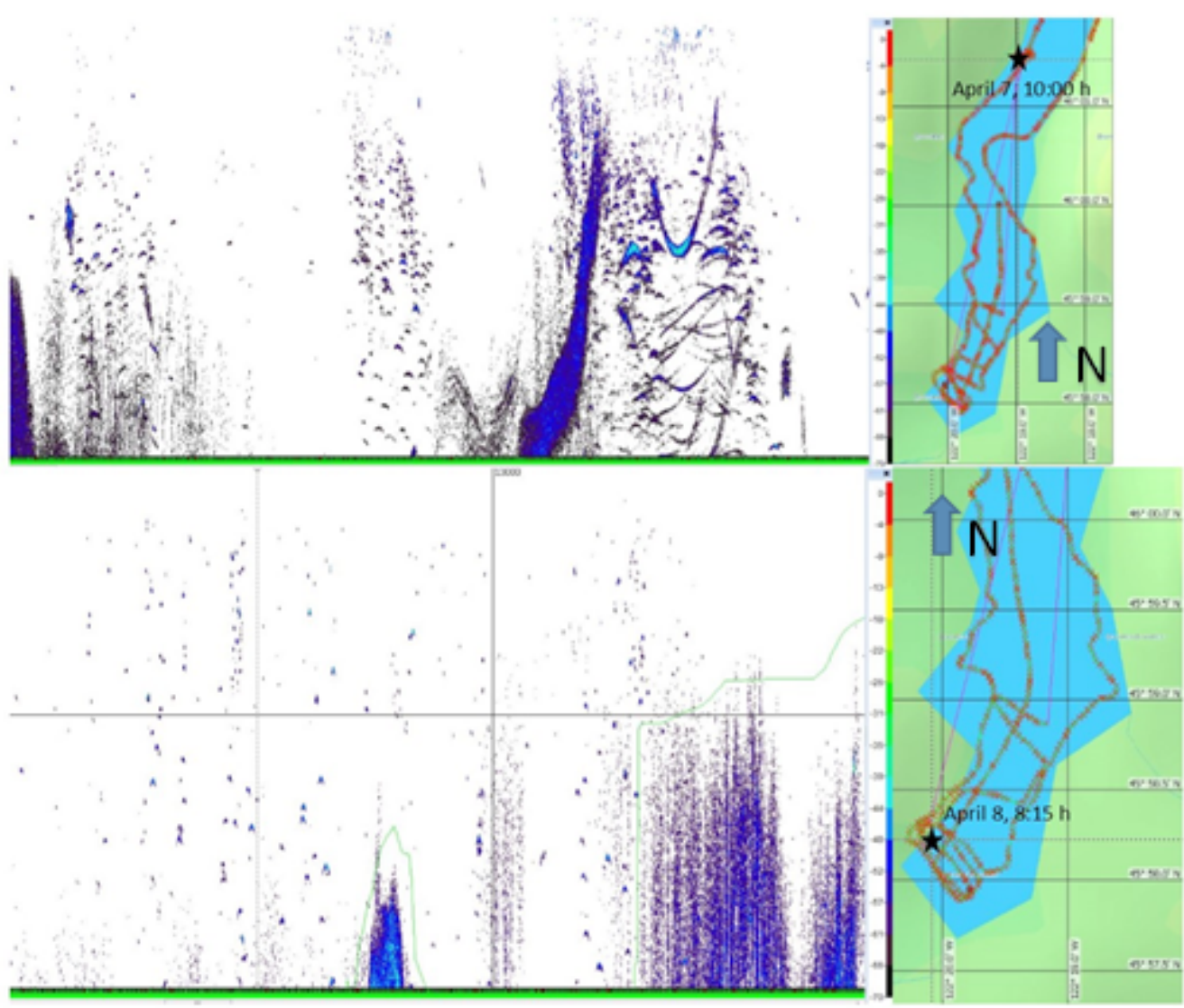

Figure D4. Echograms from the sidelooking transducer showing smolt-sized targets in Yale Lake, Washington. Top panel shows the boat launch while fish were being released, and the bottom panel shows an aggregation of smolt-sized targets seen along the west side of Yale Dam in a zig-zag transect. 

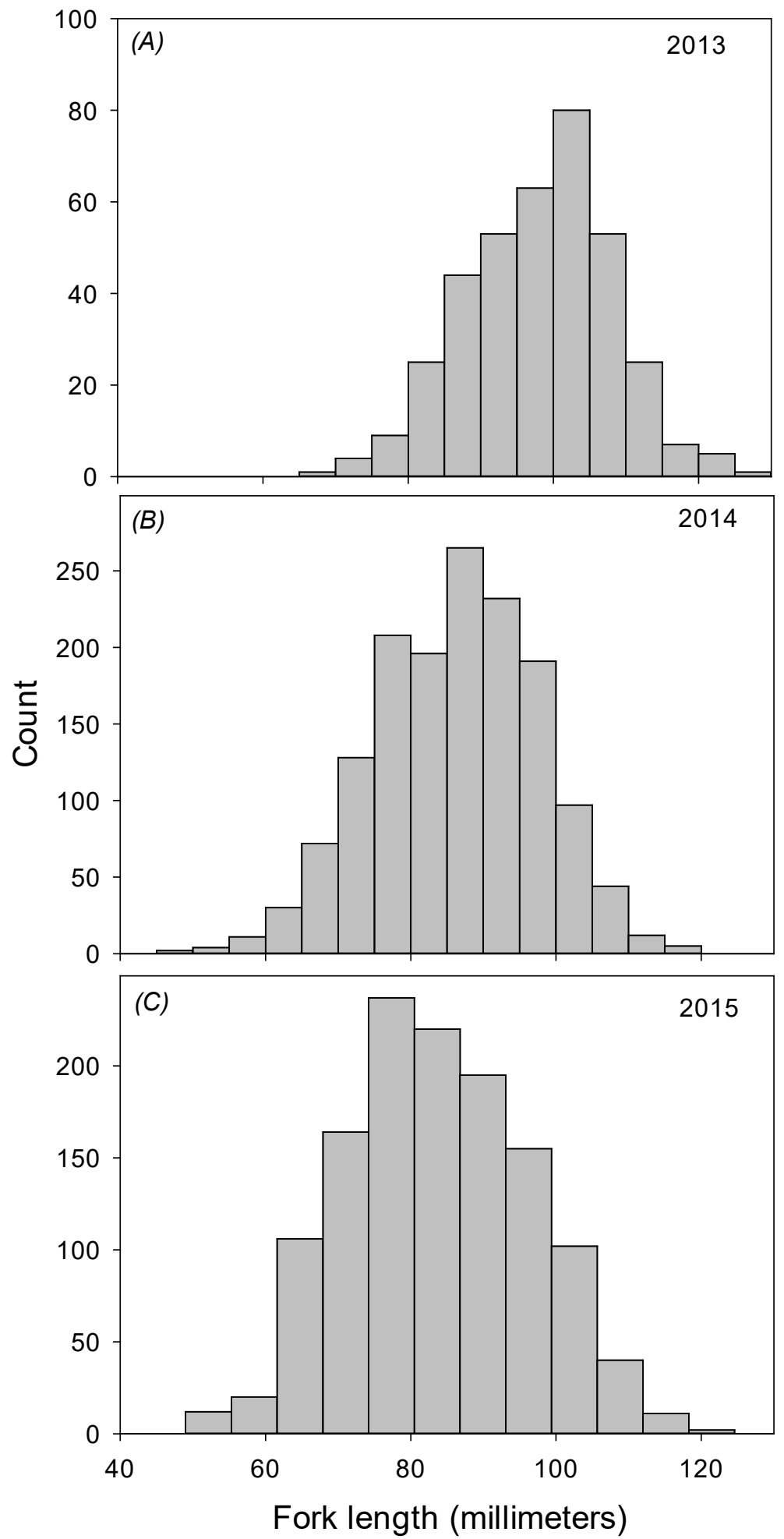

Figure D5. Length frequency of coho salmon captured in Clear Creek, Washington, during surveys in A, 2013, B, 2014, and C, 2015 (combined sampling from summer and fall surveys). 


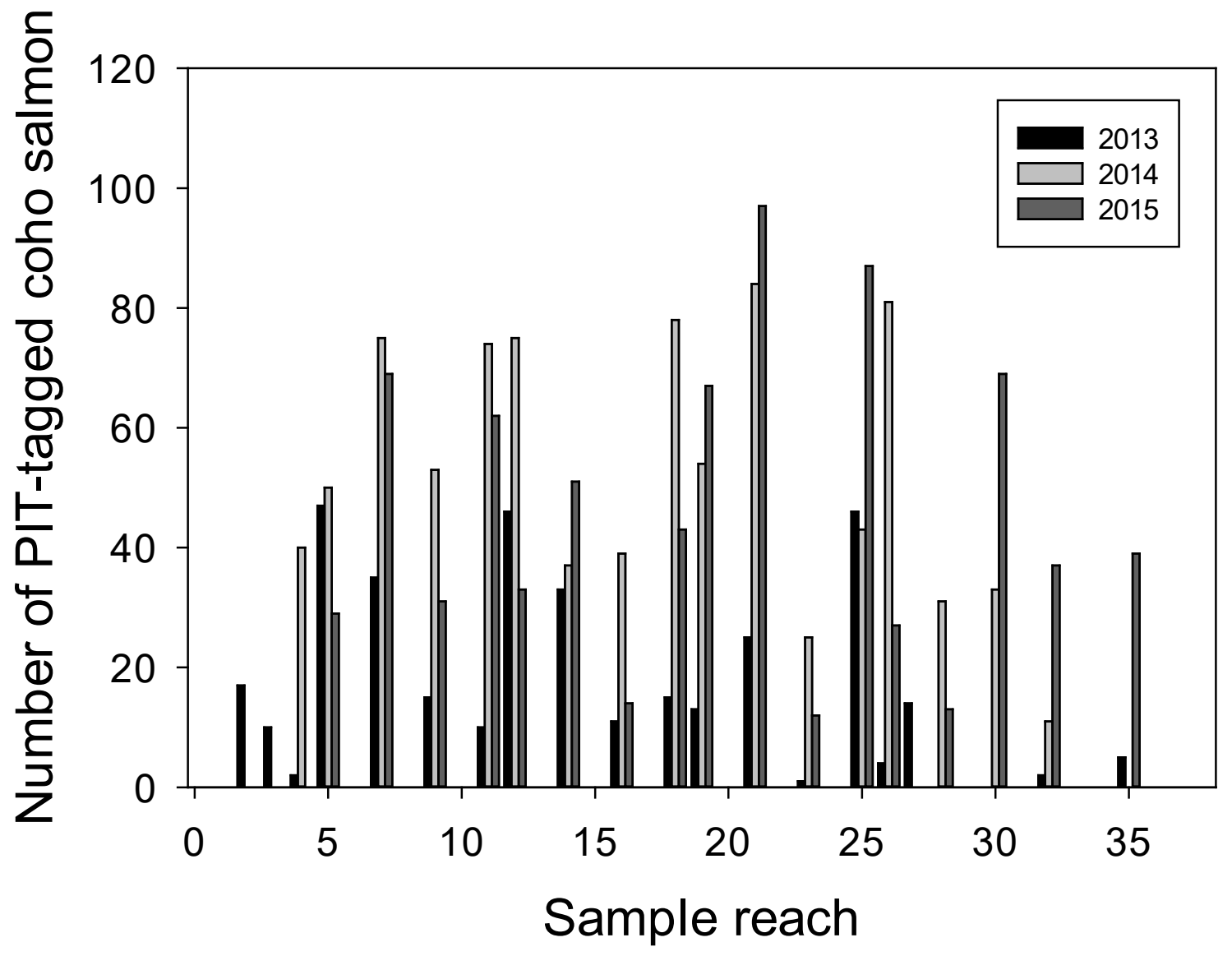

Figure D6. The number of passive integrated transponder (PIT)-tagged coho salmon by reach in 2013 (black), 2014 (light gray), and 2015 (dark gray). Refer to fig. D2 for location of sample sites, but sites progress from downstream to upstream (left to right). 


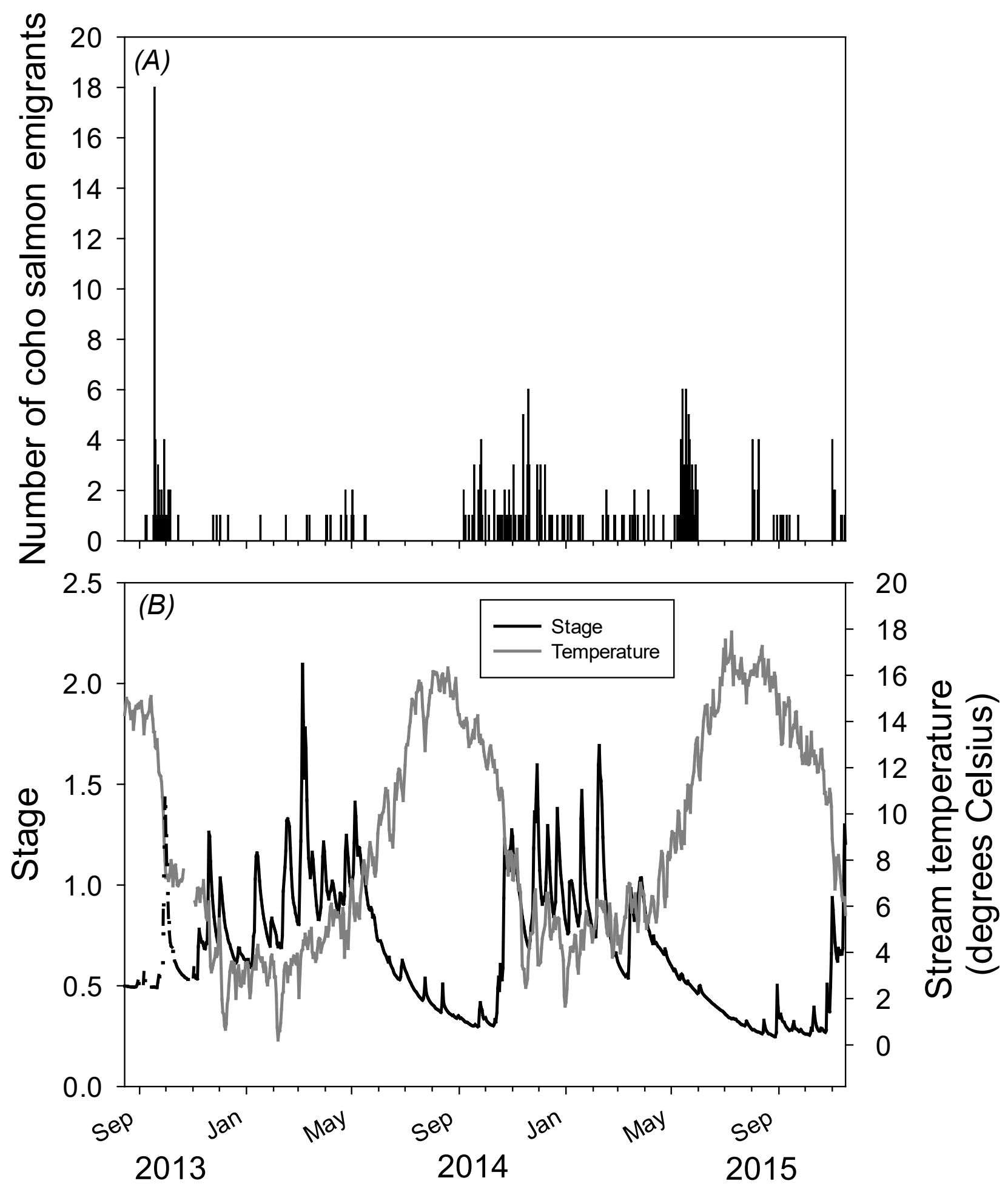

Date

Figure D7. A, Outmigration timing of passive integrated transponder (PIT)-tagged wild coho salmon downstream past the fixed antennas on Clear Creek, Washington. B, Average daily hydrologic stage (black line) and stream temperature at the Clear Creek antennas (2013-2015). Note: the dashed line is estimated from correlations with the Muddy River U.S. Geological Survey gaging station as the pressure transducer in Clear Creek was not initially operational. 


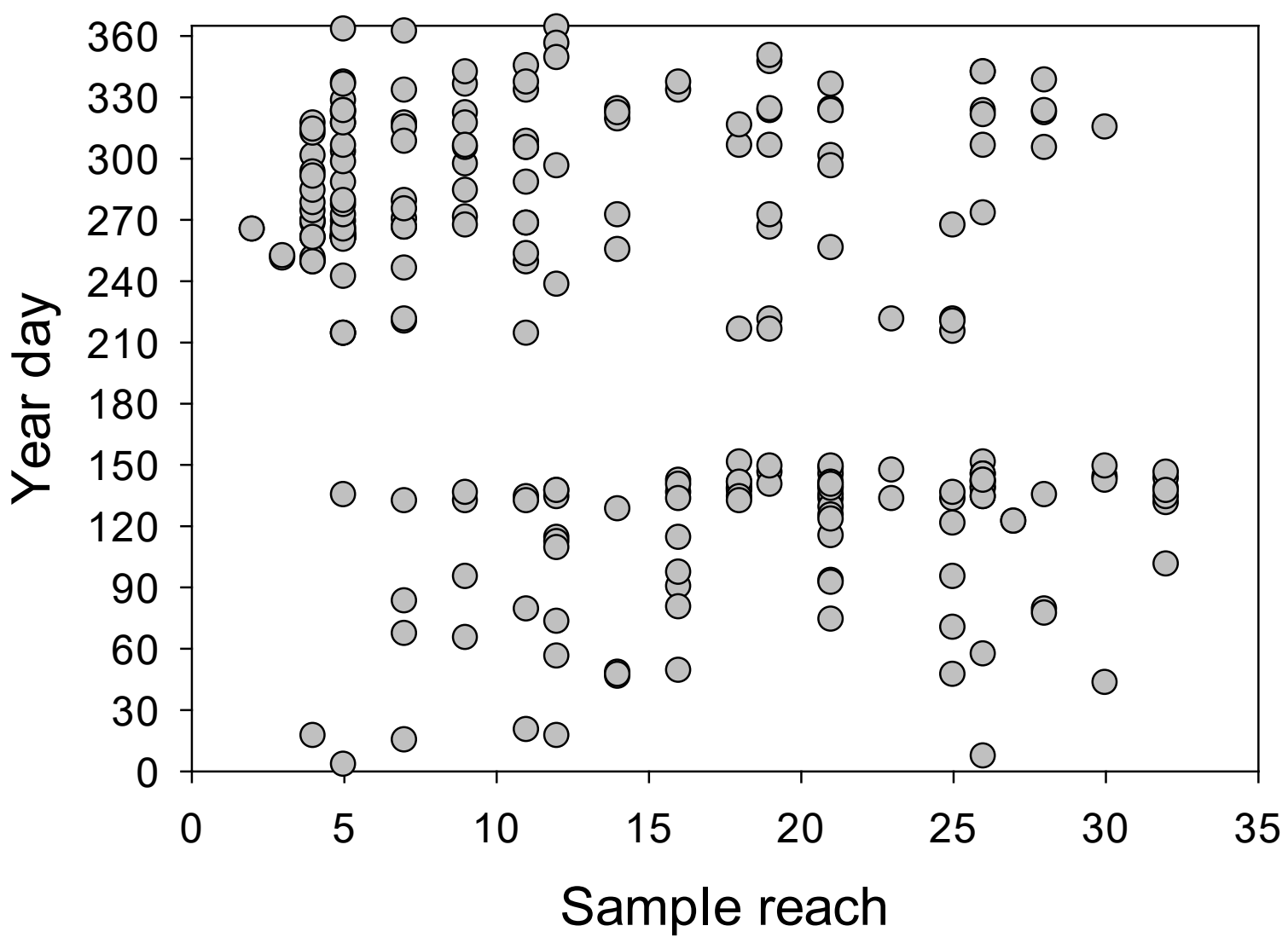

Figure D8. Julian day of outmigration (1=January 1,365=December 31$)$ past the fixed passive integrated transponder (PIT)-tag antenna for wild coho salmon marked with PIT-tags in different sampling reaches. Note the increasing reach number corresponds to distance from river mouth (see fig. D2). 


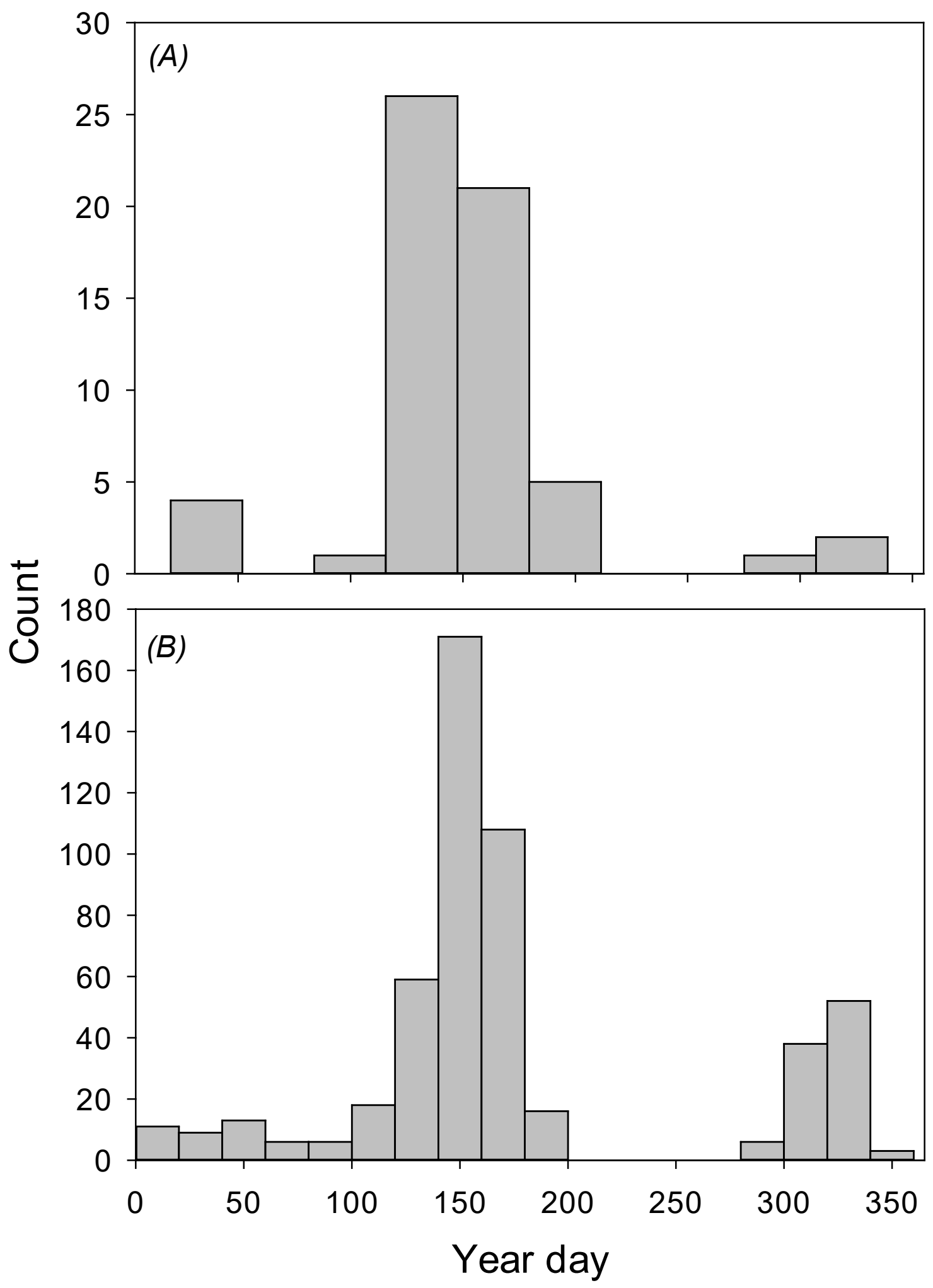

Figure D9. Year day that $A$, passive integrated transponder-tagged individual wild coho salmon (2013-2015) and $B$, hatchery-reared coho salmon (2013 only) juveniles were collected at the floating surface collector in Swift Reservoir, Washington. 


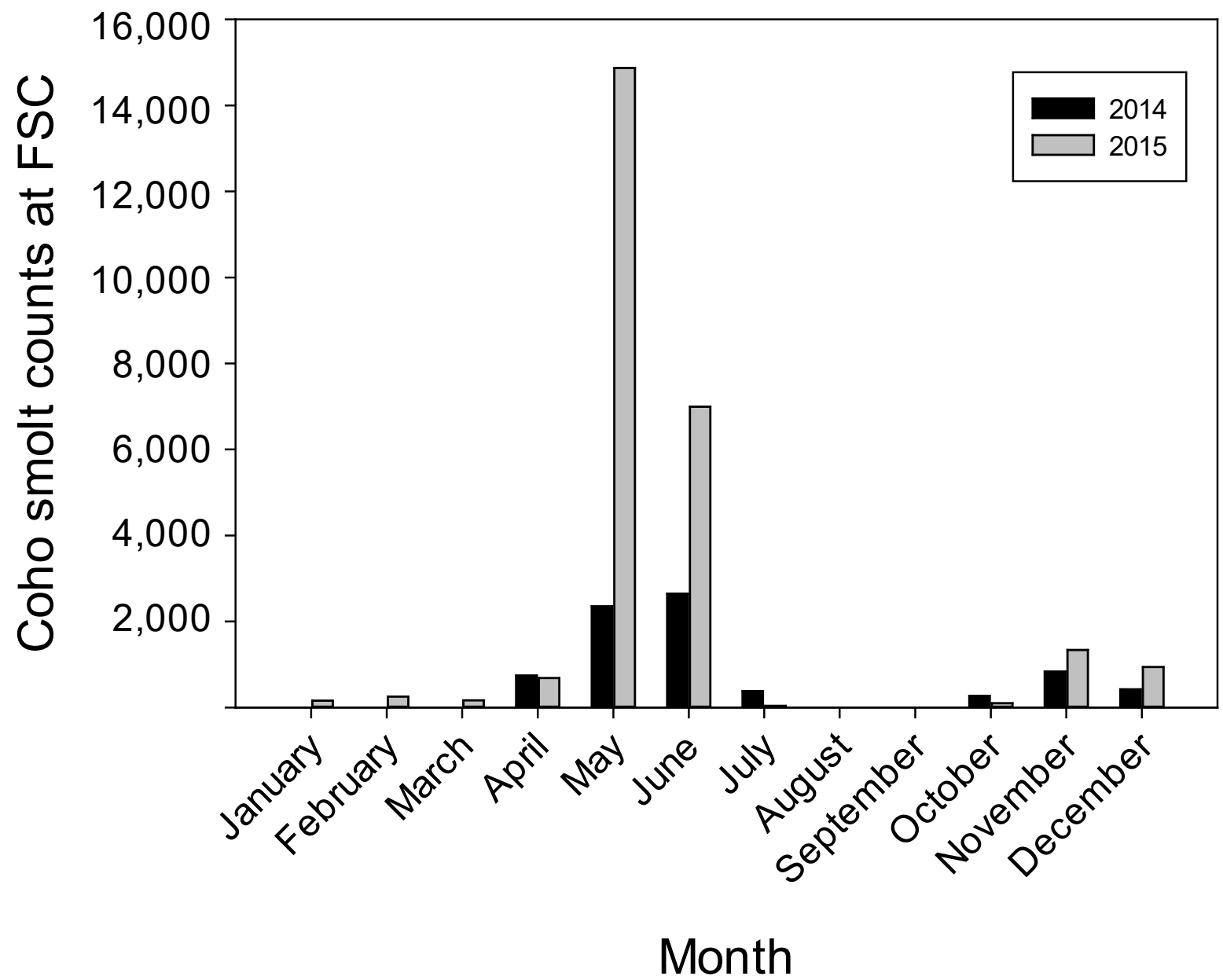

Figure D10. Total coho salmon smolt counts by month for 2014 (black) and 2015 (gray) at the floating surface collector (FSC) in Swift Reservoir, Lewis River, Washington. 


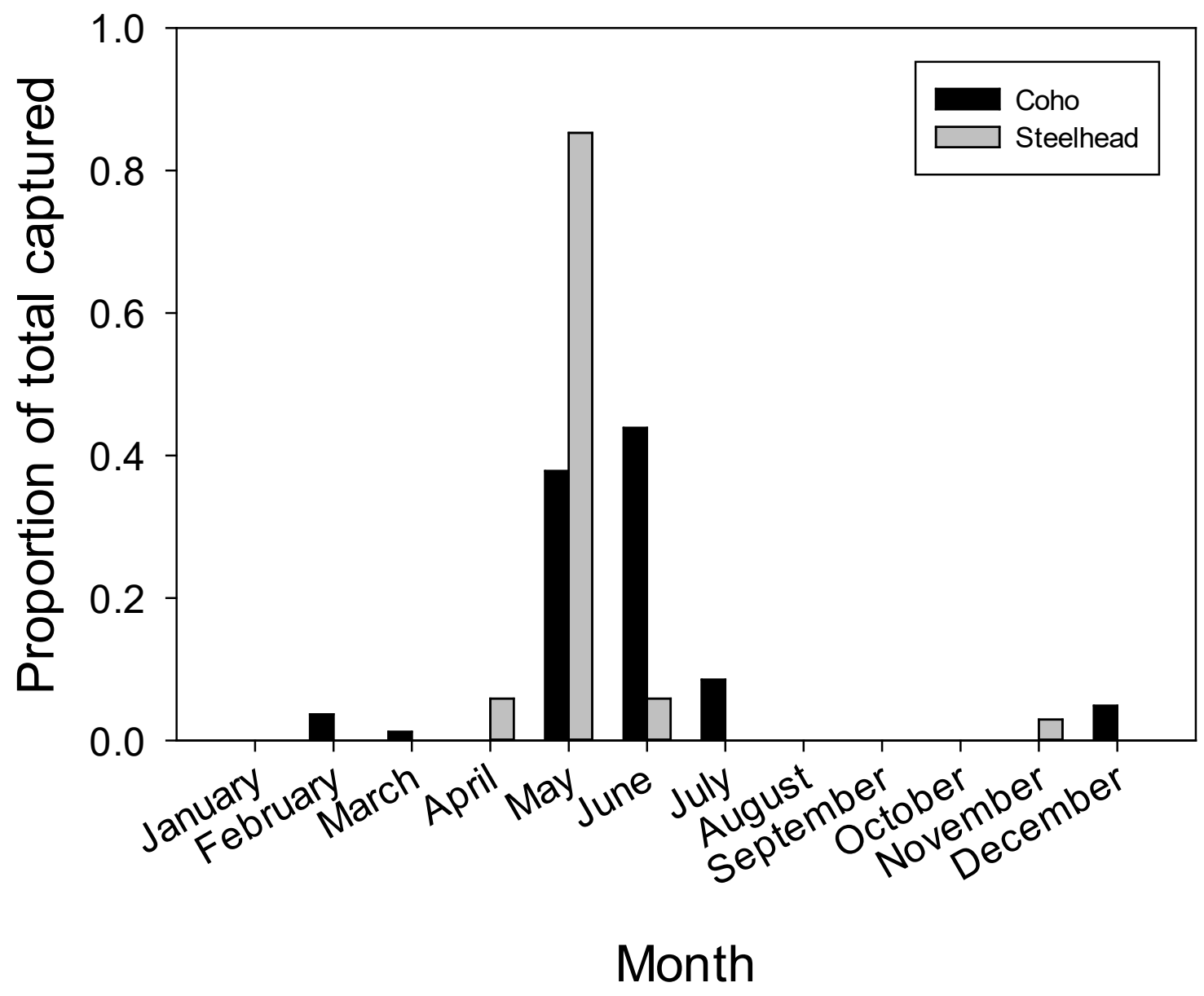

Figure D11. Proportion of coho salmon (black) and steelhead (gray) passive integrated transponder-tagged at the Eagle Cliff Screw trap collected at the floating surface collector by month. 

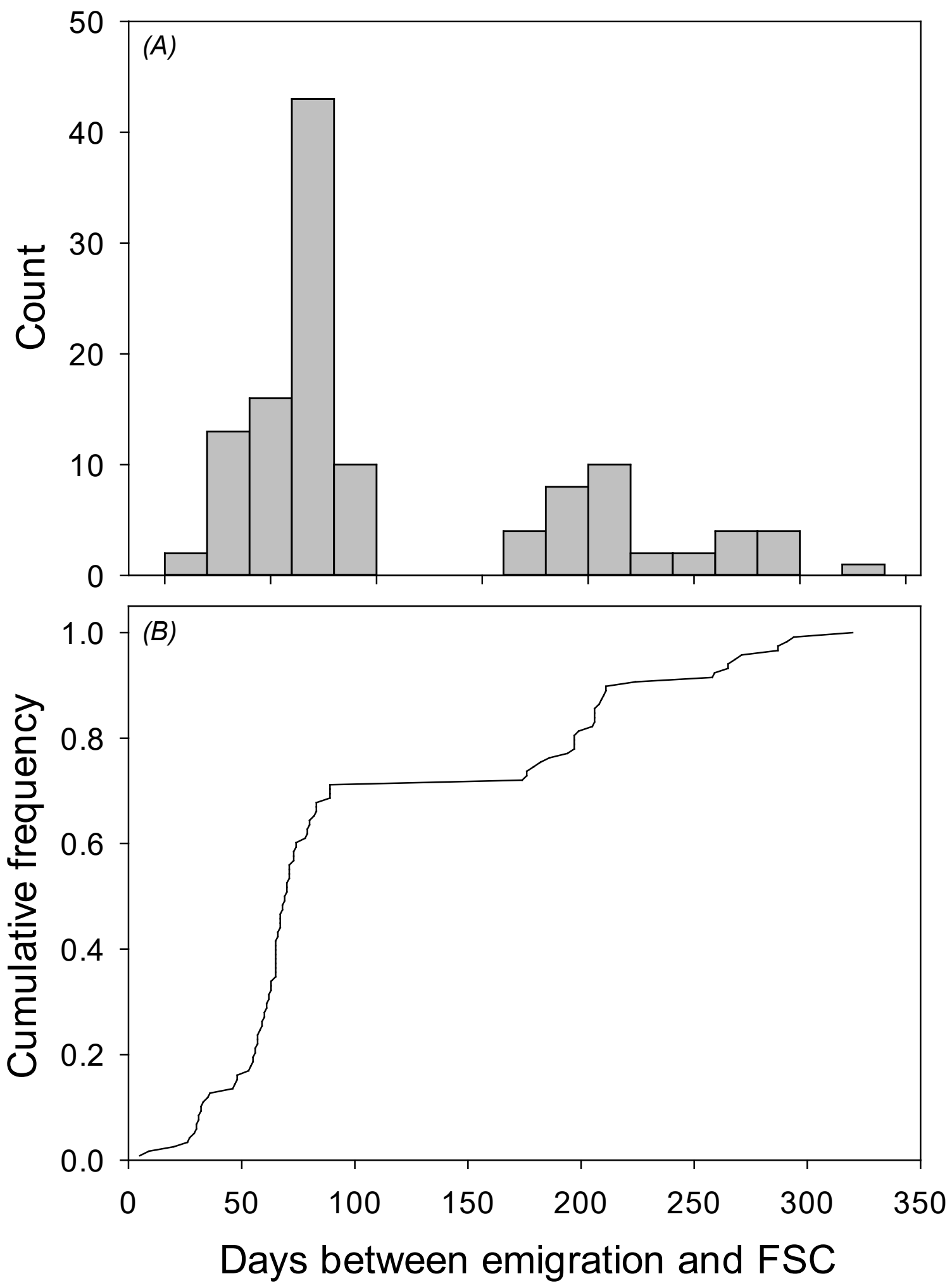

Figure D12. A frequency distribution $A$, and a cumulative frequency $B$, of the number of days passive integrated transponder (PIT)tagged spring Chinook salmon (acclimation) spent between outmigration past the fixed PIT-tag antenna on Clear Creek (likely in Swift Reservoir) and collection at the floating surface collector (FSC) in Swift Reservoir, Washington (2013-2015). 


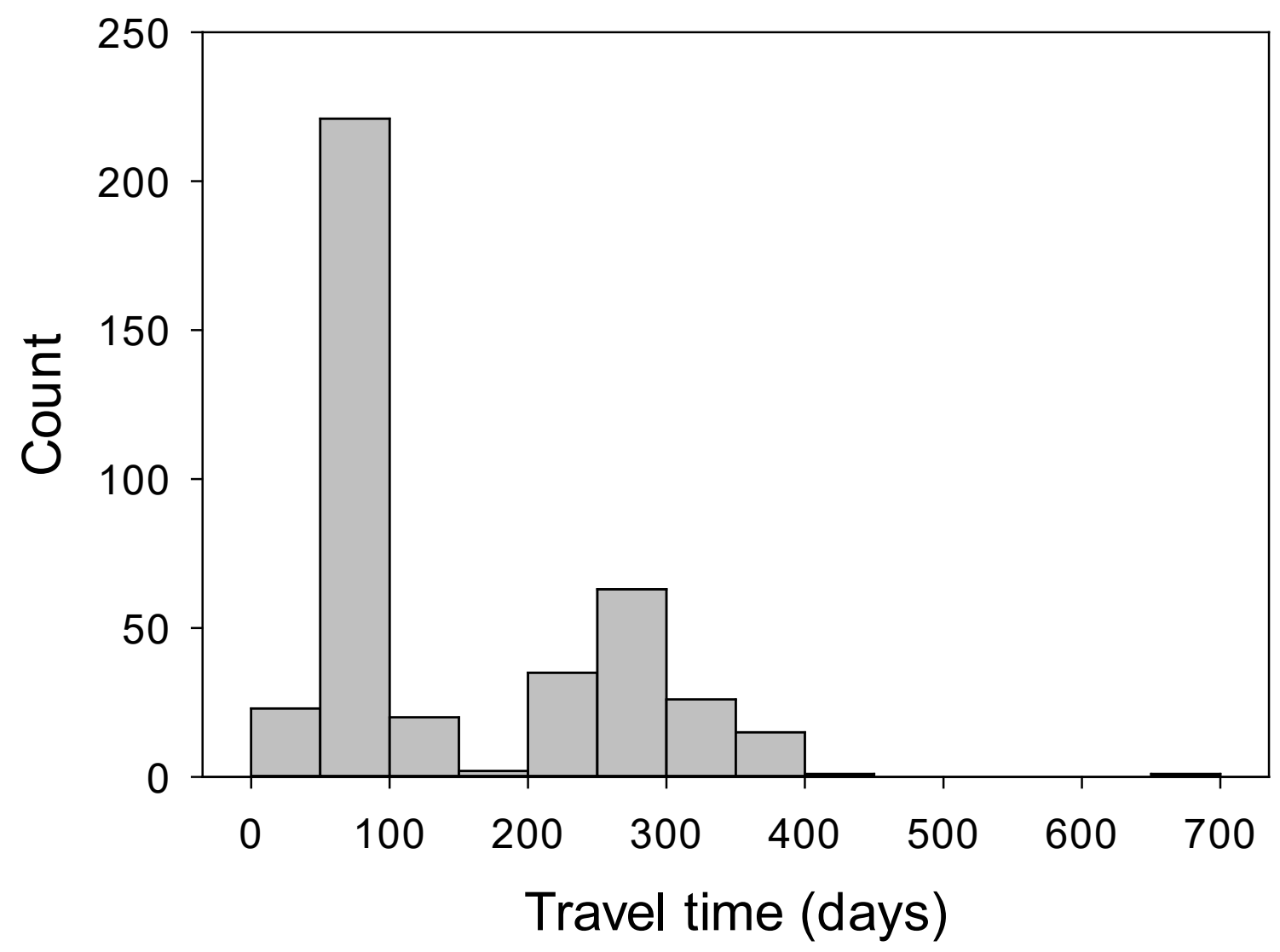

Figure D13. Total travel time for acclimation spring Chinook salmon (passive integrated transponder-tagged) from release in tributaries (Crab Creek, Muddy River, Clear Creek) to the floating surface collector in Swift Reservoir, Washington (2013-2015). 


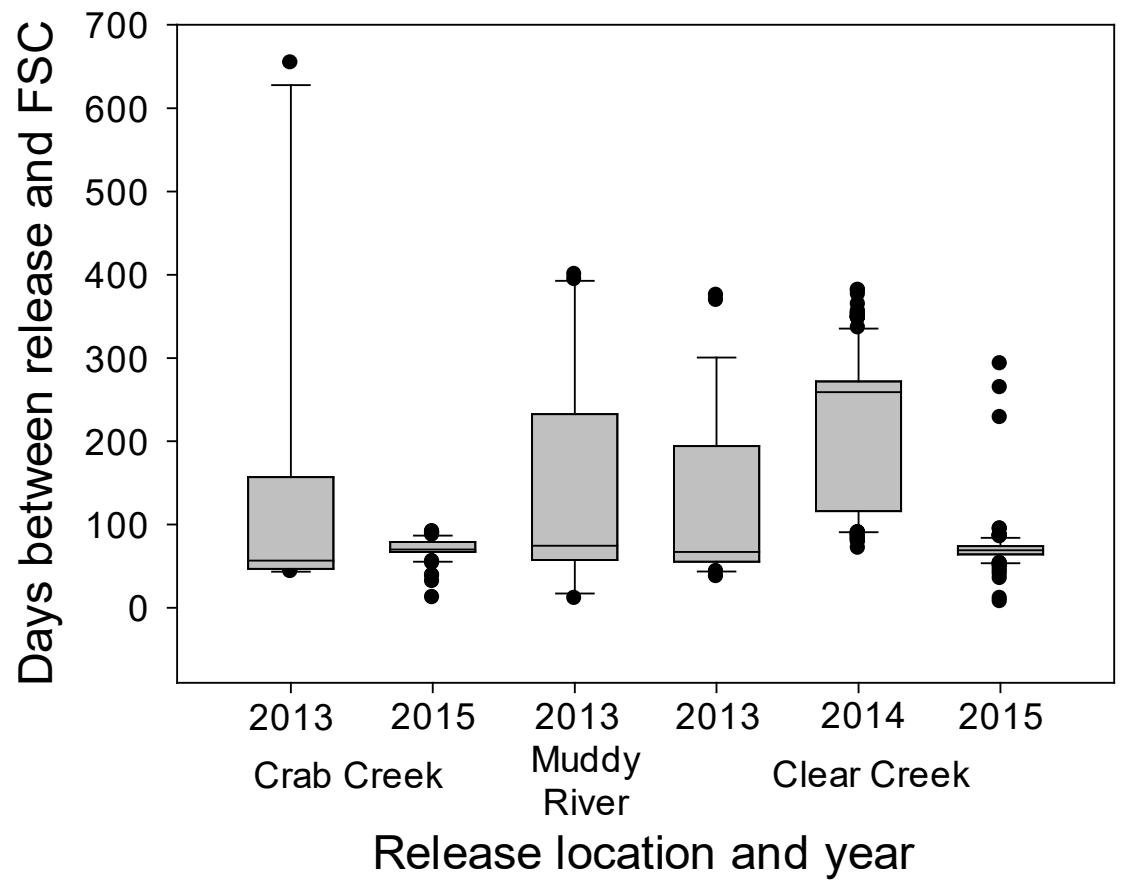

Figure D14. Total number of days between release and collection at the floating surface collector (FSC) in Swift Reservoir for acclimation spring Chinook salmon (passive integrated transponder-tagged only) across different release locations and years. Note: multiple years of releases were only available for Clear Creek and Crab Creek, Washington, and the 2015 data are incomplete. 

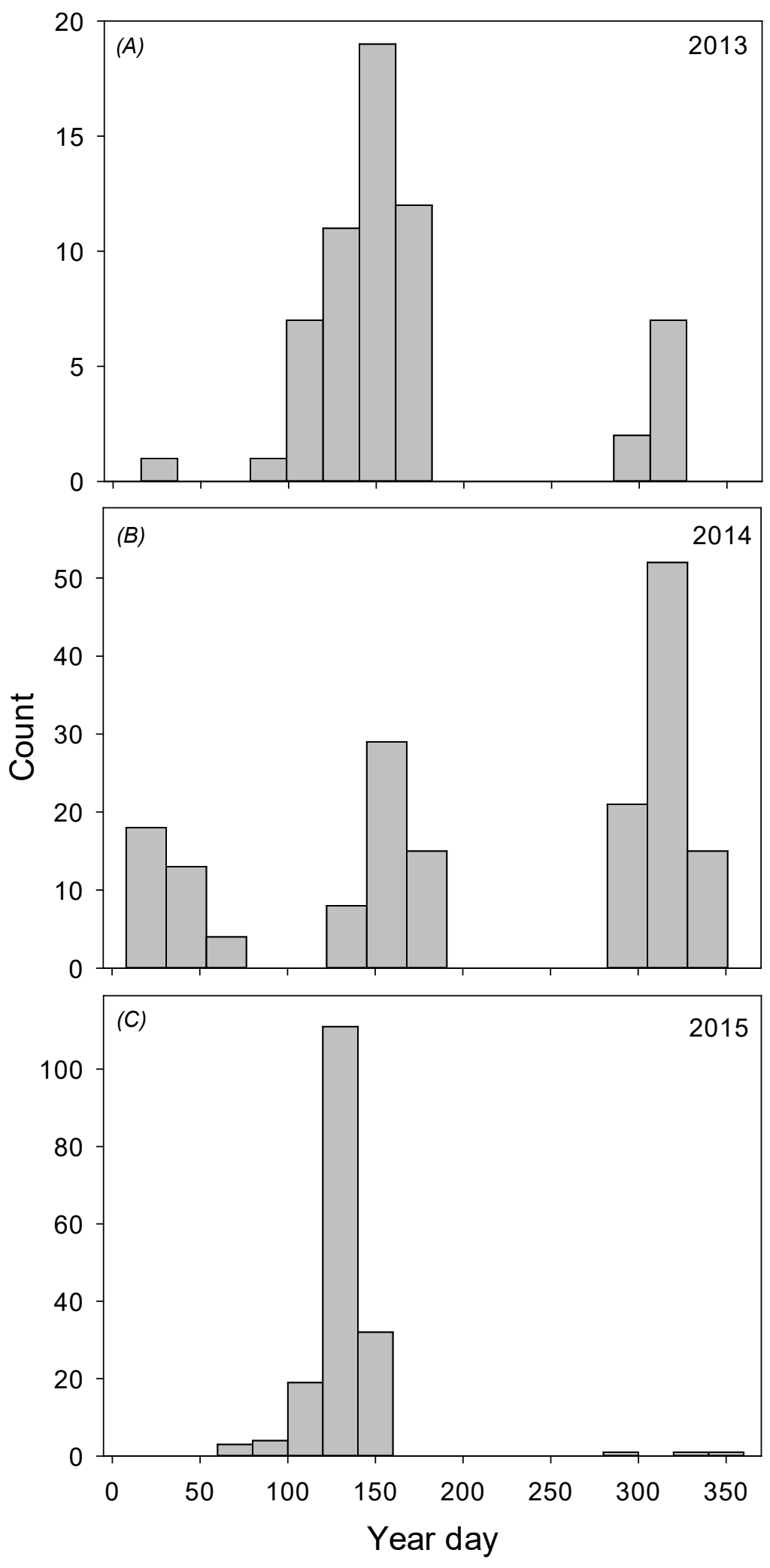

Figure D15. Year day that acclimation spring Chinook salmon released in $A, 2013, B, 2014$, and $C, 2015$ were captured at the floating surface collector in Swift Reservoir, Washington. 


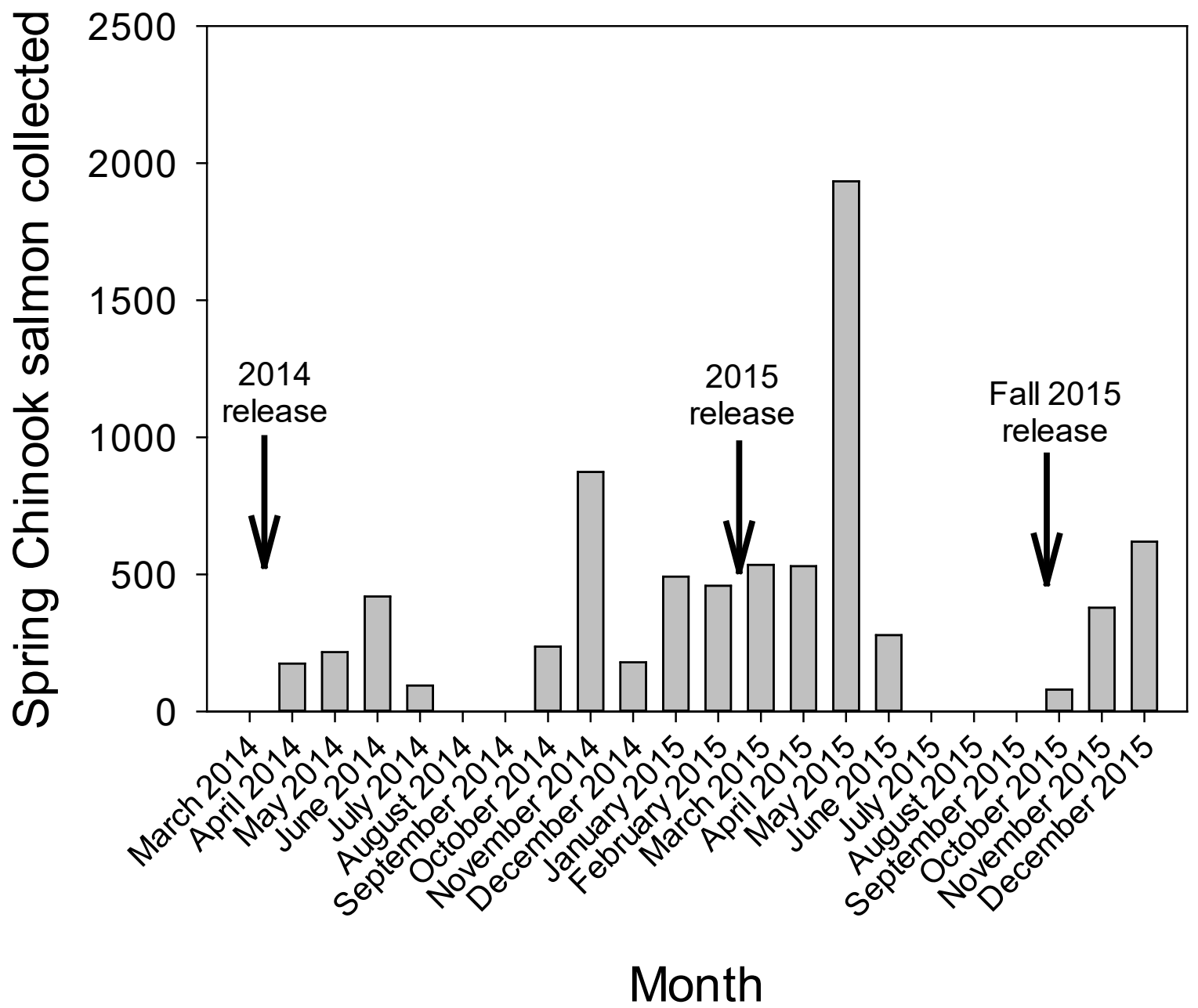

Figure D16. The total number of spring Chinook salmon collected at the floating surface collector in Swift Reservoir, Washington, from a 2014 spring release (45,000 individuals), 2015 spring release (109,666 individuals), and a fall 2015 release (48,000 individuals). 


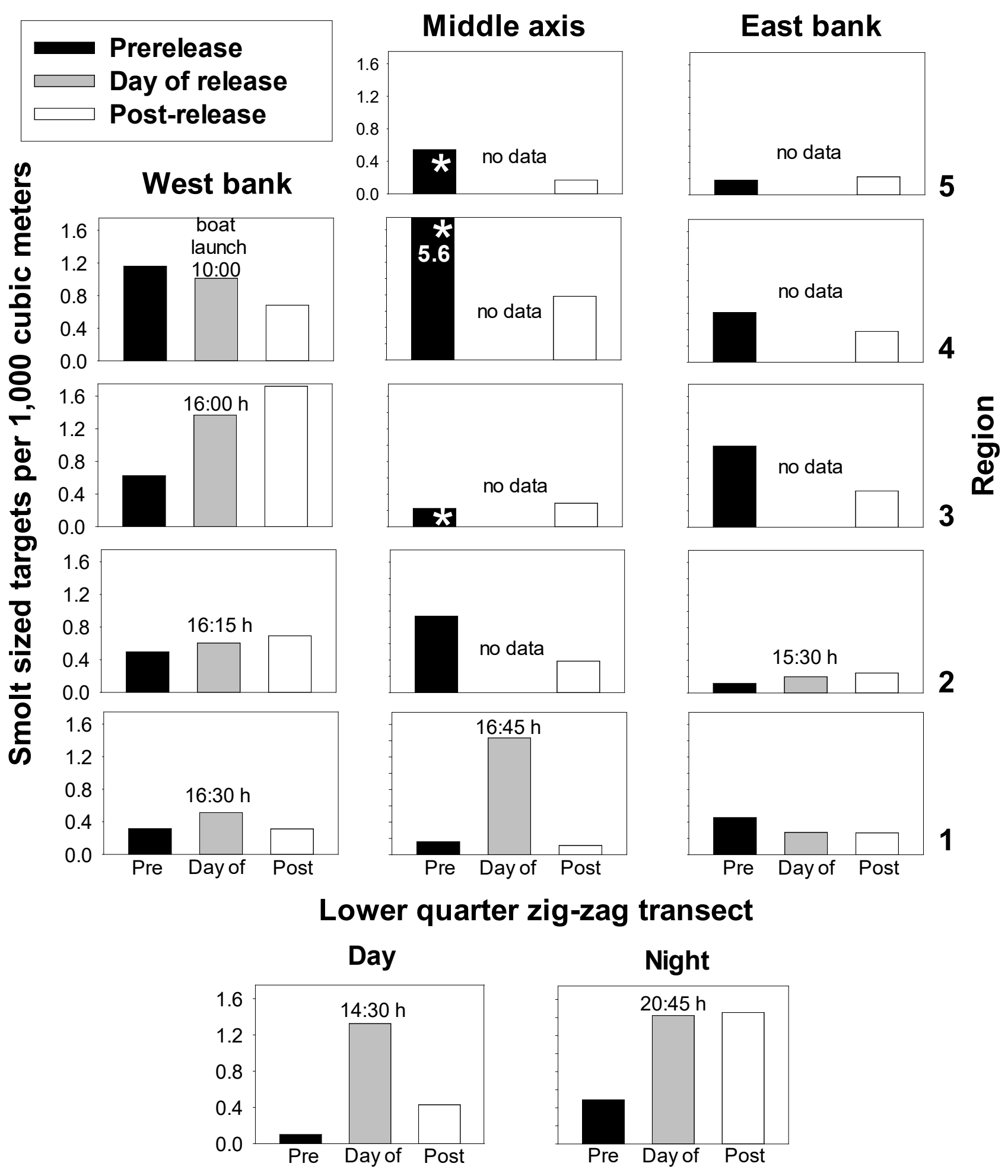

Figure D17. Coho salmon smolt sized targets per 1,000 cubic meters $\left(\mathrm{m}^{3}\right)$ observed by the sidelooking transducer in Yale Lake, Washington, prerelease (pre), the day of release (day of), and postrelease (post) of 5,000 test smolts. Panels are arranged in columns from west (left), mid-axis (center) and east (right) transects (labeled on top), and rows based on upper to lower regions in the reservoir (Region 1 closest to the dam). The lower quarter zig-zag transect panels represent densities estimated from a series of zig-zag transects within the lower quarter of the reservoir. Bars with an asterisk represent prerelease surveys conducted during dusk, while all other surveys were conducted under daylight unless otherwise noted (24-hour time). (h, hour) 
Middle axis East bank

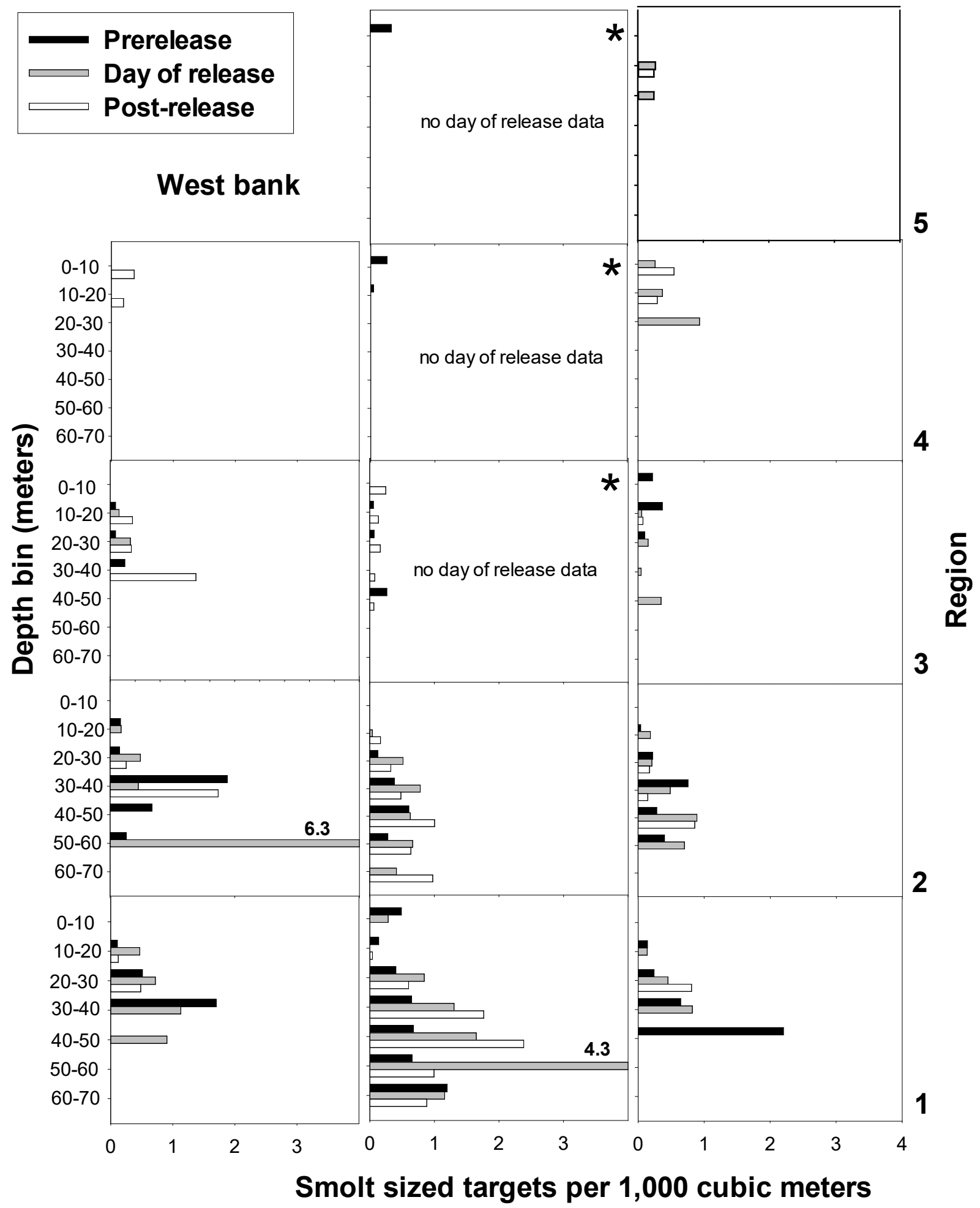

Figure D18. Smolt-sized targets per 1,000 cubic meters $\left(\mathrm{m}^{3}\right)$ observed by the downlooking transducer in 10-meter (m) depth bins in Yale Lake, Washington, prerelease (pre), the day of release (day of), and postrelease (post) of 5,000 test smolts. Panels are arranged in columns based on transects (labeled on top), and rows based on regions (labeled at lower right of row). Lower numbered regions are lower in the reservoir (that is closer to the dam). Panels with an asterisk represent prerelease surveys conducted during dusk, while all other surveys were conducted under daylight. 


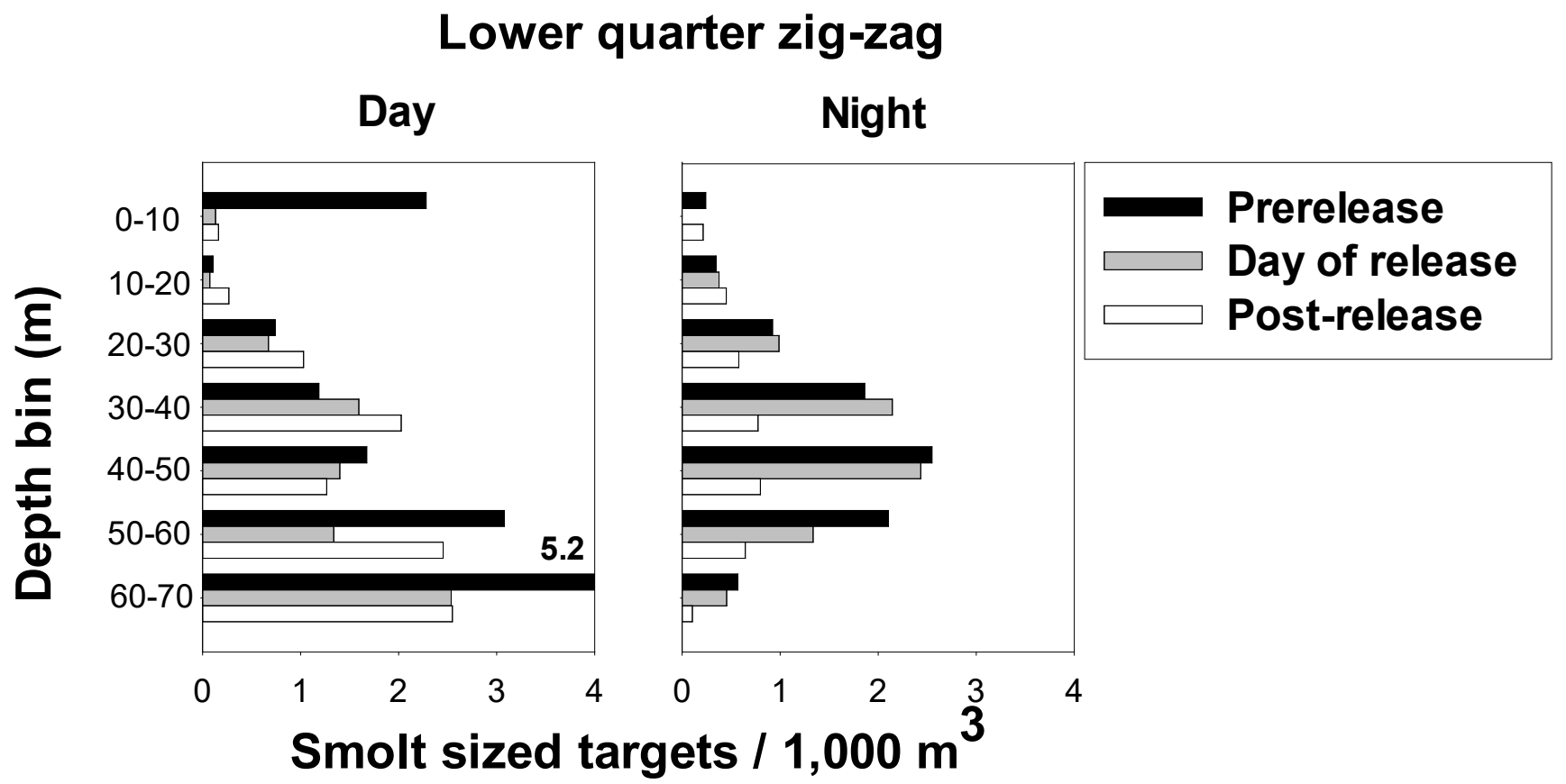

Figure D19. Coho salmon smolt-sized targets per 1,000 $\left(\mathrm{m}^{3}\right)$ observed by the downlooking transducer in 10-meter $(\mathrm{m})$ depth bins during zig-zag transects conducted in the lower quarter of Yale Lake, Washington, prerelease, day of release, and postrelease of 5,000 coho salmon smolts at Yale Park. Surveys were conducted during daylight (left panel) and night (right panel) surveys (no crepuscular survey conducted). 


\section{Tables}

Table D1. Number of passive integrated transponder-tagged acclimation coho salmon and spring Chinook salmon released by location and year in the Lewis River upstream from Swift Dam, Washington, 2013-2015. Note: the dashes (-) indicate no releases occurred.

\begin{tabular}{|l|l|c|c|c|}
\hline \multicolumn{1}{|c|}{ Year } & \multicolumn{1}{c|}{ Species } & Clear Creek & Crab Creek & Muddy River \\
\hline 2013 & Chinook salmon & 1,750 & 750 & 1,750 \\
\hline & Coho salmon & - & - & 2,000 \\
\hline 2014 & Chinook salmon & 17,576 & - & - \\
\hline 2015 & Chinook salmon & 3,400 & 3,300 & 3,300 \\
\hline
\end{tabular}

${ }^{1}$ Includes fish from Muddy River as acclimation ponds at the Muddy River site were unusable.

Table D2. Hydroacoustic transects conducted in 2014 to evaluate coho salmon smolt movement and distribution in Yale Lake, Washington, including release status (that is, prerelease, during, or postrelease), date, location, start and end times, and diel period.

\begin{tabular}{|c|c|c|c|c|c|}
\hline Release status & Date & Transect & Start time (24-hour) & End time (24-hour) & Period \\
\hline \multirow[t]{5}{*}{ Prerelease } & April 7, 2014 & West bank & $16: 28$ & $17: 00$ & Daylight \\
\hline & & Zig-zag & $17: 00$ & $17: 35$ & Daylight \\
\hline & & East bank & $17: 40$ & $18: 40$ & Daylight \\
\hline & & Middle axis & $18: 47$ & $19: 50$ & Dusk \\
\hline & & Zig-zag & $20: 33$ & $22: 03$ & Nighttime \\
\hline Prerelease & April 8, 2014 & Middle axis & $9: 21$ & $9: 40$ & Daylight \\
\hline Release & April 8, 2014 & Boat launch & $9: 50$ & $10: 10$ & Daylight \\
\hline \multirow[t]{5}{*}{ Postrelease } & April 8, 2014 & Zig-zag & $13: 58$ & $14: 35$ & Daylight \\
\hline & & East bank & $14: 35$ & $15: 35$ & Daylight \\
\hline & & West bank & $15: 35$ & $16: 40$ & Daylight \\
\hline & & Middle axis & $16: 41$ & $17: 06$ & Daylight \\
\hline & & Zig-zag & $20: 31$ & 21:01 & Nighttime \\
\hline \multirow[t]{6}{*}{ Postrelease } & April 9, 2014 & West bank & $7: 25$ & $8: 18$ & Daylight \\
\hline & & Zig-zag & $8: 18$ & $8: 36$ & Daylight \\
\hline & & East bank & $8: 37$ & $9: 39$ & Daylight \\
\hline & & Middle axis & $9: 40$ & $10: 43$ & Daylight \\
\hline & & Middle axis & $19: 28$ & $19: 47$ & Dusk \\
\hline & & Zig-zag & $20: 45$ & $21: 18$ & Nighttime \\
\hline
\end{tabular}

Table D3. Total numbers of spring Chinook salmon, coho salmon, and juvenile steelhead passive integrated transponder-tagged by year at the screw trap at Eagle Cliff on the Lewis River at the head of Swift Reservoir, Washington, and the percent (\%) of fish collected (across years) at the floating surface collector (FSC), 2014-2015.

\begin{tabular}{|l|c|c|c|}
\hline \multirow{2}{*}{\multicolumn{1}{c|}{ Species }} & \multicolumn{2}{c|}{ Year emigrated } & \multirow{2}{*}{ \% collected at FSC } \\
\cline { 2 - 3 } & $\mathbf{2 0 1 4}$ & $\mathbf{2 0 1 5}$ & 0.3 \\
\hline Chinook salmon & 276 & 38 & 15.2 \\
\hline Coho salmon & 147 & 394 & 18.7 \\
\hline Steelhead & 60 & 122 & \\
\hline
\end{tabular}





\section{Chapter E. Piscine Predator Impact Studies 2013-2014, Lake Merwin, Washington}

\section{Introduction}

Ptychocheilus oregonensis (northern pikeminnow) were identified as an abundant predator of juvenile salmon in Lake Merwin, Washington, in the 1950s and 1960s, during an experiment to determine if the reservoir could be used to rear juvenile hatchery-reared Oncorhynchus kisutch (coho salmon) (Hamilton and others, 1970). The abundance of predatory-sized northern pikeminnow (greater than or equal to $[\geq] 200$ millimeters [mm]) was estimated around 350,000 in 1961; however, the population has not since been assessed. Additionally, the Washington Department of Fish and Wildlife began stocking approximately 1,000 Esox masquinongy x E. lucius (tiger muskellunge) annually beginning in 1995 to limit the population of northern pikeminnow, but the efficacy of this program has not been formally evaluated. Here, we evaluate the contemporary abundance, diet, growth, and temporal-spatial distribution of northern pikeminnow, O. nerka (kokanee salmon), and tiger muskellunge to gauge how northern pikeminnow might affect populations of reintroduced anadromous salmonids. To achieve this objective, we characterized the temporal-spatial dimensions of the thermal environment, food supply, and the distribution, size, age, and diet of key predators and prey, and mapped the overall trophic structure of the food web through stable isotope analysis for 2013-2014. We then used these empirical data to inform bioenergetics simulations to estimate the seasonal and size-specific consumption rates and predation impact of northern pikeminnow on salmonids and alternative prey fish species (Beauchamp and others, 1997).

\section{Methods}

\section{Site Description}

Lake Merwin, a waterbody impounded by the Merwin Dam on the Lewis River, southeastern Washington, is approximately 23.3 kilometers (km) long (PacifiCorp and Cowlitz Public Utility District [PUD], 2004) with a maximum depth of 73 meters (m) and a mean depth of $31 \mathrm{~m}$ (Sorel and others, 2016). It is oligotrophic, with a mean secchi depth of roughly $5 \mathrm{~m}$ and total phosphorus of less than $(<) 0.02$ milligram per liter (mg/l) (PacifiCorp and Cowlitz PUD, 2004) (table E1). Stream habitat surveys in the Lake Merwin basin (see "Habitat Assessment of Tributaries to Swift Reservoir, Yale Lake, and Lake Merwin, Washington" chap. B of this report) suggest that there is a total of $8.2 \mathrm{~km}$ of anadromous salmonid habitat available in tributaries to Lake Merwin (Al-Chokhachy and others, 2014).

The fish assemblage in Lake Merwin consists of a large population of northern pikeminnow, Catostomus macrocheilus (largescale suckers), and moderate to small populations of kokanee salmon, $O$. clarkii (cutthroat trout), O. mykiss (rainbow trout), Cottus spp. (sculpin), Lepomis gibbosus (pumpkinseed sunfish), coho salmon, O. tshawytscha (Chinook salmon), Salvelinus confluentus (bull trout), tiger muskellunge, and Micropterus salmoides (largemouth bass) (table E2). The kokanee salmon population is supplemented with approximately 45,000 hatchery-reared fingerlings in October of each year and 48,000 yearlings in spring (PacifiCorp and Cowlitz PUD, 2014). Natural production of kokanee salmon from tributaries of Lake Merwin is likely minimal. However, recruitment from Yale Lake (directly upstream of Lake Merwin) is probably the primary source of recruitment of naturally produced kokanee salmon (PacifiCorp and Cowlitz PUD, 2004). Large (300-600 mm) hatchery-reared rainbow trout are stocked periodically, as available, to provide fishing opportunities, and juvenile coho salmon and Chinook salmon are also present through spill from Yale Lake. 


\section{Approach}

We characterized the temporal-spatial dimensions of the thermal environment, and the abundance, distribution, size- and age-structure, and diet of northern pikeminnow and tiger muskellunge in Lake Merwin. We examined the food web structure of Lake Merwin through stable isotope analysis and higher resolution data on seasonal and size-specific diet composition by these piscivores to identify potential prey of piscivorous fishes and examine their ontogenetic shifts in predatory behavior. These data informed bioenergetics model simulations constructed to estimate the seasonal and size-specific consumption rates and predation impact of northern pikeminnow on resident salmonids and alternative prey fish species, and of tiger muskellunge on northern pikeminnow and salmonids. We used the results from the bioenergetics simulations to infer the likely predation impact by northern pikeminnow on reintroduced anadromous salmonids under different scenarios regarding rearing in versus migrating through the reservoir by subyearling and yearling fish. We also used the results of the bioenergetics simulations to evaluate the cannibalism of smaller northern pikeminnow and predation of northern pikeminnow by tiger muskellunge.

\section{Fish Sampling}

Fish were systematically sampled in June, late July, and November of 2013 to obtain biological samples, seasonal fish distribution data, and population size structure. Paired small mesh $(2.5,3.2,3.8,5.1,6.4$, and 7.6 centimeter [cm] stretched mesh) and large mesh $(8.9,10.2,11.4,12.7$, and $15.2 \mathrm{~cm}$ stretched mesh) sinking gill nets were fished perpendicular to shore for approximately 24 hours. Nets were set at a minimum of three sites around the reservoir per season and were fished in three depth strata: 1-15 m; 16-30 m; and greater than (>) $30 \mathrm{~m}$ deep to approximate the depths above, within, and below the thermocline during peak summer stratification. In addition to systematic gillnetting in 2013, samples were gathered opportunistically by gill nets targeting northern pikeminnow as part of the mark-recapture study and other approaches including the use of Merwin traps (Hamilton and others, 1970; Lynch 1993), angling, dip net, minnow trap, angler surveys, and snorkeler hand collection in 2013 and 2014.

We identified and counted fish by species and recorded a subset of fork lengths to the nearest millimeter and body mass to the nearest gram. Living fish were either released or euthanized according to the University of Washington Institutional Animal Care and Use Committee (UW-IACUC; http://oaw.washington.edu/) protocol \#3286-21. Stomachs, scales from the preferred region, fin tissue, and some whole bodies were collected, placed on ice in the field, and later frozen for preservation and analysis.

Northern pikeminnow mark-recapture.-Northern pikeminnow of $200 \mathrm{~mm}$ or more were tagged with 2.54-cm numbered Floy tags. In 2013, we captured fish for tagging using short-term gill nets (5.1, 6.4, and $7.6 \mathrm{~cm}$ stretched mesh) set nearshore for 30 minutes to 2 hours. Catch rates were greatest at dusk on the north side of the reservoir around Indian George Creek and Speelyai Bay (fig. E1), so we preferentially netted in these areas at dusk. Our initial goal was to tag and release 2,000-5,000 marked fish in one season; however, difficulty in capturing sufficient numbers necessitated an extended tagging period over two years: June-August 2013 through May-July 2014. In 2014, we exclusively used Merwin traps to capture fish for tagging (number of samples $[\mathrm{n}]=4$ locations; fig. E2). Northern pikeminnow were anesthetized in Tricaine Methanesulfonate (MS-222) buffered with baking soda and tagged.

A total of 98 tagged northern pikeminnow were held in net pens for 48 hours to evaluate short-term tagging mortality and tag retention. No tag loss and approximately 10 percent mortality was observed in the holding trials. However, lacerations and evisceration observed on the carcasses of mortalities and the presence of otters and piscivorous birds in the area suggested that these fish may have been predated upon rather than injured in the capture and tagging process. Therefore, short-term tagging mortality was assumed negligible.

Northern pikeminnow were captured and examined for tags in August and September 2014 with small mesh (5.1, 6.4, $7.6 \mathrm{~cm}$ stretched mesh) and large mesh $(8.9,10.2,11.4,12.7$, and $15.2 \mathrm{~cm}$ stretched mesh) gill nets set methodically around the perimeter of the reservoir and soaked for approximately 24 hours. We assumed that fish captured and tagged during their spawning migration in June 2014 had redistributed throughout the reservoir by August. All captured individuals were measured for fork length and examined for tags, and a subset were weighed. Another subset of stomach samples (including some bycatch) were frozen for analysis. Additional individuals were captured by a separate investigator from Meridian Environmental Inc. using an electrofishing boat in the inlet to the reservoir during August 2014.

We explored multiple models and ad-hoc approaches to estimate the abundance of northern pikeminnow $\geq 200 \mathrm{~mm}$, given potential biases that emerged during the recapture phase of the study. First, the sizes of recaptured fish were biased toward $\geq 300 \mathrm{~mm}$ individuals, which was likely the result of greater natural mortality and gill net selectivity against 200-299 mm fish (Beamesderfer and Rieman, 1988). Therefore, we estimated the abundance of the 200-299 mm population of northern pikeminnow and the population of the $300 \mathrm{~mm}$ separately. Second, the majority of tags were recovered within the upper quadrant of the reservoir, where most were initially released, suggesting that tagged fish were not randomly mixed with the rest of the 
population. Consequently, we estimated abundance in the upper quadrant of the reservoir alone, in addition to our analysis of the entire reservoir. We divided the reservoir into quadrants based on geographic features such as points and bends (fig. E2), then proportionally expanded estimates from the upper quadrant (quadrant 1; fig. E2) to the remaining quadrants based on relative catch-per-unit effort (CPUE) in gill nets and the shoreline length of each quadrant.

Ultimately, the least biased and most precise abundance estimate used the Chapman estimator (Chapman, 1948; Seber, 1982) for northern pikeminnow $\geq 300 \mathrm{~mm}$ in the upper quadrant of the reservoir, expanded based on length and CPUE in the other quadrants of the reservoir. We used just the upper quadrant of the reservoir for our estimate because four of six recovered tags were captured in this quadrant. All six recovered tags occurred within the same quadrant where they were released, suggesting considerable site fidelity for this size class. The Chapman estimator was used because mortality was expected to be negligible for these large individuals during this study.

We estimated the abundance of the smaller 200-299 mm northern pikeminnow using the POPAN formulation of the JollySeber model in the program MARK (White and Burnham, 1999), based on encounters from the entire reservoir. We used the POPAN estimator because we expected significant mortality for these smaller fish over the 13-month study. We used a fixed annual survival rate (39.7 percent) based on a catch-curve analysis (Miranda and Bettoli, 2007) with the most support based on Akaike Information Criteria corrected for small sample size (AICc) ( $\mathrm{AICc}=138.4$, table E3). We used data from the entire reservoir because one of the seven recovered tags was recovered on the other side of the reservoir from where it was released. This suggested that tagged fish of this size class had mixed between regions.

Northern pikeminnow size distribution.-The length frequency distribution of northern pikeminnow captured in gill nets was adjusted for effort by mesh size, size-dependent encounter probability (Spangler and Collins, 1992), and gill-net sizeselectivity (Hansen and others, 1997) to estimate an unbiased size distribution. Only catches from mesh sizes $5.1 \mathrm{~cm}$ (2-inch stretch mesh) and above were used, as these meshes contributed the majority of the effort and catch of fish $\geq 200 \mathrm{~mm}$. We only adjusted catches from Merwin traps for the probability of encounter, as these large nets allow for equal retention of fish across a wide range of sizes. The adjusted length distribution from the Merwin traps were averaged with those from gill nets, and separate size distributions were created for male and female northern pikeminnow to account for sex-specific differences in growth and survival. Chi-square $\left(\chi^{2}\right)$ tests were used to compare the adjusted and unadjusted length distributions to examine the degree of size selection by each gear type. We also compared length distributions between this study and the 1958-1963 study (Hamilton and others, 1970) using gill-net data from similar mesh sizes and Merwin traps set in similar areas to test whether the size structure of northern pikeminnow had changed and thus changed predation risk to juvenile salmonids. Length frequency data from both study periods were adjusted for gear selectivity.

Tiger muskellunge survival and abundance.-Using stocking records and a catch-curve analysis, we constructed an age-structured population estimate. Records of the number, size, and release dates of tiger muskellunge in Lake Merwin from 1995 to 2013 were provided by the Washington Department of Fish and Wildlife. Since 2004, fish have been reared at Ringold Springs Hatchery at a constant water temperature of approximately 16 degrees Celsius $\left({ }^{\circ} \mathrm{C}\right)$ (Sorel and others, 2016), and an average of 1,340 fish (range $=500-2,220$ ) have been stocked at age-1 year in April-June. The large size at which tiger muskellunge are stocked (range $=14-348 \mathrm{~mm}$, mean $=302 \mathrm{~mm}$, standard error $=12.1 \mathrm{~mm}$ ), and the cool surface water temperatures and seemingly abundant forage base of small northern pikeminnow in spring suggest that stocking mortality is negligible.

Annual survival for tiger muskellunge at 1-10 years old (the range of ages observed) was estimated with a catch-curve analysis and is likely a function of natural and overwinter mortality. To estimate annual survival, we used cleithra-based ages and the observed size distribution to estimate a population age structure. We then used a catch-curve analysis (Miranda and Bettoli, 2007) to estimate an annual survival rate based on the estimated age-structure of the population. We estimated annual survival to be 71 percent for fish ages 1-10 years in Lake Merwin. The minimum size for legal retention of tiger muskellunge in Lake Merwin was above the maximum size observed in field efforts; therefore, the majority of angling was likely catch-and-release. Postrelease survival has been observed to be very high (approaching 100 percent) in Canadian rivers using a variety of handling procedures (Landsman and others, 2011). Thus, we assumed fishing pressure does not significantly reduce survival rates.

Bioenergetics model inputs. - Bioenergetics models are energy balance equations that operate on a daily time-step and estimate the feeding rate (percent of maximum theoretical consumption; percent $\mathrm{C}_{\max }$ ) and the consumption rate (grams of prey/day) needed to achieve the growth observed by different age classes of consumers over the time interval of the simulations. These estimates depend on the energy densities of predators and prey and species-specific parameters that describe the temperaturedependent and allometric effects of body mass on consumption, metabolism, and waste (Hanson and others, 1997). We collected field data on thermal experience (daily temperature experienced by the consumer), seasonal diet composition, and growth to construct a set of model inputs specific to northern pikeminnow and tiger muskellunge in Lake Merwin.

Thermal experience and depth distribution.-Seasonal thermal experience of different size classes of northern pikeminnow and tiger muskellunge were estimated using thermal profiles and depth-distribution patterns inferred from catches in gill and trap nets (table E4; Beauchamp and others, 2007). Temperature profiles were recorded monthly from April to November at 1-m intervals from the surface to $30 \mathrm{~m}$ and every $2 \mathrm{~m}$ thereafter down to $60 \mathrm{~m}$ at two limnology stations located at one-third and two-thirds of the length of the reservoir (fig. E1). Depth-specific measurements were averaged between stations in each 
month. Temperature profiles recorded by PacifiCorp in November 1999, January 2000, and March 2000 in the lower half of the reservoir were used to fill data gaps and complete the characterization of the seasonal thermal regime for periods outside of our monthly sampling (April to November, 2013 and 2014; Sorel and others, 2016). Relative catch at different depths in different seasons was then used to calculate the average daily thermal experience for each species and size class of consumer.

Stable isotope analysis. - Stable isotopes provide an integrative signal of diet over several months to a year, providing a valuable complement to and corroboration for stomach content analysis. We used stable isotopes to identify the length at which northern pikeminnow transition to piscivory in order to delineate size classes for calculating diet composition and thermal experience. We examined delta c $13\left(\delta^{13} \mathrm{C}\right.$, ratio of carbon isotopes ${ }^{12} \mathrm{C}$ and $\left.{ }^{13} \mathrm{C}\right)$ and delta $\mathrm{n} 15\left(\delta^{15} \mathrm{~N}\right.$, ratio of nitrogen isotopes ${ }^{15} \mathrm{~N}$ and ${ }^{14} \mathrm{~N}$ ) for a range of sizes of northern pikeminnow and tiger muskellunge and representative samples of benthic invertebrates, terrestrial insects, zooplankton, zooplanktivores, pelagic- and benthic-feeding fishes, and piscivores (Vander Zanden and Rasmussen, 1999; McIntyre and others, 2006). Fin tissue from fish, muscle tissue from clams and crayfish, and whole bodies of insects were placed on ice in the field and then frozen within 12 hours for storage. Samples were dried at $60{ }^{\circ} \mathrm{C}$ for 48 hours and homogenized with mortar and pestle, and 0.4-0.6 milligrams of material were weighed in aluminum capsules for analysis. Isotope samples were analyzed by the University of Washington IsoLab using a Costech Elemental Analyzer, Conflo III, MAT253 for continuous flow-based measurement of solid organic material. The reference material was Vienna Pee Dee belemnite for carbon and atmospheric nitrogen for nitrogen.

Diet composition and energy density of prey.-We analyzed the seasonal diets for different sizes of northern pikeminnow and tiger muskellunge as inputs for the bioenergetics model. Stomach contents were identified under a dissecting microscope to species for prey fish and to order for invertebrates. Lengths of prey fish were measured or reconstructed from diagnostic bones (Hansel and others, 1988). We calculated diet proportions by blotted wet weight (Chipps and Garvey, 2007) for two size classes of northern pikeminnow (200-299 $\mathrm{mm}$ and $\geq 300 \mathrm{~mm}$ ), the breakpoint reflecting a transition to piscivory based on $\delta^{15} \mathrm{~N}$ signatures. One set of seasonal diet proportions was calculated for all sizes of tiger muskellunge given their similarity in diet across sizes and the limited number of non-empty stomachs obtained. The energy densities of prey items were taken from literature values (table E5).

Age and growth of Northern pikeminnow.-We measured the age and growth of northern pikeminnow from scales to obtain annual growth increments for different age-classes in the bioenergetics model (table E6). Opercles were also analyzed for a subset of individuals to corroborate the scale-based ages. Scales were measured along a transect 20 degrees $\left(^{\circ}\right)$ off the scale's anterior-posterior axis from the center of the focus out to the edge of each annulus and additionally to the margin of the scale using a dissecting microscope with a digital camera and Image Pro analysis software. Fork length-at-age was back-calculated using the Fraser-Lee $(F L)$ method with a biological intercept of zero (FL Range: $34-560 \mathrm{~mm}, \mathrm{n}=101$, coefficient of determination $\left[\mathrm{R}^{2}\right]=0.97, P<0.001$; Isely and Grabowski, 2007). Growth in length was characterized by a von Bertalanffy growth function fit to length-at-age data using the back-calculated length at final annulus for each fish (von Bertalanffy, 1938). A likelihood ratio test using the "vblrt" function in the fish methods package in R (Nelson, 2014) indicated that a sex-specific von Bertalanffy growth model described the length-at-age data significantly better than a single model for both sexes where $\left(\chi^{2}=12.88\right.$, degrees of freedom $[d f]=3, P<0.005)$. The greatest scale-based age observed was 14 years ( $431 \mathrm{~mm}$ male, and $542 \mathrm{~mm}$ female). While scales often underestimate ages of northern pikeminnow $>350 \mathrm{~mm}$ (Takata and others, 2007), our analysis of opercles helped reduce this bias by identifying older annuli on $>350 \mathrm{~mm}$ fish. The von Bertalanffy growth parameters fit for females $(\mathrm{n}=34)$ were $L_{\infty}=786.64 \mathrm{~mm}, K=0.0665$, and $t_{0}=-2.0139(\mathrm{n}=34)$. Corresponding parameters for the male model $(\mathrm{n}=38)$ were $L_{\infty}=689.23$, $K=0.0599$, and $t_{0}=-3.0117$ (see fig. E3), where $L_{\infty}$ is the asymptotic average length, $K$ is the growth rate coefficient, and $t_{0}$ is the age when average length is zero.

Fork length-at-age was converted to weight-at-age $(W)$ using length-weight relationships developed for northern pikeminnow in Lake Merwin. Separate relationships were developed for individuals $<300 \mathrm{~mm}$ and $\geq 300 \mathrm{~mm}$ due to the inability of a single power function to fit length and weight measurements over the entire range of $F L$ 's observed. The $F L$ to $W$ relationship for northern pikeminnow $<300 \mathrm{~mm}$ was $\left(\mathrm{n}=2,941\right.$, range: $\left.34-299 \mathrm{~mm} ; R^{2}=0.972, P<0.001\right)$ :

$$
W=0.000012 \times F L^{2.979}
$$

The $F L$ to $W$ relationship for individuals $\geq 300 \mathrm{~mm}$ was ( $=354$, range: $300-574 \mathrm{~mm} ; \mathrm{R}^{2}=0.974, P<0.001$ ):

$$
W=0.0000015 \times F L^{3.348}
$$

Spawning losses were calculated separately for each sex by subtracting the average gonadosomatic index (100×gonad mass/body mass) of spent from ripe fish in the spring. Spawning losses were then phased in over two age classes for bioenergetics model simulations to represent an increase in the proportion of mature fish. Spawning losses were 3.9 percent of body mass for males age 3-14 years, 6 percent for age 3 females, and 8.9 percent for age 4-14 years females. 
Age and growth of tiger muskellunge.-Annual growth increments for bioenergetics simulations of tiger muskellunge consumption were determined with age-to-length and weight-length relationships (table E7). Age was determined using standard cleithra aging procedures for a parent species, northern pike (E. lucius of tiger muskellunge [Euchner, 1988]). Fish are routinely implanted with blank wire tags in various locations on their bodies that indicated their brood year, and a number of individuals were aged based on the location of these tags to corroborate cleithra-based ages. We used the proportional linear relationship derived for the other parent species, muskellunge (E. masquinongy) (Harrison and Hadley, 1979) to estimate total length at age.

$$
T L i=(T L-1.41) \times(C L i / C L)+1.41
$$

where

$T L \quad$ is total length at age $i$, and

$T L i \quad$ is back calculated based on measurements from the origin of the cleithra to each annuli $(C L i)$, the origin to edge of the cleithra $(C L)$, and $T L$ is the total length at capture.

A von Bertalanffy growth curve was fit to total length at age $\left(K=0.389, L_{\infty}=1023.9, t_{0}=0.00\right)$. Total Length $(T L, \mathrm{~mm})$ was converted to $F L$ with the relationship $F L=0.959 \times(\mathrm{TL})-0.654$ (Gayzusuzl and others, 2006). Fork length-at-age was then converted to weight-at-age with the equation ( $\mathrm{n}=62$, range: $324-975 \mathrm{~mm}, \mathrm{R}^{2}=0.988, P<0.001$ )

$$
W=0.00000037 \times F L^{3.479}
$$

Bioenergetics modeling simulations of seasonal predation.-The Wisconsin bioenergetics model (Hanson and others, 1997) parameterized for northern pikeminnow (Petersen and Ward, 1999) was used to estimate seasonal consumption demand. The bioenergetics model fit the daily per-capita consumption, starting April 1, needed to satisfy the observed growth of an average northern pikeminnow from each age class and sex over an annual cycle. Age-frequency distributions within each size class were developed from the unbiased size distribution estimated above and an age-length key (Isely and Grabowski, 2007). Per-capita consumption demand was expanded to separate age-structured populations of 1,000 northern pikeminnow of the 200-299 mm and $\geq 300 \mathrm{~mm}$ size classes, assuming an even sex ratio. We then used estimates of consumption of resident salmonids to gauge potential predation on reintroduced anadromous salmonids under different reservoir use scenarios (for example, rearing in versus migrating through the reservoir).

Estimating predation on reintroduced salmon.-In order to estimate maximum potential predation on reintroduced anadromous salmonids, we assumed that all observed predation on resident kokanee salmon would switch to anadromous fish once they were reintroduced. Thus the monthly biomass of kokanee salmon that was consumed from April 1 to March 30 based on bioenergetics simulations was divided by the estimated monthly weight of an age- 0 (that is, during the year after fish emerged) Chinook salmon that adopted a year-round reservoir rearing strategy to estimate how many individuals could be lost to predation. We divided northern pikeminnow monthly consumption of salmonid biomass by the monthly mass of individual age- 0 stream-type Chinook salmon estimated using a bioenergetics simulation. For the bioenergetics simulation of age- 0 Chinook salmon growth in the reservoir, we assumed that kokanee salmon and subyearling stream-type Chinook salmon used similar depth distributions during thermally stratified periods and would therefore share similar thermal experiences in reservoirs. This assumption, based on observations of Chinook salmon in North Fork Reservoir on the Clackamas River, Oregon (Lowery and Beauchamp, 2010), also suggests similar overlap with predatory northern pikeminnow, supporting our use of kokanee salmon as a surrogate to estimate predation on juvenile anadromous salmonids. For the bioenergetics simulation of age- 0 Chinook salmon in the reservoir, we assumed a diet of zooplankton and insects, and a feeding rate of 93 percent $\mathrm{C}_{\max }$, as informed by observations of age-1 Chinook salmon smolts that residualized in Swift Reservoir (also on the North Fork of the Lewis River) following releases in 2013. Information on the diet and feeding rate of age-0 Chinook salmon in Swift Reservoir was not available. Fish in this simulation grew from 0.36 grams $(\mathrm{g})(35 \mathrm{~mm})$ on April 1 to $45 \mathrm{~g}(155 \mathrm{~mm})$ on November 15, after which their mass was held constant through March 30 to simulate reduced prey supply and growth during winter. The mass that smolts achieved in this simulation was similar to the upper end of the length distribution of smolts outmigrating from the Columbia River that survived at least 30 days in the marine environment (Tomaro and others, 2012). We also simulated the magnitude of predation on reservoir-rearing Chinook salmon that achieved a mass of only $18 \mathrm{~g}$ (about120 mm), at the lower end of the length distribution of smolts that survived at least 30 days in the marine environment (Tomaro and others, 2012). A different predation scenario was used to simulate potential predation on age-1 smolts migrating through Lake Merwin.

Some juvenile stream-type Chinook salmon smolts might only use the reservoir as a migration corridor after rearing in tributaries. Therefore, we estimated potential predation on smolts traversing the reservoir to evaluate the effect of northern pikeminnow on their survival during the spring outmigration period. As before, our estimate of population-level consumption demand of kokanee salmon by northern pikeminnow was divided by the mass of individual stream-type Chinook salmon. However, to estimate predation on smolts, we restricted the simulation to April through June, spanning the months for peak outmigration as indicated from field efforts in Swift Reservoir (see "Assessment of Juvenile Fish Production and Outmigration Success 
on the Lewis River, Washington," chap. D of this report). We used the same potential average smolt sizes (18 and $45 \mathrm{~g}$ ), which were held constant for the 3-month simulations. Unlike in the reservoirs on the mainstem Columbia River, there is no tailrace area in Lake Merwin where disoriented salmon smolts would concentrate and provide easy access for northern pikeminnow (for example, Rieman and others, 1991), so we did not simulate northern pikeminnow feeding at an elevated rate in response to a smolt outmigration.

Our estimates of predation on age- 0 or age- 1 Chinook salmon represent the maximum potential predation on either age class because the same population-level consumption by northern pikeminnow was used to estimate losses in both scenarios. However, we expect that this overall consumption rate of salmonids by northern pikeminnow would be partitioned among the two age classes of anadromous salmonids as well as resident salmonids, so the realized predation should be lower than our estimates.

We calculated predation rates for size-structured population units of 1,000 predators for each of the two size classes of northern pikeminnow rather than for the entire populations, given the uncertainty surrounding our population abundance estimates. Our predation estimates contain all the major variability related to seasonal and size-specific diet, consumption rates, and relative abundance of each size/age class. These estimates of predation by size-structured units of 1,000 predators can be multiplied by however many thousands of predators of that size range are in the population (or however many might be prescribed in different predation scenarios) to generate full population-level predation estimates.

Estimating predation by tiger muskellunge on northern pikeminnow.-Thermal experience, size-specific diet composition, and growth estimates were used as inputs in a bioenergetics model (Bevelhimer and others, 1985) to determine annual consumption rates for each age class of tiger muskellunge. Individual consumption demands were then expanded by the respective abundance estimates of each age-class to determine the total consumption by the population.

We used the biomass of northern pikeminnow consumed by tiger muskellunge estimated with bioenergetics combined with the lengths of northern pikeminnow found in stomachs to estimate the number and size distribution of prey consumed by the predator population. For age-1 and age 2-10 predator size classes, we converted the lengths of northern pikeminnow found in tiger muskellunge diets to masses with a weight-length regression. We then used the masses of individual prey fish and the estimates of consumption demand to calculate how many of each size class of northern pikeminnow were consumed by each size class of tiger muskellunge.

\section{Results and Discussion}

\section{Seasonal Distribution and Thermal Experience of Northern Pikeminnow}

Thermal stratification in Lake Merwin began in May and peaked in August in 2014; rapid destratification occurred during October and the water column became isothermal in November of that year (fig. E4). The epilimnion encompassed the upper $8 \mathrm{~m}$ of the water column, the metalimnion ranged from roughly $8-18 \mathrm{~m}$, and the hypolimnion was approximately $18 \mathrm{~m}$ and deeper. Epilimnetic temperatures began at $14-16^{\circ} \mathrm{C}$ in May and peaked at $22-24{ }^{\circ} \mathrm{C}$ in August and July 2014 . The hypolimnion warmed from 5-6 ${ }^{\circ} \mathrm{C}$ in April to $15-18^{\circ} \mathrm{C}$ in October 2014.

The depth-distribution of northern pikeminnow varied by season. Most northern pikeminnow and especially those $\geq 300 \mathrm{~mm}$ occupied warmer surface waters nearshore in 0-15 $\mathrm{m}$ depths during thermally stratified periods in June-September but were deeper during isothermal conditions in November (figs. E1, E4). The CPUE of the larger fish was significantly higher in $0-15 \mathrm{~m}$ depths ( $4.8 \mathrm{fish} / \mathrm{net}$ ) than deeper ( $0.3 \mathrm{fish} / \mathrm{net}$ ) in spring-summer (two-sided t-test: $d f=18, t=5.11, P<0.001$ ). This pattern reversed as the reservoir became isothermal in November, and CPUE was higher in 16-30 m depths (5.7 fish/net) than in 0-15 m ( $1.5 \mathrm{fish} / \mathrm{net}$ ), although this difference was not significant (two-sided t-test: $t=1.89, d f=3, P=0.155$ ). The CPUE of northern pikeminnow 200-299 $\mathrm{mm}$ was also significantly higher at $0-15 \mathrm{~m}$ depths (24.3 fish/net), but a sizeable number also occupied $15-30 \mathrm{~m}$ (13.4 fish/net) during stratification (two-sided t-test: $d f=14, t=3.42, P=0.004)$. Northern pikeminnow 200-299 mm exhibited the same distribution pattern as fish $\geq 300 \mathrm{~mm}$ in November, and their CPUE in 16-30 m depths (15 fish/net) was also not significantly higher than in $0-15 \mathrm{~m}$ (10.4 fish/net) (two-sided t-test: $d f=3, t=0.65, P=0.561)$. Tiger muskellunge remained in the epilimnion in spring-fall similarly to large northern pikeminnow.

The density of northern pikeminnow was greater in the lower half of the reservoir (quadrants 3-4) in summer based on CPUE (fig. E5). Although not significant, the CPUE of northern pikeminnow $\geq 300 \mathrm{~mm}$ in gill nets was greater in the lower half ( 6 fish/net) than the upper half of the reservoir ( $3.1 \mathrm{fish} / \mathrm{net}$ ) during the recapture phase of the mark-recapture study in August-September 2014 (two-sided t-test; $d f=6, t=2.08, P=0.083$ ). The CPUE of 200-299 mm northern pikeminnow during this recapture effort was also significantly higher in the lower half of the reservoir (27.1 fish/net) than the upper half (17.8 fish/ net; two-sided t-test; $d f=58, t=2.79, P=0.007$ ). This pattern suggests that northern pikeminnow redistributed to the lower half of the reservoir by August after spawning in Canyon Creek (at the top of the reservoir; fig. E2), or a segment also spawned near tributaries in the lower reservoir as also observed by Hamilton and others, (1970). 


\section{Bioenergetics Inputs and Model Simulations}

Stable isotopes.-Northern pikeminnow became increasingly piscivorous and reliant on benthically derived energy with increasing size (table E8, fig. E6). The trophic position of medium-sized northern pikeminnow (200-299 mm) suggested that they fed primarily on a mix of pelagic and benthic invertebrates and increasing proportions of benthic fish or Pacifastacus leniusculus (signal crayfish) (figs. E6, E7). The isotopic signatures for large northern pikeminnow $\geq 300 \mathrm{~mm}$ suggested that they consumed a combination of pelagic and benthic fish and signal crayfish.

Stable isotope analysis suggested that tiger muskellunge fed on a mix of pelagically and bentho-littorally derived carbon and increased in trophic level steadily throughout their lives (fig. E6). Tiger muskellunge increased by nearly one trophic level $\left(\sim 3.4\right.$ per thousand increase in $\delta^{15} \mathrm{~N}$ ) (Minagawa and Wada, 1984; Hussey and others, 2014) over the range of 450-1,050 mm FL (figs. E6, E7). Thus stable isotopes suggest that tiger muskellunge initially consumed invertebrate-feeding fish but progressed to consuming partially-piscivorous fish as they grew.

Stomach content analysis. - Northern pikeminnow exhibited seasonal and ontogenetic shifts in diet composition, with an increasing reliance on prey fish at larger sizes, in agreement with the stable isotope patterns (table E8). The spring and summer of 2013-2014 diets of 200-299 mm northern pikeminnow contained 72-88 percent zooplankton and smaller proportions of sculpins, signal crayfish, insects, and plant matter, but no salmonids were detected (table E9). Diets of the $\geq 300 \mathrm{~mm}$ northern pikeminnow contained primarily fish and signal crayfish. During spring, signal crayfish (45 percent) and smaller northern pikeminnow (43 percent) were the predominant prey, indicating a high proportion of cannibalism. During summer, signal crayfish still represented 51 percent of the diet, but northern pikeminnow declined to 19 percent, whereas sculpins ( 9 percent) and kokanee salmon (14 percent) increased. The diet proportions of sculpins (14 percent) and kokanee salmon (28 percent) continued increasing during fall, while signal crayfish (29 percent) and smaller northern pikeminnow (10 percent) declined.

The maximum $F L$ of prey fish consumed increased with the $F L$ of northern pikeminnow (fig. E8). Kokanee salmon were the largest prey fish consumed by northern pikeminnow and occasionally exceeded 50 percent of the predator's $F L$ when reconstructed from diagnostic bones. Four 100-150 mm kokanee salmon were found in the guts of 300-399 mm northern pikeminnow. Six 200-325 mm kokanee salmon were observed in $\geq 400 \mathrm{~mm}$ northern pikeminnow. As prey, the average $F L$ of northern pikeminnow eaten by $300-399 \mathrm{~mm}$ cannibals was $143 \mathrm{~mm}$ (range: $143-144, \mathrm{n}=2$ ) and $164 \mathrm{~mm}$ for $\geq 400 \mathrm{~mm}$ predators (range: $101-252 \mathrm{~mm}, \mathrm{n}=10$ ).

The diet composition of tiger muskellunge remained relatively constant from spring through fall 2014 (table E10) and was corroborated by stable isotopes (table E8). Northern pikeminnow were the largest component (65-80 percent of diet across seasons) followed by largescale suckers (10-25 percent), while kokanee salmon, pumpkinseed sunfish, signal crayfish, and aquatic insects represented smaller proportions (table E5). Only one salmonid ( $F L=451 \mathrm{~mm}$ ) was found in a tiger muskellunge's stomach, a $920 \mathrm{~mm}$ fish captured in September 2014 when kokanee salmon were using the littoral zone as a spawning-migration corridor. Tiger muskellunge consumed a wide size range of northern pikeminnow (57-394 mm) (fig. E9). The majority of prey fish were approximately 40 percent of their consumers' fork lengths, but a significant number of northern pikeminnow were also $<20$ percent of the predators' lengths. Age-1 tiger muskellunge $(F L>300 \mathrm{~mm})$ began feeding on northern pikeminnow that were $\geq 200 \mathrm{~mm}$. The mean fork length of northern pikeminnow consumed was not significantly different between age-1 (152 plus or minus $[ \pm] 49 \mathrm{~mm}$ [mean \pm standard deviation]) and ages $2-10$ tiger muskellunge $(195 \pm 97 \mathrm{~mm})(\mathrm{t}$-test: $D F=19, T=-1.354, P=0.192)$. The mean fork length of northern pikeminnow consumed was also not significantly different among spring $(181 \pm 76 \mathrm{~mm})$, summer $(182 \pm 92 \mathrm{~mm})$, and fall $(163 \pm 81 \mathrm{~mm}$ ) (one-factor analysis of variance [ANOVA]: $F=0.087, P=0.917, d f=21$ ).

Size distribution.-The length frequency distributions of male and female northern pikeminnow were skewed toward smaller (200-299 mm) individuals and exhibited a decrease in modal $F L$ from 1958-1963 to 2013-2014 (fig. E10). Fish 200-299 mm made up 83 percent of the population of $\geq 200 \mathrm{~mm}$ northern pikeminnow in the present study. The adjusted length distribution was significantly different than the unadjusted distribution for fish captured in gill nets $\left(\chi^{2}=336, d f=224, P=0.003\right)$, but not in Merwin traps $\left(\chi^{2}=456, d f=418, P=0.097\right)$, suggesting that the degree of selectivity was greater for gill nets (fig. E11). Males in the present study were significantly smaller on average than in the historic study $\left(\chi^{2}=192, d f=80, P<0.001\right)$ as were females $\left(\chi^{2}=336, d f=224, P<0.001\right)$, whereas the current female size structure was significantly larger than males. The contemporary size distribution of northern pikeminnow is likely driven by predation by large northern pikeminnow and tiger muskellunge (Baker and others, 2015) on smaller northern pikeminnow (see the "Population-level consumption demand" sections in this chapter).

Mark recapture.-Estimates of northern pikeminnow abundance (table E3) were imprecise due to the relatively few recaptures $(<1$ percent). It was difficult to estimate the abundance of fish because they were only vulnerable to capture in certain locations and exhibited considerable site fidelity, eliminating much of the mixing of marked and unmarked fish necessary for accurate estimation in this type of study design. The estimate of the fully piscivorous $\geq 300 \mathrm{~mm}$ northern pikeminnow was 11,240 fish (95 percent confidence interval [CI]: 3,730-39,880), and 544,259 (95 percent CI: 190,609-1,554,062) 200-299 mm northern pikeminnow. This abundance estimate of smaller northern pikeminnow was roughly 50 times greater than the abundance of 
$\geq 300 \mathrm{~mm}$ fish whereas the observed size distribution of the population suggests that this ratio should be only approximately six times greater. This disagreement suggests some bias in the estimates of the population size distribution or abundance.

Tiger Muskellunge abundance.-The population abundance of tiger muskellunge based on the 71 percent annual survival rate was 5,488 fish. We expanded individual consumption demands by the population abundance estimate as well as calculating predation by a size-structured population of 1,000 tiger muskellunge for comparison with northern pikeminnow.

Population-level consumption demand of northern pikeminnow.-Our estimates of consumption varied by month, a pattern consistent with previous studies of northern pikeminnow predation rates (Rieman and others, 1991). Total monthly consumption demand by northern pikeminnow on salmonids and other fish was highly influenced by the size structure and abundance of the population, as well as the thermal regime, which influenced metabolic rates and seasonal diet compositions. Overall consumption started low in spring and increased as the water warmed. The greatest consumption of fish biomass was estimated in October, when relatively warm water allowed a high metabolic rate, and predators ate a higher proportion of fish (fig. E9). Consumption demand was very low in winter when water temperature limited metabolic rates. The feeding rate of the 200-299 mm northern pikeminnow was 55-65 percent $C_{\max }$, but declined to $22-25$ percent $C_{\max }$ for the $\geq 300$ mm size class (table E6). Consumption by a size-structured population of 1,000 northern pikeminnow 200-299 $\mathrm{mm}$ over the entire year totaled 1,069 kilogram ( $\mathrm{kg}$ ) of zooplankton (peak=August), $382 \mathrm{~kg}$ of plant matter (peak=August), and $159 \mathrm{~kg}$ of sculpins (peak=October) (fig. E12). Consumption by $1,000 \geq 300 \mathrm{~mm}$ fish over the entire year totaled $1,118 \mathrm{~kg}$ of crayfish (peak=August), $557 \mathrm{~kg}$ of northern pikeminnow (peak=June), and $384 \mathrm{~kg}$ of salmonids (peak=October).

Dividing monthly consumption demand of salmonids by the mass of an age- 0 stream-type Chinook salmon in the corresponding month and summing across months provided an estimate of 39,250 Chinook salmon consumed from April 1 to March 31 (peak September) by a size-structured population of 1,000 northern pikeminnow $\geq 300 \mathrm{~mm}$, if the salmon reached $18 \mathrm{~g}$ by November, and 16,022 fish if they reached a mass of $45 \mathrm{~g}$ by November (fig. E13). Our estimates of potential predation on age-1 Chinook salmon smolts was considerably less than on age-0's.

Consumption of salmonids by a size-structured population of 1,000 large northern pikeminnow during peak months of smolt outmigration in April-June was only $17 \mathrm{~kg}$ (fig. E12). This biomass was equivalent to 969 salmon smolts weighing $18 \mathrm{~g}$ each, or 388 smolts weighing $45 \mathrm{~g}$ each. These numerical estimates of smolt consumption were driven in large part by the lack of salmonids observed in northern pikeminnow diets during spring, and low overall consumption rates due to cold water in the spring months. Cannibalism on smaller northern pikeminnow by 1,000 predators $\geq 300 \mathrm{~mm}$ was $557 \mathrm{~kg}$ (fig. E12). Using the length distribution of cannibalized individuals and a length-weight regression, we estimated that this was equivalent to 1,862 fish of 200-299 $\mathrm{mm}$ and 9,311 northern pikeminnow $<200 \mathrm{~mm}$. The observed cannibalism on smaller northern pikeminnow likely reduces the overall predation pressure on salmonids.

In lieu of our estimates of salmonid consumption, we acknowledge that salmonid consumption may vary as a function of migration timing and reservoir thermal regimes (Vigg and others, 1991; Petersen and Kitchell, 2001). In Swift Reservoir, hatchery-reared spring Chinook salmon smolts rapidly emigrate to the reservoir environment (see "Assessment of Juvenile Fish Production and Outmigration Success on the Lewis River, Washington," chap. D of this report) and the median rearing time in the reservoir is approximately 2 months, but considerable variability in rearing time is possible (for example, Romer and others, 2014). For coho salmon, rearing in the reservoir environment has been approximately 4 months (see "Assessment of Juvenile Fish Production and Outmigration Success on the Lewis River, Washington," chap. D of this report). Despite the longer period of rearing for coho salmon smolts, estimates of predation by month suggest predation on spring Chinook salmon is likely to be higher given the overlap in distribution during the periods of relatively high predation (for example, spring-early summer). Further, the fact that nearly 30 percent of Chinook salmon are rearing in the reservoir for more than 9 months suggest the exposure to northern pikeminnow predation may be relatively high. However, rearing in reservoirs can be highly variable for juvenile salmon (Vendetti and others, 2000; Smith and others, 2003), which can affect the predicted patterns of predation.

Population-level consumption demand of tiger muskellunge.-We estimated that 29,069 northern pikeminnow $\geq 300 \mathrm{~mm}, 142,508$ of $200-299 \mathrm{~mm}$, and 247,736 of $<200 \mathrm{~mm}$ were consumed by the population of tiger muskellunge (ranged 316-970 mm) over the course of a year (fig. E14). The equivalent consumption by a size-structured population unit of 1,000 tiger muskellunge was 5,296 northern pikeminnow $\geq 300 \mathrm{~mm}, 25,967$ of $200-299 \mathrm{~mm}$, and 45,141 of $<200 \mathrm{~mm}$ (table E7). The numerical estimate of northern pikeminnow $\geq 300 \mathrm{~mm}$ consumed was based on a single observation of a $394 \mathrm{~mm}$ northern pikeminnow in one tiger muskellunge's stomach, which could have been an anomaly that skewed our data due to our relatively small sample size of prey fish examined. Despite this limitation, the predation of smaller northern pikeminnow by tiger muskellunge likely further reduces the overall predation of juvenile salmonids. 


\section{Conclusions}

We found large northern pikeminnow represent a substantial predation threat to anadromous smolts in Lake Merwin. Our simulations indicated that yearly consumption by a population of 1,000 large northern pikeminnow would be approximately 16,000-40,000 age- 0 spring Chinook salmon rearing in the reservoir based on their current feeding rate, consumption of resident salmonids, and the size distribution of the population. Concomitantly, our predation studies suggest the overall northern pikeminnow predation on salmonids was considerably reduced due to predation on smaller northern pikeminnow by larger conspecifics and tiger muskellunge.

Continued monitoring of reservoir rearing as efficiencies improve at juvenile collection facilities (for example, floating surface collector on Swift Reservoir) will ultimately provide further insight into the likelihood of predation for fish with varying periods of reservoir rearing. Ultimately, considering such predation rates in the context of robust productivity measures can provide key insights into the ramifications of different predation levels on long-term persistence of reintroduced anadromous species into Lake Merwin.

\section{References}

Al-Chokhachy, R., Sorel, M., Beauchamp, D.A., Clark, C., and Lowery, E.D., 2014, Development of new information to inform fish passage decision at the Yale and Merwin hydro projects on the Lewis River-Annual report: PacifiCorp, prepared by U.S. Geological Survey, Bozeman, Mont.

Baker, W.P., Divens, M.J., and Bolding, B.D., 2015, Tiger muskellunge growth, condition, diet, and effect on target prey species in two Eastern Washington lakes-Progress report 2001-2006: Olympia, Wash., Washington Department of Fish and Wildlife, FPT 15-02.

Beamesderfer, R.C., and Rieman, B.E., 1988, Size selectivity and bias in estimates of population statistics of smallmouth bass, walleye, and northern squawfish in a Columbia River reservoir: North American Journal of Fisheries Management, v. 8, p. $505-510$.

Beauchamp, D.A., Luecke, C., Wurtsbaugh, W.A., Gross, H.G., Budy, P., Spaulding, S., Dillenger, R., and Gubala, C.P., 1997, Hydroacoustic assessment of abundance and diel distribution of sockeye salmon and kokanee in the Sawtooth Valley Lakes, Idaho: North American Journal of Fisheries Management, v. 17, p. 253-267.

Beauchamp, D.A., Wahl, D.H., and Johnson, B.M., 2007, Predator \& prey interactions, chap. 16 of Guy, C.S., and Brown, M.L., eds., Analysis and interpretation of freshwater fisheries data: Bethesda, Md., American Fisheries Society, p. 765-842.

Bevelhimer, M.S., Stein, R.A., and Carline, R.F., 1985, Assessing significance of physiological differences among three esocids with a bioenergetics model: Canadian Journal of Fisheries and Aquatic Sciences, v. 42, p. 57-69.

Chapman, D.G., 1948, A mathematical study of confidence limits of salmon populations calculated from sample tag ratios: Fisheries Commission Bulletin, v. 2, p. 69-85.

Chipps, S.R., and Garvey, J.E., 2007, Assessment of diets and feeding patterns, chap. 11 of Guy, C.S., and Brown, M.L., eds., Analysis and interpretation of freshwater fisheries data: Bethesda, Md., American Fisheries Society, p. 473-514.

Euchner, R.B., 1988, Collection, preparation and use of northern pike (Esox lucius) cleithra for age determination: Fort St. John, Canada, Ministry of Environment and Park, Recreational Fisheries Branch.

Gayzusuzl, O., Gursoy, C., Ozulug, M., Tarkan, A.S., Acipinar, H., Bilge, G., and Filiz, H., 2006, Conversions of total, fork and standard length measurements based on 42 marine and freshwater species (from Turkish waters): Turkish Journal of Fisheries and Aquatic Sciences, v. 6, p. 79-84.

Hamilton, J.A.R., Rothfus, L.O., Erho, M.W., and Remington, J.D., 1970, Use of a hydroelectric reservoir for rearing of coho salmon (Oncorhynchus kisutch): Olympia, Wash., Washington Department of Fisheries, Research Bulletin no 9.

Hansel, H.C., Duke, S.D., Lofy, P.T., and Gray, G.A., 1988, Use of diagnostic bones to identify and estimate original lengths of ingested prey fishes: Transactions of the American Fisheries Society, v. 117, no. 1, p. 55-62. 
Hansen, M.J., Madenjian, C.P., Selgeby, J.H., and Helser, T.E., 1997, Gillnet selectivity for lake trout (Salvelinus namaycush) in Lake Superior: Canadian Journal of Fisheries and Aquatic Sciences, v. 54, no. 11, p. 2483-2490.

Hanson, P.C., Johnson, T.B., Schindler, D.E., and Kitchell, J.F., 1997, Fish bioenergetics 3.0 [software]: Madison, Wis., University of Wisconsin Sea Grant Institute, Technical Report WISCU-T-97-001.

Harrison, E.J., and Hadley, W.F., 1979, A comparison of the use of cleithra to the use of scales for age and growth studies: Transactions of the American Fisheries Society, v. 108, p. 452-456.

Hussey, N.E., MacNeil, M.A., McMeans, B.C., Olin, J.A., Dudley, S.F.J., Cliff, G., Wintner, S.P., Fennessy, S.T., and Fisk, A.T., 2014, Rescaling the trophic structure of marine food webs: Ecology Letters, v. 17, no. 2, p. 239-250.

Isely, J.J., and Grabowski, T.B., 2007, Age and growth, chap. 5 of Guy, C.S., and Brown, M.L., eds., Analysis and interpretation of freshwater fisheries data: Bethesda, Md., American Fisheries Society, p. 187-228.

Landsman, S.J., Wachelka, H.J., Suski, C.D., and Cooke, S.J., 2011, Evaluation of the physiology, behaviour, and survival of adult muskellunge (Esox masquinongy) captured and released by specialized anglers: Fisheries Research, v. 110, no. 2 , p. $377-386$.

Lowery, E., and Beauchamp, D.A., 2010, Baseline food web assessment of the upper Clackamas River basin prior to reintroduction of bull trout-Final report to the Upper Clackamas Bull Trout Working Group: Seattle, Wash., Washington Cooperative Fish and Wildlife Research Unit, report number WACFWRU-010-02.

Lynch, J.M., 1993, Evaluation of the Merwin trap as a means of northern squawfish (Ptychocheilus oregonensis) control in the Columbia River: Seattle, Wash., University of Washington, M.S. thesis, 114 p.

Mazur, M., 2004, Linking visual foraging with temporal prey distribution to model trophic interactions in Lake Washington: Seattle, Wash., University of Washington, Ph.D. dissertation, 186 p.

McIntyre, J.K., Beauchamp, D.A., Mazur, M.M., and Overman, N.C., 2006, Ontogenetic trophic interactions and benthopelagic coupling in Lake Washington-Evidence from stable isotopes and diet analysis: Transactions of the American Fisheries Society, v. 135, no. 5, p. 1312-1328.

Minagawa, M., and Wada, E., 1984, Stepwise enrichment of ${ }^{15} \mathrm{~N}$ along food chains-Further evidence and the relation between delta ${ }^{15} \mathrm{~N}$ and animal age: Geochimica Et Cosmochimica Acta, v. 48, p. 1135-1140.

Miranda, L.E., and Bettoli, P.W., 2007, Mortality, chap. 6 of Guy, C.S., and Brown, M.L., eds., Analysis and interpretation of freshwater fisheries data: Bethesda, Md., American Fisheries Society, p. 229-278.

Nelson, G.A., 2014, Fishery science methods and models in R-Package 'fishmethods': R Foundation for Statistical Computing, Comprehensive R Archive Network (CRAN), 142 p.

PacifiCorp and Cowlitz Public Utility District [PUD], 2004, Report on life history, habitat requirements, and distribution of aquatic analysis species: Portland, Oreg., PacifiCorp and Cowlitz Public Utility District, report AQU 1.

PacifiCorp and Cowlitz Public Utility District [PUD], 2014, Lewis River hatachery and supplementation plan: Portland, Oreg., PacifiCorp and Cowlitz Public Utility District.

Petersen, J.H., and Kitchell, J.F., 2001, Climate regimes and water temperature changes in the Columbia River-Bioenergetic implications for predators of juvenile salmon: Canadian Journal of Fisheries and Aquatic Sciences, v. 58, no. 9, p. 1831-1841.

Petersen, J.H., and Ward, D.L., 1999, Development and corroboration of a bioenergetics model for northern pikeminnow feeding on juvenile salmonids in the Columbia River: Transactions of the American Fisheries Society, v. 128, no. 5, p. 784-801.

Rieman, B.E., Beamesderfer, R.C., Vigg, S., and Poe, T.P., 1991, Estimated loss of jvenile salmonids to predation by northern squawfish, walleyes, and smallmouth bass in John Day Reservoir, Columbia River: Transactions of the American Fisheries Society, v. 120, no. 4, p. 448-458.

Romer, J.D., Monzyk, F.R., Emig, R., and Friesen, T.A., 2014, Juvenile salmonid outmigration monitoring at Willamette Valley project reservoirs: Corvallis, Oreg., Oregon Department of Fish and Wildlife, task order number W9127N-10-2-0008-0019.

Seber, G.A.F., 1982, The estimation of animal abundance (2d ed.): London, Griffin, 654 p. 
Smith, S.G., Muir, W.D., Hockersmith, E.E., Zabel, R.W., Graves, R.J., Ross, C.V., Conner, W.P., and Arnsber, B.D., 2003, Influence of river conditions on survival and travel time of Snake River subyearling fall Chinook salmon: North American Journal of Fisheries Management, v. 23, p. 939-961.

Sorel, M.H., Hansen, A.G., Connelly, K.A., Wilson, A.C., and Lowery, E.D., 2016, Predation by northern pikeminnow and tiger muskellunge on juvenile salmonids in a high-head reservoir-Implications for anadromous fish reintroductions: Transactions of the American Fisheries Society, v. 145, p. 521-536.

Spangler, G.R., and Collins, J.J., 1992, Lake Huron fish community structure based on gill-net catches corrected for selectivity and encounter probability: North American Journal of Fisheries Management, v. 12, p. 585-597.

Takata, H.K., Reesman, M.J., Reed, G.E., Layng, L.D., and Jones, T.A., 2007, Development of a system-wide predator control program - Indexing fisheries evaluations: Portland, Oreg., Oregon Department of Fish and Wildlife, Columbia River Investigations.

Tomaro, L.M., Teel, D.J., Peterson, W.T., and Miller, J.A., 2012, When is bigger better? -Early marine residence of middle and upper Columbia River spring Chinook salmon: Marine Ecology Progress Series, v. 452, p. 237-252.

Vander Zanden, M.J., and Rasmussen, J.B., 1999, Primary consumer $\delta^{13} \mathrm{C}$ and $\delta^{15} \mathrm{~N}$ and the trophic position of aquatic consumers: Ecology, v. 80, no. 4, p. 1395-1404.

Vendetti, D.A., Rondorf, D.W., and Kraut, J.M., 2000, Migratory behavior and forebay delay of radio-tagged juvenile fall Chinook salmon in a lower Snake River impoundment: North American Journal of Fisheries Management, v. 20, p. 41-52.

Vigg, S., Poe, T.P., Prendergast, L.A., and Hansel, H.C., 1991, Rates of consumption of juvenile salmonids and alternative prey fish by northern squawfish, walleyes, smallmouth bass, and channel catfish in John Day Reservoir, Columbia River: Transactions of the American Fisheries Society, v. 120, no. 4, p. 421-438.

von Bertalanffy, L., 1938, A quantitative theory of organic growth: Human Biology, v. 10, p. 181-213.

White, G.C., and Burnham, K.P., 1999, Program MARK—Survival estimation from populations of marked animals: Bird Study, v. 46 , p. $120-139$. 


\section{Figures}

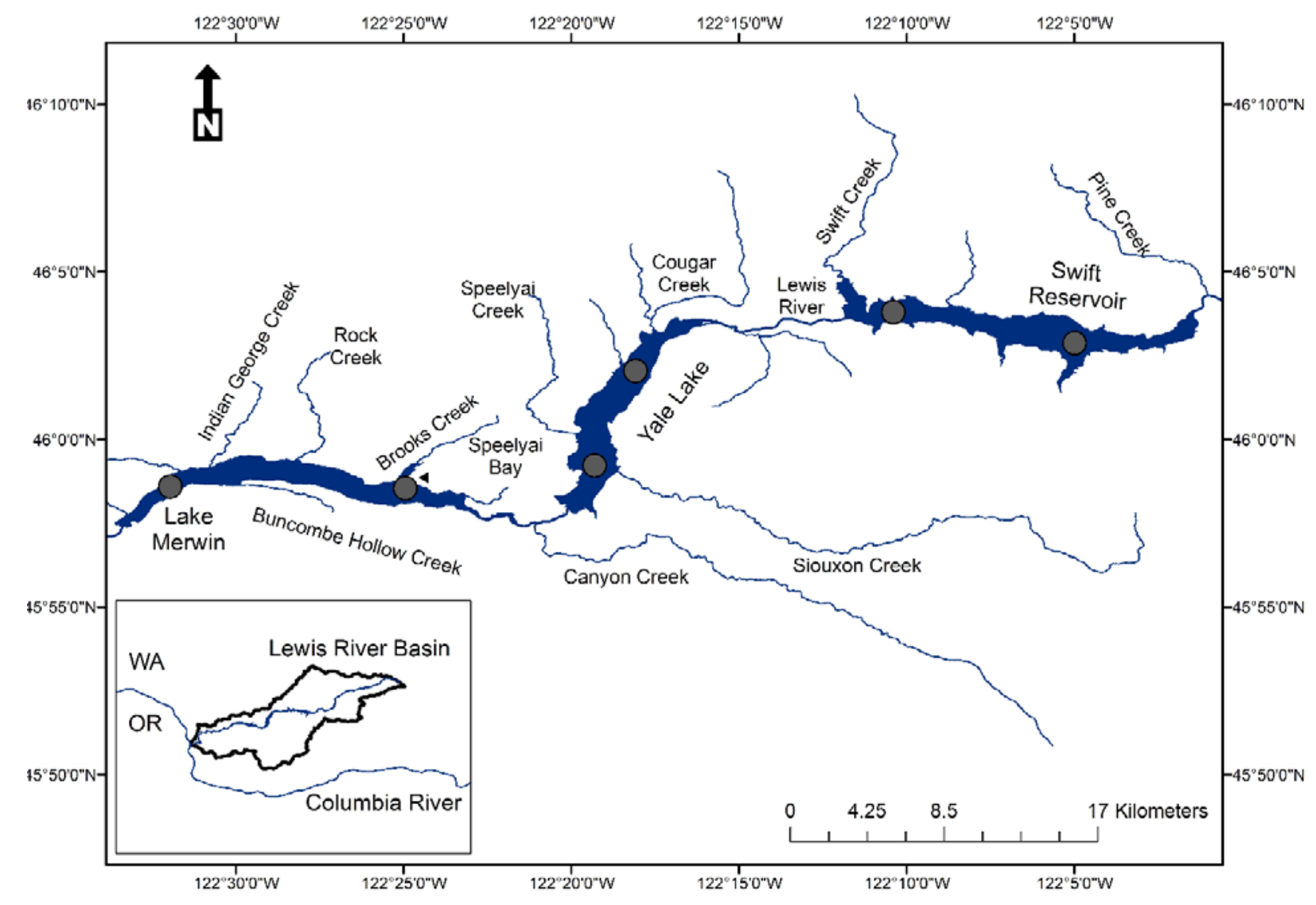

Figure E1. Study area map showing the reservoirs in the North Fork of the Lewis River, Washington, illustrating the location of limnology stations (gray circles) where temperature and zooplankton were sampled. Inset shows the location of the reservoirs and the Lewis River Basin in relation to the Columbia River in Washington (WA) and Oregon (OR). 


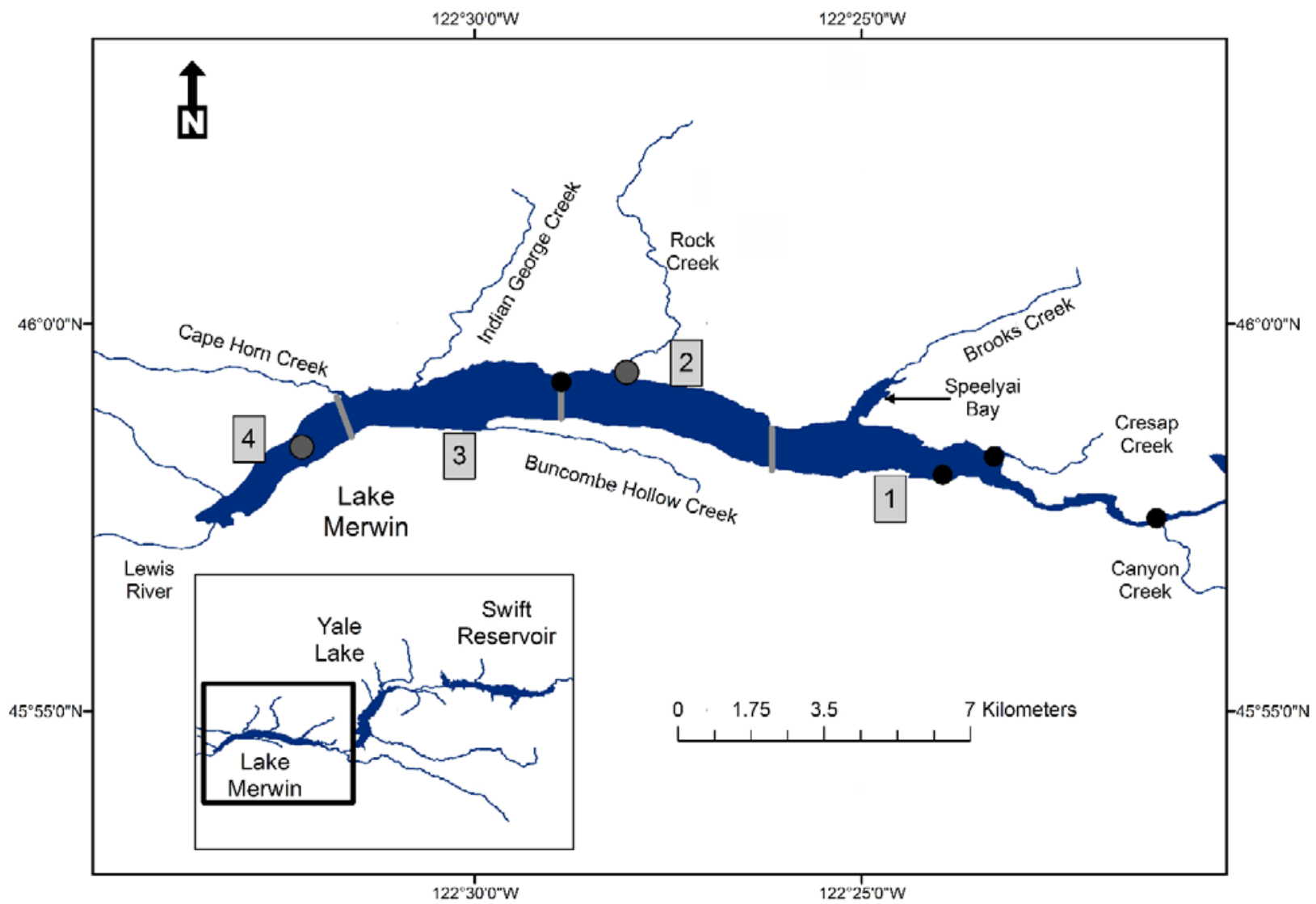

Figure E2. Limnological stations (gray circles) and Merwin trap locations (black circles) within Lake Merwin, Washington, with numbers categorizing the different quadrants. The reservoir was divided into quadrants based on geographic features such as points and bends and the quadrants are delineated by the light gray lines. Inset shows location of the Lake Merwin study area (in box) in the upper Lewis River. 


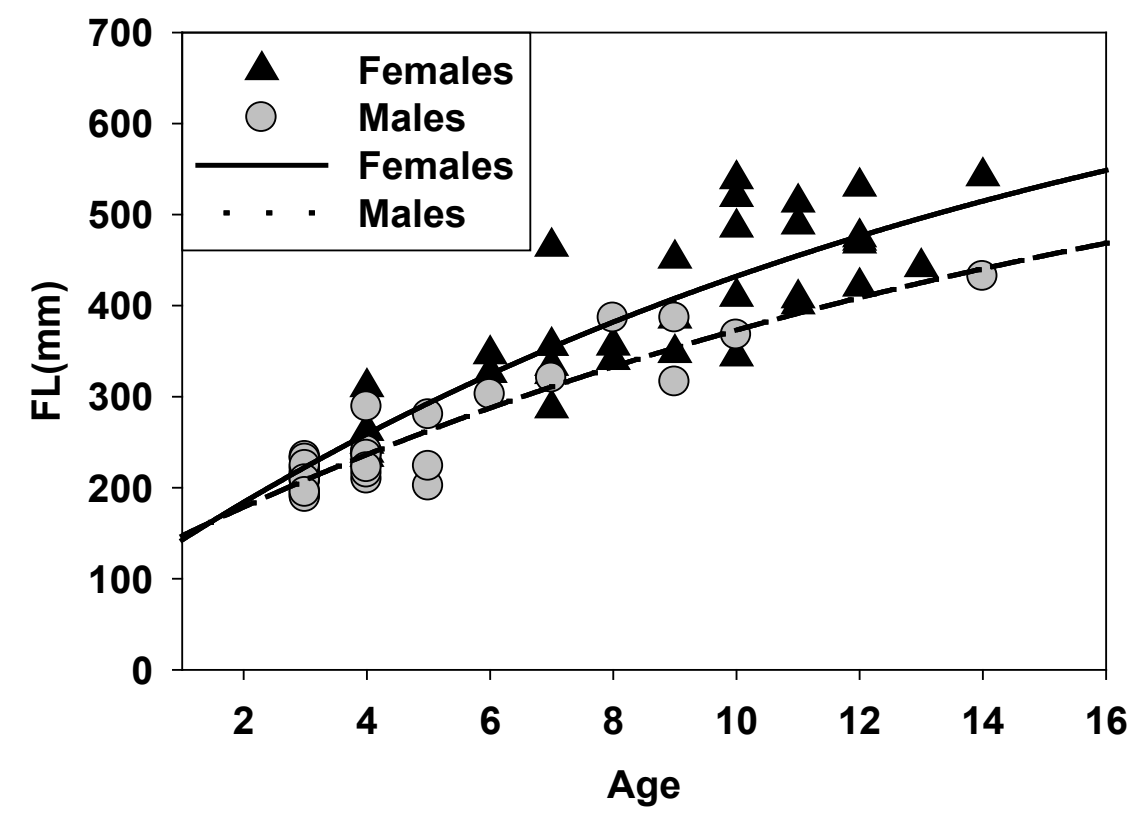

Figure E3. Length at age in years and von Bertalanffy growth curves for male (gray circles, dashed line) and female (black triangles, solid line) Ptychocheilus oregonensis (northern pikeminnow) in Lake Merwin, Washington for years 2013-2014. (FL, fork length; mm, millimeter)

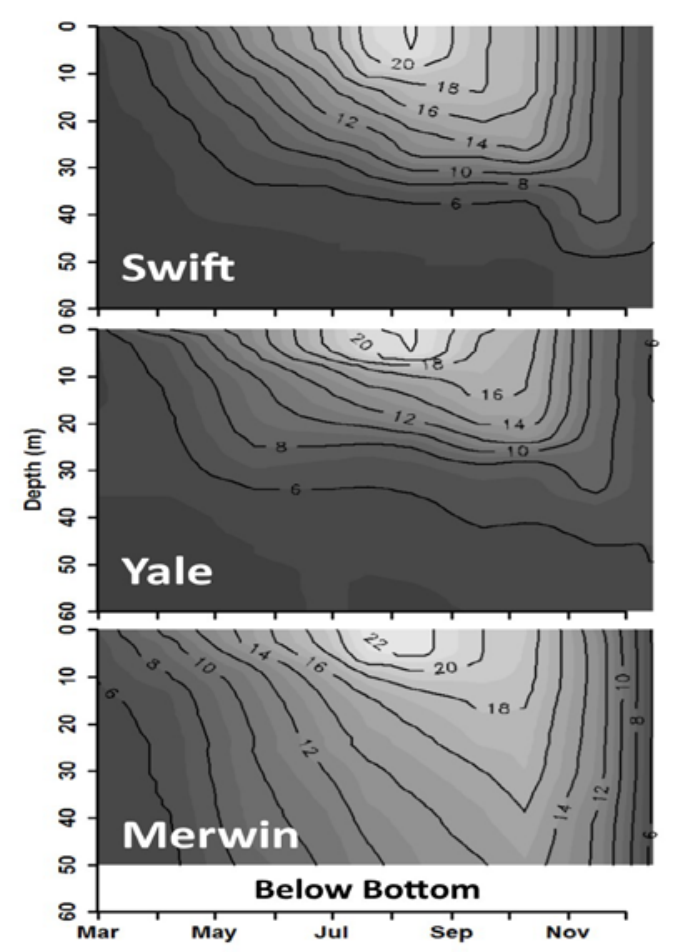

Figure E4. Isoclines of average water temperature (in degrees Celsius) from two stations one-third and two-thirds along the length of Swift Reservoir and Lake Merwin during April-0ctober 2014, and November 2014 in Yale Lake, Washington. In Swift Reservoir, a profile from November 1999 and a partial profile from November 2013 were combined, and profiles inferred from tailrace temperatures from February and December 2013 were incorporated to fill out the seasonal thermal regime. Supplementary profiles from the lower half of Yale Lake from December 1997 and February 1998, and a composite of a November 1999 and a partial profile from November 2013, and January and March 2000 from the lower half of Lake Merwin were also included. (m, meter) 


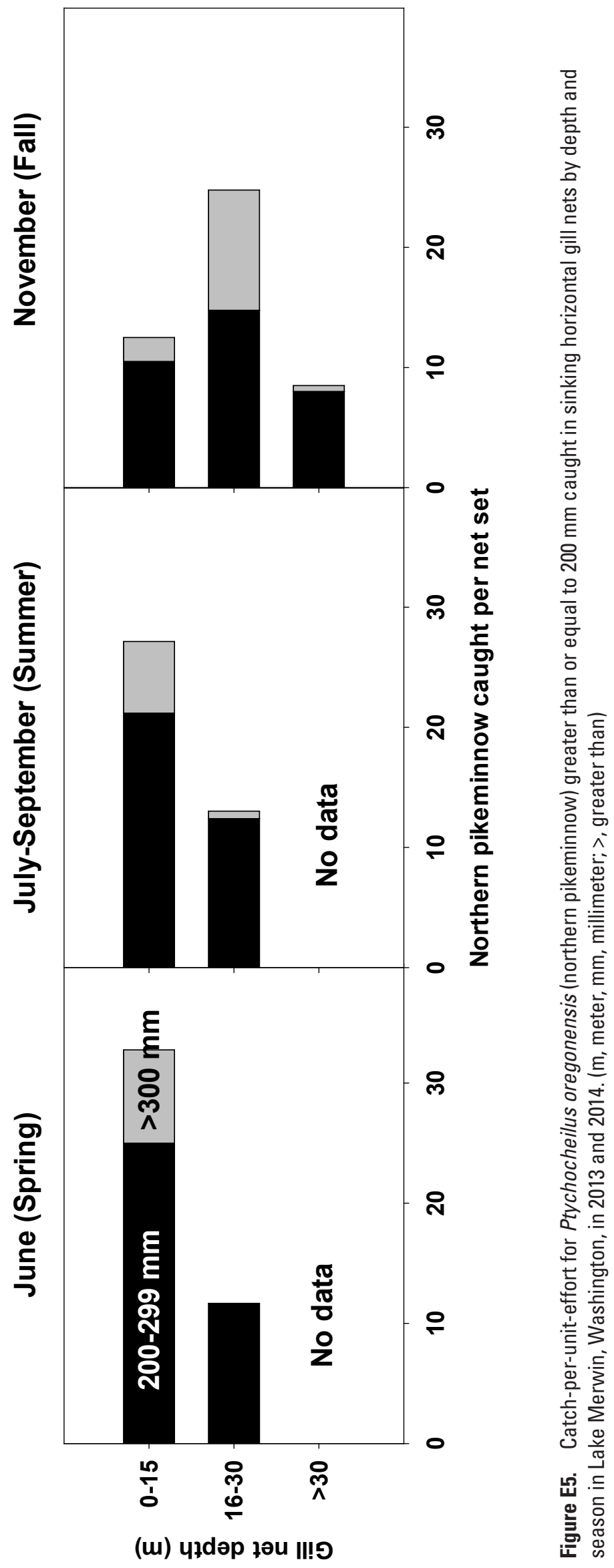




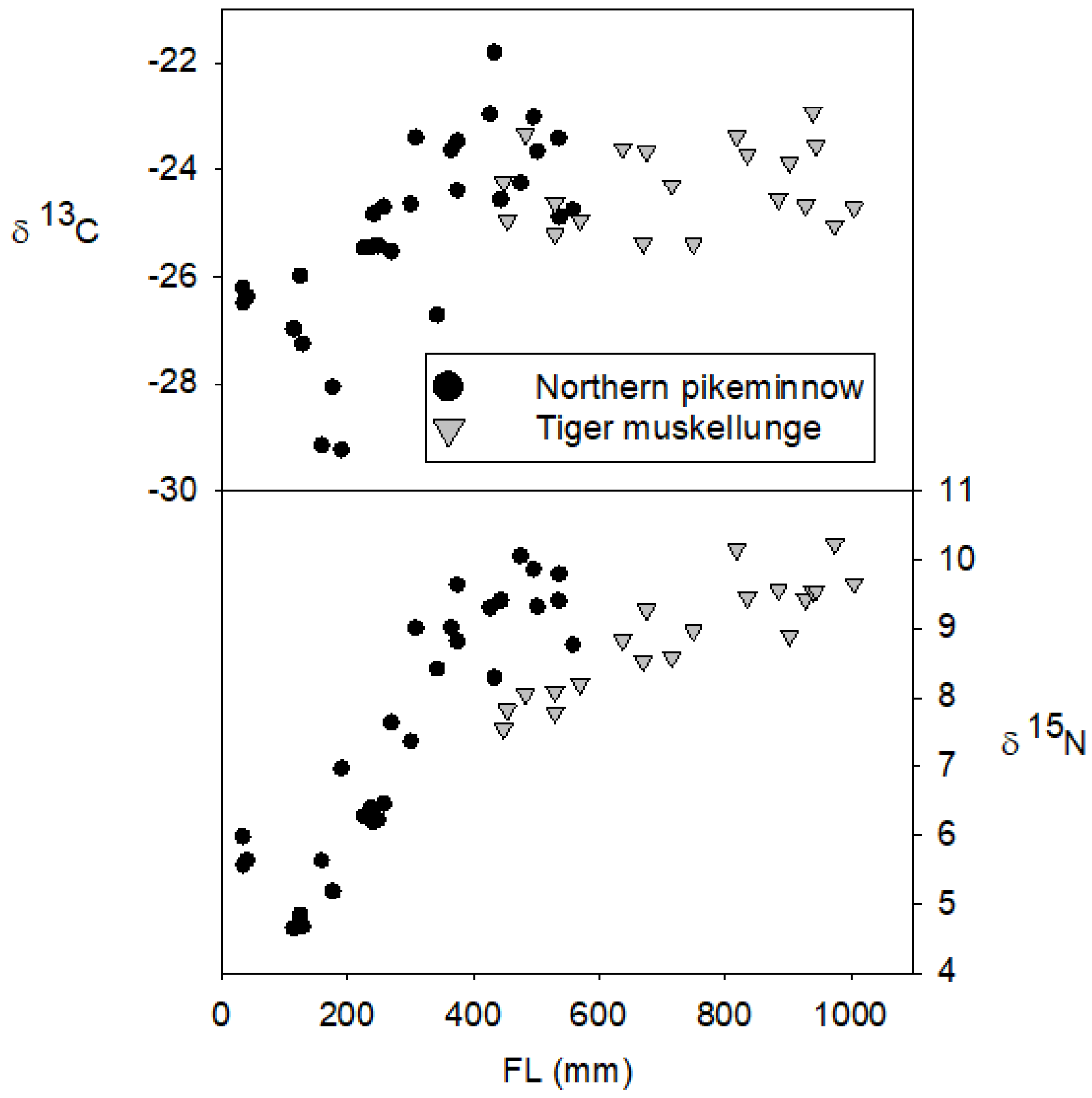

Figure E6. Stable isotope values of individual Ptychocheilus oregonensis (northern pikeminnow) and Esox lucius $\mathrm{x}$ masquinong (tiger muskellunge) as a function of fork length, Lake Merwin, Washington, for $2013-2014 .\left(\delta^{13} \mathrm{C}\right.$; delta c 13, ratio of carbon isotopes ${ }^{12} \mathrm{C}$ and ${ }^{13} \mathrm{C}$; $\delta^{15} \mathrm{~N}$, delta $\mathrm{n} 15$, ratio of nitrogen isotopes ${ }^{15} \mathrm{~N}$ and ${ }^{14} \mathrm{~N}$; FL, fork length; mm, millimeter) 


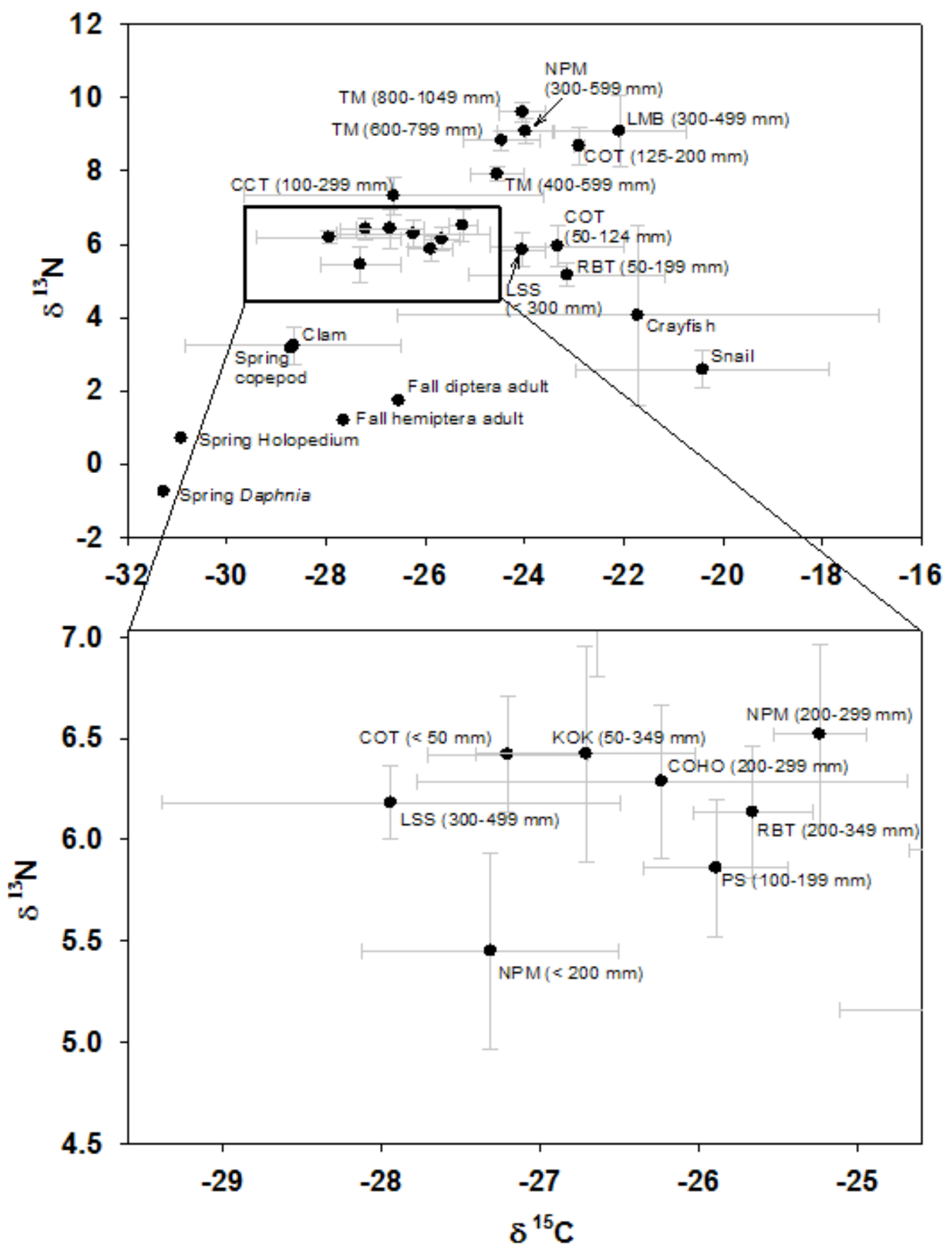

Figure E7. Average $\delta^{15} \mathrm{~N}$ and $\delta^{13} \mathrm{C}$ signatures (error bars=2 standard errors) for organisms sampled in Lake Merwin, Washington, in 2013-2014. $\left(\delta^{13} \mathrm{C}\right.$; delta c 13, ratio of carbon isotopes ${ }^{12} \mathrm{C}$ and ${ }^{13} \mathrm{C}$; NPM, Ptychocheilus oregonensis [northern pikeminnow]; TM, Esox lucius x masquinongy [tiger muskellunge]; LMB, Micropterus salmoides [largemouth bass]; COT, Cottus spp. [sculpin]; CCT, Oncorhynchus clarkii [coastal cutthroat trout]; RBT, Oncorhynchus mykiss [rainbow trout]; LSS, Catostomus macrocheilus [large-scale sucker]; crayfish, Pacifastacus leniusculus [signal crayfish]; <, less than; mm, millimeter; KOK, Oncorhynchus nerka [kokanee salmon]; $\mathrm{COHO}$, Oncorhynchus kisutch [coho Salmon]; PS, Lepomis gibbosus [pumpkinseed sunfish]; $\delta^{15} \mathrm{~N}$, delta $\mathrm{n} 15$, ratio of nitrogen isotopes ${ }^{15} \mathrm{~N}$ and $\left.{ }^{14} \mathrm{~N}\right)$ 


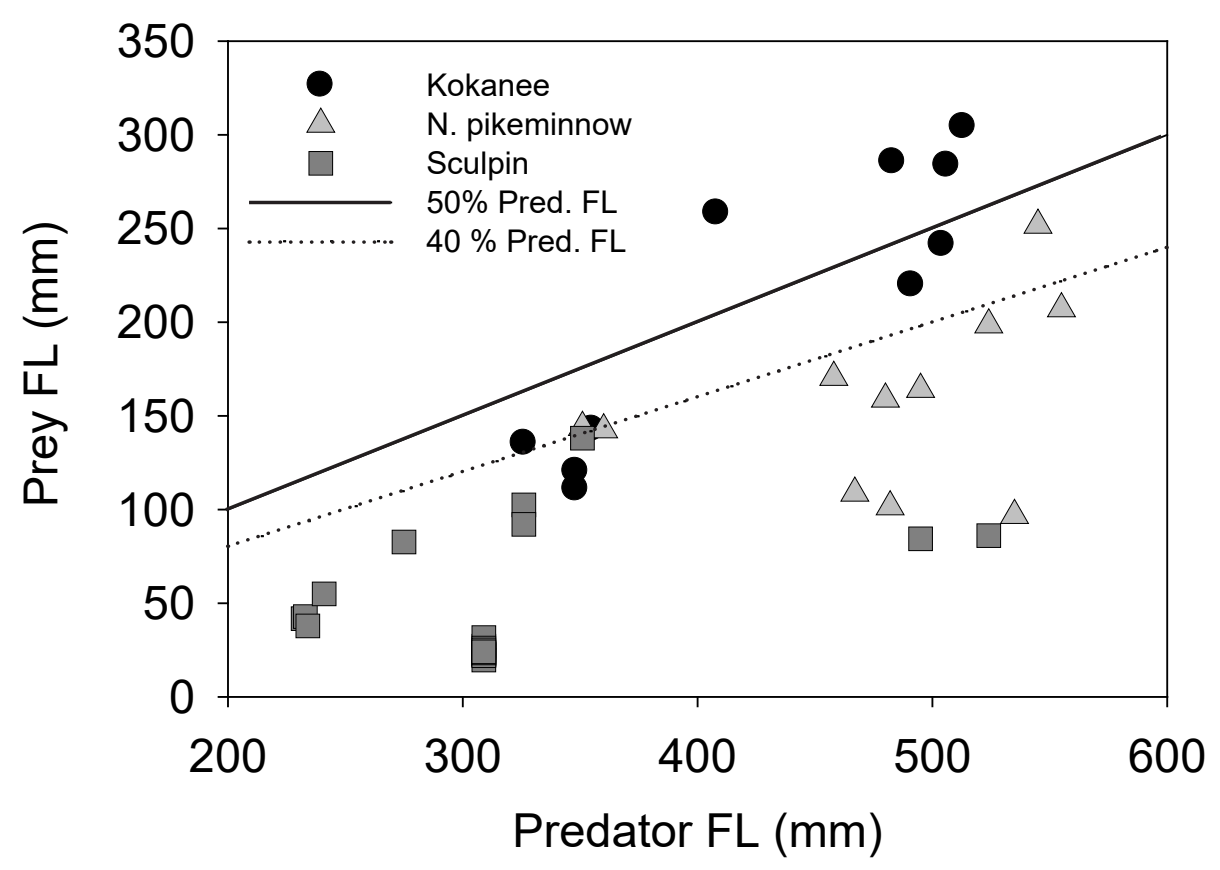

Figure E8. The relationship between Ptychocheilus oregonensis (northern pikeminnow) fork length and prey fish fork length for 2013-2014, Lake Merwin, Washington. (FL, fork length; mm, millimeter; Kokanee, Oncorhynchus nerka [kokanee salmon]; sculpin, Cottus spp. [signal crayfish]; \%, percent; Pred., predator)

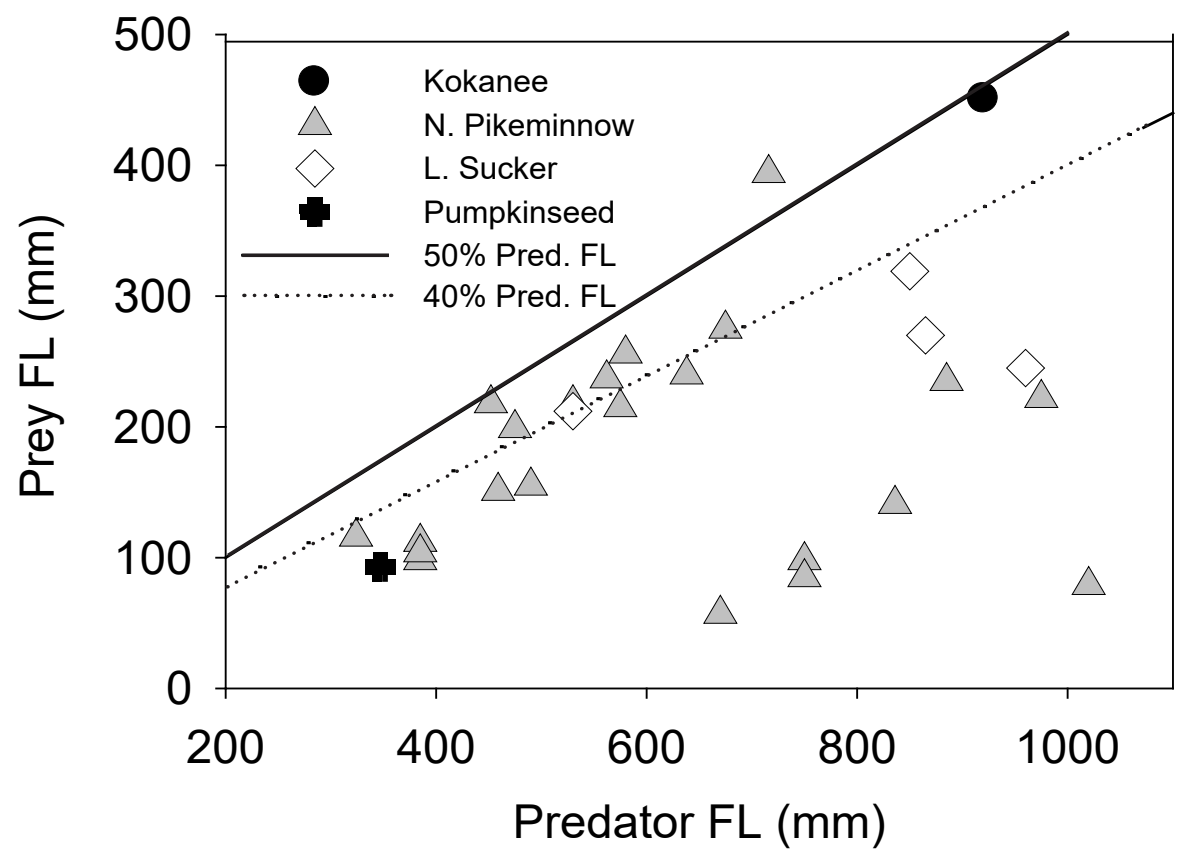

Figure E9. The relationship between Esox lucius x masquinongy (tiger muskellunge's) fork length and prey fish's fork length for 2013-2014, Lake Merwin, Washington. (Kokanee, Oncorhynchus nerka [kokanee salmon]; N. Pikeminnow, Ptychocheilus oregonensis [northern pikeminnow]; L. Sucker, Catostomus macrocheilus [largescale suckers]; Pumpkinseed, Lepomis gibbosus [pumpkinseed sunfish]; mm, millimeter; \%. percent; FL, fork length) 


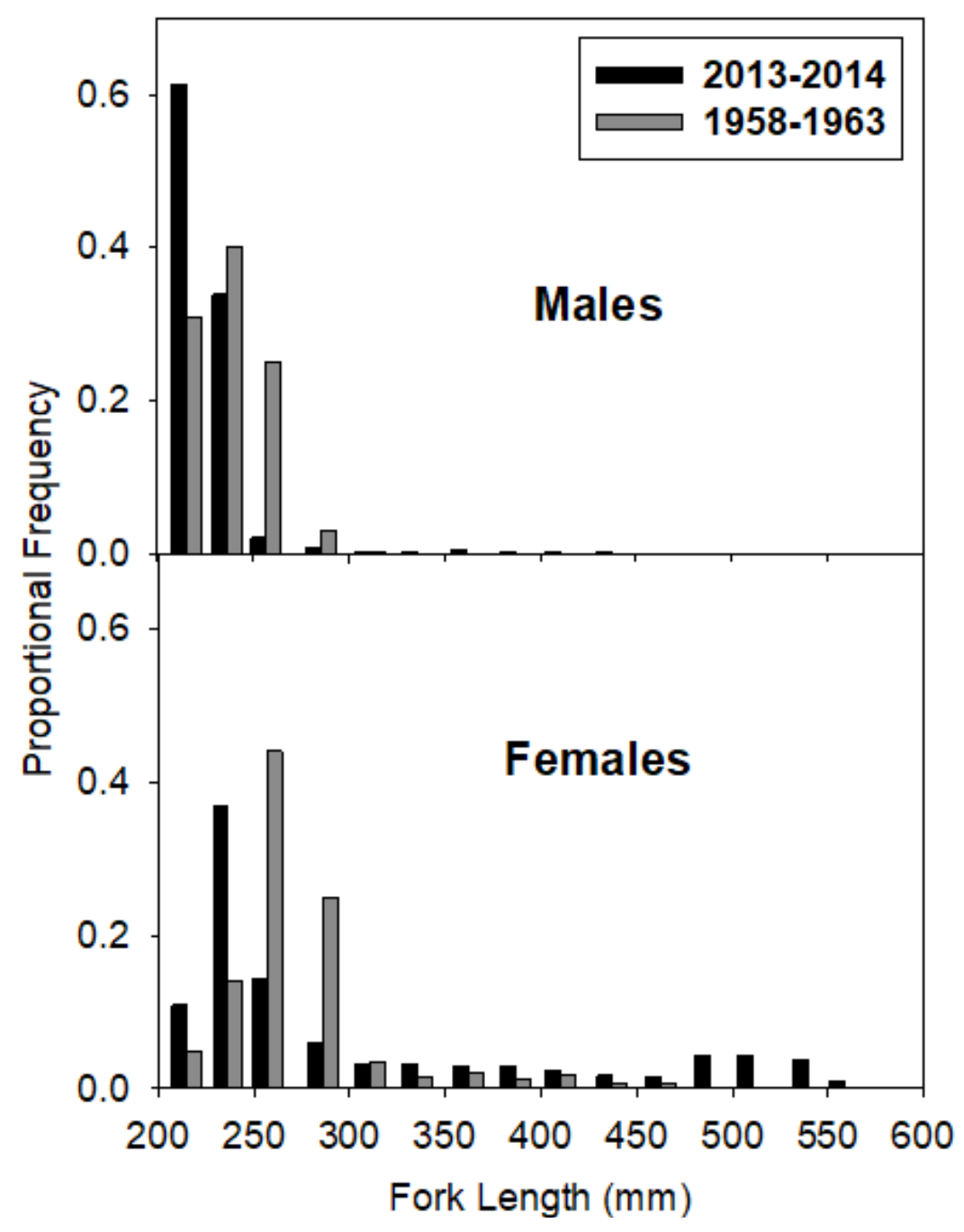

Figure E10. Length frequency distributions of male and female Ptychocheilus oregonensis (northern pikeminnow) captured in gill nets and traps in Lake Merwin, Washington, between 2013-2014 and 1958-1963 (Hamilton and others, 1970). The contemporary gill netting included larger mesh sizes than the historical data, which were included in the 2013-2014 distribution to best characterize the size distribution of the population. Efforts were made to standardize the length frequencies by including only fish captured in similar areas. (FL, fork length, mm, millimeters) 


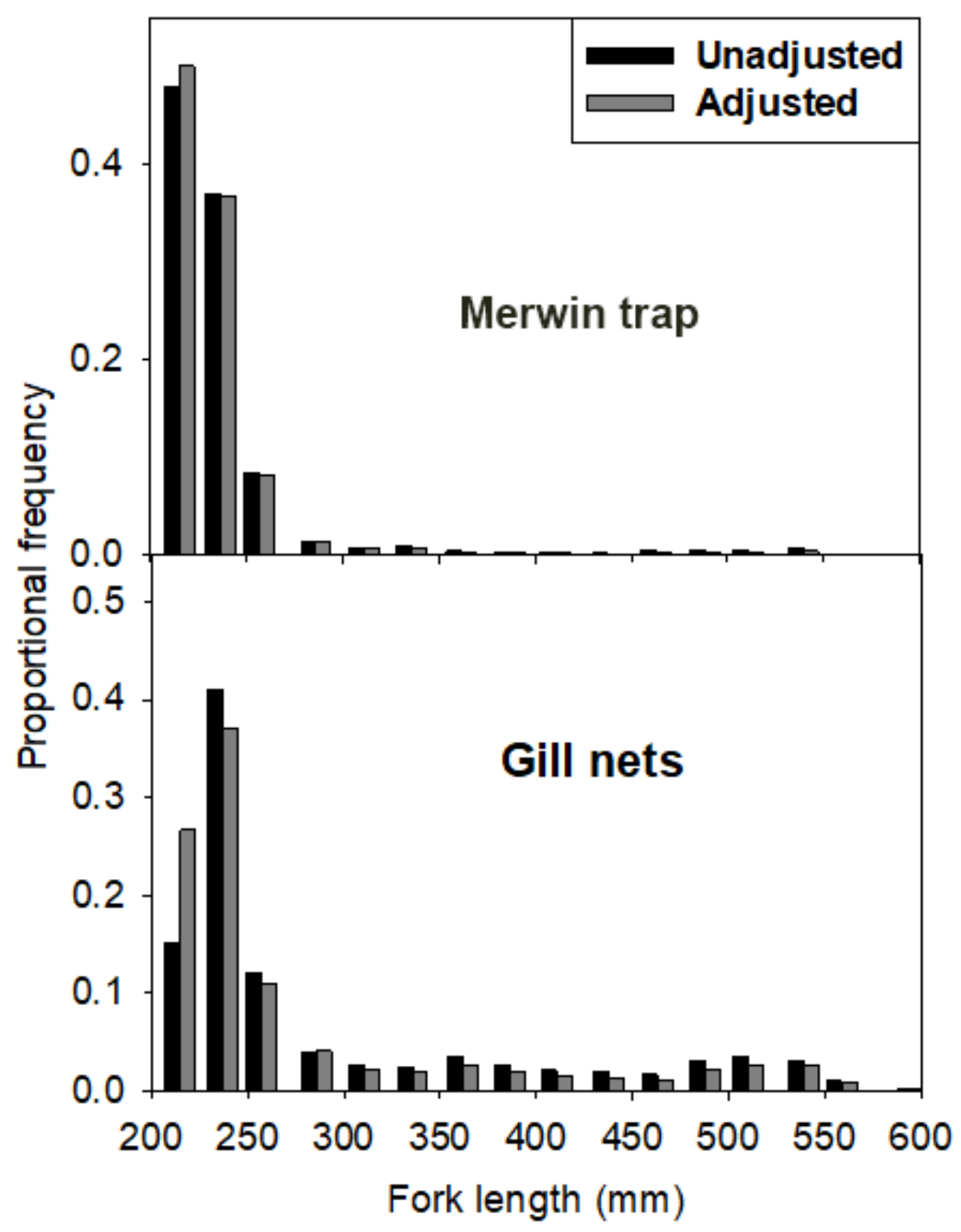

Figure E11. Length frequency histograms from raw catch data and data adjusted for size selectivity, Lake Merwin, Washington, from 2013-2014. The top panel shows Merwin trap data which was adjusted for size-selective encounter rate only, and the bottom panel shows gill net data that was adjusted for size selectivity of gill-net mesh sizes as well as encounter rate. Adjusted and unadjusted size distributions were not statistically significant for both gear types. (FL, fork length, mm, millimeters) 


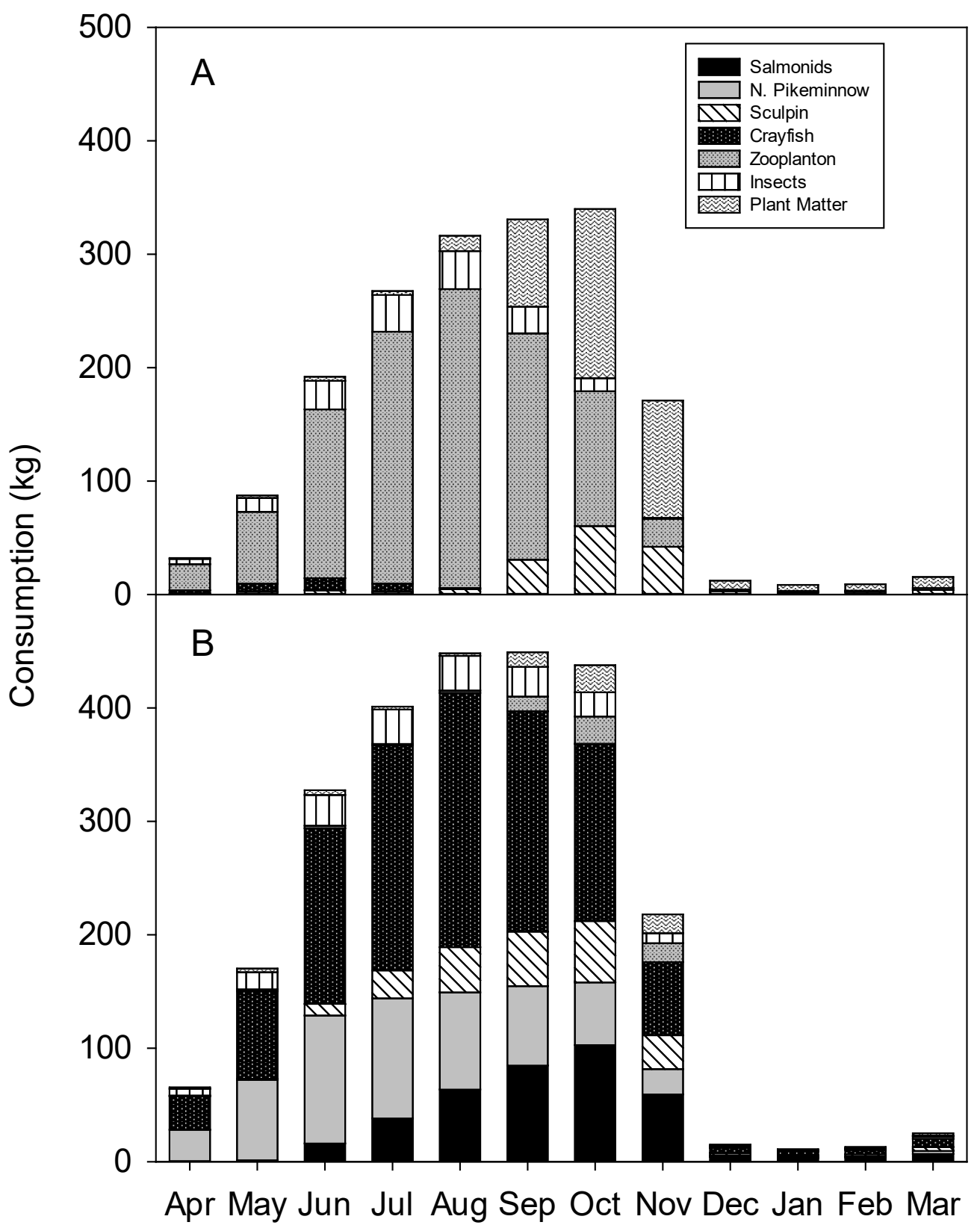

Figure E12. Estimates from bioenergetics simulations of monthly biomass of prey consumed by size-structured populations of 1,000 Ptychocheilus oregonensis (northern pikeminnow): $A, 200-299$ millimeters $(\mathrm{mm})$ and $B$, greater than or equal to $300 \mathrm{~mm}$, Lake Merwin, Washington. (kg, kilogram; sculpin, Cottus species; Crayfish, Pacifastacus leniusculus [signal crayfish]) 


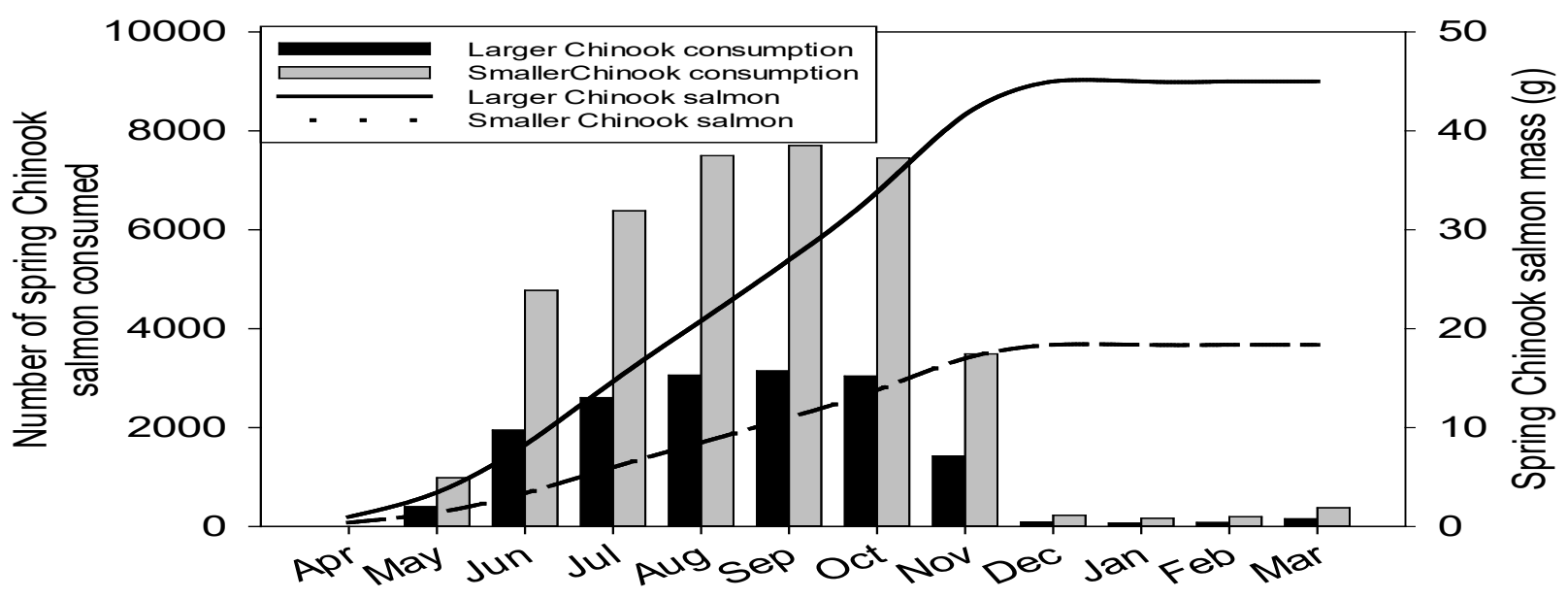

Figure E13. Estimate of potential number of subyearling spring Oncorhynchus tshawytscha (Chinook salmon) that could be consumed monthly by a size-structured population unit of 1,000 large Ptychocheilus oregonensis (northern pikeminnow), and the expected monthly masses of reservoir-rearing subyearling Chinook salmon (shown as lines and secondary y-axis), Lake Merwin, Washington, for year(s). (g, gram)

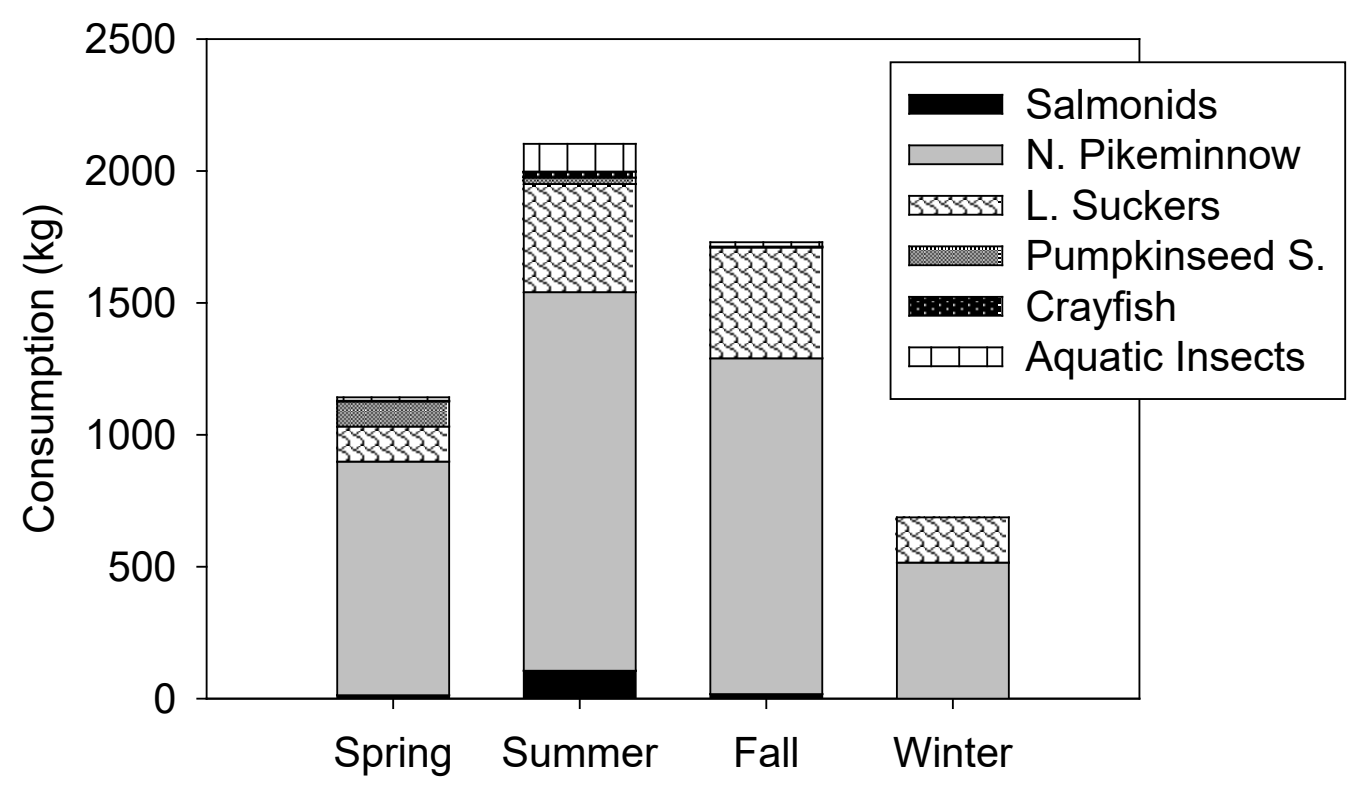

Figure E14. Estimates from bioenergetics simulations of seasonal biomass of prey consumed by an age-structured population of 5,488 Esox lucius x masquinongy (tiger muskellunge), Lake Merwin, Washington. (kg, kilogram; L. Suckers, Catostomus macrocheilus [largescale suckers]; Pumpkinseed S., Lepomis gibbosus [pumpkinseed sunfish]; Crayfish, Pacifastacus leniusculus [signal crayfish]) 


\section{Tables}

Table E1. Limnological and morphometric characteristics of Lake Merwin, Washington (PacifiCorp and Cowlitz Public Utility District, 2004; Sorel and others, 2016).

$\left[\mathrm{km}^{2}\right.$, square kilometer; km, kilometer; m, meter; $\sim$, approximately; mg/L, milligram per liter; <, less than; ${ }^{\circ} \mathrm{C}$, degree Celsius; msl, mean sea level]

\begin{tabular}{|l|c|}
\hline \multicolumn{1}{|c|}{ Attribute } & Estimate \\
\hline Surface area $\left(\mathrm{km}^{2}\right)$ & 15.7 \\
\hline Length $(\mathrm{km})$ & 23.3 \\
\hline Mean depth $(\mathrm{m})$ & 31 \\
\hline Maximum depth $(\mathrm{m})$ & 73 \\
\hline Mean Secchi depth transparency $(\mathrm{m})$ & $\sim 5$ \\
\hline Water residence time $($ days $)$ & 118.4 \\
\hline Total phosphorus $(\mathrm{mg} / \mathrm{L})$ & $<.02$ \\
\hline Trophic status & Oligotrophic \\
\hline Months stratified & April-Sept. \\
\hline Depth of thermocline $(\mathrm{m})$ & $\sim 8$ \\
\hline Ice cover & None \\
\hline Range of surface temps $\left({ }^{\circ} \mathrm{C}\right)$ & $23-5$ \\
\hline Maximum surface elevation $(\mathrm{m} \mathrm{msl})$ & 73 \\
\hline
\end{tabular}

Table E2. Relative abundance of fish species present in Lake Merwin, Washington, based on field sampling.

\begin{tabular}{|l|l|l|}
\hline \multicolumn{1}{|c|}{ Common name } & \multicolumn{1}{c|}{ Scientific name } & \multicolumn{1}{c|}{$\begin{array}{c}\text { Relative } \\
\text { abundance }\end{array}$} \\
\hline Kokanee salmon & Oncorhynchus nerka & High* \\
\hline Rainbow/steelhead trout & Oncorhynchus mykiss & Moderate* \\
\hline Bull trout & Salvelinus confluentus & Rare \\
\hline Cutthroat trout & Oncorhynchus clarkii & Rare \\
\hline Juvenile coho salmon & Oncorhynchus kisutch & Rare* \\
\hline Juvenile Chinook salmon & Oncorhynchus tshawytscha & Rare* \\
\hline Northern pikeminnow & Ptychocheilus oregonensis & High \\
\hline Largescale suckers & Catostomus macrocheilus & High \\
\hline Sculpin & Cottus spp. & High \\
\hline Threespine stickleback & Gasterosteus aculeatus & Rare \\
\hline Tiger muskellunge & Esox lucius x masquinongy & Moderate* \\
\hline Pumpkinseed sunfish & Lepomis gibbosus & High \\
\hline Largemouth bass & Micropterus salmoides & Rare \\
\hline
\end{tabular}

*Hatchery supplemented population. 
Table E3. Abundance estimates of Ptychocheilus oregonensis (northern pikeminnow) by size class in Lake Merwin, Washington for 2013.

[\%, percent; AICc, Akaike Information Criteria corrected for small sample size; $\hat{\mathrm{N}}$, abundance estimate; PJS, POPAN formulation of the Jolly-Seber model; Y, yes; N, no; LP, Chapman estimator for the Lincoln-Peterson model; NA, not applicable]

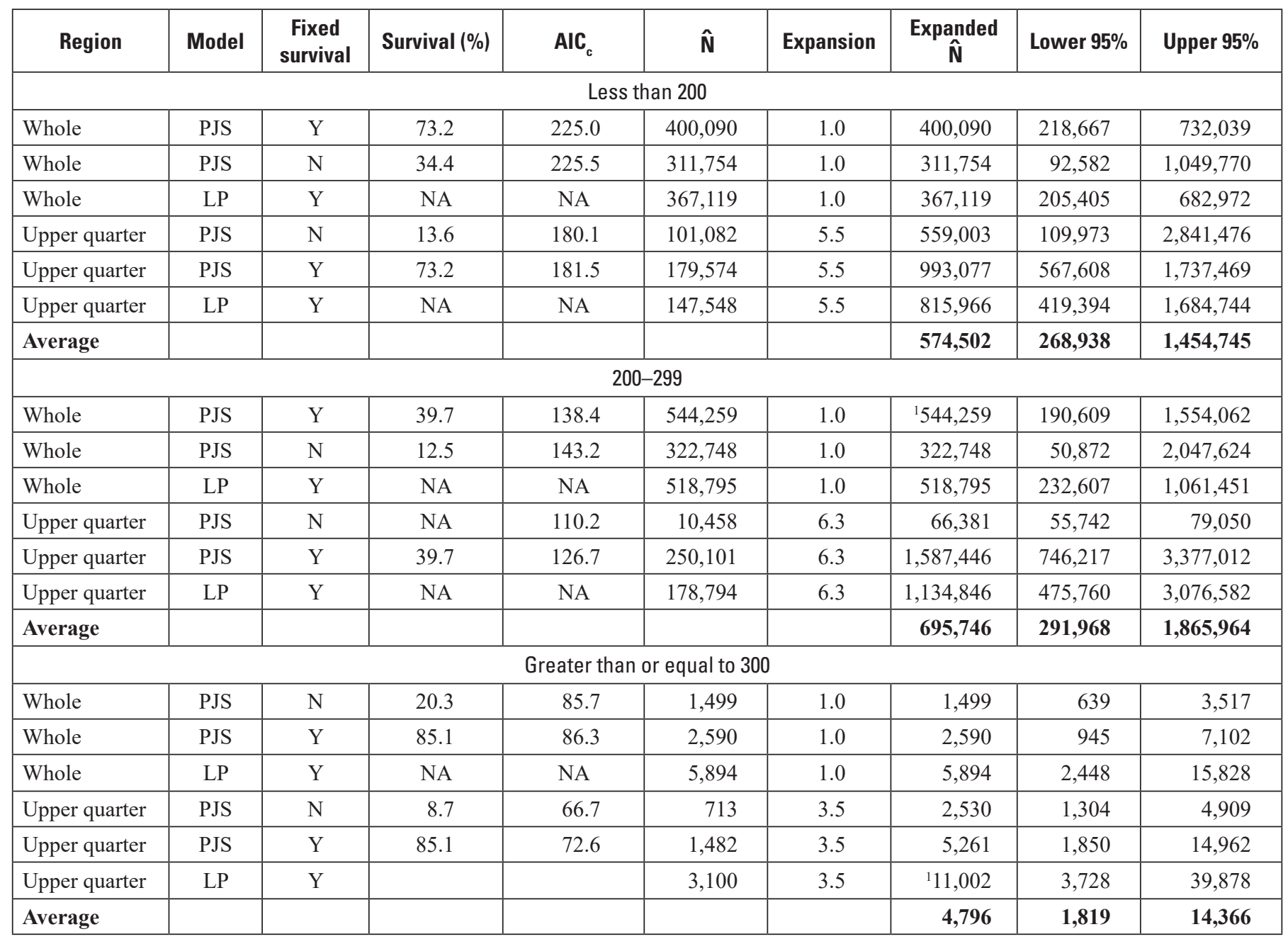

${ }^{1}$ Best estimate. 
Table E4. Thermal experiences of Ptychocheilus oregonensis (northern pikeminnow) and Esox lucius $\mathrm{x}$ masquinongy (tiger muskellunge) in Lake Merwin, Washington.

$\left[{ }^{\circ} \mathrm{C}\right.$, degree Celsius; $\mathrm{mm}$, millimeter; $\geq$, greater than or equal to $]$

\begin{tabular}{|c|c|c|c|c|}
\hline \multirow{2}{*}{ Date } & \multirow{2}{*}{ Simulation day } & \multicolumn{3}{|c|}{ Thermal experience $\left({ }^{\circ} \mathrm{C}\right)$} \\
\hline & & $200-299 \mathrm{~mm}$ & $\geq 300 \mathrm{~mm}$ & Tiger muskellunge \\
\hline 1-April & 1 & 7.7 & 8.3 & 8.3 \\
\hline 15-May & 45 & 11.5 & 12.3 & 12.3 \\
\hline 30-June & 89 & 15.6 & 16.7 & 16.7 \\
\hline 19-July & 109 & 17.8 & 19.1 & 19.1 \\
\hline 15-August & 135 & 18.8 & 20.1 & 20.1 \\
\hline 18-September & 168 & 18.6 & 19.3 & 19.3 \\
\hline 10-October & 190 & 17.5 & 17.6 & 17.6 \\
\hline 18-November & 228 & 12.5 & 12.7 & 12.7 \\
\hline 15-December & 255 & 5.8 & 5.8 & 5.8 \\
\hline 1-January & 270 & 5.5 & 5.5 & 5.5 \\
\hline 1-March & 330 & 6 & 6.3 & 6.3 \\
\hline 31-March & 365 & 7.7 & 8.3 & 8.3 \\
\hline
\end{tabular}

Table E5. Energy density (joules/gram) of prey items of Ptychocheilus oregonensis (northern pikeminnow) and Esox lucius $\mathrm{x}$ masquinongy (tiger muskellunge) in Lake Merwin, Washington.

[ZOOP, zooplankton; TERR, terrestrial insects; Inverts, aquatic invertebrates; Sal, salmonids; COT, Cottus spp.(sculpin); NPM, northern pikeminnow; LSS, Catostomus macrocheilus (largescale sucker), PUM, Lepomis gibbosus (pumpkinseed sunfish); CRAY, Pacifastacus leniusculus (signal crayfish); Plant, miscellaneous vegetation]

\begin{tabular}{|c|c|c|c|c|c|c|c|c|c|c|}
\hline Day & ZOOP & TERR & Inverts & Sal & COT & NPM & LSS & PUM & CRAY & Plant \\
\hline 1 & $1950^{\mathrm{a}}$ & 5000 & $3400^{\mathrm{b}}$ & 5200 & $4305^{\mathrm{c}}$ & $6703^{\mathrm{d}}$ & 3641 & 4186 & $3318^{\mathrm{c}}$ & 1435 \\
\hline 365 & 1950 & 5000 & 3400 & 5200 & 4305 & 6703 & 3641 & 4186 & 3318 & 1435 \\
\hline
\end{tabular}

${ }^{\text {aS }}$ Sorel and others, 2016.

bHansen and others, 1997.

'Mazur, 2004.

dPetersen and Ward, 1999. 
Table E6. Bioenergetics inputs of growth and spawning losses and output of percent maximum consumption and total Oncorhynchus nerka (Kokanee salmon), Ptychocheilus oregonensis (northern pikeminnow), Cottus spp. (sculpins); and Pacifastacus leniusculus (signal crayfish) annual consumption by individual male and female northern pikeminnow age 3-14 years, Lake Merwin, Washington. Spawning losses were 3.9 percent of body mass for males age 3-14 years, 6 percent for females age 3 years, and 8.9 percent for females age 4-14 years.

[mm, millimeter; g, gram; \%, percent; $\% \mathrm{C}_{\max }$, percent maximum consumption]

\begin{tabular}{|c|c|c|c|c|c|c|c|c|c|}
\hline \multirow{2}{*}{$\begin{array}{c}\text { Age } \\
\text { (years) }\end{array}$} & \multirow{2}{*}{$\begin{array}{l}\text { Initial fork } \\
\text { length }(\mathrm{mm})\end{array}$} & \multirow{2}{*}{$\begin{array}{c}\text { Initial } \\
\text { weight }(g)\end{array}$} & \multirow{2}{*}{$\begin{array}{c}\text { Final } \\
\text { weight }(g)\end{array}$} & \multirow[b]{2}{*}{$\% C_{\max }$} & \multicolumn{5}{|c|}{ Annual consumption (g) } \\
\hline & & & & & Total & Kokanee & $\begin{array}{c}\text { Northern } \\
\text { pikeminnow }\end{array}$ & Sculpins & $\begin{array}{c}\text { Signal } \\
\text { crayfish }\end{array}$ \\
\hline \multicolumn{10}{|c|}{ Males } \\
\hline 3 & 208 & 94 & 137 & 60 & 1,290 & 0 & 0 & 90 & 21 \\
\hline 4 & 236 & 137 & 188 & 57 & 1,622 & 0 & 0 & 116 & 26 \\
\hline 5 & 263 & 188 & 258 & 57 & 2,060 & 0 & 0 & 144 & 34 \\
\hline 6 & 288 & 258 & 335 & 55 & 2,480 & 0 & 0 & 182 & 42 \\
\hline 7 & 311 & 335 & 421 & 25 & 1,481 & 0 & 0 & 215 & 640 \\
\hline 8 & 333 & 421 & 515 & 24 & 1,708 & 223 & 316 & 126 & 739 \\
\hline 9 & 354 & 515 & 617 & 23 & 1,939 & 256 & 366 & 144 & 839 \\
\hline 10 & 373 & 617 & 724 & 23 & 2,170 & 289 & 417 & 163 & 940 \\
\hline 11 & 392 & 724 & 837 & 23 & 2,401 & 323 & 468 & 182 & 1,041 \\
\hline 12 & 409 & 837 & 954 & 22 & 2,630 & 356 & 519 & 201 & 1,140 \\
\hline 13 & 425 & 954 & 1,075 & 22 & 2,856 & 389 & 570 & 219 & 1,239 \\
\hline 14 & 440 & 1,075 & 1,197 & 22 & 3,078 & 421 & 620 & 238 & 1,336 \\
\hline \multicolumn{10}{|c|}{ Females } \\
\hline 3 & 223 & 115 & 180 & 62 & 1,602 & 0 & 0 & 107 & 25 \\
\hline 4 & 259 & 180 & 259 & 60 & 2,107 & 0 & 0 & 146 & 34 \\
\hline 5 & 293 & 259 & 387 & 28 & 1,456 & 0 & 0 & 189 & 625 \\
\hline 6 & 325 & 387 & 518 & 26 & 1,784 & 224 & 305 & 126 & 768 \\
\hline 7 & 354 & 518 & 667 & 25 & 2,139 & 271 & 379 & 152 & 923 \\
\hline 8 & 382 & 667 & 831 & 25 & 2,504 & 322 & 457 & 181 & 1,082 \\
\hline 9 & 408 & 831 & 1,009 & 24 & 2,873 & 375 & 537 & 211 & 1,243 \\
\hline 10 & 432 & 1,009 & 1,198 & 23 & 3,244 & 428 & 619 & 241 & 1,404 \\
\hline 11 & 455 & 1,198 & 1,396 & 23 & 3,614 & 482 & 701 & 272 & 1,565 \\
\hline 12 & 476 & 1,396 & 1,601 & 23 & 3,978 & 535 & 783 & 302 & 1,724 \\
\hline 13 & 496 & 1,601 & 1,811 & 22 & 4,337 & 587 & 864 & 331 & 1,880 \\
\hline 14 & 515 & 1,811 & 2,025 & 22 & 4,687 & 639 & 944 & 360 & 2,033 \\
\hline
\end{tabular}


Table E7. Bioenergetics inputs of growth-at-age and outputs of percent maximum consumption $\left(\% \mathrm{C}_{\max }\right)$, total consumption, and growth efficiency by individual Esox lucius x masquinongy (tiger muskellunge) aged 1-10 years, Lake Merwin, Washington.

[mm, millimeter; g, gram; $\% \mathrm{C}_{\max }$, percent maximum consumption; \%, percent]

\begin{tabular}{|c|c|c|c|c|c|c|c|}
\hline $\begin{array}{c}\text { Age } \\
\text { (years) }\end{array}$ & $\begin{array}{c}\text { Initial fork } \\
\text { length (mm) }\end{array}$ & $\begin{array}{c}\text { Initial } \\
\text { weight } \\
\mathbf{( g )}\end{array}$ & $\begin{array}{c}\text { Final } \\
\mathbf{w e i g h t} \\
\mathbf{( g )}\end{array}$ & $\begin{array}{c}\text { Growth } \\
\mathbf{( g )}\end{array}$ & $\mathbf{\%}_{\text {max }}$ & $\begin{array}{c}\text { Total } \\
\text { consumption } \\
\text { (g) }\end{array}$ & $\begin{array}{c}\text { Growth } \\
\text { efficiency } \\
\text { (\%) }\end{array}$ \\
\hline 1 & 316 & 184 & 1,116 & 932 & 48.85 & 2,213 & 42.13 \\
\hline 2 & 531 & 1,116 & 2,592 & 1,475 & 40.28 & 4,772 & 30.92 \\
\hline 3 & 676 & 2,592 & 4,159 & 1,568 & 35.74 & 7,049 & 22.24 \\
\hline 4 & 774 & 4,159 & 5,545 & 1,385 & 33.01 & 8,798 & 15.74 \\
\hline 5 & 841 & 5,545 & 6,652 & 1,107 & 31.29 & 10,060 & 11.01 \\
\hline 6 & 886 & 6,652 & 7,486 & 834 & 30.18 & 10,944 & 7.62 \\
\hline 7 & 917 & 7,486 & 8,092 & 606 & 29.44 & 11,553 & 5.24 \\
\hline 8 & 938 & 8,092 & 8,521 & 430 & 28.95 & 11,971 & 3.59 \\
\hline 9 & 952 & 8,521 & 8,821 & 300 & 28.62 & 12,255 & 2.45 \\
\hline 10 & 961 & 8,821 & 9,029 & 208 & 28.40 & 12,448 & 1.67 \\
\hline
\end{tabular}

Table E8. Mean $\delta^{15} \mathrm{~N}$ and $\delta^{13} \mathrm{C}$ stable isotope values for fish and invertebrates sampled in the Lake Merwin, Yale Lake, and Swift Reservoir of the Lewis River, Washington, by location and size class for years 2013-2014.

$\left[\mathrm{mm}\right.$, millimeter; n, number of samples; $\delta^{15} \mathrm{~N}$, delta $\mathrm{n} 15$, ratio of nitrogen isotopes ${ }^{15} \mathrm{~N}$ and ${ }^{14} \mathrm{~N} ; \delta^{13} \mathrm{C}$; delta c 13 , ratio of carbon isotopes ${ }^{12} \mathrm{C}$ and ${ }^{13} \mathrm{C}$; PS, pumpkinseed sunfish (Lepomis gibbosu); NA, not applicable; Coho, Oncorhynchus kisutch (coho salmon); sculpin, Cottus spp.; <, less than; CCT, O. clarkii (coastal cutthroat trout); RBT, Os. mykiss (rainbow trout); kokanee, O. nerka (kokanee salmon); LMB, Micropterus salmoides (largemouth bass); LSS, Catostomus macrocheilus (largescale sucker); NPM, Ptychocheilus oregonensis (northern pikeminnow); tiger muskie, Esox lucius x masquinongy (tiger muskellunge); SB, Gasterosteus aculeatus (threespine stickleback)]

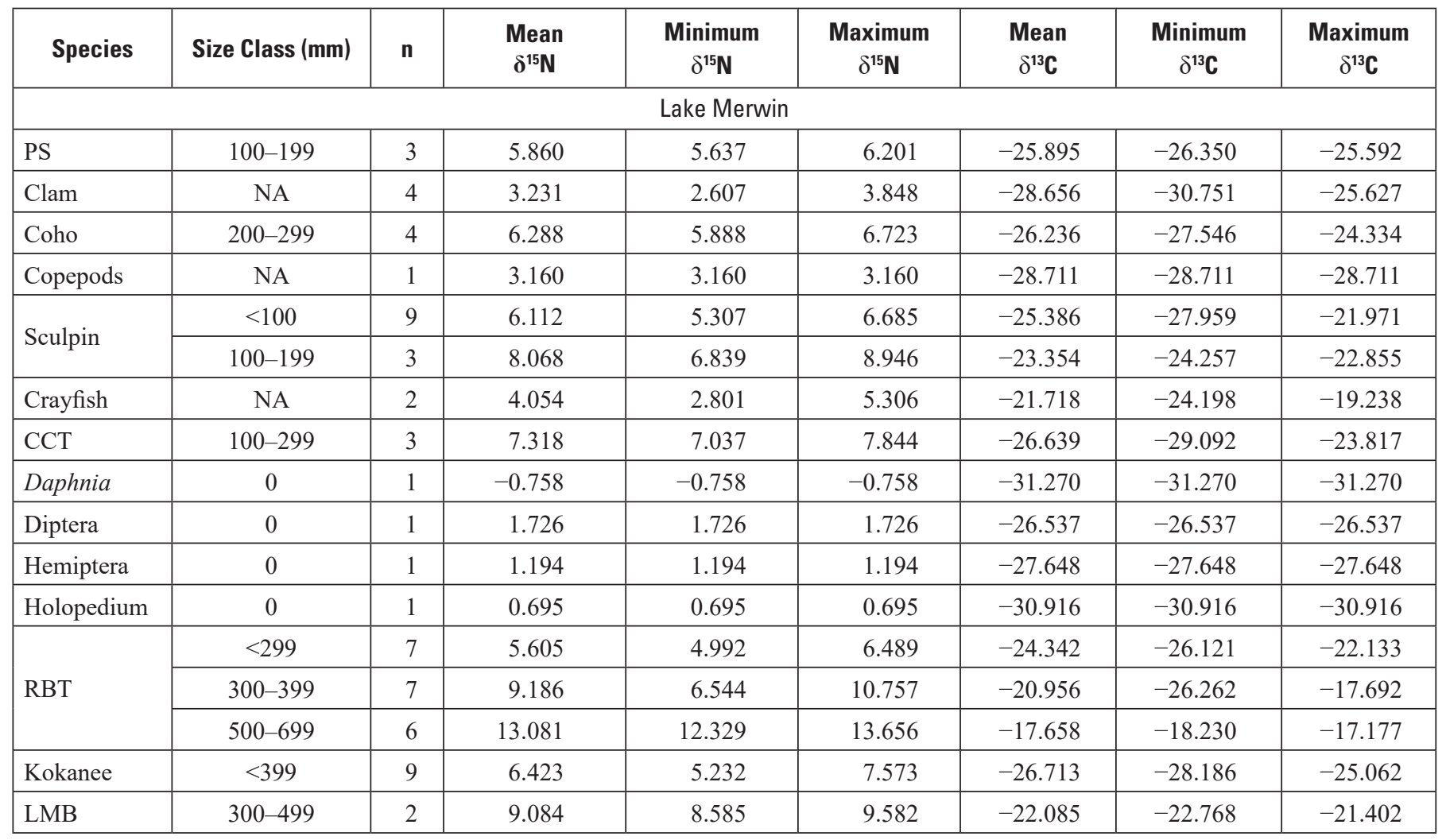


Table E8. Mean $\delta^{15} \mathrm{~N}$ and $\delta^{13} \mathrm{C}$ stable isotope values for fish and invertebrates sampled in the Lake Merwin, Yale Lake, and Swift Reservoir of the Lewis River, Washington, by location and size class for years 2013-2014.-Continued

$\left[\mathrm{mm}\right.$, millimeter; $\mathrm{n}$, number of samples; $\delta^{15} \mathrm{~N}$, delta $\mathrm{n} 15$, ratio of nitrogen isotopes ${ }^{15} \mathrm{~N}$ and ${ }^{14} \mathrm{~N} ;{ }^{13} \mathrm{C}$; delta c 13 , ratio of carbon isotopes ${ }^{12} \mathrm{C}$ and ${ }^{13} \mathrm{C}$; PS, pumpkinseed sunfish (Lepomis gibbosu); NA, not applicable; Coho, Oncorhynchus kisutch (coho salmon); sculpin, Cottus spp.; <, less than; CCT, O. clarkii (coastal cutthroat trout); RBT, O. mykiss (rainbow trout); kokanee, O. nerka (kokanee salmon); LMB, Micropterus salmoides (largemouth bass); LSS, Catostomus macrocheilus (largescale sucker); NPM, Ptychocheilus oregonensis (northern pikeminnow); tiger muskie, Esox lucius x masquinongy (tiger muskellunge); SB, Gasterosteus aculeatus (threespine stickleback)]

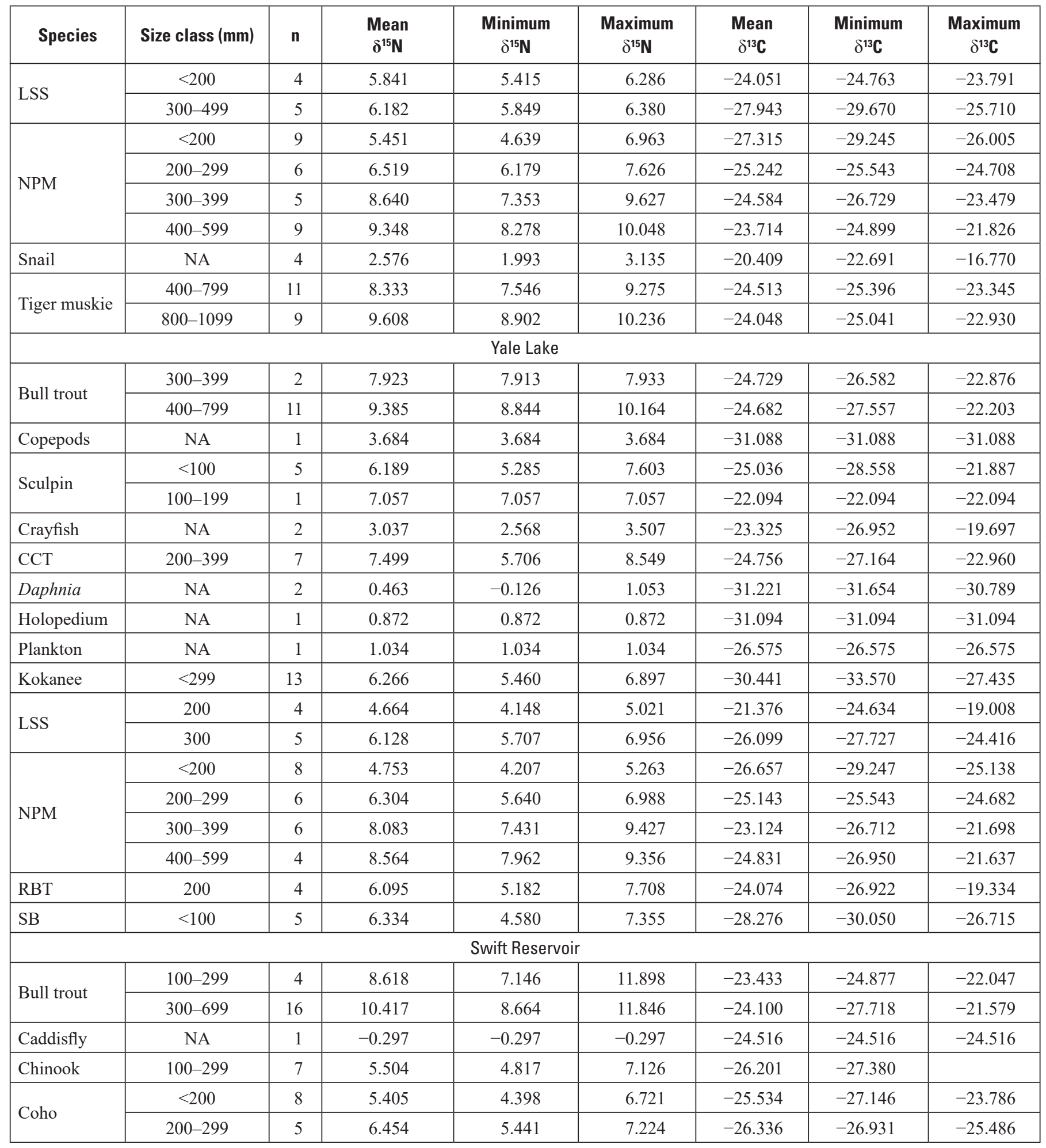


Table E8. Mean $\delta^{15} \mathrm{~N}$ and $\delta^{13} \mathrm{C}$ stable isotope values for fish and invertebrates sampled in the Lake Merwin, Yale Lake, and Swift Reservoir of the Lewis River, Washington, by location and size class for years 2013-2014. - Continued

$\left[\mathrm{mm}\right.$, millimeter; $\mathrm{n}$, number of samples; $\delta^{15} \mathrm{~N}$, delta $\mathrm{n} 15$, ratio of nitrogen isotopes ${ }^{15} \mathrm{~N}$ and ${ }^{14} \mathrm{~N} ; \delta^{13} \mathrm{C}$; delta c 13 , ratio of carbon isotopes ${ }^{12} \mathrm{C}$ and ${ }^{13} \mathrm{C}$; PS, pumpkinseed sunfish (Lepomis gibbosu); NA, not applicable; Coho, Oncorhynchus kisutch (coho salmon); sculpin, Cottus spp.; <, less than; CCT, O. clarkii (coastal cutthroat trout); RBT, O. mykiss (rainbow trout); kokanee, O. nerka (kokanee salmon); LMB, Micropterus salmoides (largemouth bass); LSS, Catostomus macrocheilus (largescale sucker); NPM, Ptychocheilus oregonensis (northern pikeminnow); tiger muskie, Esox lucius x masquinongy (tiger muskellunge); SB, Gasterosteus aculeatus (threespine stickleback)]

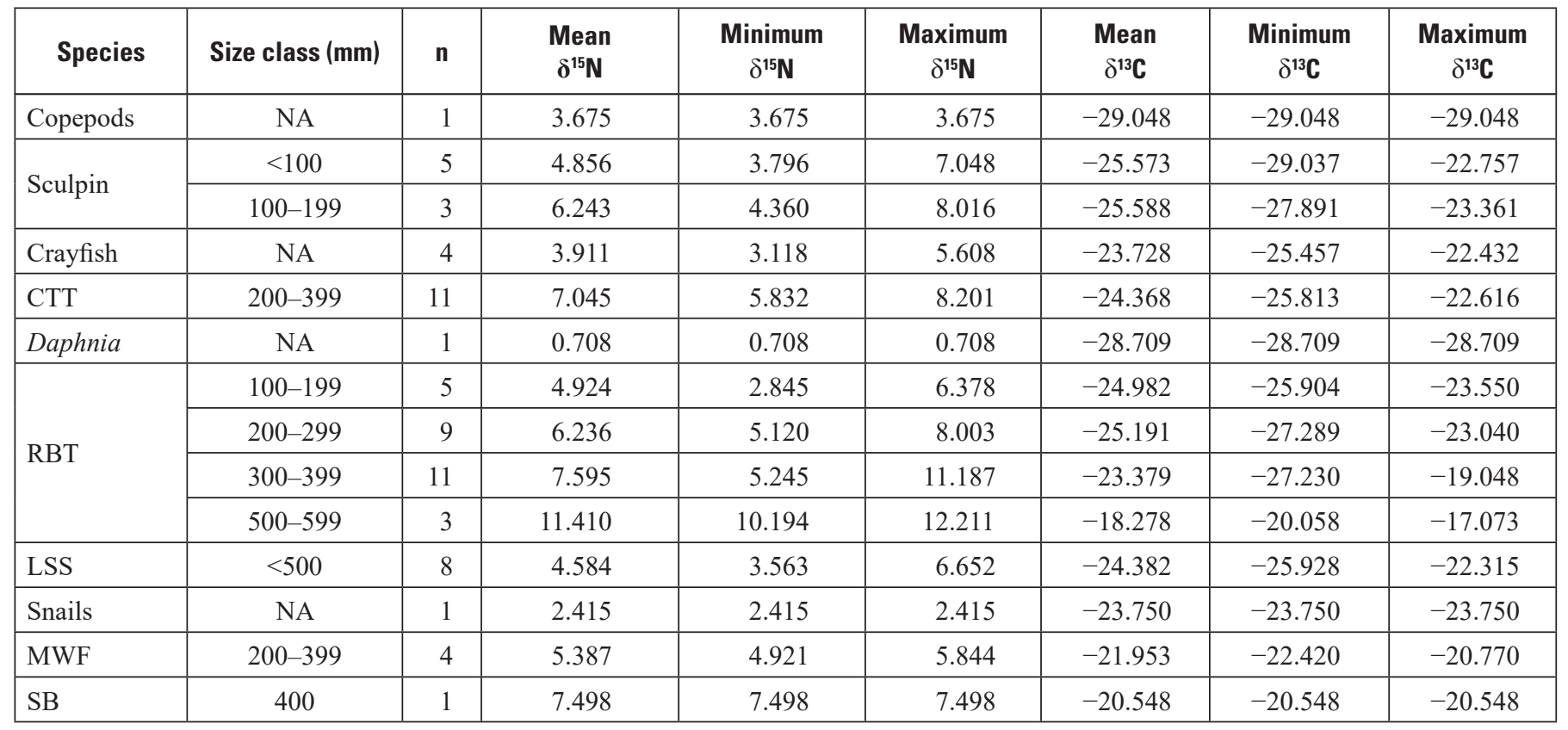

Table E9. Diet proportions of two size classes of Ptychocheilus oregonensis (northern pikeminnow) (200-299 millimeters [mm] and greater than [>] $300 \mathrm{~mm}$ ) in Lake Merwin, Washington, for the years 2013-2014.

[n, sample size; ZOOP, zooplankton; Inverts, aquatic invertebrates; KOK, Oncorhynchus nerka (kokanee salmon); COT, Cottus, spp. (sculpin); NPM, Ptychocheilus oregonensis (northern pikeminnow); CRAY, Pacifastacus leniusculus (signal crayfish); Plant, miscellaneous vegetation]

\begin{tabular}{|c|c|c|c|c|c|c|c|c|c|c|}
\hline \multirow[b]{2}{*}{ Day } & \multirow[b]{2}{*}{ Season } & \multirow[b]{2}{*}{$\mathbf{n}$} & \multicolumn{8}{|c|}{ Diet proportions } \\
\hline & & & ZOOP & $\begin{array}{c}\text { Terrestrial } \\
\text { insects }\end{array}$ & Inverts & КОК & COT & NPM & CRAY & Plant \\
\hline 45 & Spring & 23 & 0.72 & 0.05 & 0.10 & 0 & 0.03 & 0 & 0.09 & 0.03 \\
\hline 135 & Summer & 9 & 0.88 & 0 & 0.11 & 0 & 0 & 0 & 0 & 0.01 \\
\hline \multicolumn{11}{|c|}{$>300$ millimeters } \\
\hline 45 & Spring & 11 & 0.01 & 0 & 0.09 & 0 & 0 & 0.43 & 0.45 & 0.02 \\
\hline 135 & Summer & 29 & 0 & 0 & 0.07 & 0.14 & 0.09 & 0.19 & 0.51 & 0 \\
\hline 225 & Fall & 26 & 0.08 & 0.04 & 0 & 0.28 & 0.14 & 0.10 & 0.29 & 0.08 \\
\hline
\end{tabular}


Table E10. Diet proportions of tiger muskellunge including Ptychocheilus oregonensis (northern pikeminnow) in Lake Merwin, Washington, for years 2013-2014.

[n, sample size; FL, fork length; mm, millimeter; NPM, northern pikeminnow; LSS, Catostomus macrocheilus (largescale suckers), PKS, Lepomis gibbosus (pumpkinseed sunfish); KOK, Oncorhynchus nerka (kokanee); Inverts, aquatic macroinvertebrates]

\begin{tabular}{|c|c|c|c|c|c|c|c|}
\hline \multirow{2}{*}{ Season } & \multirow{2}{*}{$\mathbf{n}$} & \multirow{2}{*}{ FL range (mm) } & \multicolumn{5}{|c|}{ Diet proportions } \\
\hline & & & NPM & LSS & PKS & КОК & Inverts. \\
\hline Spring & 10 & $300-975$ & 0.80 & 0.10 & 0.10 & 0 & 0 \\
\hline Fall & 4 & $525-850$ & 0.75 & 0.25 & 0 & 0 & 0 \\
\hline
\end{tabular}




\section{Chapter F. Studies Assessing Anadromous-Resident Fish Interactions in the Lewis River, Washington}

\section{Introduction}

Understanding interspecific interactions and likely risks of such interactions is considered an important part of anadromous salmon reintroductions (Anderson and others, 2014). The goal of this report is to assess the effects of anadromous fish reintroduction on resident species, and, conversely, assess the effects of resident fish on the reintroduced anadromous fish. Juvenile anadromous salmonids will utilize tributary habitat and reservoirs as migratory corridors and rearing habitats to varying degrees. Therefore, the magnitude and duration of interactions with resident species will vary among anadromous salmonids. Although Salvelinus confluentus (bull trout), Oncorhynchus kisutch (coho salmon), O. mykiss (steelhead), O.tshawytscha (Chinook salmon), and $O$. clarki (coastal cutthroat trout) all historically occurred sympatrically in the upper part of the Lewis River in Washington (for example, Lowery and Beauchamp, 2015), the physical template has changed dramatically since anadromous species were abundant in the upper Lewis River.

This report focuses on evaluating interactions between newly reintroduced salmon and resident fish in tributary and reservoir environments. To address this objective, we specifically evaluated the distribution, behavior, and community interactions of anadromous salmon and resident fish at different life stages. Within the reservoir environment, we also assessed the forage base and capacity of reservoirs to support juvenile salmon. Since reintroduction of anadromous salmonids is already underway in Swift Reservoir on the Lewis River, we focused most of these efforts in the Swift Reservoir and its tributaries. Our specific objectives included (1) conducting a review of the existing literature and potential community-level interactions among reintroduced anadromous species and native and nonnative taxa and (2) conducting a suite of field studies in the upper Lewis River (a) assessing the distributional overlap between coho salmon and bull trout at different life stages and environments, (b) quantifying the extent of diet overlap between resident fish and anadromous species in tributary and reservoir habitats and potential shifts since reintroduction was initiated, (c) determining the forage capacity of the reservoir environments, and (d) integrating field results into a modeling framework to assess the potential effects of anadromous reintroductions on extant bull trout populations. We present the results of objective 1 first and follow with field studies and field results.

\section{Literature Review and Potential Community-Level Interactions}

\section{Review of Anadromous-Resident Interactions}

This review focused on the effects of anadromous reintroductions on native bull trout and coastal cutthroat trout but did not cover interactions between hatchery and wild fish of anadromous species, which have been extensively studied. Given a report describing habitat use and the overlap between anadromous species and resident trout (PacifiCorp and Cowlitz Public Utility District [PUD], 2002), we constrained our synthesis to new information describing niche overlap, the known effects of anadromous species on coastal cutthroat trout and bull trout from empirical studies, information describing known effects of native taxa (bull trout, coastal cutthroat trout) on anadromous reintroductions, and predation by nonnative predators and management considerations.

\section{Effects of Anadromous Reintroductions on Native Species}

Distributional overlap.-Overlap in distribution of bull trout and juvenile Chinook salmon, coho salmon, steelhead, and coastal cutthroat trout is likely to be dictated by thermal requirements, local habitat quality, and life-history expressions and vary across life-stage and species. The fact that both coastal cutthroat trout and bull trout can exhibit complex life histories with 
movements from headwaters to reservoir environments suggests the potential for overlap with anadromous species within the upper Lewis River. Indeed, overlap between coastal cutthroat trout with coho salmon (for example, Glova, 1987; Trotter, 1989; Sabo and Pauley, 1997; Pess and others, 2011) and steelhead (for example, Trotter, 1989; Slaney and others, 1996; Heath and others, 2010) is extensive and well established in the literature. Bull trout overlap with Chinook salmon, steelhead, and coho salmon has also been demonstrated, particularly in headwater streams and reservoirs (for example, Thurow and others, 1997; Taylor and others, 1999; Lowery, 2009; Schoby and Keeley, 2011; Portland General Electric Company, 2013).

Formal assessments of overlap during anadromous species supplementation programs in the Yakima River Basin in Washington have found considerable overlap between spring Chinook salmon, steelhead, and coastal cutthroat trout, but limited overlap with bull trout (Pearsons and Temple, 2007). The greatest amount of overlap found by Pearsons and Temple (2007) occurred in mainstem reaches with mixed results for smaller tributaries. Interestingly, the changes in distributional overlap have not occurred during large increases in abundance of anadromous stocks. Within the Yakima River Basin, however, supplementation release sites were specifically targeted to minimize impacts to nontarget taxa (Pearsons, 2008, 2010).

Where native bull trout distributions are proximate to supplementation sites, distributional overlap may be considerably higher, particularly during later phases of reintroductions and where supplementation fish demonstrate large upstream movements (McMichael and Pearsons, 2001). The extent of distributional overlap is also likely to be affected by native trout life-history strategies, abundance, distribution, and thermal and habitat characteristics of the basins (that is, are habitat conditions suitable for overlap?). In the Lewis River, upstream of Swift Reservoir, overlap in distribution has been observed between wild (that is, naturally produced) juvenile coho salmon and juvenile bull trout (see distributional overlap parts of the "Results and Discussion" section of this report). Although, densities of juvenile coho salmon are currently relatively low, recent diet studies indicate considerable overlap in trophic level and likely forage resources (see "Food web and community interactions" in this chapter).

Overlap in thermal requirements.-The importance of temperature in determining seasonal species distribution patterns and mediating interspecific interactions has been well documented (for example, Fausch, 1989; Wenger and others, 2011). When compared, the temperatures needed for maximum growth of bull trout are considerably lower than for juvenile Chinook salmon, coho salmon, and steelhead (the maximum is unknown for coastal cutthroat trout). However, the range for optimum growth is relatively similar (little information on thermal suitability for coastal cutthroat trout currently exists; table F1) with considerable opportunities for overlap. Ultimately, information regarding the thermal suitability from lab studies and those observed in different portions of species ranges (Spina, 2007) may not be appropriate to determine in situ thermal preferences for the upper Lewis River. Interspecific interactions may shift the thermal ranges used by individual species (for example, Hutchinson, 1978). In addition, it is unclear to what extent there is local variation in species thermal tolerances (meaning, Eliason and others, 2011).

Overlap in spawning habitat for fall spawners. - Overlap in spawning habitat can be substantial for bull trout, coho salmon, and Chinook salmon, all spawn during the late summer to early autumn. Across their native range, bull trout typically spawn from August through late October (Fraley and Shepard, 1989; Rieman and McIntyre, 1993; Howell and Sankovich, 2012). In the upper Lewis River Basin, bull trout spawning in Cougar Creek and Pine Creek and tributaries has been documented as early as the end of July through late October (PacifiCorp and Cowlitz PUD, 2002). Recent redd data (2012-2015) suggest that bull trout spawning in Swift Reservoir tributaries typically ends in mid-October (see "Redd superimposition" in this chapter). Based on information in the Lewis River below Merwin Dam, spring Chinook salmon typically spawn during September and October; fall Chinook salmon spawn during mid-October through early November; and coho salmon spawn during mid-October through early December (PacifiCorp and Cowlitz PUD, 2002), typically after bull trout. During the first 3 years of coho salmon reintroduction above Swift Dam (2012-2015), redd surveys indicate that most spawning occurred between early October and late November.

Of greatest concern during the fall spawning period is the risk of redd superimposition. Superimposition by coho salmon on top of bull trout has been documented in tributaries to Swift Reservoir (see "Redd superimposition" in this chapter).

Superimposition is likely to be most pronounced as densities of Chinook and coho salmon increase and saturate habitat. Recent survey information suggests the later timing of coho salmon spawning and the fact that coho salmon spawn in smaller streams more similar to those where bull trout spawn than do Chinook salmon suggest a higher likelihood of coho salmon superimposition on bull trout redds. Where the species are naturally sympatric (that is, wild populations; Lowery and Beauchamp, 2015), superimposition is likely to be substantially lower (that is, minimizing contemporary competitive interactions; Connell, 1980) than during reintroduction phases when hatchery-reared individuals that lack natal homing are released (Quinn, 1993).

Coho and Chinook salmon and bull trout are all generally considered to spawn in pool-tail/riffle-crest habitat (Kondolf, 2000), but spawning generally extends to areas with high intragravel flows. These intragravel flows include both upwelling (positive vertical hydraulic gradient) and downwelling (negative vertical hydraulic gradient), and use of both types of intragravel flows has been observed for bull trout (Baxter and McPhail, 1999; Baxter and Hauer, 2000; Bean, 2012), Chinook salmon (Vronskii and Leman, 1991; Geist and Dauble, 1998), and coho salmon (Mull and Wilzbach, 2007). The selection of areas with positive or negative hydraulic gradients is likely to allow for the exchange between subsurface and surface flows (Baxter and Hauer, 2000) or to moderate temperatures.

Like most salmonids, high levels of fine sediment can have detrimental effects on egg survival (Tappel and Bjornn, 1983), and spawning gravel is an additional habitat metric that may facilitate overlap between the three species. Although some overlap 
is likely across each of the species, substrate use for spawning Chinook salmon (mean $=47$ millimeters [mm]; range $=1-175 \mathrm{~mm}$; as reviewed in Kondolf, 2000) is considerably larger than observed for bull trout (mean $=29 \mathrm{~mm}$; range $=3-58 \mathrm{~mm}$; as reviewed in Baxter and McPhail, 1996) and coho salmon (mean=20 mm; range=5-35 mm; as reviewed in Kondolf, 2000; Mull, 2005). The similarity in spawning substrate used by bull trout and coho salmon further suggests a high potential for overlap during the spawning period, particularly when densities are high.

The current understanding of Chinook salmon, coho salmon, and bull trout spawning periods in the upper Lewis River suggests redd depth may also be an important factor in considering interspecific interactions. Redd depths are typically shallower for bull trout (mean $=16.5$ centimeters [cm], mean range $=11-17 \mathrm{~cm}$; Weeber and others, 2010) than reported for Chinook salmon (mean=28.4 cm, mean range $=24.2-43.9 \mathrm{~cm}$; DeVries, 1997), and coho salmon (mean range $=21.1 \mathrm{~cm}$, mean range=12.3-31.6 cm; Devries, 1997; Mull, 2005). Deeper redds from anadromous species are likely to have the greatest superimposition impacts on bull trout redds where anadromous species spawn later than bull trout (Weeber and others, 2010). Monitoring the potential for superimposition is warranted in the upper Lewis River, particularly if Chinook and coho salmon spawning extends to the core bull trout spawning areas (for example, Pine Creek and its tributaries).

Population-level effects.-The effects of anadromous reintroductions on native bull trout and coastal cutthroat trout populations are relatively limited, so other native salmonids need to be considered for this review. Riley and others (2004) found no significant effects of Chinook and coho salmon supplementation releases on rainbow trout and coastal cutthroat trout fry densities. In a review of existing studies, Naman and Sharpe (2012) found hatchery-reared predation of native fish to be relatively low. At an individual level, predation of fry may be relatively low, but population-level predation may be high, particularly where large supplementation releases occur and considerable overlap in distribution occurs with fry rearing (Naman and Sharpe, 2012; Tabor and others, 2012).

Additional concerns exist surrounding the competitive interactions between coastal cutthroat trout and juvenile coho salmon. Some competition studies have shown that coho salmon exhibit dominance over juvenile coastal cutthroat trout (Sabo and Pauley, 1997) often resulting in displacement of coastal cutthroat trout (Glova, 1987). However, these results are not consistent across studies (Kiffney and others, 2009), and the effects of interspecific competition has not been documented to have fitness or population-level effects for coastal cutthroat trout (Kiffney and others, 2009; Pess and others, 2011).

Early research in headwater streams in the Yakima River Basin, however, suggested large increases in wild Chinook salmon abundance did not lead to significant changes in rainbow trout growth, abundance, and biomass (McMichael and Pearsons, 1998). Long-term assessments, however, found hatchery-reared Chinook salmon supplementation has led to significant declines in rainbow trout abundance, which is likely attributable to the cumulative effects of increases in both hatchery supplementation and increases in wild Chinook salmon abundance (that is, replacement; Pearsons and Temple, 2010). Continued monitoring in the Yakima River, however, has found differential results, as mean abundance of coastal cutthroat trout and Prosopium williamsoni (mountain whitefish) increased during hatchery supplementation (Temple and Pearsons, 2012). Although Temple and Pearsons (2012) continued to find significant decreases in rainbow trout size structure during supplementation (that is, similar to Pearsons and Temple, 2010), the authors did not find evidence that supplementation caused such patterns.

The effects of reintroductions can also have genetic consequences for closely related steelhead and coastal cutthroat trout. Of particular concern is the effect of residualized hatchery-reared steelhead (that is, fish that remain as residents), which typically is greater than (>) 5 percent but can approach levels as high as 17 percent (Hausch and Melnychuk, 2012). Residualized steelhead can make relatively long upstream movements ( $>12$ kilometers [km]; McMichael and Pearsons, 2001). The presence of residualized steelhead can also lead to significantly reduced growth in wild rainbow trout (McMichael and others, 1997). The presence of steelhead residuals is likely to lead to erosion of reproductive barriers with native coastal cutthroat trout (Docker and others, 2003) and increase hybridization levels above that observed in wild populations (for example, Ostberg and others, 2004; Heath and others, 2010). Although relatively close to the Pacific Ocean, the presence of reservoir habitat may lead to increased residualization in the upper Lewis River (Hausch and Melnychuk, 2012), suggesting the need to develop monitoring programs to account for the extent of residualization and potential hybridization.

In general, detecting the effects of anadromous reintroductions on bull trout and coastal cutthroat trout populations is likely to be challenging (Ham and Pearsons, 2000; Weber and Fausch, 2003). The difficulties of detecting changes in abundance of salmonids (Ham and Pearsons, 2000) and relating these changes to reintroduction actions (for example, Temple and Pearsons, 2012) suggests the need to consider multiple metrics to quantify the effects of management actions. Given that the effects to resident trout species may differ within and across streams (Kiffney and others, 2009), caution should be urged in extrapolating results where data are limited. Furthermore, in many cases, the effects may either be unknown or cumulative, which can be challenging to identify (Pearsons, 2008). Ultimately implementing an approach as outlined in Pearsons (2002 and 2010), with long-term monitoring sites (for example, Temple and Pearsons, 2012) and adaptive management practices, is likely to provide feedback to managers on how to limit negative effects on native taxa. 


\section{Effects of Native Species on Anadromous Reintroductions}

The occurrence of native bull trout, coastal cutthroat trout, and Ptychocheilus oregonensis (northern pikeminnow) in the upper Lewis River highlights the need for consideration of the effects these native fish have on anadromous reintroductions. This review specifically focuses on predation effects on anadromous stocks.

Coastal cutthroat trout. - Although generally not considered a top predator, coastal cutthroat trout predation on juvenile anadromous species can be substantial (Gregory and Levings, 1996). In an extensive diet study in the lower Cedar River in Washington, juvenile Chinook salmon represented up to 30 percent of winter/spring diets for juveniles/small adult coastal cutthroat trout (Tabor and others, 2012). Although diets consisted primarily of aquatic insects during the summer, when year-round predation was linked with population estimates, Tabor and others (2012) found annual predation to be 66,000 Chinook salmon in a given year. Although seemingly high, these predation rates appear to be relatively consistent with coho salmon predation of $O$. nerka (sockeye salmon) in the lower Cedar River, suggesting relatively high predation rates on newly emerged salmon is common even among anadromous salmon species. Despite high predation estimates, mortality during emergence is likely to be naturally high, and no studies have evaluated predation effects on adult salmon returns or smolt-to-adult survival. Given the relatively ubiquitous distribution of coastal cutthroat trout in the upper Lewis River, there is a potential for predation of anadromous fry/parr (for example, Tabor and others, 2012); however, densities of coastal cutthroat trout from field sampling in the upper Lewis River are relatively low, suggesting limited population-level effects.

Bull trout.-Across the Pacific Northwest, bull trout are one of the top native freshwater predators. Bull trout are considered to be highly piscivorous, particularly with increasing size (Rieman and McIntyre, 1993; Wilhelm and others, 1999; Beauchamp and Van Tassell, 2001; Lowery and Beauchamp, 2015). When comparing bull trout diets among studies, the proportion of fish in bull trout diets averaged 44.7 percent (range $=01-100$ percent; table F2). When considering only large bull trout (that is, where size is identified including adults, $>500$ grams, and $>300 \mathrm{~mm}$ ), the mean proportion of fish in diets increased to 68.2 percent (range $=22-100$ percent). Where sympatric with $O$. nerka (kokanee) in reservoir/lake systems, kokanee tend to be the dominant prey (Clarke and others, 2005; Guy and others, 2011; Hill and others, 2014); this pattern is consistent (albeit limited-time series) in Lake Billy Chinook in Oregon where no identified Chinook salmon juveniles were found in bull trout diets during spring or fall sampling events (Hill and others, 2014). Few studies have evaluated bull trout predation on anadromous species within fluvial systems. Lowery (2009) found consistent predation of anadromous smolts but considerable variability in the extent of predation of any prey species (coho salmon, Chinook salmon, and steelhead) within and across seasons/years. Lowery and Beauchamp (2015) also found substantial differences in the proportion of predation across tributary and mainstem habitat for bull trout 96-300 mm. Budy and others (2012) found bull trout diets in the Sout Fork Walla Walla River in Oregon varied across years (2002-2012), with Oncorhynchus spp. (Chinook salmon, steelhead, and rainbow trout) making up anywhere between 0 percent and $>95$ percent of diets. Ultimately, these studies suggest bull trout are opportunistic feeders in fluvial environments.

A recent expert panel found bull trout impacts to anadromous populations in a fluvial population (Clackamas River in Oregon) to be predominantly characterized as moderately low to none (Marcot and others, 2012). Although predation impacts to kokanee salmon can be relatively high in fluvial environments (Beauchamp and Van Tassell, 2001), there remains considerable uncertainty of predation effects on coho salmon, Chinook salmon, and steelhead. There is a relatively high potential for bull trout predation of anadromous smolts within mainstem Lewis River (Lowery and Beauchamp, 2015), in reservoirs where thermal conditions are suitable, and in tributary streams where overlap occurs. Increases in fish abundance and distribution through the reintroduction process may increase the extent of predation. Within reservoir systems, predation will likely be dictated by thermal profiles, smolt migration timing and routes, smolt delays at collection facilities, and abundance of bull trout. Ultimately, monitoring bull trout and anadromous species distribution, abundance, and diet patterns will be needed to accurately understand bull trout predation effects.

\section{Effects of Native and Nonnative Salmonid Predators to Reintroduction Efforts}

Considerable populations of native and nonnative predators exist within the upper Lewis River Basin as a result of fisheries management objectives and native species distributions. Within the upper Lewis River Basin, the main native predator is the northern pikeminnow. Nonnative predators include Esox masquinongy $\times$ lucius or Esox lucius $\times$ masquinongy (tiger muskellunge), Lepomis macrochirus (bluegill), Micropterus salmoides (largemouth bass), and Ameiurus nebulosus (brown bullheads).

Given the extensive review of the effects of nonnative species on anadromous species (PacifiCorp and Cowlitz PUD, 2002), this review only included new literature related to existing native and nonnative predators within the upper Lewis River Basin. Of particular concern in the upper Lewis River Basin is the uncertainty of predation rates of tiger muskellunge, the relative strength of predation of largemouth bass, and the need for potential management actions to control native and nonnative predators.

Tiger muskellunge.-Data related to tiger muskellunge predation on salmonids continues to be rare in the literature (see PacifiCorp and Cowlitz PUD, 2002), raising the need to consider diet data for closely related Esox lucius (northern pike). Recent diet studies have indicated considerably higher northern pike predation of salmonids than observed in Schmetterling (2001). In Alaska, Sepulveda and others (2013) found the extent of predation to vary based on suitable habitat. In streams with high 
overlap in distribution (suitable thermal and physical habitat) for both salmon and northern pike, salmonids were the dominant prey across sample sites and spring and summer months (48-70 percent diet mass). In an adjacent stream, with habitat only suitable for salmon and northern pike in the lower reaches, the proportion of salmonids in diets was relatively high (31 percent) in the suitable lower reaches but no salmon were found in northern pike diets in reaches with unsuitable habitat. Interestingly, Sepulveda and others (2013) found no correlation in the proportion of Chinook or coho salmon in diets with increasing size of northern pike $($ range $=25-100 \mathrm{~cm}$ ). Northern pike predation across size classes suggests relatively high predation potential for similar predators like tiger muskellunge on salmon at the population level.

Other studies have also found considerable northern pike predation on salmonids (Muhlfeld and others, 2008; Spens and Ball, 2008). In Montana, Muhlfeld and others (2008) observed northern pike consuming a mixture of soft-rayed and bonyrayed fish. Northern pike diets varied across seasons with predation on bull trout and $O$. clarkii lewisi (westslope cutthroat trout) to be relatively high during winter and spring, and their proportion of diet was $>50$ percent during the spring months. Summer predation was minimal, likely because of thermal segregation of the species. Northern pike predation on salmonids was generally higher for large fish $(>600 \mathrm{~mm})$, but some predation did occur for smaller fish with apparently greater selection of westslope cutthroat trout over bull trout at smaller size classes, suggesting that size classes affect predation levels. These recent results suggest salmonids are extremely vulnerable to northern pike (Haught and von Hippel, 2011) and that predation on salmonids from species with similar life histories and morphologies such as tiger muskellunge, is likely to be high in the upper Lewis River. Although field estimates from Lake Merwin suggest salmonid predation by tiger muskellunge is relatively low (see "Piscine Predator Impact Studies 2013-2014, Lake Merwin, Washington," chap. E of this report), such patterns may change considerably if the density and distribution of coho salmon, Chinook salmon, and steelhead increases substantially during and after reintroduction. However, given the potential for tiger muskellunge to be long-lived ( $>8$ years; Schmuck and Petersen, 2006), total predation estimates will require extensive diet data, which can vary across individuals (PacifiCorp and Cowlitz PUD, 2002), and population estimates across size classes.

Other nonnatives. - Empirical data continue to be relatively limited for predation of juvenile salmon and steelhead by brown bullheads and largemouth bass. Where formally evaluated, brown bullhead predation potential appears to be low (Tabor and others, 2004), which is consistent with other studies in the Pacific Northwest (Bonar, and others 2005; Gray, 2005), suggesting population-level effects are limited. Largemouth bass are considered to be a relatively warm-water fish, but predation on juvenile salmonids suggests this species has relatively high predation potential (Bonar, and others 2005). In Lake Washington in Washington, Tabor and others (2004) found salmonids in the diets of largemouth bass ranging from 100 to $>300 \mathrm{~mm}$ in length. Although diet varied, salmonids made up approximately 50 percent of small $(100-199 \mathrm{~mm})$ largemouth bass diets in some habitats (for example, a shipping canal). Given the relative abundance of smaller-sized largemouth bass, population-level predation may be an additional limitation to reintroduction efforts where present (for example, Karchesky and Bennett, 1999). Such results highlight the need for in situ studies of largemouth bass predation, and indicate a potential for competition effects between juvenile bass and juvenile salmonids (Gray, 2005).

Management considerations. - Given the relative abundance of northern pikeminnow and the presence of additional nonnative predators in the upper Lewis River, there continues to be concern about the need for management actions to control predation and enhance recovery efforts. Management efforts to control populations of predators have been in place for decades on the Columbia River (Beamesderfer and others, 1996; Porter, 2012). The effects of predator controls, however, are complex (Harvey and Kareiva, 2005; Carey and others, 2012). During 22 years, more than 2 million northern pikeminnow have been removed from the Columbia River in Oregon and Washington under the Columbia River Northern Sport Reward Fishery Program, but there appears to be no apparent trends in annual harvest and catch-per-unit-effort appears to be increasing (Porter, 2012), raising questions regarding the population-level impacts of the management efforts. However, within certain reservoirs (for example, John Day Reservoir in Oregon), populations of northern pikeminnow are decreasing as a result of management actions, but nonnative populations of Sander vitreus (walleye) have increased.

Management actions in the Columbia River have met target goals of reducing predator numbers, but there is uncertainty as to how these actions have affected overall salmon survival rates (Beamsderfer and others, 1996). The challenges associated with management actions also stem from the potential indirect effects that management actions for one population may have on sympatric predator populations (Harvey and Kareiva, 2005) or compensatory mechanisms within the source population (Beamsderfer and others, 1996). Prior to implementing management actions to control native and nonnative predator populations, considerable data related to species distribution, abundance, and community interactions (for example, food web) are needed to avoid unintended consequences and improve the efficiency of management actions (if needed). Ultimately, with ample data, scenario modeling should be completed (for example, Harvey and Kareiva, 2005) and an adaptive management framework should be established with iterative analyses of monitoring and evaluation data. 


\section{Field Studies and Methods}

\section{Stream Habitat}

During 2014, we continued studies to quantify interactions between resident and anadromous species in the study area. Given that reintroductions have not occurred in Lake Merwin and Yale Lake in Washington, efforts were largely focused in tributaries to Swift Reservoir.

Distributional overlap.-We quantified the distributional overlap of bull trout and juvenile coho salmon using snorkel surveys each year. During 2013-2014, we focused efforts on Pine Creek and P8, a tributary to Pine Creek because the creek's tributaries are considered to contain the highest densities of bull trout in the Swift Basin. Snorkeling studies can substantially underestimate the abundance of bull trout, particularly during daytime studies (Thurow and others, 2006). However, we selected daytime snorkeling surveys because bull trout commonly use Pine Creek and P8, and we needed to quantify juvenile coho salmon distribution, which is unlikely to have pronounced diel bias. To assess the distribution of both species, we used a systematic sampling approach (random start), as both species are likely clumped in distribution (for example, Al-Chokhachy and others, 2009, fig. 4). Reach lengths varied by stream, with reaches of at least 200 meters (m) used in Pine Creek and $150 \mathrm{~m}$ used in P8. We partitioned the entire study area into reaches and sampled every third reach from the random start for an approximate annual sample rate of 33 percent. From 2013-2015, we systematically sampled adjacent reaches to effectively sample the entire study area during the duration of this study. Each year, we conducted all surveys during late August and early September.

In 2015, we expanded our snorkel surveys both spatially and temporally. In addition to Pine Creek and P8, we also included Rush Creek, a tributary considered to be a historical stronghold for bull trout in the Swift Basin, and P3, a tributary to Pine Creek, within the known distribution of bull trout. In Rush Creek, we sampled two reaches at least $250 \mathrm{~m}$ long and systematically spaced within the first kilometer of stream habitat. Given the relatively short amount of habitat available in P3, we sampled all of the existing habitat (400 m). For all four tributaries (Pine Creek, P8, P3, and Rush Creek), we conducted two sets of surveys including an early survey (mid-June through mid-July) and a late survey (mid-late August).

During snorkel surveys, two individuals progressed upstream (except at P3, where only one individual conducted the surveys because of the narrow channel width, mean=1.7 m), remaining relatively parallel to each other; all side channels and channel units were included in each survey. All fish were recorded, placed into 50-mm categories, and reported to a third individual on the streambank. Snorkelers carried flashlights to improve visibility in shaded areas and in sections with large amounts of woody debris.

In addition to overlap with bull trout, we considered the potential interactions between acclimation juvenile Chinook and wild coho salmon in Clear Creek, a tributary in the upper Lewis River. Specifically, we evaluated the spatial and temporal overlap of acclimation for juvenile Chinook salmon using tagging location data and emigration patterns, based on recapture events at the fixed antennae on Clear Creek, of tagged coho salmon (see "Assessment of Juvenile Fish Production and Outmigration Success on the Lewis River, Washington," chap. D of this report) and passive integrated transponder (PIT)-tagged acclimation Chinook salmon. The data on migration timing will help also identify "residence" time of acclimation Chinook salmon and migration patterns of wild coho salmon in response to the population of acclimation juvenile Chinook salmon.

Food web and community interactions.--During 2014, we continued to assess community interactions using a combination of stable isotope data and diet analyses. We revisited sites previously sampled during 2009 as part of a "baseline" assessment of community interactions prior to large-scale anadromous reintroductions in the upper Lewis River. Because anadromous reintroductions have been initiated at Swift Reservoir, this food web and community interaction study focused on tributaries to the reservoir, including Swift Creek, Drift Creek, Rush Creek, P8, and Cussed Hollow Creek. In addition, we also collected diet and isotope data at two sites on Clear Creek upstream of the confluence with Muddy River. At each site, we collected samples during June-July, mid-late August, and early November, after the onset of coho salmon spawning.

At each site, we sampled macroinvertebrates were sampled tissue samples and diets were collected from fish. We used a variety of sampling techniques to capture fish including backpack electrofishing, electrofishing down to a seine, snorkel herding fish into a seine, and minnow traps. We did not constrain our sampling approaches to only electrofishing methods, which were used during baseline efforts, as we were interested in the most efficient methods for capturing appropriate sample sizes across species, and the use of different sampling methods were unlikely to affect results from isotopic and diet studies.

Captured fish were anesthetized with AQUI-S, weighed, and measured; tissue and diet samples were also collected. Tissue samples were taken from the anal fin $(3 \mathrm{~mm} \times 3 \mathrm{~mm})$ and frozen for isotopic analyses. To collect diet data, we used gastric lavage methods for all fish $>60 \mathrm{~mm}$. After sampling, we placed fish in flow-through recovery tanks within the sample stream; upon full recovery of equilibrium, fish were released at their point of capture. We sampled for macroinvertebrates using drift nets set in the thalweg at the bottom of each reach with set times of approximately 1 hour. We elected not to use Serber approaches, which were used in the 2009 baseline surveys, to minimize sorting of items because most fish in the food web study are drift feeders, and differences in methods are unlikely to influence interpretations of isotopic analyses. 
All drift, diet, and fin clips for isotope samples were placed in water and frozen until analyzed. All diet and isotope samples were sorted by family and grouped based on functional feeding groups. Isotopic samples were prepared following the University of California, Davis Stable Isotope Facility protocol (http://stableisotopefacility.ucdavis.edu/). Briefly, fin samples were dried at temperatures between 50-60 degrees Celsius $\left({ }^{\circ} \mathrm{C}\right)$, set aside in a dehydrator to allow for acclimation to humidity, ground using a mortar and pestle, and encapsulated in a tin capsule for analyses. All isotopic analyses were conducted by the University of California, Davis Stable Isotope Facility.

Redd superimposition.-Given that both bull trout and coho salmon are fall spawners, we investigated the potential for and evidence of superimposition of spawning redds (for example, Weeber and others, 2010). First, we evaluated the potential for superimposition using existing redd data for bull trout and coho salmon. We quantified the date of each redd observed for each species during recent (2012-2014) surveys in the upper Lewis River and calculated the proportion of coho salmon redds that occurred after the onset of bull trout spawning.

In 2014 and 2015, we assessed superimposition in P8 given the abundance of bull trout and the abundance of reintroduced adult coho salmon in the Swift Reservoir. Because of the potential for misidentifying bull trout redds (Dunham and others, 2001), we used existing redd location information provided by local biologists for bull trout (that is, flagged redds). Starting in mid-October of 2014 and 2015, we conducted biweekly redd surveys to identify coho salmon redds superimposed on bull trout redds. Because of high levels of suspended and delivered fine sediment in Pine Creek during the fall, accurately identifying the boundaries of existing bull trout redds can be challenging. As such, we delineated the boundaries of each redd using rocks that were painted white and identified instances of superimposition where coho salmon redds overlapped within the perimeter of bull trout redds.

\section{Reservoir Habitat}

Carrying capacity of reservoir habitat: zooplankton supply and demand.-To assess the ability of the reservoirs on the Lewis River to support additional salmonids, we examined the annual progression of vertical temperature profiles, seasonal cycles of zooplankton production and distribution, and consumption demand of the existing resident planktivore community. Our approach to assess potential interactions between anadromous and resident fish and carrying capacity in each reservoir is similar to that used in our Lake Merwin predator impact studies (see "Piscine Predator Impact Studies 2013-2014, Lake Merwin, Washington," chap. E of this report), so some methods and data presented previously overlap with the studies herein. The fish communities of each reservoir were sampled, and stable isotope analysis was used to evaluate the trophic structure of the food webs. We measured seasonal depth distribution, diets, growth, and abundance of key planktivore populations in each reservoir and used bioenergetics models to estimate their monthly consumption demand. Ultimately, planktivore consumption demand was compared to prey supply to evaluate the availability of food to support reintroduced populations of age- 0 salmonids. We assumed that the carrying capacity of a reservoir had been reached if the current consumption demand exceeded 50 percent of the prey supply in any month of the growing season. To estimate how many additional subyearlings could be supported in each reservoir, we divided any surplus prey that could have been consumed without exceeding this arbitrary estimate of maximum sustainable prey exploitation by the consumption demand of individual age- 0 salmonids. These estimates could be added to expected production from lotic habitats to evaluate the feasibility of reintroductions and weigh the costs against the benefits.

Fish sampling.-We deployed short-term gill nets for 30 minutes to 24 hours in Swift Reservoir, Yale Lake, and Lake Merwin in June-November 2013 and May-November 2014 to obtain biological samples and information on relative abundance and seasonal depth distribution of resident fish. We only set gill nets for short periods in Swift Reservoir and Yale Lake to reduce the risk of harming bull trout, which are listed as threatened under the Endangered Species Act, and all sampling was conducted through U.S. Fish and Wildlife Permit TE08551B-0. Creel sampling and trolling were also used to sample fish. Midwater trawling was conducted in June and August 2013 but was restricted to the epilimnion because of submerged standing timber in Swift Reservoir, Yale Lake, and Lake Merwin.

In the field, we identified, sexed, and counted fish by species and recorded a subset of fork lengths in millimeters and wet weights in grams. Stomachs were collected for diet analysis and scales from the preferred region were taken for age and growth analysis. In addition, fin tissue was sampled for stable isotope analysis, placed on ice in the field, and later frozen for preservation and analysis in the laboratory. Fish were handled and released or euthanized according to the University of WashingtonInstitutional Animal Care and Use Committee (UW-IACUC) protocol \#3286-21.

Hydroacoustics.-Mobile hydroacoustic surveys were conducted in June, August, and November 2013 to evaluate the abundance and depth distribution of small, limnetic fish in Swift Reservoir, Yale Lake, and Lake Merwin. The hydroacoustics system used for all surveys consisted of two multiplexed split-beam 200 kilohertz transducers towed in sidelooking (12.8-degree full-beam angle) and downlooking (6.8-degree full-beam angle) orientations. The transducers were connected to a Biosonics DE-6000 scientific echosounder with the following data acquisition parameters: bottom threshold $=70 \mathrm{~m}$, ping rate $=2-3$ pulses per second, pulse width $=0.3$ millisecond, and target strength detection threshold $=-65$ decibels. 
Quantitative surveys of fish abundance and distribution in August 2013 consisted of 15-18 zigzag transects across and one long transect along the center axis of Swift Reservoir, Yale Lake, and Lake Merwin (fig. F1). Qualitative surveys in June and November 2013 consisted of fewer (5-15) loose zigzag transects across, and one transect along, the center axis of each body of water to examine the vertical distribution of fish. A portion of each of the surveys was repeated during daytime, at dusk, and at night to evaluate diel vertical migrations, and to more accurately estimate mean daily thermal temperatures that could be used in bioenergetics models.

The abundance of kokanee salmon and the distribution of limnetic fish were estimated from hydroacoustic survey data using standard echo-counting techniques (Beauchamp and others, 1997) with EchoView 5.4 software from Myriax Pty, Ltd. (https://www.echoview.com/). All targets in Yale Lake and Lake Merwin within the main target strength modes corresponding with expected fish lengths (30-275 $\mathrm{mm}$ in Yale Lake and 30-400 $\mathrm{mm}$ in Lake Merwin) were assumed to be kokanee salmon, given that the composition of angler catches was nearly 100 percent kokanee salmon in Yale Lake (with one account of a bull trout hooked) and 80 percent kokanee salmon in Lake Merwin. The other approximately 20 percent of angler catches in Lake Merwin consisted of larger, hatchery-reared rainbow trout and an occasional northern pikeminnow. Catches in midwater trawls were 92 percent age-0 kokanee salmon in Yale Lake. It is likely that some Gasterosteus aculeatus (threespine stickleback), pelagic fish in the superfamily Cottoidea (sculpins), and other fish species were allocated to kokanee salmon population estimates. However, this creates a conservative population estimate for the purpose of comparing zooplankton consumption demand and supply, as all planktivore species draw from the same zooplankton resources.

Targets measured by the sidelooking transducer were used to estimate fish densities within the upper $20 \mathrm{~m}$ of the water column over a range of 0-40 m from the survey vessel; however, side-aspect target strengths cannot be reliably converted to fish lengths. Therefore, the observed densities of side-aspect targets represented the total within a range of sizes and were used to estimate depth distributions of thermal experience (daily temperature experienced by fish), but not of size-specific densities for abundance and age-structure calculations.

Fish target strengths measured by the downlooking transducer were converted to fork lengths based on Love's (1977) equation and used to estimate size-specific target densities. Size-specific target density estimates were calculated for each 5-m depth interval. Targets were summed within specific size modes in the target strength frequency distribution that corresponded with the expected lengths of each size class of fish, and the sums were divided by the volume of water examined by the acoustic beam to calculate density. Targets outside the range of strengths associated with kokanee salmon were assumed to be background noise caused by debris and noise (if smaller), or branches of standing timber and large piscivorous fish (if larger).

Abundance and survival. - Abundances of age-0-2 kokanee salmon were estimated by expanding density estimates from quantitative hydroacoustic surveys by the volume of water in the corresponding depth layers. Constant recruitment and survival between years and age classes was assumed to simplify the calculations. Annual survival rate was fit to the abundance of age- 0 fish and to fish age-1 to age-2, assuming there were no age-3 or older fish because the majority of kokanee salmon in Lake Merwin and Yale Lake die after the second year of life (PacifiCorp and Cowlitz PUD, 2004). We used annual survival rates to allocate the estimated abundance of age-1-2 kokanee salmon to each age class. Daily population abundances for the rest of the year were calculated using daily survival rates, derived with an exponential relationship, survival $=e^{(-r t)}$, where $r=$ instantaneous mortality rate and $t=$ time.

In Swift Reservoir, kokanee salmon were not present, and hydroacoustic surveys could not reliably estimate the abundance and survival rate of the primary planktivore, rainbow trout, because of their nearshore and surface orientation. Instead, we relied on stocking and catch data to estimate the abundance and age structure of rainbow trout. Catches from the main reservoir were entirely rainbow trout from the current year's stocking event, based on scale and length frequency analysis. We modeled consumption demand for a single cohort of 50,000 hatchery-reared rainbow trout with zero mortality from the date of stocking (June 1) to the end of November of that year. The approach of not incorporating mortality provided a maximum possible estimate of the consumption demand by rainbow trout. The total fish abundance in August, estimated by hydroacoustics, was 40,500 fish in the size class 20-200 $\mathrm{mm}$ and 9,800 fish in the size class 200-400 $\mathrm{mm}$. The low number of rainbow trout-sized targets observed suggested that some may have been undetectable because of a nearshore or surface orientation. However, given that there were less than $(<)$ 50,000 total fish observed by hydroacoustics in August, and the majority of these fish appeared to be $<200 \mathrm{~mm}$, our estimate of the consumption demand of 50,000 hatchery-reared rainbow trout was likely a reasonable approximation of the consumption demand of all planktivorous fish in Swift Reservoir.

\section{Bioenergetics Model Inputs}

Bioenergetics models are energy balance equations that are commonly used to estimate the feeding rate (percent of maximum theoretical consumption; percent $\mathrm{C}_{\max }$ ) and the consumption rate (amount consumed in a given time; grams of prey/day) needed to achieve the growth observed by different age classes of consumers while accounting for variability in body size, thermal experience (daily temperature experienced by the consumer), and diet of the consumer (Ney, 1993). The models operate on 
a daily time step that enables simulations to track processes with high temporal resolution. These estimates depend on the energy densities of predators and prey and species-specific parameters that describe the temperature-dependent and allometric effects of body mass on consumption, metabolism, and waste (Hanson and others, 1997). We collected field data on growth, seasonal diet composition, and thermal experience to construct a set of model inputs specific to each planktivore population. We used stable isotope analysis to corroborate the diet composition data.

Temperature profiles and thermal experience.-Temperature profiles were recorded monthly from April to November at 1-m intervals from the surface to $30 \mathrm{~m}$ and at 2-m intervals from 30-60 $\mathrm{m}$ at two limnology stations located at one-third and two-thirds of the length of Swift Reservoir, Yale Lake, and Lake Merwin (fig. F1), and measurements were averaged between stations. Temperature profiles recorded by PacifiCorp in the late fall and winter months were used to complete the characterization of the seasonal thermal regime (PacifiCorp, 1999; PacifiCorp and Cowlitz, 2004). For each reservoir, vertical temperature profiles (fig. F2, table F3) were combined with the depth distribution of pelagic fish from hydroacoustics to calculate time- and depth-weighted thermal experience for bioenergetics simulations (Beauchamp and others, 2007).

Stable isotope analysis. - We characterized the food web structure of Swift Reservoir, Yale Lake, and Lake Merwin with stable isotopes to identify key trophic pathways that support or potentially limit juvenile salmonids and the other abundant resident fish that are likely to overlap in time and space with juvenile salmon (McIntyre and others, 2006). Stable isotope values of fin tissue provide an integrative signal of diet for several months to 1 year (depending on the growth rate of the consumer), providing a valuable complement to the instantaneous snapshot of diet composition provided by stomach content analysis. Tissue from representative samples of benthic invertebrates, terrestrial insects, zooplankton, planktivores, benthic feeding fish, and piscivores were immediately placed on ice in the field and then frozen within 12 hours for storage (Vander Zanden and Rasmussen, 2001). Zooplankton were identified to genus, and muscle tissue was removed from snails, Corbicula fluminea (Asian clams), and Pacifastacus leniusculus (signal crayfish). For fish samples, caudal fin tissue was clipped from three individuals within each 50-mm size class of each population. Samples were then dried at $60{ }^{\circ} \mathrm{C}$ for 48 hours, homogenized with mortar and pestle, and $0.4-0.6 \mathrm{mg}$ of the resulting material was weighed in aluminum capsules for analysis. Isotope samples were analyzed by the University of Washington IsoLab using a Costech Elemental Analyzer, Conflo III, and MAT253 for continuous flow-based measurement of solid organic material. The reference material was Vienna Pee Dee Belemnite for carbon $\left(\delta^{13} \mathrm{C}\right)$ and atmospheric $\mathrm{N}_{2}$ for nitrogen $\left(\delta^{15} \mathrm{~N}\right)$.

Diet composition and energy density of prey.-Seasonal diet analysis was conducted for key pelagic species to provide input data for bioenergetics modeling and to evaluate trophic interactions between resident and anadromous fish. Diet composition was evaluated for a range of sizes of the primary pelagic planktivores in each reservoir. Using a dissecting microscope, we identified stomach contents to taxonomic order for invertebrates and calculated diet proportions by wet weight (Chipps and Garvey, 2007). The energy densities of prey items were taken from literature values (table F4).

Age and growth.-A fish's mass at the beginning and ending dates of bioenergetics simulations was estimated based on scale analysis and observations of length and weight. Hatchery-reared rainbow trout and yearling Chinook salmon were weighed at the hatchery before stocking in Swift Reservoir (PacifiCorp and Cowlitz PUD, 2014) and again during sampling at the end of the growing season in November, providing an estimate of growth over this interval. Growth intervals for yearling Chinook salmon were used to generate feeding rates ( $p$-values or percent $\mathrm{C}_{\max }\left[\% \mathrm{C}_{\max }\right]$ ) which were then used to simulate the growth and consumption demand of subyearlings. For kokanee salmon, the length-at-age was back calculated from scale measurements using the Fraser-Lee method (Isely and Grabowski, 2007). Images and measurements of scales were recorded with Image-Pro ${ }^{\circ}$ digital image-analysis software and a digital camera mounted on a dissecting microscope [1.6-3.2 times magnification].

Kokanee salmon lengths were converted to weight based on weight-to-fork length (FL) regressions for Yale Lake (sample size $[\mathrm{n}]=36$, range $\left.=46-281 \mathrm{~mm} ; R^{2}=0.996, P<0.001\right)$ :

$$
\text { Weight }=0.00000198 \times F L^{3.3259}
$$

The same procedure was used for Lake Merwin ( $\mathrm{n}=179$, range=91-400 mm; $\left.R^{2} 0.964, P<0.001\right)$ :

$$
\text { Weight }=0.0000263 \times F L^{2.868}
$$

Seasonal-size frequency distributions were examined to partition growth, and thus fitted-consumption estimates, into fastand slow-growth seasons. The age-1 kokanee salmon captured in Lake Merwin in November 2013 had a mean fork length of $279 \mathrm{~mm}$, equal to the mean back-calculated length at second annulus for the previous year's cohort. Assuming approximately equal growth conditions between years, this suggests that the majority of annual growth occurred before December 1, which is consistent with cycles of zooplankton abundance. Therefore, we allocated 90 percent of annual growth in mass to the period between April 1 and November 30. Given that the thermal regimes and zooplankton cycles followed similar patterns between reservoirs, we allocated growth in this manner for all our simulations (table F5). 
Zooplankton.-We measured the monthly densities of crustacean zooplankton in Swift Reservoir, Yale Lake, and Lake Merwin to estimate the available food supply for resident and reintroduced anadromous salmonids. We conducted oblique tows with a Clarke-Bumpus sampler fitted with a 153-micrometer $(\mu \mathrm{m})$ mesh conical net at depths that represented the epilimnion, metalimnion, and hypolimnion during stratification and similar depths during spring and fall at both limnology stations in each body of water (fig. F1). We identified and enumerated $>50$ crustacean zooplankton in subsamples of each tow and measured the body length of the first 30 individuals of each genus encountered in each subsample using Image-Pro digital image-analysis software. Daphnia spp. were the most abundant zooplankton species found and the most significant prey item for juvenile salmonids during the growing season, so we focused our analysis on their abundance and distribution. Daphnia were measured from the top of the helmet to the base of the tail spine, and body length (in millimeters) was converted to wet mass (in micrograms) with the equation wet mass $=50 \times$ body length ${ }^{2.88}$ (Kuns and Sprules, 2000). Standing stock biomass was then calculated by multiplying the density of Daphnia by the mean wet mass and expanded based on the volume of water within each corresponding depth layer (Sorel and others, 2016). The number of Daphnia eggs was also enumerated in each sample to estimate zooplankton production rates with the egg-ratio method (Kuns and Sprules, 2000). We were not able to estimate production in November without data on zooplankton density in December.

Consumption demand to prey supply ratio and carrying capacity calculation.-The Wisconsin Bioenergetics Model (Hanson, 1997) parameterized for rainbow trout (Rand and others, 1993), kokanee salmon (Beauchamp and others, 1989), and Chinook salmon (Stewart and Ibarra, 1991) was used to estimate consumption demand. The bioenergetics model estimates the daily per-capita consumption needed to satisfy the observed growth of a mean individual from each population and age class. Simulations started at the beginning of the growing season (April 1) for kokanee salmon in Yale Lake and Lake Merwin and on the approximate stocking date (June 1) for hatchery-reared rainbow trout in Swift Reservoir (table F5). Simulations ended on November 30, which we considered as the end of the high-growth season, or at spawning. In each body of water, per-capita consumption by salmonids was expanded to the population level to reflect the abundance, size distribution, and seasonal consumption by each age class of consumer. Population-level consumption was compared to prey supply to evaluate potential food limitations.

The monthly ratio of consumption demand to prey supply was examined to estimate carrying capacity. We designated consumption demand equal to 50 percent of prey supply in the single month of greatest prey demand and supply during the growing season as a conservative estimate for carrying capacity, because the remaining 50 percent of the biomass could absorb additional sources of mortality and produce future generations of Daphnia (Beauchamp and others, 1995; Baldwin and others, 2000).

Daphnia consumption demand:supply ratios were created using both (1) standing stock biomass as a lower estimate of prey supply and (2) standing stock biomass plus production within depths thermally accessible to salmonids as a higher estimate of prey supply. Using only the standing stock biomass throughout the growing season represents the lowest, most restrictive estimate of food supply because production by Daphnia can provide significant additional biomass. Daphnia standing stock biomass plus production was used to represent higher (but still conservative) estimates of carrying capacity, again assuming that carrying capacity was exceeded when the demand:supply ratio was $>50$ percent in any month of the growing season. To simulate the ratio of prey demand and supply assuming salmonids were thermally excluded from the epilimnion during stratification (JulySeptember in Swift Reservoir and Yale Lake, July-October in Lake Merwin), we additionally compared consumption demand to prey supply below the epilimnion (metalimnion and hypolimnion) in those months.

For the month with the greatest demand:supply ratios, the difference between resident salmonids' consumption demand and 50 percent of prey supply was divided by the consumption demand of individual age- 0 salmonids to estimate the number of additional subyearling salmonids that could have been supported in each reservoir for 1 year. We used the estimate of prey supply that included standing stock biomass and production, but it was restricted to the metalimnion and hypolimnion during stratification as our best estimate for these calculations, as it created a moderate estimate of carrying capacity by including Daphnia production but excluded epilimnetic prey during stratification. Consumption demand of age-0 spring Chinook salmon in Swift Reservoir was estimated with a bioenergetics simulation using the feeding rate ( $p$-value or $\mathrm{C}_{\max }$ ) observed for age-1 fish during the growing season (104 percent) and an estimated starting mass of 0.2 grams on April 1. Age-0 kokanee salmon were used as a surrogate for estimating the consumption demand of subyearling salmon in Yale Lake and Lake Merwin. We consider kokanee salmon as a reasonable surrogate for Chinook salmon because both species feed on Daphnia in the limnetic zone and exhibit diet-vertical migrations (Koehler and others, 2006; Lowery and Beauchamp, 2010).

\section{Assessing the Effects of Anadromous Reintroductions on Bull Trout—Simulation Analyses}

We assessed the potential effects of anadromous reintroductions on bull trout in the upper Lewis River using an individualbased modeling framework (for example, Al-Chokhachy and others, 2015). We integrated field data from ongoing research and monitoring conducted by the Washington Department of Fish \& Wildlife and PacifiCorp and the results of this study with existing data for bull populations in the Pacific Northwest to parameterize the model. We used the modeling framework to evaluate 
three main objectives to assess to the effects of anadromous salmon reintroduction including: (1) reductions in the carrying capacity for juvenile bull trout because of overlap in resources; (2) potential effects of coho salmon redd superimposition on existing bull trout redds; and (3) reductions in subadult and adult bull trout carrying capacity, growth, and survival in reservoir environments as a result of residualized salmon. Our goal with this modeling effort was to evaluate the relative importance of each of these factors on the extant bull trout population. We focused our efforts on the bull trout population in Swift Reservoir given the current state of anadromous reintroductions in the upper Lewis River Basin.

\section{Model Inputs}

Growth.-We used individual-specific information from PIT-tagged subadult and adult bull trout (age-3 and above) for estimates of annual growth. We only included growth estimates where the interval between marking and recapture was $>300$ days to avoid the potential effects of handling on growth (for example, McCormick and others, 1998) and (or) erroneous extrapolations of annual growth estimates from short-interval recaptures. We accounted for differences in the interval between marking and recapture across individuals by scaling growth to 365 days. We assumed age- 3 bull trout were approximately $350 \mathrm{~mm}$ based on feedback from the Lewis River Bull Trout Working Group. We then used the individual growth data (fig. F3) to estimate size for subsequent ages using an exponential decay model of decreasing growth with age. Given that few estimates of age-2 adfluvial bull trout are available, we used this model to also estimate age- 2 growth within the reservoir environment. We used the size-atage information in Al-Chokhachy and others (2015) for age-1 fish (table F6) and considered bull trout emigration to the reservoir to occur primarily at age-2 (Fraley and Shepard, 1989; Downs and others, 2006; Johnston and others, 2007).

Survival.-We used a combination of local and literature-based estimates of survival at different ages of bull trout. For eggto-fry survival and age-1 survival, we relied on estimates from the literature (Bowerman and Budy, 2012; Bowerman and others, 2014) as no empirical data in the Lewis River was available for these age classes. Next, we used estimates of reservoir survival for age-6 and older from Swift Reservoir PIT-tag analyses (Stillwater Sciences, 2016). No estimates of juvenile through early adult (age-2-5) survival are currently available for adfluvial bull trout populations. As such, we linearly interpolated estimates for age-2- 5 between known estimates (age-1 and age-6, table F6).

Sexual maturity, frequency of spawning, and fecundity.-We relied on literature estimates of sexual maturity and the professional opinions of local biologists. Sexual maturity in adfluvial bull trout populations has been found to occur between age-4 and age-6 (Fraley and Shepard, 1989; Hagen and Taylor, 2001; Downs and others, 2006; Johnston and others, 2007). For this study, we assumed 25 percent of age- 4 fish, 50 percent of age- 5 fish, and 100 percent of fish older than age- 5 to be mature.

The spawning frequency of bull trout populations is largely considered to be annual unless populations are at extremely high densities (Johnston and Post, 2009). As such, we modeled bull trout spawning annually for bull trout in the Swift Reservoir population. Finally, we used an empirical, literature-based estimate of adfluvial bull trout length-fecundity from Johnston and others (2007) where fecundity $=0.00172 \times$ Fork Length ${ }^{2.31}$.

Baseline population model.-For each model run, we simulated bull trout population dynamics for 50 total years. To avoid bias associated with transient dynamics, we did not begin recording results until year 31 of each simulation ( $\mathrm{n}=20$ years per simulation). We used the IBM framework to identify and track individual outcomes of growth, survival, sex, and sexual maturity. For each individual, we randomly selected growth, survival, and sexual maturity values for each year based on the model inputs (for example, table F6). Given the high variability in egg survival (Bowerman and others, 2014), we modeled egg-to-fry survival using a beta distribution $(\alpha=21.33 ; \beta=2.99)$ based on field estimates of survival at this life stage. For all other age classes, we selected survival estimates from normal distributions based on the empirical and literature-derived estimates of the mean and variance of the normal distributions for each estimate. For maturity, we determined sexual maturity of individuals within an age class using proportional sampling methods.

We assumed density-dependent mechanisms would control survival and consequently, population size. We assumed density-dependent effects would occur at the emergence-to-juvenile stage consistent with previous studies (that is, fry to age-1; Van Kirk and Hill, 2007; Johnston and Post, 2009). We adjusted the carrying capacity of the population to where the mean annual adult population was approximately 275 adults based on existing redd count and abundance estimates and feedback from the Lewis River Bull Trout Working Group.

Model perturbations.-We assessed each of our objectives by perturbing the model based on likely interactions between anadromous salmon and bull trout (see the "Stream habitat" and "Reservoir habitat" sections in this chapter). First, we assessed the effects of changes in carrying capacity of early life-history stages as a result of substantial increases in the biomass represented in juvenile salmon. We reduced the carrying capacity by 20 percent given the overlap observed in juvenile-rearing habitat and diets in tributary habitat (see the "Distributional overlap" sections in this chapter).

To address the second objective (to assess distributional overlap between coho salmon and bull trout at different life stages and environments), we evaluated the effects of coho salmon redd superimposition by assuming 20 percent of the annual bull 
trout redds would be superimposed by adult coho salmon. Although this proportion is higher than observed in field studies, we used this estimate to quantify the population-level effects relative to other perturbations.

Our third perturbation focused on changes in the reservoir environment resulting from substantial increases in the biomass of juvenile salmon. Juvenile Chinook and coho salmon are spending considerable portions of the year in the reservoir environment (see "Assessment of Juvenile Fish Production and Outmigration Success on the Lewis River, Washington," chap. D of this report). In addition, collection information suggests a large portion of juvenile salmon are residualizing in Swift Reservoir either volitionally (for example, Perales and others, 2015) or because it is difficult to find the juvenile collection facility (for example, Beeman and others, 2016). Large increases in salmon densities may increase potential competition in the reservoir environment, thus increasing density-dependent mechanisms such as reduced growth and survival. Indeed, Johnston and Post (2009) found large changes in bull trout abundance led to substantial compensatory changes in population demographics and vital rates. For this study, we evaluated such effects by independently and simultaneously reducing growth and survival of all reservoir age classes by 20 percent.

\section{Results and Discussion}

\section{Stream Habitat}

Distributional overlap of bull trout and coho salmon.-During 2015, we snorkeled a total of $10.57 \mathrm{~km}$ of stream during two survey periods. The extent of sampling in 2015 was greater than during $2013(3.25 \mathrm{~km})$ and 2014 (2.86 km), largely because of spatial expansion of surveys, but also because multiple surveys were used in 2015. Surveys from 2013 and 2014 indicated relatively few coho salmon in the Pine Creek drainage (fig. F4). Although we observed considerably more juvenile coho salmon in $2014(\mathrm{n}=40)$ than in $2013(\mathrm{n}=2)$, the density of coho salmon appeared to be relatively low. However, in 2015, we found higher numbers of juvenile coho salmon in Pine Creek than observed in previous years (figs. F5, F6, F7). The largest densities of coho salmon were found in P3, in reaches of Pine Creek adjacent to P3, and in Rush Creek. Relatively few coho salmon were observed in P8 and the upper reaches of Pine Creek (figs. F5, F6). The apparent differences between the 2013 and 2014 data and the 2015 data in Pine Creek and tributaries are likely because of the timing of surveys, as the 2015 surveys were conducted earlier in the summer months, and sampling was done in different reaches (that is, clumped distribution of salmonids; Al-Chokhachy and others, 2009). The lower numbers of juvenile coho salmon during the later surveys in 2015 in Pine Creek and P8 suggest that juvenile coho salmon may emigrate downstream in the Pine Creek system during the summer, likely because of cold stream temperatures (Ebersole and others, 2006b) and the lack of slow-water habitat with cover (Bisson and others, 1988; Giannico and Healey, 1999). These conclusions are supported by the finding of the highest densities of juvenile coho salmon in and around P3, a tributary with habitat linked with high densities of juvenile coho salmon, and observations of juvenile coho salmon in side channel habitats.

The relative abundance of juvenile coho salmon varied substantially between tributaries. Despite the higher densities in 2015, juvenile coho salmon represent a low proportion of the total number of fish observed in the majority of reaches in 2013 (fig. F8A), 2014 (fig. F8B), and 2015 (fig. F9). This pattern is considerably different than results from P3 and Rush Creek (fig. F10), where juvenile coho salmon represent a large portion of the total number of fish observed. Together, these results suggest that overlap between juvenile coho salmon and bull trout does occur in key bull trout spawning and rearing tributaries, a pattern not surprising given recent results from Lowery and Beauchamp (2015). However, our results also suggest the degree of overlap varies considerably between streams.

Distributional overlap of acclimation spring Chinook and juvenile coho salmon.-The temporal overlap between acclimation spring Chinook and juvenile coho salmon and other species during 2014 and 2015 appears to be relatively short (fig. F11). The median days between release and emigration past the antenna in Clear Creek is 6 days (range $=3-362$ days). Approximately 60 percent of the 6,150 fish observed at the antenna emigrated by 7 days. However, more than 35 percent of the fish remained in Clear Creek for more than 50 days. These juvenile Chinook salmon that resided for more than 50 days in Clear Creek all occurred during 2014. It is unknown if fish that resided longer in Clear Creek were released directly into the river or were placed into the acclimation ponds adjacent to Clear Creek. The short time that hatchery-reared spring Chinook salmon remained within the tributaries suggests that the potential impacts on native fish within tributaries is likely to be minimal; however, the impacts may be more severe within the reservoir environment (see "Assessing the Effects of Anadromous Reintroductions on Bull Trout" section in this chapter).

Food web and community interactions.-Anadromous species can bring substantial nutrient resources to freshwater ecosystems (Gresh and others, 2000; Scheuerell and others, 2005). The influx of nutrients and forage can alter food webs (Bilby and others, 1998; Scheuerell and others, 2007) and, in some cases, enhance the growth and condition of freshwater fish (Wipfli and others, 2004; Guyette and others, 2013; Swain and others, 2014). In the upper Lewis River Basin, our results suggest small, 
subtle shifts in the community food webs as a result of the reintroduction and presence of wild juvenile coho salmon. Contrasting stable isotope data from pre-reintroduction (2009) and post-reintroduction in P8 suggests only small shifts in the forage resources utilized by juvenile bull trout (figs. F12A, F12B) but no shifts in trophic position (DeNiro and Epstein, 1978). This pattern is consistent with results for small $(<150 \mathrm{~mm})$ and larger $(>150 \mathrm{~mm})$ coastal cutthroat trout. Diet signatures from 2014 indicate coho salmon juveniles overlapped with coastal cutthroat trout in both trophic position and carbon sources, a pattern consistent with previous studies (Bilby and others, 1996). Our results from 2014 also suggest that juvenile bull trout and coho salmon feed at similar trophic levels and utilize generally similar prey resources. All three species appear to utilize a variety of food resources including larvae of Diptera (true flies) and Trichoptera (caddisflies), Lepidoptera spp., and adult Plecoptera (stoneflies). We found similar patterns for Rush Creek with coho salmon, coastal cutthroat trout, and bull trout demonstrating generally consistent trophic position and using similar forage resources (fig. F13). Our results are consistent with a recent study by Lowery and Beauchamp (2015) in the Skagit River, Washington, where juvenile bull trout in tributary habitats demonstrated high overlap in forage niche with coho salmon and juvenile steelhead.

Diet data for the different tributaries indicate seasonal differences in diet resources and diet overlap among the different species. During the late spring-summer, there was much diet overlap between juvenile coho salmon, bull trout, sculpin, and coastal cutthroat trout (fig. F14) with a high proportion of diets comprised of Ephemeroptera (mayflies), caddisflies, and true flies. In addition, diet data from all species except for sculpins suggest the importance of terrestrial inputs. Fall diet data suggest considerable shifts with diets of coastal cutthroat trout $<150$ millimeters shifting to a greater proportion of caddisflies (fig. F15). Data from juvenile coho salmon and coastal cutthroat trout (>150 millimeters) indicate continued use of terrestrial prey items. Together, these data suggest considerable overlap in diets from late spring through the summer, a period with typically low streamflow, but this overlap appears to attenuate during the fall.

We found surprisingly little evidence of predation on young-of-year anadromous salmon in our spring-summer isotope data. This is somewhat surprising given the extent of predation on young-of-year that can occur in tributaries with anadromous fish spawning (Tabor and others, 2012). The lack of evidence of predation on young-of-year coho salmon is likely because of the relatively low densities of juvenile coho salmon within bull trout distribution areas compared to tributaries with high densities of coho salmon redds (for example, Clear Creek; fig. F16).

Redd superimposition.-Between 2012 and 2014, adult coho salmon demonstrated a broad distribution of spawning activity and substantial evidence of spawning in Pine Creek and P8 (fig. F16). Based on existing redd data, we found that coho salmon spawning initiated after the majority of bull trout spawning occurred each year (60 percent of total bull trout spawning activity; fig. F17). Furthermore, we found that 80 percent of the coho salmon spawning occurred after the end of documented bull trout spawning. The observed timing is consistent or earlier than reported for many populations of coho salmon in the Pacific Northwest (Weitkamp and others, 1995), suggesting a high potential for temporal overlap and superimposition.

During 2014 and 2015, we conducted multiple redd surveys per week beginning in early October and continuing through mid-late November. Based on existing bull trout redd information and coho salmon spawning surveys, we confirmed evidence of coho salmon redd superimposition on existing bull trout redds in $2014(\mathrm{n}=2)$ but no evidence of coho salmon spawning in 2015. The lack of coho salmon spawning in 2015 , however, is likely driven by the fact that fewer adult coho salmon $(3,754)$ were transported to Swift Reservoir in 2015 than in previous years (for example, in 2014, when 9,179 fish were transported) and relative to recovery planning and yearly management targets of 7,500 fish (Lesko, 2018). Furthermore, 2015 represented anomalous in-tributary conditions with extremely low stream flows, which likely altered coho salmon spawn timing and site selection. Although bull trout and coho salmon historically overlapped across much of their distributions in the Pacific Northwest (Cavender, 1978) and redd superimposition was likely to be minimal to minimize the negative effects (Connell, 1980), the lack of natal homing for transported hatchery-reared coho salmon adults and variable stray rates (Keefer and Caudill, 2012) suggests the need for further monitoring.

\section{Reservoir Habitat}

Relative abundance of fish.-The primary pelagic planktivores captured by anglers and our sampling in Swift Reservoir were hatchery-reared rainbow trout and Chinook salmon smolts. Each spring, Swift Reservoir is stocked with approximately 50,000 hatchery-reared rainbow trout of catchable size (200-250 mm; PacifiCorp and Cowlitz, 2014). Stocking has historically been in April but was delayed until early June in 2013-2016 to avoid interactions with outmigrating anadromous salmonid smolts. Several thousand hatchery-reared yearling Chinook salmon smolts were released in tributaries of the Swift Reservoir in 2013 and 2014 as part of the reintroduction (PacifiCorp and Cowlitz, 2014). Fish typically migrate out in late spring and early summer, but some of these fish were captured in the reservoir in the fall (see "Assessment of Juvenile Fish Production and Outmigration Success on the Lewis River, Washington," chap. D of this report). Three-spine stickleback were another planktivore observed in 2013 and 2014. A relatively small population of adfluvial bull trout residing in Swift Reservoir consume invertebrates when they are juveniles but become highly piscivorous as adults (Lowery and Beauchamp, 2015). 
Kokanee salmon were the primary pelagic planktivore captured in Yale Lake in 2013 and 2014 (table F7). The population of kokanee salmon in Yale Lake is self-sustaining and spawns in October at age-3 (after their third growing season) in Cougar Creek, a tributary of the upper portion of the reservoir (fig. F1; PacifiCorp and Cowlitz, 2004). Other planktivores in Yale Lake include three-spine stickleback and juvenile sculpin. There is also a small population of adfluvial bull trout.

Kokanee salmon were the primary pelagic planktivore in Lake Merwin in 2013 and 2014 (table F7). Annually, approximately 45,000 kokanee salmon are stocked as fingerlings in fall, and another 48,000 are stocked as yearlings in spring (PacifiCorp and Cowlitz PUD, 2014). Natural production of kokanee salmon is considered minimal from Lake Merwin tributaries, although most fish mature at age-3 and attempt to spawn in September-October. The primary source of natural recruitment of kokanee salmon into Lake Merwin is considered to be entrainment from Yale Lake (PacifiCorp and Cowlitz, 2004. Northern pikeminnow are abundant in Lake Merwin and can be planktivorous as juveniles and piscivorous as adults (Petersen and Kitchell, 2001); however, they generally occupy nearshore habitats during spring and warmer shallower water in the limnetic zone during the summer, thus reducing the likelihood of predation on or competition with salmon (Brown and Moyle, 1981). The consumption demand of the northern pikeminnow population is examined in detail in this report (see "Piscine Predator Impact Studies 2013-2014, Lake Merwin, Washington," chap. E of this report) and is, therefore, not covered here.

Thermal experience and depth distribution of pelagic planktivores.-Thermal regimes and stratification have a large effect on metabolic rates driving consumption demand, vertical distribution of fish, and rates of zooplankton production. Epilimnetic temperatures in Swift Reservoir and Yale Lake fluctuated between $13-14{ }^{\circ} \mathrm{C}$ in May, and $22-23{ }^{\circ} \mathrm{C}$ in August. The hypolimnion remained at approximately $5{ }^{\circ} \mathrm{C}$ throughout the year (fig. F2). Epilimnetic temperatures were slightly warmer in Lake Merwin at $14-16{ }^{\circ} \mathrm{C}$ in May and peaked at $22-24{ }^{\circ} \mathrm{C}$ in July and August. Unlike Swift Reservoir and Yale Lake, the hypolimnion in Lake Merwin warmed from $5-6{ }^{\circ} \mathrm{C}$ in April to $15-18^{\circ} \mathrm{C}$ in October.

Vertical distributions of fish are influenced by a number of factors including prey density and distribution, temperature, and predation risk (Clark and Levy, 1988; Scheuerell and Schindler, 2003; Hardiman and others, 2004). Stratification strongly influenced the vertical distributions of salmonids during summer (Quinn and others, 2012). Epilimnetic temperatures exceeded the thermal preferences of salmonids during peak summer stratification in all three bodies of water, but the metalimnion remained usable year-round (fig. F2). In general, fish occupied the upper $40 \mathrm{~m}$ of the water column in Swift Reservoir during daylight in spring-fall (fig. F18). During dusk and night, most fish were in the upper 0-15 $\mathrm{m}$ of the water column in spring and fall and from 10-30 m during summer. A similar seasonal pattern was observed for kokanee salmon in Yale Lake and Lake Merwin; however, fish in Lake Merwin were more shallowly distributed in spring and fall, and deeply distributed in summer than in Yale Lake (figs. F19, F20). We expect that Chinook salmon would exhibit similar patterns of seasonal depth distribution and diel vertical migrations as do kokanee salmon (for example, Clark and Levy, 1988; Lowery and Beauchamp, 2010). In Yale Lake at night, fish were distributed at 5-40 m during spring and fall but were more concentrated at 15-30 m during the summer. In Lake Merwin, nighttime fish distribution was bimodal in spring and fall with a larger mode at $0-10 \mathrm{~m}$ and a smaller mode at $20-40 \mathrm{~m}$. As expected (Quinn and others, 2012), fish were distributed in a single deeper mode at 20-50 $\mathrm{m}$ in the summer in Lake Merwin. The deeper fish distributions observed in August relative to June in all three bodies of water suggest decreased access to epilimnetic prey resources during the peak of summer stratification.

Stable isotope analysis. - Diet and stable isotope analyses revealed that salmonids in these reservoirs depend on zooplankton and specifically Daphnia during the growing season, as expected (Spencer and others, 1991; Tabor and others, 1996), but insects provided a significant supplementary source of energy when Daphnia were less abundant. Fluctuations in water level, often resulting from drawdown, can alter the species composition of littoral and benthic macroinvertebrate communities, productivity, and timing of prey production (for example, Furey and others, 2006). Stable isotope analysis clarified the trophic levels and relative dependence on pelagic and benthic energy pathways of the species and size classes of organisms within the food webs and corroborated our identification of the key pelagic planktivore populations. Organisms representing bentho-littoral energy pathways, such as snails and small Catostomus macrocheilus (largescale suckers), were enriched in $\delta^{13} \mathrm{C}\left(\delta^{13} \mathrm{C}\right.$; delta c 13 , ratio of carbon isotopes ${ }^{12} \mathrm{C}$ and ${ }^{13} \mathrm{C}$ ), and organisms representing pelagic energy pathways, such as filter-feeding Daphnia were depleted of $\delta^{13} \mathrm{C}$ in all three bodies of water (figs. F21, F22, F23). Stable isotope signatures varied within and among species, size classes, trophic levels, and reservoirs according to position in and structure of each food web.

Three-spine sticklebacks and Chinook and coho salmon were the most dependent on pelagically derived nutrients in Swift Reservoir, whereas rainbow trout exhibited more benthic influence (fig. F21). Larger sculpin also appeared to rely on pelagic nutrients but could have been feeding on consumers of limnetically derived detritus. Kokanee salmon were the primary pelagic planktivores in Yale Lake and Lake Merwin (fig. F22; fig. 7 in "Piscine Predator Impact Studies 2013-2014, Lake Merwin, Washington," chap. E of this report.). As expected, kokanee salmon showed the greatest dependence on pelagic prey of all fish in Yale Lake as evidenced by their $\delta^{15} \mathrm{~N}>3 \%\left(\delta^{15} \mathrm{~N}\right.$, delta 15 , ratio of nitrogen isotopes ${ }^{15} \mathrm{~N}$ and $\left.{ }^{14} \mathrm{~N}\right)$ above zooplankton and $\delta^{13} \mathrm{C}$ values similar to Daphnia and other zooplankton (Stockwell and others, 1999). However, $\delta^{13} \mathrm{C}$ values for Daphnia and kokanee salmon were surprisingly dissimilar in Lake Merwin, a pattern warranting further investigation. Small northern pikeminnow also relied on pelagic prey to a large degree in Lake Merwin (see "Piscine Predator Impact Studies 2013-2014, Lake 
Merwin, Washington," chap. E of this report). Secondary consumers (that is, invertivores) were enriched in $\delta^{15} \mathrm{~N}$ relative to primary consumers, and piscivores were further enriched relative to invertivores.

Bull trout are often considered the top predator in natural lakes and reservoirs (Beauchamp and Van Tassell, 2001; Clarke and others, 2005; Guy and others, 2011). Our stable isotope results are consistent with that conclusion as we found bull trout at the top of the food web in both Yale Lake and Swift Reservoir (figs. F21, F22). In Yale Lake, bull trout $\delta^{13} \mathrm{C}$ values suggest coastal cutthroat trout, rainbow trout, and sculpin are likely prey resources. In Swift Reservoir, we evaluated bull trout forage resources using isotope samples collected prior to and after anadromous fish reintroductions (2013-2014). Our results indicate a considerable shift in prey resources with more benthically derived $\delta^{13} \mathrm{C}$ (fig. F21) for smaller (100-400 mm) and larger $(>400 \mathrm{~mm})$ bull trout since salmon reintroduction began. The $\delta^{13} \mathrm{C}$ values suggest bull trout are not utilizing coho or Chinook salmon as a food resource, a pattern which may be because of the large volume of water and low encounter rates with juvenile salmon. Concomitantly, recent results by Lowery and Beauchamp (2015) suggest the majority of fish consumed by even very large bull trout (for example, $700 \mathrm{~mm}$ ) are $<150 \mathrm{~mm}$. Given that acclimation Chinook salmon have been released at mean lengths of $134 \mathrm{~mm}$ (in 2015) and $148 \mathrm{~mm}$ (in 2014), our results suggest that even minimal growth would likely exceed the predation window by most bull trout. Juvenile coho salmon emigration, however, has been extremely variable across years (see "Assessment of Juvenile Fish Production and Outmigration Success on the Lewis River, Washington," chap. D of this report) and juvenile coho salmon emigrants collected at the Eagle Cliff screw trap during the spring (March-May) have been considerably smaller ( 2015 mean=102 mm; standard deviation=39). Together these data suggest that a substantial portion of juvenile coho salmon are likely available as prey resources for larger bull trout.

Diet analysis.-The results of stable isotope analyses were largely corroborated by stomach contents analyses. Stomach contents of age- $0-2$ kokanee salmon, age- 1 hatchery-reared rainbow trout, and age- 1 Chinook salmon (the primary pelagic planktivores based on relative abundance and stable isotope analysis) all exhibited similar seasonal trends in the proportions of zooplankton and insects consumed (table F8). Cladocera (clodocerans), including mostly Daphnia, represented 54-68 percent of diets in spring, with immature aquatic insects (10-36 percent) contributing most of the remainder. Cladocerans increased to 76-100 percent of diets during summer when they were more available and 86-89 percent in fall. Overall, Daphnia was the most common prey consumed, which emphasized the focus on Daphnia abundance and distribution as a determining factor in carrying capacity. In Swift Reservoir and Lake Merwin, Copepoda (copepods) represented $<2$ percent of stomach contents across species and seasons but were somewhat more prevalent in diets of kokanee salmon in Yale Lake (6-16 percent). Holopedium gibberum, a Cladoceran encased in a gelatinous shell, were common in zooplankton samples from Yale Lake (mean=3.5 per liter (L), standard error[SE] $=2.1 / \mathrm{L}$ in the epilimnion), and Swift Reservoir and Lake Merwin to a lesser degree but were rare in diets. Terrestrial insects represented a small proportion of diets (mean $=5.0$ percent, $\mathrm{SE}=1.5$ percent) compared to aquatic insects (mean=11.5 percent, $\mathrm{SE}=3.5$ percent), and both were most common in spring followed by fall. Rainbow trout diets suggested a higher reliance on zooplankton (59-100 percent during spring and fall), a pattern contrary to previous studies (Clarke and others, 2005) and our isotope results. However, only four samples of rainbow trout diets were analyzed to represent the summer season and some sampling occurred during the peak of a Daphnia bloom, so the results of stomach contents analysis likely underrepresent the true proportion of benthic invertebrates in their summer diet composition. Although rainbow trout had nearly doubled in weight since release, the isotopic signature of their hatchery feed (enriched in $\delta^{15} \mathrm{~N}$ and $\delta^{13} \mathrm{C}$ ) may have influenced their values, further contributing to their seemingly benthic signature.

Zooplankton.-A strong late spring peak in Daphnia occurred in all reservoirs between May and July, with a smaller secondary peak in September-October (fig. F24). In general, moderate to high densities of Daphnia (2.5-26 individuals per L) persisted in the epilimnion and metalimnion throughout the growing season in all three reservoirs. Densities in the epilimnion were generally twice that of the metalimnion, and hypolimnetic densities were always below 1 Daphnia/L with the exception of May-August in Lake Merwin.

Daphnia standing stock biomass followed similar patterns as density in all three reservoirs but was somewhat less variable than density across months as Daphnia production was highly variable and did not follow the same progression as density. The dampening of the fluctuation in Daphnia biomass relative to density resulted from the inverse relationship between mean individual mass and density because of increased production of smaller individuals when density increased (fig. F24). Production varied widely among months from a fraction to an order of magnitude greater than standing stock biomass (figs. F25, F26, F27). Many months of high production translated into increases in biomass the following month (and vice versa) as expected; however, significant production did not always translate into increased biomass. This could have been a result of a few different potential sources of error in our estimates of production. Monthly sampling events were too far apart to capture all the dynamics of egg ratios, which are notoriously variable across short intervals. Further, we did not account for entrainment out of reservoirs, and our egg-production model did not incorporate information on Daphnia age structure (Lynch, 1982; Brett and others, 1992). Despite these potential sources of bias, we consider our production estimates to be reliable for estimating salmon carrying capacity.

Kokanee salmon abundance.-Kokanee salmon were approximately one-half as abundant in Lake Merwin as in Yale Lake, but annual survival rates were similar. We estimated that the annual survival rate for kokanee salmon in Yale Lake was 
35 percent and that in August there were 396,262 age-0; 139,847 age-1; and 49,354 age-2 kokanee salmon. We estimated that the annual survival rate for kokanee salmon in Lake Merwin was 32 percent and that in August there were 189,937 age-0; 60,105 age-1; and 19,020 age-2 kokanee salmon. The observed survival rates are considerably higher than estimated from a study using similar sampling methods in Lake Billy Chinook (25 percent; Beauchamp and Van Tassell, 2001); the difference may be attributed to differences in angling pressure.

Resident planktivore consumption demand to prey supply ratios and carrying capacity.-Exceeding the carrying capacity of the reservoir for pelagic planktivores would have pronounced negative effects on juvenile growth, survival, and production of salmon smolts (Hyatt and Stockner, 1985; Edmundson and Mazumder, 2001). Even in a month of high prey availability, fish can only consume a limited amount of prey as dictated by stomach volume and digestion rate. In months of low prey availability, consumption by juvenile salmon is limited by the rate at which they can capture prey (as a function of their size-dependent foraging abilities and prey densities), and antipredation behaviors that limit their ability to forage at certain times of day or at certain depths (Stockwell and Johnson, 1999; Koski and Johnson, 2002). Significant prey shortages during the growing season would hinder their ability to acquire enough energy to effectively fight disease and survive. Furthermore, food limitation and size-dependent predation risk promotes risk-taking behavior, which increases predation mortality (Biro and others, 2005).

Our simulations indicated that the Swift Reservoir, Yale Lake, and Lake Merwin all offered a moderate surplus of Daphnia to support consumption demand by reintroduced anadromous salmonids, but a significant portion of prey was also consumed by resident pelagic planktivores. Resident pelagic planktivores fed at moderate to high levels relative to their potential maximum in all three reservoirs (59-123\% $\mathrm{C}_{\max }$; table $\left.\mathrm{F} 5\right)$, but their consumption demand fluctuated based on ambient water temperatures and mean fish size (figs. F25, F26, F27). Kokanee salmon in Lake Merwin had the highest feeding rate $\left(92-123 \% \mathrm{C}_{\max }\right)$ across age classes from April through November, while the feeding rate was intermediate for hatchery-reared rainbow trout in Swift Reservoir $\left(78 \% \mathrm{C}_{\max ) \text { and }}\right.$ kokanee salmon in Yale Lake $\left(59-86 \% \mathrm{C}_{\max }\right.$ table F5). Consumption demand on Daphnia by the planktivore populations in all three reservoirs started at moderate levels in April-June (20-34 metric tons [MT]/month), peaked in AugustOctober (33-55 MT]/month), then declined with decreasing water temperatures (figs. F25, F26, F27).

Swift Reservoir had ample prey resources available from May to August, as well as October-November, suggesting that fish would have access to plenty of food during the critical stages of reservoir entry in spring, and again in late summer-fall when fish store energy before winter. The ratio of consumption demand by rainbow trout to prey supply in Swift Reservoir started low in June and increased through September (fig. F25A). Consumption demand to Daphnia supply ratios were $<10$ percent of standing stock biomass in June (prior to stratification and with all depth layers summed) but increased in July-September once stratification was established. Consumption demand was 21 percent of the most conservative estimate of prey supply. Biomass within the metalimnion and hypolimnion (epilimnion thermally inaccessible) in July and increased to 56 percent in August and 96 percent in September (figs. 25B,C). However, consumption demand was $<30$ percent of biomass in all months examined if the whole water column was considered. The demand:supply ratio if production was also included was $<30$ percent in the metalimnion and hypolimnion and $<15$ percent in the whole water column in all months examined (fig. 25D). Biomass was 5-10 times greater than consumption demand in October-November. These ratios suggest that a moderate amount of surplus prey would be available to support additional demand if production is considered in addition to biomass. There was a surplus of approximately 9 MT of Daphnia biomass and production in the metalimnion and hypolimnion in September, the month of highest demand:supply ratios, which could have been consumed without exceeding the 50 percent carrying capacity cutoff according to our simulations. Dividing this biomass by the estimated consumption demand of a subyearling spring Chinook salmon in Swift Reservoir yielded an estimate of roughly 150,000 juvenile salmon that could have been supported during their first year by the surplus prey in the month of greatest prey demand:supply ratio.

Simulated consumption demand of kokanee salmon in Yale Lake (fig. F26A) exceeded Daphnia standing stock biomass plus production in April and May, as Daphnia did not begin to bloom until June (figs. F26B, C). Consumption demand in June was 61 percent of biomass but only 16 percent of biomass plus production. During peak thermal stratification from July through September, consumption demand was 39-122 percent of biomass in the metalimnion and hypolimnion or 22-37 percent of biomass plus production. Across the entire water column from July through September, consumption demand was 22-65 percent of biomass or 7-15 percent of biomass plus production (fig. F26D). Simulated consumption was 66-70 percent of the biomass in October-November and 17 percent of biomass plus production in October (production data not available for November). These ratios suggest that a moderate amount of surplus prey would be available during the summer growing season to support additional demand in Yale Lake if production is considered in addition to standing stock biomass. The surplus biomass and production of Daphnia in the metalimnion and hypolimnion in September, the month of highest demand:supply ratio during the summer growing season, without exceeding 50 percent of prey supply, was roughly 14 MT. Dividing this by the consumption demand of an individual age- 0 kokanee salmon (as a surrogate for a subyearling salmon) yielded an estimate of an additional 330,000 fish that could have been supported in the reservoir through their first year.

In April, before the major bloom of Daphnia was established, our simulated consumption demand for kokanee salmon in Lake Merwin (fig. F27A) was 143 percent of standing stock biomass of Daphnia but only 8 percent of biomass plus production (fig. F27B, C). The consumption demand to prey supply ratio dropped significantly when the spring Daphnia bloom was 
established in May and was $<15$ percent of biomass in May-June (fig. F27D). Consumption demand increased, and prey supply began to decrease in July-August, with demand:supply ratios increasing to 33-36 percent of biomass in the metalimnion and hypolimnion, and 20-28 percent in the whole water column. Consumption demand began to outstrip prey supply in September-October when it was 94-125 percent of biomass and 37-65 percent of biomass plus production in the metalimnion and hypolimnion. The demand:supply ratio in September-October, when Daphnia in the entire water column was included, was 41-57 percent biomass and 18-22 percent biomass plus production. Simulated prey demand was approximately twice the biomass in November. Overall, Lake Merwin also offers a moderate surplus of prey resources from April through September. Dividing surplus biomass and production in the metalimnion and hypolimnion in September, the month of highest demand:supply ratios during the summer growing season, by the consumption demand of an individual age- 0 kokanee salmon yielded an estimate of 130,000 additional subyearling fish that could have been supported in the reservoir for their first year.

Overall, our results indicate that the seasonal supply and depth-distribution of Daphnia are important factors regulating the carrying capacity of Swift Reservoir, Yale Lake, and Lake Merwin for resident and juvenile anadromous Salmonids, which will share zooplankton resources. Our findings are particularly relevant given the effects of size on juvenile salmon survival (Ebersole and others, 2006a; Pess and others, 2011). Furthermore, Zabel and Achord (2004) found that the length of juvenile Chinook salmon in the Salmon River Basin had a strong effect on survival to outmigration within populations but not across multiple populations. The results from Zabel and Achord (2004) also suggest the potential for size-dependent survival advantages at subsequent life stages. Size has also been found important for juvenile Chinook salmon in California's Central Valley, where larger and faster growing individuals at marine entry exhibited greater survival during years when overall survival to adulthood was lower for their cohort (Woodson and others, 2013). Together, these results illustrate the importance of considering the capacity of the reservoir and lake environments, which likely will influence the overall success of anadromous reintroductions.

\section{Assessing the Effects of Anadromous Reintroductions on Bull Trout}

We employed the use of a population model, not to necessarily emulate the "truth" but to consider potential effects of reintroduced salmon populations on extant bull trout populations and also identify potential data gaps in our current knowledge (Conroy and Moore, 2001). The "baseline" population model with demographic stochasticity alone resulted in a simulated population of more than 250 adult bull trout (fig. F28). Within any simulation run, our models demonstrated considerable interannual variability, a result that is consistent with long-term monitoring of bull trout populations (Kovach and others, 2017).

Results from our perturbations (fig. F29) indicate that reductions in the carrying capacity of bull trout tributary streams (fig. F30) and reservoir and lake survival (fig. F31) would likely have the largest effects on the existing bull trout population. We considered reductions in carrying capacity to be relevant given the considerable overlap in distribution and forage patterns observed in our field studies and the effects of reduced carrying capacity on bull trout recruitment (Ratliff and others, 2015). In general, there is a paucity of information describing salmon-bull trout interactions, particularly at the early life stages. Lowery and Beauchamp (2015) found considerable overlap in both distribution and forage. Under such natural conditions, competitive interactions are likely minimized as a result of niche segregation. However, the bull trout population in the upper Lewis River Basin remains relatively small with limited distribution. While adult anadromous salmon can contribute substantial nutrient inputs to headwater environments (Naiman and others, 2002; Kohler and others, 2012), juvenile salmon can also represent substantial vectors for exporting nutrients (Kohler and others, 2013). Indeed, the net benefit to freshwater ecosystems can be highly variable (Janetski and others, 2009). Our results suggest any considerable reductions in carrying capacity within this distribution may lead to declines in the abundance of bull trout populations.

In general, adfluvial bull trout populations demonstrate considerable resiliency, likely because of the stability of lentic habitat (Kovach and others, 2017). While bull trout populations sympatric with kokanee salmon can be particularly robust with large, adfluvial spawners (Johnston and others, 2007; Ratliff and others, 2015), there remains a paucity of information regarding anadromous salmon and steelhead reintroductions. Our results indicate the greatest risk to bull trout in the upper Lewis River Basin would occur through reductions in bull trout survival in the reservoir environment (fig. F31) and the populationlevel consequences of such changes. For example, in addition to reductions in abundance, our results also indicate reductions in survival may lead to considerably higher interannual variations in abundance (coefficient of variation $[\mathrm{CV}]=0.22$ ) relative to baseline conditions $(\mathrm{CV}=0.17)$. A concern for bull trout in the studied environments is the seemingly large number of residualized salmon (see "Assessment of Juvenile Fish Production and Outmigration Success on the Lewis River, Washington," chap. D of this report) that, with even moderate growth, may escape predation by most bull trout (Lowery and Beauchamp, 2015). Increased density dependence can have significant effects on bull trout survival and recruitment (Johnston and Post, 2009; Ratliff and others, 2015). However, our early food web results suggest that bull trout and coho and Chinook salmon in Swift Reservoir are utilizing different forage resources. It remains unclear how the forage base and food web will change throughout the reintroduction, but if competition for forage increases, particularly if residualized salmon exceed bull trout gape limitations of bull trout (Lowery and Beauchamp, 2015) and yearly smolts exceed capacity, there may be negative consequences for bull trout. 
Our simulations indicate even moderate levels of redd superimposition are unlikely to have large effects on bull trout. These results are likely driven by compensatory mechanisms associated with density dependence (for example, Paul and others, 2000). It is unclear, however, how superimposition may exacerbate variability in population abundance when coupled with substantial environmental stochastic events.

\section{Conclusions}

Historically, bull trout and coho and Chinook salmon likely occurred sympatrically in the upper Lewis River Basin with varying degrees of overlap across different life stages. Since the 1930s, however, there have been large changes in the upper Lewis River Basin resulting from habitat alterations (for example, building of hydropower facilities, the eruption of Mount Saint Helens), which have altered the physical and hydrologic template. As such, there continues to be uncertainty in how anadromous reintroductions will influence native salmonids such as bull trout. Further, there remains very limited information about bull trout interactions with juvenile salmon in reservoirs and lakes across the Pacific Northwest to provide guidance. In the upper Lewis River, the low proportion of juvenile salmon at downstream collectors and bull trout forage patterns (see the "Assessing the Effects of Anadromous Reintroductions on Bull Trout" section in this chapter) suggest a large portion of juvenile salmon may not be available as prey to all but the largest bull trout. Because it is unclear how increases in biomass in the reservoir and lake environments will alter the existing food webs and, concomitantly, affect bull trout at different life stages, continued monitoring is needed.

\section{References}

Al-Chokhachy, R., and Budy, P., 2008, Demographic characteristics, population structure, and vital rates of a fluvial population of bull trout in Oregon: Transactions of the American Fisheries Society, v. 137, no. 6, p. 1709-1722.

Al-Chokhachy, R., Budy, P., and Conner, M., 2009, Detecting declines in the abundance of a bull trout (Salvelinus confluentus) population-Understanding the accuracy, precision, and costs of our efforts: Canadian Journal of Fisheries and Aquatic Sciences, v. 66, no. 4, p. 649-658.

Al-Chokhachy, R., Moran, S., McHugh, P.A., Bernall, S.R., Fredenberg, W., and DosSantos, J.M., 2015, Consequences of actively managing a small bull trout population in a fragmented landscape: Transactions of the American Fisheries Society, v. 144 , p. $515-531$.

Anderson, J.H., Pess, G.R., Carmichael, R.W., Ford, M.J., Cooney, T.D., Baldwin, C.M., and McClure, M.M., 2014, Planning Pacific salmon and steelhead reintroductions aimed at long-term viability and recovery: North American Journal of Fisheries Management, v. 34, no. 1, p. 72-93.

Baldwin, C.M., Beauchamp, D.A.and Van Tassell, J.J., 2000, Bioenergetic assessment of temporal food supply and consumption demand by salmonids in the Strawberry Reservoir food web: Transactions of the American Fisheries Society, v. 129, no. 2, p. $429-450$.

Baxter, C.V., and Hauer, F.R., 2000, Geomorphology, hyporheic exchange, and selection of spawning habitat by bull trout (Salvelinus confluentus): Canadian Journal of Fisheries and Aquatic Sciences, v. 57, no. 7, p. 1470-1481.

Baxter, J.S., and McPhail, J.D., 1996, Bull trout spawning and rearing habitat requirements-Summary of the literature: [British Columbia] Ministry of Environment, Lands and Parks, Fisheries Technical Circular 98, 25 p.

Baxter, J.S., and McPhail, J.D., 1999, The influence of redd site selection, groundwater upwelling, and over-winter incubation temperature on survival of bull trout (Salvelinus confluentus) from egg to alevin: Canadian Journal of Zoology (Revue Canadienne De Zoologie), v. 77, no. 8, p. 1233-1239.

Baxter, J.S., and Westover, W.T., 2000, An overview of the Wigwam River bull trout program (1995-1999)—Habitat Conservation Trust Fund final report: [British Columbia] Ministry of Water, Land, and Air Protection, Fisheries Project Report KO58, 23 p.

Beamesderfer, R.C., and Rieman, B.E., 1988, Size selectivity and bias in estimates of population statistics of smallmouth bass, walleye, and northern squawfish in a Columbia River reservoir: North American Journal of Fisheries Management, v. 8, p. 505-510. 
Beamesderfer, R.C.P., Ward, D.L., and Nigro, A.A., 1996, Evaluation of the biological basis for a predator control program on northern squawfish (Ptychocheilus oregonensis) in the Columbia and Snake rivers: Canadian Journal of Fisheries and Aquatic Sciences, v. 53, no. 12, p. 2898-2908.

Bean, J., 2012, Multi-scale hydrogeomorphic influences on bull trout spawning habitat in snowmelt-dominated headwater streams: Missoula, Mont., University of Montana, M.S. thesis, 100 p.

Bear, E.A., McMahon, T.E., and Zale, A.V., 2007, Comparative thermal requirements of westslope cutthroat trout and rainbow trout-Implications for species interactions and development of Thermal protection standards: Transactions of the American Fisheries Society, v. 136, no. 4, p. 1113-1121.

Beauchamp, D.A., Luecke, C., Wurtsbaugh, W.A., Gross, H.G., Budy, P.E., Spaulding, S., Dillenger, R., and Gubala, C.P., 1997, Hydroacoustic assessment of abundance and diel distribution of sockeye salmon and kokanee in the Sawtooth Valley Lakes, Idaho: North American Journal of Fisheries Management, v. 17, p. 253-267.

Beauchamp, D.A., Stewart, D.J., and Thomas, G.L., 1989, Corroboration of a bioenergetics model for sockeye salmon: Transactions of the American Fisheries Society, v. 118, p. 597-607.

Beauchamp, D.A., LaRiviere, M.G., and Thomas G.L., 1995, Evaluation of competition and predation as limits to juvenile Kokanee and sockeye salmon production in Lake Ozette, Washington: North American Journal of Fisheries Management, v. 15 , no. 1 , p. $121-135$.

Beauchamp, D.A., and Van Tassell, J.J., 2001, Modeling seasonal trophic interactions of adfluvial bull trout in Lake Billy Chinook, Oregon: Transactions of the American Fisheries Society, v. 130, no. 2, p. 204-216.

Beauchamp, D.A., Wahl, D.H., and Johnson, B.M., 2007, Predator \& prey interactions, chap. 16 of Guy, C.S., and Brown, M.L., eds., Analysis and interpretation of freshwater fisheries data: Bethesda, Md., American Fisheries Society, p. 765-842.

Beeman, J.W., Evans, R.E., Haner, P.V., Hansel, H.C., Hansen, A.C., Hansen, G.S., Hatton, T.W., Sprando, J.M., Smith, C.D., and Adams, N.S., 2016, Evalauation of the biological and hydraulic performance of the portable floating fish collector at Cougar Reservoir and Dam, Oregon, 2014: U.S. Geological Survey Open-File Report 2016-1003, 143 p., accessed November 8, 2018, at https://pubs.usgs.gov/of/2016/1003/ofr20161003.pdf.

Bilby, R.E., Fransen, B.R., and Bisson, P.A., 1996, Incorporation of nitrogen and carbon from spawning coho salmon into the trophic system of small streams-Evidence from stable isotopes: Canadian Journal of Fisheries and Aquatic Sciences, v. 53, p. 164-173.

Bilby, R.E., Fransen, B.R., Bisson, P.A., and Walter, J.K., 1998, Response of juvenile coho salmon (Oncorhynchus kisutch) and steelhead (Oncorhynchus mykiss) to the addition of salmon carcasses to two streams in southwestern Washington, USA: Canadian Journal of Fisheries and Aquatic Sciences, v. 55, no. 8, p. 1909-1918.

Biro, P.A., Post, J.R., and Abrahams, M.V., 2005, Ontogeny of energy allocation reveals selective pressure promoting risk-taking behaviour in young fish cohorts: Proceedings of the Royal Society-B. Biological Sciences, v. 272, no. 1571, p. 1443-1448.

Bisson, P.A., Sullivan, K., and Nielsen, J.L., 1988, Channel hydraulics, habitat use, and body form of juvenile coho salmon, steelhead, and cutthroat trout in streams: Transactions of the American Fisheries Society, v. 117, p. 262-273.

Bonar, S.A., Bolding, B.D., Divens, M., and Meyer, W., 2005, Effects of introduced fishes [sic] on wild juvenile coho salmon in three shallow Pacific Northwest lakes: Transactions of the American Fisheries Society, v. 134, no. 3, p. 641-652.

Bowerman, T., and Budy, P., 2012, Incorporating movement patterns to improve survival estimates for juvenile bull trout: North American Journal of Fisheries Management, v. 32, no. 6, p. 1123-1136.

Bowerman, T., Neilson, B.T., and Budy, P., 2014, Effects of fine sediment, hyporheic flow, and spawning site characteristics on survival and development of bull trout embryos: Canadian Journal of Fisheries and Aquatic Sciences, v. 71, no. 7, p. $1059-1071$.

Brett, M.T., Martin, T.L., and Kaweck, T.J., 1992, An experimental test of the egg-ratio method-Estimated versus observed death rates: Freshwater Biology, v. 28, p. 237-248.

Brown, L.R., and Moyle, P.B., 1981, The impact of squawfish on salmonid populations-A review: North American Journal of Fisheries Management, v. 1, p. 772-782. 
Budy, P., Al-Chokhachy, R., and Thiede, G.P., 2004, Bull trout population assessment and life-history characteristics in association with habitat quality and land use-A template for recovery planning: Logan, Utah, U.S. Geological Survey Utah Cooperative Fish and Wildlife Research Unit.

Budy, P., Al-Chokhachy, R., and Thiede, G.P., 2007, Bull trout population assessment in northeastern Oregon—A template for recovery planning: Logan, Utah, U.S. Geological Survey Utah Cooperative Fish and Wildlife Research Unit.

Budy, P., Epstein, D., Bowerman, T., and Thiede, G., 2012, Bull trout population assessment in northeastern Oregon-A template for recovery planning: Logan, Utah, U.S. Geological Survey Utah Cooperative Fish and Wildlife Research Unit.

Carey, M.P., Sanderson, B.L., Barnas, K.A., and Olden, J.D., 2012, Native invaders-Challenges for science, management, policy, and society: Frontiers in Ecology and the Environment, v. 10, no. 7, p. 373-381.

Cavender, T.M., 1978, Taxonomy and distribution of bull trout, Salvelinus confluentus (Suckley), from the American Northwest: California Fish and Game, v. 64, p. 139-174.

Chipps, S.R., and Garvey, J.E., 2007, Assessment of diets and feeding patterns, chap. 11 of Guy, C.S., and Brown, M.L., eds., Analysis and interpretation of freshwater fisheries data: Bethesda, Md., American Fisheries Society, p. 473-514.

Clark, C.W., and Levy, D.A., 1988, Diel vertical migrations by juvenile sockeye salmon and the antipredation window: The American Naturalist, v. 131, p. 271-290.

Clarke, L.R., Vidergar, D.T., and Bennett, D.H., 2005, Stable isotopes and gut content show diet overlap among native and introduced piscivores in a large oligotrophic lake: Ecology of Freshwater Fish, v. 14, no. 3, p. 267-277.

Connell, J.H., 1980, Diversity and the coevolution of competitors, or the ghost of competition past: Oikos, v. 35, p. 131-138.

Conroy, M.J., and Moore, C.T., 2001, Simulation models and optimal decision making in natural resource management, in Shenk, T.M., and Franklin, A.B., eds., Resource management—Development, interpretation, and applications: Washington, D.C., Island Press, p. 91-104.

DeNiro, M.J., and Epstein, S., 1978, Influence of diet on the distribution of carbon isotopes in animals: Geochimica et Cosmochimica Acta, v. 42, p. 495-506.

DeVries, P., 1997, Riverine salmonid egg burial depths—-Review of published data and implications for scour studies: Canadian Journal of Fisheries and Aquatic Sciences, v. 54, no. 8, p. 1685-1698.

Docker, M.F., Dale, A., and Heath, D.D., 2003, Erosion of interspecific reproductive barriers resulting from hatchery supplementation of rainbow trout sympatric with cutthroat trout: Molecular Ecology, v. 12, no. 12, p. 3515-3521.

Donald, D.B., and Alger, D.J., 1993, Geographic-distribution, species displacement, and niche overlap for lake trout and bull trout in mountain lakes: Canadian Journal of Zoology (Revue Canadienne De Zoologie), v. 71, no. 2, p. 238-247.

Downs, C.C., Horan, D., Morgan-Harris, E., and Jakubowski, R., 2006, Spawning demographics and juvenile dispersal of an adfluvial bull trout population in Trestle Creek, Idaho: North American Journal of Fisheries Management, v. 26, no. 1, p. 190-200.

Dunham, J., Rieman, B., and Davis, K., 2001, Sources and magnitude of sampling error in redd counts for bull trout: North American Journal of Fisheries Management, v. 21, no. 2, p. 343-352.

Ebersole, J.L., Wigington, P.J., Jr., Baker, J.P., Cairns, M.A., and Church, M.R., 2006a, Juvenile coho salmon growth and survival across stream network seasonal habitats: Transactions of the American Fisheries Society, v. 135, p. 1681-1697.

Ebersole, J.L., Wigington, P.J., Jr., Baker, J.P., Cairns, M.A., Church, M.R., Hansen, B.P., Miller, B.A., LaVigne, H.R., Compton, J.E., and Leibowitz, S.G., 2006b, Juvenile coho salmon growth and survival across stream network seasonal habitats: Transactions of the American Fisheries Society, v. 135, no. 6, p. 1681-1697.

Edmundson, J.A., and Mazumder, A., 2001, Linking growth of juvenile sockeye salmon to habitat temperature in Alaskan lakes: Transactions of the American Fisheries Society, v. 130, no. 4, p. 644-662.

Eliason, E.J., Clark, T.D., Hague, M.J., Hanson, L.M., Gallagher, Z.S., Jeffries, K.M., Gale, M.K., Patterson, D.A., Hinch, S.G., and Farrell, A.P., 2011, Differences in thermal tolerance among socke salmon populations: Science, v. 332, no. 6025, p. 109-112. 
Fausch, K.D., 1989, Do gradient and temperature affect distributions of, and interactions between, brook charr (Salvelinus fontinalis) and other resident salmonids in streams?: Physiology and Ecology Japan, Special Volume 1, p. 303-322.

Fraley, J.J., and Shepard, B.B., 1989, Life history, ecology and population status of migratory bull trout (Salvelinus confluentus) in the Flathead Lake and river system, Montana: Northwest Science, v. 63, no. 4, p. 133-143.

Furey, P.C., Nordin, R.N., and Mazumder, A., 2006, Littoral benthic macroinvertebrates under contrasting drawdown in a reservoir and a natural lake: Journal of the North American Benthological Society, v. 25, no. 1, p. 19-31.

Geist, D.R., and Dauble, D.D., 1998, Redd site selection and spawning habitat use by fall chinook salmon-The importance of geomorphic features in large rivers: Environmental Management, v. 22, no. 5, p. 655-669.

Giannico, G.R., and Healey, M.C., 1999, Ideal free distribution theory as a tool to examine juvenile coho salmon (Oncorhynchus kisutch) habitat choice under different conditions of food abundance and cover: Canadian Journal of Fisheries and Aquatic Sciences, v. 56, no. 12, p. 2362-2373.

Glova, G.J., 1987, Comparison of allopatric cutthroat trout stocks with those sympatric with coho salmon and sculpins in small streams: Environmental Biology of Fishes, v. 20, no. 4, p. 275-284.

Gray, M., 2005, Oregon plan for salmon and watersheds-Oregon coastal coho assessment—Introduced fishes impacts: Salem, Oreg., Oregon Department of Fish and Wildlife, 31 p.

Gregory, R.S., and Levings, C.D., 1996, The effects of turbidity and vegetation on the risk of juvenile salmonids, Oncorhynchus spp., to predation by adult cutthroat trout, O. clarkii: Environmental Biology of Fish, v. 47, no. 3, p. 279-288.

Gresh, T., Lichatowich, J.A., and Schoonmaker, P., 2000, An estimation of historic and current levels of salmon production in the northeast Pacific ecosystem-Evidence of a nutrient deficit in the freshwater systems of the Pacific Northwest: Fisheries, v. 25 , p. $15-21$.

Gunckel, S.L., 2001, Feeding behavior and diet of native bull trout Salvelinus confluentus and introduced brook trout S. fontinalis in two eastern Oregon streams: Corvallis, Oreg., Oregon State University, M.S. thesis, 97 p.

Guy, C.S., McMahon, T.E., Fredenberg, W.A., Smith, C.J., Garfield, D.W., and Cox, B.S., 2011, Diet overlap of top-level predators in recent sympatry-Bull trout and nonnative lake trout: Journal of Fish and Wildlife Management, v. 2, no. 2, p. $183-189$.

Guyette, M.Q., Loftin, C.S., and Zydlewski, J., 2013, Carcass analog addition enhances juvenile Atlantic salmon (Salmo salar) growth and condition: Canadian Journal of Fisheries and Aquatic Sciences, v. 70, no. 6, p. 860-870.

Hagen, J., and Taylor, E.B., 2001, Resource partitioning as a factor limiting gene flow in hybridizing populations of Dolly Varden char (Salvelinus malma) and bull trout (Salvelinus confluentus): Canadian Journal of Fisheries and Aquatic Sciences, v. 58, no. 10, p. 2037-2047.

Ham, K.D., and Pearsons, T.N., 2000, Can reduced salmonid population abundance be detected in time to limit management impacts?: Canadian Journal of Fisheries and Aquatic Sciences, v. 57, no. 1, p. 17-24.

Hanson, P.C., Johnson, T.B., Schindler, D.E., and Kitchell, J.F., 1997, Fish bioenergetics 3.0 [software]: Madison, Wis., University of Wisconsin Sea Grant Institute, Technical Report WISCU-T-97-001.

Hardiman, J.M., Johnson, B.M., and Martinez, P.J., 2004, Do predators influence the distribution of age-0 kokanee in a Colorado reservoir?: Transactions of the American Fisheries Society, v. 133, no. 6, p. 1366-1378.

Harvey, C.J., and Kareiva, P.M., 2005, Community context and the influence of non-indigenous species on juvenile salmon survival in a Columbia River reservoir: Biological Invasions, v. 7, no. 4, p. 651-663.

Haught, S., and von Hippel, F.A., 2011, Invasive pike establishment in Cook Inlet Basin lakes, Alaska-Diet, native fish abundance and lake environment: Biological Invasions, v. 13, no. 9, p. 2103-2114.

Hausch, S.J., and Melnychuk, M.C., 2012, Residualization of hatchery steelhead-A meta-analysis of hatchery practices: North American Journal of Fisheries Management, v. 32, no. 5, p. 905-921.

Heath, D., Bettles, C.M., and Roff, D., 2010, Environmental factors associated with reproductive barrier breakdown in sympatric trout populations on Vancouver Island: Evolutionary Applications, v. 3, no. 1, p. 77-90. 
Hill, M., Quesada, C., and Bennett, M., 2014, Reservoir survival/predation, fishery and disease test and verification study 2013 annual report: Portland, Oreg., Portland General Electric and the Confederated Tribes of the Warm Springs Reservation of Oregon, $79 \mathrm{p}$.

Hokanson, K.E.F., Kleiner, C.F., and Thorslund, T.W., 1977, Effects of constant temperatures and diel temperature fluctuations on specific growth and moratlity-rates and yeild of juvenile rainbow trout: Journal of the Fisheries Research Board of Canada, v. 34 , no. 5 , p. $639-648$.

Howell, P.J., and Sankovich, P.M., 2012, An evaluation of redd counts as a measure of bull trout population size and trend: North American Journal of Fisheries Management, v. 32, no. 1, p. 1-13.

Hutchinson, G.E., 1978, An introduction to population ecology: New Haven, Conn., Yale University Press, 260 p.

Hyatt, K.D., and Stockner, J.G., 1985, Responses of sockeye salmon (Oncorhynchus nerka) to fertilization of British Columbia coastal lakes: Canadian Journal of Fisheries and Aquatic Sciences, v. 42, no. 2, p. 320-331.

Isely, J.J., and Grabowski T.B., 2007, Age and growth, chap. 5 of Guy, C.S. and Brown, M.J., eds., Analysis and interpretation of freshwater fisheries data: Bethesda, Md., American Fisheries Society, p. 187-228.

Janetski, D.J., Chaloner, D.T., Tiegs, S.D., and Lamberti, G.A., 2009, Pacific salmon effects on stream ecosystems-A quantitative synthesis: Oecologia, v. 159, p. 583-595.

Johnson, O.W., Ruckelshaus, M.H., Grant, W.S., Waknitz, F.W., Garrett, A.M., Bryant, G.J., Neely, K., and Hard, J.J., 1999, Status review of coastal cutthroat trout from Washington, Oregon, and California: Seattle, Wash., National Oceanic and Atmospheric Administration, National Marine Fisheries Service, Technical Memorandum NMFS-NWFSC-37.

Johnston, F.D., and Post, J.R., 2009, Density-dependent life-history compensation of an iteroparous salmonid: Ecological Applications, v. 19 , no. 2 , p. $449-467$.

Johnston, F.D., Post, J.R., Mushens, C.J., Stelfox, J.D., Paul, A.J., and Lajeunesse, B., 2007, The demography of recovery of an overexploited bull trout, Salvelinus confluentus, population: Canadian Journal of Fisheries and Aquatic Sciences, v. 64, no. 1, p. 113-126.

Karchesky, C., and Bennett, D.H., 1999, Dietary overlap between introduced fishes and juvenile salmonids in Lower Granite Reservoir, Idaho-Washington: Portland, Oreg., National Oceanic and Atmospheric Administration, National Marine Fisheries Service.

Keefer, M.L., and Caudill, C.C., 2012, A review of adult salmon and steelhead straying with an emphasis on Columbia River populations: Moscow, Idaho, University of Idaho, contract number W912EF-08-D-0007.

Kiffney, P.M., Pess, G.R., Anderson, J.H., Faulds, P., Burton, K., and Riley, S.C., 2009, Changes in fish communities following recolonization of the Cedar River, WA, USA by Pacific salmon after 103 years of local extirpation: River Research and Applications, v. 25 , no. 4 , p. $438-452$.

Koehler, M.E., Fresh, K.L., Beauchamp, D.A., Cordell, J.R., Simenstad, C.A., and Seiler, D.E., 2006, Diet and bioenergetics of lake-rearing juvenile Chinook salmon in Lake Washington: Transactions of the American Fisheries Society, v. 135, no. 6, p. $1580-1591$.

Kohler, A.E., Kusnierz, P.C., Copeland, T., Venditti, D.A., Denny, L., Gable, J., Lewis, B.A., Kinzer, R., Barnett, B., and Wipfli, M.S., 2013, Salmon-mediated nutrient flux in selected streams of the Columbia River Basin, USA: Canadian Journal of Fisheries and Aquatic Sciences, v. 70, no. 3, p. 502-512.

Kohler, A.E., Pearsons, T.N., Zendt, J.S., Mesa, M.G., Johnson, C.L., and Connolly, P.J., 2012, Nutrient enrichment with salmon carcass analogs in the Columbia River Basin, USA-A stream food web analysis: Transactions of the American Fisheries Society, v. 141 , no. 3 , p. $802-824$.

Kondolf, G.M., 2000, Assessing salmonid spawning gravel quality: Transactions of the American Fisheries Society, v. 129, no. 1, p. $262-281$.

Konecki, J.T., Woody, C.A., and Quinn, T.P., 1995, Temperature preference in two populations of juvenile coho salmon, Oncorhynchus kisutch: Environmental Biology of Fishes, v. 44, no. 4, p. 417-421. 
Koski, M.L., and Johnson, B.M., 2002, Functional response of kokanee salmon Oncorhynchus nerka to Daphnia at different light levels: Canadian Journal of Fisheries and Aquatic Sciences, v. 56, p. 707-716.

Kovach, R.P., Al-Chokhachy, R., Whited, D.C., Schmetterling, D., Dux, A., and Muhlfeld, C.C., 2017, A threat assemblage drives population dynamics of a cold-water specialist: Journal of Applied Ecology, v. 54, p. 638-647.

Kuns, M.M., and Sprules, W.G., 2000, Zooplankton production in Lake Ontario-A multistrata approach: Canadian Journal of Fisheries and Aquatic Sciences, v. 57, p. 2240-2247.

Leathe, S.A., and Graham, P.J., 1982, Flathead Lake fish food habits study: Kalispell, Mont., Montana Fish, Wildlife and Parks, contract ROO8224-01-4.

Lesko, E., 2018, Hatchery and supplementation program, 2017 annual report, Final: Portland, Oreg., PacifiCorp, p. 1-22.

Love, R.H., 1977, Target strength of an individual fish from any aspect: Journal of the Acoustical Society of America, v. 62, p. $1397-1403$.

Lowery, E., 2009, Trophic relations and seasonal effects of predation on Pacific salmon by fluvial bull trout in a riverine food web: Seattle, Wash., University of Washington, M.S. thesis, 85 p.

Lowery, E., and Beauchamp, D.A., 2010, Baseline food web assessment of the upper Clackamas River Basin prior to reintroduction of bull trout - Final Report to the Upper Clackamas Bull Trout Working Group: Seattle, Wash., Washington Cooperative Fish and Wildlife Research Unit, report number WACFWRU-010-02.

Lowery, E.D., and Beauchamp, D.A., 2015, Trophic ontogeny of fluvial bull trout and seasonal predation on Pacific salmon in a riverine food web: Transactions of the American Fisheries Society, v. 144, no. 4, p. 724-741.

Luecke, C., and Brandt, D., 1993, Estimating the energy density of Daphnid prey for the use with rainbow trout bioenergetics models: Transactions of the American Fisheries Society, v. 122, p. 386-389.

Lynch, J.M., 1993, Evaluation of the Merwin trap as a means of northern squawfish (Ptychocheilus oregonensis) control in the Columbia River: Seattle, Wash., University of Washington, M.S. thesis, 114 p.

Lynch, M., 1982, How well does the Edmonson-Paloheimo model approximate instantaneous birth rates?: Ecology, v. 63, p. 12-18.

Marcot, B.G., Allen, C.S., Morey, S., Shively, D., and White, R., 2012, An expert panel approach to assessing potential effects of bull trout reintroduction on federally listed salmonids in the Clackamas River, Oregon: North American Journal of Fisheries Management, v. 32, no. 3, p. 450-465.

Martin, S.W., Schuck, M.A., Underwood, K.D., and Scholz, A.T., 1992, Investigations of bull trout (Salvelinus confluentus), steelhead trout (Onorhynchus mykiss), and spring Chinook salmon (O. tshawytscha) interactions in southeast Washington streams: Spokane, Wash., Eastern Washington University, project number 90-53, contract number DE-BI79-91BP17758.

McCormick, S.D., Shrimpton, J.M., Carey, J.B., O’Dea, M.F., Sloan, K.E., Moriyama, S., and Bjornsson, B.T., 1998, Repeated acute stress reduces growth rate of Atlantic salmon parr and alters plasma levels of growth hormone, insulin-like growth factor I and cortisol: Aquaculture, v. 168, no. (1-4), p. 221-235.

McIntyre, J.K., Beauchamp, D.A., Mazur, M.M., and Overman, N.C., 2006. Ontogenetic trophic interactions and benthopelagic coupling in Lake Washington-Evidence from stable isotopes and diet analysis: Transactions of the American Fisheries Society, v. 135, p. 1312-1328.

McMahon, T.E., Zale, A.V., Barrows, F.T., Selong, J.H., and Danehy, R.J., 2007, Temperature and competition between bull trout and brook trout-A test of the elevation refuge hypothesis: Transactions of the American Fisheries Society, v. 136, no. 5, p. 1313-1326.

McMichael, G.A., and Pearsons, T.N., 1998, Effects of wild juvenile spring Chinook salmon on growth and abundance of wild rainbow trout: Transactions of the American Fisheries Society, v. 127, no. 2, p. 261-274.

McMichael, G.A., and Pearsons, T.N., 2001, Upstream movement of residual hatchery steelhead into areas containing bull trout and cutthroat trout: North American Journal of Fisheries Management, v. 21, no. 4, p. 943-946. 
McMichael, G.A., Sharpe, C.S., and Pearsons, T.N., 1997, Effects of residual hatchery-reared steelhead on growth of wild rainbow trout and spring Chinook salmon: Transactions of the American Fisheries Society, v. 126, no. 2, p. 230-239.

Muhlfeld, C.C., Bennett, D.H., Steinhorst, R.K., Marotz, B., and Boyer, M., 2008, Using bioenergetics modeling to estimate consumption of native juvenile salmonids by nonnative northern pike in the upper Flathead River System, Montana: North American Journal of Fisheries Management, v. 28, no. 3, p. 636-648.

Mull, K.E., 2005, Selection of spawning sites by coho salmon (Oncorhynchus kisutch) in Freshwater Creek, California: Arcata, Calif., Humboldt State University, M.S. thesis, 48 p.

Mull, K.E., and Wilzbach, M.A., 2007, Selection of spawning sites by coho salmon in a northern California stream: North American Journal of Fisheries Management, v. 27, no. 4, p. 1343-1354.

Naiman, R.J., Bilby, R.E., Schindler, D.E., and Helfield, J.M., 2002, Pacific salmon, nutrients, and the dynamics of freshwater and riparian ecosystems: Ecosystems, v. 5, no. 4, p. 399-417.

Naman, S.W., and Sharpe, C.S., 2012, Predation by hatchery yearling salmonids on wild subyearling salmonids in the freshwater environment - A review of studies, two case histories, and implications for management: Environmental Biology of Fishes, v. 94 , no. 1 , p. $21-28$.

Ney, J.J., 1993, Bioenergetics modeling today-Growing pains on the cutting edge: Transactions of the American Fisheries Society, v. 122, p. 736-748.

Oregon Department of Environmental Quality [ODEQ], 1995, Temperature, in 1992-1994 water quality standards review: Oregon Department of Environmental Quality, final issue paper, variously paged.

Ostberg, C.O., Slatton, S.L., and Rodriguez, R.J., 2004, Spatial partitioning and asymmetric hybridization among sympatric coastal steelhead trout (Oncorhynchus mykiss irideus), coastal cutthroat trout (O. clarki clarki) and interspecific hybrids: Molecular Ecology, v. 13, no. 9, p. 2773-2788.

PacifiCorp, 1999, Final technical report—Aquatic resources, Yale Hydroelectric Project: Portland, Oreg., PacifiCorp, Project No. 20871, appendix 2.1-1.

PacifiCorp and Cowlitz Public Utility District [PUD], 2002, Summary of information available to assess potential aquatic species interactions in the Lewis River Basin: Portland, Oreg., PacifiCorp and Cowlitz Public Utility District report, AQU 16.

PacifiCorp and Cowlitz Public Utility District [PUD], 2004, Behavior of salmonid smolts at Swift Dam using 3-dimensional tracking with acoustic tags: Portland, Oreg., PacifiCorp and Cowlitz Public Utility District, AQU 17.

PacifiCorp and Cowlitz Public Utility District [PUD], 2014, Lewis River hatchery and supplementation plan: Portland, Oreg., PacifiCorp and Cowlitz Public Utility District.

PacifiCorp and Cowlitz Public Utility District [PUD], 2015, Lewis River fish passage program 2014 annual report: Portland, Oreg., PacifiCorp and Cowlitz Public Utility District.

Paul, A.J., Post, J.R., Sterling, G.L., and Hunt, C., 2000, Density-dependent intercohort interactions and recruitment dynamicsModels and a bull trout (Salvelinus confluentus) time series: Canadian Journal of Fisheries and Aquatic Sciences, v. 57, no. 6, p. $1220-1231$.

Pearsons, T.N., 2002, Chronology of ecological interactions associated with the life-span of salmon supplementation programs: Fisheries, v. 27, no. 12, p. 10-15.

Pearsons, T.N., 2008, Misconception, reality, and uncertainty about ecological interactions and risks between hatchery and wild salmonids: Fisheries, v. 33, no. 6, p. 278-290.

Pearsons, T.N., 2010, Operating hatcheries within an ecosystem context using the adaptive stocking concept: Fisheries, v. 35, no. 1, p. 23-31.

Pearsons, T.N., and Temple, G.M., 2007, Impacts of early stages of salmon supplementation and reintroduction programs on three trout species: North American Journal of Fisheries Management, v. 27, no. 1, p. 1-20. 
Pearsons, T.N., and Temple, G.M., 2010, Changes to rainbow trout abundance and salmonid biomass in a Washington watershed as related to hatchery salmon supplementation: Transactions of the American Fisheries Society, v. 139, no. 2, p. 502-520.

Perales, K.M., Rowan, J., and Moyle, P.B., 2015, Evidence of landlocked Chinook salmon populations in California: North American Journal of Fisheries Management, v. 35, no. 6, p. 1101-1105.

Pess, G.R., Kiffney, P.M., Liermann, M.C., Bennett, T.R., Anderson, J.H., and Quinn, T.P., 2011, The influences of body size, habitat quality, and competition on the movement and survival of juvenile coho salmon during the early stages of stream recolonization: Transactions of the American Fisheries Society, v. 140, no. 4, p. 883-897.

Petersen, J.H., and Kitchell, J.F., 2001, Climate regimes and water temperature changes in the Columbia River-Bioenergetic implications for predators of juvenile salmon: Canadian Journal of Fisheries and Aquatic Sciences, v. 58, no. 9, p. 1831-1841.

Portland General Electric Company, 2013, Pelton Round Butte project (FERC 2030) 2012 fish passage annual report: Portland, Oreg., Portland General Electric Company and Confederated Tribes of the Warms Springs Reservation.

Porter, R., 2012, Report on the predation index, predator control fisheries, and program evaluation for the Columbia River Basin experimental northern pikeminnow management program: Portland, Oreg., Pacific State Marine Fisheries Commission.

Quinn, T.P., 1993, A review of homing and straying of wild and hatchery-produced salmon: Fisheries Research, v. 18, p. $29-44$.

Quinn, T.P., Sergeant, C.J., Beaudreau, A.H., and Beauchamp, D.A., 2012, Spatial and temporal patterns of vertical distribution for three planktivorous fishes in Lake Washington: Ecology of Freshwater Fish, v. 21, no. 3, p. 337-348.

Rand, R.S., Stewart, D.J., Seelbach, P.W., Jones, M.L., and Wedge, L.R., 1993, Modeling steelhead population energetics in Lakes Michigan and Ontario: Transactions of the American Fisheries Society, v. 122, p. 977-1001.

Ratliff, D., Spateholts, R., Hill, M., and Schulz, E., 2015, Recruitment of young bull trout into the Metolius River and Lake Billy Chinook, Oregon: North American Journal of Fisheries Management, v. 35, no. 6, p. 1077-1089.

Rieman, B., and McIntyre, J.D., 1993, Demographic and habitat requirements for conservation of bull trout: U.S. Department of Agriculture, Forest Service, General Technical Report INT-302.

Riley, S.C., Fuss, H.J., and LeClair, L.L., 2004, Ecological effects of hatchery-reared juvenile Chinook and coho salmon on wild juvenile salmonids in two Washington streams: North American Journal of Fisheries Management, v. 24, no. 2, p. 506-517.

Sabo, J.L., and Pauley, G.B., 1997, Competition between stream-dwelling cutthroat trout (Oncorhynchus clarki) and coho salmon (Oncorhynchus kisutch) -Effects of relative size and population origin: Canadian Journal of Fisheries and Aquatic Sciences, v. 54, no. 11, p. 2609-2617.

Scheuerell, M.D., Levin, P.S., Zabel, R.W., Williams, J.G., and Sanderson, B.L., 2005, A new perspective on the importance of marine-derived nutrients to threatened stocks of Pacific salmon (Oncorhynchus spp.): Canadian Journal of Fisheries and Aquatic Sciences, v. 62, p. 961-964.

Scheuerell, M.D., Moore, J.W., Schindler, D.E., and Harvey, C.J., 2007, Varying effects of anadromous sockeye salmon on the trophic ecology of two species of resident salmonids in southwest Alaska: Freshwater Biology, v. 52, no. 10, p. 1944-1956.

Scheuerell, M.D., and Schindler, D.E., 2003, Diel vertical migration by juvenile sockeye salmon—Empirical evidence for the antipredation window: Ecology, v. 84, no. 7, p. 1713-1720.

Schmetterling, D., 2001, Northern pike investigations in Milltown Reservoir: Missoula, Mont., Montana Fish, Wildlife and Parks.

Schmuck, M.R., and Petersen, M.R., 2006, Warmwater fisheries survey of Evergreen Reservoir, Grant County, Washington: Ephrata, Wash., Washington Department of Fish \& Wildlife.

Schoby, G.P., and Keeley, E.R., 2011, Home range size and foraging ecology of bull trout and westslope cutthroat trout in the upper Salmon River Basin, Idaho: Transactions of the American Fisheries Society, v. 140, no. 3, p. 636-645.

Selong, J.H., McMahon, T.E., Zale, A.V., and Barrows, F.T., 2001, Effect of temperature on growth and survival of bull trout, with application of an improved method for determining thermal tolerance in fishes: Transactions of the American Fisheries Society, v. 130, no. 6, p. 1026-1037. 
Sepulveda, A.J., Rutz, D.S., Ivey, S.S., Dunker, K.J., and Gross, J.A., 2013, Introduced northern pike predation on salmonids in southcentral Alaska: Ecology of Freshwater Fish, v. 22, no. 2, p. 268-279.

Slaney, T.L., Hyatt, K.D., Northcote, T.G., and Fielden, R.J., 1996, Status of anadromous salmon and trout in British Columbia and Yukon: Fisheries, v. 21, no. 10, p. 20-35.

Sorel, M.H., Hansen, A.G., Connelly, K.A., and Beauchamp, D.A., 2016, Trophic feasibility of reintroducing anadromous salmonids in three reservoirs on the North Fork Lewis River, Washington: prey supply and consumption demand of resident fishes: Transactions of the American Fisheries Soceity v. 145, p. 1331-1347.

Stillwater Sciences, 2016, Technical memorandum-Analysis of bull trout PIT tag and detection data: Arcata, Calif., Stillwater Sciences, $8 \mathrm{p}$.

Spencer, C.N., McClelland, B.R., and Stanford, J.A., 1991, Shrimp stocking, salmon collapse, and eagle displacement: Bioscience, v. 41, no. 1, p. 14-21.

Spens, J., and Ball, J.P., 2008, Salmonid or nonsalmonid lakes—Predicting the fate of northern boreal fish communities with hierarchical filters relating to a keystone piscivore: Canadian Journal of Fisheries and Aquatic Sciences, v. 65, no. 9, p. 1945-1955.

Spina, A.P., 2007, Thermal ecology of juvenile steelhead in a warm-water environment: Environmental Biology of Fishes, v. 80, no. 1, p. 23-34.

Stewart, D.J., and Ibarra, M., 1991, Preadation and production of salmonine fishes in Lake Michigan: Canadian Journal of Fisheries and Aquatic Sciences, v. 48, p. 909-922.

Stockwell, J.D., Bonfantine, K.L., and Johnson, B.M., 1999, Kokanee foraging-A Daphnia in the stomach's worth two in the lake: Transactions of the American Fisheries Society, v. 128, p. 169-174.

Stockwell, J.D., and Johnson, B.M., 1999, Field evaluation of a bioenergetics-based foraging model for kokanee Oncorhynchus nerka: Canadian Journal of Fisheries and Aquatic Sciences, v. 56, p. 140-151.

Swain, N.R., Hocking, M.D., Harding, J.N., and Reynolds, J.D., 2014, Effects of salmon on the diet and condition of streamresident sculpins: Canadian Journal of Fisheries and Aquatic Sciences, v. 71, no. 4, p. 521-532.

Tabor, R., Luecke, C., and Wurtsbaugh, W.A., 1996, Effects of Daphnia availability on growth and food consumption of rainbow trout in two Utah reservoirs: North American Journal of Fisheries Management, v. 16, p. 591-599.

Tabor, R.A., Berge, H.B., Klunge, M.M., Thompson, B.E., Lantz, D.W., and Price, B.E., 2012, Predation of juvenile salmonids by resident trout and other fishes in the lower Cedar River, Washington: Lacy, Wash., U.S. Fish and Wildlife Service.

Tabor, R.A., Caledonia, M.T., Mejia, F., Piaskowski, R.M., Low, D.L., Footen, B.A., and Park, L., 2004, Predation of juvenile Chinook salmon by predatory fishes in three areas of the Lake Washington Basin: Lacey, Wash., U.S. Fish and Wildlife Service.

Tappel, P.D., and Bjornn, T.C., 1983, A new method of relating size of spawning gravel to salmonid embryo survival: North American Journal of Fisheries Management, v. 3, p. 123-135.

Taylor, E.B., Pollard, S., and Louie, D., 1999, Mitochondrial DNA variation in bull trout (Salvelinus confluentus) from northwestern North America-Implications for zoogeography and conservation: Molecular Ecology, v. 8, no. 7, p. 1155-1170.

Temple, G.M., and Pearsons, T.N., 2012, Risk management of non-target fish taxa in the Yakima River Watershed associated with hatchery salmon supplementation: Environmental Biology of Fishes, v. 94, no. 1, p. 67-86.

Thurow, R., Lee, D.C., and Rieman, B.E., 1997, Distribution and status of seven native salmonids in the interior Columbia River Basin and portions of the Klamath River and Great Basins: North American Journal of Fisheries Management, v. 17, p. 1094-1110.

Thurow, R.F., Peterson, J.T., and Guzevich, J.W., 2006, Utility and validation of day and night snorkel counts for estimating bull trout abundance in first- to third-order streams: North American Journal of Fisheries Management, v. 26, no. 1, p. $217-232$.

Trotter, P.C., 1989, Coastal cutthroat trout—A life-history compedium: Transactions of the American Fisheries Society, v. 118, no. 5, p. 463-473. 
Underwood, K.D., Martin, S.W., Schuck, M.L., and Scholz, A.T., 1995, Investigations of bull trout (Salvinus confluentus), steelhead trout (Oncorhynchus mykiss), and spring Chinook salmon (O. tshawytscha) interactions in southeast Washington streams: Portland, Oreg., Bonneville Power Administration, project number 90-053, contract number DE-B179-9 IBP 177858.

U.S. Environmental Protection Agency [EPA], 2001, Summary of technical literature examining the effects of temperature on salmonids: U.S. Environmental Protection Agency, EPA 910-D-01-005.

Van Kirk, R.W., and Hill, S.L., 2007, Demographic model predicts trout population response to selenium based on individuallevel toxicity: Ecological Modelling, v. 206, no. 3-4, p. 407-420.

Vander Zanden, M.J., and Rasmussen, J.B., 2001, Variation in $\delta^{15} \mathrm{~N}$ and $\delta^{13} \mathrm{C}$ trophic fractionation-Implications for aquatic food web studies: Limnology and Oceanography, v. 46, no. 8, p. 2061-2066.

Vronskii, B.B., and Leman, V.N., 1991, Spawning stations, hydrologic regime, and survival of progeny in nests of Chinook salmon, Oncorhynchus tshawytscha: Journal of Icthyology, v. 31, p. 91-102.

Ward, D.M., Nislow, K.H., and Folt, C.L., 2008, Do native species limit survival of reintroduced Atlantic salmon in historic rearing streams?: Biological Conservation, v. 141, no. 1, p. 146-152.

Washington State Department of Ecology, 2002, Evaluating standards for protecting aquatic life in Washington's surface water quality standards - Temperature criteria: Washington State Department of Ecology, publication number 00-10-070.

Weber, E.D., and Fausch, K.D., 2003, Interactions between hatchery and wild salmonids in streams-Differences in biology and evidence for competition: Canadian Journal of Fisheries and Aquatic Sciences, v. 60, no. 8, p. 1018-1036.

Weeber, M.A., Giannico, G.R., and Jacobs, S.E., 2010, Effects of redd superimposition by introduced kokanee on the spawning success of native bull trout: North American Journal of Fisheries Management, v. 30, no. 1, p. 47-54.

Weitkamp, L.A., Wainwright, T.C., Bryant, G.J., Milner, G.B., Teel, D.J., Kope, R.G., and Waples, R.S., 1995, Status review of coho salmon from Washington, Oregon, and California: National Oceanic and Atmospheric Administration, National Marine Fisheries Service, Technical Memorandum NMFS-NWFSC-24.

Wenger, S.J., Isaak, D.J., Luce, C.H., Nevile, H.M., Fausch, K.D., Dunham, J.B., Dauwalter, D.C., Young, M.K., Elsner, M.M., Rieman, B.E., Hamlet, A.F., and Williams, J.E., 2011, Flow regime, temperature, and biotic interactions drive differential declines of trout species under climate change: Proceedings of the National Academy of Sciences of the United States of America, v. 108, p. 14175-14180.

Wilhelm, F.M., Parker, B.R., Schindler, D.W., and Donald, D.B., 1999, Seasonal food habits of bull trout from a small alpine lake in the Canadian Rocky Mountains: Transactions of the American Fisheries Society, v. 128, no. 6, p. 1176-1192.

Wipfli, M.S., Hudson, J.P., and Caouette, J.P., 2004, Restoring productivity of salmon-based food webs-Contrasting effects of salmon carcass and salmon carcass analog additions on stream-resident salmonids: Transactions of the American Fisheries Society, v. 133, no. 6, p. 1440-1454.

Woodson, L.E., Wells, B.K., Weber, P.K., MacFarlane, R.B., Whitman, G.E., and Johnson, R.C., 2013, Size, growth, and origindependent mortality of juvenile Chinook salmon Oncorhynchus tshawytscha during early ocean residence: Marine Ecology Progress Series, v. 487, p. 163-175.

Zabel, R.W., and Achord, S., 2004, Relating size of juveniles to survival within and among populations of Chinook salmon: Ecology, v. 85, no. 3, p. 795-806. 


\section{Figures}

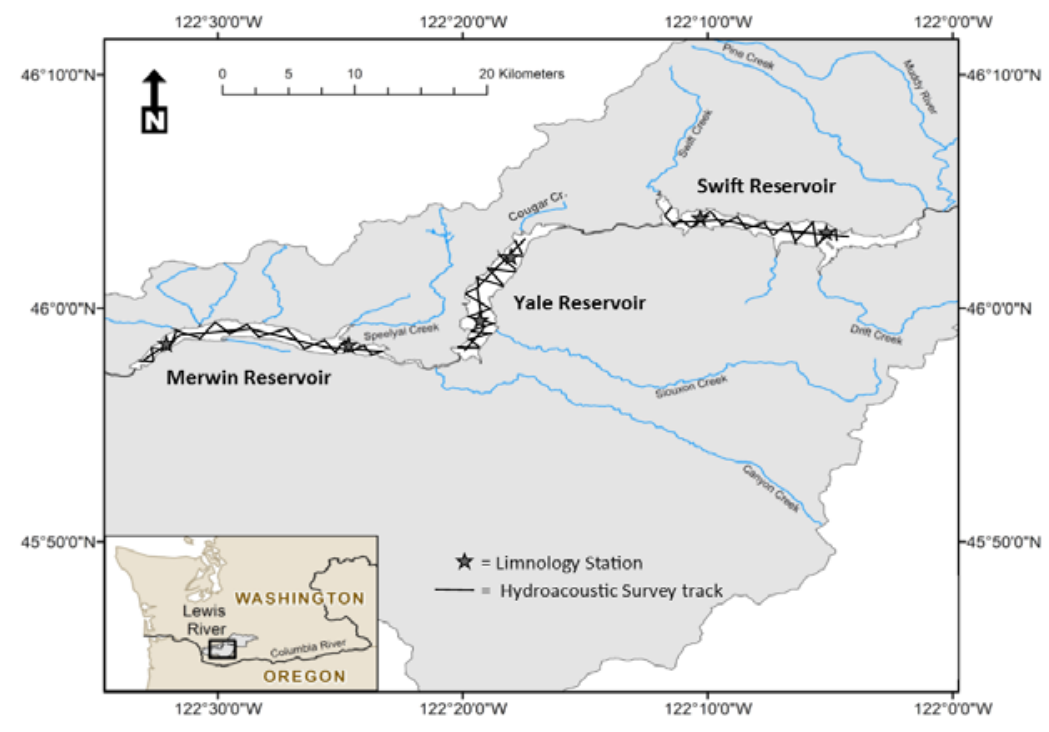

Figure F1. Map of North Fork of the Lewis River reservoirs in Washington with respect to the hydrologic basin (gray shaded area). Stars show the temperature and zooplankton sampling locations; solid lines indicate the hydroacoustic survey tracks. (Cr., creek) 


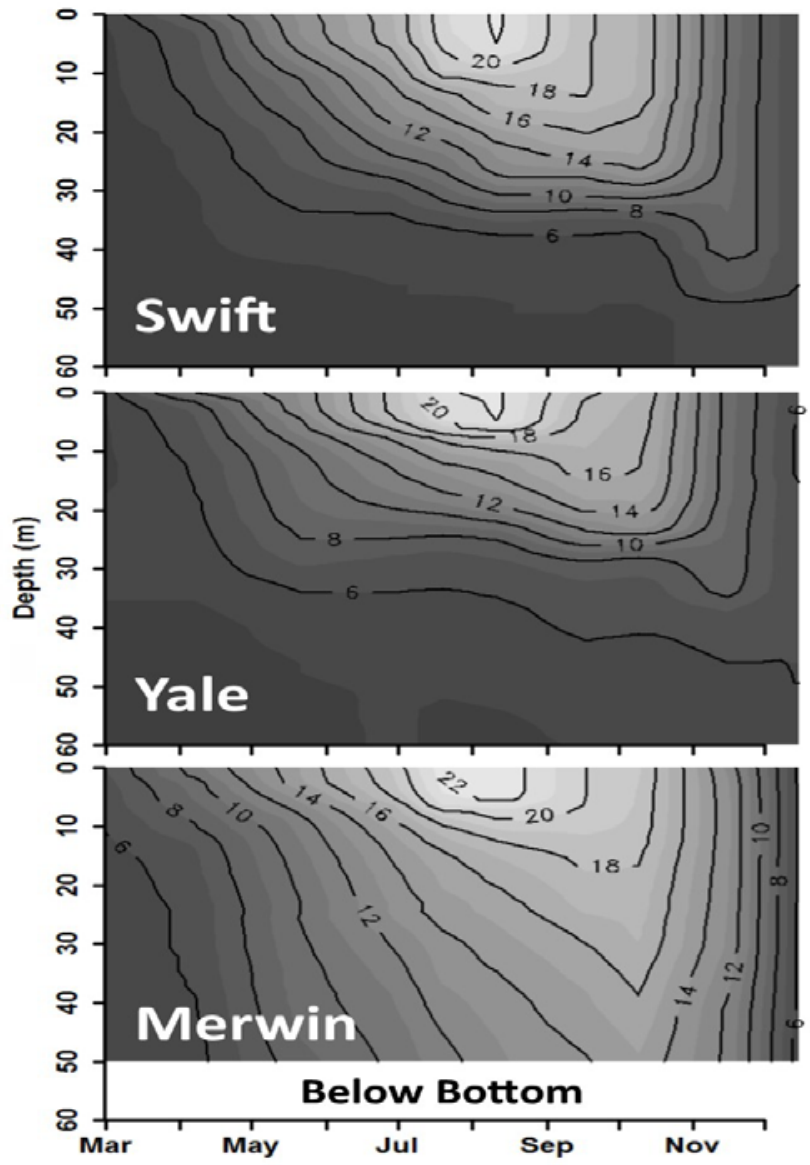

Figure F2. Isoclines of mean water temperature (degrees Celsius) with respect to depth (in meters [m]) in Swift Reservoir, Yale Lake, and Lake Merwin, Washington, during March-December 2013. 


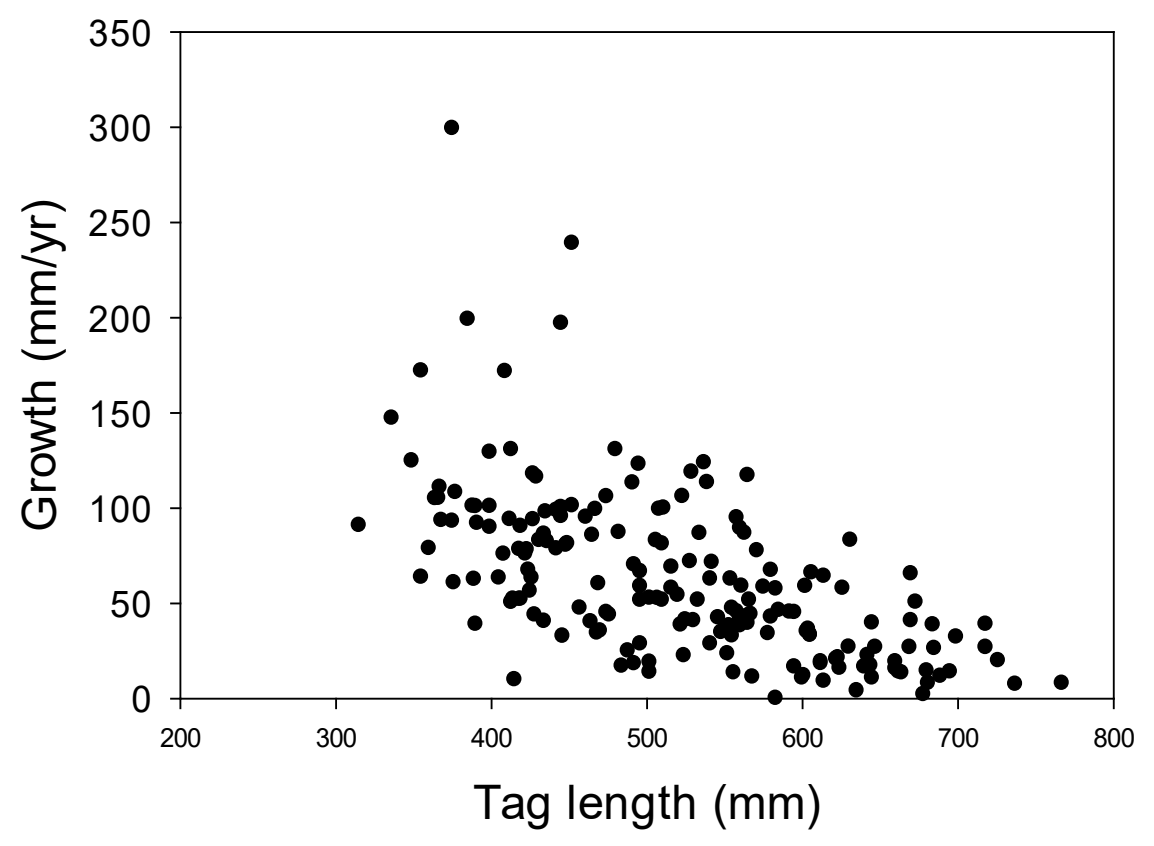

Figure F3. Annual individual bull trout growth data for fish individually marked by Washington Department of Fish and Wildlife and PacifiCorp biologists from 1993-2016 in Swift Reservoir, Washington, in relation to length at tagging (in millimeters). Growth rate is in millimeters per year (mm/yr). 


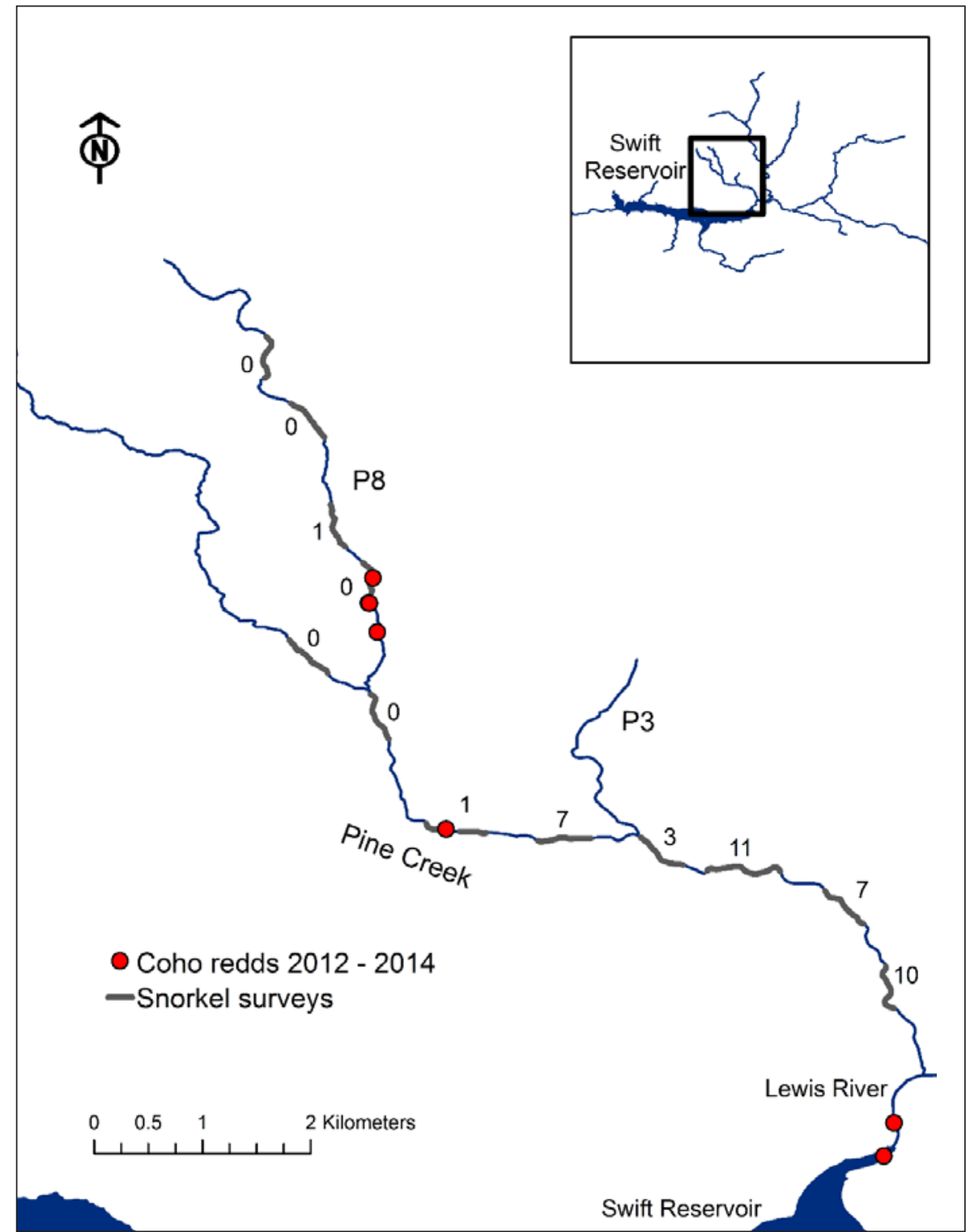

Figure F4. The location of coho salmon redds (red circles; 2012-2014) in Pine Creek and a tributary to Pine Creek (P8), Washington; snorkel surveys used to identify overlap in the distribution of bull trout and coho salmon during 2013 and 2014 (gray); and the number of juvenile coho salmon (number by reach) detected in surveys. The black box in the inset map shows the location of Pine Creek and tributaries within Swift Reservoir. 


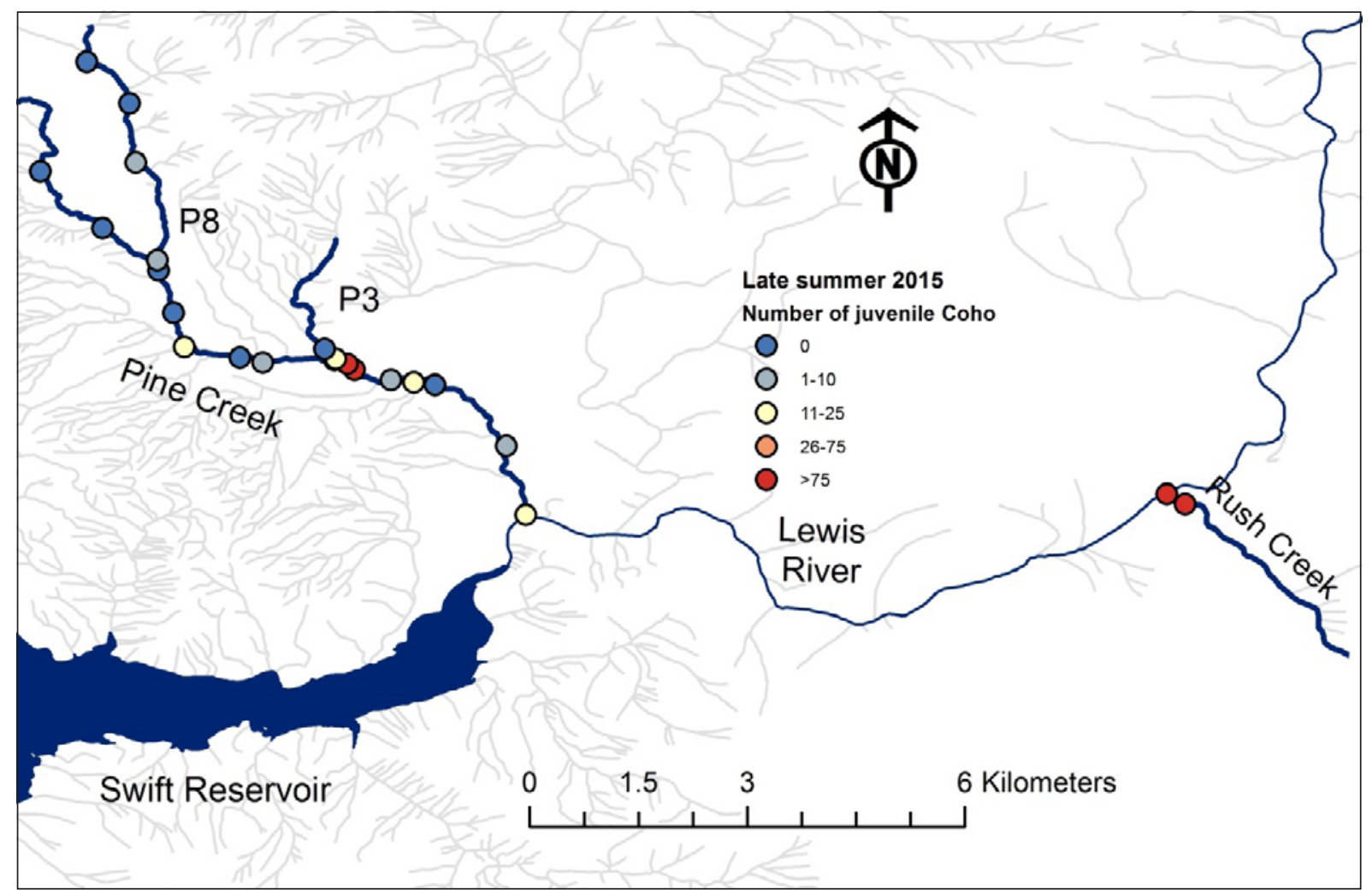

Figure F5. The number of juvenile coho salmon observed during daytime snorkel surveys during early summer (June 2015) in Pine Creek, P8, P3, and Rush Creek, Washington. 


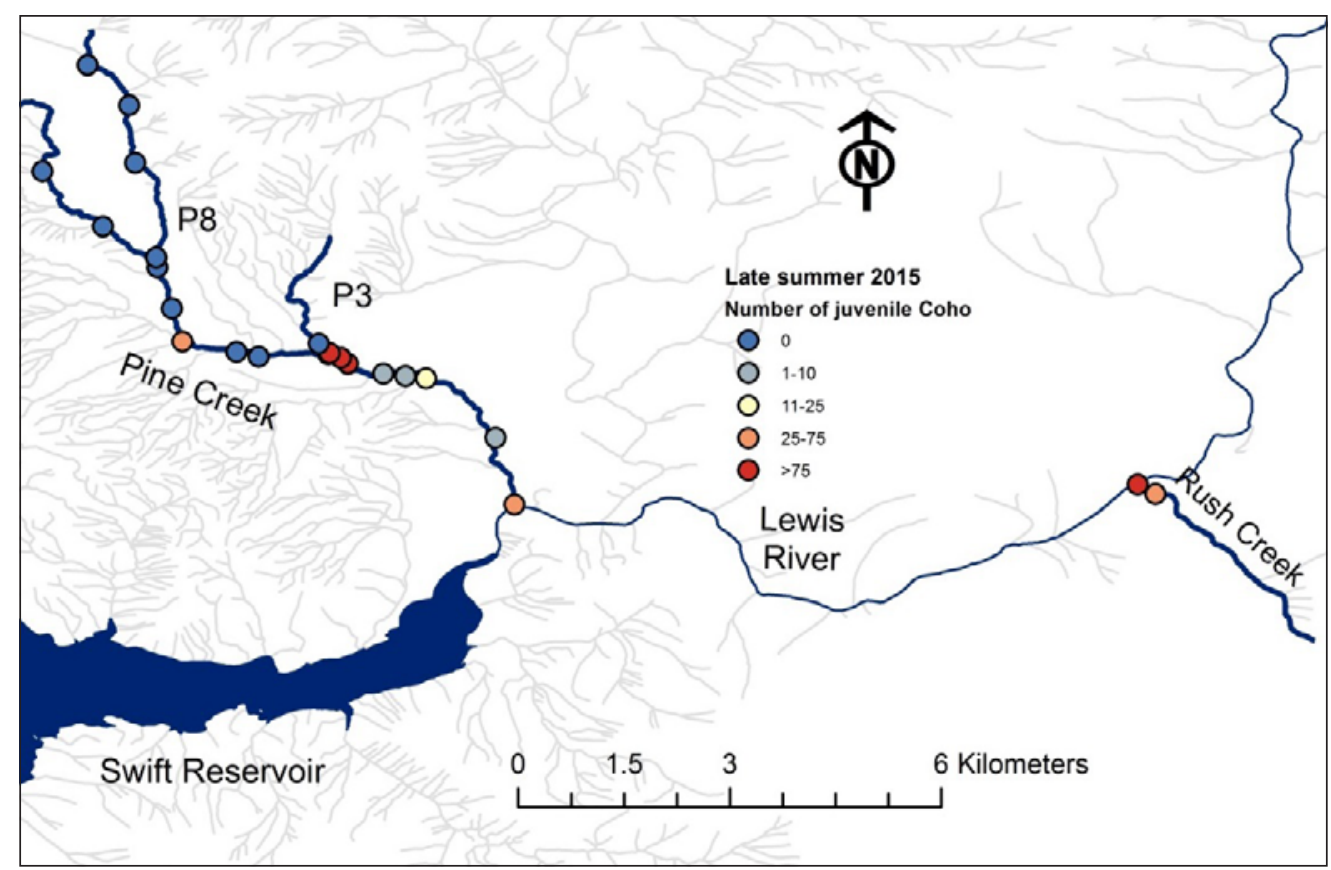

Figure F6. The number of juvenile coho salmon observed during daytime snorkel surveys during mid-late summer (July-September 2015) in Pine Creek, P8, P3, and Rush Creek, Washington.

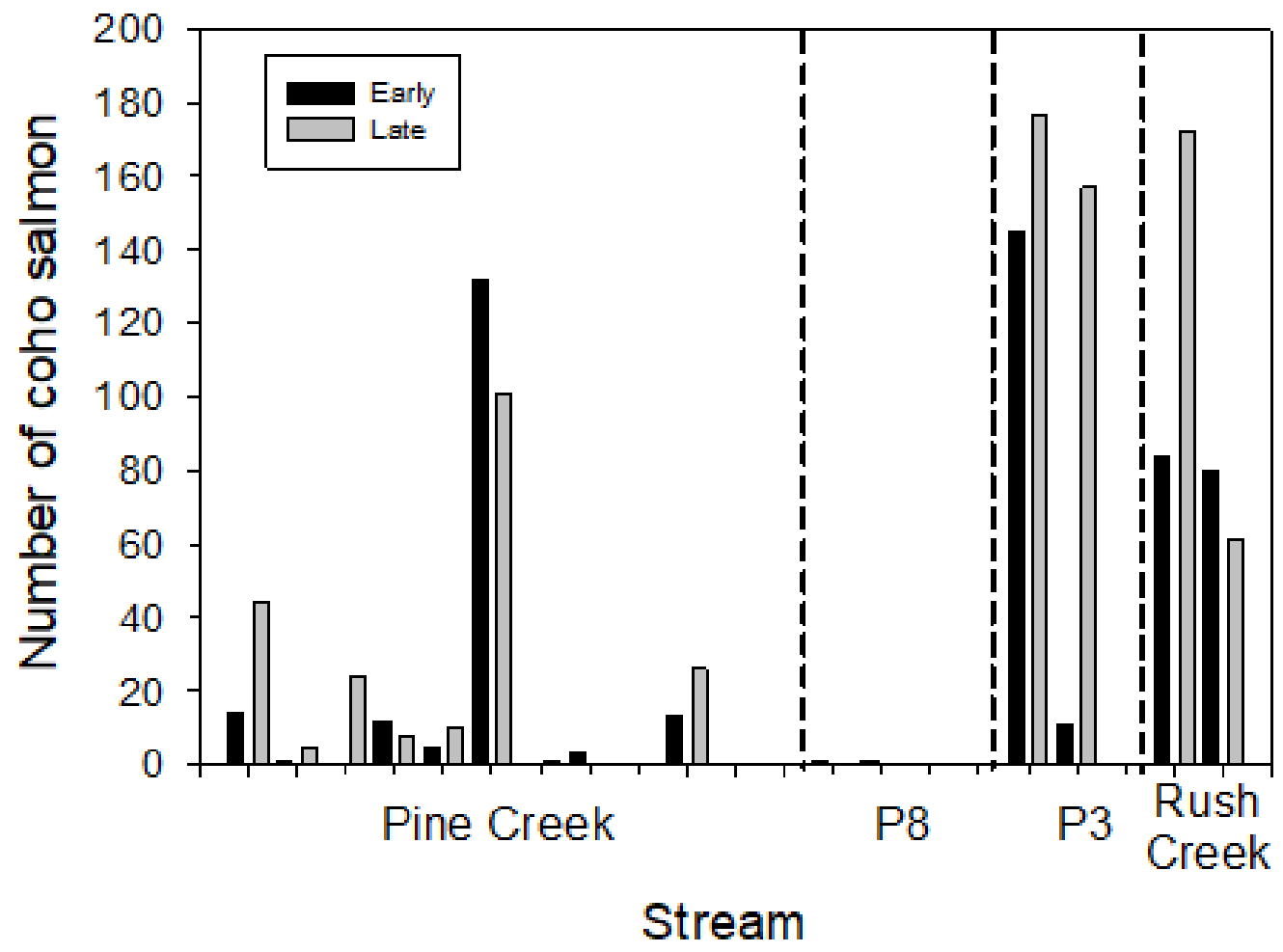

Figure F7. The number of juvenile coho salmon observed during snorkel surveys in early (June 2015) and mid-late summer (JulySeptember 2015) in Pine Creek, P8, P3, and Rush Creek, Washington. Study areas are separated by dashed lines. 


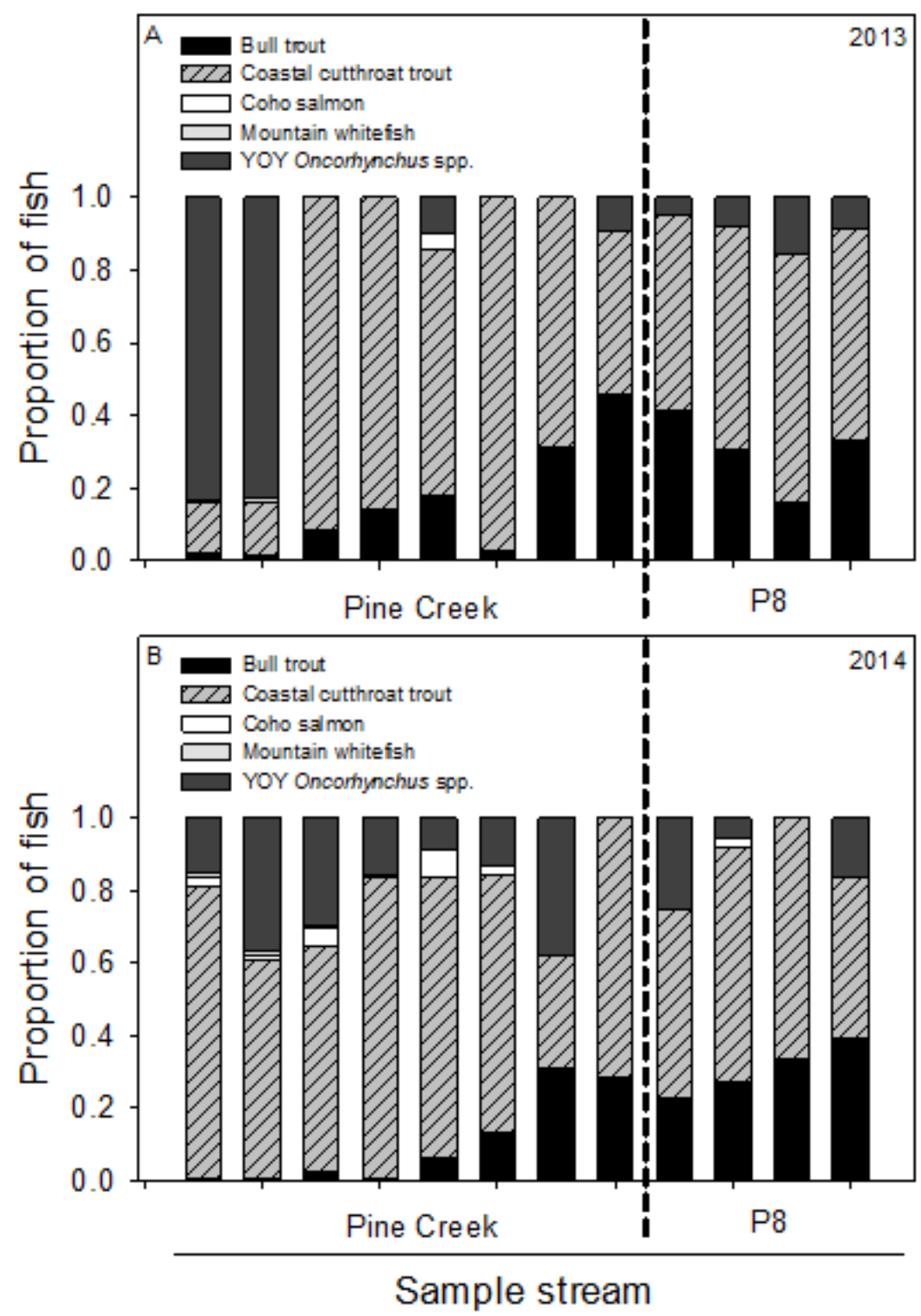

Figure F8. The proportion of bull trout, coastal cutthroat trout, young-of-year (YOY) coho salmon, mountain whitefish, and YOY Oncorhynchus spp. (rainbow trout/steelhead and coastal cutthroat trout) observed during snorkel surveys in different reaches (denoted by column) in Pine Creek and P8 during 2013 and 2014. Reaches are ordered in a downstream to upstream manner; left-to-right, and 2013 and 2014. Reaches are not the same-see methods; study areas are separated by dashed lines. 

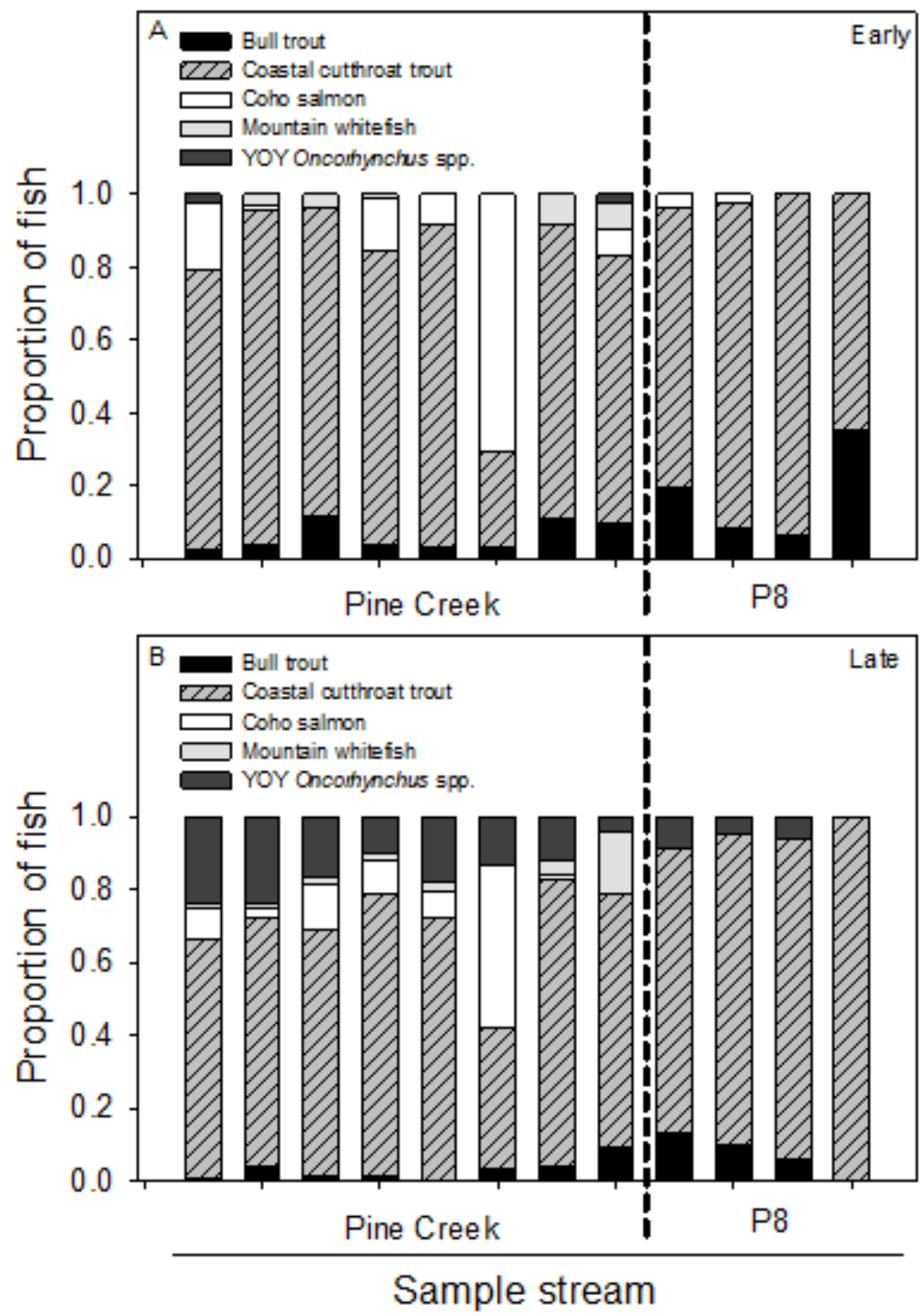

Figure F9. The proportion of bull trout (black), coastal cutthroat trout (gray hatched), young-of-year (YOY) coho salmon (white), mountain whitefish (light gray), and Oncorhynchus spp. (rainbow trout/steelhead and coastal cutthroat trout; dark gray) observed during snorkel surveys in different reaches (denoted by column) in Pine Creek and P8 during A, early 2015 (June-July) and B, late 2015 (August). Reaches are ordered in a downstream to upstream manner (left-to-right); study areas are separated by dashed lines. 


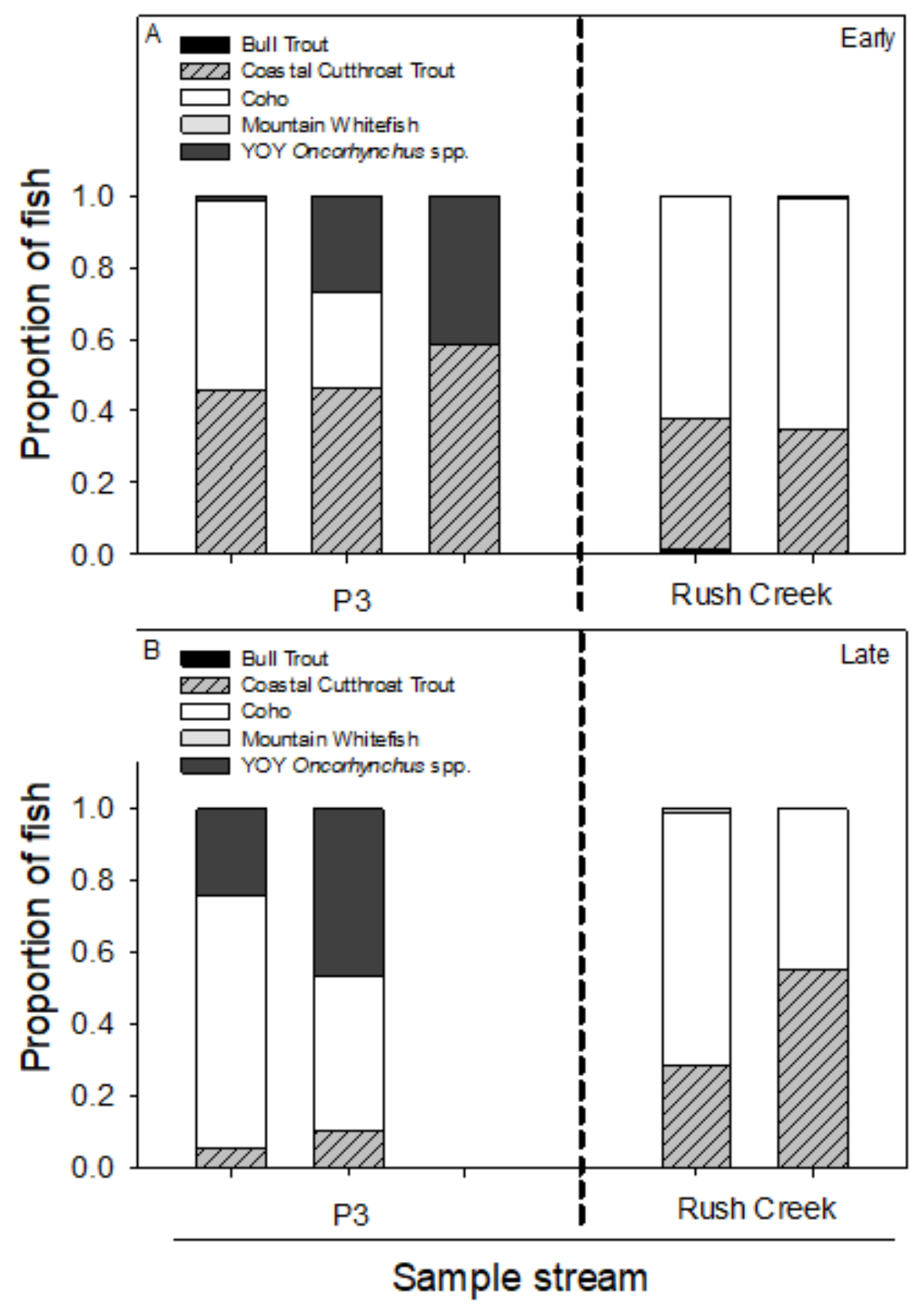

Figure F10. The proportion of bull trout (black), coastal cutthroat trout (gray hatched), young-of-year (YOY) coho salmon (white), mountain whitefish (light gray), and YOY Oncorhynchus spp. (rainbow trout/steelhead and coastal cutthroat trout; dark gray) observed during snorkel surveys in different reaches (denoted by column) in P3 (small tributary to Pine Creek) and Rush Creek during $A$, early 2015 (June-July) and $B$, late 2015 (August). Reaches are ordered in a downstream to upstream manner (left-to-right); the downstream reach on P3 was sampled during late July and the most upstream reach was not resampled as very few fish were observed. Study areas are separated by dashed lines. 


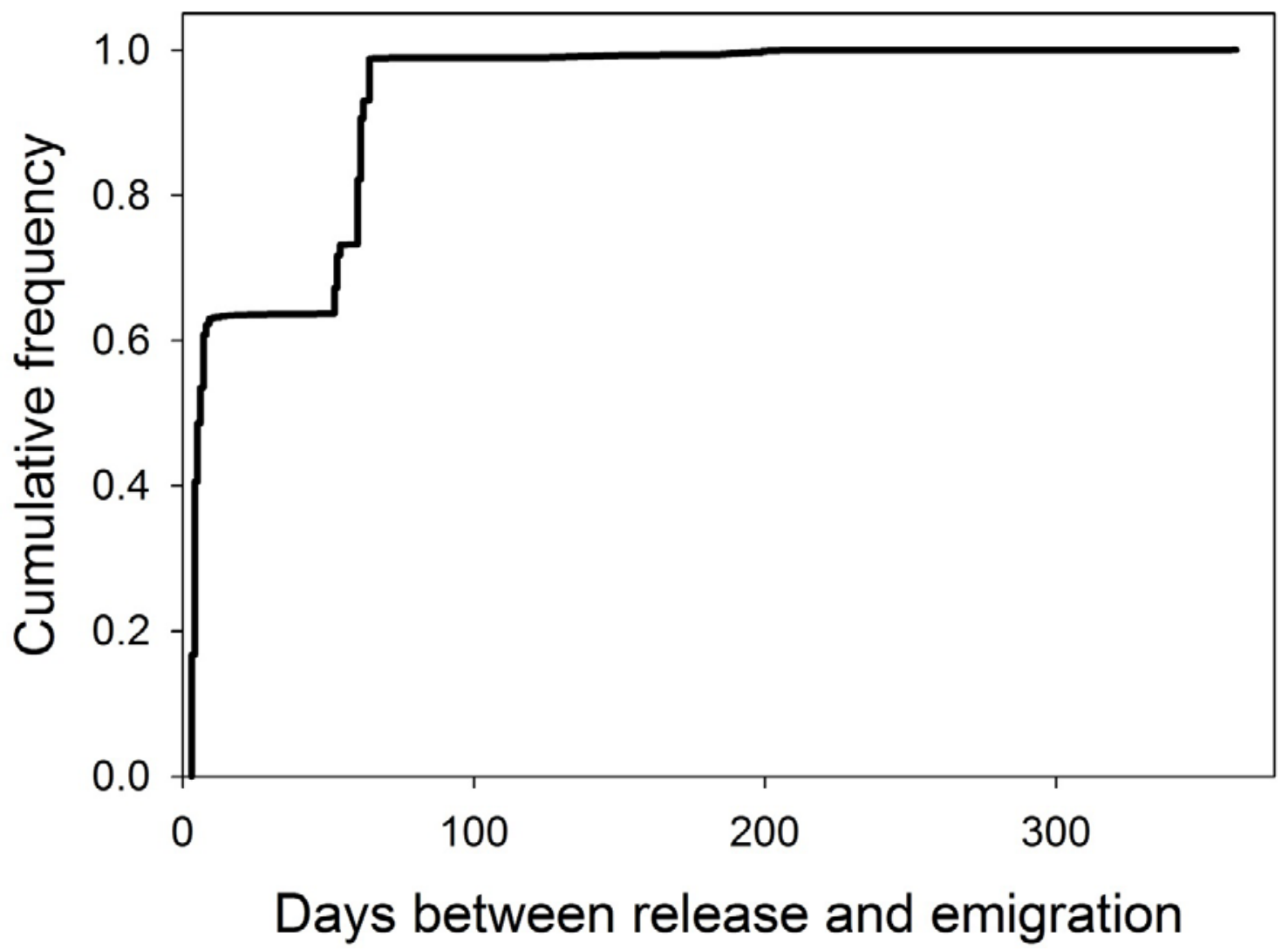

Figure F11. The cumulative frequency of the days between release and emigration past the Clear Creek, Washington, passive integrated transponder-tag antenna for acclimation spring Chinook salmon released during the spring of 2014 and 2015. 

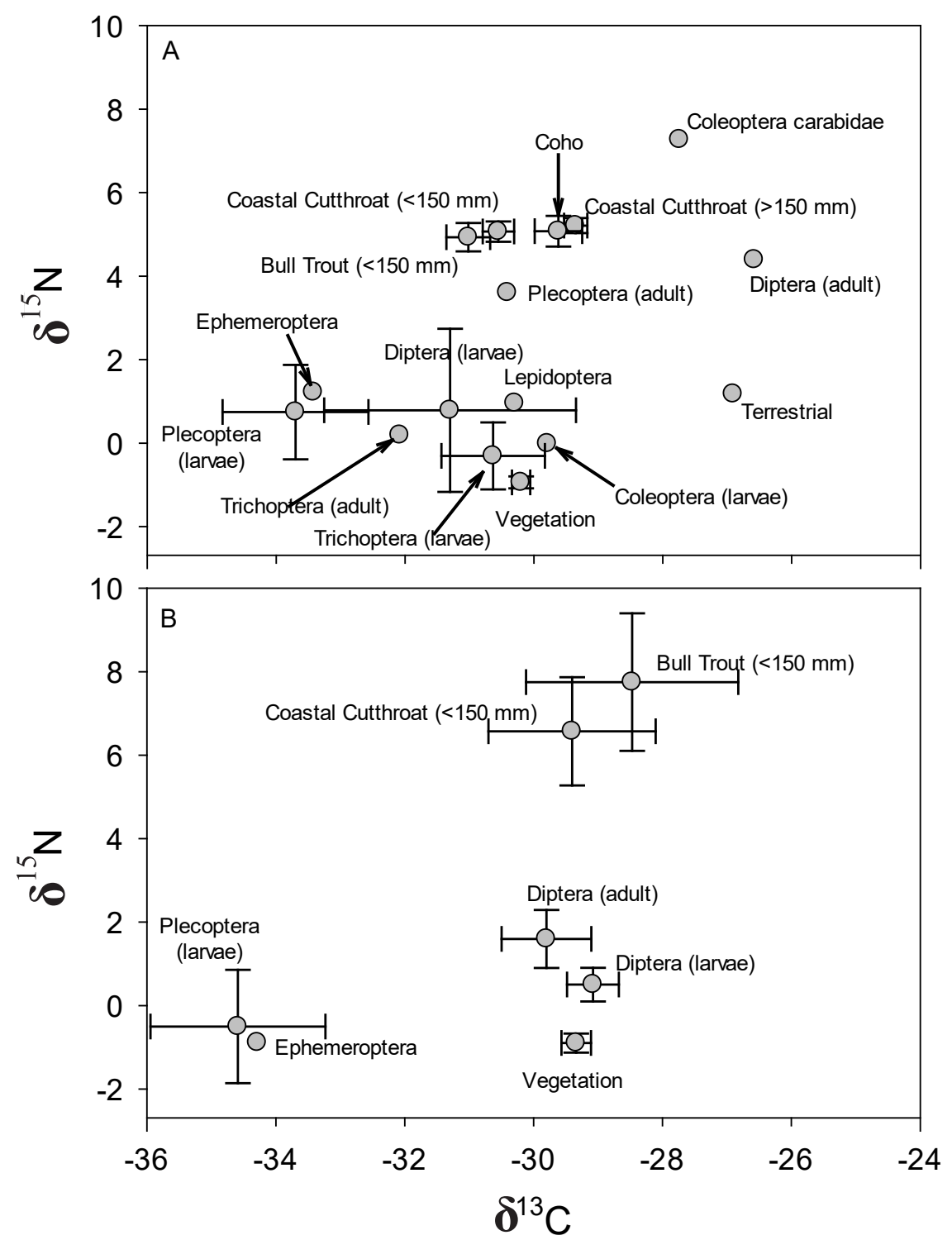

Figure F12. Stable isotope results with 95-percent confidence intervals during late spring-summer in $A, 2009$ (prior to anadromous reintroductions) and $B, 2014$ (after the onset of anadromous reintroductions) for different size classes (measured in millimeters; $\mathrm{mm}$ ) of fish from P8, a main tributary to Pine Creek and the major bull trout spawning tributary in the Swift Reservoir portion of the upper Lewis River, Washington. 

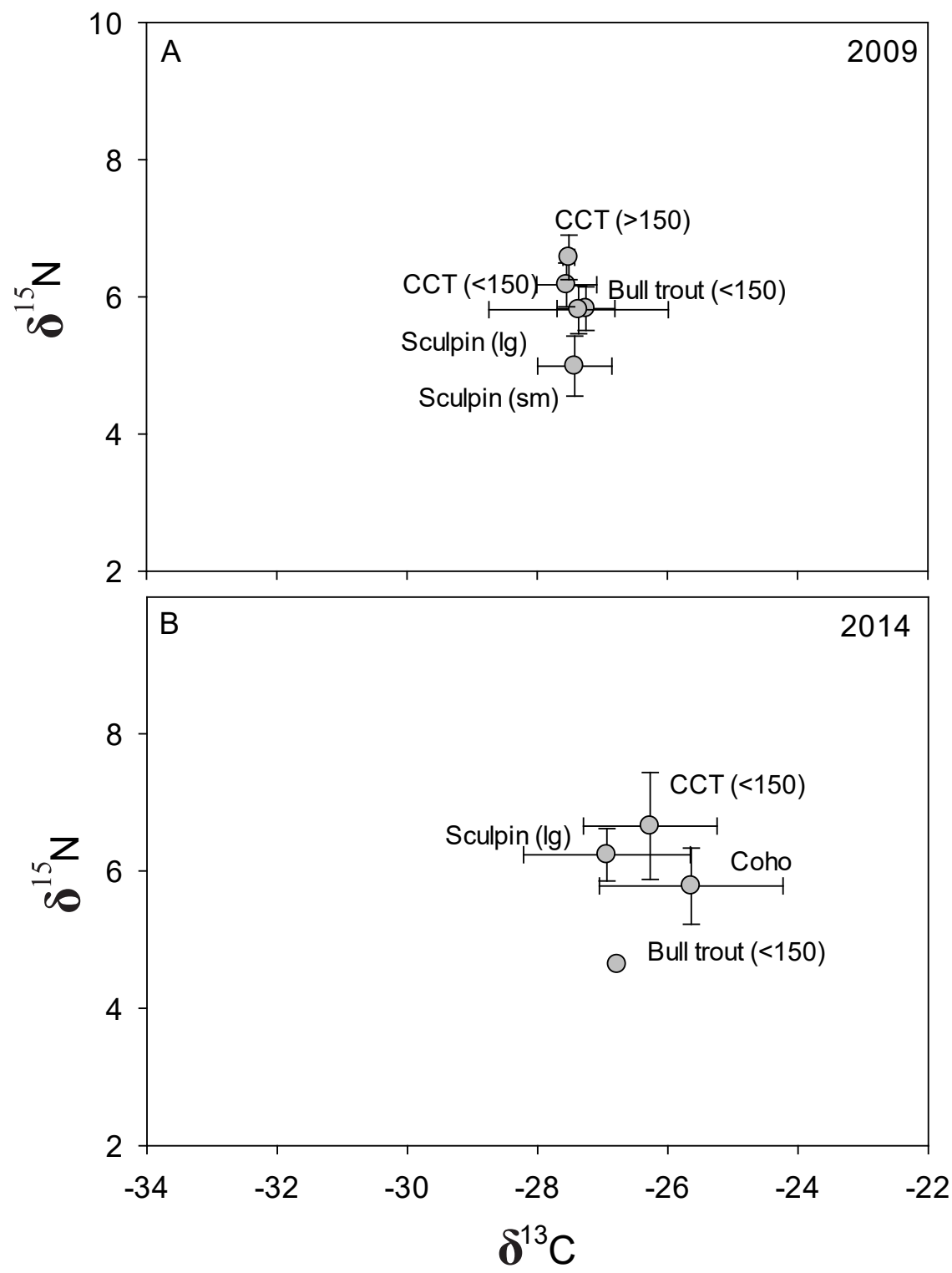

Figure F13. Stable isotope results with 95-percent confidence intervals during late spring-summer in $A, 2009$ (prior to anadromous reintroductions) and $B, 2014$ (after the onset of anadromous reintroductions) for different size classes (large [lg] $\geq 80 \mathrm{~mm}$; small [sm], $<80 \mathrm{~mm}$ ) of fish from Rush Creek, one of the major bull trout spawning tributaries in the Swift Reservoir portion of the upper Lewis River, Washington. (mm, millimeter; CCT, coastal cutthroat trout; $\geq$, greater than or equal to; $<$, less than) 


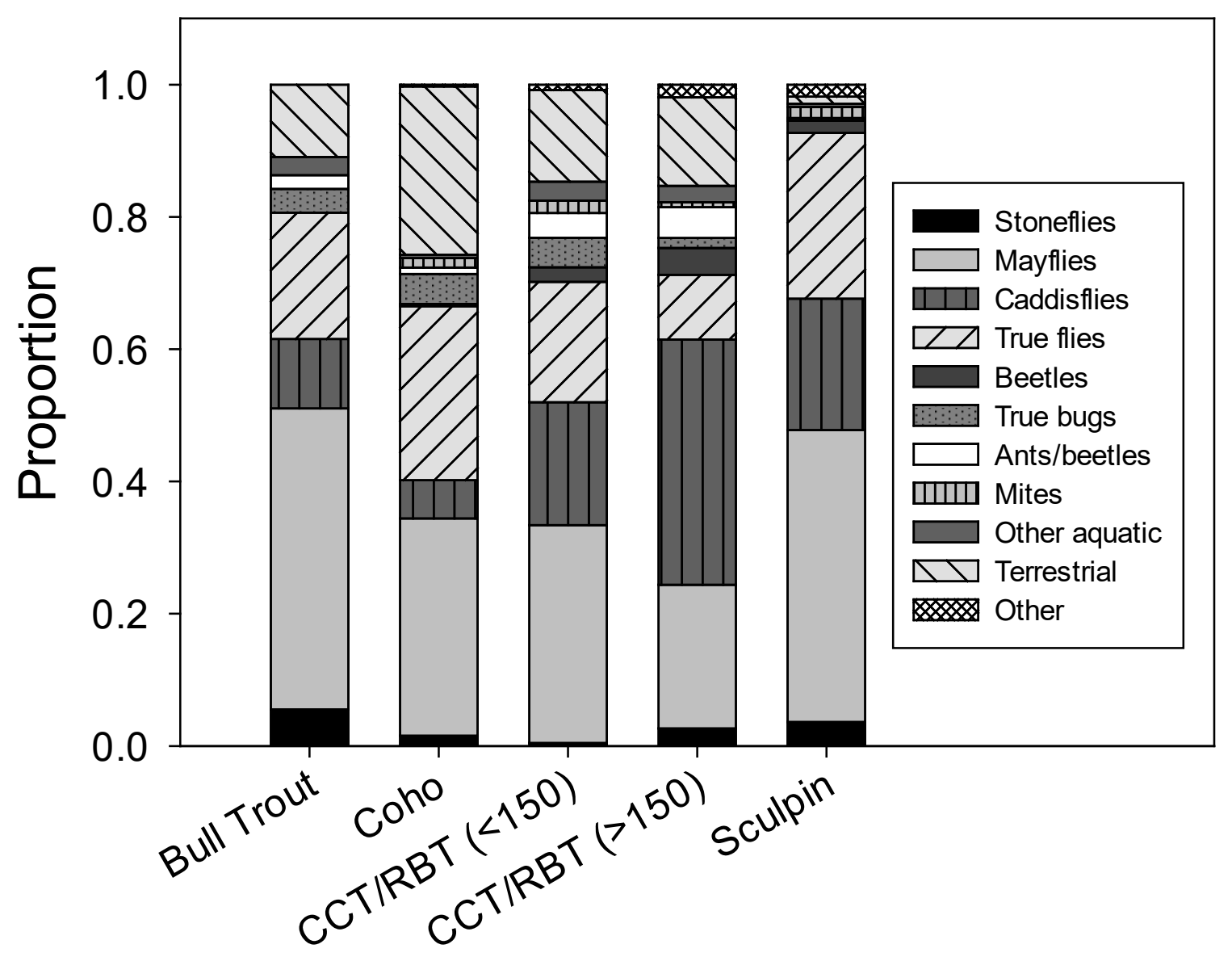

Species

Figure F14. The proportion of bull trout, juvenile coho salmon, coastal cutthroat trout (CCT)/rainbow trout (RBT) by size class as measured in millimeters [mm]) and sculpin diets as Plecoptera (stoneflies), Ephemeroptera (mayflies), Trichoptera (caddisflies), Diptera (true flies), Coleoptera (beetles), Hemiptera (true bugs), Hymenoptera (ants and bees), Trombidiformes (mites), other aquatic insects, terrestrial insects, and other prey items (fish, fish eggs, worms, snails) during the late spring-summer (June-August). 


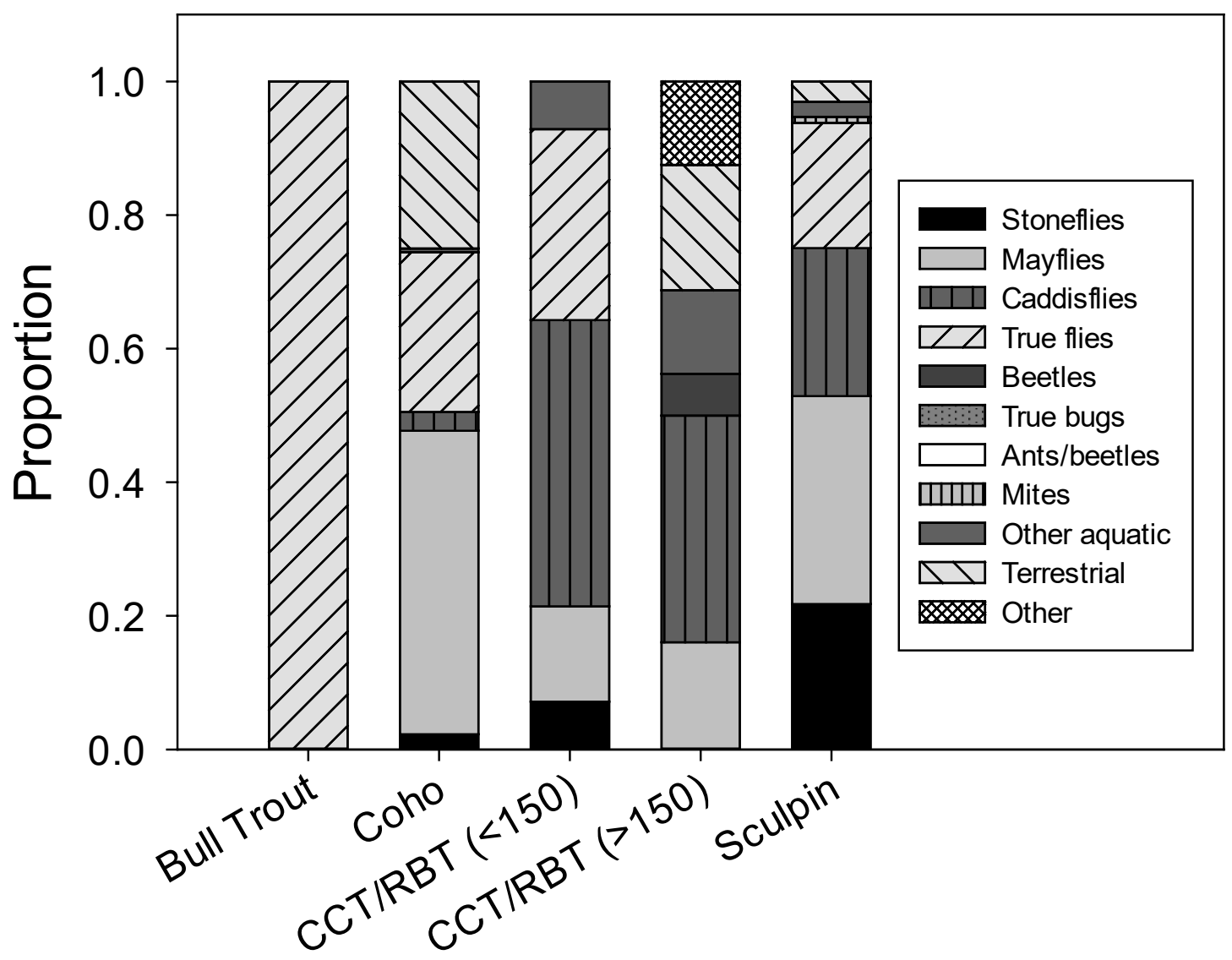

\section{Species}

Figure F15. The proportion of bull trout, juvenile coho salmon, coastal cutthroat trout (CCT)/rainbow trout (RBT to by size class as measured in millimeters [mm]), and sculpin diets as Plecoptera (stoneflies), Ephemeroptera (mayflies), Trichoptera (caddisflies), Diptera (true flies), Coleoptera (beetles), Hemiptera (true bugs), Hymenoptera (ants and bees), Trombidiformes (mites), other aquatic insects, terrestrial insects, and other prey items (fish, fish eggs, worms, snails) during the late fall (November). Only one bull trout diet was collected during the fall sampling. 


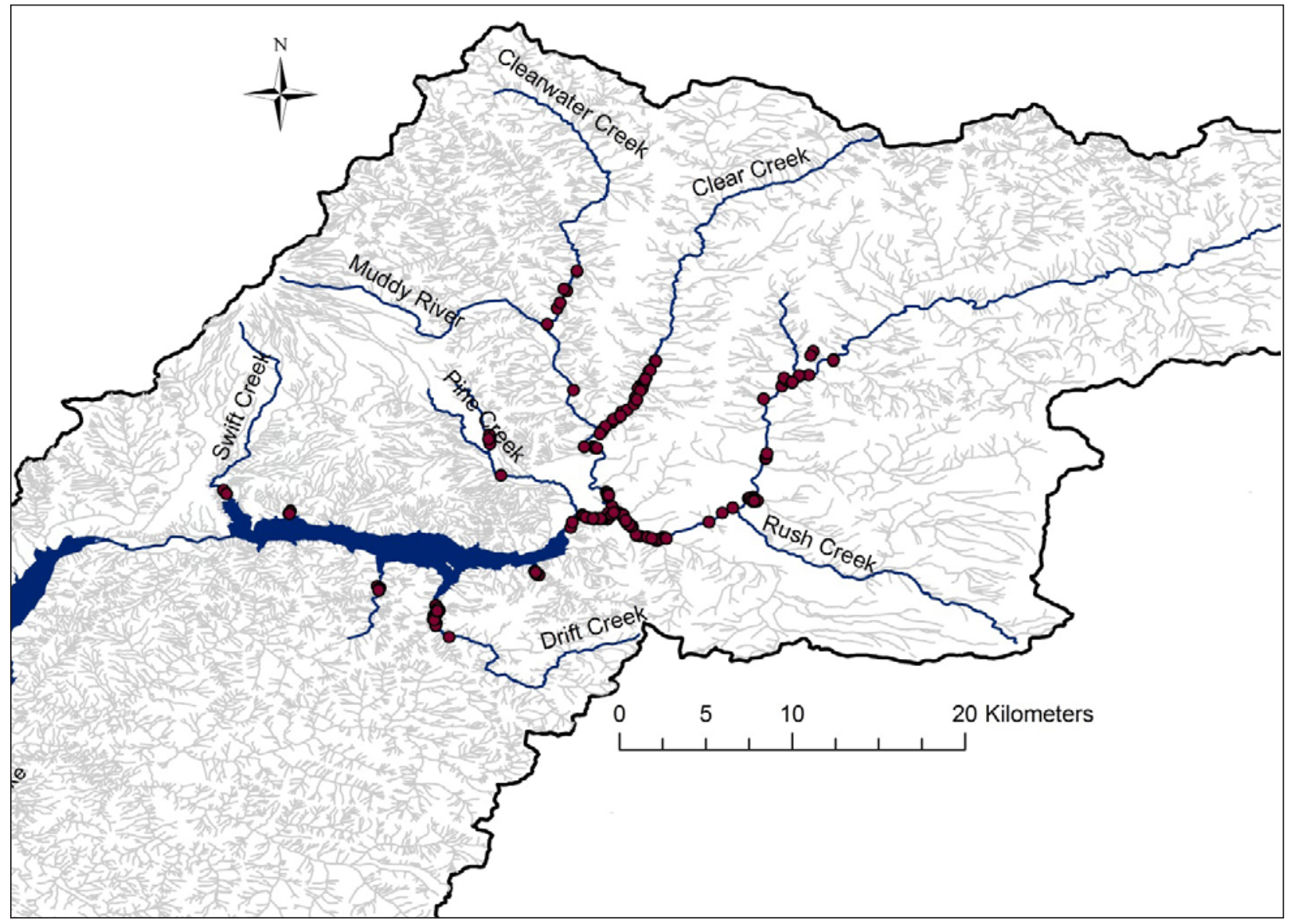

Figure F16. The distribution of coho salmon redds in the upper Lewis River, Washington, from surveys conducted in 2012 to 2014. 


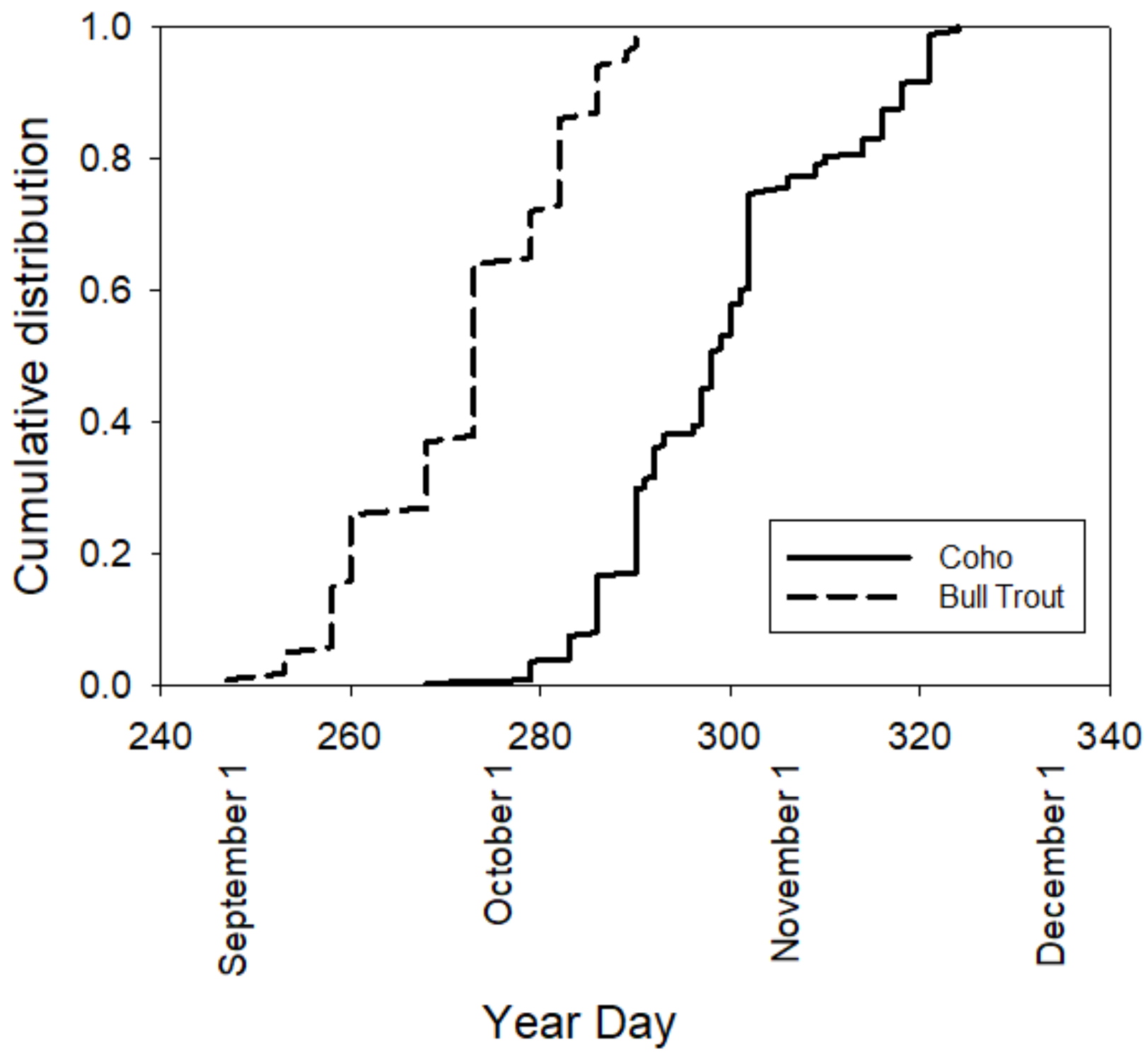

Figure F17. The cumulative frequency distributions of the timing (shown as year day) of coho salmon (solid) and bull trout (dashed) redds in the upper Lewis River, Washington. 


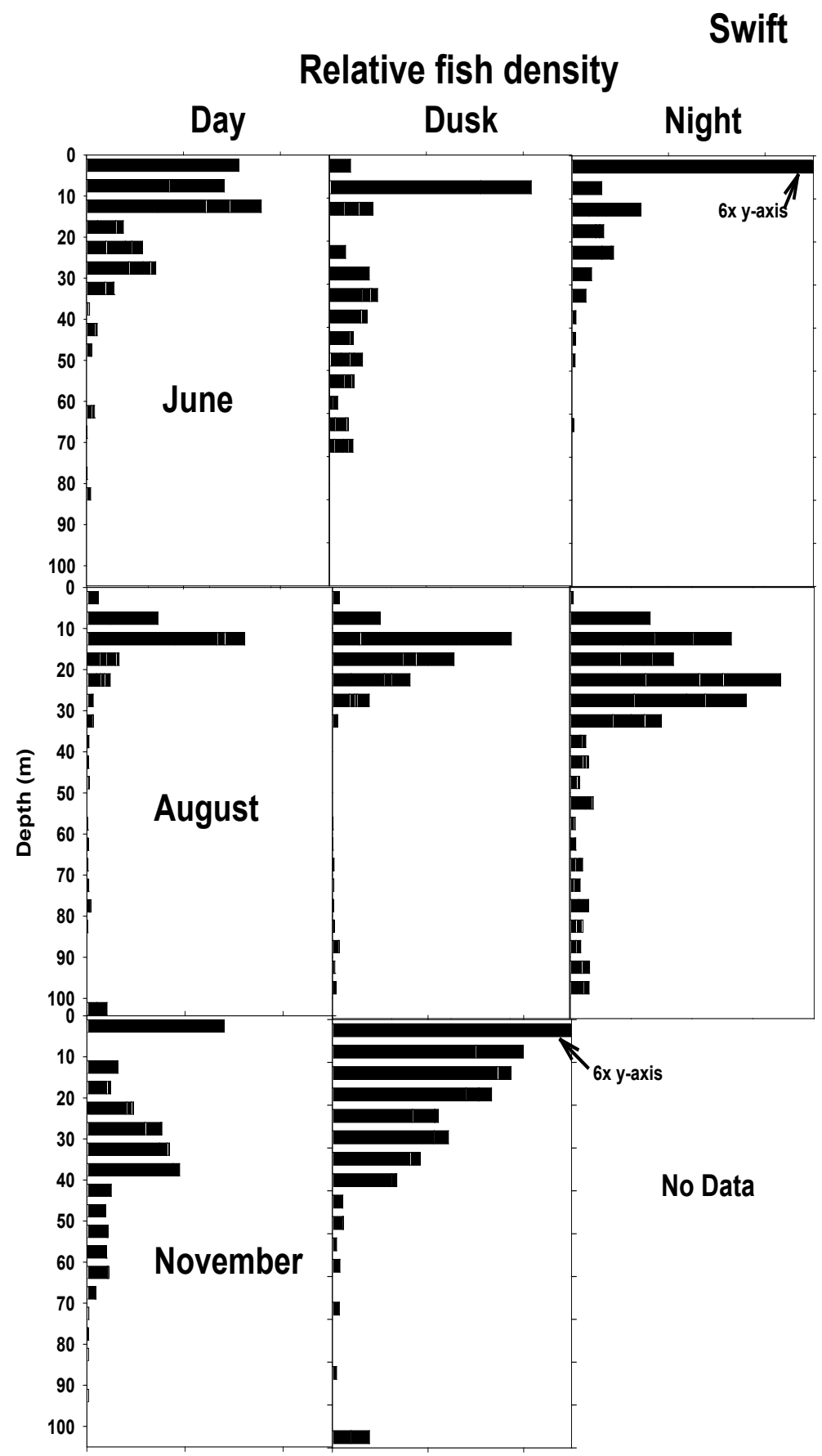

Figure F18. Day, dusk, and night sequence of hydroacoustic target depth distributions (measured in meters [m]) in June, August, and November 2013 in Swift Reservoir, Washington. 


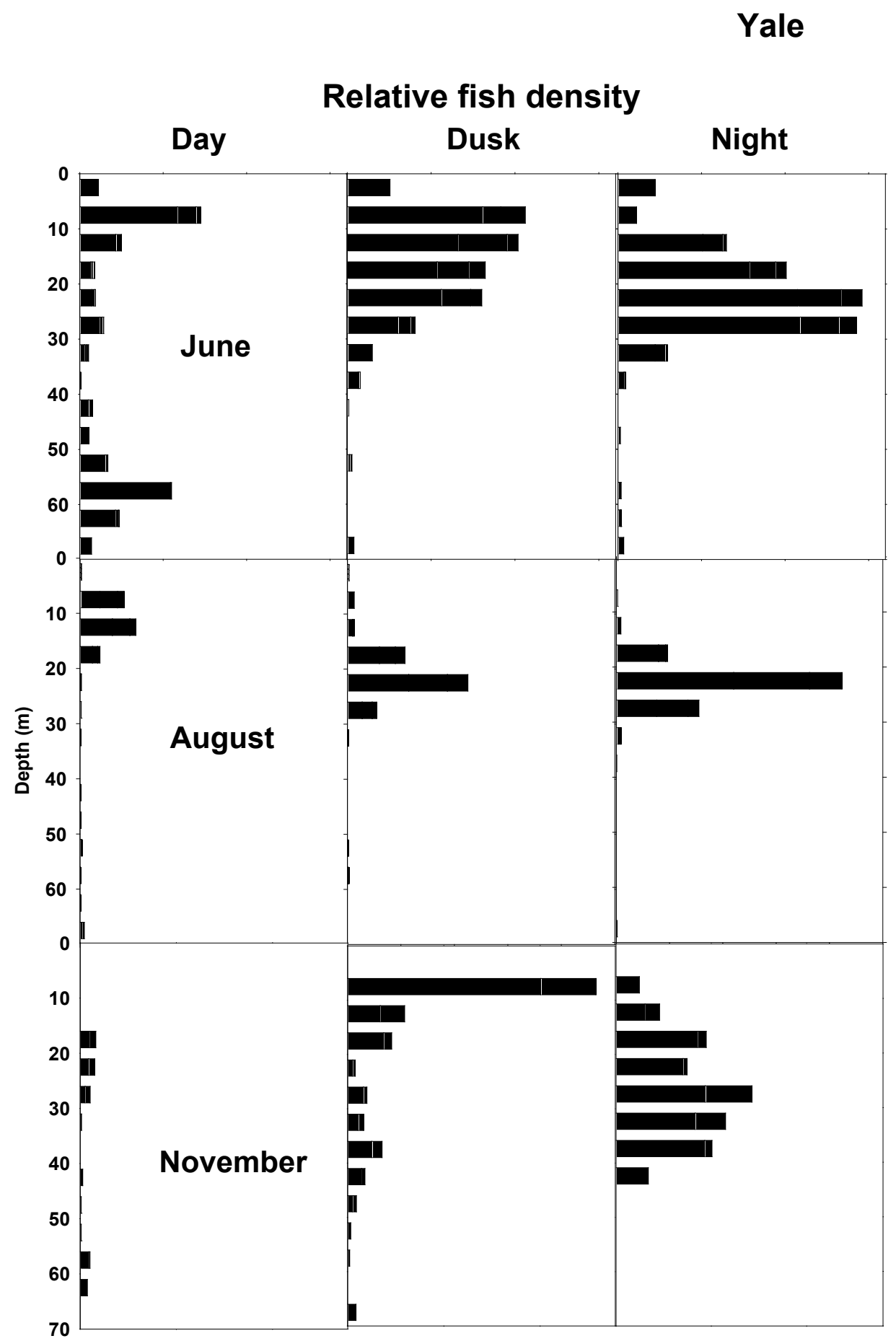

Figure F19. Day, dusk, and night sequence of hydroacoustic target depth distributions (measured in meters; $m$ ) in June, August, and November 2013 in Yale Lake, Washington. 


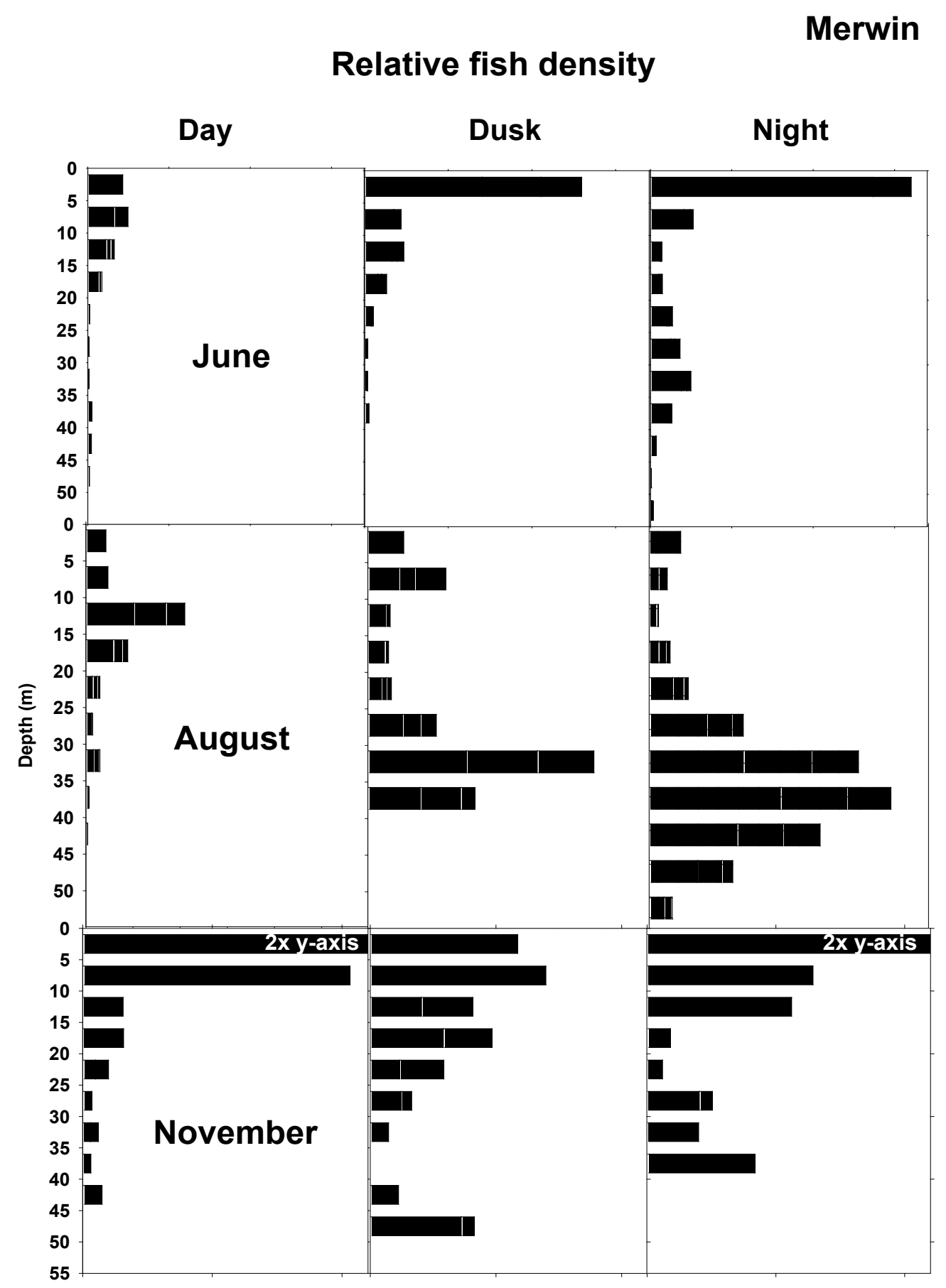

Figure F20. Day, dusk, and night sequence of hydroacoustic target depth distributions (measured in meters [m]) in June, August, and November 2013 in Lake Merwin, Washington. 


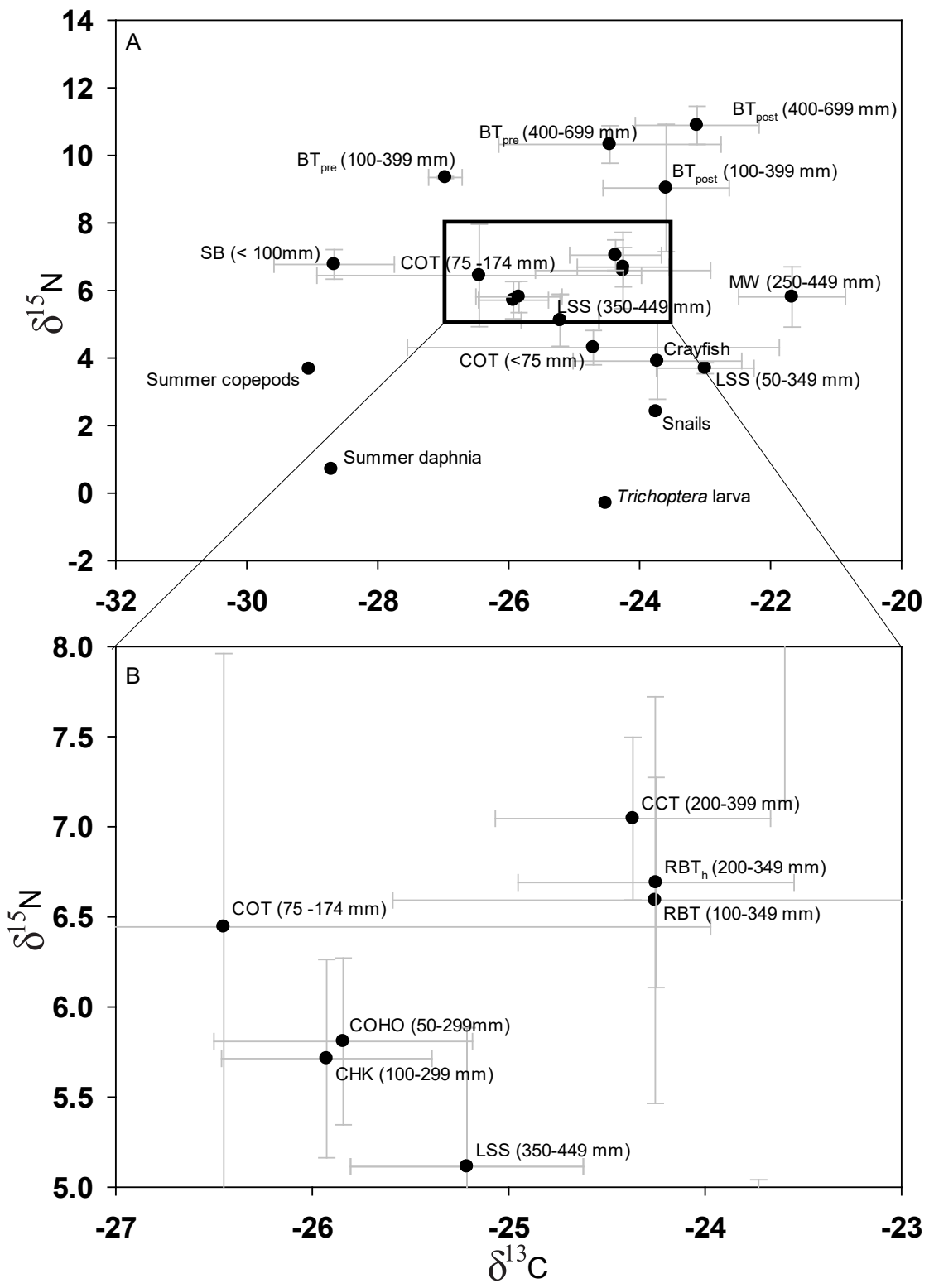

Figure F21. Mean $\delta^{15} \mathrm{~N}$ and $\delta^{13} \mathrm{C}$ values (plus or minus two standard error) for organisms sampled (lengths measured in millimeters; $\mathrm{mm}$ ) in Swift Reservoir, Washington; $B$, is an enlarged inset of $A$, for ease of interpretation. ( $\mathrm{BT}_{\text {pre }}$, bull trout sampled in 2010 before significant reintroduction of anadromous salmonids; $\mathrm{BT}_{{ }_{\text {post }}}$, bull trout sampled in 2013-2014 after the reintroduction had begun; CCT, coastal cutthroat trout; CHK, spring Chinook salmon; COHO, coho salmon; COT, sculpin; LSS, large-scale sucker; MW, mountain whitefish; $\mathrm{RBT}$, rainbow trout; $\mathrm{RBT}_{\mathrm{h}^{\prime}}$, hatchery-reared rainbow trout; $\mathrm{SB}$, three-spine stickleback) 


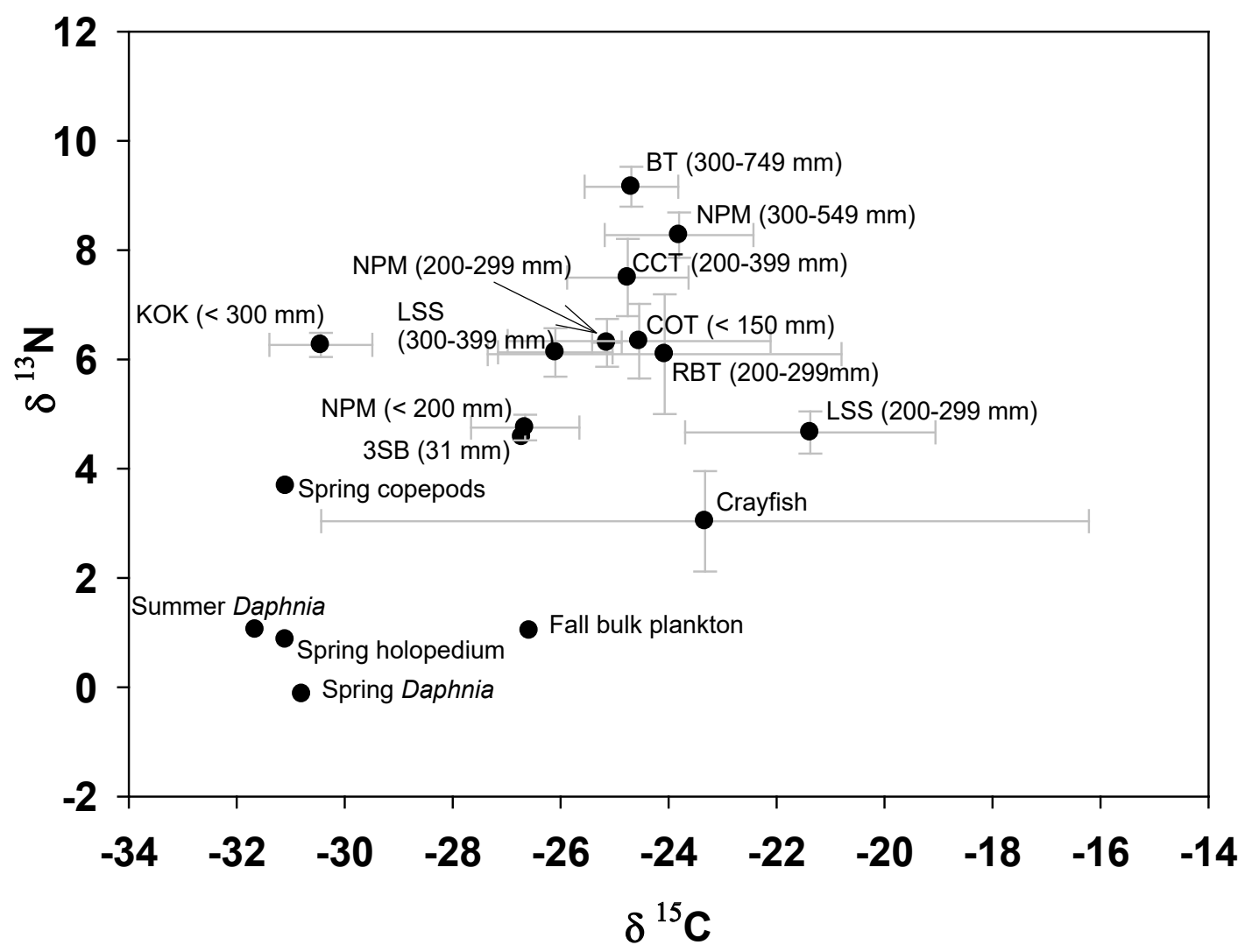

Figure F22. Mean $\delta^{15} \mathrm{~N}$ versus $\delta^{13} \mathrm{C}$ (plus or minus two standard error) for organisms sampled (lengths measured in millimeters; $\mathrm{mm}$ ) in Yale Lake, Washington. (BT, bull trout; CCT, coastal cutthroat trout; COT, sculpin; KOK, kokanee; LSS, large-scale sucker; NPM, northern pikeminnow; RBT, rainbow trout; SB, three-spine stickleback; sampling occurred during 2013-2014) 


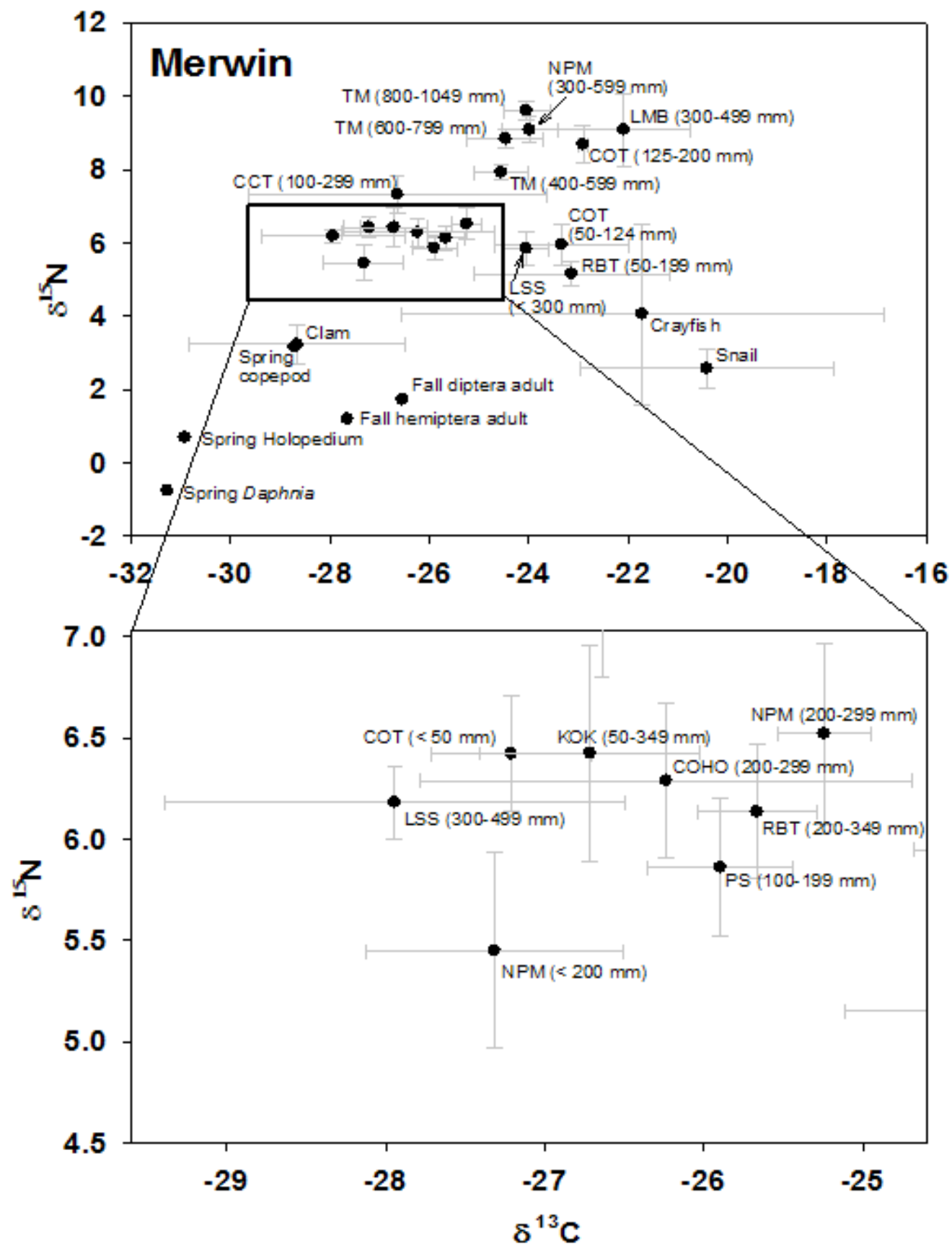

Figure F23. Mean $\delta^{15} \mathrm{~N}$ and $\delta^{13} \mathrm{C}$ (plus or minus two standard error) for organisms sampled (lengths measured in millimeters; $\mathrm{mm}$ ) in Lake Merwin. (CCT, coastal cutthroat trout; COHO, coho salmon; COT, sculpins; crayfish, signal crayfish; KOK, kokanee; LMB, largemouth bass; LSS, largescale sucker; NPM, northern pikeminnow; RBT, rainbow trout; PS, pumpkinseed sunfish; snail, juga snail; TM, tiger muskelunge; sampling occurred during 2013-2014) 


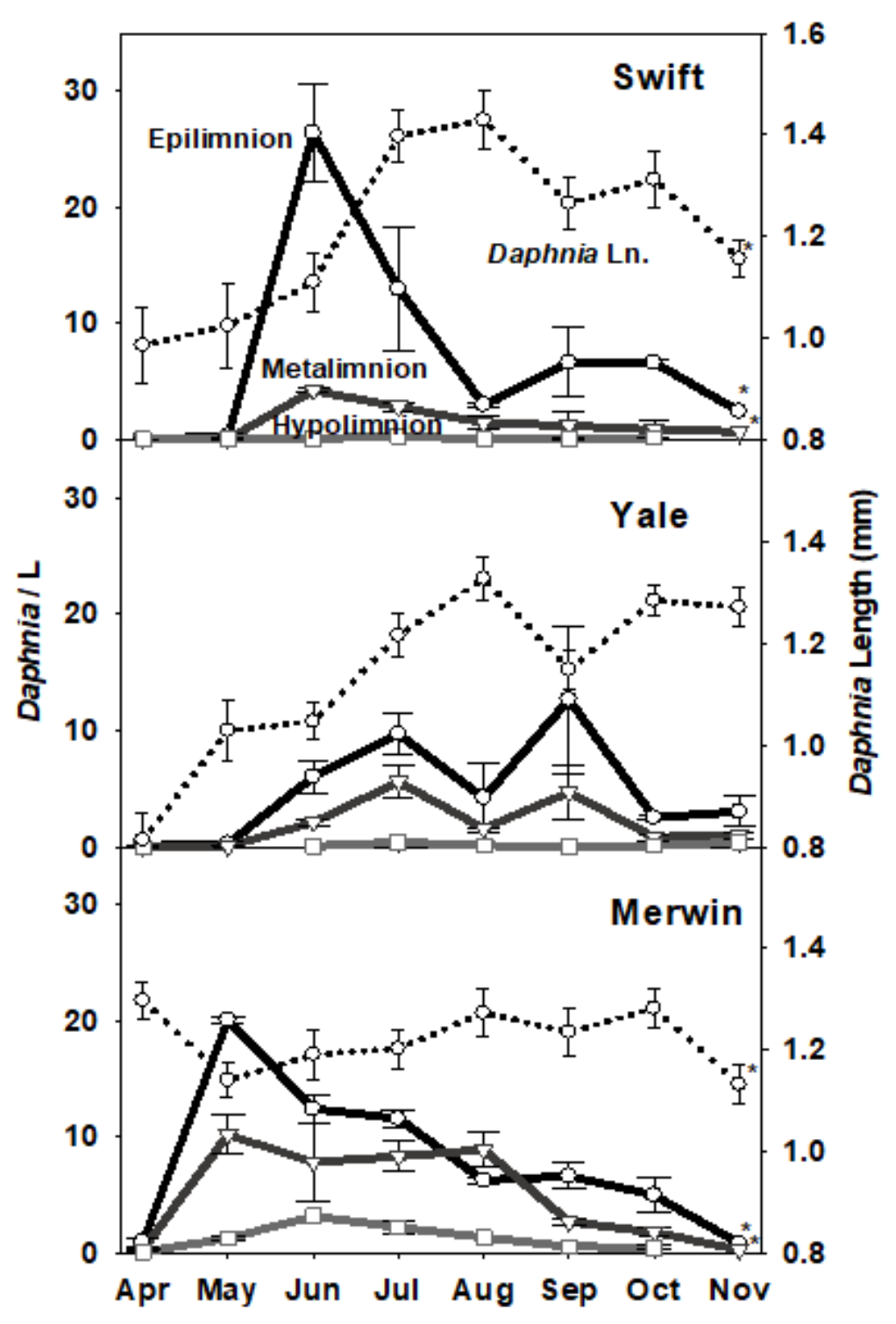

Figure F24. The mean monthly density of Daphnia (plus or minus [ \pm ] one standard error [SE]) in three depth strata from two stations located approximately one-third and two-thirds along the length (measured in meters [m]) of Swift Reservoir, Yale Lake, and Lake Merwin between April and November 2014. The mean length (measured in millimeters [mm]) of Daphnia ( \pm 1 SE) measured each month is also included. *Data from the lower sampling site in Swift Reservoir and Lake Merwin collected in November 2013 are included to show a complete growing season, as samples could not be collected in these reservoirs in November 2014. 


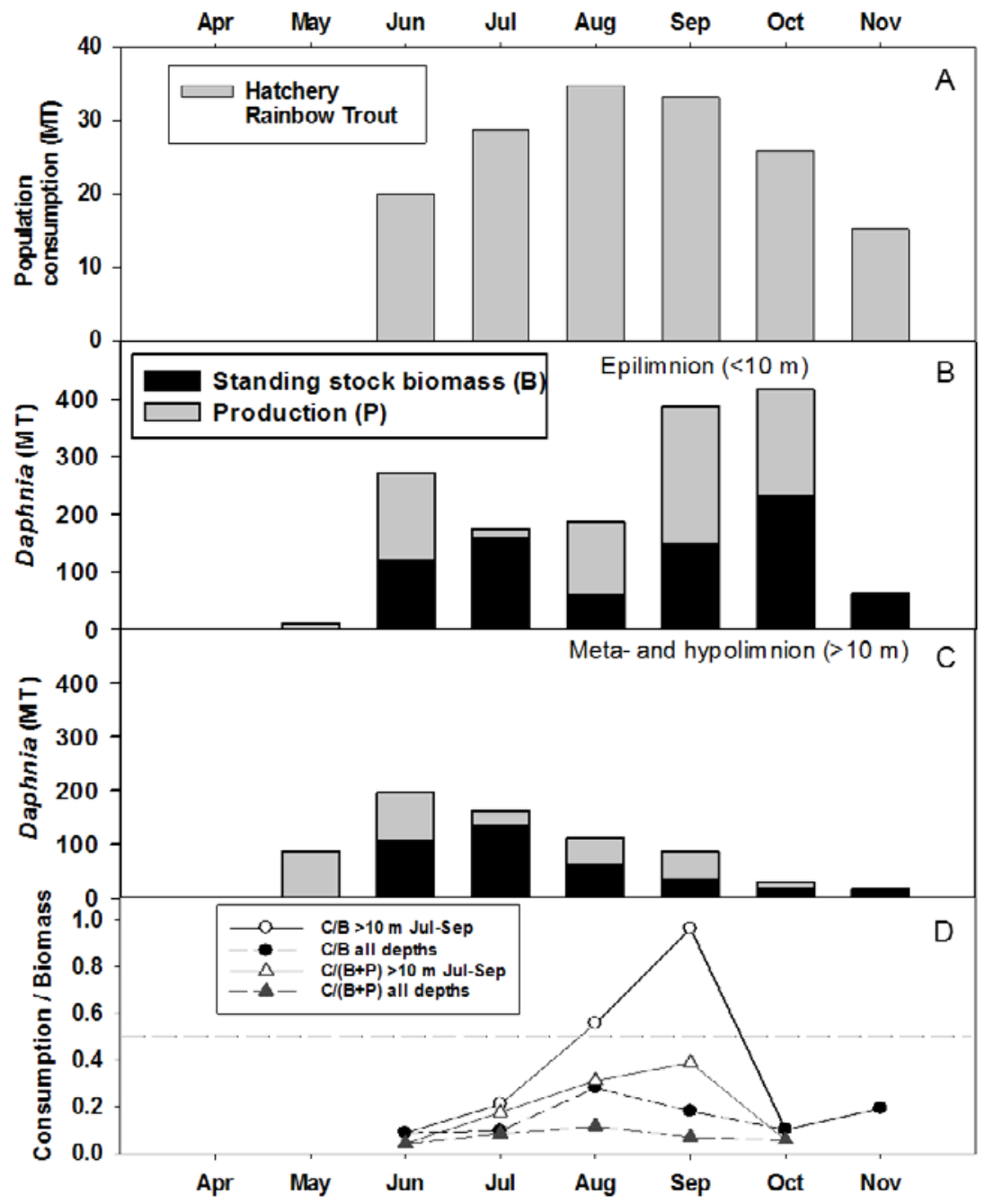

Figure F25. Estimates for Swift Reservoir, Washington, of $A$, monthly consumption (in metric tons; MT) demand of Daphnia by a cohort of hatchery-reared rainbow trout; $B$, Daphnia supply from April through November, stratified by depth in the epilimnion (the warm upper layer in which temperatures were relatively constant across depths [approximately 5-10 $\mathrm{m}$ deep from April through September and 10-18 $\mathrm{m}$ deep in October and November]); C, Daphnia supply stratified by depth in the metalimnion (the middle layer in which temperatures decreased significantly with depth [approximately 15-35 $\mathrm{m}$ deep in April-September]) and hypolimnion (the deepest layer in which temperatures were more stable and cooler than the metalimnion; and $D$, the ratio of consumption demand to prey supply for a range of prey supply scenarios. Dotted line represents 50 percent consumption over biomass ratio at which carrying capacity is reached. (m, meter) 


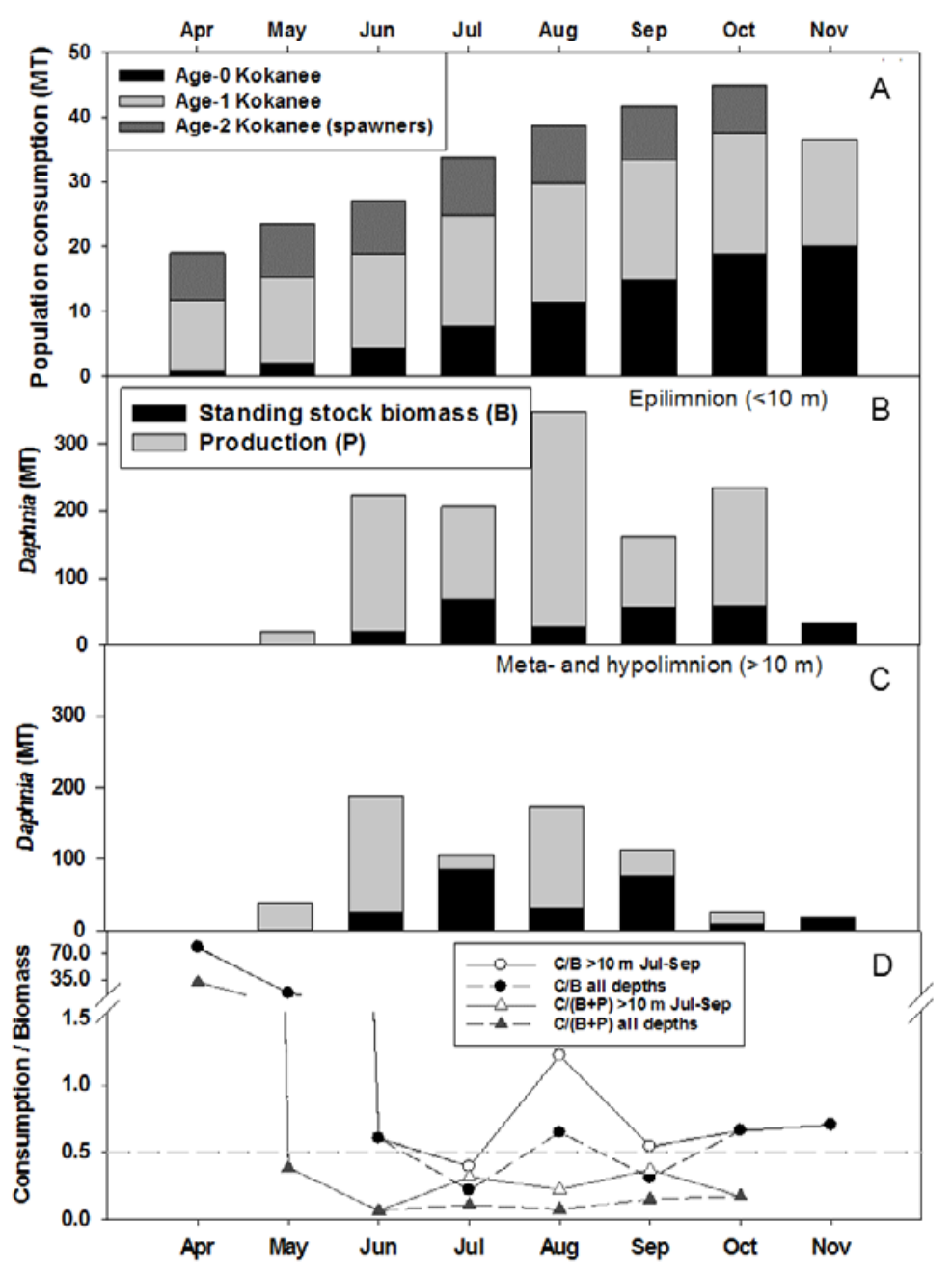

Figure F26. Estimates for Yale Lake, Washington of the $A$, monthly consumption (in metric tons [MT]) demand of Daphnia by three ageclasses of kokanee salmon; $B$, Daphnia supply from April through November, stratified by depth in the epilimnion (the warm upper layer in which temperatures were relatively constant across depths [approximately 5-10 m deep from April through September and 10-18 $\mathrm{m}$ deep in October and November]); C, Daphnia supply in the metalimnion (the middle layer in which temperatures decreased significantly with depth [approximately 15-35 m deep in April-September]) and hypolimnion (the deepest layer in which temperatures were more stable and cooler than the metalimnion; and $D$, the ratio of consumption demand to prey supply for a range of prey supply scenarios. Dotted line represents 50 percent consumption over biomass ratio at which carrying capacity is reached. (m, meter) 


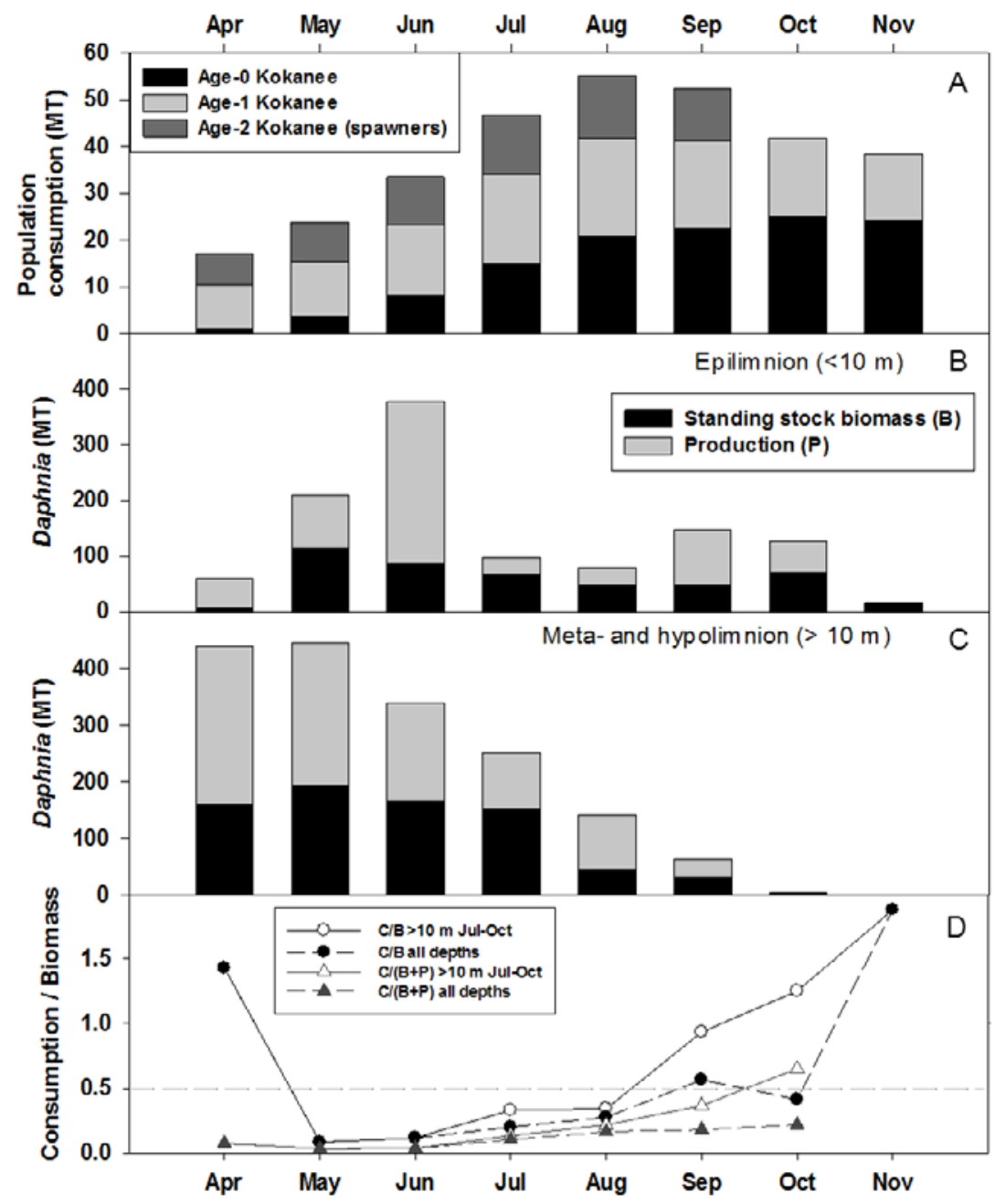

Figure F27. Estimates for Lake Merwin, Washington of $A$, monthly consumption (in metric tons [MT]) demand of Daphnia by three ageclasses of kokanee salmon: $B$, Daphnia supply from April through November, stratified by depth in the epilimnion (the warm upper layer in which temperatures were relatively constant across depths [approximately 5-10 $\mathrm{m}$ deep from April through September and 10-18 $\mathrm{m}$ deep in October and November]); C, Daphnia supply stratified by depth in the metalimnion (the middle layer in which temperatures decreased significantly with depth [approximately $15-35 \mathrm{~m}$ deep in April-September]) and hypolimnion (the deepest layer in which temperatures were more stable and cooler than the metalimnion; and $D$, the ratio of consumption demand to prey supply for a range of prey supply scenarios. Dotted line represents 50 percent consumption over biomass ratio, at which carrying capacity is reached. 


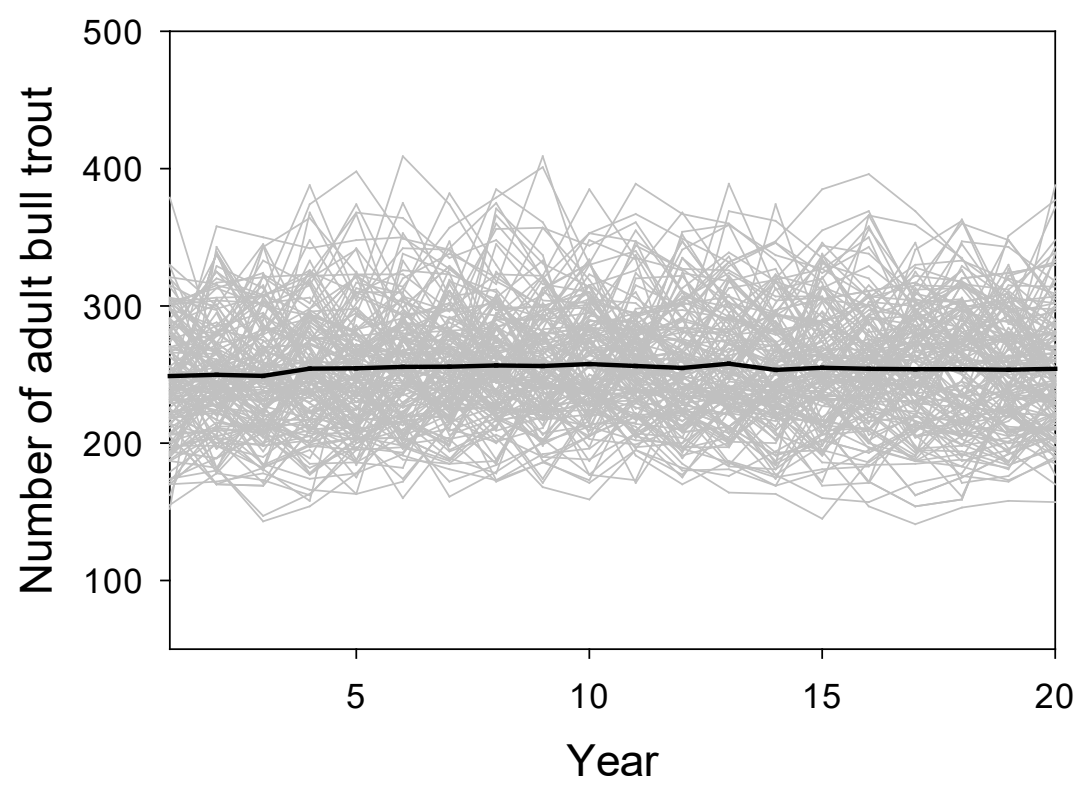

Figure F28. Simulation results indicating the number of adult bull trout in each simulation (gray) and the mean (black) for the 20 years of model runs under the baseline bull trout population model with no effects of salmon reintroductions.

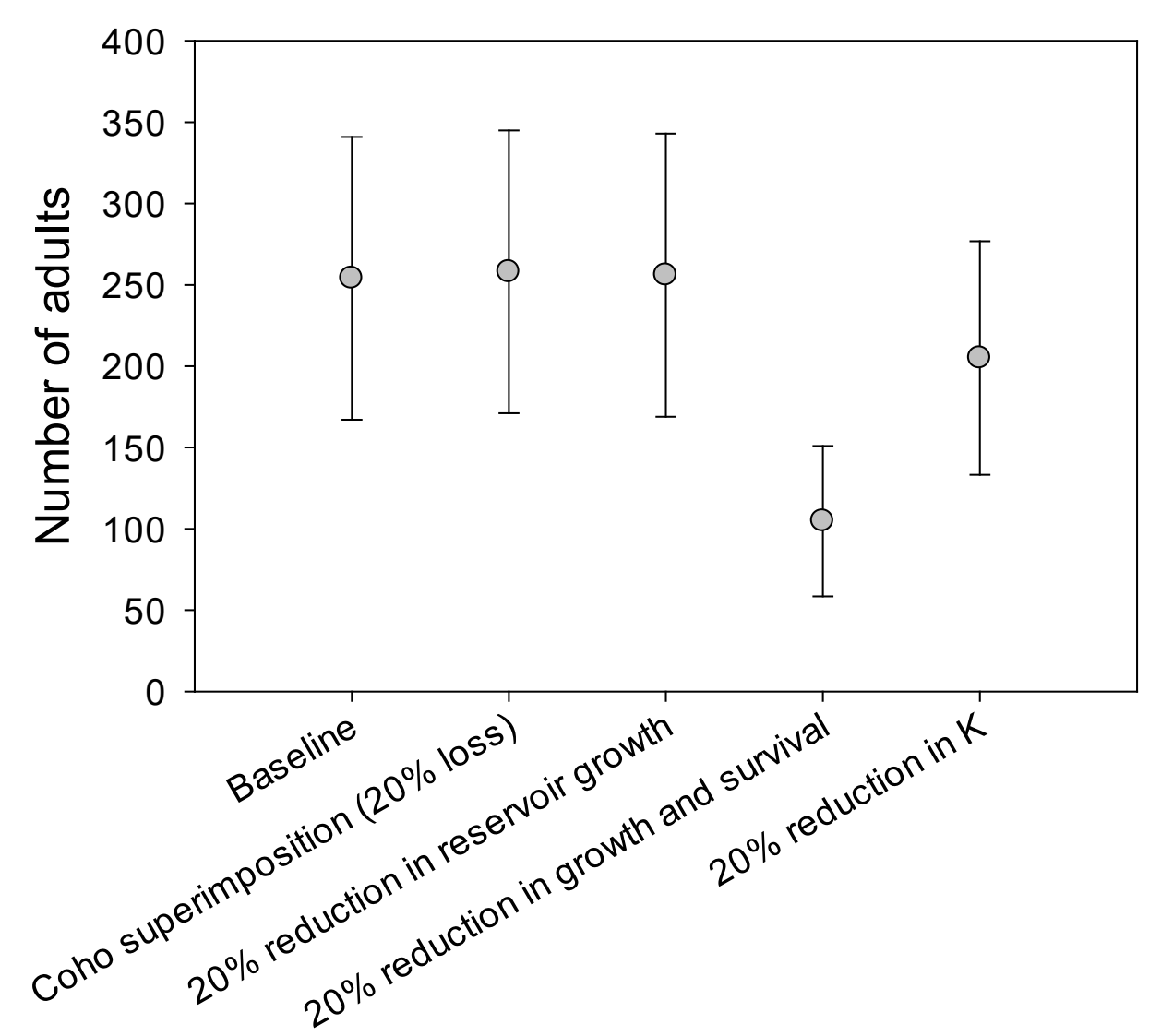

Figure F29. The mean (95-percent confidence interval) number of adult bull trout across all simulation years $(n=6,000)$ under the baseline population model (no perturbations, current population), where a 20 percent loss of egg survival occurs through coho salmon redd superimposition, a 20 percent reduction in reservoir growth, a 20 percent reduction in reservoir growth and survival, and a 20 percent reduction in bull trout tributary carrying capacity (K). (\%, percent) 


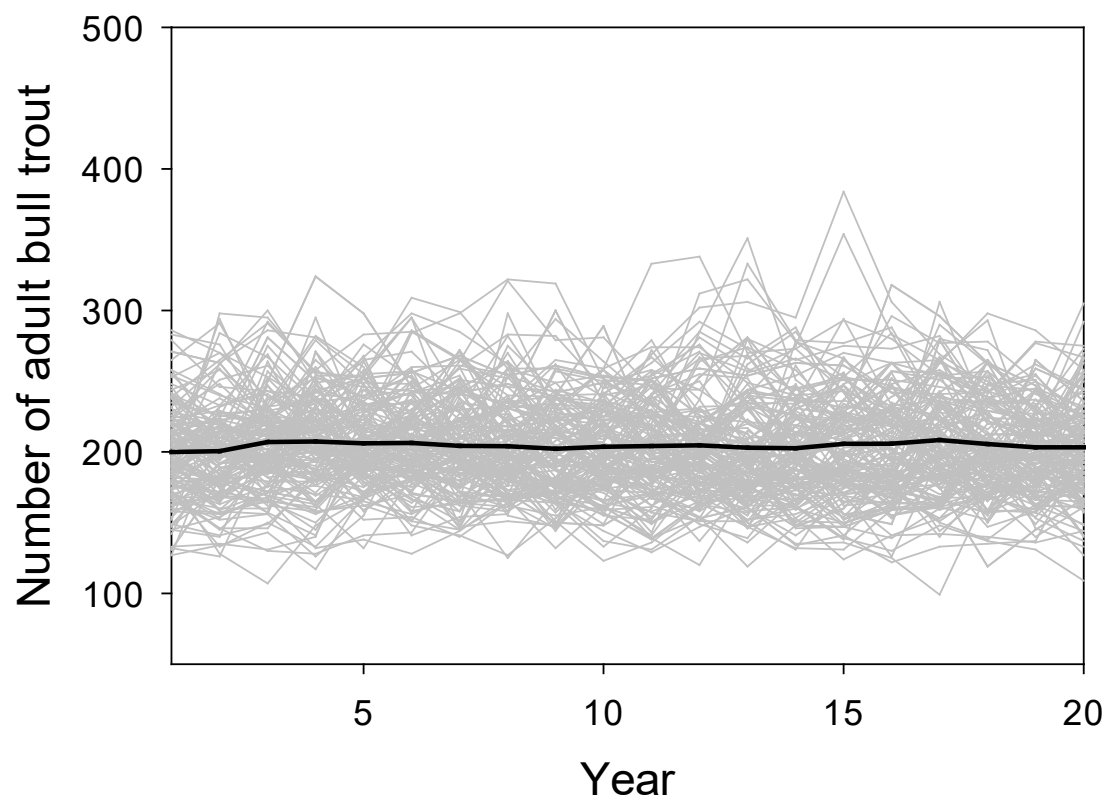

Figure F30. Simulation results indicating the number of adult bull trout in each simulation (gray) and the mean (black) for the 20 years of model runs where carrying capacity of tributary juvenile bull trout rearing is reduced by 20 percent of the baseline levels as a result of increased distribution and abundance of juvenile salmon.

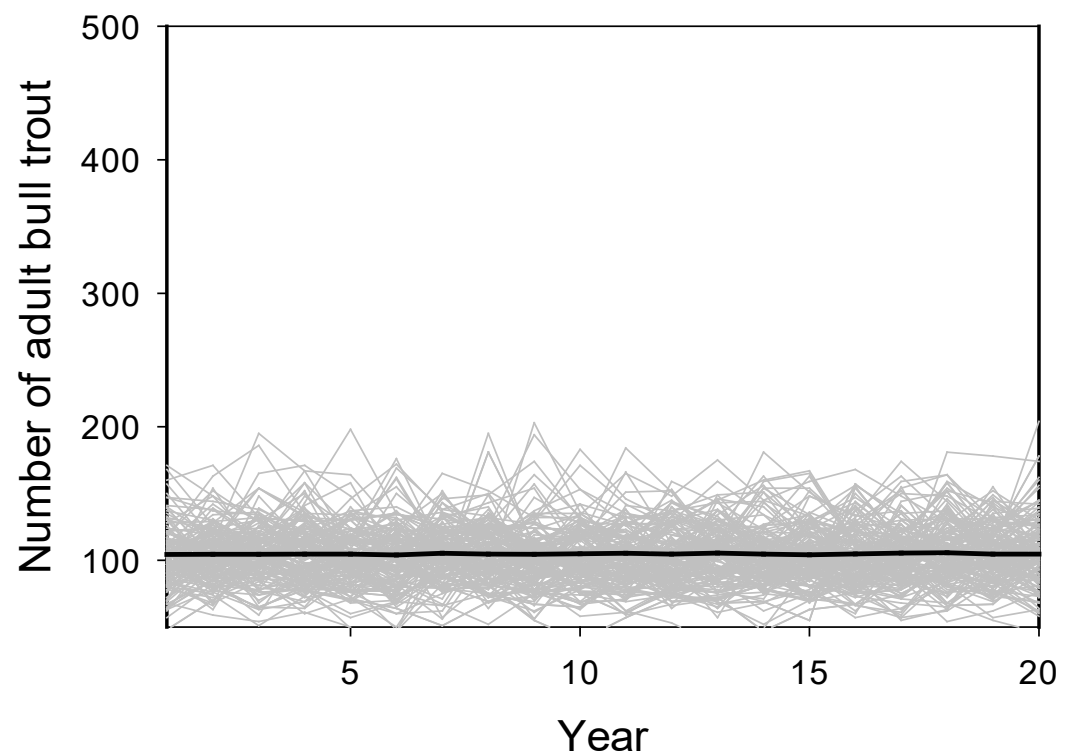

Figure F31. Simulation results indicating the number of adult bull trout in each simulation (gray) and the mean (black) for the 20 years of model runs where bull trout growth and survival in the reservoir are reduced by 20 percent through reductions in reservoir carrying capacity as a result of increased abundance of juvenile and residualized salmon. 


\section{Tables}

Table F1. Estimates of water temperatures for optimum and maximum growth from existing literature for bull trout, Chinook salmon, coho salmon, steelhead, and coastal cutthroat trout.

[ODEQ, Oregon Department of Environmental Quality; EPA, U.S. Environmental Protection Agency; NA, not available]

\begin{tabular}{|c|c|c|c|c|}
\hline Species & $\begin{array}{l}\text { Optimum growth } \\
\text { range } \\
\text { (degrees Celsius) }\end{array}$ & $\begin{array}{l}\text { Maximum } \\
\text { growth } \\
\text { (degrees Cel- } \\
\text { sius) }\end{array}$ & Reference optimum & Reference maximum \\
\hline Bull trout & $10-15$ & 13.2 & $\begin{array}{l}\text { Selong and others, 2001; McMahon and } \\
\text { others, } 2007\end{array}$ & Selong and others, 2001 \\
\hline Chinook salmon & $10-15.6$ & $16,18.9-20.5$ & ODEQ, 1995; EPA, 2001 & $\begin{array}{l}\text { Washington State Deparment } \\
\text { of Ecology, } 2002\end{array}$ \\
\hline Steelhead & $9.8-22$ & $13.1,17.2$ & Bear and others, 2007; Spina, 2007 & $\begin{array}{l}\text { Hokanson and others, 1977; } \\
\text { Bear and others, } 2007\end{array}$ \\
\hline Coastal cutthroat trout & 15 & NA & Johnson and others, 1999 & - \\
\hline
\end{tabular}


Table F2. Percent of fish, including sculpin, in bull trout diets by lake or river, life-history forms, and size classes.

[OR, Oregon; <, less than; mm, millimeter; WA, Washington; >, greater than; AB, Alberta, Canada; NA, not applicable; MT, Montana; g, gram; ID, Idaho]

\begin{tabular}{|c|c|c|c|c|c|}
\hline Lake/river & Location & Life-history & $\begin{array}{l}\text { Percent fish } \\
\text { in diet }\end{array}$ & $\begin{array}{c}\text { Size } \\
\text { classes }\end{array}$ & Source \\
\hline Walla Walla River & OR & Fluvial & 96.0 & Mixed & Budy and others, 2012 \\
\hline Walla Walla River & OR & Fluvial & 57.0 & Mixed & Budy and others, 2012 \\
\hline Walla Walla River & OR & Fluvial & 90.1 & Mixed & Budy and others, 2012 \\
\hline Walla Walla River & OR & Fluvial & 85.7 & Mixed & Budy and others, 2012 \\
\hline Walla Walla River & OR & Fluvial & 62.9 & Mixed & Budy and others, 2012 \\
\hline North Fork John Day River & OR & Fluvial & 16.6 & mixed; $<475 \mathrm{~mm}$ & Budy and others, 2007 \\
\hline North Fork John Day River & OR & Fluvial & 13.9 & mixed; $<450 \mathrm{~mm}$ & Budy and others, 2007 \\
\hline Skagit River & WA & Fluvial & $89.9^{1}$ & $301-450 \mathrm{~mm}$ & Lowery and Beauchamp, 2015 \\
\hline Skagit River & WA & Fluvial & $86.7^{1}$ & $>450 \mathrm{~mm}$ & Lowery and Beauchamp, 2015 \\
\hline Miette Lake & $\mathrm{AB}$ & Adfluvial & 0 & NA & Donald and Alger, 1993 \\
\hline Southesk Lake & $\mathrm{AB}$ & Adfluvial & 27.0 & NA & Donald and Alger, 1993 \\
\hline Flathead Lake & MT & Adfluvial & 77.9 & NA & Fraley and Shepard, 1989 \\
\hline Flathead Lake & MT & Adfluvial & 100 & NA & Leathe and Graham, 1982 \\
\hline Skagit River & WA & Fluvial & 58.8 & Adult & Lowery, 2009 \\
\hline Skagit River & WA & Fluvial & 61.5 & Adult & Lowery, 2009 \\
\hline Lake Billy Chinook & OR & Adfluvial & 28.6 & $<300 \mathrm{~mm}$ & Beauchamp and Van Tassel, 2001 \\
\hline Meadow Fork Creek & OR & Resident/fluvial & 0 & $<300 \mathrm{~mm}$ & Gunckel, 2001 \\
\hline North Powder River & OR & Resident/fluvial & 0 & $<300 \mathrm{~mm}$ & Gunckel, 2001 \\
\hline Swan Lake & MT & Adfluvial & 12.0 & $<301 \mathrm{~mm}$ & Guy and others, 2011 \\
\hline Swan Lake & MT & Adfluvial & 22.0 & $301-500 \mathrm{~mm}$ & Guy and others, 2011 \\
\hline Swan Lake & MT & Adfluvial & 94.0 & $502-700 \mathrm{~mm}$ & Guy and others, 2011 \\
\hline Harrison Lake & $\mathrm{AB}$ & Adfluvial & 0 & $<250 \mathrm{~mm}$ & Wilhelm and others, 1999 \\
\hline Harrison Lake & $\mathrm{AB}$ & Adfluvial & 1.5 & $>250 \mathrm{~mm}$ & Wilhelm and others, 1999 \\
\hline Mill Creek & WA & Resident/fluvial & 12.3 & $<270 \mathrm{~mm}$ & Underwood and others, 1995 \\
\hline Tucannon River & WA & Resident/fluvial & 0 & $<225 \mathrm{~mm}$ & Underwood and others, 1995 \\
\hline Mill Creek & WA & Resident/fluvial & 50.4 & $100-250 \mathrm{~mm}$ & Martin and others, 1992 \\
\hline Lake Pend Oreille & ID & Adfluvial & 100 & $>400 \mathrm{~mm}$ & Clarke and others, 2005 \\
\hline Wolf Fork & WA & Resident/fluvial & 23.5 & $100-250 \mathrm{~mm}$ & Martin and others, 1992 \\
\hline Wolf Fork Creek & WA & Resident/fluvial & 9.0 & $100-250 \mathrm{~mm}$ & Martin and others, 1992 \\
\hline
\end{tabular}

${ }^{1}$ Estimates are a weighted mean across sample sizes and include both tributary and mainstem habitat (where applicable). Estimates include fish, fish eggs, and carcasses and are the mean across seasons. 
Table F3. Thermal experience of pelagic planktivore consumption demands across different dates and simulation days within a bioenergetics modeling framework in Swift Reservoir, Yale Lake, and Lake Merwin, Washington. $\left({ }^{\circ} \mathrm{C}\right.$, degree Celsius)

\begin{tabular}{|c|c|c|c|c|}
\hline \multirow[b]{2}{*}{ Date } & \multirow{2}{*}{$\begin{array}{c}\text { Simulation } \\
\text { day }\end{array}$} & \multicolumn{3}{|c|}{ Thermal experience $\left({ }^{\circ} \mathrm{C}\right)$} \\
\hline & & $\begin{array}{c}\text { Swift } \\
\text { Reservoir }\end{array}$ & $\begin{array}{l}\text { Yale } \\
\text { Lake }\end{array}$ & $\begin{array}{c}\text { Lake } \\
\text { Merwin }\end{array}$ \\
\hline 1-April & 1 & 6.6 & 5.6 & 7.8 \\
\hline 18-May & 49 & 10.8 & 8.2 & 11.9 \\
\hline 30-June & 89 & 14.3 & 9.5 & 13.5 \\
\hline 19-July & 109 & 14.7 & 11.7 & 15 \\
\hline 15-August & 135 & 15.1 & 12.5 & 16.2 \\
\hline 17-September & 167 & 14.6 & 14 & 17 \\
\hline 13-October & 193 & 9.1 & 11.5 & 17.3 \\
\hline 15-November & 225 & 8.2 & 8 & 12.7 \\
\hline 15-December & 255 & 5.9 & 5.9 & 5.8 \\
\hline 1-January & 270 & 5.2 & 5.4 & 5.9 \\
\hline 1-March & 330 & 4.5 & 4.9 & 6.1 \\
\hline 31-March & 365 & 6.6 & 5.6 & 7.8 \\
\hline
\end{tabular}

Table F4. Energy density (joules/gram) of prey items consumed by pelagic planktivores. An indigestible fraction of 17 percent was assessed on all invertebrate prey consumed (Beauchamp and others, 2007).

\begin{tabular}{|c|c|c|c|c|}
\hline Day & Daphnia & $\begin{array}{c}\text { Copepoda } \\
\text { (Copepod) }\end{array}$ & Terrestrial insects & Aquatic insects \\
\hline 1 & $1,950^{1}$ & 2,260 & 5,000 & 3,400 \\
\hline 365 & 1,950 & 2,260 & 5,000 & 3,400 \\
\hline
\end{tabular}

${ }^{1}$ Energy density for Daphnia taken from Luecke and Brandt (1993) and Stockwell and others (1999).

Table F5. Starting and ending dates and consumer weights used in bioenergetics simulations and outputs of feeding rate $\left(\% \mathrm{C}_{\max }\right)$ and consumption in biomass.

[mm, millimeter; wt., weight; g, gram; $\mathrm{RBT}_{\mathrm{h}}$, hatchery-reared rainbow trout]

\begin{tabular}{|c|c|c|c|c|c|c|c|c|c|c|}
\hline \multicolumn{11}{|c|}{ Swift Reservoir } \\
\hline $\mathrm{RBT}_{\mathrm{h}}$ & 1 & 78 & 1-June & 20-November & 258 & 200.0 & 362.0 & 162 & 3,499 & 3,149 \\
\hline Chinook salmon & 0 & 104 & 1-April & 30-November & 25 & 0.2 & 30.5 & 30.3 & 331 & 299 \\
\hline \multirow[t]{3}{*}{ Kokanee salmon } & 0 & 86 & 1-April & 30-November & 25 & 0.1 & 40.6 & 41 & 288 & 234 \\
\hline & 1 & 74 & 1-April & 30-November & 163 & 45.0 & 143.5 & 98 & 1,227 & 952 \\
\hline & 2 & 59 & 1-April & 30-October & 236 & 154.0 & 219 & 64 & 1,498 & 1,124 \\
\hline \multicolumn{11}{|c|}{ Lake Merwin } \\
\hline
\end{tabular}


Table F6. Model inputs of survival, growth, and percent maturity by age for bull trout in the Swift Reservoir, Washington.

[NA, not available]

\begin{tabular}{|c|c|c|c|}
\hline Age & $\begin{array}{c}\text { Survival } \\
\text { (standard deviation) }\end{array}$ & $\begin{array}{c}\text { Growth } \\
\text { (standard deviation, } \\
\text { millimeter) }\end{array}$ & $\begin{array}{c}\text { Percent (\%) } \\
\text { mature }\end{array}$ \\
\hline egg-fry $^{1}$ & $0.44(34)^{1}$ & NA & 0 \\
\hline fry-age-1 $^{1}$ & $0.21(0.12)^{1}$ & $85(20)$ & 0 \\
\hline 2 & $0.21(0.12)^{2,4}$ & $152(74)$ & 0 \\
\hline 3 & $0.37(0.13)^{2,4}$ & $112(54)$ & 0 \\
\hline 4 & $0.54(0.19)^{2,4}$ & $80(43)$ & 25 \\
\hline 5 & $0.70(0.25)^{2,4}$ & $59(30)$ & 50 \\
\hline 6 & $0.84(0.30)^{3,4}$ & $36(19)$ & 100 \\
\hline 7 & $0.84(0.30)^{3,4}$ & $26(19)$ & 100 \\
\hline 8 & $0.84(0.30)^{3,4}$ & $25(17)$ & 100 \\
\hline 9 & $0.84(0.30)^{3,4}$ & $21(12)$ & 100 \\
\hline 10 & $0.84(0.30)^{3,4}$ & $17(10)$ & 100 \\
\hline 11 & $0.84(0.30)^{3,4}$ & $16(9)$ & 100 \\
\hline 12 & $0.84(0.30)^{3,4}$ & $15(8)$ & 100 \\
\hline 13 & $0.84(0.30)^{3,4}$ & $14(8)$ & 100 \\
\hline 14 & $0.84(0.30)^{3,4}$ & $13(7)$ & 100 \\
\hline 15 & $0.84(0.30)^{3,4}$ & $13(7)$ & 100 \\
\hline 16 & $0.84(0.30)^{3,4}$ & $12(7)$ & 100 \\
\hline
\end{tabular}

${ }^{1}$ Bowerman and Budy, 2012; Bowerman and others, 2014.

${ }^{2}$ No data for subadult adfluvial exist. Estimates are estimated by linear increase from age-1 estimates to age-6 estimates.

${ }^{3}$ Stillwater Sciences, 2016.

${ }^{4}$ Standard deviation estimated as proportional to adults (Al-Chokhachy and Budy, 2008).

Table F7. Relative abundance of fish species observed in sampling conducted in this study and predominant summer crustacean zooplankton found in Swift Reservoir, Yale Lake, and Lake Merwin, Washington.

[NA, species absent]

\begin{tabular}{|c|c|c|c|}
\hline & Swift Reservoir & Yale Lake & Lake Merwin \\
\hline Northern pikeminnow & $\mathrm{NA}$ & Moderate & High \\
\hline Largescale sucker & High & High & High \\
\hline Kokanee salmon & NA & High & High \\
\hline Rainbow trout & High & Rare & Moderate \\
\hline Coastal cutthroat trout & Rare & Rare & Rare \\
\hline Bull trout & Rare & Rare & NA \\
\hline Tiger muskellunge & NA & NA & Moderate \\
\hline Sculpins & Moderate & Moderate & Moderate \\
\hline Three-spine stickleback & Moderate & Rare & Rare \\
\hline Mountain whitefish & Rare & NA & NA \\
\hline Largemouth bass & NA & NA & Rare \\
\hline Juvenile coho salmon & Rare & NA & Rare \\
\hline Juvenile Chinook salmon & Moderate & NA & NA \\
\hline Predominant summer zooplankton & Daphnia & Daphnia & Daphnia \\
\hline
\end{tabular}


Table F8. Seasonal diet proportions (n=total number of diets) for invertebrate-feeding salmonids including spring Chinook salmon, hatchery-reared rainbow trout, and kokanee salmon captured in Swift Reservoir, Yale Lake, and Lake Merwin, June-November 2013 and May-November 2014, and also including Cladocerans, Copepoda, terrestrial insects, aquatic insects, and fish.

\begin{tabular}{|c|c|c|c|c|c|c|c|}
\hline Size class & Season & $\mathbf{n}$ & Cladocerans & Copepoda & $\begin{array}{l}\text { Terrestrial } \\
\text { insects }\end{array}$ & $\begin{array}{l}\text { Aquatic } \\
\text { insects }\end{array}$ & Fish \\
\hline \multicolumn{8}{|c|}{ Swift Reservoir_-Spring Chinook Salmon } \\
\hline $100-299$ & Summer & 7 & 1.000 & 0 & 0 & 0 & 0 \\
\hline $100-299$ & Fall & 11 & 0.855 & 0 & 0.009 & 0.136 & 0 \\
\hline $200-399$ & Spring & 10 & 0.593 & 0 & 0.046 & 0.360 & 0 \\
\hline $200-299$ & Summer & 4 & 0.999 & 0.001 & 0 & 0 & 0 \\
\hline $200-399$ & Fall & 13 & 0.893 & 0.002 & 0.049 & 0.056 & 0 \\
\hline \multicolumn{8}{|c|}{ Yale Lake-Kokanee Salmon } \\
\hline \multicolumn{8}{|c|}{ Lake Merwin-Kokanee Salmon } \\
\hline $0-399$ & Spring & 17 & 0.536 & 0.018 & 0.202 & 0.243 & 0 \\
\hline 200-399 & Summer & 11 & 0.908 & 0.001 & 0.018 & 0.073 & 0 \\
\hline $100-399$ & Fall & 13 & 0.857 & 0 & 0.043 & 0.100 & 0 \\
\hline
\end{tabular}



